\title{
ATENUACIÓN SÍSMICA EN LA REGIÓN DE NUEVO CUYO
}

\author{
Autor \\ Gabriela Alejandra Badi
}

Directores

Jesús Miguel Ibáñez Godoy

Nora Cristina Sabbione

Tesis presentada para optar por el grado de DOCTOR EN GEOFÍSICA

Facultad de Ciencias Astronómicas y Geofísicas Universidad Nacional de La Plata 
A César, Euge y Romi 


\section{Agradecimientos}

En primer lugar deseo agradecer a mis directores, Jesús y Nora, quienes me han acompañado y alentado para que esta tesis llegara a buen puerto. Sé que hubieran deseado que fuera más breve, en tiempo y hojas, pero es así como soy. A Jesús, por sobre todo amigo, especialmente porque creyó en mí hace tantos años cuando nos conocimos en I. Decepción, y desde entonces ha puesto paciencia y afecto para tratar de hacerme optimista. A Nora, porque me presentó a la sismología, me dio la oportunidad de dedicarme a ella y hacer y deshacer mis pensamientos todos estos años siempre con un buen consejo de su parte. A ambos, por todo lo que me han enseñado y por las inquietudes que me han planteado.

A la Universidad Nacional de La Plata, que me ha hecho lo que soy a nivel profesional. A ella le debo mi carrera entera y espero que este aporte retribuya en algo todo su esfuerzo.

A la Universidad de Granada, que a través de convenios y proyectos me ha permitido vivir un poco de la maravillosa Granada en varias oportunidades, mientras aprendía y trabajaba en esta tesis.

Al INPRES, en particular a Mario Araujo y al personal, que además de proveer generosamente los datos para esta tesis me brindaron su asesoramiento en el manejo de los mismos y la localización.

A Edoardo Del Pezzo, Francesca Bianco, Gerardo Alguacil y Carmen Martínez Arévalo quienes me pasaron parte de su experiencia y estuvieron dispuestos a colaborar. En especial a Edoardo, con toda su sabiduría y su carácter afable y a Gerardo, por traducirme la electrónica y ser tan buena persona.

A Victor Ramos por enseñarme prácticamente todo lo que aprendí de geotectónica y a Andrés Tassara porque casi sin darse cuenta me ayudó a sacar las conclusiones. Espero no haber tergiversado sus expresiones.

A mis compañeros del Departamento de Sismología que me han acompañado y soportado todos estos años. A Laura y Carolina, en especial a Lau por cubrirme tantas veces y estar cerca y a Caro, por sus aportes con el VELEST y la gastronomía. Amigas y tesistas como yo, compañeras de catarsis, ¡Vamos chicas, que llegamos! A los técnicos que tantas veces nos han solucionado inconvenientes aportando buen humor, apapachamientos y un mate, en especial a Miguel, Néstor, Jorge y Alberto. A Andrea, que mientras estudiaba su carrera de geología compartió conmigo lo aprendido y hasta lo tradujo a mi vocabulario o me enseñó a hablarlo. A Ruben, quien me quiso hacer astrónoma mientras me daba una mano con la informática y su buen criterio en el diseño. Siempre con su predisposición para ayudar en lo que fuera. A Cecilia y Milton, con quienes compartí escritorio y computadora cuando recién empezábamos.

A Claudia Tocho y Graciela Font, por sus palabras de aliento, por sus detalles, por traerme la calma en más de un momento, por dar siempre el primer paso en venir a saludar. A Daniel Lorenzo, quién se iba a imaginar que su intuición acerca de mi interés por la sismología me llevaría a donde estoy ahora.

A los técnicos y administrativos del IAG, que facilitaron trámites durante mis estancias en Granada. En especial a Benito quien me ayudó a dar los primeros pasos con el SEISAN.

A Alberto Caselli y toda su gente, por la confianza, la paciencia y el aliento. Por ese mail preguntando $y$ ?? Cómo vas? Por esperarme y guardarme ese lugarcito en la volcanología.

A mis tesistas, Luciana y Marcela, por haberme confiado el final de sus carreras y ser pacientes cuando hizo falta para que yo trabajara en esta tesis. A todos mis alumnos, porque a través de sus inquietudes, planteos y discusiones he aprendido un montón y creo que esta tesis ha integrado varios de esos conceptos. Espero que también les sea de utilidad.

A mis compañeros de campañas antárticas, son tantos, que temo nombrarlos porque no quiero olvidarme a ninguno, todos saben que los tengo en el corazón. Puedo dar algunos nombres: Enrique, Javi, Flor, Mauri, Cintia y Araceli esperando que los demás no se ofendan. Pero hay muchos más, de aquí y de allá. En especial a aquellos que han estado en Granada durante mis estancias y me han hecho sentir como en casa. Sin ustedes no habría sido nada fácil.

A mis amigos de aquí, amigos de la vida. Por tantos años compartidos, por las reuniones relajadas, por estar ahí cada vez que nos necesitamos, por ser más hermanos que amigos. Por las charlas de temas varios y todos entremezclados que nos transforman a cada uno en un poco del otro. Por entretener a mi familia cuando yo necesitaba tiempo para escribir y por comprenderme en mis locuras. A Chacho, Ana, Silva, Billy, Andrea, Lupe y Marianela.

A mis amigas y profesoras de Pilates, por conseguir que las contracturas disminuyeran, al menos hasta hace un tiempo, y por lograr que esperará la hora de ir al gimnasio con ganas. Por hacerme reír.

A Yoli por hacer que mi casa anduviera mientras yo permanecía sentada frente a la laptop y por ser madre sustituta o abuela de corazón de mis hijas desde antes que nacieran.

A mi familia, mis padres, Piry y Pedro, por hacerme y darme la oportunidad de ser. Por la paciencia ante mi carácter y por entender mis ausencias y aceleres. En especial a mi mamá por preocuparse y ocuparse, por estar atenta y por hacer de soporte tantas veces. A mi papá por contagiarme su pasión por las ciencias y en especial por los terremotos. A Poupèe, más madre que suegra, siempre lista para cambiar sus planes y colaborar en lo 
que fuera para darme ese espacio de tranquilidad para la concentración. A mis hermanos y sobrinos, por estar ahí, cerca o lejos, por su colaboración como tíos y primos de mis hijas. Por aguantar mis largas narraciones geocientistas, por acompañar y alegrarse conmigo y preocuparse cuando hizo falta, por comprender y aceptar mis ausencias. A los que ya no están, pero han sido una parte muy importante en esta historia, Moni y Juan, ojalá se me haya pegado algo de su coraje y optimismo.

A César, Euge y Romi, desde el fondo de mi alma y con toda la fuerza que puedo reunir en este instante. Por alentarme, soportarme, sostenerme y esperarme. Por amarme como soy y a pesar de eso. Por ser independientes cuando así lo necesitaba y estar encima cuando hacía falta. Por los mimos, las cartitas, los tes de madrugada, los scones de los viernes, las provisiones de chocolates y gomitas para distraer al sueño, los chistes y las canciones para arrancarme una sonrisa en los momentos de desesperación, las horas de juegos tranquilos para no molestar a mamá. Son muchos los años que me ha tomado terminar. En casa, todos hemos sido un poco tesistas en sismología. Pero sin lugar a dudas, quien me hizo llegar hasta aquí es César, mi mayor apoyo, el que me ha entendido desde un principio, me ha impulsado a emprender esta aventura, ha asumido tareas para darme tiempo, ha desenmarañado la lógica de los primeros códigos, ha sido mi primer evaluador y mi asesor y auxilio de edición. Pero sobre todo, paciente y compañero, mi otro yo, me ha traído la calma y me ha puesto los pies en la tierra. A los tres les debo todo y espero poder compensarlos al menos en parte. Mi primer propósito es comenzar a disfrutar juntos y sin culpa, el tiempo libre.

Agradezco al jurado por aceptar leer esta tesis con atención, a los que quieran leerla por cariño o curiosidad y a los que mi memoria no pudo recordar.

A todos muchísimas gracias.

Esta tesis ha sido parcialmente financiada por el proyecto "Modelos Sísmicos De Alta Resolución De Volúmenes Sismogenéticos De Volcanes Activos, Islas De Tenerife Y Decepción, Y Su Impacto En La Valoración Del Peligro Volcánico. HISS”. CGL2008-01660.

Gabriela Badi

La Plata, 01 de junio de 2011 
Índice

Prólogo

\section{CAPÍTULO I}

Introducción. 7

1.1 DE LA TIERRA ELÁSTICA, HOMOGÉNEA E ISÓTROPA A LA TIERRA

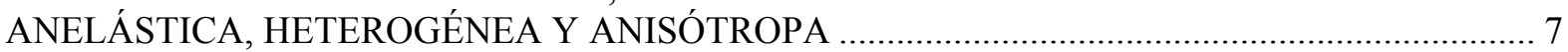

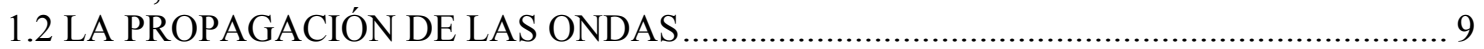

1.3 LA REGIÓN DE NUEVO CUYO Y LA ATENUACIÓN SÍSMICA.................................... 18

\section{CAPÍTULO II}

Atenuación de Ondas Sísmicas: Modelos y Métodos de determinación .... 21

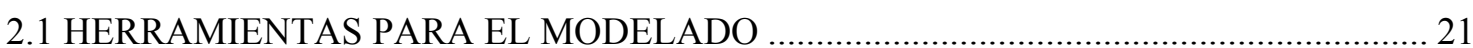

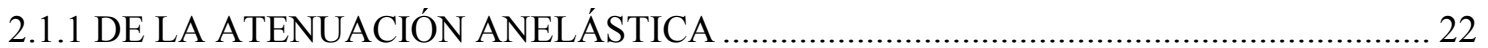

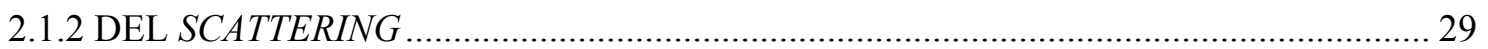

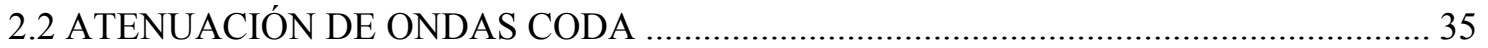

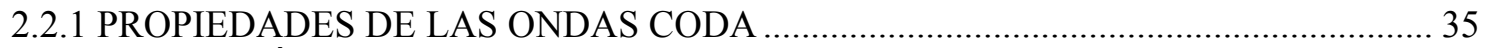

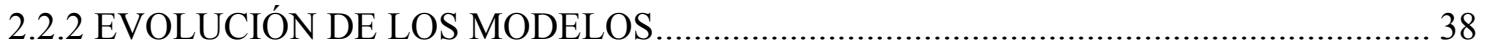

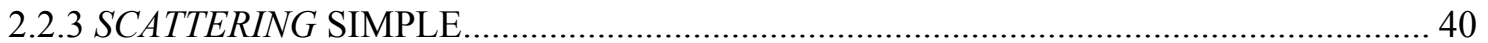

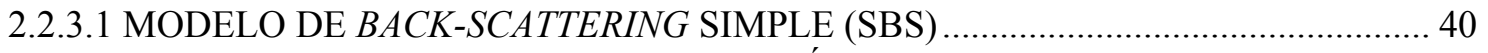

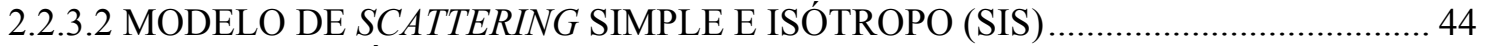

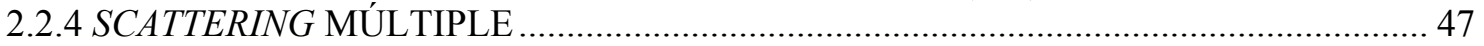

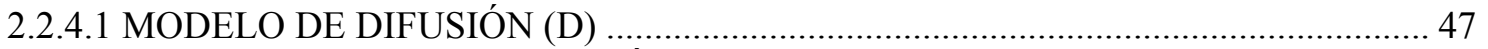

2.2.4.2 MODELO DE SCATTERING MÚLTIPLE DE GAO (SMG) ....................................... 49

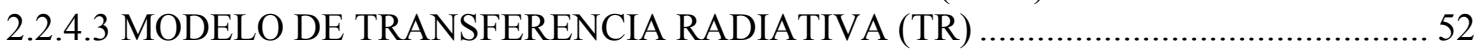

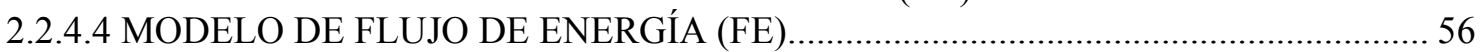

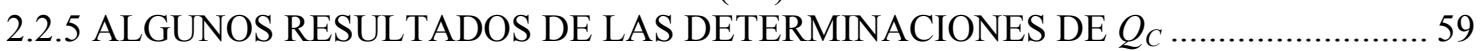

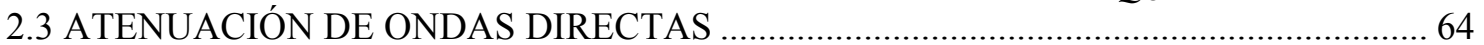

2.3.1 ALGUNAS PROPIEDADES DE LA ATENUACIÓN DE ONDAS DIRECTAS............ 64

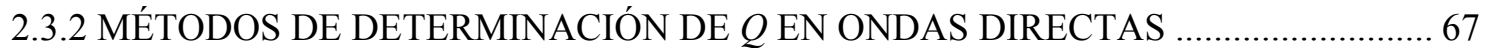

2.3.2.1 MÉTODO DEL ENSANCHAMIENTO DEL PRIMER PULSO DE P (APP) ............... 67

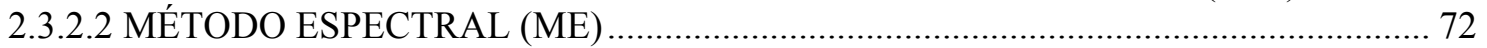

2.3.2.3 MÉTODOS DE LA SIMPLE y DOBLE RAZÓN ESPECTRAL (SRE y DRE)............ 77

2.3.2.4 MÉTODO DE NORMALIZACIÓN DE LA CODA (NC) ........................................... 80

2.3.3 ALGUNOS RESULTADOS DE LA DETERMINACIÓN DE LA ATENUACIÓN DE

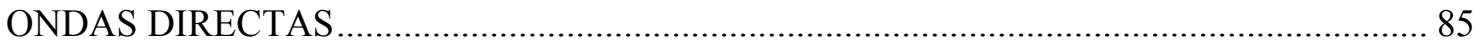

2.4 SEPARACIÓN DE LA ATENUACIÓN INTRÍNSECA y DE SCATTERING …..................... 90

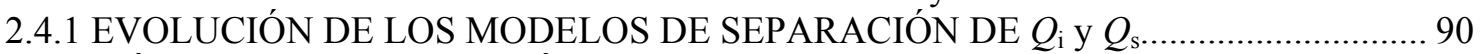

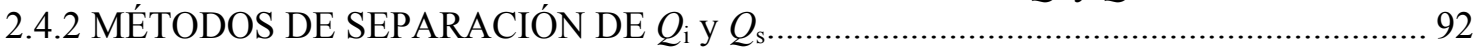

2.4.2.1 MÉTODO DE VENTANAS TEMPORALES MÚLTIPLES O MULTIPLE LAPSE TIME

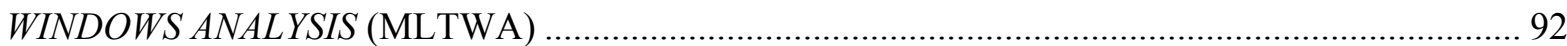

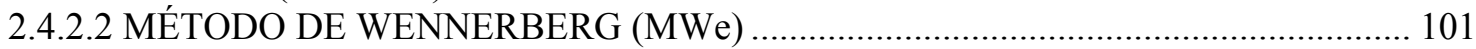

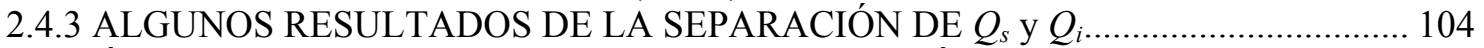

2.5 MÉTODOS SELECCIONADOS PARA SU APLICACIÓN .............................................. 109

\section{CAPÍTULO III}

\section{Región de Nuevo Cuyo: Marco Sismotectónico y Datos Sismológicos..... 111}

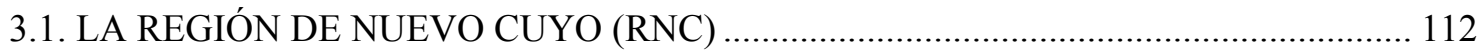

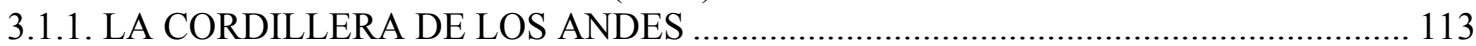

3.1.2. PROVINCIAS GEOLÓGICAS EN LA REGIÓN DE NUEVO CUYO ........................ 117 
3.1.2.1. LLANURA CHACOBONAERENSE O CHACOPAMPEANA ….............................. 118

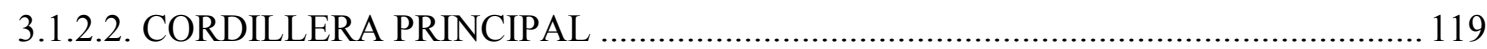

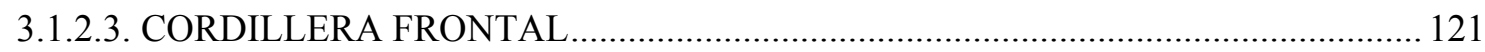

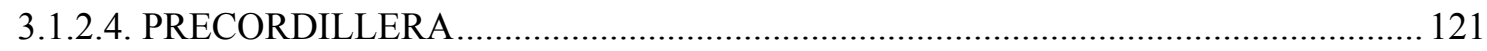

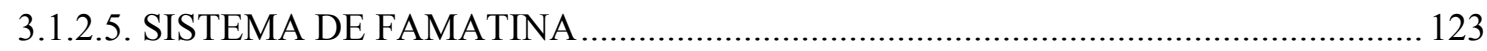

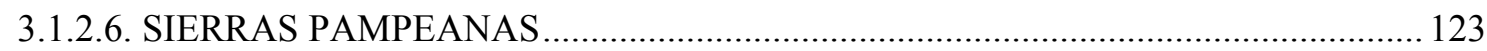

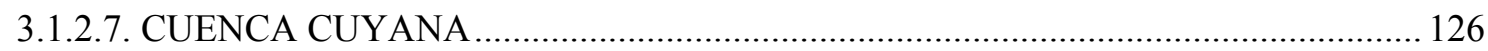

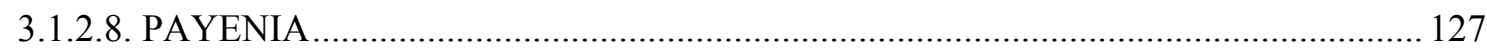

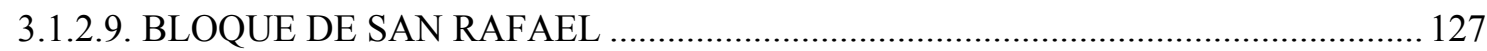

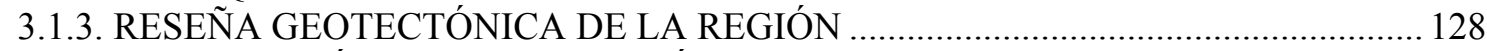

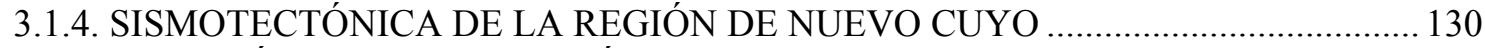

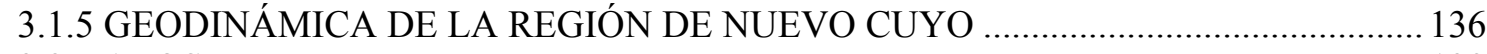

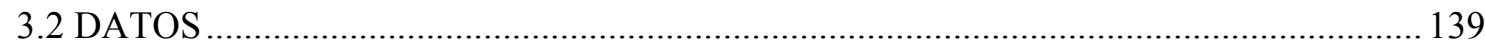

3.3 ESTRUCTURA UNIDIMENSIONAL DE VELOCIDAD DE ONDAS P........................ 145

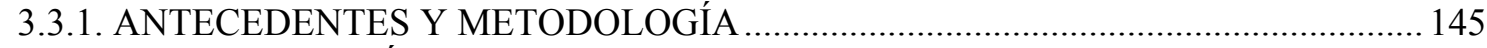

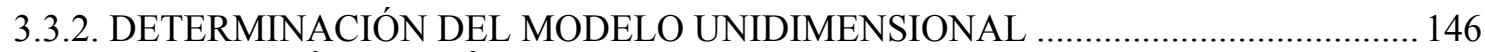

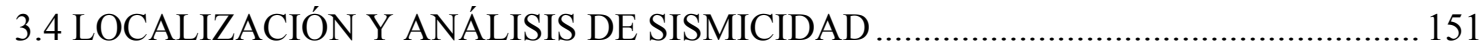

\section{CAPÍTULO IV}

\section{Determinación de Q en ondas coda, ondas P y S: Aplicación, resultados e}

interpretación............................................................................................................163

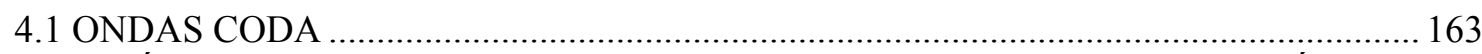

4.1.1 MÉTODOS DE BACK-SCATTERING SIMPLE (SBS) Y SCATTERING ISÓTROPO

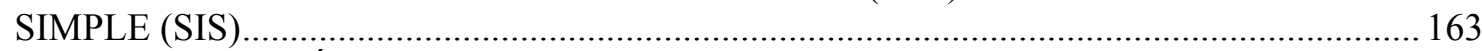

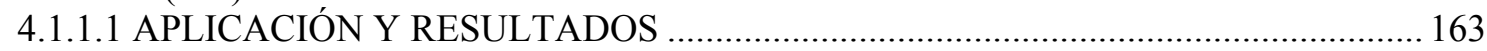

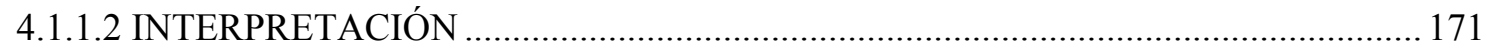

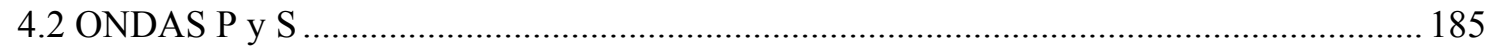

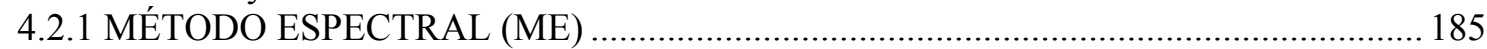

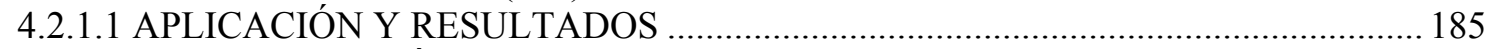

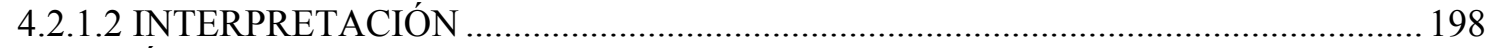

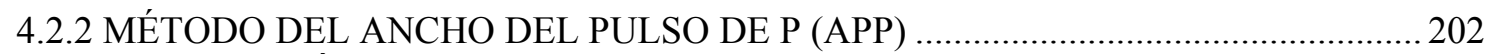

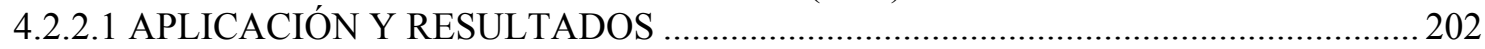

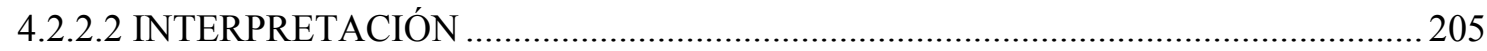

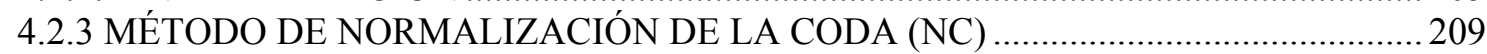

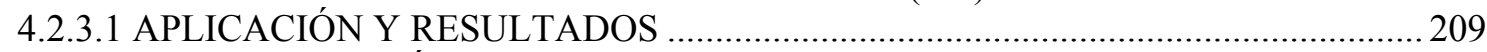

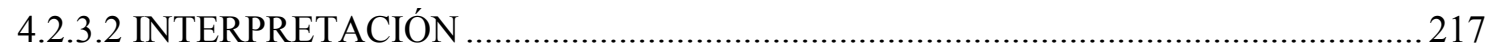

4.2.4 MÉTODO DE LAS DOBLES RAZONES ESPECTRALES (DRE) ................................. 220

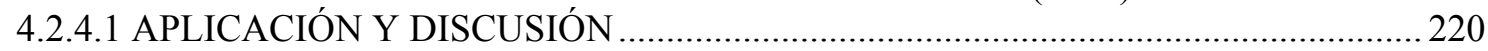

4.3 SEPARACIÓN DE LA ATENUACIÓN INTRÍNSECA y DE SCATTERING ................. 222

4.3.1 MÉTODO DE LAS VENTANAS TEMPORALES MÚLTIPLES (MLTWA) ................222

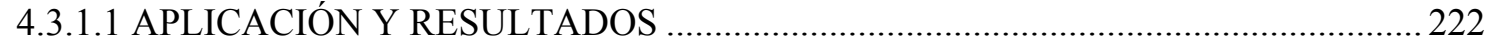

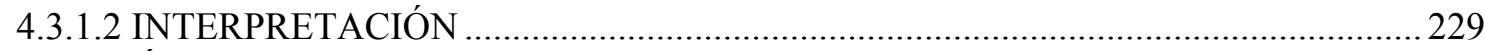

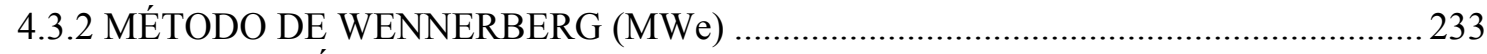

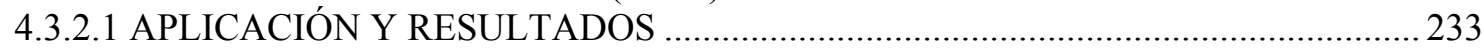

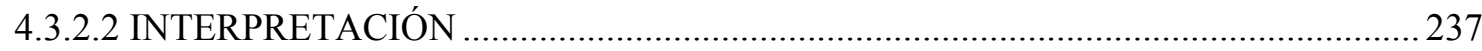

\section{CAPÍTULO V}

Análisis comparativo de los resultados........................................................ 239

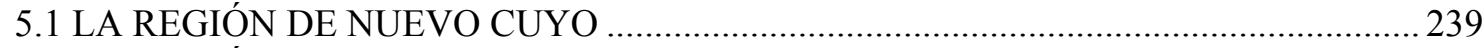

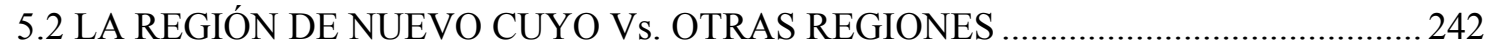

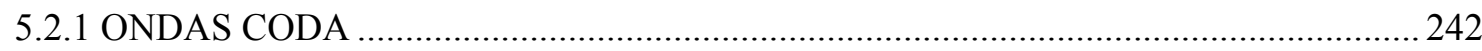

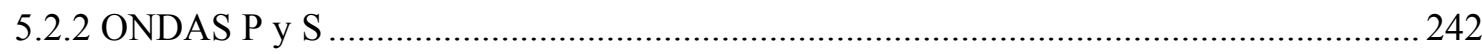




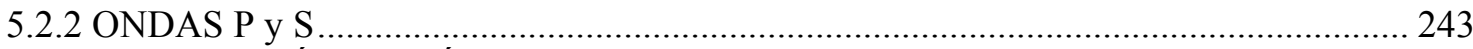

5.2.3 ATENUACIÓN INTRÍNSECA y DE SCATTERING ............................................... 245

\section{CAPÍTULO VI}

Resumen y Conclusiones.................................................................................... 247

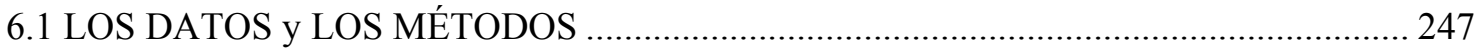

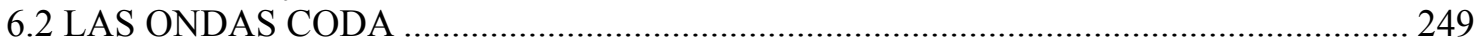

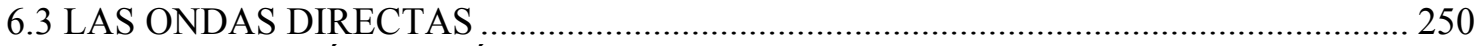

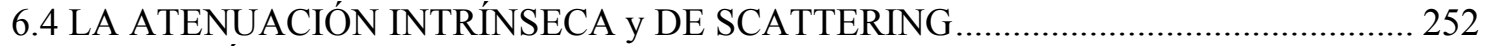

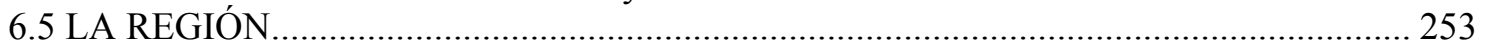

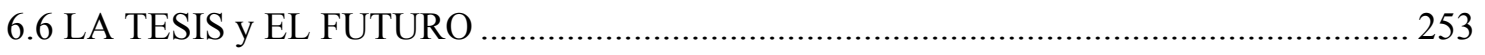

Apéndice A

Detalle de condiciones y parámetros de registración de las estaciones

utilizadas de la Red Sismológica Zonal Nuevo Cuyo (RSZNC -

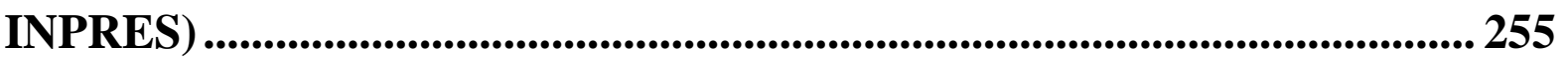

Apéndice B

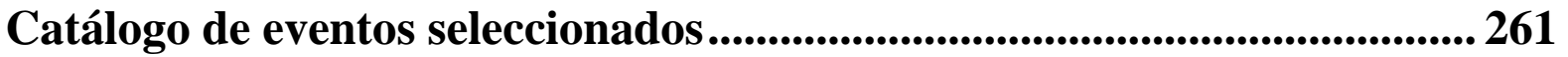

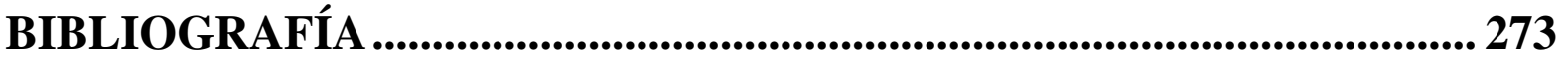




\section{Prólogo}

La Región de Nuevo Cuyo (RNC) ha sido calificada como la de más alta peligrosidad sísmica en la República Argentina (Giardini et al., 1999).

Este es el primer trabajo de atenuación sísmica con datos digitales sobre ondas $\mathrm{P}, \mathrm{S}$ y coda en la región de Nuevo Cuyo utilizando datos de estaciones permanentes argentinas. Es además, el resultado de la colaboración entre varias instituciones, del país y del exterior, ya que los datos han sido provistos por el INPRES (Instituto Nacional de Prevención Símica, San Juan) y parte del trabajo se ha realizado en colaboración con investigadores de España: del Instituto Andaluz de Geofísica (IAG), Univ. de Granada y de Italia: del Osservatorio Vesuviano, Istituto Nazionale di Geofisica e Vulcanologia, Nápoles y de la Universidad de Salerno.

La caracterización del comportamiento de la región ante la acción de las ondas sísmicas es un dato indispensable para la prevención. La mayoría de los análisis sismológicos que extraen información de las formas de onda registradas requieren identificar las pérdidas y redistribuciones de energía en las ondas, que resumen el efecto de la atenuación, a lo largo de la trayectoria. Estos efectos se originan en la heterogeneidad y en la anelasticidad del medio y pueden estudiarse sobre distintos tipos de ondas sísmicas, en función de la frecuencia o independientemente de ella. Cada resultado por separado, sus variaciones y las relaciones que pueden establecerse entre los mismos dan una visión integrada de la interacción de las ondas con el medio dinámico donde se propagan. La caracterización de la atenuación relacionada a los parámetros reológicos que la definen, brinda además un mejor conocimiento de los procesos geodinámicos de la región.

Mediante el estudio de las variaciones de la energía para ondas directas y dispersadas en los sismogramas se determinan los factores de calidad del medio, $Q$, cuya inversa permite cuantificar el efecto de la atenuación y efectuar regionalizaciones de acuerdo al modelo empleado. Vinculando los valores de $Q$ de ondas directas y dispersadas mediante métodos numéricos y analíticos se separan la proporción anelástica, $Q_{\mathrm{i}}$, y de scattering, $Q_{\mathrm{s}}$, de la atenuación en la región. El valor de $Q_{\mathrm{s}}$ se relaciona con la densidad de heterogeneidades y el de $Q_{\mathrm{i}}$ con la anelasticidad del medio, vinculada a su vez a la temperatura, la presión, el grado de fluidez y el contenido de fluidos. Por lo anteriormente expuesto es que $Q$ es más sensible a los parámetros reológicos que la velocidad de las ondas.

Los valores de la atenuación obtenidos para la RNC son una clara manifestación de su estado de actividad tectónica. La RNC se caracteriza por una alta atenuación de ondas coda con variaciones laterales a profundidades corticales, estabilizándose su valor cuando los volúmenes de scattering alcanzan profundidades más allá de la zona sismogénica en la placa subducida. Las determinaciones de la atenuación de ondas directas, realizadas por los distintos métodos muestran cierta convergencia en frecuencia y profundidad. Los valores medios de atenuación de ondas $\mathrm{P}$ y $\mathrm{S}$ muestran los diferentes efectos de la atenuación de corteza y manto y mantienen una relación entre sí que ubica a la atenuación en la RNC dentro de los valores promedio para regiones de actividad tectónica similar.

La separación de la atenuación intrínseca y de scattering ha mostrado que no son las heterogeneidades estructurales superficiales las que dominan la atenuación sino que la absorción anelástica es el efecto predominante, más aún cuanto mayor sea la profundidad de validez de los resultados. 
Esta tesis consta de seis capítulos:

En el Capítulo I se hace una introducción planteando los avances de la sismología que llevaron al estudio de la atenuación y se presentan los conceptos de scattering y de absorción anelástica que finalmente serán los protagonistas de este trabajo junto a la región de estudio.

En el Capítulo II, se describen los modelos básicos del scattering y de la atenuación intrínseca, las propiedades de las diversas ondas estudiadas así como la evolución de los modelos y métodos empleados a tal fin. Finalmente, se presenta un conjunto de métodos seleccionados para estimar la atenuación tanto de ondas coda como de ondas directas y la metodología utilizada para la separación de la atenuación intrínseca y de scattering.

En el Capítulo III se caracteriza a la región desde la geología, la sismicidad asociada y la geodinámica. Se presenta un nuevo modelo unidimensional de velocidad de ondas $\mathrm{P}$ y se describe la distribución de la sismicidad obtenida para este trabajo.

En el Capítulo IV se detallan los criterios de aplicación utilizados en cada método, se presentan los resultados obtenidos y se interpretan en planta y/o profundidad a fin de relacionarlos con la geodinámica regional.

En el Capítulo V se comparan los resultados obtenidos para la región de Nuevo Cuyo con los distintos métodos y ondas empleados y con resultados obtenidos por otros trabajos en la misma región u otras de actividad tectónica similar.

En el Capítulo VI se presentan las conclusiones tanto en relación a los métodos e implementaciones aplicados como a sus resultados para la región de Nuevo Cuyo, planteándose las implicancias de esta tesis en los trabajos a futuro. 


\section{CAPÍTULO I}

\section{Introducción}

El presente capítulo es un resumen de los avances de la sismología que llevaron a la concepción de una Tierra más real que puede considerarse como un sistema de generación y transmisión de energía. Las ondas registradas en los sismogramas son interpretadas como el producto resultante de diferentes etapas de transformación introduciendo los conceptos físicos y las definiciones fundamentales que serán de utilidad para el modelado matemático. Es así como se presentan los conceptos de scattering y absorción anelástica que finalmente serán los protagonistas de esta tesis junto a la región de estudio. Tratar de entender estos procesos en la región de Nuevo Cuyo es uno de los motivos que dan lugar a este trabajo.

\subsection{DE LA TIERRA ELÁSTICA, HOMOGÉNEA E ISÓTROPA A LA TIERRA ANELÁSTICA, HETEROGÉNEA Y ANISÓTROPA}

Las ondas generadas en un terremoto y registradas en estaciones sismológicas, después de haber viajado a través de la Tierra, tienen formas complejas. Cualquier onda puede representarse por la suma de infinitas ondas de frecuencias diferentes que con diversas amplitudes conformarán la señal registrada. El estudio de esas señales permite conocer características de la región donde se emplaza la estación, del camino por donde se han propagado las ondas y de la fuente de donde surgieron. La sismología plantea modelos de cada una de estas etapas y sus efectos sobre las ondas.

El desarrollo de las formulaciones de la generación y propagación de ondas de la sismología clásica se basa en la ley de Hooke de la elasticidad lineal. Haciendo consideraciones de conservación de energía, homogeneidad e isotropía se llega a relacionar las tensiones con las deformaciones a través de sólo dos parámetros elásticos que a su vez, son constantes en el medio. Según la elasticidad de Hooke, si un cuerpo es sometido a tensión se deforma proporcionalmente a ella y si se la quita, entonces vuelve a su estado inicial. Esto es válido si las tensiones tienen valores muy por debajo de las que puede soportar un material sin romperse para deformaciones infinitesimales o a temperaturas lo suficientemente bajas como 
para que el material no comience a fundirse y fluya. En casos más reales, los parámetros elásticos no serán constantes y su relación con los esfuerzos no será lineal. Y además, al quitar la tensión, los materiales tratarán de recuperarse pero conservarán cierta deformación residual. Se habrá pasado de la elasticidad a la anelasticidad. Deberá tenerse en cuenta también que la energía ya no se conservará como energía elástica. La fricción en la ruptura frágil o los requerimientos de los cambios de estado, por ejemplo, harán que parte de la energía elástica se transforme en calor. La energía distribuida en el frente de onda ya no será constante. Los modelos planteados por Maxwell y Kelvin en el siglo XIX sentaron las bases para el tratamiento de los materiales viscoelásticos, permitiendo describir mediante análogos mecánicos la respuesta de estos medios a las tensiones y deformaciones.

Según sea el período de las ondas, distintas deben ser las consideraciones al modelarlas. Por ejemplo, las ondas de períodos largos que se utilizan para estudios globales de la estructura terrestre, las ondas directas que se analizan para la determinación de parámetros de la fuente o las ondas superficiales en el modelado de la estructura cortical, admiten habitualmente un modelo de tierra lateralmente homogéneo, donde la velocidad varía solamente con la profundidad. En cambio, en el tratamiento de ondas de corto período (menos de 1s) este modelo ideal no alcanza a reproducir las formas de onda registradas. Fue en la década de los años '60 cuando comenzaron a surgir estas preocupaciones. Por esos años se empezó a generalizar el uso de las denominadas Redes Sismológicas Locales de corto período. Esta nueva instrumentación incorporaba un nuevo rango de frecuencias en los registros sismológicos. La observación de los datos puso en evidencia que los modelos sísmicos usados hasta el momento no eran capaces de explicar las señales registradas. Una Tierra con una estructura en capas lateralmente homogéneas era totalmente insuficiente. El aumento del número de redes de este tipo en todo el planeta, la aparición del registro digital, la ampliación del rango dinámico del registro y sobre todo la mayor cantidad de observaciones pusieron de manifiesto la necesidad y dieron la posibilidad de plantear nuevos modelos más complejos. Estos modelos deberían ser capaces de explicar fenómenos como la forma en que la amplitud de los sismogramas decae, la amplificación local en las distintas estaciones, la disminución de la señal con la distancia que no podía ser explicadas por el modelo lateralmente homogéneo, el aumento en la duración de los primeros paquetes de ondas internas con la distancia y otros. La base de los mismos sería la hipótesis de una Tierra lateralmente heterogénea, con presencia de fuertes contrastes de velocidad. La realización de modelos tomográficos (Nakanishi y Anderson, 1983; Woodhouse y Dziewonski, 1984, entre otros) confirmaron esta complejidad estructural y esta misma dio lugar a la elaboración de 
numerosas hipótesis para nuevos modelos y técnicas de cálculo.

En la Tierra existen heterogeneidades en una amplia variedad de escalas, desde los defectos cristalinos que modifican los módulos elásticos de los minerales formadores de rocas hasta los procesos tectónicos involucrados en los movimientos relativos de las placas litosféricas o inclusive las heterogeneidades sobre el límite manto-núcleo terrestre, la capa D”, que serían la base de las plumas, si es que existen (Anderson, 2000, Lay, 2005). Uno de los principales objetivos de la sismología es la caracterización de esas heterogeneidades. Hacia ese objetivo convergen también otras disciplinas de las geociencias. Esta interdisciplinariedad ha permitido por ejemplo, un mejor entendimiento de los mecanismos que han formado la corteza terrestre, los procesos volcánicos y la naturaleza de las zonas sísmicamente activas, por citar solo unos pocos.

Por último, el pasaje de la isotropía a la anisotropía. En un material isótropo las propiedades físico-químicas que caracterizan su comportamiento son constantes en todas las direcciones. Por ejemplo en un material con simetría uniaxial, como podría ser un medio estratificado, las propiedades se consideran idénticas en direcciones paralelas al plano de la estratificación. Mientras en un medio isótropo hacen falta sólo dos parámetros elásticos, en un medio uniaxial se requieren cinco parámetros para definir las velocidades de las ondas sísmicas y sus variaciones según la dirección de propagación. A diferentes escalas, ya sea en los minerales por la estructura de sus redes cristalinas, en las rocas por la disposición de los granos o inclusive en las estructuras geológicas por la presencia de fracturas, existen direcciones preferenciales de propagación. Las consideraciones de anisotropía a la vez que requieren de mayor cantidad de parámetros en su tratamiento, reducen la necesidad de incorporar numerosas hipótesis como se hace en los modelos de la Tierra isótropa, como por ejemplo, las capas de baja velocidad necesarias para definir el límite litósfera-astenósfera. Un efecto de la anisotropía sobre las ondas puede ser el desdoblamiento o la redistribución de la energía de la onda en pulsos de diferentes polaridades y velocidades de propagación (Stein y Wysession, 2003).

\subsection{LA PROPAGACIÓN DE LAS ONDAS}

Como ya se ha dicho, las ondas que se registran en una estación sismológica difieren de las que se originaron en la fuente y de las registradas en otras estaciones aún cuando estén a la misma distancia epicentral. Esa señal registrada puede entenderse como el resultado de varias etapas de transformación tanto de la amplitud como del contenido en frecuencias. Dichas etapas, por orden de aparición, son: el mecanismo de la fuente, la expansión de la energía en 
el frente de onda, las propiedades del camino sísmico, las estructuras superficiales en la estación y las limitaciones instrumentales de la estación con que se efectúa el registro. Algunas características de cada una de ellas son:

\section{- El Patrón de Radiación y la Función Fuente:}

La fuente considerada originalmente para el planteo de las ecuaciones de ondas en un medio elástico, isótropo y homogéneo es puntual. De este modo las ondas $\mathrm{P}$ y S, obtenidas como solución para una tierra esférica, resultan tener frentes esféricos. A grandes distancias, es decir, cuando se trabaja con telesismos, estos frentes pueden aproximarse como planos, lo que simplifica mucho el tratamiento. $\mathrm{Si}$, a partir de una fuente puntual se propaga una onda con un frente de onda esférico en un medio como el planteado idealmente, las polaridades de las señales registradas en cualquier estación, independientemente de su ubicación relativa serían idénticas. Como esto no ocurre en la realidad, se han diseñado diferentes modelos de fuente que tratan de reproducir el patrón de amplitudes real. Resolviendo nuevamente las ecuaciones de movimiento pero con un modelo de mecanismo de fuente, se obtienen las soluciones del campo de ondas longitudinales y transversales dependientes de la distancia, del azimut y de la frecuencia (Aki y Richards, 2002). Si el evento en cuestión incorpora la presencia de fluidos, con cambios de presión o de fase y circulación, el patrón se complica aún más (Foulger et al., 2004). Es decir, que a priori, no se puede suponer un patrón de radiación para los datos registrados de un evento, hasta tanto no se hagan estudios específicos. En el caso de eventos tectónicos, una solución práctica para eliminar el patrón de radiación ante esta falta de conocimiento, consiste en realizar cocientes entre amplitudes tomadas a distintos tiempos dentro de un mismo registro y para la misma frecuencia, esto es lo que se define como cocientes espectrales.

Las ondas irradiadas desde la fuente son el resultado del producto del momento sísmico escalar $\left(M_{0}=\mu A D\right.$, con $\mu$, el coeficiente de rigidez; $A$, el área de la falla y $D$, la dislocación o deslizamiento) con la función fuente que depende del tiempo en que dicha dislocación se produce. El modelo más simple de función fuente es la convolución de dos funciones "cajón" temporales que permiten representar la duración de la fuente y la longitud de la ruptura (Stein y Wysession, 2003). La transformada de Fourier de la convolución de funciones es el producto de las transformadas de cada función que en este caso resultan iguales a una función de la forma $\operatorname{sinc}(x)=\sin x / x$. Esta función es comúnmente utilizada en la modelización de procesos de longitud finita. Una aproximación que suele hacerse a la función $\operatorname{sinc}(x)$ es otra función que toma un valor constante e igual a 1 para $x<1$ y decae como $1 / x$ para $x>1$ (Fig. 
1.1a). Un gráfico logarítmico de la densidad de amplitud de desplazamiento espectral $(\log |A(\omega)|$ vs. $\log \omega)$ muestra tres segmentos con comportamientos diferentes según la frecuencia (Fig. 1.1b). Las frecuencias $2 / T_{\mathrm{R}}$ y $2 / T_{\mathrm{D}}$ se denominan frecuencias de corte y se relacionan con la duración de la fuente, mientras que la amplitud de la parte plana permite estimar el momento sísmico, $M_{0}$. A $T_{\mathrm{R}}$ se le denomina tiempo de ruptura y es el tiempo que tarda la ruptura en propagarse por la falla, $T_{\mathrm{D}}$ es el rise time o lo que tarda la dislocación en alcanzar su valor máximo en cualquier punto de la falla y depende de la caída de esfuerzo ocurrida durante el terremoto y $M_{0}$ depende del tamaño de la falla, la dislocación y el coeficiente de rigidez en la fuente. En algunos casos puede agregarse una etapa más a la envolvente del espectro de desplazamiento con una pendiente $\omega^{-3}$, pero el modelo $\omega^{-2}$ es el que mejor ajusta a las magnitudes de ondas internas y superficiales (mb, Ms) para grandes terremotos (Aki, 1967, Hanks, 1979). Puede utilizarse entonces una única frecuencia de corte región plana. La frecuencia de corte de las ondas $\mathrm{S}$ suele ser inferior a la de las ondas $\mathrm{P}$ en la mayoría de los modelos. A altas frecuencias puede evitarse la dependencia azimutal del
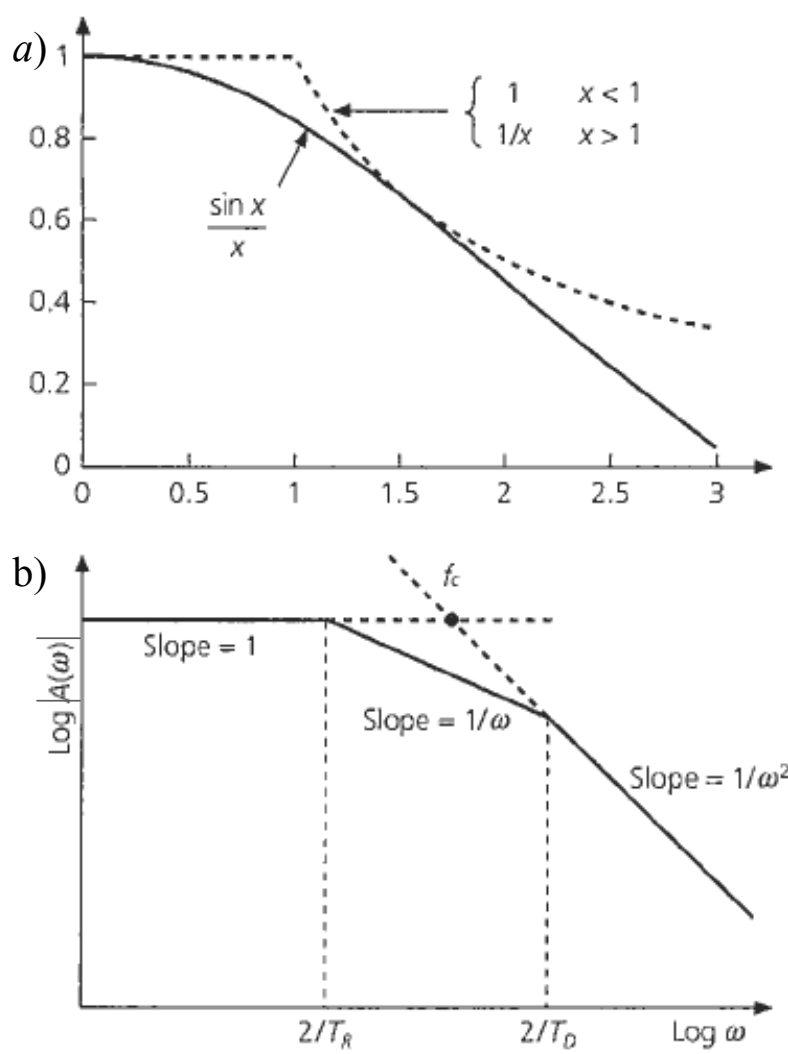

Fig. 1.1.- a) Aproximación a la función sin x / x utilizada para modelar el espectro de la fuente. b) Espectro teórico de un terremoto modelado en tres regiones con pendientes $1, \omega^{-1}$ y $\omega^{-2}$ delimitadas por las frecuencias angulares que corresponden a los tiempos de ruptura y rise time en la fuente, $T_{R}$ y $T_{D}$ respectivamente. Una aproximación simplificada utiliza una única frecuencia de corte $f_{c}$ ubicada en la intersección entre las extrapolaciones de la primera y la tercera etapa. (Extractada de Stein y Wysession, 2003). 
espectro de la fuente, promediando los espectros registrados en diversas direcciones con respecto a la fuente. (Cormier, 1982). La caída de tensión en la fuente es aproximadamente independiente de $M_{0}$ (Stein y Wysession, 2003). Puede probarse que para una dada caída de tensión, a mayor longitud de la falla, mayores serán $M_{0}, T_{\mathrm{R}}$ y $T_{\mathrm{D}}$, por lo que menores serán las frecuencias de corte. Esto equivale a decir que cuanto mayor sea la magnitud de un evento menor será el ancho de banda de su espectro.

\section{- La Expansión Geométrica:}

La energía irradiada en la fuente establece la magnitud del terremoto. Si se considera un medio elástico, isótropo y homogéneo, esta energía se conserva distribuyéndose sobre el frente de onda para dar la densidad de energía. Si las ondas registradas corresponden a ondas internas de eventos locales el frente de onda se considera esférico. En ese caso la densidad vendrá dada por el cociente entre la energía irradiada y el área de la esfera de radio igual a la distancia hipocentral, $R, E=I / 4 \pi R^{2}$, como es el caso de una onda interna o de cuerpo, $\mathrm{P}$ o S. En cambio, en una onda superficial, donde la propagación es paralela a la superficie, el frente de onda puede aproximarse por un cilindro y la densidad de energía se expresa como $E=I / 2 \pi h R$, donde $h$ es el espesor de la capa donde esta onda se propaga. Según la teoría de la elasticidad, la densidad de energía es proporcional al cuadrado de la deformación y esta a su vez se relaciona con el desplazamiento, por lo que la densidad de energía en una estación es proporcional al cuadrado de la amplitud del desplazamiento registrado, $E \propto A^{2}$. La amplitud del desplazamiento a una distancia $R$, se aproxima entonces por $A_{0} R^{-m}$, donde $A_{0}$ es la amplitud en la fuente y $m$ es el coeficiente de expansión que puede ser igual a $0.5,1 \mathrm{o}$ incluso otros valores si el medio es heterogéneo.

En el caso de considerar ondas internas telesísmicas propagándose en un medio con velocidad variando suavemente con la profundidad, la distribución de energía sobre los frentes de onda esféricos lleva a una relación $E \propto\left(R_{T}{ }^{2} r \sin \Delta\right)^{-1} d^{2} t / d \Delta^{2}$ donde $R_{T}$ es el radio terrestre, $r$ es el radio en la fuente, $\Delta$ es la distancia epicentral en radianes y $d^{2} t / d \Delta^{2}$ es la variación en la pendiente de las curvas de tiempo de recorrido (Udías Vallina, 1999). Si las ondas telesísmicas son superficiales, puede considerarse su propagación en un anillo alrededor de la Tierra con centro en la fuente de las ondas considerada superficial. De este modo, la energía se distribuye en un anillo de longitud $2 \pi R_{T} \sin \Delta$, por lo que la densidad de energía decrecerá de la forma $E \propto\left(R_{T} \sin \Delta\right)^{-1}$ (Stein y Wysession, 2003).

Para poder comparar señales registradas a diferentes distancias de la fuente, debe 
corregirse el efecto de la expansión del frente, a la que se llama geométrica porque sólo se debe a su forma.

\section{- El Efecto de Sitio en la estación}

Las características geológicas del lugar donde se instala una estación afectan de manera particular a todas las señales registradas. Este efecto se considera dependiente de la frecuencia y también del backazimut, (la dirección desde la que provienen las ondas que llegan a la estación). El efecto de sitio depende también del hombre, ya que este es el que determina las condiciones de la instalación de la estación. Puede causar reverberaciones, amplificaciones locales de la señal o incluso alguna complejidad de la forma de onda que no puede ser modelada por métodos determinísticos (Sato y Fehler, 1998). Para conocer este efecto deben hacerse estudios sobre un gran número de señales registradas bajo diversas condiciones, que permitan modelar una expresión que lo represente y así corregir los registros sismológicos para hacerlos comparables a los de otras estaciones en diferentes emplazamientos. Además la determinación del efecto de sitio en función de la frecuencia es esencial para el establecimiento de códigos edilicios y la estimación del peligro sísmico.

\section{- La Respuesta Instrumental}

Para poder conocer el movimiento del suelo en la estación, es necesario poder eliminar el efecto de la registración. Este efecto suele darse en la forma de una función de la frecuencia que se conoce como respuesta instrumental. Esta respuesta, además de amplificar la señal del suelo para hacerla medible, modifica el contenido de frecuencia de la misma debido a las limitaciones propias de la construcción del equipo que se esté utilizando. La variedad de instrumental sismológico existente hoy en día hace indispensable el conocimiento de las características de la registración. De otro modo sería imposible compartir datos de diferentes estaciones. También es importante la actualización periódica de las expresiones de la respuesta, dado que pueden variar con el tiempo por el desgaste o desbalance del instrumental. Una forma de eliminar su efecto en el sismograma, sin conocer su expresión matemática, es haciendo cocientes espectrales del mismo modo que se hace con el patrón de radiación de la fuente. Conocida la respuesta instrumental, pueden implementarse la deconvolución o la división espectral, contemplando siempre los errores que se pueden incorporar.

\section{- El Efecto del Medio de Propagación}

Como se ha dicho anteriormente, del análisis de ondas de corto período muchos autores concluyeron que el medio por el que se propagan las ondas debe considerarse heterogéneo, anelástico y anisótropo (Herraiz y Espinosa, 1986). Cuanto mayor sea el volumen de Tierra 
que haya afectado a las ondas registradas, las características del medio se verán promediadas, tendiendo al medio ideal. A menor escala los efectos de estas propiedades se harán evidentes pero superpuestos unos sobre otros, haciéndose muy difícil distinguirlos, ya que las heterogeneidades a su vez probablemente sean anelásticas y anisótropas. Los efectos de las heterogeneidades y de la anisotropía se relacionan más con las modificaciones de las trayectorias de las ondas y la redistribución de la energía en el medio, en cambio los efectos de la anelasticidad se vinculan al intercambio de energía entre la onda y el medio.

Surge entonces el concepto de atenuación sísmica para describir la disminución de la densidad de energía en el frente de una onda sísmica y el lento decaimiento de la amplitud en los sismogramas que no llegan a ser explicados a través de los fenómenos conocidos en medios elásticos y homogéneos

A continuación se describen algunos de los fenómenos relacionados a la propagación de ondas en medios heterogéneos y anelásticos, los conceptos básicos para su tratamiento y algunos efectos observados sobre las ondas que permitieron establecer metodologías de análisis de los datos.

\section{O Interacción con heterogeneidades:}

Ya se ha presentado a las heterogeneidades como fuertes contrastes de velocidad que encuentran las ondas en su camino entre la fuente y la estación. En este encuentro entre la onda elástica y la partícula, vale el principio de Huygens por el que todo punto perturbado se transforma en una fuente de perturbaciones al producirse un intercambio de cantidad de movimiento entre la onda incidente y la heterogeneidad. De este modo quedan definidas las ondas primarias, que inciden en la heterogeneidad desde la fuente y las ondas secundarias que salen de la heterogeneidad que es perturbada. En general, las ondas secundarias no saldrán en la misma dirección que la onda primaria y además existe la posibilidad de que por cada onda primaria surjan varias ondas secundarias. Es así que la energía incidente deberá redistribuirse y la amplitud de la onda registrada será generalmente menor que la amplitud de la onda que partió de la fuente. La energía, aún conservada, se habrá dispersado y es por eso que a las heterogeneidades también se las llama dispersores. Las características de este fenómeno dependen de la relación existente entre la longitud de onda y las dimensiones de la heterogeneidad. En un esquema muy simplificado, existen tres grandes grupos de heterogeneidades:

Heterogeneidades de dimensiones mucho más grandes que la longitud de onda:

Si la longitud de onda es mucho menor que la extensión del dispersor, el efecto sobre el frente de onda será el que resulte al tratar de pasar de un medio a otro con parámetros 
elásticos diferentes. En este caso, la teoría de rayos permite hallar la dirección de propagación de las ondas secundarias, su naturaleza y la cantidad de energía que llevarán luego de la redistribución. Esta es la escala donde los procesos característicos son la reflexión y la refracción y se aplica en medios estratificados con dimensiones laterales que pueden considerarse como infinitas y saltos de velocidad bien definidos en las discontinuidades que separan las capas.

\section{Heterogeneidades de dimensiones muy inferiores a la longitud de onda}

Si la onda encuentra en su trayectoria una heterogeneidad mucho más pequeña que su longitud de onda, probablemente no ocurra intercambio de energía y continúe su trayectoria sin ser afectada. Esto puede compararse al efecto de un medio homogéneo o con contrastes de velocidad muy leves.

\section{Heterogeneidades de dimensiones cercanas a la longitud de onda}

Si la heterogeneidad es un cuerpo de dimensiones finitas dentro de un rango centrado en la longitud de onda, por el principio de Huygens, las ondas secundarias formarán un patrón de radiación complejo. La energía se redistribuirá de maneras diferentes dependiendo de la forma de la heterogeneidad y del contraste que represente. Los procesos que ocurren ante esta relación de escala se denominan en general procesos de scattering ${ }^{*}$ y puede tener efectos muy diferentes dependiendo de la distancia recorrida por las ondas, la longitud de onda y la distancia entre las heterogeneidades o las dimensiones de las mismas. Un caso particular es la difracción, que ocurre si la heterogeneidad tiene la misma escala que la longitud de onda.

La terminología usada en el scattering de las ondas sísmicas proviene de la física de partículas. De esta manera, el medio puede tratarse entonces como un medio homogéneo con heterogeneidades distribuidas según una densidad $\rho_{n}$ superficial o volumétrica. A su vez, la

* Tanto el vocablo inglés scattering como dispersion se traducen al español como dispersión. Este término es reservado en la sismología de habla hispana, para el proceso que sufren las ondas superficiales debido a su propia naturaleza y difiere ampliamente de este cuyo origen es la naturaleza heterogénea del medio. Si bien existen otros sinónimos, ninguno de los hallados parece adaptarse sin causar confusión. Varios de ellos, como difusión, disipación, irradiación y propagación, ya se asocian a otros procesos físicos. No parece que sea conveniente hablar de desparramo, disgregación o esparcimiento de las ondas como si se tratara de partículas, tampoco de separación o bifurcación que darían a pensar en sólo el camino recorrido, menos aún de una desbandada o fuga. Es por ello que se ha optado por conservar el término scattering para denominar este proceso. A las ondas afectadas por él se las llamará ondas dispersadas sin ánimo de confundir con la teoría de ondas superficiales.

distribución de los dispersores puede considerarse homogénea o heterogénea haciendo que las ondas sean afectadas en ciertas direcciones de propagación más que en otras. Se define: 
- forward-scattering o scattering hacia adelante, si las ondas secundarias tienen una dirección y sentido promedio similares a los de las ondas primarias.

- $\quad$ back-scattering o scattering hacia atrás, cuando la dirección y sentido promedio de las ondas secundarias son contrarios a los de las ondas primarias.

- $\quad$ scattering isótropo, si no existe una dirección promedio preferencial en las ondas secundarias.

- scattering simple, cuando la densidad de dispersores es tan baja que puede considerarse que la onda en su trayectoria ha sido afectada por un único dispersor.

- $\quad$ scattering múltiple si la onda se ha encontrado con más de un dispersor antes de llegar al receptor y el caso extremo será la difusión donde la energía secundaria se distribuye en todas las direcciones de propagación.

- $\quad$ scattering débil, si la energía de la onda varía muy poco en la interacción, es decir si la energía de la onda secundaria es mucho menor que la de la primaria.

- $\quad$ scattering fuerte, cuando la energía de la onda secundaria es similar a la de la onda primaria.

\section{oAbsorción anelástica}

El comportamiento de los materiales dentro de la Tierra depende de varios factores como por ejemplo composición, presión, temperatura, porosidad, contenido de agua y porcentaje de fusión. Cuando las rocas son sometidas a presiones confinantes bajas su comportamiento puede asumirse como frágil o elástico. Estos son los mecanismos principales en los procesos tectónicos que ocurren en los primeros 5-10 km de profundidad. Byerlee (1978) comprobó empíricamente que si la tensión de cizalla supera el 85\% de la tensión normal, a presiones confinantes inferiores a $200 \mathrm{MPa}$, la roca se fracturará. Por encima de los $200 \mathrm{MPa}$, la relación entre tensiones es algo menor. Por debajo de la tensión de fractura los materiales serán elásticos, deformándose proporcionalmente a la tensión aplicada según la ley de Hooke.

A mayor profundidad, el comportamiento del medio puede cambiar de frágil a dúctil si la tensión aplicada supera una tensión límite elástico que depende de la temperatura y la presión. Para representar el comportamiento de estos medios se plantean modelos mecánicos, siendo los más sencillos aquellos que superponen la elasticidad lineal con la viscosidad lineal de Stokes dando cuerpos linealmente viscoelásticos (Ben-Menahem y Singh, 1998). Un cuerpo elástico se deforma instantáneamente al aplicarle una tensión y esa deformación se recupera totalmente y en forma instantánea cuando la tensión cede. Un cuerpo viscoso lineal sometido a una deformación constante, sufrirá un aumento súbito de la tensión que luego irá 
relajando conforme el cuerpo alcanza la deformación impuesta. Un modelo mecánico ampliamente utilizado es el sólido lineal estándar, que es una combinación de los modelos de Maxwell y Kelvin-Voigt. Cuando se aplica una tensión sobre el mismo, este sufre una deformación instantánea que depende de su elasticidad y una deformación continua retrasada debido a su viscosidad en un proceso que se denomina reptación. Al quitar la tensión, la deformación completa se recupera gradualmente (reptación elástica). En algunos sólidos viscoelásticos puede quedar una cierta deformación permanente (flujo elástico). Para mejores aproximaciones a la realidad se utilizan combinaciones de sólidos lineal estándar (Liu et al., 1976). A partir de un medio anelástico lineal, Kjartansson (1979) explicó el aumento de la duración del pulso y la disminución de la amplitud de las ondas con la distancia.

Los procesos de deformación dominantes en la Tierra, dependen de la profundidad y la temperatura consideradas. En la corteza, a temperaturas bajas, domina la reptación por presión ya que las rocas pueden plegarse dúctilmente ante un exceso de tensión. En la litósfera inferior, a mayor temperatura, domina la relajación de tensiones por un comportamiento viscoelástico. En el manto, con un gradiente de temperaturas constante y pequeño, la convección y la deriva continental se atribuyen a procesos de reptación.

La reptación puede entenderse mejor, vista a escala de estructura cristalina (Turcotte y Schubert, 2002). Los átomos que forman la red cristalina se mantienen con un espaciamiento que permite el balance de las fuerzas interatómicas de Coulomb. Ese equilibrio corresponde a un nivel de energía mínimo de la red denominado energía de cohesión que deberá superarse si se quiere romper la estructura cristalina. Los átomos oscilan alrededor de sus posiciones de equilibrio y la energía cinética y potencial de esa oscilación determina la temperatura del sólido. Los parámetros elásticos controlan las fuerzas restauradoras de esa oscilación y la consecuencia de que esas oscilaciones no sean armónicas es la expansión térmica.

La reptación puede darse a bajas tensiones y altas temperaturas, por la difusión de un defecto cristalino dentro de la red. Si los átomos adquieren suficiente energía cinética como para vencer su energía de cohesión, irán intercambiando posiciones con el defecto dentro de la red y el grano se deformará. A veces los defectos cristalinos no están aislados sino que forman una cadena que se llama dislocación. Esta dislocación aumenta la tensión local interna de la red, por lo que la energía externa necesaria para vencer la cohesión y movilizar la dislocación será menor que en el caso de los defectos aislados. Esta migración de dislocaciones puede ocurrir entonces a temperaturas más bajas que la difusión. Si al migrar, las dislocaciones interfieren entre sí y se bloquean, se requerirá una tensión mayor que la 
anterior para moverlas resultando en un endurecimiento plástico. Si se deja la estructura en reposo, las dislocaciones tienden a redistribuirse en una configuración de menor energía a través de la recuperación. A bajas temperaturas $\left(0^{\circ} \mathrm{C}\right.$ a $350{ }^{\circ} \mathrm{C}$ aproximadamente $)$ y grandes esfuerzos diferenciales predomina la migración de dislocaciones, acompañada de endurecimiento por deformación. A temperaturas mayores $\left(350{ }^{\circ} \mathrm{C}\right.$ a $\left.550{ }^{\circ} \mathrm{C}\right)$ predomina la migración de dislocaciones acompañada de recuperación y si las temperaturas son aún mayores $\left(550{ }^{\circ} \mathrm{C}\right.$ a $\left.800{ }^{\circ} \mathrm{C}\right)$, la difusión por el interior del cristal pasa a ser dominante (Martínez Catalán, 2002). Existen además otros tipos de reptación que aquí no se mencionan.

Cuando una onda atraviesa un medio viscoelástico intentando imponerle su energía, este reptará del modo en que sus condiciones reológicas definan y luego se recuperará en un tiempo que depende de la relación entre sus parámetros elásticos y su viscosidad. En ese proceso puede haber absorbido parte de esa energía en una deformación permanente, aumentado su temperatura o incluso cambiado de fase. La onda incidente seguirá su viaje habiendo disminuido su carga energética. Al llegar a la estación, la amplitud de la onda registrada deberá corregirse del efecto de absorción anelástica del medio por el que se propagó. Este efecto se expresa a través de un parámetro que se define como la atenuación intrínseca.

\subsection{LA REGIÓN DE NUEVO CUYO Y LA ATENUACIÓN SÍSMICA}

La subducción de la Placa de Nazca bajo la Placa Sudamericana presenta una geometría segmentada, según se infiere de la distribución de sismos intermedios, en zonas de subducción normal y subhorizontal (Stauder, 1973, Barazangi y Isacks, 1976). La Región de Nuevo Cuyo ha sido calificada como la de más alta peligrosidad sísmica en la República Argentina (Giardini et al., 1999). Cuenta en su historia reciente con sismos destructivos como los que tuvieron epicentro en las ciudades de Mendoza (1861, Ms 7.0), San Juan (1944, Ms 7.4) y Caucete (1977, Ms 7.4). La energía sísmica liberada sobre este segmento de subducción horizontal es en promedio 3 a 5 veces mayor que en las áreas de subducción normal adyacentes (Gutscher y Malavieille, 1999).

Esta región ha sido objeto de numerosos estudios relacionados con su geodinámica y en particular, en el área de sismología, con análisis de sismicidad, mecanismos de fuente, modelos de velocidad, modelado cortical a través de inversión de onda, tomografía de ondas internas y otros (por ejemplo: Barazangi y Isacks, 1976; Triep y de Cardinali, 1984; Smalley y Isacks, 1987; Kadinsky-Cade, 1985; Bollinger y Langer, 1988; Pujol et al., 1991; Smalley et al., 1993; Regnier et al., 1994; Beck et al., 1996; Gutscher et al., 2000; Pardo et al., 2002, 
2004; Fromm et al., 2004; Alvarado et al., 2005, 2009; Gilbert et al., 2006).

El análisis de sismicidad cortical en la región de Nuevo Cuyo realizado por Smalley et al. (1990) determinó profundidades focales centradas en corteza media e inferior. Esto era coincidente con lo hallado por Suárez et al. (1983) para la sismicidad cortical en la zona de subducción plana peruana y difería con las profundidades determinadas por Cahill y Isacks (1992) en zonas de subducción normal. La variación de las profundidades focales fue entonces atribuida a diferencias regionales de las características termodinámicas y reológicas de la litósfera. Por otro lado, la sismicidad intermedia analizada en la región de Nuevo Cuyo es intraplaca y muestra mecanismos de extensión debidos a la flexión de la Placa de Nazca (Stauder, 1973; Triep y de Cardinali, 1984; Anderson et al., 2007).

Giroldi (1990) y Alvarado (1992) realizaron las primeras determinaciones de la atenuación sísmica en la región utilizando codas de registros analógicos, estimando valores promedio regionales del factor de calidad, $Q$, a frecuencias de $1 \mathrm{~Hz}$ que indicaban un efecto de la atenuación consistente con un área de alta actividad tectónica. Deshayes et al. (2008) presentaron resultados preliminares de una tomografía de atenuación de ondas $\mathrm{P}$ y S con datos de estaciones temporales de proyectos internacionales (proyectos OVA99 y CHARSME) y estaciones permanentes chilenas, estimando una atenuación altamente heterogénea por encima de los $20 \mathrm{~km}$ pero que se homogeniza a mayores profundidades.

La ausencia o acumulación de sismos en una región se da por diferentes combinaciones de los parámetros geodinámicos, densidad, rigidez, temperatura, presencia de fluidos, tensiones tectónicas, etc. Mediante el estudio de las variaciones de la energía en ondas directas y dispersadas en los sismogramas pueden determinarse los factores de calidad del medio, $Q$. A través de relaciones establecidas entre los valores de $Q$ de ondas directas y dispersadas puede separarse la proporción anelástica, $Q_{\mathrm{i}}$, y de scattering, $Q_{s}$, de la región. El valor de $Q_{s}$ se relaciona con la densidad de heterogeneidades y el de $Q_{\mathrm{i}}$ con la anelasticidad del medio, esta última vinculada a la temperatura, la presión, el grado de fluidez y el contenido de fluidos. Por esto es que $Q$ es más sensible a los parámetros reológicos que la velocidad de las ondas. 


\section{Atenuación de Ondas Sísmicas: Modelos y Métodos de determinación}

Se presentan las herramientas físico-matemáticas básicas del tratamiento del scattering y los modelos mecánicos de la absorción anelástica que se reúnen en el concepto de la atenuación sísmica Las ondas coda con sus características particulares han sido el primer objeto en los estudios de atenuación sísmica. Un resumen casi cronológico de la evolución de los modelos permite seguir el hilo de las ideas que se fueron imponiendo en la sismología observacional desde los años '60 y de cómo las expresiones matemáticas que modelan la forma de las ondas que se propagan se vieron modificadas para aproximarse a los valores observados. Finalmente se describe una selección de métodos de determinación de la atenuación de ondas coda y de ondas directas a través de sus principios, ecuaciones, criterios generales de aplicación y algunos resultados de aplicaciones a los dos tipos de ondas. Se presentan dos métodos de separación de la atenuación intrínseca y de scattering a partir de la determinación de la atenuación total y de ondas coda.

\subsection{HERRAMIENTAS PARA EL MODELADO}

La atenuación reúne los efectos de la absorción anelástica y del scattering y expresa el decaimiento de la amplitud de las ondas cuando se propagan. Un sismograma local puede considerarse en un principio, compuesto de ondas directas que han sufrido absorción y ondas secundarias que además de absorción han sufrido desviaciones de sus trayectorias con la consecuente redistribución de energía. En el medio real, el proceso de la atenuación comienza entonces con la anelasticidad, por lo que se hace prioritario darle una expresión matemática que ajuste a los datos. Después de ella pueden considerarse las direcciones de propagación resultantes y la consecuente división de energía. Si bien este modelo es muy rudimentario, puede ser de utilidad en un comienzo. 


\subsubsection{DE LA ATENUACIÓN ANELÁSTICA}

Se ha mencionado al sólido lineal estándar como un análogo mecánico sencillo de la viscoelasticidad (Fig. 1.1). Este cuerpo se representa mediante la asociación de un resorte y un pistón en paralelo (un sólido de Kelvin-Voigt) a los que se agrega otro resorte en serie o

a)

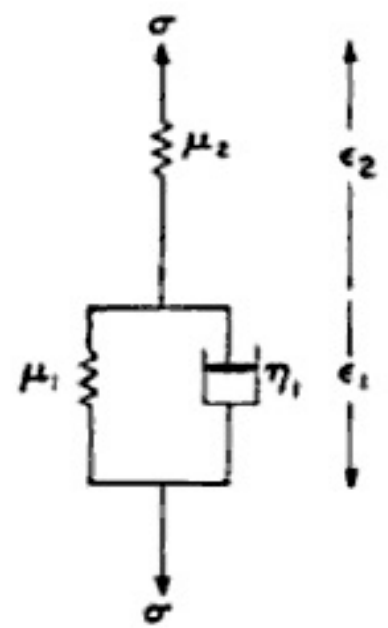

b)

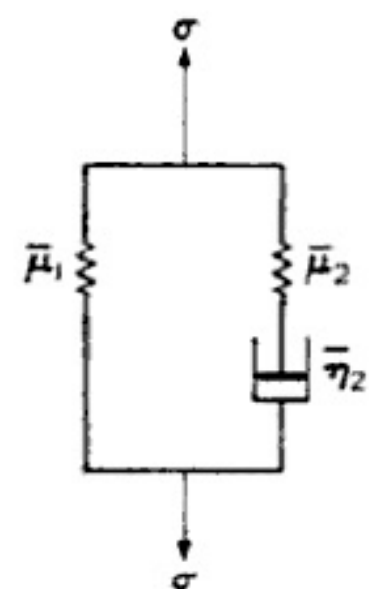

c)
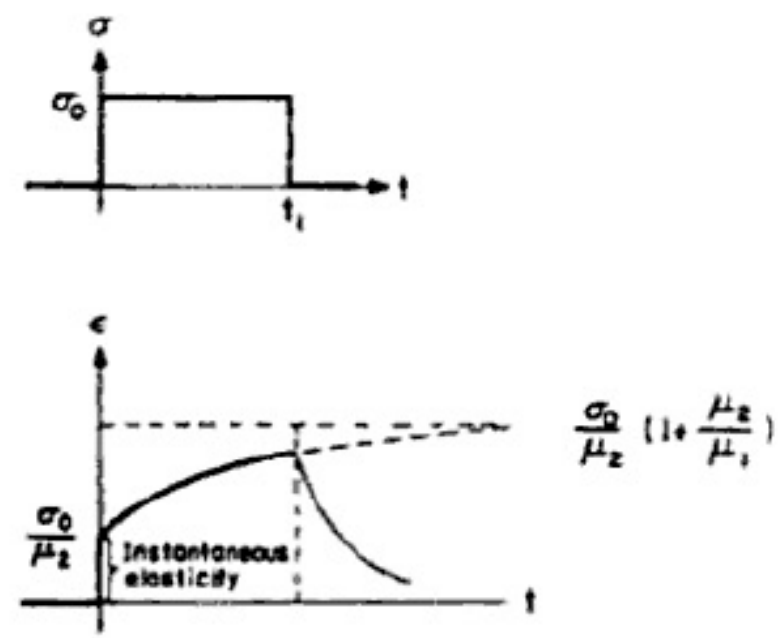

Fig. 2.1.- a) Modelo mecánico del sólido lineal estándar. b) Representación análoga que demuestra la no unicidad de la solución. c) Experimento de reptación y recuperación. Extractada de BenMenahem y Singh (1998.)

paralelo con el primero ya que la solución no es biunívoca. El sólido lineal estándar permite fácilmente entender la mecánica de la reptación, la recuperación y la relajación. Observando el modelo (Fig. 2.1.a), puede intuirse que al aplicar una tensión, esta es soportada tanto por el resorte de constante elástica $\mu_{2}$ como por el sistema resorte-pistón, de constante elástica $\mu_{1} \mathrm{y}$ viscosidad $\eta_{1}$. Si se aplica una deformación, esta se distribuye entre el resorte $\mu_{2}$ y el sistema resorte-pistón $\mu_{1}-\eta_{1}$, en el que ambos deben experimentar la misma deformación. Con estos conceptos se construye la ecuación constitutiva del sólido lineal estándar (Ben Menahem y Singh, 1998). Sea $\sigma$ la tensión aplicada exterior y $\varepsilon$ la deformación que experimenta el 
cuerpo, sus distribuciones en los elementos que componen el cuerpo deben verificar

$$
\begin{aligned}
& \sigma=\mu_{1} \varepsilon_{1}+\eta_{1} \dot{\varepsilon}_{1}=\mu_{2} \varepsilon_{2} \\
& \varepsilon=\varepsilon_{1}+\varepsilon_{2}
\end{aligned}
$$

Eliminando $\varepsilon_{1}$ y $\varepsilon_{2}$ queda la ecuación constitutiva

$$
\sigma+\tau_{\sigma} \dot{\sigma}=M_{R}\left[\varepsilon+\tau_{\varepsilon} \dot{\varepsilon}\right]
$$

donde

$$
\tau_{\sigma}=\frac{\eta_{1}}{\mu_{1}+\mu_{2}}, \quad \tau_{\varepsilon}=\frac{\eta_{1}}{\mu_{1}}>\tau_{\sigma}, \quad M_{R}=\frac{\mu_{1} \mu_{2}}{\mu_{1}+\mu_{2}}
$$

La solución para un escalón de tensión $\sigma=\sigma_{0} H(t)$ donde $\sigma_{0}$ es una constante y $H(t)$ es la función escalón de amplitud unitaria para tiempos mayores o iguales que 0 y de amplitud nula para tiempos menores que 0 .

$$
\begin{aligned}
\varepsilon(t) & =\frac{\sigma_{0}}{M_{R}}\left[1-\left(1-\frac{\tau_{\sigma}}{\tau_{\varepsilon}}\right) e^{-t / \tau_{\varepsilon}}\right] H(t) \\
& =\frac{\sigma_{0}}{\mu_{2}}\left[1+\left(\frac{\tau_{\varepsilon}}{\tau_{\sigma}}-1\right)\left(1-e^{-t / \tau_{\varepsilon}}\right)\right] H(t)
\end{aligned}
$$

Si $\mu_{2} \rightarrow \infty$ el sólido se reduce a un sólido de Kelvin-Voigt. Las expresiones de la Ec. 2.3 permiten ver los valores límites rápidamente $\varepsilon(0)=\frac{\sigma_{0}}{\mu_{2}}, \varepsilon(\infty)=\frac{\sigma_{0}}{M_{R}}$. El cuerpo experimenta una tensión inicial elástica instantánea y luego una reptación hasta alcanzar el valor límite de deformación a un tiempo infinito. Se puede observar que a un tiempo muy grande, la relación tensión/deformación está dada por $M_{R}$.

Si se le aplica ahora una deformación escalón $\varepsilon=\varepsilon_{0} H(t)$, la expresión de la tensión queda

$$
\sigma(t)=M_{R} \varepsilon_{0}\left[1+\left(\frac{\tau_{\varepsilon}}{\tau_{\sigma}}-1\right) e^{-t / \tau_{\sigma}}\right] H(t)
$$

con valores límites $\sigma(0)=\mu_{2} \varepsilon_{0}, \sigma(\infty)=M_{R} \varepsilon_{0}$. El resorte soporta una tensión inicial instantánea y que luego se relaja en el medio viscoelástico hasta alcanzar un valor límite donde la relación tensión/deformación nuevamente es $M_{R}$. De aquí que a $M_{R}$ se lo denomina módulo elástico relajado. 
Si a un sólido lineal estándar se le aplica una tensión o una deformación armónicas, similar al efecto de una componente armónica de una onda elástica, la respuesta del medio es otra onda armónica retrasada y con amplitud modulada por un efecto transitorio de forma exponencial. La forma de la envolvente del transitorio queda definida por los parámetros del medio. La amplitud de la respuesta depende de la frecuencia y amplitud de la onda incidente así como de los parámetros del medio. Para el desarrollo de la solución analítica se utilizan

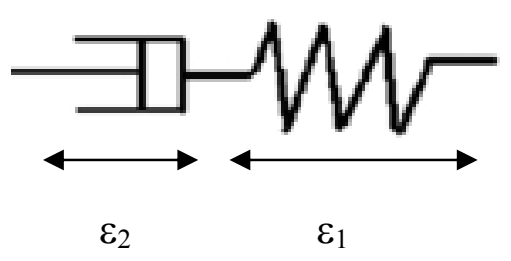

Fig. 2.2.- Sólido de Maxwell

módulos elásticos relajados complejos. También puede entenderse el efecto analizándolo sobre un modelo más sencillo como el de Maxwell. La Fig. 2.2 muestra un líquido viscoelástico de Maxwell, formado por un resorte y un pistón en serie. Este es el principio del oscilador amortiguado.

La deformación del sistema es la suma de las deformaciones del pistón y el resorte, $\varepsilon=\varepsilon_{1}+\varepsilon_{2}$, mientras ambos soportan la misma tensión. La ecuación constitutiva es

$$
\dot{\varepsilon}=\dot{\varepsilon}_{1}+\dot{\varepsilon}_{2}=\frac{d}{d t}\left(\frac{\sigma}{\mu}\right)+\frac{\sigma}{\eta}
$$

Si se le aplica una deformación constante para $t>0$, entonces $\dot{\varepsilon}=0$. La solución de la ecuación homogénea tiene la forma

$$
\sigma(t)=\sigma_{0} e^{\left(-t / \tau_{0}\right)}
$$

donde $\tau_{0}=\eta / \mu$, tiempo de relajación, es el tiempo en que la tensión decae a $e^{-1}$ de su máximo. Ante una tensión $\sigma(t)$, la solución general para la deformación puede integrarse de la Ec. 2.5

$$
\varepsilon(t)=\frac{\sigma(t)}{\mu}+\frac{1}{\eta} \int_{0}^{t} \sigma(\theta) d \theta
$$

En el caso de una tensión armónica aplicada, $\sigma(t)=\sigma_{0} \sin \omega_{0} t$, la deformación en el cuerpo es 


$$
\begin{aligned}
\varepsilon(t) & =\frac{\sigma_{0}}{\mu}\left[\sin \omega_{0} t-\frac{1}{\tau_{0} \omega_{0}} \cos \omega_{0} t+\frac{1}{\tau_{0} \omega_{0}}\right]= \\
& =\frac{\sigma_{0}}{\mu}\left\{\left[1+\frac{1}{\left(\tau_{0} \omega_{0}\right)^{2}}\right]^{1 / 2} \sin \left[\omega_{0} t-\chi_{0}\right]+\frac{1}{\left(\tau_{0} \omega_{0}\right)}\right\}
\end{aligned}
$$

donde se ha definido $\tan \chi_{0}=1 /\left(\tau_{0} \omega_{0}\right)$. Esto significa que el cuerpo oscila con una frecuencia igual a la de la tensión aplicada pero con un retraso de fase igual a $\tan ^{-1}\left(1 /\left(\tau_{0} \omega_{0}\right)\right) \approx 1 /\left(\tau_{0} \omega_{0}\right)$, si $\tau_{0} \omega_{0} \gg 1$.

Un sólido de Kelvin-Voigt con el agregado de una masa $m$, representa el modelo mecánico del oscilador armónico amortiguado. Si la constante del resorte es $k$ y el coeficiente de amortiguación del pistón es $\gamma$, la ecuación de movimiento unidimensional queda

$$
m \frac{d^{2} u(t)}{d t^{2}}+\gamma m \frac{d u(t)}{d t}+k u(t)=0
$$

pudiendo obtenerse una expresión del desplazamiento armónico amortiguado en respuesta a un impulso en $t=0$

$$
u(t)=A_{0} e^{-\omega_{0} t /(2 Q)} \cos (\omega t)
$$

donde la frecuencia natural del resorte es $\omega_{0}=(k / m)^{1 / 2}$ (Stein y Wysession, 2003). Se define el factor de calidad del oscilador como $Q=\omega_{0} / \gamma$ y la frecuencia de la oscilación armónica $\omega=\omega_{0}\left(1-1 /\left(4 Q^{2}\right)\right)^{1 / 2}$. El inverso de $Q$ es la función disipativa, $Q^{-1}$. La amplitud del desplazamiento (2.10) decae exponencialmente con el tiempo (Fig. 2.3) y su envolvente está dada por

$$
A(t)=A_{0} e^{-\omega_{0} t /(2 Q)}
$$

Comparando con la Ec 2.6, la envolvente de la oscilación representa la relajación del impulso $A_{0}$. El tiempo de relajación, $\tau_{0}$, se relaciona con el factor de calidad del oscilador armónico mediante la relación $Q=\omega_{0} \tau_{0} / 2$ y con el coeficiente de amortiguación, según $\gamma=2 / \tau_{0}$. Si $Q$ es constante, la forma del decaimiento depende sólo de los parámetros del medio.

El período del oscilador es mayor que su período natural y la amplitud decae exponencialmente, como puede verse en la Fig. 2.3. El cálculo del decrecimiento logarítmico 


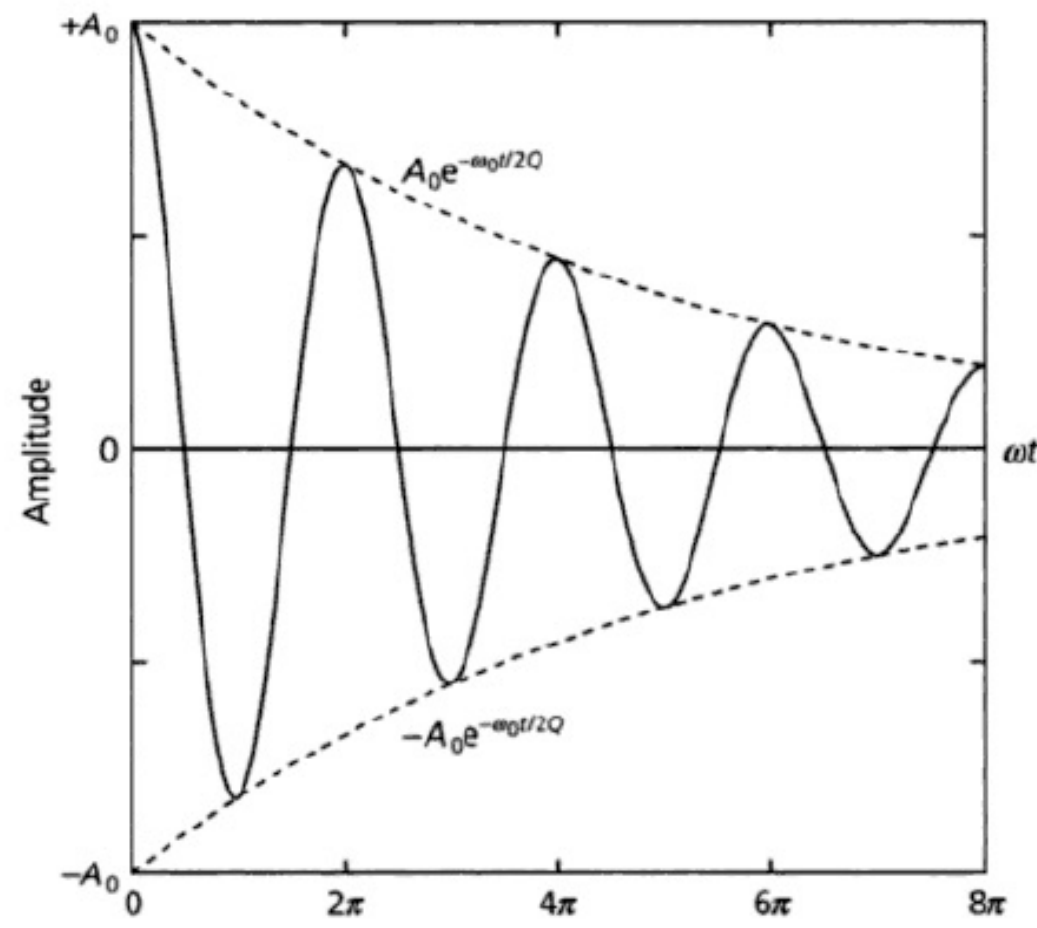

Fig. 2.3.- Respuesta impulsiva del oscilador armónico amortiguado. (Stein y Wysession, 2003).

de la oscilación amortiguada permite hallar una relación entre la amplitud de la oscilación y el factor de calidad. La relación de amplitudes entre dos máximos sucesivos cualesquiera de la oscilación da

$$
\ln \left(A(t) / A\left(t+2 \pi / \omega_{0}\right)\right)=\pi / Q
$$

y dado que la energía se relaciona con la amplitud por $E \propto A^{2}$, entonces la pérdida relativa de energía en un ciclo será

$$
\frac{|\Delta E|}{2 \pi E}=\frac{1}{Q}
$$

Si la perturbación es una onda que incide en un oscilador, se puede estimar el decaimiento temporal de su oscilación en función de sus parámetros viscoelásticos. Si una onda se propaga una distancia dada por $v t$, valdrá el mismo análisis, pudiendo estimarse su disminución de amplitud con la distancia a través de la expresión

$$
A(r)=A_{0} e^{-\omega_{0} r /(2 v Q)}
$$

donde $r$ es la distancia hipocentral y $v$ es la velocidad de propagación de la onda. A los valores $\omega_{0} / 2 Q$ y $\omega_{0} / 2 v Q$ se los suele llamar coeficientes de atenuación temporal y espacial de la amplitud, respectivamente y se los denomina $\gamma(\omega)$. Para la densidad de energía el coeficiente de atenuación intrínseca queda expresado como $\eta_{i}=2 \pi f v^{-1} Q_{i}^{-1}$.

Los primeros modelos de la atenuación sísmica trataban de explicar la independencia 
aparente de $Q$ con la frecuencia para largos períodos, fundamentalmente en el caso de ondas internas $\left(Q_{\alpha}\right.$ para ondas $\mathrm{P}$ y $Q_{\beta}$ para ondas $\left.\mathrm{S}\right)$. Muchos de los modelos de atenuación anelástica propuestos eran mecanismos de relajación con tiempos característicos que dependían de las dimensiones de los granos en la roca. Cada grano tendría su valor máximo de $Q^{-1}$ a una dada frecuencia y en una roca compuesta de granos de diversos tamaños, la atenuación promedio de la misma sería constante en un cierto rango de frecuencia. Sin embargo esta suposición provocaba una aceleración de ciertas componentes de las ondas por lo que debía haber una dependencia de la atenuación con la frecuencia en amplitud y fase (Aki y Richards, 1980).

Siguiendo a Sato y Fehler (1998) y a Aki (1980a) se describen algunos de los mecanismos de atenuación intrínseca propuestos cuyas frecuencias características caen en el rango de las frecuencias de interés de la sismicidad local o regional. Según los autores, cualquier modelo viable ha de ser consistente con la dependencia frecuencial observada de $Q_{\beta}$, teniendo un máximo del orden de 100 a una frecuencia alrededor de los $0.5 \mathrm{~Hz}$. Determinaciones de $Q_{\beta}$ para diferentes tipos de ondas en la tierra han mostrado que puede considerarse independiente de la frecuencia en el intervalo $0.001 \mathrm{~Hz}$ a $1.0 \mathrm{~Hz}$ (Lay y Wallace, 1995).

Numerosos mecanismos de atenuación intrínseca se fundamentan en la presencia de microfracturas y poros en las rocas, rellenos total o parcialmente con fluidos, afectando a las velocidades de propagación de las ondas P y S. Las dimensiones de los mismos han de ser muy inferiores a las longitudes de onda de las fases sísmicas regionales. Las curvas estáticas de tensión-deformación de las rocas tienen histéresis y el área debajo del ciclo de histéresis es la pérdida de energía del campo elástico durante el ciclo tensión-deformación. McCall y Guyer (1994) plantearon un modelo de comportamiento dinámico de las rocas, donde la apertura, cierre y deslizamiento de las fracturas durante la carga elástica era la causa de la atenuación que a su vez era independiente de la frecuencia. Aunque su modelo no explicaba el proceso de histéresis, cuantificaba exitosamente la diferencia entre el régimen estático y dinámico de la roca, las medidas de histéresis y el comportamiento no lineal de las rocas.

Un parámetro dominante en muchos modelos de atenuación planteados, era la relación entre el ancho y el largo de las fracturas. Walsh $(1966,1969)$ propuso dos mecanismos de atenuación intrínseca, el primero basado en el deslizamiento con fricción sobre las paredes secas de fracturas delgadas y el segundo, en la disipación viscosa de energía debida al movimiento de líquido a través de las fracturas. El primero resultaba en una atenuación 
independiente de la frecuencia, mientras que el segundo presentaba un máximo a una frecuencia dependiente de la fracturación, del coeficiente de rigidez de la roca de caja y de la viscosidad del fluido. Aki (1980a) descartó en principio este modelo ya que conducía a valores constantes de $Q$ y sus estimaciones de la dimensión de las fracturas eran inconsistentes con los obtenidos por Hadley (1976).

Nur (1971) propuso que la capa de baja velocidad y alta atenuación en la base de la litósfera se explicaba por la disipación viscosa en zonas de rocas parcialmente fundidas. La presencia de agua reduce la temperatura de fusión de las rocas, sin embargo las temperaturas estimadas en algunas regiones a la profundidad del Moho son inferiores al punto de fusión y además es probable que no existan rocas fundidas en muchas regiones de la litósfera. A partir de la frecuencia correspondiente al máximo de atenuación encontrada, Aki (1980a) analizó varios modelos con fracturas total o parcialmente saturadas y con gas, obteniendo valores de viscosidad extremadamente altos e irreales.

Anderson y Hart (1978) asociaron la atenuación en el manto superior a procesos activados termalmente en los bordes de granos. Como ya se ha visto en el Cap. I, la reptación por difusión domina los procesos anelásticos a las temperaturas correspondientes al manto. La difusión térmica reduce los gradientes de temperatura inducidos por el pasaje de las ondas compresivas removiendo parte de la energía de las ondas. El intercambio de calor entre granos adyacentes juega un papel importante en el valor de $Q_{\alpha}$. Savage (1966) investigó la termo-elasticidad causada por la concentración de tensiones inducida por la presencia de fracturas sin fluidos, hallando una relación $Q_{\alpha}<Q_{\beta}$ para una longitud de fractura del orden de $1 \mathrm{~mm}$ a una frecuencia de $0.5 \mathrm{~Hz}$.

Como aclaran Sato y Fehler (1998), la importancia de los mecanismos arriba enunciados varía con la profundidad, la temperatura, la presencia de fracturas, la geometría de las mismas y la presencia de fluidos. A partir de valores de $Q$ obtenidos para ondas $\mathrm{S}$, Aki (1980a) otorgó mayor credibilidad a la termoelasticidad de Savage (1966), cuya escala de heterogeneidad para granos de roca y fracturas, resultaba en frecuencias dominantes para la atenuación del orden de las observadas.

Solomon (1972a) enunció las tres dependencias principales de la distribución de $Q_{i}$ en la Tierra:

- $Q_{i}$ varía con la profundidad, generalmente el $Q$ en la astenósfera es un orden mayor que el de la litósfera suprayacente. 
- $Q_{i}$ varía lateralmente, por gradientes de temperatura relativamente bajos que suelen asociarse a actividad tectónica.

- $Q_{i}$ varía con la frecuencia, en ese entonces se suponía solo en la astenósfera.

\subsubsection{DEL SCATTERING}

Knopoff y Hudson $(1964,1967)$ mostraron que, en el análisis del scattering en corto período, las conversiones $\mathrm{P} \rightarrow \mathrm{S}$ y $\mathrm{S} \rightarrow \mathrm{P}$ pueden despreciarse. Dada la complejidad de las ondas vectoriales, esta simplificación se sigue usando al estudiar codas de terremotos locales y fluctuaciones de velocidad, aunque deberían ser consideradas en un tratamiento completo (Sato, 1994). Si se supone un medio inhomogéneo, entonces el análisis del scattering debe hacerse a partir de las ecuaciones inhomogéneas de la propagación de ondas (Sato, 1979). En la escala de observación y el intervalo de frecuencias utilizados en este trabajo y según las metodologías empleadas, basta con considerar un medio homogéneo con una distribución uniforme y aleatoria de heterogeneidades y a las ondas primarias y secundarias con idéntica naturaleza.

Mediante el uso de tres parámetros puede caracterizarse el proceso de scattering dominante y definirse el tratamiento matemático conveniente: $L$, la dimensión del medio o distancia recorrida por la onda hasta la heterogeneidad; $\alpha$, la distancia de correlación o distancia entre las heterogeneidades a partir de la cual las ondas secundarias que emerjan de ellas puede suponerse incoherentes y $\kappa$, el número de onda. Aki y Richards (1980) realizan un análisis detallado del patrón de radiación de las ondas secundarias donde se distinguen los efectos que producen las heterogeneidades representadas por cambios en los parámetros elásticos y los efectos de los cambios de velocidad puros. En la Fig. 2.4 se muestra un esquema para el caso $\mathrm{L}>>>\alpha$. Si $1<\kappa \alpha<10$, la escala de la heterogeneidad es similar a la longitud de onda, por lo que el scattering puede ser fuerte.

En el caso de las ondas sísmicas, las heterogeneidades pueden ser variaciones de los parámetros elásticos y la densidad y si dichas fluctuaciones son infinitesimales, se tratará de un scattering débil. Las fluctuaciones en la impedancia sísmica $(\rho v)$ tienden a producir backscattering, mientras que las fluctuaciones de la velocidad sin cambio de la impedancia producen forward-scattering. En el análisis de las ondas coda se supone $\kappa \alpha \approx 1$ (Wu y Aki, 1985b). Hong et al. (2004) indicaron que las variaciones de densidad producen más scattering que las variaciones puras de velocidad, con un consecuente aumento del nivel de la coda, una disminución en su decaimiento y por lo tanto una mayor duración. 
Sea un medio homogéneo con velocidad de propagación $v_{0} \mathrm{y}$ una densidad $\rho_{n}$ de heterogeneidades puntuales que se suponen distribuidas en forma aleatoria e isótropa. Sato y Fehler (1998) explican este fenómeno como un proceso estacionario y definen la densidad de flujo de energía como la cantidad de energía que atraviesa un área unitaria transversal a la dirección de propagación en la unidad de tiempo. El scattering estará caracterizado por la sección transversal diferencial de scattering $d \sigma / d \Omega$ donde $\sigma$ es la sección transversal de

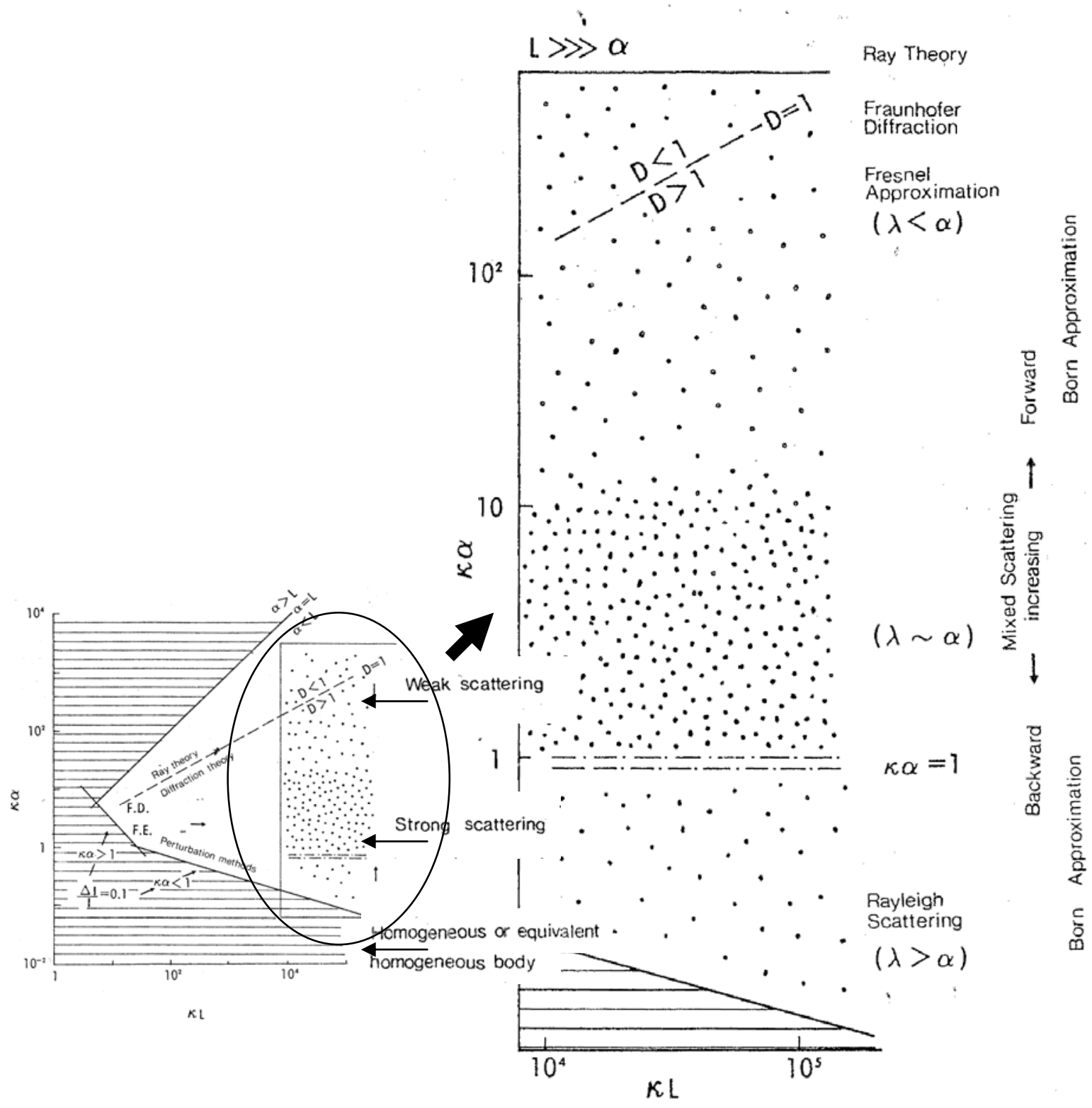

Fig. 2.4.- Diagrama de escalas del scattering. L es la distancia recorrida por las ondas en el medio heterogéneo, $\alpha$ da idea del tamaño de la heterogeneidad y $\kappa$ es el número de onda. Detalle de la región de interés $L>>>\alpha$. (Adaptación de Aki y Richards, 1980 y Herraiz y Espinosa, 1986). 
scattering (equivalente a la sección eficaz de la física de partículas) y $\Omega$ es un ángulo sólido. En este modelo (Fig. 2.5), una onda incidente con densidad de flujo de energía $J^{0}$ interactúa con una heterogeneidad y allí se generan ondas esféricas emergentes con una densidad de flujo de energía $J^{1}$. Entonces la cantidad de energía dispersada por unidad de tiempo en un diferencial de ángulo sólido $(d \Omega)$ dado es $J^{1} r^{2} d \Omega$.

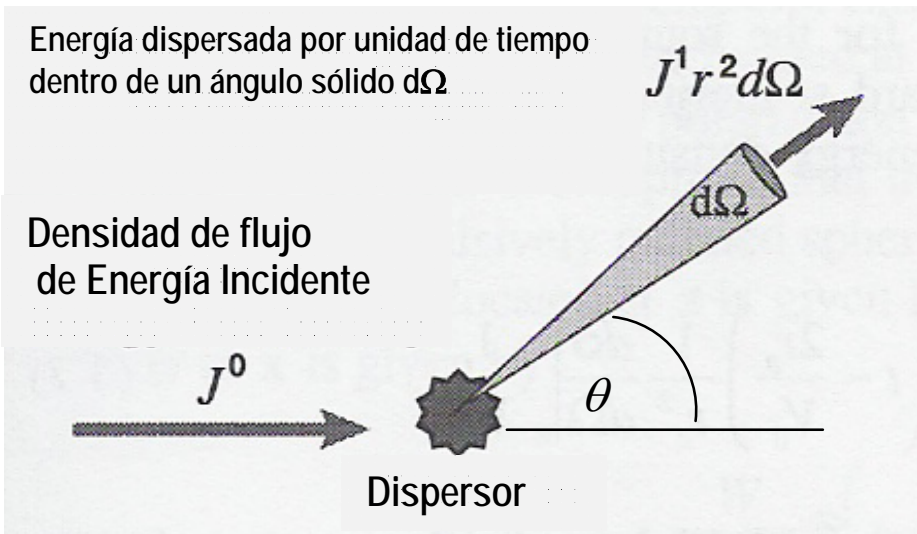

Fig. 2.5.- Concepto de una sección transversal de scattering diferencial para una heterogeneidad simple. (Adaptada de Sato y Fehler, 1998)

Se define entonces la sección transversal diferencial de scattering como

$$
\frac{d \sigma}{d \Omega}=\frac{J^{1} r^{2}}{J^{0}}
$$

El coeficiente de scattering para el caso de un scattering isótropo se define como

$$
g=4 \pi \rho_{n} \frac{d \sigma}{d \Omega}
$$

$g$ tiene dimensiones de inversa de longitud y representa la pérdida de energía relativa por unidad de distancia en un volumen unitario. Si el scattering no es isótropo, $g / 4 \pi$ representa la pérdida fraccional de energía por scattering en un ángulo sólido unitario alrededor de una dirección de radiación $(\theta)$. Si $\theta<\pi / 2$ se dice que es un proceso de forward-scattering y si $\theta \pi$ se habla de backscattering. El coeficiente total de scattering, $g_{0,}$, vendrá dado por el promedio sobre todas las direcciones y es igual a

$$
g_{0}=\frac{1}{4 \pi} \oint g d \Omega=\rho_{n} \sigma_{0}=\ell^{-1}
$$

donde $\sigma_{0}$, la sección transversal total de scattering, representa la integral del diferencial de sección transversal de scattering sobre un ángulo sólido (Sato y Fehler, 1998). El inverso del coeficiente total de scattering, $\ell$, es el camino libre medio. El coeficiente $g_{0}$ también es 
llamado coeficiente de turbidez (Chernov, 1960) y se define como

$$
g_{0}=\frac{\Delta E}{E L}
$$

donde $\Delta E$ representa la pérdida de energía por scattering de la onda primaria con una energía $E$ que atraviesa una capa de espesor $L$. Si $g$ «1 se aplica la aproximación de Born (Lifshitz et al., 1986), la pérdida de energía de la onda primaria será despreciable y el scattering será débil. En el caso contrario, el scattering será fuerte, debiendo considerarse scattering múltiple.

Recordando de la (2.13) la definición del factor de calidad, puede asociarse

$$
g_{0}=\frac{\Delta E}{E L}=\frac{2 \pi}{Q L}=Q_{s}^{-1} k=Q_{s}^{-1} \frac{\omega}{v}
$$

donde $k$ es el número de onda, $\omega$ es la frecuencia angular y $v$ la velocidad de propagación. Como la energía de las ondas decrece con la distancia debido al scattering, $Q_{s}^{-1}$ se define como el factor de disipación por scattering y $g_{0}$ es la atenuación de la energía dispersada.

El camino libre medio controla la transferencia de energía de la onda primaria a la onda secundaria en la trayectoria a través del medio heterogéneo. Del segundo miembro de la (2.19) se deduce que dada una onda plana propagándose en $x$, la presencia de heterogeneidades reduce su densidad de flujo de energía en la forma

$$
e^{-x / \ell}=e^{-g_{0} x}=e^{-k x / Q_{s}}=e^{-\omega x /\left(Q_{s} \nu\right)}
$$

Es decir que la energía de la onda primaria se reduce por scattering a $e^{-1}$ de su máximo en una distancia igual a $\ell$ (se ve aquí la equivalencia entre el camino libre medio en el scattering y el tiempo de relajación en la absorción anelástica). Según sea la relación $\ell / L$ menor o mayor que la unidad, el scattering podrá tratarse como simple o múltiple (Gao et al., 1983). En el caso de las ondas coda, en general se ha utilizado mayormente el scattering simple en la bibliografía. El caso extremo del scattering múltiple es el modelo de difusión, que ha sido aplicado para estudiar sismogramas lunares (Nakamura, 1977 y Dainty y Toksöz, 1981). Trabajos realizados por Sato (1984) y Wu y Aki (1985a) han demostrado que la parte inicial de la coda es más sensible a la asimetría del patrón de radiación y del scattering que la parte final, donde es mayor la influencia del scattering múltiple. En un scattering isótropo y aleatorio, las ondas dispersadas son incoherentes entre sí por lo que se pueden despreciar los retrasos de fase y considerar al scattering como un proceso lineal. Por lo tanto cada onda 


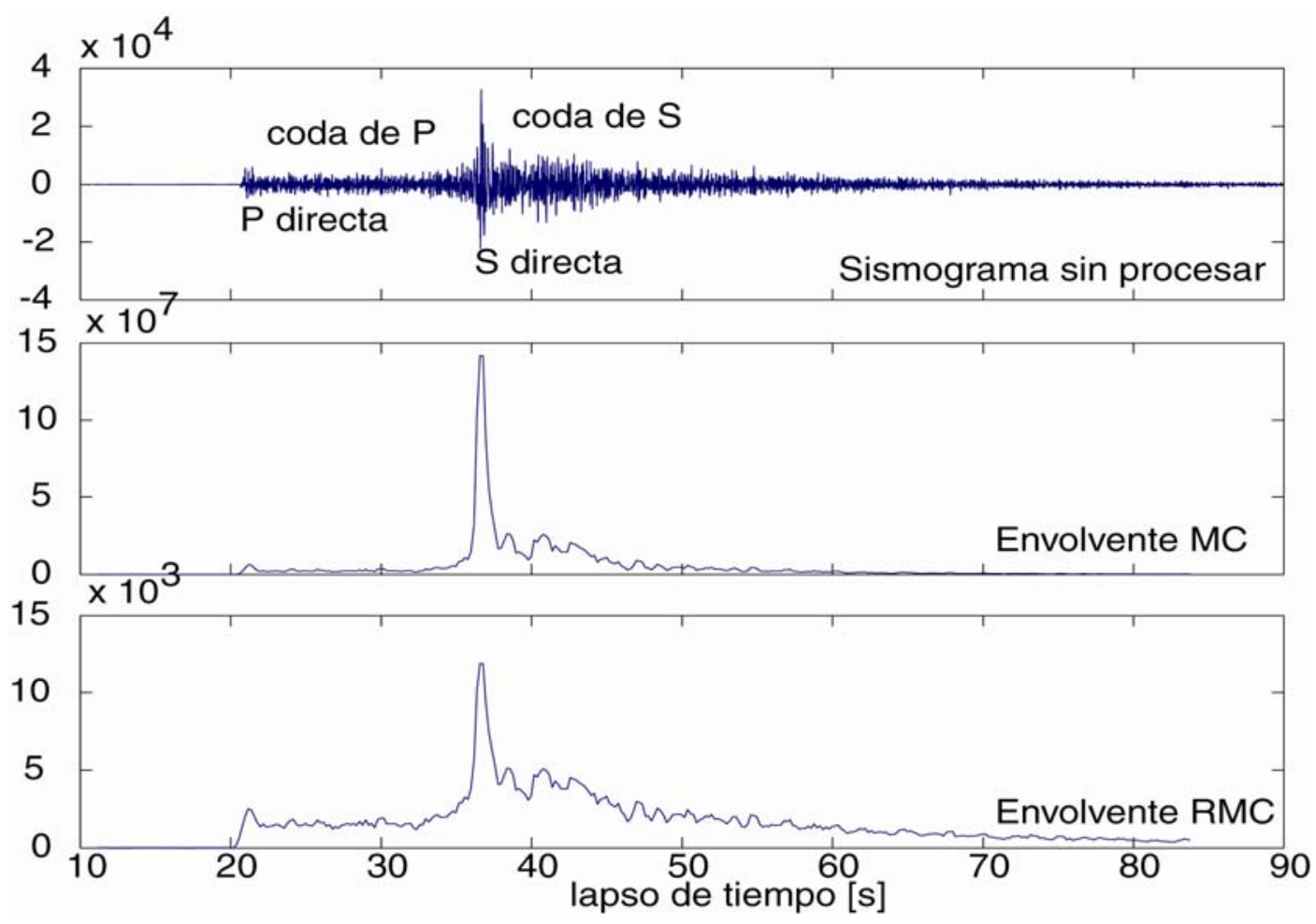

Fig. 2.6.- Ejemplo de sismograma de velocidad registrado en la estación CH11 de la Red Sismológica Zonal Nuevo Cuyo, correspondiente a un evento a $24.4 \mathrm{~km}$ de profundidad focal y $124 \mathrm{~km}$ de distancia epicentral.

primaria disminuirá su densidad de flujo de energía exponencialmente con la distancia al haberla transferido a la onda secundaria en el scattering.

La densidad de energía de las ondas es proporcional al espectro de densidad de potencia de la señal registrada (EDP) (Aki, 1969). La relación entre amplitudes espectrales y temporales se da a través de la amplitud media cuadrática de la señal (MC) que por el teorema de Parseval es la integral del espectro de densidad de potencia (EDP) en el intervalo de frecuencias considerado (Havskov y Alguacil, 2004). La raíz cuadrada de la MC (RMC) estima la envolvente del sismograma en amplitud y forma y el ajuste de la envolvente del sismograma permite estimar el factor de calidad $Q$. Fedotov y Boldyrev (1969) asumieron una dependencia del factor de calidad de ondas internas con la frecuencia de la forma $Q=c f^{n}$ y determinaron los valores de las constantes a partir de las observaciones. Aki y Chouet (1975) observaron que el factor de calidad $Q$, estimado a partir de las envolventes RMC de codas (Fig. 2.6), crecía con la frecuencia y lo interpretaron como un efecto de la variación de la atenuación con la profundidad. Rautian y Khalturin (1978) aproximaron una relación $Q(f) \propto f^{0.5}$. Aki (1980b) observó que la relación con la frecuencia podía variar de acuerdo al predominio del scattering o de la absorción anelástica. Numerosas simulaciones numéricas y físicas de la propagación de ondas en medios inhomogéneos (Menke y Chen, 
1984, Frankel y Clayton, 1986; entre otros) han hallado una fuerte relación entre la distribución de tamaños de las heterogeneidades (Fig. 2.7) y las características de la envolvente del sismograma. Wu y Aki (1985b) propusieron una relación de la forma

$$
Q_{s}=Q_{s o}\left(\frac{f}{f_{0}}\right)^{n}
$$

donde $f_{0}$ es una frecuencia de referencia, generalmente igual a $1 \mathrm{~Hz}$ y $Q_{s 0}=Q_{s}\left(f_{0}\right)$. El valor del exponente $n$ dará la relación con la distribución de dimensiones de las heterogeneidades. Por ejemplo, Wu y Aki (1985b) consideraron una distribución de heterogeneidades unidimensional aproximada por una función fractal (Fig. 2.7), con un espectro de densidad de potencia (EDP) $P(k)=k^{-m_{n}}$, donde $k$ es el número de onda de las heterogeneidades, en ese caso encontraron que la teoría del scattering débil predice $n=m_{n}-1$.

Frankel (1991) observó que el efecto del scattering variaba con la frecuencia dependiendo de la distribución de tamaños de las heterogeneidades. A partir de diferentes modelos de distribuciones tridimensionales y aleatorias de heterogeneidades (Fig. 2.7) se han estimado las envolventes sintéticas correspondientes al scattering comprobándose que una distribución de tipo von Kármán es la que mejor ajusta la dependencia de la envolvente de los
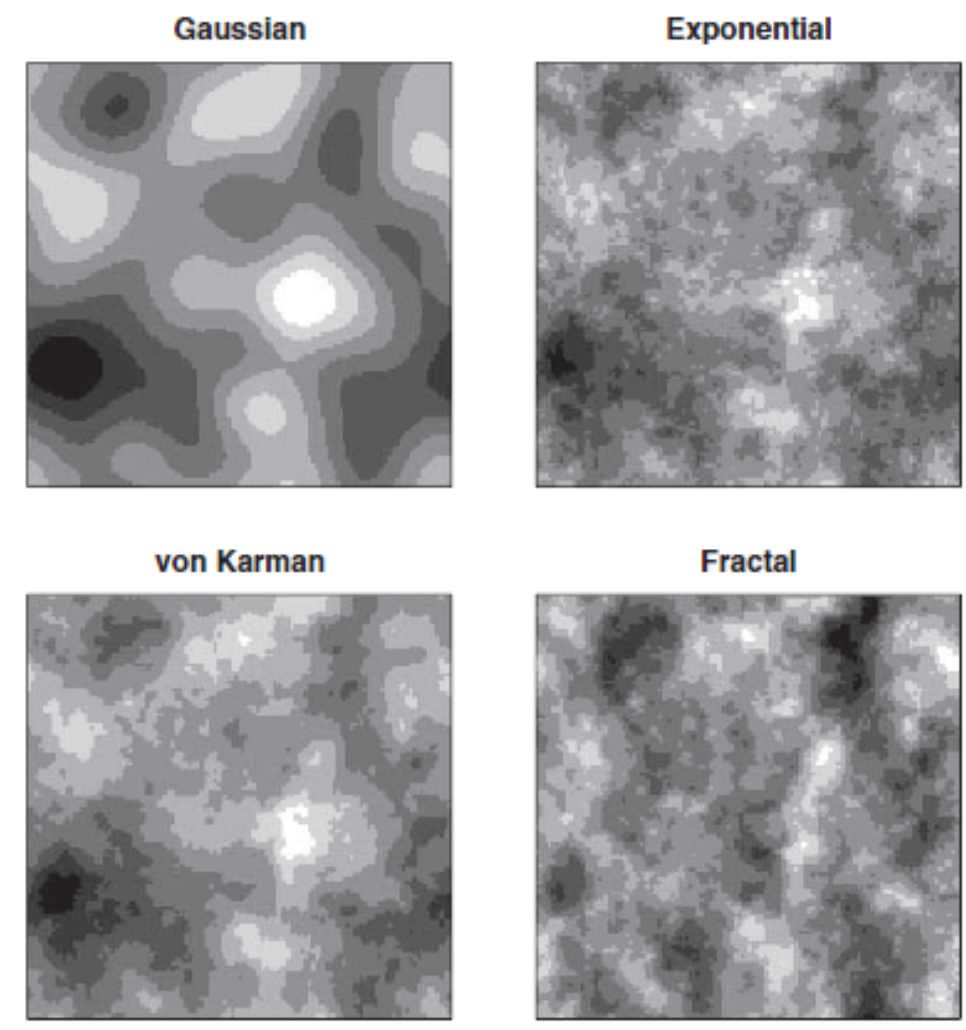

Fig. 2.7.- Medios descriptos por funciones de distribución de fluctuaciones de velocidad tipo Gaussiana, Exponencial, von Kármán y Fractal. Extractada de Mai y Beroza (2006) 
sismogramas con la distancia y la frecuencia en un medio caracterizado por heterogeneidades de escalas múltiples (Fig. 2.8). (Tripathi y Ram, 1997, Saito et al., 2002; Mai y Beroza, 2002, Tripathi, 2002, Fehler y Sato, 2003, Saito et al., 2005).

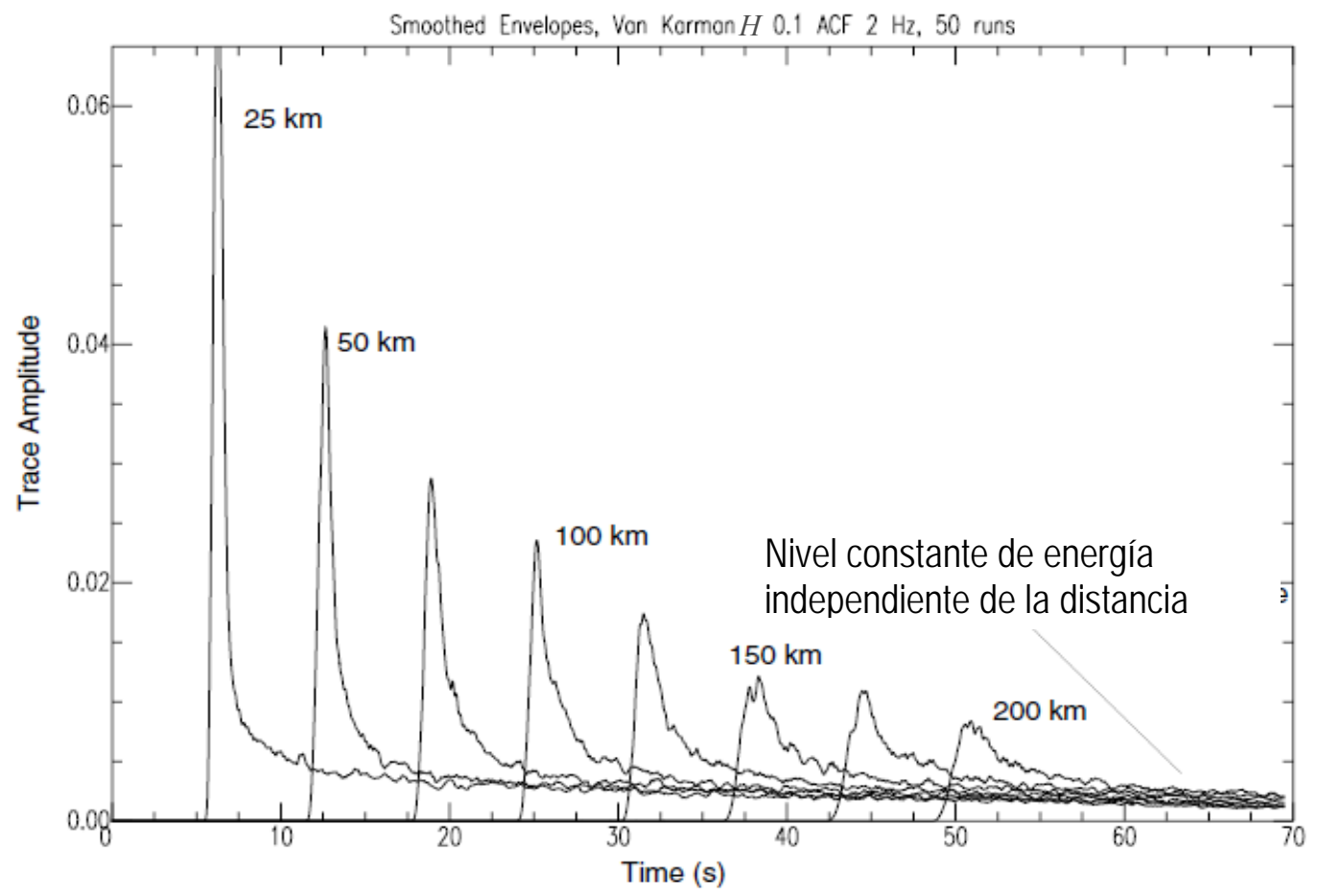

Fig. 2.8.- Envolventes RMC calculadas para un medio aleatorio con distribución de heterogeneidades tipo von Kármán. La frecuencia de la fuente es de $2 \mathrm{~Hz}$ por lo que pueden compararse con envolventes de sismogramas filtrados entre 1 y $4 \mathrm{~Hz}$. El exponente Hurst (H),en este caso igual a 0.1, determina el decaimiento espectral para números de onda grandes en la expresión de la distribución de Von Kármán. Notar la igualdad de amplitudes de coda para lapsos de tiempo grandes independientemente de la distancia a la fuente (indicada en $\mathrm{km}$ ). (Extractada de Fehler y Sato, 2003)

\subsection{ATENUACIÓN DE ONDAS CODA}

\subsubsection{PROPIEDADES DE LAS ONDAS CODA}

La palabra "coda" proviene del latín "cauda", que podría traducirse al español también como cola. Originalmente fue utilizada para referirse al movimiento del suelo después del paso de las ondas superficiales más lentas (Jeffreys, 1929, 1959; Ewing et al., 1957) o inclusive al movimiento posterior a la amplitud máxima de las ondas superficiales. Aki (1969) fue el primero que asoció la palabra coda a la parte final de los sismogramas de terremotos locales (distancia $<\sim 100 \mathrm{~km}$ ), discutió su origen e intentó modelarla. Aki y Chouet (1975) definieron la "coda" como la parte final de un sismograma local, la señal que se registra después de la llegada de las ondas de mayor amplitud (P, S y superficiales). En eventos regionales o telesismos puede hablarse también de la coda de $\mathrm{S}$ o de $\mathrm{P}$, refiriéndose a 
la energía que llega después de los primeros pulsos de esas ondas más energéticos que han recorrido el camino directo entre la fuente y la estación (Gir et al., 1978, Mohan et al., 1979, Lay, 1987). En cualquier caso, la coda tiene una forma característica, su amplitud disminuye en forma suave y uniforme hasta confundirse con el ruido.

Las codas de los terremotos locales tienen ciertas características fundamentales que han sido analizadas en numerosas regiones del mundo. Las observaciones de Bisztricsany (1958) indicaron que la duración de los sismogramas locales es independiente de la naturaleza de la fuente, la distancia epicentral y la geología regional, pero depende fuertemente de la magnitud de los eventos. La amplitud de las ondas directas disminuye en función de la distancia, en cambio, la amplitud de la coda en función de la distancia es casi constante si se mide a un tiempo mayor que dos veces el tiempo de viaje de la onda S (Rautian y Khalturin, 1978). Se llama lapso de tiempo al intervalo de tiempo desde el origen del evento hasta el instante en que se mide la amplitud de la coda. Es decir que la energía de la coda tras el frente de onda inicial de la $\mathrm{S}$, a un lapso de tiempo $t>2 t_{\mathrm{S}}$ es aproximadamente homogénea en el espacio. Esta observación es utilizada para calcular la magnitud de terremotos con escalas basadas en la duración de la coda, las escalas de Magnitud Local.

A través de análisis $f-k$ de datos obtenidos con antenas sísmicas de pequeña apertura en Japón, Aki y Tsujiura (1959) observaron que las ondas coda no mostraban una dirección consistente de llegada a la estación y por lo tanto no se podían interpretar como ondas planas provenientes directamente desde la fuente. Aki (1969) supuso a las ondas coda como ondas superficiales dispersadas por heterogeneidades distribuidas bidimensionalmente en forma uniforme y aleatoria. Utilizó un tratamiento estadístico de la coda suponiendo que la naturaleza de las ondas no cambiaba en la interacción con ellas y que la distancia entre la fuente y el dispersor era mucho más grande que la distancia epicentral. Observó que los espectros de las codas corregidos por expansión geométrica y dispersión mantenían un decaimiento temporal similar independientemente de la localización y del tamaño de la fuente. Aki y Chouet (1975) extendieron la aproximación de Aki (1969) a una distribución tridimensional de heterogeneidades, concluyendo que las ondas coda estaban compuestas por ondas internas dispersadas a frecuencias por encima de los $10 \mathrm{~Hz}$ y por ondas superficiales dispersadas a frecuencias cercanas a $1 \mathrm{~Hz}$. En su trabajo, sentaron las bases del estudio de la atenuación de ondas coda describiendo dos modelos extremos de scattering y diseñando un método que sería empleado hasta hoy en día como la herramienta básica en cualquier análisis de atenuación sísmica. En un trabajo sobre atenuación de ondas $\mathrm{S}$ de corto período, Aki(1980) concluyó que a frecuencias entre 1 y $25 \mathrm{~Hz}$, las codas de sismogramas locales 
estaban compuestas por ondas $\mathrm{S}$ dispersadas y sin cambio de naturaleza.

Scherbaum et al. (1991) analizando enjambres de microterremotos, llegaron a la conclusión de que la coda de $\mathrm{S}$ tenía dos patrones diferentes de comportamiento. El inicio de la coda, después de la onda $\mathrm{S}$ directa, estaba compuesto por ondículas con el mismo vector lentitud que las ondas S directas, mientras que el final de la coda de S, estaba compuesto por ondículas que abandonaban la fuente en una variedad de direcciones. La transición entre los dos comportamientos se daba a lapsos de tiempo iguales a 1.5-2 veces el tiempo de viaje de $\mathrm{S}$. Kuwahara et al. (1997) mediante análisis estadísticos sobre datos de antenas sísmicas, observaron que mientras las ondas directas, $\mathrm{P}$ y S, mostraban direcciones de propagación que provenían de una fuente única, las ondas coda provenían de fuentes múltiples con velocidades aparentes diversas formando un paquete de ondas incoherentes.

Numerosos trabajos han demostrado que las determinaciones del efecto de sitio, el factor de calidad y la magnitud resultan más estables si se hacen usando ondas coda que con ondas S directas (Tsujiura, 1978, Rautian y Khalturin, 1978, Aki, 1980, Phillips y Aki, 1986, Margheriti et al., 1994). Aki et al. (1977) hallaron en el análisis del decaimiento de la coda de pequeños terremotos $(M<6)$ una herramienta útil para la predicción del movimiento fuerte de un gran terremoto en una región sísmica dada. Sato (1988) observó que el valor medio y la dispersión del gradiente de la envolvente de la coda muestran variaciones temporales que pueden utilizarse en la predicción de terremotos al relacionarlas con el aumento inhomogéneo de heterogeneidades dadas por la microfracturación.

Todas estas observaciones se pueden resumir en las siguientes propiedades de las codas en sismogramas de terremotos locales:

- el contenido espectral de la porción final de la coda de $\mathrm{S}$ es similar en todas las estaciones de una región.

- la duración de la coda es una medida confiable de la magnitud.

- la forma del decaimiento temporal de la coda a una dada frecuencia es independiente de la distancia y de la naturaleza del camino sísmico directo entre la fuente y la estación.

- la forma del decaimiento temporal de la coda a una dada frecuencia es independiente de la magnitud al menos para $\mathrm{M}<6$.

- la amplitud de la coda de S depende de la geología de la estación. 
- en análisis de datos de array, las ondas coda no muestran direcciones de propagación que provengan de la fuente.

\subsubsection{EVOLUCIÓN DE LOS MODELOS}

Entre las primeras evidencias observadas del efecto de scattering sobre las ondas, se encuentran los cambios de polaridad en las ondas directas registradas. La polarización lineal ideal en un medio elástico, isótropo y homogéneo se observaba en la realidad elipsóidica y hasta esférica. Aki (1956) observó que los espectros de ondas P y S de terremotos locales eran fuertemente afectados por el camino sísmico pero la parte final de los sismogramas era menos sensible. Más tarde Aki (1969) volvió a prestar atención a las ondas en la parte final del sismograma con la intención de desarrollar un método de determinación de los parámetros de la fuente que tuviera en cuenta la propagación en medios heterogéneos. Las llamó "ondas coda" y supuso que su origen estaba en la suma de las ondas superficiales que eran desviadas de sus trayectorias hacia atrás por las heterogeneidades distribuidas aleatoria $\mathrm{y}$ bidimensionalmente en el medio entre la fuente y el receptor. Esas heterogeneidades podían ser la topografía irregular, las estructuras geológicas complejas, las propiedades elásticas heterogéneas de las rocas y la presencia de pequeñas fracturas y fallas. Para poder corregir el efecto de la propagación, Aki (1969) modeló el espectro de potencia de las ondas utilizando un tratamiento estadístico del backscattering. A partir de sus observaciones, Aki y Chouet (1975) desarrollaron el modelo de Back-Scattering Simple, donde se considera una distribución tridimensional de heterogeneidades y se desprecian las pérdidas de energía del scattering. Sato (1977) lo adaptó para el caso en que la distancia fuente-receptor no era despreciable y el scattering fuera isótropo, resultando el modelo de Scattering Isótropo Simple.

Aki y Chouet (1975) también consideraron un modelo opuesto al del Back-Scattering Simple, donde la energía sísmica se transfería por un proceso de difusión, llegando a la conclusión de que ambos modelos propuestos eran extremadamente simplistas y no permitían distinguir entre el efecto de scattering y el efecto de la absorción. Por otra parte Kopnichev (1977) mostró que el scattering por difusión no era válido en la Tierra. Hudson y Heritage (1981) comprobaron que la aproximación de Born era válida en medios con scattering débil. De este modo, el método de Back-Scattering Simple permitía una descripción cuantitativa de la pérdida de energía en las ondas coda que, dada la sencillez de su implementación, lo transformaría en el modelo más ampliamente usado. 
En un análisis sobre el scattering de codas de $\mathrm{P}$ telesísmicas y codas de $\mathrm{S}$ locales, tomados como extremos de la atenuación, Aki (1982) observó discrepancias que lo llevaron a remarcar la necesidad del tratamiento del scattering múltiple de ondas vectoriales en un medio estratificado con propiedades estadísticas anisótropas. Gao et al. (1983a, b) retomaron la teoría del scattering simple para transformarlo en un scattering múltiple e isótropo, extendiendo las expresiones hasta un orden m. En sus desarrollos concluyeron que el efecto del scattering múltiple era predominante cuando los tiempos medidos en las codas eran largos y que la energía del scattering de órdenes mayores que 7 era despreciable. Wu (1985) adaptó la teoría de la transferencia radiativa a las ondas sísmicas para intentar separar los efectos del scattering y la absorción anelástica, consideró la posibilidad del scattering fuerte e isótropo. Al aplicar el modelo de $\mathrm{Wu}$ (1985) a datos reales, Mayeda et al. (1991) observaron que el ajuste no era del todo perfecto y que haría falta considerar el scattering inhomogéneo y anisótropo.

Frankel y Wennerberg (1987) propusieron el modelo de flujo de energía que permitía separar los efectos de la absorción y del scattering a la vez que ajustaba mejor el decaimiento de las amplitudes de los sismogramas. Este era un modelo fenomenológico basado en la homogeneidad observada de la energía en la coda y la hipótesis de la conservación de la energía.

Sato (1989) observó que la duración de la envolvente de las ondas S registradas aumentaba con la distancia epicentral, concluyendo que era efecto de lentas variaciones de la estructura de velocidad y no de la fuente ni de la estación. Mediante análisis estadísticos del patrón de radiación en sismogramas locales, Sato (1991a) comprobó que la duración de la S en las componentes horizontales era mayor que la duración de la fuente y que la amplitud máxima de la fase $\mathrm{S}$ en la componente vertical estaba retrasada respecto de las componentes horizontales. Del Pezzo et al. (1990) discutieron el origen de las variaciones observadas en el decaimiento de las ondas coda en función del lapso de tiempo, concluyendo que más que el modelo empleado podía ser efecto de variaciones de la atenuación con la profundidad.

Ante la confusión reinante en la literatura acerca de la dependencia o independencia con la frecuencia de los efectos de la atenuación anelástica y de scattering sobre un pulso, Wennerberg y Frankel (1989) analizaron en paralelo ambos procesos concluyendo que la variación del $Q$ anelástico con la frecuencia depende de la distribución de los tiempos de relajación del mismo modo que la variación del $Q$ de scattering con la frecuencia se relaciona con la distribución de tamaños de las heterogeneidades. 
Los siguientes esfuerzos fueron puestos en tratar de identificar el significado del decaimiento de la energía en función de los procesos físicos asociados a ella. Con ese fin, Hoshiba et al. (1991) desarrollaron un método numérico sobre la base de la teoría de la transferencia radiativa de $\mathrm{Wu}$ (1985). Zeng et al. (1991) lograron una expresión teórica de la densidad de energía que reunía los modelos del scattering simple y múltiple con el de la transferencia radiativa. Una expresión compacta de la misma (Zeng, 1991) sería de gran utilidad al método de separación de los efectos de absorción intrínseca y de scattering que desarrollaría Wennerberg (1993).

\subsubsection{SCATTERING SIMPLE}

En primer lugar se presenta el modelo de Back-Scattering Simple de Aki y Chouet (1975) para el caso de un medio homogéneo con una densidad baja de heterogeneidades y donde predomina el scattering hacia atrás y puede aplicarse la aproximación de Born (Fig. 2.4). Seguidamente se presenta el modelo de Sato (1977) para el caso del scattering isótropo simple.

\subsubsection{MODELO DE BACK-SCATTERING SIMPLE (SBS)}

Las características observadas de las ondas coda en terremotos locales llevaron a Aki (1969) a suponer que la coda estaba formada por ondas secundarias que provenían del backscattering generado en numerosas heterogeneidades distribuidas bidimensionalmente. Supuso que podía tratarse como un proceso de scattering simple y que las ondas secundarias eran incoherentes entre sí, permitiendo un análisis estadístico.

Aki y Chouet (1975) retomaron el modelo planteado por Aki (1969) pero considerando que las heterogeneidades estaban distribuidas tridimensionalmente. De los dos modelos opuestos que plantearon para la generación de las ondas coda, uno sería el de Back-Scattering Simple (SBS).

Las hipótesis fundamentales del modelo de

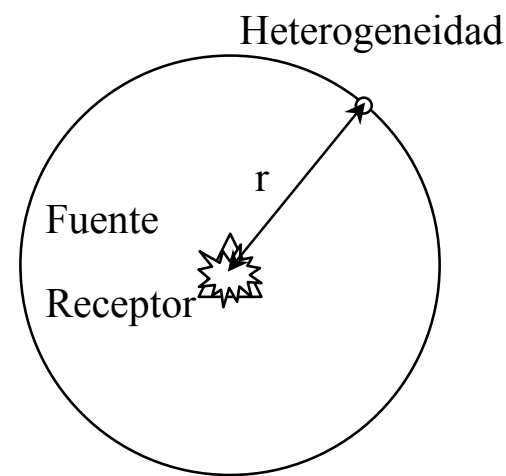

Fig. 2.9. Geometría del modelo de SBS

SBS son las siguientes: 
- las heterogeneidades se suponen distribuidas aleatoria y uniformemente en el espacio;

- el medio que contiene a las heterogeneidades es homogéneo;

- las ondas primarias y secundarias son de la misma naturaleza;

- las distancias dispersor-epicentro y dispersor-estación han de ser aproximadamente iguales y mucho más grandes que la distancia epicentral. Por simplicidad se supone a la fuente y a la estación en un mismo punto (Fig. 2.9);

- cada onda que arriba a la estación es debida a una única heterogeneidad presente en el medio, $\lambda<<\ell$ (donde $\lambda$ es la longitud de onda y $\ell$ es el camino libre medio) por lo que el scattering es un proceso débil pudiendo usarse la aproximación de Born despreciándose las pérdidas de energía de la onda primaria y el scattering múltiple. Esta sobresimplificación viola la ley de conservación de la energía.

En el desarrollo del SBS no se considera la polarización de las ondas ni la partición de la energía en tres componentes (Sato y Fehler, 1998). La ecuación de la densidad de energía de ondas coda en función de la frecuencia para cada lapso de tiempo $t,(\omega \mid t)$, es

$$
E(\omega \mid t)=S(\omega) C(\omega \mid t)
$$

donde $S(\omega)$ refleja la función fuente que no depende del lapso de tiempo registrado y $C(\omega \mid t)$ representa el efecto de un área extensa que incluye a la fuente y al receptor y varía con el lapso de tiempo más que con la distancia o la naturaleza del camino sísmico directo.

Sea una fuente puntual ubicada en el origen de coordenadas. Si $W(\omega)$ es la energía total irradiada en una banda de frecuencia unitaria alrededor de $\omega$, entonces la densidad de flujo de energía a una distancia $r$ para tiempos $t>r / v$, con $v$ la velocidad de propagación de la onda, puede expresarse como

$$
J^{0}(r, t \mid \omega)=\frac{W(\omega)}{4 \pi r^{2}} \delta\left(t-\frac{r}{v}\right)
$$

donde $r^{2}$ representa el efecto de la expansión geométrica de las ondas esféricas y la función $\delta$, delta de Dirac, provee el retraso de tiempo de la energía en la estación debido a la propagación.

Si en el punto denotado por $r$ existe una única heterogeneidad, la energía que retorna 
hacia la fuente $(r=0)$ debido al scattering según (2.15) es

$$
J^{1}(0, t \mid \omega)=\left.\frac{W(\omega)}{4 \pi r^{2}} \delta\left(t-\frac{2 r}{v}\right) \frac{1}{r^{2}} \frac{d \sigma}{d \Omega}\right|_{\pi}
$$

donde se ha tenido en cuenta que el scattering es hacia atrás, evaluando la sección transversal diferencial de scattering (2.15) para una dirección $\theta=\pi$. Planteando la coincidencia de la fuente y el receptor en el espacio se verifica que la llegada de la onda secundaria es a un lapso de tiempo $t>2 r / v$.

Se supone que tanto la onda primaria, que parte de la fuente, como la onda secundaria, generada en la heterogeneidad, son de la misma naturaleza y su velocidad es $v$. Las ondas que arriben a la estación en un intervalo de tiempo $[t, t+\Delta t]$ medido desde el tiempo origen, donde $\Delta t$ es más largo que la duración de cada pulso de onda secundaria, provienen de las heterogeneidades en la zona encerrada por dos elipses (en el caso de ondas superficiales y elipsoides en el caso de ondas internas) con focos en la estación y el epicentro y ejes mayores de longitudes $v t$ y $v(t+\Delta t)$, respectivamente. Dividiendo la energía por su velocidad de propagación, $v$, se obtiene la densidad de energía. Sumando las energías provenientes de todas las heterogeneidades presentes en el medio queda la expresión

$$
E^{1}(0, t \mid \omega)=\left.\sum_{\text {Heterogeneidades }} \frac{W(\omega)}{4 \pi r^{2}} \delta\left(t-\frac{2 r}{v}\right) \frac{1}{r^{2}} \frac{d \sigma}{d \Omega}\right|_{\pi} \frac{1}{v}
$$

en la que el supraíndice 1 indica que cada onda ha sido afectada por una única heterogeneidad. Al considerar todas las heterogeneidades distribuidas en el espacio se reemplaza la sumatoria por una integral cuya solución es

$$
E^{1}(0, t \mid \omega)=\frac{W(\omega) g_{\pi}}{2 \pi v^{2} t^{2}} H(t)
$$

donde $\mathrm{H}(\mathrm{t})$ es la función escalón y $g_{\pi}=\left.4 \pi \rho_{n}(d \sigma / d \Omega)\right|_{\pi}$ es el coeficiente de backscattering. En la expresión (2.26) puede verse que la densidad de energía de una onda afectada de scattering simple, decrece con $t^{-2}$, o lo que es lo mismo, la RMC decrece con $t^{-1}$.

Teniendo en cuenta que cada onda pierde energía en la propagación debido a la anelasticidad del medio (2.14) y que esta pérdida depende del intervalo de tiempo medido y de la frecuencia angular, $\omega$, de la forma $\exp \left(-\omega t / Q_{c}\right)$ con $Q_{\mathrm{c}}$ el factor de calidad de las ondas coda, queda la siguiente expresión para la densidad de energía de ondas internas que han sufrido SBS 


$$
E^{1}(0, t \mid \omega)=\frac{W(\omega) g_{\pi}}{2 \pi v^{2} t^{2}} H(t) e^{-\frac{\omega t}{Q_{c}}}
$$

Por lo tanto la densidad de energía registrada para ondas esféricas, es proporcional a $t^{-2}$. El análisis para ondas superficiales es similar, salvo por la geometría bidimensional de la propagación. En ese caso se llega a que la densidad de energía es proporcional a $t^{-1}$.

Una expresión que representa la densidad de energía de las ondas afectadas por SBS tanto en el caso de ondas internas como superficiales es

$$
E^{1}(t \mid \omega)=E_{0}(\omega) t^{-m} e^{-\frac{\omega t}{Q_{c}}}
$$

donde $m$ es el factor de expansión geométrica de la energía $(m=1,2)$ que depende de la naturaleza de las ondas y $E_{0}(\omega)$ es la densidad de energía en la fuente de ondas coda que representa tanto el efecto de la fuente primaria como la secundaria. La fuente secundaria está representada por las heterogeneidades del medio y será común a todos los terremotos de una región siempre que las ondas primarias sean las mismas. Se espera por lo tanto, que el factor fuente dependa únicamente de la fuente primaria. Es decir que una vez que se corrige el efecto de la expansión geométrica y se filtra el sismograma en un ancho de banda angosto alrededor de una dada frecuencia, el gradiente de la envolvente de la coda depende únicamente de la atenuación a través del factor $Q_{\mathrm{c}}{ }^{-1}$ (Fig. 2.10).

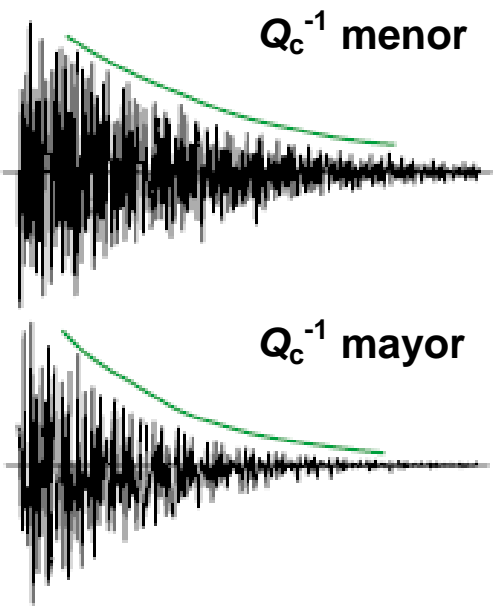

Fig. 2.10.- Ejemplos de sismogramas de regiones con atenuación baja (arriba) y alta (abajo) relativamente. Se observa la pendiente más pronunciada en el caso de mayor atenuación.

Los resultados de Aki y Chouet (1975) mostraron que a frecuencias cercanas a 1Hz, las ondas dispersadas eran superficiales, mientras que a frecuencias mayores resultaban ser ondas internas. Originalmente, Aki y Chouet (1975) asociaron el factor de calidad $Q_{\mathrm{c}}$ únicamente a la anelasticidad del medio. El modelo SBS, considera inicialmente la acción sobre las ondas de una única heterogeneidad, pero luego atribuye también al scattering parte de la pérdida de energía en el trayecto entre la heterogeneidad y el receptor (Frankel and Wennerberg, 1987). En un desarrollo de la expresión del backscattering simple, Dainty (1984) evidenció que el scattering es una parte importante del efecto de atenuación para altas frecuencias. Aki (1980a) realizó una interpretación del origen del $Q$ de ondas $\mathrm{S}$ directas, $\left(Q_{\mathrm{d}}\right)$, llegando a la 
conclusión de que la causa más probable de la atenuación era el scattering de las ondas en el camino fuente-receptor. Asumiendo una relación $Q_{d}=\omega / g v$ de acuerdo a la expresión (2.19) y remplazando el coeficiente de turbidez en la (2.27) queda

$$
E^{1}(0, t \mid \omega) \propto \frac{\omega}{Q_{d} v} t^{-m} e^{-\frac{\omega t}{Q_{c}}}
$$

donde puede asociarse el origen de $Q_{\mathrm{d}}$ al proceso de scattering. Sin embargo, continuaba sin aclararse por ese entonces la parte de atenuación que representaba el $Q_{c}$.

\subsubsection{MODELO DE SCATTERING SIMPLE E ISÓTROPO (SIS)}

Sato (1977a) amplía el modelo de Aki y Chouet (1975) al caso donde la energía elástica se propaga en frentes esféricos desde una fuente puntual a través de un medio elástico tridimensional e infinito donde hay numerosas heterogeneidades distribuidas homogénea y aleatoriamente. Además, restringe su tratamiento a ondas internas asumiendo un scattering simple e isótropo en un medio originalmente elástico, $\left(g=g_{0}\right)$.

En la aproximación del scattering simple e isótropo, para una fuente y observador separados una distancia $r$, el camino libre medio, $\ell$, es mucho mayor que $r$. Por lo tanto $t<<\ell / v$ puesto que se supone al scattering como un proceso débil donde la onda es afectada por una única heterogeneidad situada a una distancia $r_{i}$ de la fuente y a una distancia $r_{i}{ }^{\prime}$ del observador (Fig. 2.11). El desarrollo se basa en considerar sólo la estimación lineal de la sección transversal del scattering al plantear las fórmulas de la densidad media de energía directa y dispersada. Para una onda plana que se propaga, la densidad media de energía es dos veces la densidad media de energía cinética. Si la fuente se ubica en el origen de coordenadas y se considera un área pequeña

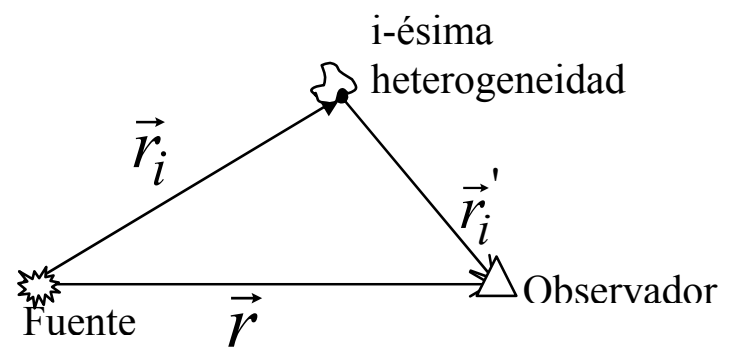

Fig. 2.11. Geometría del modelo de SIS del frente de ondas a una distancia $\vec{r}=r \cdot \hat{r}$ con $r>>\lambda$, entonces, la densidad media de energía $E$ se relaciona con la densidad media del flujo de energía $J$ según

$$
\vec{J}(\vec{r}, t \mid \omega)=E(\vec{r}, t \mid \omega) \cdot v \cdot \hat{r}
$$

La energía generada en la fuente en un intervalo de tiempo $u$ mucho menor que $t$ es $W_{u}(t \mid \omega)$ y tiene simetría esférica. La densidad media del flujo de energía directa a una 
distancia $\vec{r}=\vec{r}_{i}+\vec{r}_{i}^{\prime}$ será

$$
\vec{J}_{d}(\vec{r}, t \mid \omega)=\frac{e^{-n \sigma_{0} r}}{4 \pi r^{2}} W_{u}\left(t-\frac{r}{v} \mid \omega\right) \hat{r} \approx \frac{\left(1-n \sigma_{0} r\right)}{4 \pi r^{2}} W_{u}\left(t-\frac{r}{v} \mid \omega\right) \hat{r}
$$

donde el subíndice $d$ denota a la onda directa y se considera la pérdida de energía debida al scattering y al efecto de la expansión geométrica. Se observa que la energía de la onda directa decrece monótonamente a medida que se transfiere a las ondas dispersadas.

Considerando el caso de una única heterogeneidad, la densidad media de energía $E$ que llega al observador puede expresarse como la suma de la energía directa, $E_{d}$, y la densidad media de energía dispersada por dicha heterogeneidad, $E_{s}$. De acuerdo a las posiciones de la heterogeneidad y el observador (Fig. 2.11) el flujo de densidad de energía visto por el observador proveniente de la heterogeneidad vendrá dado por

$$
\vec{J}_{s}^{i}(\vec{r}, t \mid \omega)=\frac{\left(1-n \sigma_{0} r_{i}^{\prime}\right)}{4 \pi r_{i}^{\prime 2}} \sigma_{0}\left|\vec{J}_{d}\left(\vec{r}_{i}, t-\frac{r_{i}^{\prime}}{v} \mid \omega\right)\right| \hat{r}_{i}^{\prime}
$$

La densidad de energía dispersada por cada heterogeneidad se despeja a partir de (2.30) y (2.32). Para obtener la densidad de energía dispersada total que llega al observador se multiplica la densidad de energía dispersada por cada heterogeneidad por la densidad de heterogeneidades, $n$, y se integra sobre elipsoide con focos en la fuente y el receptor que verifica $r_{i}+r_{i}^{\prime}=r v$. La variable $v=v t / r=$ cte define una cáscara isócrona de scattering para un lapso de tiempo dado. Teniendo en cuenta la duración de la fuente, $u$, el análisis debe hacerse en un intervalo de tiempo $\Delta T>u$ tal que además $v \Delta T$ sea mayor que el tamaño de las heterogeneidades. De este modo, se obtienen las expresiones

$$
\begin{aligned}
E_{d}(\vec{r}, t \mid \omega) & =\frac{W\left(1-g_{0} r\right)}{4 \pi r^{2}} \frac{1}{v u} S_{u}\left(t-\frac{r}{v}\right) \\
E_{s}^{1}(\vec{r}, t \mid \omega) & =\frac{W g_{0}}{4 \pi r^{2}} K\left(\frac{v t}{r}\right) H\left(\frac{v t}{r}-1\right)
\end{aligned}
$$

que representan las energías directa y dispersada respectivamente, donde $S_{u}(t-r / v)$ es una función cajón de ancho $u$ desplazada, el supraíndice 1 indica que la energía ha sufrido un único scattering, $H(v t / r-1)$ es la función escalón desplazada y $K(v)$ es un factor de corrección por expansión geométrica de la forma 


$$
K(v)=\frac{1}{v} \ln \frac{v+1}{v-1} \text { para } v>1
$$

En la Fig. 2.12 puede verse que $K(v) \approx 2 / v^{2}$ cuando $v » 1$, por lo que (2.34) coincide con (2.26) si $g_{0}=g_{\pi .} . \quad K(v)$ diverge logarítmicamente cuando $v \rightarrow 1_{+}$pero dado que $E_{s}$ es una densidad, su integral en el espacio, la energía total, es finita. La densidad de energía media en la fuente se obtiene haciendo el límite de la (2.34) para $\vec{r} \rightarrow 0$. Cerca de la fuente, la energía puede considerarse proporcional a $t^{-2}$, pero a medida que $t$ crece, su variación se hace más lenta.

Una ventaja del método de Sato (1977a) sobre el de Aki y Chouet (1975) es que puede aplicarse inmediatamente después de la llegada de las ondas $\mathrm{S}$ directas. Los resultados de análisis adimensionales de la

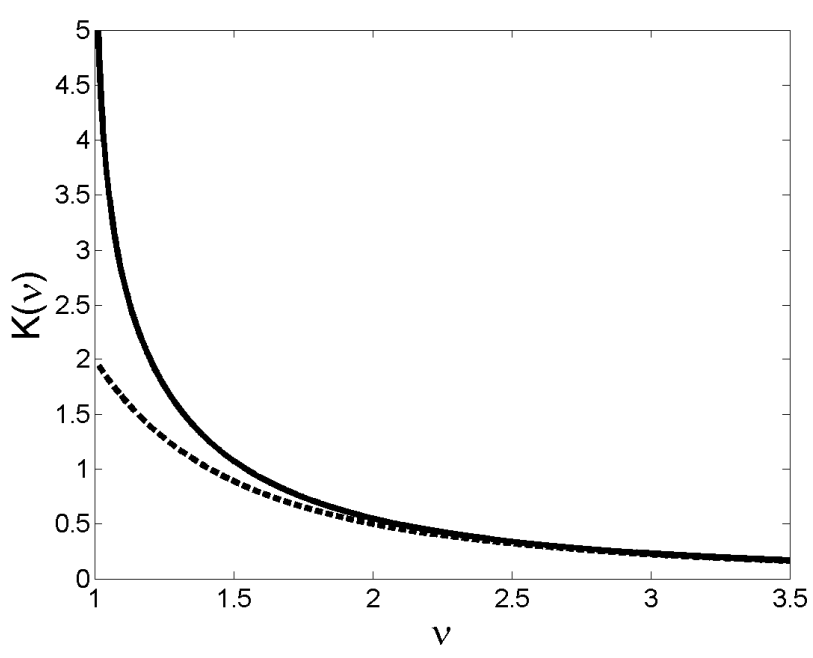

Fig. 2.12.- Factor de corrección de la expansión geométrica según Sato (1977a), K(v), para v mayor que 1 en línea sólida y asíntota, $2 v^{-2}$, en línea punteada. energía a diferentes distancias y tiempos mediante la teoría de SIS permitieron a Sato (1977a) explicar cualitativamente las principales propiedades de las ondas coda. En trabajos posteriores, consideró la posibilidad de conversión de ondas en el scattering $\mathrm{S} \rightarrow \mathrm{P}$ y $\mathrm{P} \rightarrow \mathrm{S}$ (Sato, 1977b) y el scattering múltiple (Sato, 1988, 1993, 1994).

Incorporando el efecto de la atenuación anelástica y trabajando las (2.33) y (2.34), la relación de energías entre la onda directa y la coda permite conocer el factor de calidad de las ondas coda en función del tiempo de $\mathrm{S}$ y el lapso de tiempo de coda

$$
\frac{E_{d}(\omega)}{E_{s(\omega)}}=\frac{1}{g_{0} \nu u} K(v)^{-1} e^{-\frac{\omega\left(t-t_{S}\right)}{Q_{c}}}
$$

donde $t$ es el lapso de tiempo de la coda y $t_{\mathrm{S}}$ es el tiempo de viaje de la onda $\mathrm{S}$ directa.

Novelo-Casanova y Lee (1991) usando codas sintéticas, compararon la aplicabilidad de las técnicas de SBS y SIS. Encontraron que ambos métodos aproximaban razonablemente los modelos de entrada propuestos al analizar las mismas ventanas de coda y que los valores bajos de $Q_{c}$ eran mejor estimados en la parte inicial y final de la coda mientras que valores 
altos de $Q_{c}$ se extraían más precisamente para lapsos de tiempo largos. Además, la incorporación de $K(v)$ en el método de SIS parecía trabajar mejor con codas altamente atenuadas, esto es $Q_{c}$ bajos, mientras que su influencia no se notaba para $Q_{c}$ altos ni para lapsos de tiempo largos.

Según Frankel y Wennerberg (1987), el tratamiento similar del scattering y la anelasticidad en el SS parece contradecir la física del problema ya que dichos procesos afectan de manera distinta al decaimiento de coda. Mientras que un aumento de la anelasticidad conlleva a la disminución de energía tanto en las ondas directas como en la coda, un aumento del scattering, desviará energía de las ondas directas hacia la coda.

Debido a todas estas consideraciones, diversos autores propusieron diferentes modelos para la generación de ondas coda y el tratamiento de la atenuación, como veremos más adelante. Sin embargo, el SBS sigue siendo utilizado dada la sencillez de su aplicación y porque la información que brinda resulta suficiente para algunos fines como por ejemplo, las determinaciones de peligrosidad sísmica.

\subsubsection{SCATTERING MÚLTIPLE}

En regiones con mayor densidad de heterogeneidades surge la necesidad de incluir los efectos del scattering múltiple, es así que comienzan a desarrollarse nuevos modelos con bases teóricas y/o fenomenológicas. En primer lugar el modelo extremo de Difusión de la energía (Aki y Chouet, 1975), luego el modelo de Scattering Múltiple de Gao et al. (1983a,b) que parte del scattering simple agregando órdenes de dispersión; el modelo de Transferencia de Energía (Wu, 1985) que parte de las ecuación de Boltzmann de la teoría cinética de los gases y el de Flujo de Energía (Frankel y Wennerberg, 1987) que se basa en el balance de energía entre las ondas directas y la coda. Si bien estos métodos no se implementan en forma directa en esta tesis al ajuste de las codas, ellos han sentado las bases de los métodos de separación de los efectos de absorción anelástica y de scattering que sí se aplican.

\subsubsection{MODELO DE DIFUSIÓN (D)}

Dado que el SBS viola el balance energético, Aki y Chouet (1975) consideraron también el modelo de difusión. En este caso, plantearon la transferencia de energía sísmica como un scattering fuerte donde la energía se fracciona de diferente manera hacia adelante y hacia atrás al encontrar una heterogeneidad e incluye los efectos de la transformación de la energía en calor. Es lógico pensar que a medida que el lapso de tiempo de propagación aumenta, el efecto del scattering múltiple domina frente al scattering simple produciendo una 
distribución espacial de energía suavizada.

Sea $E^{D}(\vec{r}, t \mid \omega)$ la energía sísmica por unidad de volumen en una banda de frecuencia unitaria alrededor de $\omega$. El flujo y el gradiente de la energía cambian con el tiempo, por lo que se aplica la segunda ley de la difusión para el estado no estacionario (Morse e Ingard, 1986) incluyendo un término de disipación lineal debido a la consideración de conservación de la energía

$$
\frac{\partial E^{D}}{\partial t}=D \nabla^{2} E^{D}-\frac{\omega}{Q} E^{D}
$$

donde $D$ es el coeficiente de difusión y el segundo término representa la transformación de energía en calor, es decir que $Q$ corresponde solo a la atenuación anelástica. Solucionando la (2.37) para una fuente puntual e instantánea que emite una energía sísmica total $W(\omega)$ en una banda de frecuencia unitaria alrededor de $\omega$, se obtiene

$$
E^{D}(\omega \mid x, t)=\frac{W(\omega)}{(4 \pi D)^{3 / 2}} t^{-3 / 2} e^{-\frac{x^{2}+y^{2}+z^{2}}{4 D t}} e^{-\frac{\omega t}{Q}}
$$

Suponiendo distancias cortas y tiempos largos, a partir de la definición de la densidad de potencia espectral (EDP) y teniendo en cuenta que la densidad de energía sísmica es dos veces la energía cinética de las ondas que se propagan o de ondas estacionarias en un medio de densidad $\rho$, se llega a la función EDP

$$
P^{D}(\omega \mid t)=E^{D}(\omega \mid 0, t) \frac{1}{\rho \omega^{2}}=\frac{W(\omega)}{\rho \omega^{2}(4 \pi D)^{3 / 2}} t^{-3 / 2} e^{-\frac{\omega t}{Q}}
$$

Por lo tanto el modelo de difusión plantea una dependencia de la energía con el lapso de tiempo intermedia entre los casos de ondas internas y superficiales del modelo de SBS. En el análisis espacio-temporal parametrizado de la ecuación de difusión mediante curvas normalizadas de energía (Fig. 2.13) se ve que la energía viaja por delante del frente de ondas violando el principio de causalidad.

Dainty et al. (1974b) obtuvieron la relación entre $D$ y el camino libre medio $\ell$ de la forma $D=v \ell / 3$ donde $v$ es la velocidad de propagación. Según Aki y Chouet (1975) las ondas que sufren scattering hacia adelante se comportan más como ondas viajeras que como afectadas por difusión, por lo que este proceso se aplica sólo a la parte de la energía 

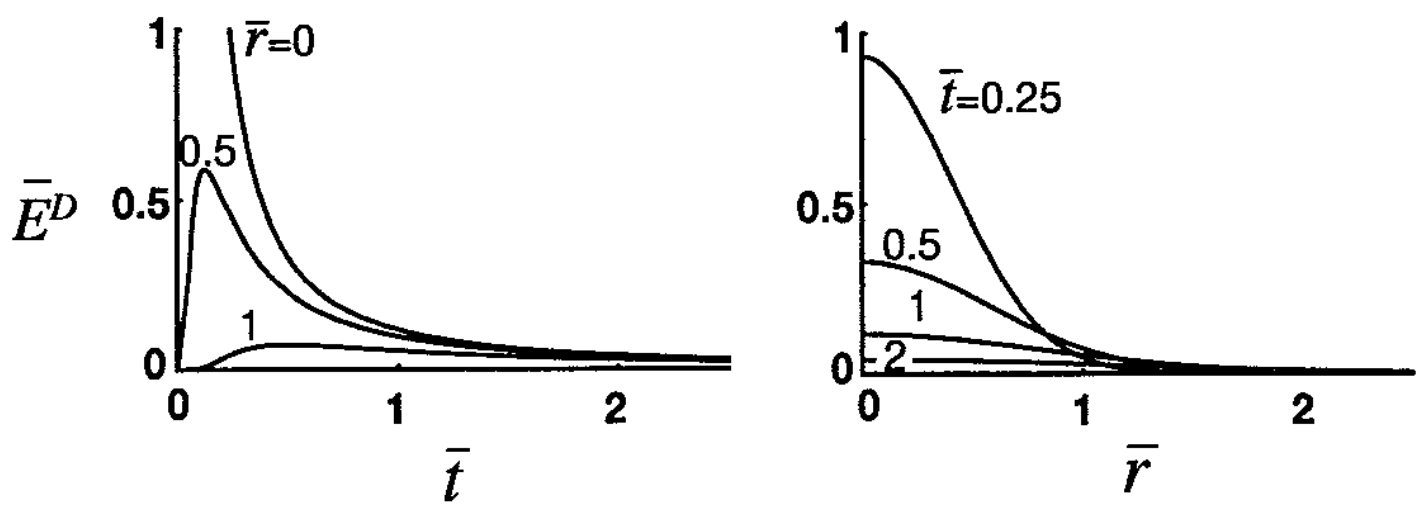

Fig. 2.13. Modelo de difusión: (a) densidad de energía vs. tiempo a diferentes distancias y (b) densidad de energía vs. distancia con el tiempo como parámetro (Extractada de Sato y Fehler, 1998)

dispersada hacia atrás. Por eso en las expresiones de difusión, se incorpora $g(\pi)$ en lugar del promedio de $g(\theta)$ para todas las direcciones.

Más adelante, en la teoría de transferencia radiativa se verá que el scattering múltiple isótropo converge a la difusión para lapsos de tiempo largos.

\subsubsection{MODELO DE SCATTERING MÚLTIPLE DE GAO (SMG)}

Según se ha visto, la teoría del scattering simple es válida solo si el camino libre medio de las ondas es mucho más grande que la distancia de la heterogeneidad al receptor. Sin embargo existen numerosas situaciones donde esta condición no se cumple y entonces debe considerarse la contribución del scattering múltiple a la coda. Kopnichev (1977) desarrolló las expresiones del scattering doble y triple y la difusión para un medio aleatorio, uniforme, isótropo y normal asumiendo además, que la distancia recorrida por las ondas es mucho mayor que la distancia epicentral. Nuevamente la difusión fue descartada para aplicaciones terrestres. En los casos de doble y triple scattering consideró ondas internas y superficiales con la condición de que las ondas primarias y secundarias fuesen de la misma naturaleza. Los resultados indicaron que la parte inicial de la coda estaba compuesta principalmente de ondas afectadas de scattering simple, mientras que la contribución del scattering múltiple aumentaba con el correr del tiempo. De este modo se establecía una limitación en las duraciones de codas consideradas según el modelo empleado.

Gao et al. (1983a,b) continuaron el estudio del scattering múltiple a partir de los desarrollos de scattering simple de Aki y Chouet (1975). Inicialmente desarrollaron la teoría para un medio bidimensional Gao et al. (1983a) y luego la extendieron al caso tridimensional Gao et al. (1983b). Para un medio tridimensional caracterizado por scattering múltiple e isótropo, suponiendo que no se producen cambios de naturaleza y que las energías de las 
ondas afectadas son aditivas, el EDP a un lapso de tiempo $t$ puede expresarse de la forma

$$
P(\omega \mid t)=\sum_{k} P_{k}(\omega \mid t)
$$

donde el índice $k$ indica la contribución energética de la onda generada en la $k$-ésima heterogeneidad.

Para obtener las expresiones correspondientes, partiendo de las expresiones del SBS desarrollaron el scattering doble, triple y su extensión a órdenes superiores. Suponiendo a la fuente y el receptor coincidentes $(\mathrm{F} \equiv \mathrm{R})$ en el espacio y una primera heterogeneidad $\left(\mathrm{H}_{1}\right)$ a distancia $r_{1}$. Las segundas heterogeneidades se plantean con una densidad igual a $n$, sección transversal $\sigma_{0}$ y distribuidas en un anillo elipsoidal con focos en $\mathrm{H}_{1}$ y $\mathrm{F}$. Los ejes mayores de las elipsoides interna y externa son $2 r-r_{1} \quad y \quad 2(r+d r)-r_{1}$ con $d r=v d t / 2$. Las segundas heterogeneidades recibirán ondas provenientes de $\mathrm{H}_{1}$ y $\mathrm{F}$, a distancias $\mathrm{r}_{2} \mathrm{y}_{3}$ respectivamente. A cierta distancia de referencia de la fuente, $r_{0}$, el espectro de amplitud de las ondas será $\mid \phi\left(\omega \mid r_{0}\right)$. Para el espectro de amplitud de las ondas afectadas por las dos heterogeneidades se tiene la expresión

$$
\left|\phi_{2}(\omega \mid t)\right|=\frac{\sigma_{0}}{4 \pi r_{2} r_{3}}\left(\frac{r_{0}}{r_{1}}\right) \phi\left(\omega \mid r_{0}\right) e^{-\frac{\omega}{2 Q}\left(t-t_{0}\right)}
$$

si se suman las contribuciones de todas las heterogeneidades en el elemento de volumen entre los dos elipsoides para hallar el EDP que llega al receptor entre el lapso de tiempo $t$ y $t+d t$, se obtiene

$$
P_{2}(\omega \mid t)=K_{2} \frac{n^{2} \sigma_{0}{ }^{2} v S(\omega)}{r} e^{-\frac{\omega t}{Q}}
$$

donde

$$
S(\omega)=r_{0}{ }^{2} \mid \phi\left(\omega \mid r_{0}\right)^{2} e^{\frac{\omega t_{0}}{Q}}
$$

representa la energía a la distancia de referencia $r_{0}, K_{2}$ representa la geometría del problema y su valor, hallado numéricamente, es igual a $\pi^{2} / 8$. Gao et al. (1983b) hallaron del mismo modo las contribuciones de los scattering de orden tres, cuatro y hasta siete, obteniendo la siguiente generalización para órdenes superiores 


$$
P(\omega \mid t)=v\left(n \sigma_{0}\right)^{3} S(\omega) e^{-\frac{\omega t}{Q}} \sum_{i=1}^{\infty}\left(n \sigma_{0} r\right)^{i-3} K_{i}
$$

donde el índice $i$ indica el orden del scattering. Resulta fácil entonces, separar el efecto del scattering simple del múltiple

$$
P(\omega \mid t)=P_{S}(\omega \mid t)+P_{m}(\omega \mid t)
$$

donde

$$
\begin{gathered}
P_{S}(\omega \mid t)=\frac{v n \sigma_{0} S(\omega)}{2 r^{2}} e^{-\frac{\omega t}{Q}} \\
P_{m}(\omega \mid t)=\frac{v\left(n \sigma_{0}\right)^{2} S(\omega)}{r} e^{-\frac{\omega t}{Q} \sum_{i=2}^{m}\left(n \sigma_{0} r\right)^{i-2} K_{i}}
\end{gathered}
$$

Como puede verse, de acuerdo a la dependencia de $P_{S}$ y $P_{m}$ respecto de $r$ o su equivalente $v t / 2$, la contribución del scattering múltiple para tiempos largos es mayor que la del scattering simple. Aunque, integrando los valores de $K_{i}$, Gao et al. (1983a, b) comprobaron que la contribución del scattering de orden $i>7$ resultaba ser prácticamente nula.

Teniendo en cuenta la (2.19) se puede expresar

$$
Q_{s}=\frac{\omega t}{2 n \sigma_{0} r}=\frac{\omega t}{\gamma}
$$

donde la cantidad $\gamma=2 \mathrm{n}_{0} r$ es adimensional y representa la pérdida de potencia debida al scattering simple. Dainty y Toksöz (1981) plantearon que el factor de calidad del medio, $Q$, podía expresarse a través de la contribución de los factores de calidad correspondientes a los dos procesos que causaban la atenuación, esto es la anelasticidad del medio $\left(Q_{i}\right)$ y el scattering $\left(Q_{s}\right)$ en la forma

$$
\frac{1}{Q}=\frac{1}{Q_{i}}+\frac{1}{Q_{S}}
$$

Integrando hasta el séptimo orden de scattering, Gao et al (1983b) establecieron la siguiente expresión para el EDP del scattering múltiple

$$
P_{m}(\omega \mid t)=1.23 \gamma^{-1} 2 v\left(n \sigma_{0}\right)^{3} S(\omega) e^{-\omega t\left(\frac{0.67}{Q_{s}}+\frac{1}{Q_{i}}\right)}
$$


Analizando los valores de $P_{s}$ y $P_{m}$ en función de $\gamma$, observaron que se intersecan para $\gamma=\gamma_{c}=0.65=t_{c} n \sigma_{0} v$. Para $t>t_{c}=0.65\left(n \sigma_{0} v\right)^{-1}$, el scattering múltiple domina el EDP mientras que para tiempos menores el SBS permite un buen ajuste. De la (2.50) se deduce que para $t$ $>>\mathrm{t}_{\mathrm{c}}$, los valores de $Q_{s}$ son sobreestimados en un factor 1/0.67 en el análisis del scattering simple.

Gao (1984) mostró que el análisis de las ondas coda podía ser una herramienta matemática útil para distinguir ambas contribuciones. Integrando la expresión del scattering múltiple hasta el séptimo orden con la incorporación de (2.48) y (2.49) obtuvo la siguiente expresión para el espectro de potencia

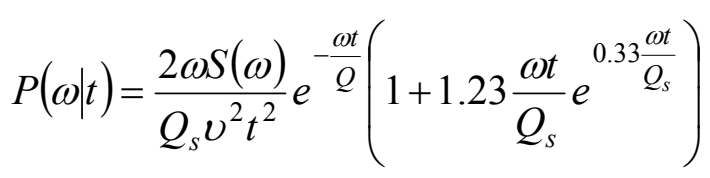

que puede solucionarse usando un método de ajuste no lineal para determinar los valores de $Q_{i}$ y $Q_{s}$. El primer término representa la contribución del scattering simple y el segundo término representa la contribución del scattering múltiple. Puede verse que en la estimación de $Q_{c}$ para lapsos de tiempo largos con el modelo de Aki y Chouet, se estaría sobreestimando su valor en un factor de 1.23 para el caso tridimensional si se considera despreciable la absorción intrínseca del medio.

\subsubsection{MODELO DE TRANSFERENCIA RADIATIVA (TR)}

El modelo de Gao et al (1983b) permitía un mejor ajuste de la envolvente media cuadrática del sismograma, pero aún quedaba por resolver el problema del tratamiento de la conservación de la energía total. Una aproximación sistemática al modelado del scattering múltiple consiste en considerar la teoría de la transferencia radiativa (teoría del transporte de energía) para la densidad de energía. Los modelos iniciales que usaban esta teoría no incluían la conversión de ondas en el scattering. Wu (1985) y Wu y Aki (1988a) fueron los primeros en explorar su aplicación en el modelado de envolventes de sismogramas de alta frecuencia. Wu (1985) consideró el caso del scattering isótropo y del scattering fuerte hacia adelante para ondas $\mathrm{S}$ y coda de pequeños terremotos locales.

La ecuación básica es la llamada ecuación de transferencia o transporte y es equivalente a la ecuación de Boltzmann usada en la teoría cinética de los gases, en la óptica astrofísica y en la teoría del transporte de neutrones. Esta teoría ha sido aplicada con éxito, por ejemplo, para modelar visibilidad atmosférica, acústica oceánica y la propagación de la luz en atmósferas planetarias (Chandrasekhar, 1960). Asumiendo que la energía total se conserva y 
sin considerar la interferencia de ondas, el desarrollo de fórmulas es más bien heurístico, perdiendo rigurosidad en el tratamiento de la ecuación de onda. De este modo se simplifican las matemáticas a riesgo de hallar algunos problemas de balance energético local por el scattering simple.

Se presenta aquí un resumen que sirve de introducción a métodos cuyos desarrollos se fundan en este modelo y que se aplican en este trabajo.

En primer lugar el análisis de la energía se realiza para una frecuencia fija, por lo que las expresiones no dependerán de $\omega$. La intensidad media, $\bar{I}(r)$, es el promedio de la integral sobre todas las direcciones de la intensidad específica o direccional $I(r, \hat{\Omega})$. Esta última es la cantidad fundamental de la teoría, ya que da el flujo de potencia en un ángulo sólido unitario en la dirección $\hat{\Omega}$. La densidad de energía para una onda de velocidad $v$, será

$$
E(r)=\frac{1}{v} \int_{4 \pi} I(r, \hat{\Omega}) d \Omega=\frac{4 \pi}{v} \bar{I}(r)
$$

Se define el albedo sísmico, $B_{0,}$, como

$$
B_{0}=\frac{\eta_{s}}{\eta_{s}+\eta_{i}}
$$

donde $\eta_{s} \equiv g_{0}$ es el coeficiente total de scattering definido a partir de (2.17) y (2.19) salvo que en este caso el coeficiente de scattering, $g$, es direccional; $\eta_{i}$ es el coeficiente de absorción intrínseca del medio y $\eta_{e}$, el coeficiente de extinción del medio, es $\eta_{e}=\eta_{s+} \eta_{i}$. La directividad del scattering está dada por la relación $D\left(\hat{\Omega}, \hat{\Omega}_{0}\right)=g\left(\hat{\Omega}, \hat{\Omega}_{0}\right) / \eta_{s}$.

La ecuación diferencial para la intensidad específica, o ecuación de transferencia, será

$$
\frac{d I(r, \hat{\Omega})}{d l}=-\eta_{e} I(r, \hat{\Omega})+\frac{\eta_{s}}{4 \pi} \int_{4 \pi} D\left(\hat{\Omega}, \hat{\Omega}_{0}\right) I\left(r, \hat{\Omega}_{0}\right) d \Omega_{0}+W(r, \hat{\Omega})
$$

donde $d l$ es la longitud de un cilindro elemental de sección transversal unitaria, alrededor de la dirección $\hat{\Omega}$ y $W(r, \hat{\Omega})$ es la función intensidad de la fuente, que define la cantidad de potencia emitida en la dirección $\hat{\Omega}$ por unidad de ángulo sólido. El primer término de la derecha de la ecuación (2.54) representa la pérdida de potencia en la dirección $\hat{\Omega}$ por absorción y scattering, el segundo término da la ganancia de potencia en dicha dirección por intensidad incidente que sufrió scattering desde todas las direcciones y el tercer término es la contribución directa de la fuente. 
No existe una solución analítica general para la ecuación de transferencia pero pueden hallarse soluciones numéricas. Las soluciones se plantean reemplazando la (2.52) en (2.54). El caso más sencillo es el correspondiente al scattering isótropo, es decir que $D\left(\hat{\Omega}, \hat{\Omega}_{0}\right) \equiv 1$. Si se integra sobre todas las direcciones, para obtener la densidad de energía se llega a una ecuación diferencial de primer orden de la forma

$$
\frac{d E(r)}{d l}=-\eta_{e} E(r)+Q(l)
$$

con

$$
Q(l)=\frac{1}{C} \int_{4 \pi}\left[\frac{\eta_{s}}{4 \pi} \int_{4 \pi} I\left(r, \hat{\Omega}_{0}\right) d \Omega_{0}+W(r, \hat{\Omega})\right] d \Omega
$$

La solución general de la (2.55) es

$$
E(r)=A e^{-\eta_{e} l}+\int_{0}^{l} Q\left(l_{1}\right) e^{-\eta_{e}\left(l-l_{1}\right)} d l_{1}
$$

donde $A$ es una constante. Puede observarse que la densidad de energía contiene un primer término denominado densidad de energía coherente, $E_{c}$, o densidad de energía reducida, que decae exponencialmente con el coeficiente de extinción, $\eta_{e}$, como coeficiente de atenuación. El segundo término se denomina densidad de energía difusa, $E_{d}$, producida por el scattering. Queda por determinar la función $I\left(r, \hat{\Omega}_{0}\right)$, para lo cual se deben imponer condiciones de contorno. Wu (1985) supuso una fuente puntual en el origen irradiando una densidad de energía $E_{0}$ en un medio infinito aleatorio e inhomogéneo, obteniendo la siguiente solución

$$
\begin{aligned}
4 \pi r^{2} E(r) & =\eta_{e} P_{d} r e^{-\eta_{e} d_{0} r}+\eta_{e} r \int_{0}^{1} f\left(\xi, B_{0}\right) e^{-\frac{\eta_{e} r}{\xi}} \frac{d \xi}{\xi^{2}} \\
& =4 \pi r^{2}\left(E_{d}+E_{c}\right)
\end{aligned}
$$

donde la energía de difusión, afectada por el scattering múltiple, está caracterizada por

$$
P_{d}=\frac{2 d_{0}^{2}\left(1-d_{0}^{2}\right)}{B_{0}\left(d_{0}^{2}+B_{0}-1\right)}
$$

y $d_{0}$, que se define como el multiplicador difusivo se determina a partir de su relación con $B_{0}$ 


$$
\frac{B_{0}}{2 d_{0}} \ln \left(\frac{1+d_{0}}{1-d_{0}}\right)=1
$$

Por último, la función que caracteriza a la energía coherente puede escribirse como

$$
f\left(\xi, B_{0}\right)=\left[\left(1-B_{0} \xi \tanh ^{-1}(\xi)\right)^{2}+\left(\frac{\pi}{2} B_{0} \xi\right)^{2}\right]^{-1}
$$

El término difusor también puede escribirse discriminando en su exponente el efecto de la absorción y el scattering

$$
\begin{gathered}
E_{d}(r)=\eta_{e} P_{d} r e^{\left[-\left(\eta_{i}+d_{s} \eta_{s}\right) r\right]} \\
d_{s}=\frac{d_{0}-\left(1-B_{0}\right)}{B_{0}}
\end{gathered}
$$

Cuando la distancia es grande, especialmente para $B_{0}$ grandes, el término de difusión es dominante y la $E(r)$ tendrá aproximadamente un decaimiento exponencial con un coeficiente de atenuación aparente $d_{0} \eta_{e}$ que es menor que $\eta_{e}$, ya que $d_{0}$ es siempre menor que 1 .

Wu (1985) analizó también el caso de heterogeneidades de gran escala con un scattering fuerte hacia adelante, tal como se habían encontrado en las grandes antenas sísmicas de LASA y NORSAR, dibujando las curvas normalizadas de densidad flujo de energía $\left(4 \pi r^{2} E(r)\right)$ en función de $r \eta$, la distancia de extinción, absorción o scattering según el caso (Fig. 2.14). Para el scattering isótropo o aproximadamente isótropo, halló que las curvas dependían fuertemente del albedo sísmico, $B_{0}$, manteniendo una forma de arco con su máximo dependiendo del coeficiente de extinción del medio, $\eta_{e}$, para el caso $B_{0}>0.5$. A partir del

Fig. 2.14. Curvas de distribución de densidad de energía normalizada $4 \pi r^{2} E(r)$, donde $r$ es la distancia de propagación desde la fuente puntual, $D_{e}=\eta_{e}$ es la distancia de extinción y $\eta_{e}=\eta_{s^{+}} \eta_{i}$ es el coeficiente de extinción. $B_{o}$ es el albedo sísmico del medio. (extractada de Wu, 1985).

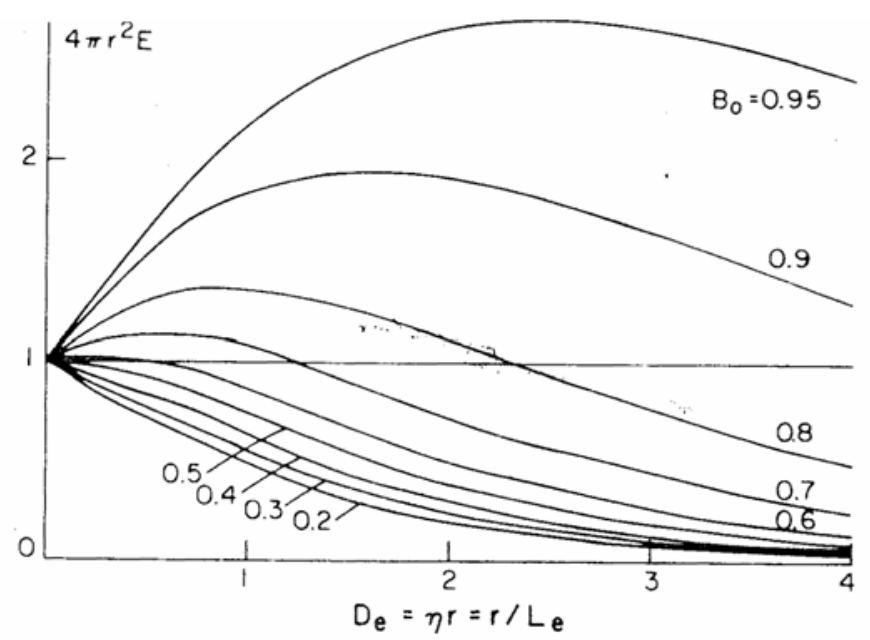


valor del albedo, podrían separarse las contribuciones de la absorción y el scattering a la atenuación (Fig. 2.15). Por otra parte, para el scattering fuerte, la forma de la curva de energía vs. distancia es insensible al albedo, por lo que la separación de los efectos de absorción y scattering se vuelve más difícil.

La implementación de este modelo requería la comparación visual de curvas experimentales con curvas sintéticas. En el momento que fue desarrollado esto demandaba un tiempo de cálculo que no era justificado por la fiabilidad del ajuste y el margen de error de los datos. Unos años

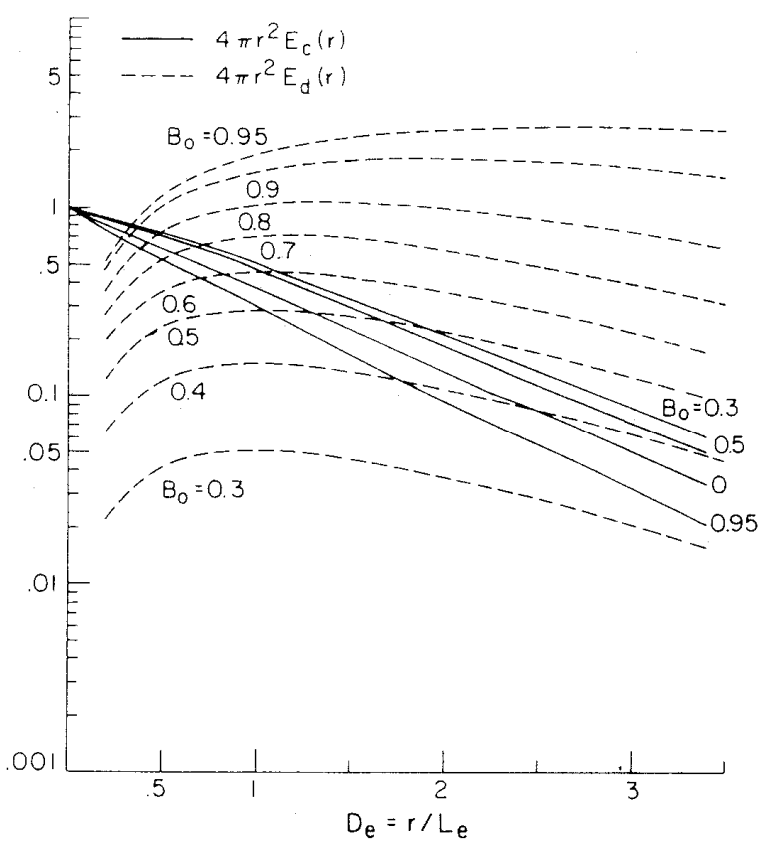

Fig. 2.15. Amplitudes relativas de la energía difusa $E_{d}$ y la energía coherente $E_{c}$ en función de la distancia de extinción $D_{e} y$ diferentes albedos sísmicos $B_{o}$. $(W u, 1985)$ más tarde Hoshiba et al. (1991) retomarían este modelo para mejorarlo.

\subsubsection{MODELO DE FLUJO DE ENERGÍA (FE)}

Frankel y Clayton (1984), comprobaron que la atenuación aparente es mayor cuando la heterogeneidad tiene dimensiones comparables a la longitud de onda. Analizando sismogramas sintéticos en un medio aleatorio bidimensional observaron que el contenido en frecuencias de la coda aumenta cuando disminuye el tamaño de la heterogeneidad.

Posteriormente (Frankel y Clayton, 1986), observaron la excitación de ondas coda al analizar simulaciones de propagación de ondas en medios inhomogéneos y encontraron que el decaimiento de la coda en sismogramas sintéticos era insensible al $Q$ de scattering del medio.

Los resultados previos condujeron a Frankel y Wennerberg (1987) a la conclusión de que, debido al scattering, las ondas se dispersan rápidamente en un volumen esférico por detrás del frente de onda. Entre sus observaciones cabe destacar, que el scattering y la atenuación intrínseca deberían causar diferentes efectos sobre el decaimiento de la coda. Mientras un aumento del scattering causa una mayor distribución de la energía directa hacia la coda a medida que aumenta el tiempo, un aumento de la atenuación intrínseca produce una mayor pérdida de energía con el tiempo tanto en la coda como en la onda directa.

El modelo propuesto es consistente con las observaciones de las envolventes de sismogramas a diferentes distancias y con las amplitudes de codas de $\mathrm{S}$ para lapsos de tiempo 
largos. Por otra parte permite diferenciar explícitamente entre la atenuación anelástica y la atenuación por scattering, siendo válido tanto para el scattering débil como para el fuerte. Demostraron, mediante la aplicación sobre sismogramas sintéticos que el decaimiento de la coda es sensible al $Q$ intrínseco del medio y que el cociente entre la amplitud de la coda y la energía de la onda directa registrada sirve como medida de la atenuación por scattering. Mediante pruebas sobre datos reales concluyeron que los valores de $Q$ obtenidos para ondas coda en la litósfera se aproximan al efecto de la atenuación intrínseca y no se relacionan con la atenuación por scattering.

Al igual que el modelo de transferencia radiativa, el modelo de flujo de energía es un modelo fenomenológico y no hace intentos de explicar el proceso de scattering. Considera que la energía fluye entre el frente de onda inicial y la coda, que la energía de la coda es aproximadamente homogénea en el espacio y aplica el principio de conservación de la energía. Las fórmulas locales de magnitud que dependen únicamente de la duración del registro son válidas por la propiedad de homogeneidad de la coda.

En el desarrollo del método, Frankel y Wennerberg (1987) plantearon ondas acústicas propagándose desde una fuente explosiva a través de dos clases de medios diferentes según el tamaño dominante de sus heterogeneidades. En principio simularon sismogramas donde las ondas directas eran afectadas por scattering y expansión geométrica, tal como lo plantearon Aki y Chouet (1975), pero sin incluir el efecto de la atenuación intrínseca. La naturaleza de las ondas podía ser tanto de ondas superficiales como internas. Luego determinaron el $Q$ de scattering a partir del decaimiento de la amplitud máxima de las envolventes corregidas por divergencia geométrica y en función de las distancias fuente-receptor. Demostraron que el $Q$ de scattering es menor cuando las heterogeneidades son de tamaño similar a la longitud de onda sísmica que cuando las heterogeneidades son mucho más pequeñas que la longitud de onda. En el primer caso, la amplitud promedio de las ondas coda es mayor y el decaimiento con la distancia de la amplitud de la onda $\mathrm{S}$ directa es más notorio, demostrando la homogeneidad de la coda mencionada anteriormente. Para cualquier distancia de correlación, $Q_{s}^{-1}$ es casi proporcional a la varianza $\left(\sigma^{2}\right)$ de la velocidad sísmica del medio.

Suponiendo un medio sin atenuación intrínseca, la energía de la onda directa es dispersada en forma continua hacia la coda a medida que el frente de onda avanza. Si $W_{T}$ es la energía total, $W_{D}$ la energía de la onda directa y $W_{C}$ la energía de la coda, entonces a un lapso de tiempo $t$, la energía de la onda directa será 


$$
W_{D}=W_{T} e^{-\omega t / Q_{S}}
$$

donde $\omega$ es la frecuencia angular y $Q_{S}$ representa el scattering. La energía de la coda puede expresarse como $W_{C}=W_{T}-W_{D}$ y dado que $W_{D}$ se mide en cierta ventana temporal que se inicia en el instante de arribo de la onda, puede ser que en ella se incluya algo de energía afectada de forward-scattering. Estas consideraciones conducen a

$$
W_{C}=W_{T}\left(1-e^{-\omega t / Q_{s}}\right)
$$

Como la coda es homogénea, su densidad de energía vendrá dada por

$$
E_{C}^{F E}(\omega \mid r, t)=W_{C} /\left(4 / 3 \pi t^{3} v^{3}\right)
$$

siempre que se tome la ventana temporal de la coda a $v t \gg>r$ (Frankel y Wennerberg, 1987). Reemplazando esta expresión en (2.65) e integrando para todas las heterogeneidades del medio tridimensional (Sato y Fehler, 1998), se obtiene

$$
\begin{aligned}
E_{C}^{F E}(\omega \mid r, t) & =\frac{3 W_{T}\left(1-e^{-\omega t / Q_{s}}\right)}{4 \pi v^{3} t^{3}} H\left(t-\frac{r}{v}\right) \\
& \approx \frac{3 W_{T} \omega Q_{s}^{-1}}{4 \pi v^{3} t^{2}} H\left(t-\frac{r}{v}\right)
\end{aligned}
$$

Según el modelo de flujo de energía las envolventes de coda convergen para medios con diferentes valores de $Q_{s}$ y lapsos de tiempo largos, mientras que en el modelo de scattering simple las amplitudes de coda serán proporcionales a los valores de $Q_{s}$ (Fig. 2.16).

Incorporando ahora al medio la atenuación intrínseca a través de $Q_{i}$, según la relación $W_{T}=W e^{-\omega t / Q_{i}}$, se obtiene

$$
E_{C}^{F E}(\omega \mid r, t)=\frac{3 W\left(1-e^{-\omega t / Q_{s}}\right) e^{-\omega t / Q_{i}}}{4 \pi v^{3} t^{3}} H\left(t-\frac{r}{v}\right)
$$

En este caso, como en los otros métodos, $W$ es la energía elástica radiada originalmente en la fuente. La atenuación intrínseca causa un decaimiento exponencial de la amplitud en la coda que se superpone a los efectos del scattering y la expansión geométrica. El decaimiento de la coda con ambos tipos de atenuación es más pronunciado y es bastante similar a los observados. El scattering simple sobreestima la velocidad de decaimiento de la coda en un medio con atenuación intrínseca y moderada atenuación de scattering.

Aplicando la expresión (2.49) de Dainty y Toksöz (1981), puede evaluarse la contribución del scattering y la absorción anelástica a la atenuación de ondas coda. Frankel y 
Wennerberg (1987) demostraron mediante simulaciones que el decaimiento de la coda es más sensible al $Q_{i}$ que al $Q_{s}$. Como puede verse en la (2.68), si el scattering es muy débil, el modelo de flujo de energía se aproxima al del SBS (2.28). En ese caso habrá que tener en cuenta que $Q_{c}$ deberá ser igual a $Q_{i}, Q_{s}$ será muy grande y los lapsos de tiempo $t$, muy cortos de modo que pueda aproximarse $e^{-\omega t / Q s} \approx 1-\omega t / Q_{s}$. La gran diferencia con el modelo de SBS es la suposición de que $Q_{c}$ representa solo a $Q_{i}$. Esta era la suposición original de Aki y Chouet (1975), aunque Aki (1980a) concluyera que el $Q_{c}$ obtenido por SBS, era más bien la atenuación total del medio e incluía tanto la atenuación intrínseca como la de scattering.

Finalmente, Frankel y Wennerberg (1987) mostraron que los valores de $Q_{c}$ calculados por el método de SBS, aproximan en el mejor caso al $Q_{i}$ de la litósfera y no se
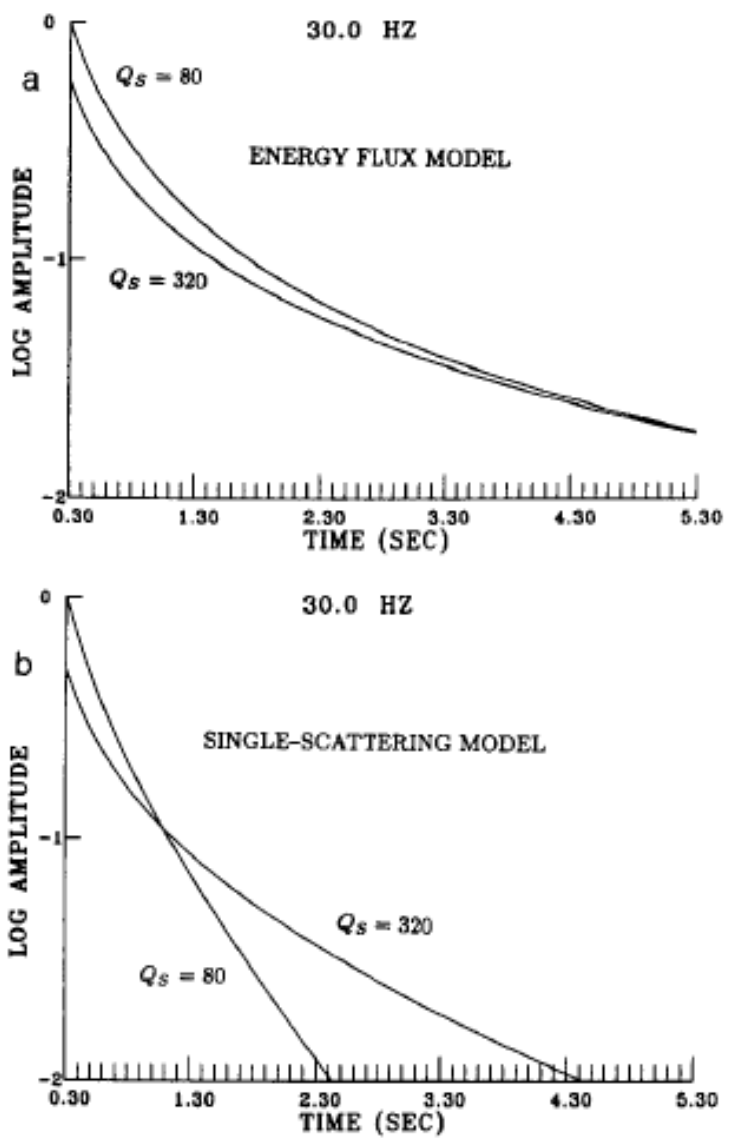

Fig. 2.16. Decaimiento teórico de la coda según (a) el modelo de flujo de energía y (b) el modelo de scattering simple para medios con $Q_{s}$ entre $80 y$ 320 en función del lapso de tiempo. (Extractada de Frankel y Wennerberg, 1987) relacionan con $Q_{s}$.

\subsubsection{ALGUNOS RESULTADOS DE LAS DETERMINACIONES DE $Q_{C}$}

Los modelos de SBS y SIS han resultado ser los más utilizados en todas las regiones donde se han hecho determinaciones de atenuación sísmica. Si bien los mismos Aki y Chouet (1975) calificaron a su modelo de simplista, su expresión de la envolvente de coda ajusta de buen modo en la mayoría de los marcos tectónicos en los que se ha aplicado. Conforme el método SBS se ponía en práctica, los resultados obtenidos sobre los datos reales permitían redefinir los criterios de aplicación y mostraban la variación de $Q_{\mathrm{c}}$ en una misma región induciendo a interpretaciones geodinámicas. Para una discriminación de las posibles causas de la forma de decaimiento de la coda, habría que valerse de modelos más sofisticados como los descriptos en las secciones anteriores. En algunas aplicaciones se han realizado modificaciones al método SBS como la propuesta por Herrmann (1980) quien consideró el 
efecto que agregaba la respuesta instrumental al ajuste de $Q_{c}$ (Canas et al., 1991; Singh y Herrmann, 1983). Entre las regiones que han sido estudiadas mediante el modelo de scattering simple pueden mencionarse: Japón (Aki y Chouet, 1975); Afganistán (Rautian y Khalturin, 1978); varias regiones de EEUU (Chouet, 1979, Singh y Herrmann, 1983, Biswas y Aki, 1984, Havskov et al., 1989); Italia y sur de España (del Pezzo et al., 1983a, b y 1990); NE de España (Correig y Mitchell, 1989 y Correig et al., 1990); Noruega (Kvamme y Havskov, 1989); cuenca de Granada, España (Ibáñez et al., 1990, Morales et al., 1991); Canadá (Woodgold, 1990 y 1994); Francia (Chevrot y Cansi, 1996); Costa Rica (González y Persson, 1997); India (Gupta et al., 1998); México (Novelo Casanova y Valdés-González, 2000); NE de Brasil (Pereira Dias y de Souza, 2004); Venezuela (García y Mendoza, 2004); NO de los Himalaya (Kumar et al., 2005) y Cuba (Biescas et al., 2007). La Fig. 2.17, extractada de Sato y Fehler (1998) reúne valores de $Q_{\mathrm{c}}$ estimados en función de la frecuencia en numerosas regiones del mundo.

Una de las cuestiones que se discuten al aplicar el modelo de scattering simple es la naturaleza de las ondas que componen la coda. Rautian y Khalturin (1978) observaron que no se diferenciaba el efecto de la elección del factor de expansión geométrica, $m=1$ o 2 , excepto para las frecuencias más bajas. En su trabajo midieron envolventes de codas de terremotos locales y regionales registrados en diferentes regiones y con magnitudes diferentes, en un amplio rango de distancias epicentrales, profundidades y frecuencias. En todos los casos el modelo de scattering simple ajustó bien a los datos, mostrando que la validez de esa aproximación se extendía más allá de lo establecido por Aki y Chouet (1975). Poco después, Aki (1980a, b) planteó un método para la determinación del factor $Q$ de ondas $\mathrm{S}$ directas $\left(Q_{\mathrm{d}}\right)$ utilizando las amplitudes de coda para normalizar y las expresiones del SBS para modelar su decaimiento. Sus resultados mostraron una gran similitud entre los valores de $Q_{\mathrm{c}}$ y $Q_{\mathrm{d}}$ llegando a la conclusión de que las codas de terremotos locales en un rango de frecuencias de 1 a $25 \mathrm{~Hz}$, estaban compuestas por ondas S dispersadas por scattering.

La definición del inicio y fin de la coda resulta ser otra cuestión de importancia a la hora de interpretar la naturaleza de las ondas así como su grado de dispersión. En el desarrollo teórico de la aproximación al scattering simple, Aki y Chouet (1975) expresaron la energía de la onda secundaria en el receptor a un lapso de tiempo $t>2 t_{\mathrm{s}}$. Rautian y Khalturin (1978) recomendaron fijar el inicio de la coda en un lapso de tiempo igual a dos veces el tiempo de propagación de la onda $\mathrm{S}$. En ocasiones esta condición se torna demasiado exigente ya que puede haberse descartado gran parte de la energía de la coda o incluso medir amplitudes 


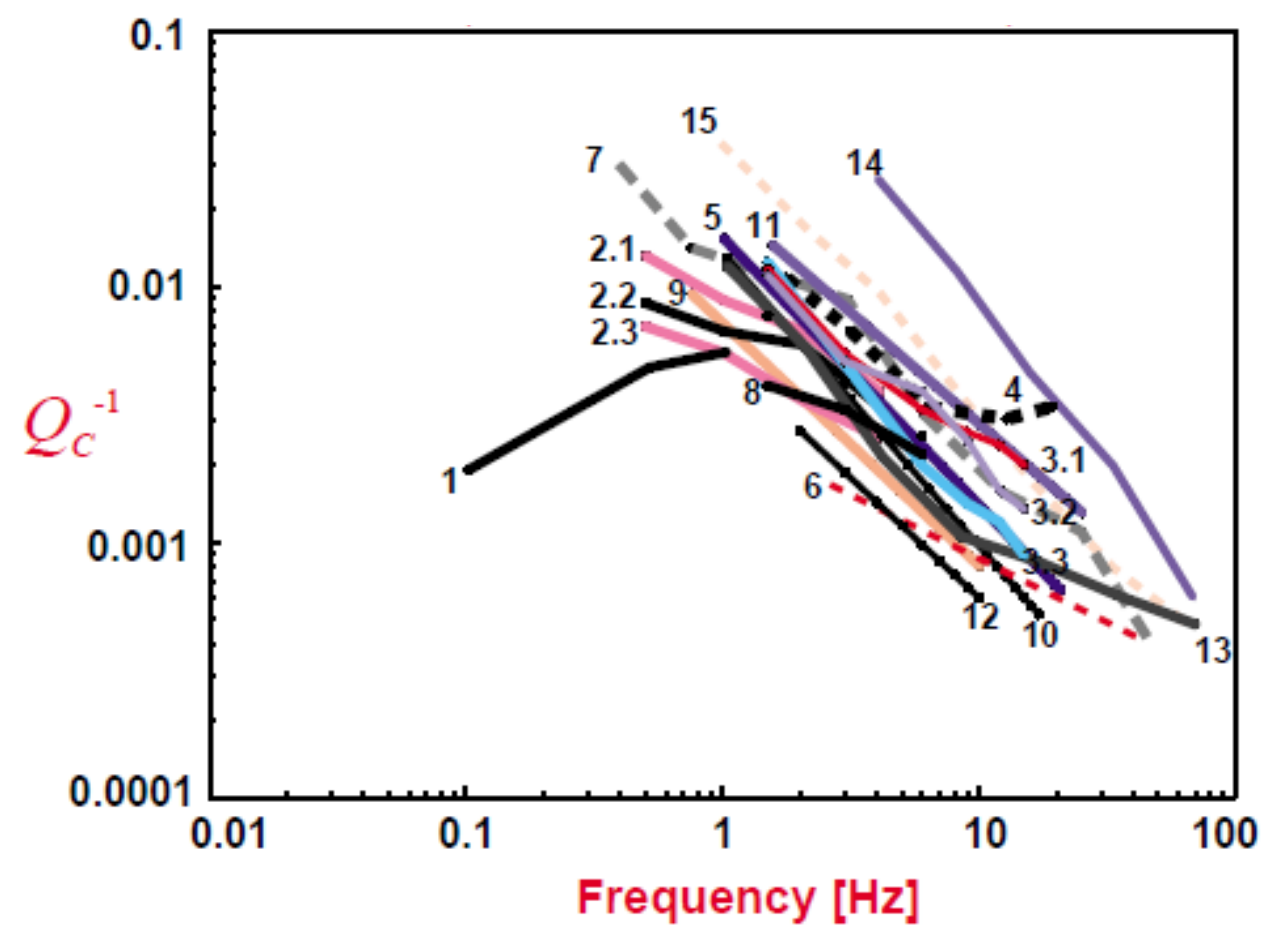

Fig. 2.17. Atenuación de coda $Q_{c}^{-1}$ en función de la frecuencia para varias regiones: 1 , centro y centro-sur de Alaska (Biswas y Aki, 1984); litósferas oceánicas en 2.1, Islandia; 2.2, Galápagos; 2.3, Guam (Jin et al., 1985); 3.1, California central, 3.2, Hawaii, 3.3, Long Valley en California (Mayeda et al., 1992); 4 región volcánica Campi Flegrei, sur de Italia (del Pezzo et al., 1985); 5 Mar Muerto, Asia (Eck, 1988); 6 Garm, Asia central (Rautian y Khalturin, 1978); 7, Indu-Kush, Asia (Roecker et al., 1982); 8, Kanto-Tokai, Japón (Fehler et al., 1992); 9, New England, U.S.A. (Pulli, 1984); 10, sur de Noruega (Kvamme and Havskov, 1989); 11, Petatlan, Guerrero, México (Rodriguez et al., 1983); 12, Carolina del Sur, U.S.A. (Reha, 1984); 13, Nagano occidental, Japón (Kosuga, 1992); 14, corteza superior en Ashio, Japón (Baskoutas and Sato, 1989); 15, corteza superior en Nagano occidental, Japón (Kosuga, 1992). Extractada de Sato y Fehler, 1998.

próximas al ruido de fondo, por lo que el inicio puede ubicarse en un tiempo anterior, tomando la precaución de evitar las ondas directas (Herraiz and Espinosa, 1986; Ibáñez et al, 1993). Ibáñez et al. (1993) observaron que basta con fijar el inicio de la coda luego del instante de máxima amplitud del sismograma donde el decaimiento se torna regular extendiéndose hasta donde la señal se confunde con el ruido de fondo. De este modo la coda constituye en general, más del $60 \%$ del sismograma en el rango de frecuencias de $2 \mathrm{~s}$ hasta $50 \mathrm{~Hz}$.

Rautian y Khalturin (1978) fueron los primeros en concluir que un único $Q_{c}$ no alcanzaba a describir la envolvente de coda completa ya que sistemáticamente cambiaba su forma de decaimiento con el lapso de tiempo. En todos los trabajos citados se ha encontrado que $Q_{c}$ crece con el lapso de tiempo, por lo que la mayoría de los investigadores han sugerido que la energía que llega hacia el final de la coda ha atravesado regiones con menor atenuación que su predecesora. De este modo se sugería una dependencia de la atenuación con la 
profundidad (Del Pezzo et al., 1990). Como ya se ha mencionado en la Sección 2.2.3.2, Novelo-Casanova y Lee (1991) concluyeron, mediante datos sintéticos, que el SBS y el SIS aproximan razonablemente bien las envolventes de coda dando valores casi iguales de $Q_{c}$ cuando los lapsos de tiempo considerados son los mismos. Ibáñez et al. (1991) observaron que ambos métodos coinciden para distancias epicentrales pequeñas $(<70 \mathrm{~km})$, pero si estas aumentan, comienzan a aparecer discrepancias en los resultados. La variación temporal de $Q_{c}$ fue reportada por Chouet (1979) a lo largo de un año de observación pero no pudo correlacionarlo con la actividad sísmica. Jin y Aki (1986), Guzev y Lemzikov (1985) entre otros, analizando variaciones de codas regionales por períodos de muchos años, detectaron un mínimo de $Q_{c}$ unos meses antes de la ocurrencia de grandes terremotos. Sin embargo no ha habido nuevos resultados que confirmen esta propiedad.

Tradicionalmente se estima el valor de $Q_{\mathrm{c}}$ mediante un ajuste lineal por cuadrados mínimos a las envolventes de coda, previa transformación logarítmica de la ecuación. Sin embargo Ibáñez (1990) e Ibáñez et al. (1993) puntualizaron que si los datos observados tienen una distribución de errores de tipo Gaussiana, la transformación de tipo log-log de la función no lineal que los representa, tendrá una distribución de errores no-Gaussiana. Esto viola una de las hipótesis del ajuste por mínimos cuadrados, pudiendo resultar en una distorsión de los resultados. Por otra parte, la linealización logarítmica no resulta ser una buena elección cuando se tiene la misma precisión en la medida de las amplitudes en función de la variable independiente, el tiempo. El logaritmo amplifica la dispersión de las amplitudes cercanas a cero. Este es el caso de la envolvente de coda, donde al aumentar el lapso de tiempo, disminuye la relación señal-ruido y por lo tanto aumenta el error en la estima del logaritmo de la amplitud. Los autores propusieron, entonces, un ajuste por un método no-lineal como puede ser Gauss-Newton, donde además puede estimarse el intervalo de confianza de la estima y el coeficiente de correlación que da la bondad del ajuste. De la comparación entre ambos métodos de ajuste, surgió que, el método no lineal aproxima mejor a los datos tanto al inicio como hacia el final de la coda (Ibáñez, 1990, Ibáñez et al., 1993).

Como sugiriera Tsujiura (1978), el valor de $Q_{c}$ es medido usualmente sobre los sismogramas filtrados a varias frecuencias seleccionadas y con un ancho de banda de una octava. Esto significa que los valores obtenidos de $Q_{c}$ representan en realidad el factor de calidad promedio en un intervalo de frecuencia. Si bien las expresiones teóricas del método son estrictamente válidas en cada onda monocromática, esto es imposible de considerar en la práctica debido a la pérdida de estabilidad en el filtrado de los datos en bandas muy estrechas. Dado que las longitudes de coda y los rangos de frecuencia varían según los trabajos y 
regiones analizados, han de tomarse las precauciones debidas al momento de comparar resultados, ya que las dependencias observadas resultan estar fuertemente relacionadas con los parámetros de la determinación (Del Pezzo et al., 1990, Ibáñez, 1990).

En el tratamiento del scattering (Sección 2.1.2) se vio que el factor de calidad se relaciona con la distribución y dimensiones de las heterogeneidades y debido a eso muestra una dependencia con la frecuencia. $Q_{c}^{-1}$ toma valores del orden de $10^{-2}$ para $1 \mathrm{~Hz}$ y decrece a $10^{-3}$ para frecuencias cercanas a $20 \mathrm{~Hz} . Q_{c}^{-1}$ es generalmente mayor en regiones volcánicas y en la corteza superior. Los ajustes realizados de $Q_{\mathrm{c}}$ en todas las regiones (Fig. 2.17) muestran una dependencia con la frecuencia que puede expresarse a través de la relación (2.21). Es común hallarla expresada de la forma

$$
Q_{c}=Q_{0 c} f^{n}
$$

a pesar de ser dimensionalmente incorrecta, donde $Q_{0 c}$ es el valor de $Q_{c}$ evaluado a la frecuencia $f_{0}=1 \mathrm{~Hz}, n$ es el exponente que define la dependencia con $f$. Esta expresión es válida para $f>1 \mathrm{~Hz}$, ya que para frecuencias menores se han encontrado valores de $Q_{\mathrm{c}}$ casi constantes. Los valores de $n$ ajustados se encuentran entre 0.5 y 1 en la mayoría de los casos estudiados (Sato y Fehler, 1998). La comparación de los valores de $Q_{0 \text { c }}$ obtenidos para diferentes regiones ha permitido ver claramente que $Q_{0 \mathrm{c}}$ es mayor en áreas tectónicamente estables y menor en áreas activas.

En algunas regiones, contando con datos de varias estaciones correspondientes a eventos bien distribuidos, se han observado variaciones laterales y en profundidad de la atenuación de ondas coda. Pueden encontrarse en la bibliografía criterios muy diferentes entre sí para la regionalización de los valores de $Q_{0 \mathrm{c}}$ estimados. Sin embargo, debe quedar claro que las ondas coda han sufrido scattering y no provienen directamente de la fuente sismogénica sino que han barrido un área más amplia que la que se encuentra entre el hipocentro y la estación. Una manera sencilla de establecer una relación entre las coordenadas de un mapa y los valores de $Q_{0 \text { c }}$ consiste en realizar un promedio de los valores obtenidos para cada estación dentro de un cierto radio de distancia epicentral. Otra forma consiste en asignar los valores obtenidos de $Q_{0 \mathrm{c}}$ al punto medio de la trayectoria. Una tercera regionalización se funda en el modelo de scattering simple de Aki (1969) y ha sido aplicada por ejemplo por Pulli (1984) y Havskov et al. (1989). En ella se supone que las ondas coda que llegan a una estación en un cierto intervalo de tiempo, han sido afectadas por las heterogeneidades que se encuentran en un volumen encerrado en el elipsoide con focos en el hipocentro y la estación y semieje mayor $v t / 2$, permitiendo además estimar la profundidad 
máxima a la que se asigna cada valor de $Q_{c}$.

Los métodos basados en el scattering múltiple han sido utilizados pero no en la misma proporción que los de scattering simple, al menos hasta hace un par de décadas cuando la mayor velocidad de cálculo de curvas teóricas agilizó su implementación. Frankel y Wennerberg (1987) probaron el método de FE en California; Wu y Aki (1988) aplicaron el método de TR para determinar la atenuación en la región del Hindu-Kush; Matsunami y Nakamura (2004) estimaron la atenuación en el sudoeste de Japón empleando el método híbrido de SDZ de Zeng (1991). Wegler (2004) propuso condiciones de contorno para la ecuación de difusión consiguiendo una teoría más simple que las existentes hasta ese momento y que resulta de utilidad especialmente en volcanes. Dada la alta heterogeneidad que caracteriza a los terrenos en zonas volcánicas, el modelo de difusión resulta en un buen ajuste del decaimiento de las ondas coda generadas en ellos (Wegler, 2004; McNutt, 2005; Yoshimoto et al., 2006; Del Pezzo, 2008). Los métodos que consideran el scattering múltiple han sido una importante contribución al entendimiento de los procesos que ocasionan la atenuación y han dado origen a nuevas técnicas que permiten separar sus efectos.

\subsection{ATENUACIÓN DE ONDAS DIRECTAS}

\subsubsection{ALGUNAS PROPIEDADES DE LA ATENUACIÓN DE ONDAS DIRECTAS}

Como se ha visto en la Sección 2.1 a medida que un pulso viaja a través de un medio heterogéneo o anelástico pierde energía que puede ser transferida hacia otras partes del campo de onda por scattering o hacia el medio en forma de calor por anelasticidad (Wennerberg y Frankel, 1989).

Las primeras observaciones de la atenuación de ondas superficiales se atribuyen a Ewing y Press (1954) cuando analizaban las ondas Rayleigh del terremoto de Kamchatka en el manto. Brune (1962) demostró que debía usarse la velocidad de grupo para la determinación del factor $Q$ lo que fue corroborado por Bloch, Hales y Landisman (1969) quienes comprobaron que las velocidades de grupo eran más sensibles que la velocidad de fase a los pequeños cambios en los parámetros físicos del medio.

Knopoff (1962) no dudó en que la pérdida de energía de las ondas sísmicas en el interior terrestre se debía a la atenuación intrínseca y analizando datos de ondas internas, ondas superficiales y oscilaciones libres encontró que el manto superior tenía mayor atenuación que 
el manto inferior.

Los valores de $Q$ de ondas internas ( $Q_{P}$ correspondiente a ondas $\mathrm{P}$ y $Q_{S}$ para ondas $\mathrm{S}$ ) en el interior terrestre pueden expresarse en función de los factores $Q_{\mu}$ y $Q_{K}$ que se relacionan a su vez, con los módulos de rigidez $(\mu)$ y compresibilidad $(K)$ en medios anelásticos (Udías Vallina, 1999)

$$
\begin{aligned}
\frac{1}{Q_{S}} & =\frac{1}{Q_{\mu}} \\
\frac{1}{Q_{P}} & =\frac{4}{3}\left(\frac{v_{S}}{v_{P}}\right)^{2} \frac{1}{Q_{\mu}}+\left[1-\frac{4}{3}\left(\frac{v_{S}}{v_{P}}\right)^{2}\right] \frac{1}{Q_{K}}
\end{aligned}
$$

Es común suponer que no hay disipación de energía en los procesos compresivos y por eso $Q_{K} \approx \infty$. En un sólido de Poisson esto significaría que $Q_{P}=9 / 4 Q_{S}$.

A partir de las observaciones de la atenuación de ondas internas telesísmicas, ondas superficiales y oscilaciones libres del planeta y con un modelo de simetría radial, se construyeron los primeros modelos de $Q$ para toda la Tierra (Anderson et al., 1965, Anderson y Hart, 1978) que se denominaron MM8 y SL8. En estos modelos se planteaba una velocidad que era independiente de la atenuación y mantenía su distribución constante. En la actualidad, se utilizan inversiones simultáneas de atenuación y de velocidad mediante técnicas tomográficas (por ej.: Roth et al., 1999, Romanowicz, 2003). El modelo SL8, se caracteriza por valores de $Q_{\mathrm{S}}$ entre 200 y 500 para la litósfera, valores más bajos, cercanos a 110, en el manto superior y un manto inferior con valores de $Q_{\text {s }}$ que crecen desde 50 hasta 500 con la profundidad. $Q_{\mathrm{S}}$ es nulo en el núcleo externo y creciente en el núcleo interno (400-800). $Q_{\mathrm{K}}$ se considera tendiendo a infinito en el manto pero igual a $Q_{\mu}$ en el núcleo interno. La atenuación en el interior terrestre se asocia principalmente a la disipación de energía en las ondas de corte. La atenuación de los modos normales de la Tierra ha mostrado que $Q_{\mathrm{K}}$ no es realmente infinito. En los modelos globales basta con suponer un $Q$ dependiente con la profundidad y constante con la frecuencia. En cambio, esta suposición no es válida en el análisis de ondas internas de corto período.

En la litósfera, se han elaborado modelos promedio de $Q$ que indican una atenuación alta en corteza $(Q \approx 160)$, una atenuación moderada en la litósfera inferior ( $Q \approx 500)$ y una mayor atenuación en la capa de baja velocidad que limita la litósfera $(Q \approx 125)$. Los valores en corteza pueden mostrar fuertes variaciones laterales dependiendo del grado de heterogeneidad $(120<Q<600)$ (Udías Vallina, 1999). En zonas volcánicas los valores de $Q$ 
pueden ser aún mucho más bajos $(12<Q<46)$ (Martínez Arévalo, 2005) lo que puede asociarse tanto a la heterogeneidad de la estructura volcánica como a la presencia de altas temperaturas y contenido de fluidos (por fusión o inserción de agua en el sistema).

En un pulso, la atenuación se manifiesta a través de una disminución de amplitud y un ensanchamiento de la forma de onda por la desigual absorción entre altas y bajas frecuencias. En un sismograma puede verse un aumento de la duración de la envolvente del sismograma con la distancia. (Sección 1.1). Comparando sismogramas de terremotos de magnitud local menor que 5 (con una duración de la fuente menor que 1 s), Sato (1989) observó que la duración del primer paquete de ondas $\mathrm{S}$ era mayor y aumentaba con la distancia. Las envolventes de sismogramas filtrados a diferentes frecuencias, mostraban un retraso entre los tiempos de arribo de la máxima amplitud y su mitad que aumentaba con la distancia. Esta variación, indicaba, no podía ser efecto de la fuente, pero tampoco podía ser efecto de sitio en la estación dado que el emplazamiento era en roca firme.

Utilizando características como el retraso de las ondas en medios con atenuación, la pérdida de contenido en frecuencia, la disminución de amplitudes con la distancia o el tiempo, se han desarrollado numerosos métodos de determinación de la atenuación sobre ondas $\mathrm{P}, \mathrm{S}$ y superficiales. Las redes globales de estaciones sismológicas facilitaron las observaciones de las ondas superficiales en su propagación, lo que dio lugar al desarrollo de métodos de determinación de la atenuación en las capas más superficiales de la estructura terrestre, entre ellos los métodos con datos de una o dos estaciones (Ben-Menahem, 1965, Tsai y Aki, 1969). Los métodos basados en la observación del decaimiento espectral de las ondas se popularizaron y diversificaron con el inicio de la registración digital. Estos métodos pueden implementarse con datos de una única estación, analizando las amplitudes en función de la frecuencia para varios terremotos o bien para al menos dos estaciones en la misma región y el mismo terremoto.

Otras medidas se basan en la observación de cambios en la amplitud de la onda directa con la distancia utilizando datos de una única estación, como es el método de Normalización de la Coda (Aki, 1980a) inspirado en mediciones hechas por Aki y Chouet (1975) sobre ondas P y por Rautian y Khalturin (1978) para ondas S. A partir de sus resultados acerca de la atenuación de ondas S y coda y sustentado en las observaciones de Tsujiura (1978) sobre determinaciones de efecto de sitio realizadas con ondas $\mathrm{P}, \mathrm{S}$ y coda, el método de la Normalización de la Coda llevó a Aki (1980a) a concluir que las ondas coda estaban compuestas por ondas $\mathrm{S}$ dispersadas y sin cambio de naturaleza. Este método resulta ser de 
gran utilidad para la corrección de la anisotropía de la radiación en la fuente y los efectos de la propagación sobre las señales registradas cuando se pretende determinar el tamaño de un terremoto a través del espectro de radiación (Aki, 1967, Brune, 1970) y también para la eliminación del patrón de radiación y el efecto de sitio en los métodos de separación de la atenuación intrínseca y de scattering (Gao, 1984, Hoshiba et al., 1991).

Las investigaciones hechas en el campo de la acústica con la propagación de ondas de alta frecuencia en diferentes materiales (Ricker, 1953, Knopoff, 1964) fueron extrapoladas a las frecuencias sísmológicas para mejorar los criterios de lectura de tiempos de llegada de las ondas y a su vez permitieron medir su atenuación (Gladwin y Stacey, 1974b).

\subsubsection{MÉTODOS DE DETERMINACIÓN DE Q EN ONDAS DIRECTAS}

En los siguientes párrafos se repasarán los fundamentos y las expresiones de algunos de los métodos más utilizados en la bibliografía para determinar la atenuación de las ondas directas en sismogramas locales y/o regionales y que serán aplicados en este trabajo. Existen sin duda otras técnicas que podrían aplicarse pero de acuerdo a la distribución de sismicidad y de estaciones y a fin de comparar con resultados obtenidos en otras regiones se ha hecho esta selección. En primer lugar se presenta el Método del Ancho del Primer Pulso de P (Gladwin y Stacey, 1974b) que observa cómo disminuye la pendiente del primer pulso del desplazamiento con la distancia (una onda impulsiva se vuelve emergente) y lo relaciona con la atenuación. Los Métodos Espectrales que analizan el decaimiento del espectro de amplitud del desplazamiento en una estación, son representados en este trabajo por un par de adaptaciones del método de Tsujiura (1966). El Método de las Dobles Razones Espectrales (Chun et al., 1987) es una modificación del Método de las Dos Estaciones (Tsai y Aki, 1969) y permite estudiar la atenuación de ondas superficiales o internas sin necesidad de conocer patrones de radiación, efectos de sitio ni respuestas instrumentales. El Método de Normalización de la Coda (Aki, 1980a) permite estimar la atenuación de las ondas directas con datos de una estación, eliminando los efectos del patrón de radiación, la respuesta instrumental y el efecto de sitio mediante la normalización por el espectro de potencia de la coda previamente calculado y efectuando promedios en distancia y azimut. Para finalizar se resumen algunos de los resultados obtenidos en distintas regiones del planeta.

\subsubsection{MÉTODO DEL ENSANCHAMIENTO DEL PRIMER PULSO DE P (APP)}

Ricker (1953) se planteó dos preguntas: “¿Cuál es la forma de una perturbación sísmica 
que parte de la explosión de una carga de dinamita en la tierra y cuáles son las leyes de la propagación de dicha perturbación? A partir de la ecuación de onda de Stokes para un sólido de Kelvin-Voigt observó que la densidad y la viscosidad afectaban la forma de la onda y determinaban su propagación. Las funciones halladas por Ricker (1953) corresponden al comportamiento de la tierra cuando el desplazamiento en la fuente es impulsivo y describen la manera en que varía su forma, ancho y amplitud con la distancia recorrida desde la fuente.

Knopoff (1964) resumió las mediciones experimentales de atenuación realizadas en diferentes tipos de medios concluyendo que para una variedad de fluidos se observaba una dependencia de la atenuación con la frecuencia del tipo $Q^{-1}(\omega) \propto \omega$, mientras que en los sólidos la atenuación resultaba prácticamente constante en el intervalo de frecuencias observadas, $Q^{-1}(\omega) \propto$ cte. A partir de la suposición de un $Q$ constante con la frecuencia se desarrollaron varios métodos para su determinación.

Mediciones precisas hechas in situ sobre pulsos de ondas de presión propagándose en rocas (Gladwin y Stacey, 1974a) mostraron que además de la atenuación de la amplitud del desplazamiento con la distancia, se producía un alargamiento temporal del pulso que causaba ambigüedad en la lectura del tiempo de llegada del mismo. En particular, observaron que el tiempo de subida (o en inglés rise time) crecía linealmente con la distancia de propagación. A partir de la tangente al pulso en el punto de mayor pendiente, definieron el tiempo de subida, $\tau$, como el intervalo entre su extrapolación al cruce por cero y a la máxima amplitud del pulso. Estas observaciones eran útiles en la detección automática de eventos ya que permitían definir los criterios de selección de los niveles de disparo. Sin embargo, debía comprobarse la veracidad de la relación para el caso de medios atenuados antes de generalizarla. Gladwin y Stacey (1974b) ampliaron el número de mediciones incorporando medios atenuados y comparándolas con modelos teóricos de propagación de pulsos como los de Ricker (1953), Azimi et al. (1968) y otros. Sus mediciones fueron hechas a menos de $10 \mathrm{~m}$ de distancia de la fuente y con tiempos de elevación menores que $60 \mu \mathrm{s}$. Las mediciones confirmaron una relación lineal entre el tiempo de subida del primer pulso de una onda de presión y su tiempo de propagación, $t_{P}$. Intuitivamente, resulta lógico pensar que a menor $Q$ el pulso se deformará más rápidamente en la propagación debido a la mayor absorción de energía. Para cuantificar esta relación entre el tiempo de viaje, el tiempo de subida y la atenuación, los autores determinaron los valores de $Q$ mediante el método de la razón espectral simple (Sección 2.3.2.4). La posibilidad de que $\tau$ aumentara en función de las variaciones de $Q$ fue descartada dado que $Q$ variaba poco en los medios observados. Planteando una variación con el tiempo 
de viaje observaron una dependencia de la forma

$$
\tau=\tau_{0}+\frac{C}{Q} t_{P}
$$

donde $\tau$ es el tiempo de subida en el registro, $\tau_{0}$ es su equivalente en la fuente, $t_{P}$ es el tiempo de viaje de la onda y $C$ es una constante cuyo valor experimental resultó igual a $0.53 \pm 0.04$. Como el efecto de la atenuación es lineal, se aplica a cualquier elemento del camino sísmico y por lo tanto se puede generalizar para un medio con atenuación variable y velocidad variable (Stacey et al., 1975)

$$
\tau=\tau_{0}+C \int_{\text {rayo }} \frac{d s}{v Q}=\tau_{0}+C \int_{\text {rayo }} \frac{d t}{Q}
$$

donde $d s$ es un elemento de la trayectoria, $v$ es la velocidad de propagación, $d t=d s / v$ y $Q$ es el factor de calidad de la onda que se asume independiente de la frecuencia.

Al reunir las mediciones de $\tau_{0}$ efectuadas en diferentes estaciones, debe asegurarse que el contenido en frecuencia del pulso no dependa de las condiciones de registro. El conocimiento de la sensibilidad del instrumento no es de importancia ya que se miden tiempos y no amplitudes. Por esta razón este método requiere como mínimo los mismos anchos de banda instrumental en las estaciones usadas y la independencia acimutal del patrón de radiación de la fuente. La ventaja del método del ancho del pulso es que sólo necesita una pequeña porción inicial de la señal evitando la contaminación por ondas secundarias pero de una longitud suficiente tal que pueda suponerse representado todo su espectro. Entre las limitaciones se cuentan la necesidad de registros con primeros pulsos muy claros que permitan estimar el tiempo de subida y el requisito de mecanismos de fuente similares para todos los eventos considerados.

Comparando con los modelos teóricos de propagación de pulsos, Gladwin y Stacey (1974b) observaron que el modelo de Azimi et al. (1968) era el que mejor ajustaba a sus observaciones. Azimi et al. (1968) consideraban medios con $Q$ y $v$ independientes de la frecuencia. El modelo de Ricker (1953) quedaba descartado por dos motivos, en primer lugar, su relación entre el ancho del pulso y el tiempo de propagación, dada por $\tau \propto t^{1 / 2}$, no ajustaba a las observaciones y en segundo lugar, resultaba ilógico aceptar que la atenuación aceleraba la propagación de una parte del pulso como su desarrollo proponía. Gladwin y Stacey (1974b) analizaron también la validez de modelos no dispersivos, con $Q$ independiente de la frecuencia y donde vale el principio de superposición lineal de los efectos de la atenuación 
sobre los armónicos que conforman un pulso. Estos modelos resultan en un ensanchamiento del pulso y en una aceleración de parte del mismo de modo que llega a la estación en un tiempo menor que el tiempo de propagación correspondiente a medios elásticos. Esta violación de la causalidad se evita introduciendo cierta dispersión y una dependencia débil de $Q$ con la frecuencia. Las ecuaciones de Kramers-Kronig permiten reconstruir la atenuación a partir del comportamiento dispersivo y viceversa ya que ambas cantidades están relacionadas por un par de transformadas de Hilbert. A tal fin es suficiente derivar las relaciones de Kramers-Kronig para el número de onda complejo de la respuesta de onda plana (Sato et al., 2009).

El valor de la constante $C$ fue determinado teórica y experimentalmente por diversos autores. Kjartansson (1979) retomaría la idea del $Q$ constante pero incorporando la dispersión y concluyendo que la relación (2.71) es válida para registros en desplazamiento, velocidad y aceleración pero con valores de $\mathrm{C}=0.485,0.298,0.217$ respectivamente. Blair y Spathis (1982) aplicando el modelo de Kjartansson observaron que la constante $C$ debía ser dependiente de la fuente y que su discrepancia con Gladwin y Stacey (1974) se basaba en las extremadamente cortas distancias usadas por estos autores así como la falta de consideración de los errores de medición. Para asegurar la validez del modelo es conveniente seleccionar eventos correspondientes a fuentes con mecanismos idénticos para asegurar un único $\tau_{0}$.

Wu y Lees (1996) sostuvieron, mediante un detallado modelado numérico, que podían utilizar un valor de $\mathrm{C}=0.5$ al tratar con registros en velocidad de microsismos cuyas fuentes se suponían desplazamiento impulsivos. Su análisis se basó en que las fuentes sísmicas suelen modelarse como un escalón de dislocación en la falla. En el campo lejano, el desplazamiento es entonces proporcional a la velocidad de partículas en el plano de falla. De este modo, puede decirse que un escalón de desplazamiento en la fuente producirá un desplazamiento impulsivo en la estación. Si no se conoce con certeza el valor de C que mejor se adapta a la fuente considerada, puede hacerse la inversión de (2.71) para $Q / C$. El valor promedio de $Q / C$ puede determinarse a través de un ajuste por mínimos cuadrados al ancho del pulso en función del tiempo de propagación. En el caso de una tierra heterogénea, Wu y Lees (1996) plantearon diferentes aproximaciones para el ajuste. Partiendo de un valor promedio del factor de calidad igual a $Q_{0}$, pueden representarse los apartamientos respecto del mismo como $\Delta Q$ de modo que $Q=Q_{0}+\Delta Q$, entonces

$$
\tau=\tau_{0}+\frac{C t}{Q_{0}}-\frac{C}{Q_{0}^{2}} \int_{\text {rayo }} \Delta Q \cdot d t
$$


de otro modo, puede usarse la (2.72) para invertir $Q$ y $\tau_{0}$, o bien $Q_{0} / C$ para luego estimar $\tau_{0}$

$$
\tau-\tau_{0}=C \int_{\text {rayo }} \frac{1}{Q v} d s
$$

Existen diferentes definiciones de lo que se entiende como $\tau$, además de la propuesta originalmente por Gladwin y Stacey (1974). Sin embargo no se han observado variaciones en los valores de Q estimados de acuerdo a la definición adoptada. Si la relación señal-ruido es baja o el ancho de banda es estrecho, el inicio del pulso puede ser difícil de determinar y se introducirá un error importante, sobre todo si los pulsos son cortos. Wu y Lees (1996) definieron el tiempo de subida como el cociente entre la máxima amplitud alcanzada y la máxima pendiente del primer cuarto de ciclo del pulso registrado en desplazamiento. El tiempo de subida en desplazamiento es aproximadamente igual al ancho del medio pulso medido en velocidad, ya que lo que tarda el desplazamiento en alcanzar su máxima amplitud es lo que tarda la velocidad en anularse luego de haber pasado por un máximo. Así, mientras para registros en desplazamiento debe usarse el tiempo entre el arribo y el primer máximo; para registros en velocidad, se usará el tiempo entre el arribo y el primer cruce por cero y para registros en aceleración, el tiempo entre el arribo y el segundo cruce por cero. De este modo Wu y Lees (1996) definieron el ancho del pulso en sismogramas en velocidad como el tiempo que se extiende entre la intersección de la extrapolación de la máxima pendiente de elevación del pulso con la línea base y el primer cruce por cero de la señal (Fig. 2.18).

Jongmans (1991) analizó numéricamente la evolución del pulso en las cercanías de la

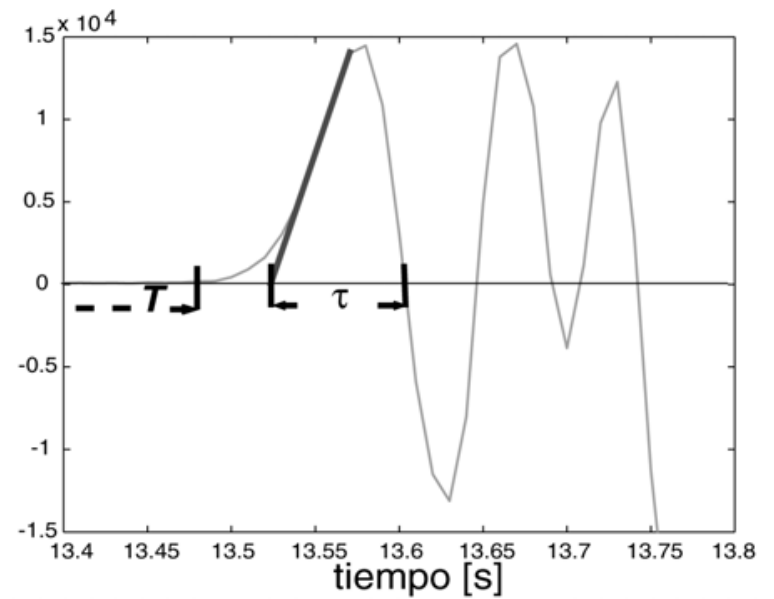

Fig. 2.18. Definición del ancho del pulso en un registro en velocidad según Wu y Lees (1996). T es el tiempo de arribo de la onda $y$ tes el ancho del pulso (ver texto para mayor explicación) fuente para estimar las limitaciones del método. Supuso una fuente en un medio infinito y en un semiespacio en ambos casos con $Q$ constante. Observó que la forma del pulso cambia cerca de la fuente independientemente de la atenuación del medio y que el tiempo de subida varía con la profundidad de la fuente debido al campo cercano y a la generación de ondas Rayleigh. De esta manera estimó las distancias hipocentrales donde la forma del pulso es controlada principalmente por el 
campo de desplazamiento cercano y/o el inicio de ondas superficiales, recomendando el uso del método del ancho del pulso para distancias mayores que 1.2 veces las longitudes de onda analizadas.

\subsubsection{MÉTODO ESPECTRAL (ME)}

Bajo la misma denominación de Método Espectral, pueden hallarse en la bibliografía diversas técnicas para calcular el valor del factor de atenuación (Tsujiura, 1966, Joyner and Boore, 1981, Cormier, 1982, Anderson and Hough, 1984, Rietbrock, 2001, Havskov, 2005, Giampiccolo et al., 2007, Mancilla et al., 2008 entre otros). Los métodos espectrales se basan en la observación de la forma característica que tiene el espectro de amplitud del desplazamiento producido por las ondas sísmicas. La interpretación de los espectros de los sismogramas depende en cierta manera del modelo de la fuente (Stein y Wysession, 2003) (Sección 1.2).

Si el medio donde se propagan las ondas es elástico, isótropo y homogéneo, la señal que llega a la estación conservará intacta la firma de la fuente, viéndose afectada únicamente por la expansión geométrica. Ya en la estación deberá considerarse el efecto de sitio y la respuesta instrumental. Si bien ambos pueden estimarse o bien eliminarse mediante cocientes espectrales, es importante tener en cuenta que la banda de paso de la estación debe ser lo suficientemente ancha como para no perder componentes espectrales debido a las limitaciones instrumentales. Asumiendo un medio heterogéneo y anelástico, debe tenerse en cuenta además el efecto de la atenuación con la distancia. El espectro de amplitud del desplazamiento de las ondas directas registradas en una estación puede expresarse mediante

$$
A(\omega, r)=R(\theta, \phi) S(\omega) G(\omega, \theta, \phi) I(\omega) r^{-1} e^{-\frac{\omega r}{2 Q v}}
$$

donde $r=v t$ es la distancia hipocentral, $\omega$ y $v$ son respectivamente la frecuencia angular y la velocidad promedio de las ondas $\mathrm{S},(\theta, \phi)$ son las coordenadas del punto de observación, $R(\theta, \phi)$ representa la dependencia del patrón de radiación con la dirección de propagación, $S(\omega)$ es la función fuente, $G(\omega, \theta, \phi)$ es el efecto de sitio en la estación, $I(\omega)$ es la respuesta de instrumento, $r^{-1}$ aproxima el efecto de la expansión geométrica por tratarse de ondas de cuerpo y $Q$ es el factor de calidad que se asigna a la trayectoria de la onda. Una vez corregida la señal por el efecto de sitio, la respuesta instrumental y la expansión geométrica, la expresión de la amplitud del desplazamiento en función del tiempo de viaje y la frecuencia tiene la forma 


$$
A(\omega, t)=R(\theta, \phi) S(\omega) e^{-\frac{\omega t}{2 Q}}
$$

Aplicando logaritmos naturales en ambos miembros de (2.76) queda

$$
\ln (A(\omega, t))=\ln (R(\theta, \phi) S(\omega))-\frac{\omega t}{2 Q}
$$

En la Sección 1.2 se ha visto que en un gráfico bi-logarítmico, la función fuente puede aproximarse en el campo lejano por una función con amplitud constante para $\omega<\omega_{\mathrm{c}}$ y una pendiente $\omega^{-2}$ para $\omega>\omega_{\mathrm{c}}$. Reemplazando $f=(\omega / 2 \pi)$, esto significa que para $f<f_{\mathrm{c}}$ y en ausencia de atenuación, la amplitud espectral debería ser constante (Fig. 2.19). Por otra parte, en un medio con atenuación, el logaritmo natural del espectro de amplitud del desplazamiento varía con la frecuencia proporcionalmente a $-t /(2 Q)$ aún para $f<f_{\mathrm{c}}$. Este decaimiento observado en los espectros de desplazamiento permite estimar un factor de calidad independiente de la frecuencia trabajando por debajo de la frecuencia de corte.

Para frecuencias mayores que la frecuencia de corte debe tenerse en cuenta el decaimiento de la función fuente, por lo que debe asumirse un modelo que la represente. Si se asume el modelo de fuente circular de Brune (1970), el espectro de desplazamiento en la fuente de la onda $\mathrm{S}$ se expresa mediante

$$
S(f)=\frac{A_{0}}{1+\left(\frac{f}{f_{c}}\right)^{2}}
$$

donde $A_{0}$ es una constante proporcional al momento sísmico y $f_{c}$ es la frecuencia de corte. La consideración de la función fuente permite determinar los parámetros de la fuente a partir del espectro de amplitud del desplazamiento corregido del efecto de la atenuación (Fig. 2.19) o bien modelar ambos efectos simultáneamente por métodos numéricos. El factor de calidad puede determinarse como un valor medio constante para todas las frecuencias o admitiendo su posible dependencia con ellas. En este trabajo se ha elegido ajustar el factor de calidad en cada frecuencia empleando el método de Tsujiura (1966) a través de cocientes de amplitudes espectrales sobre cada sismograma. Se explican a continuación la adaptación de Giampiccolo et al. (2007) al método de Tsujiura (1966) y una nueva adaptación de la misma propuesta en esta tesis. 


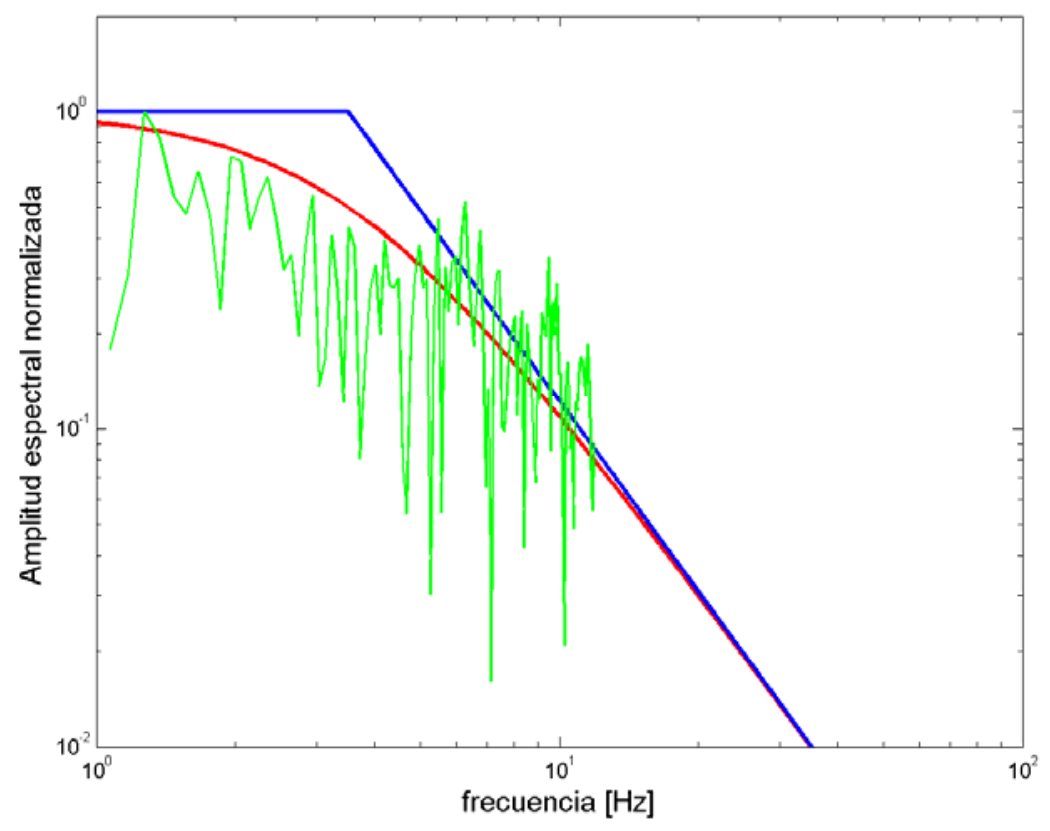

Fig. 2.19.- Aproximaciones teóricas al espectro de amplitud registrado (verde) en función de $f=\omega /(2 \pi)$. Se muestran la función fuente teórica con amplitud constante para frecuencias menores que la de corte, $f_{c} y$ pendiente $f^{2}$ para $f>f_{c}$ (azul) y el modelo de fuente de Brune (1970) (rojo). El decaimiento de la amplitud espectral para $f<f_{c}$ muestra el efecto de la atenuación.

Sea la expresión

$$
A_{k}\left(f_{i}, r_{j}\right)=R_{k}(\theta, \phi) S_{k}\left(f_{i}\right) G_{j}\left(f_{i}, \theta, \phi\right) I_{j}\left(f_{i}\right) r_{j}^{-1} e^{-\frac{\pi f_{i} t_{j}}{Q_{i}}}
$$

que representa la amplitud del espectro de desplazamiento de las ondas internas ( $\mathrm{P}$ o S) del $k$ ésimo terremoto registradas en una estación a una distancia $r_{j}$ luego de un tiempo de viaje $t_{j}=$ $r_{j} / v$ y a una frecuencia $f_{i}$. La amplitud espectral de las mismas ondas a una frecuencia de referencia $f_{1}$, distinta de $f_{\mathrm{i}}$, es

$$
A_{k}\left(f_{1}, r_{j}\right)=R_{k}(\theta, \phi) S_{k}\left(f_{1}\right) G_{j}\left(f_{1}, \theta, \phi\right) I_{j}\left(f_{1}\right) r_{j}^{-1} e^{-\frac{\pi f_{1} t_{j}}{Q_{1}}}
$$

donde se ha supuesto que los factores de calidad $Q_{i}$ y $Q_{1}$ no son necesariamente iguales. Haciendo el cociente entre (2.79) y (2.80) se eliminan el patrón de radiación y el efecto de la expansión geométrica que sólo dependen de la ubicación de la estación respecto de la fuente. Tomando logaritmos naturales se llega a

$$
\ln \frac{A_{k}\left(f_{i}, r_{j}\right)}{A_{k}\left(f_{1}, r_{j}\right)}=\ln \frac{S_{k}\left(f_{i}\right)}{S_{k}\left(f_{1}\right)}+\ln \frac{G_{j}\left(f_{i}, \theta, \phi\right)}{G_{j}\left(f_{1}, \theta, \phi\right)}-\pi\left(\frac{f_{i}}{Q_{i}}-\frac{f_{1}}{Q_{1}}\right) t_{j}
$$

donde $S_{k}\left(f_{i}\right) / S_{k}\left(f_{1}\right)$ varía con la fuente analizada y $G_{j}\left(f_{i}, \theta, \phi\right) / G_{j}\left(f_{1}, \theta, \phi\right)$ depende de la 
estación y de la dirección de propagación de las ondas, pero ambos cocientes son independientes del tiempo de viaje. El cociente $S_{k}\left(f_{i}\right) / S_{k}\left(f_{1}\right)$ puede considerarse independiente de la fuente si se admite que los eventos empleados tienen funciones fuente similares. Para ello se debe restringir la zona sismogénica y el intervalo de magnitudes considerados y seleccionar aquellos espectros de desplazamiento que presenten menos dispersión en su forma. A fin de admitir que $G_{j}\left(f_{i}, \theta, \phi\right) / G_{j}\left(f_{1}, \theta, \phi\right)$ es independiente de la dirección de propagación, puede hacerse el promedio de la expresión (2.81) para eventos distribuidos alrededor de la estación eliminando la dependencia azimutal del efecto de sitio. Una vez efectuado el promedio de la (2.81) sobre todos los $r_{j}$ disponibles para la estación $k$, se obtendrá una distribución de amplitudes en función del tiempo y para cada par de frecuencias $\left(f_{\mathrm{i}}, f_{1}\right)$, de la forma

$$
\left\langle\ln \frac{A_{k}\left(f_{i}, r_{j}\right)}{A_{k}\left(f_{1}, r_{j}\right)}\right\rangle=a-\pi\left(\frac{f_{i}}{Q_{i}}-\frac{f_{1}}{Q_{1}}\right) t_{j}
$$

que representa una recta con pendiente

$$
m_{i}=-\pi\left(f_{i} / Q_{i}-f_{1} / Q_{1}\right) \quad i=2, n
$$

La ordenada al origen, $a$, puede considerarse constante al hacer el promedio sobre todos los eventos con función fuente similar y distribuidos azimutalmente en forma homogénea. El valor de la pendiente puede obtenerse mediante un ajuste lineal por mínimos cuadrados.

Seleccionando un conjunto de frecuencias $f_{i}$ con $i=2,3, \ldots . n$, en un intervalo que depende del ancho de banda y de la frecuencia de muestreo en cada estación y evaluando la (2.82) para cada par de frecuencias $f_{i}, f_{1}$, se obtienen las pendientes $m_{i}\left(Q_{i}, Q_{1}\right)$ que forman un sistema de $n$-1 ecuaciones con $n$ incógnitas, por lo que se deberá incorporar al menos una condición sobre una de ellas.

En este paso es donde divergen las dos adaptaciones presentadas:

1) En esta aproximación el factor de calidad se considera constante en cada intervalo $\left[f_{1}-f_{\mathrm{i}}\right]$ (Giampiccolo et al., 2007). A esta aproximación se la llamará ME_L.

2) En este caso, se supone que el factor de calidad tiene una dependencia no lineal con la frecuencia del tipo de (2.21). A esta aproximación se la llamará ME_NL.

En el caso 1) suponiendo $Q_{\mathrm{i}}=Q_{1}$, el sistema de ecuaciones representado por la (2.83) se reduce a $n-1$ ecuaciones con 1 incógnita cada una 


$$
m_{i}=-\pi\left(f_{i} / Q_{i}-f_{1} / Q_{i}\right) \quad i=2, n
$$

que se resuelven despejando en forma individual cada valor de $Q_{\mathrm{i}}$

$$
Q_{i}=-\frac{\pi\left(f_{i}-f_{1}\right)}{m_{i}}
$$

Una vez estimados los factores de calidad en cada intervalo de frecuencia, los diferentes valores obtenidos permiten observar una variación del factor de calidad con la frecuencia a la que se ajusta una función $Q(f)$ dada por la relación (2.21).

En la segunda adaptación, la condición adicional impuesta sobre los valores del factor de calidad consiste en admitir, desde un principio, una relación $Q(f)$ del tipo de la (2.21). Haciendo la aproximación $Q_{i}=Q_{0}\left(f_{i} / f_{0}\right)^{\alpha}$ y $Q_{1}=Q_{0}\left(f_{1} / f_{0}\right)^{\alpha}$ el sistema de ecuaciones (2.83) queda de la forma

$$
m_{i}=-\pi Q_{0}^{-1} f_{0}^{\alpha}\left(f_{i}^{(1-\alpha)}-f_{1}^{(1-\alpha)}\right) \quad i=2, n
$$

que representa un sistema de $n$ - 1 ecuaciones no lineales con 2 incógnitas, $\left(Q_{0}, \alpha\right)$. Generando la función

$$
F\left(Q_{0}, \alpha\right)=\sum_{i=2}^{n-1}\left\{m_{i} / \pi-Q_{0}^{-1}\left[f_{1}^{(1-\alpha)}-f_{i}^{(1-\alpha)}\right]\right\}^{2}
$$

donde se ha supuesto $f_{0}=1 \mathrm{~Hz}$, la solución $\left(Q_{0}, \alpha\right)$ que minimiza la función $F\left(Q_{0}, \alpha\right)$ puede hallarse por métodos iterativos tipo gradiente (Gauss Newton) o tipo búsqueda (Búsqueda Aleatoria).

Una vez conocida la relación $Q(f)$ para cada estación, pueden corregirse los espectros de desplazamiento por atenuación y expansión geométrica para luego proceder al ajuste de la función fuente y estimar los parámetros de la misma.

Tanto en el ME_L como en el ME_NL ha debido asumirse una variación de $Q$ con la frecuencia. El ME_NL emplea una dependencia con la frecuencia (2.21) que ha sido observada en la gran mayoría de los análisis de atenuación sobre datos reales. El ME_L presupone, en cambio, una constancia de $Q$ en intervalos de frecuencia que varían su ancho a lo largo de la banda de paso de la estación $\left(\left[f_{1}-f_{2}\right],\left[f_{1}-f_{3}\right],\left[f_{1}-f_{4}\right], \ldots,\left[f_{1}-f_{n}\right]\right)$ para finalmente ajustar una dependencia de $Q$ con la frecuencia. En cada frecuencia $f_{i}$ el ME_L estima un valor promedio de $Q$ entre $f_{1}$ y $f_{i}$. Como se ha probado que $Q$ crece con la frecuencia, se supone que el ME_L subestimará los valores de $Q$ para las frecuencias más altas. 


\subsubsection{MÉTODOS DE LA SIMPLE y DOBLE RAZÓN ESPECTRAL (SRE y DRE)}

Los métodos SRE y DRE fueron diseñados originalmente para el análisis de ondas superficiales confinadas en corteza, conocidas como $L g$ que son características de los sismogramas regionales de corto período en zonas continentales. Las ondas $L g$ se observan a distancias epicentrales que varían desde $150 \mathrm{~km}$ o $500 \mathrm{~km}$ (según las observaciones realizadas por diversos autores como por ejemplo Chun et al., 1987 y Kulhánek, 2002) hasta varios miles de kilómetros. Se han modelado como ondas reflejadas múltiples veces en la corteza cuyo registro presenta una duración que depende de la estratificación de la corteza inferior (Campillo y Paul, 1992). Dainty y Toksöz (1990) observaron que la primera porción de las ondas $L g$ está compuesta por ondas que han sufrido scattering hacia adelante, mientras que el final se conforma de ondas afectadas por scattering omnidireccional. Según indican Sato y Fehler (1998) esta última característica requiere un modelo con heterogeneidad 3-D.

Utilizando amplitudes espectrales de ondas superficiales generadas por dos eventos diferentes para cada estación, Ben-Menahem (1965) propuso la determinación del coeficiente de atenuación espacial (ver ecuación 2.14) en función de la frecuencia, $\gamma(f)$, a través de la relación

$$
\gamma(f)=\left\{\ln \left[A\left(f, \Delta^{\prime}\right) / A(f, \Delta)\right]\right\} /\left(\Delta^{\prime}-\Delta\right)
$$

donde $\Delta^{\prime}$ y $\Delta$ son las distancias epicentrales y $A\left(f, \Delta^{\prime}\right), A(f, \Delta)$ son las respectivas amplitudes espectrales a la frecuencia estudiada. La expresión (2.88) debía ser modificada para compensar los efectos de la dispersión de las ondas superficiales y el valor de $Q$ podía calcularse mediante $Q=\pi f / \gamma U$ con $U$, la velocidad de grupo. La creación de la Red Global de Estaciones Sismológicas Estandarizadas (World Wide Standardized Seismographic Network, WWSSN) y las bases de datos sismológicos dieron la posibilidad de observar la atenuación de las ondas superficiales entre dos o más estaciones con suficiente distancia entre las mismas.

Tsai y Aki (1969) utilizaron el mismo método de Ben-Menahem (1965) pero extendido a dos estaciones por evento, de modo tal que permanecieran en el mismo círculo máximo para evitar la dependencia acimutal del patrón de radiación en la fuente. Lo denominaron Método de las Dos Estaciones. Al hacer los cocientes de amplitudes espectrales se elimina el patrón de radiación pudiendo obtenerse la atenuación promedio en el camino utilizado entre estaciones. La distancia entre las estaciones ha de ser relativamente grande pero manteniendo 


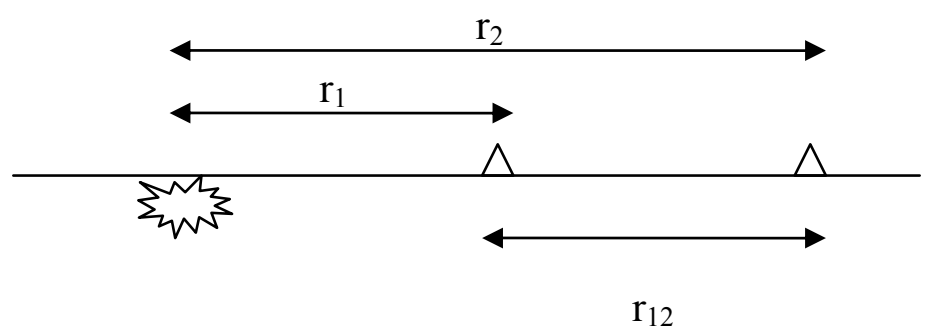

Fig. 2.20.- Diagrama de ubicación relativa de las dos estaciones (triángulos) y el epicentro utilizados en el método de la Simple Razón Espectral.

sus propiedades elásticas y anelásticas similares, sin importantes variaciones laterales, de modo que se puedan suponer efectos de sitio similares.

Si se dispone de amplitudes espectrales de un mismo terremoto observadas en dos estaciones ubicadas como en la Fig. 2.20, el espectro de amplitudes en la estación a menor distancia, $r_{1}$, estará dado por

$$
A\left(f, r_{1}\right)=A_{0}(f) R\left(\theta_{1}, \phi_{1}\right) G\left(f, \theta_{1}, \phi_{1}\right)\left(\sin \Delta_{1}\right)^{-1 / 2} I_{1}(f) e^{-\gamma_{1}(f) r_{1}}
$$

y en la estación a mayor distancia, $r_{2}$, será

$$
A\left(f, r_{2}\right)=A_{0}(f) R\left(\theta_{2}, \phi_{2}\right) G\left(f, \theta_{2}, \phi_{2}\right)\left(\sin \Delta_{2}\right)^{-1 / 2} I_{2}(f) e^{-\gamma_{2}(f) r_{2}}
$$

expresiones similares a la (2.75) donde $r_{1}, r_{2}$ son las distancias epicentrales en $\mathrm{km}$, $\Delta_{i}=r_{i} / 6371 \mathrm{~km}$ es la distancia epicentral en radianes y se ha reemplazado la función fuente $S(f)$ por $A_{0}(f)$ para denominar a la amplitud a distancia epicentral nula. La corrección por expansión geométrica queda representada a través de $(\sin \Delta)^{-1 / 2}($ ver Sección 1.2) por tratarse de ondas superficiales a distancias regionales (Tsai y Aki, 1969).

Considerando que la atenuación con la distancia es un proceso lineal puede suponerse que el efecto total en la trayectoria es igual a la suma de los efectos en los segmentos que la conforman. Es decir

$$
\gamma_{1} r_{1}+\gamma_{12} r_{12}=\gamma_{2} r_{2}
$$

donde $\gamma_{12}$ es la atenuación en el trayecto entre las estaciones que distan $r_{12}=r_{2}-r_{1}$. Esta suposición es válida si se considera que ambas trayectorias permanecen dentro del mismo círculo máximo de modo que las ondas han recorrido el mismo camino entre estaciones. Con las consideraciones anteriores, el cociente entre las amplitudes espectrales elimina el efecto de sitio, el patrón de radiación y el efecto de la atenuación en la trayectoria $r_{1}$. Tomando logaritmos naturales y despejando se llega a 


$$
\gamma_{12}(f)=\frac{\ln \left[\frac{A\left(f, r_{1}\right) I_{2}(f)\left(\sin \Delta_{1}\right)^{\frac{1}{2}}}{A\left(f, r_{2}\right) I_{1}(f)\left(\sin \Delta_{2}\right)^{\frac{1}{2}}}\right]}{r_{2}-r_{1}}
$$

Una vez conocida la atenuación entre estaciones a la frecuencia $f$, podrá calcularse el factor de calidad correspondiente mediante la relación $Q(f)=\pi f / \gamma_{12}(f) U_{12}(f)$ (Canas y Mitchell, 1981, Osagie, 1986). La velocidad de grupo entre estaciones $U_{12}(f)$ se estima mediante

$$
U_{12}(f)=\frac{r_{2}-r_{1}}{r_{2} / u_{2}-r_{1} / u_{1}}
$$

donde las velocidades de grupo para cada estación $\left(u_{2}, u_{1}\right)$ se calculan usando la técnica de filtrado múltiple (Dziewonski, Bloch y Landisman, 1969) a la vez que se estiman las amplitudes espectrales $\left[A_{1}\left(f, r_{1}\right), A_{2}\left(f, r_{2}\right)\right]$.

Existen variaciones en la implementación de este método que pueden verse en Mitchell (1995). Una de ellas es la propuesta por Chun et al. (1987) para eliminar la dependencia respecto de la respuesta instrumental y el efecto de sitio en la estación. Lo denominaron Reverse Two Stations Method (Método de las Dos Estaciones Inverso) que en español también se conoce como Método de la Doble Razón Espectral (DRE), quedando la denominación de Método de la Simple Razón Espectral (SRE) como nombre optativo del Método de las Dos Estaciones.

En la expresión (2.92) se observa que si el evento se encuentra al otro lado de las estaciones, el papel de las mismas en la ecuación puede intercambiarse. De este modo Chun et al. (1987) consideraron un par de fuentes cuyos epicentros permanezcan sobre el mismo círculo máximo que une a las estaciones y por fuera de ellas, con unos pocos grados de error, como se ilustra en la Figura 2.21. Si J y K son las estaciones y 1 y 2 las fuentes, haciendo el cociente de amplitudes espectrales registradas en ambas estaciones para cada fuente (respetando el orden lejano/cercano) y multiplicando ambos cocientes, se tiene la expresión

$$
\frac{A_{1 k}(f)}{A_{1 j}(f)} \cdot \frac{A_{2 j}(f)}{A_{2 k}(f)}=\frac{A_{01}(f) I_{k}(f) r_{1 k}^{-m} e^{-\gamma_{1 k} r_{1 k}}}{A_{01}(f) I_{j}(f) r_{1 j}^{-m} e^{-\gamma_{1 j} r_{1 j}}} \frac{A_{02}(f) I_{j}(f) r_{2 j}^{-m} e^{-\gamma_{2 j} r_{2 j}}}{A_{02}(f) I_{k}(f) r_{2 k}^{-m} e^{-\gamma_{2 k} r_{2 k}}}
$$

las distancias epicentrales se denotan $r_{s e}$ donde $s$ indica la fuente y $e$, la estación. En este caso se ha utilizado la corrección por divergencia geométrica de la forma $r^{-m}$, válida para distancias epicentrales locales o regionales. En el método de las dos estaciones, el doble cociente de 


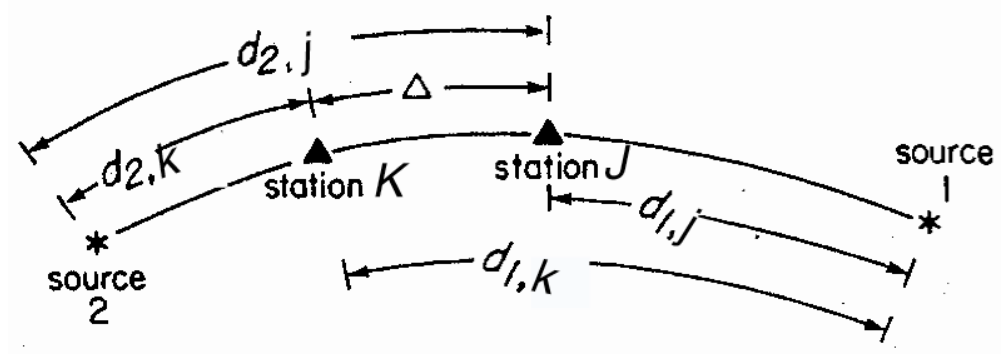

Fig. 2.21. Diagrama esquemático del Método de la Doble Razón Espectral que determina la atenuación en la trayectoria entre estaciones. Ver el texto para referencias. (Adaptada de Chun et al, 1987).

amplitudes elimina formalmente los patrones de radiación, las amplitudes espectrales en la fuente y los efectos de sitio en ambas estaciones, que no se han expresado en la fórmula por simplificación. En el DRE, se eliminan además las respuestas instrumentales de ambas estaciones. De esta manera, la (2.94) quedará

$$
\frac{A_{1 k}(f)}{A_{1 j}(f)} \cdot \frac{A_{2 j}(f)}{A_{2 k}(f)}=\frac{r_{1 k}^{-m} e^{-\gamma_{1 k} r_{1 k}}}{r_{1 j}^{-m} e^{-\gamma_{1 j} r_{1 j}}} \frac{r_{2 j}^{-m} e^{-\gamma_{2 j} r_{2 j}}}{r_{2 k}^{-m} e^{-\gamma_{2 k} r_{2 k}}}
$$

De acuerdo con la (2.91), dada la configuración de estaciones y epicentros de la Fig. 2.21, podrá escribirse

$$
\begin{aligned}
& \gamma_{1 k} r_{1 k}=\gamma_{1 j} r_{1 j}+\gamma_{j k} r_{j k} \\
& \gamma_{2 j} r_{2 j}=\gamma_{2 k} r_{2 k}+\gamma_{j k} r_{j k}
\end{aligned}
$$

Reemplazando en la (2.95) y reordenando, quedará

$$
\frac{A_{1 k}(f)}{A_{1 j}(f)} \cdot \frac{A_{2 j}(f)}{A_{2 k}(f)}=\left(\frac{r_{1 k} r_{2 j}}{r_{1 j} r_{2 k}}\right)^{-m} e^{\left(-2 \gamma_{j k} r_{j k}\right)}
$$

A partir de esta expresión puede hallarse fácilmente la atenuación promedio entre estaciones y estimar el valor de Q.

\subsubsection{MÉTODO DE NORMALIZACIÓN DE LA CODA (NC)}

El método de "Normalización de la Coda" denominado así por Frankel et al. (1990), es el que Aki (1980a) propuso como "Método de una estación". El método fue diseñado para determinar la atenuación de las ondas de corte en la litosfera evitando la influencia del efecto de sitio en la fuente y en la estación. Como la determinación de los factores de calidad se realiza para cada frecuencia en forma independiente, no necesita hacer suposiciones sobre la relación $Q(f)$ ni sobre el espectro de la fuente, ya que usa los espectros de fuente estimados 
por el SBS. A su vez, este método da la posibilidad de estimar la dependencia con la frecuencia del patrón de radiación en la fuente, de la propagación y del efecto de sitio en la estación. Usualmente esto se logra aislando uno de dichos efectos, mediante la eliminación de los otros dos, para estudiarlo en detalle.

La idea del método de NC surge de la observación empírica acerca de que la longitud de un sismograma regional es proporcional a la energía irradiada en la fuente. Es importante notar, como aclaran Sato y Fehler (1998), que el método de NC no está fundado en ningún modelo teórico de propagación de ondas y en particular no depende de la validez del scattering simple. Por el contrario sus fundamentos son empíricos. La hipótesis de la homogeneidad de la distribución de energía, para cierto lapso de tiempo en un volumen dado alrededor de la fuente, se ve limitada por las consideraciones hechas en el proceso de scattering múltiple basado en la teoría de transferencia radiativa (Sección 2.2.4.3). Sin embargo, la fiabilidad de los resultados obtenidos por el método de NC ayuda a confirmar la validez de dicha hipótesis. A su vez, es consistente con el modelo de flujo de energía, discutido en la Sección 2.2.4.4.

Aki y Chouet (1975) emplearon el SBS para determinar el espectro de la fuente en eventos de baja magnitud registrados a distancias suficientemente cortas como para despreciar el scattering pero largas como para suponer la validez del campo lejano. La exigencia de magnitud baja permite suponer frecuencias de corte más altas y por lo tanto una representación más completa del espectro de fuente. El efecto de la atenuación se corregía haciendo el cociente del EDP de la onda P y el factor fuente de la coda a cada frecuencia y estudiando su variación con el tiempo de viaje para varios eventos. Aki y Chouet (1975) plantearon la atenuación de la onda $\mathrm{P}$ como proporcional a $t^{-1} e^{-\pi f t / Q_{\alpha}}$ y describieron sucintamente la metodología, pero sería Aki (1980a) quien luego la detallaría.

Sea $A_{i j}(f, D)$ la amplitud espectral de la onda directa registrada correspondiente al $i$ ésimo evento en la estación $j$-ésima a una distancia hipocentral $D$ para la frecuencia $f$. Puede representarse aproximadamente por

$$
A_{i j}(f, D)=R_{i}(\theta, \phi) S_{i}(f) G_{j}(f, \theta, \phi) I_{j}(f) D^{-m} e^{-\frac{\pi f D}{Q_{d} v}}
$$

donde $I_{j}(f)$ es la respuesta de instrumento, $G_{j}(f, \theta, \phi)$ es el efecto de sitio en la estación, $S_{\mathrm{i}}(f)$ es la función fuente, $R_{i}(\theta, \phi)$ es el patrón de radiación, $m$ es el coeficiente de expansión geométrica ( $m=1$ o 0.5 según sean ondas internas o superficiales) y $v$ es la velocidad 
promedio de las ondas. $Q_{d}$ es el factor de calidad que se asigna a la trayectoria de las ondas.

Por otra parte, se ha visto (Aki y Chouet, 1975, Tsujiura, 1978, Rautian y Khalturin, 1978) que la amplitud espectral de la coda registrada por el mismo instrumento a un lapso de tiempo $t_{c}$ mayor que aproximadamente dos veces el tiempo de propagación de $\mathrm{S}$ puede escribirse como

$$
A_{i j}^{c}\left(f, t_{c}\right)=S_{i}^{c}(f) G_{j}^{c}(f) I_{j}(f) P\left(f, t_{c}\right)
$$

donde $P\left(f, t_{c}\right)$ es la función de decaimiento de la coda a lo largo del sismograma que será independiente de la distancia, $G_{j}^{c}(f)$ e $I_{j}(f)$ tienen el mismo significado que en la onda directa salvo que $G_{j}^{c}(f)$ no depende de la dirección y $S_{i}^{c}(f)$ es la función fuente de las ondas codas supuesta independiente de la dirección de propagación. Estas conclusiones, si bien son empíricas, se explican asumiendo la validez del scattering hacia atrás debido a heterogeneidades distribuidas aleatoriamente (Aki, 1969).

El factor de calidad se determina sobre un promedio de trayectorias en diferentes direcciones y con una variedad de distancias. Para ello es necesario suponer que el promedio de los cocientes $S_{i}(f) / S_{i}^{c}(f)$ y $G_{j}(f, \theta, \phi) / G_{j}^{c}(f)$, para muchos eventos distribuidos alrededor de la estación y considerando que no hay variaciones regionales sistemáticas, se tornan independientes de la dirección y tienden a la unidad. Haciendo el cociente de las amplitudes espectrales y tomando el logaritmo natural se obtiene una expresión de la forma

$$
\left\langle\ln \left\{D^{m} A_{i j}(f, D) / A_{i j}^{c}\left(f, t_{c}\right)\right\}\right\rangle_{D \pm \Delta D}=a-b D
$$

El símbolo $\langle X\rangle_{D \pm \Delta D}$ indica el promedio de $X$ para distancias hipocentrales alrededor de $D(D \pm \Delta D)$. Si las hipótesis establecidas son correctas, se espera obtener una dependencia lineal con la distancia para los valores promedio del miembro izquierdo de la (2.100). Los valores de $a$ y $b$ estarán dados por las siguientes relaciones independientes de $D$

$$
\begin{aligned}
& b=\pi f / Q_{d} v \\
& e^{a}=\frac{R_{i}(\theta, \phi) S_{i}(f) G_{j}(f, \theta, \phi)}{S_{i}^{c}(f) G_{j}^{c}(f) P\left(f, t_{c}\right)}
\end{aligned}
$$

Al promediar los efectos direccionales de los patrones de radiación y del efecto de sitio en la estación para eventos distribuidos alrededor de la misma podrá ajustarse una única recta cuya pendiente, $b$, dará el valor de $Q$ de las ondas directas en función de la frecuencia central 
del filtro mientras que la ordenada al origen, $a$, será solo dependiente de la frecuencia si se normalizan los tiempos de coda en todas las trazas. De este modo, el tiempo $t_{c}$ debe ser fijado para todos los eventos en un valor constante.

Aki (1980a) recomendó filtrar los sismogramas en varias bandas de frecuencia de un ancho de una octava y sobre ellos medir la amplitud pico a pico máxima de la onda $\mathrm{S}$ así como la amplitud media de las ondas coda en una ventana de tiempo alrededor de $t_{\mathrm{c}}$. Sin embargo, es muy probable, que todos los registros no tengan la misma duración ni relación señal ruido, por lo que Aki (1980a) propuso medir la amplitud de coda a un tiempo $t_{1}$ seleccionado en cada traza y luego reducirla a su valor ajustado a $t_{c}$. Para ello se multiplica por $P\left(\omega, t_{c}\right) / P\left(\omega, t_{1}\right)$, haciendo uso de la expresión del decaimiento de coda obtenida anteriormente por otro método. Es decir que la expresión de la amplitud de coda al tiempo de referencia $t_{c}$ se calcula a partir de la amplitud de coda medida a un tiempo $t_{1}$, en función del valor de $Q_{c}$ obtenido y la frecuencia. Aki $\left(1980 \mathrm{a}\right.$, b) utilizó la determinación de $Q_{c}$ mediante el modelo SBS.

Los resultados de Aki (1980a) mostraron un $Q_{\mathrm{d}}$ sorprendentemente cercano a $Q_{\mathrm{c}} \mathrm{y}$ dependiente de la frecuencia llevándolo a interpretar a las ondas coda como compuestas principalmente por ondas S dispersadas y por lo tanto afectadas por la misma dependencia. En esto, discrepaba con las conclusiones previas de Aki y Chouet (1975) quienes interpretaron la variación de $Q_{\text {c }}$ con la frecuencia como debida a la combinación de variaciones de $Q$ con la profundidad y a que la coda contenía contribución de ondas superficiales.

Si se utiliza el modelo SIS, como se hará también en este trabajo, las amplitudes de la envolvente de coda a los tiempos $t_{1}$ y $t_{c}$ se expresan mediante

$$
\begin{aligned}
& A_{i j}^{c}\left(f, t_{1}\right)=\frac{A_{0}}{t_{1}} K\left(t_{1}\right)^{1 / 2} e^{-\frac{\pi f t_{1}}{Q_{c}\left(f, t_{1}\right)}} \\
& A_{i j}^{c}\left(f, t_{c}\right)=\frac{A_{0}}{t_{c}} K\left(t_{c}\right)^{1 / 2} e^{-\frac{\pi f t_{c}}{Q_{c}\left(f, t_{c}\right)}}
\end{aligned}
$$

donde $K\left(\right.$ ) se evalúa mediante (2.35) en los lapsos de tiempo correspondientes y $Q_{c}$ toma los valores correspondientes a esos tiempos según el modelo SIS. Siendo consistentes con los criterios establecidos en la determinación de $Q_{\mathrm{c}}$, se ha supuesto que las ondas coda son ondas internas, por lo que el coeficiente de expansión geométrica se ha elegido igual a 1. Haciendo el cociente de (2.103) y (2.102) y despejando, se obtiene la expresión de la amplitud de coda 
reducida al tiempo de referencia elegido

$$
A_{i j}^{c}\left(f, t_{c}\right)=A_{i j}^{c}\left(f, t_{1}\right) \frac{t_{1}}{t_{c}}\left[\frac{K\left(t_{c}\right)}{K\left(t_{1}\right)}\right]^{1 / 2} e^{-\pi f\left(\frac{t_{c}}{Q_{c}\left(f, t_{c}\right)}-\frac{t_{1}}{Q_{c}\left(f, t_{1}\right)}\right)}
$$

que se reemplaza en la (2.100) para ajustar por mínimos cuadrados la pendiente $b$ de la recta que permite estimar el valor de $Q_{\mathrm{d}}$. En la (2.104) quedan indicadas las variaciones de $Q_{c}$ con la frecuencia y el lapso de tiempo según las observaciones realizadas por otros autores y que se mencionaran en la Sección 2.2.5.

Aki (1980b) avanzó sobre la interpretación del significado de $Q_{\mathrm{d}}$ expresándolo en función del coeficiente de turbidez $g$ y de la velocidad de las ondas $v=\beta$, en el caso de ondas $\mathrm{S}$, quedando $Q_{d}=2 \pi f / g \beta$. Supuso que las ondas coda son ondas planas afectadas por backscattering simple caracterizado por $g$ y reemplazó la función fuente de las ondas coda por la misma de las ondas primarias pero incluyendo el efecto de la absorción y del scattering antes de entrar al volumen heterogéneo. En su interpretación, $Q_{\mathrm{c}}$ debía ser considerado como un $Q$ efectivo que incluía ambos efectos, la absorción y el scattering. Utilizando estos conceptos volvió a las relaciones (2.101) para dar la expresión de la ordenada al origen del ajuste, $a$

$$
e^{a}=\frac{(\Delta f)^{1 / 2} \beta t_{c}}{2[\beta g(\pi)]^{1 / 2} e^{-\omega t_{c} /\left(2 Q_{c}\right)}}
$$

donde $\Delta f$ es el ancho de banda del filtro utilizado y $g(\pi)$ especifica el coeficiente de turbidez para ondas secundarias propagándose en la misma dirección que las ondas primarias pero en sentido contrario. Mediante la expresión (2.105) pudo estimar el valor del coeficiente de turbidez en el back-scattering.

La coincidencia general hallada entre $Q_{\mathrm{c}}$ y $Q_{\mathrm{d}}$ para varias regiones en el intervalo de frecuencias entre 1 y $25 \mathrm{~Hz}$, llevaron a Aki (1980b) a concluir que las ondas coda de terremotos locales están compuestas por scattering simple de ondas $\mathrm{S}$ a S. También notó que la incertidumbre en la estimación de los espectros y el modelo de scattering utilizado podían causar la falta de posibilidad de diferenciación entre los efectos de absorción y scattering. Sin embargo, como dijeran Sato y Fehler (2003) las ondas coda han probado ser una herramienta valiosa. El método de Normalización de la Coda, sin una teoría formal que lo explique, permite hacer buenas determinaciones de la radiación de la fuente y del efecto de sitio. Este método y el modelo de la transferencia radiativa serían reunidos luego por Hoshiba et al. (1991) para alcanzar resultados que ningún otro método había podido obtener, la separación 
de los efectos de atenuación.

\subsubsection{ALGUNOS RESULTADOS DE LA DETERMINACIÓN DE LA ATENUACIÓN DE ONDAS DIRECTAS}

La atenuación de ondas directas ha sido ampliamente estudiada en diferentes regiones del mundo, siendo mayor el número de trabajos sobre ondas $\mathrm{S}$ que sobre ondas $\mathrm{P}$. Esta preferencia resulta lógica si se piensa que las ondas S presentan mayor amplitud y duración en los sismogramas de eventos superficiales que las ondas $\mathrm{P}$ y a su vez, que la propagación de las ondas $\mathrm{S}$ es más sensible a anomalías que pueden asociarse a fusión parcial o a contenido de fluidos siendo su factor de calidad un mejor reflejo de la reología del medio.

Las Figuras 2.22 y 2.23 extractadas de Sato y Fehler (1998) resumen las estimaciones de $Q_{P}{ }^{-1}$ y $Q_{S}^{-1}$ efectuadas en diferentes regiones del planeta a profundidades litosféricas. El valor global de $Q_{\mathrm{P}}{ }^{-1}$ obtenido con ondas superficiales es menor que 0.001. En la Fig. 2.22 puede verse que se han obtenido muy pocos ajustes de la atenuación de ondas $\mathrm{P}$, aunque se sabe que varía con la frecuencia con una ley ${Q_{P}}^{-1} \propto f^{-n}$. En los últimos años se han realizado numerosas determinaciones de la atenuación mediante tomografía de ondas $\mathrm{P}$ en distintas regiones del mundo (Haberland y Rietbrock, 2001, Deshayes et al., 2008, Roth et al., 1999) aplicando métodos independientes de la frecuencia o con una dependencia preestablecida (Pozgay et al., 2009).

En la Fig. 2.23 (Sato y Fehler, 1998) se presentan estimaciones de $Q_{S}^{-1}$ a profundidades litosféricas a partir del análisis de ondas superficiales (método SRE) y de ondas S directas (métodos NC, ME y MLTWA, ver Sección 2.4.2.1).

Los resultados de $Q$ para ondas superficiales muestran valores constantes de $Q^{-1}$ entre 0.01 y 0.0005 a frecuencias por debajo de $1 \mathrm{~Hz}$, con un valor medio global de 0.002 . Las estimaciones hechas a frecuencias entre 0.01 y $10 \mathrm{~Hz}$ con el ME en México (curva 19 en línea punteada) muestran un decaimiento similar con valores intermedios entre los de dos regiones tectónicamente diferentes entre sí de Estados Unidos (curvas 11.1 y 11.2). Los métodos SRE y DRE han sido utilizados fundamentalmente sobre ondas superficiales Lg, Love y Rayleigh con datos de telesismos para trayectorias continentales y oceánicas (por ejemplo: SRE: BenMenahem, 1969; Tsai y Aki, 1969; Canas y Mitchell, 1978 y 1981; Osagie, 1986; Hwang and Mitchell, 1987; Canas et al., 1989; DRE: Chun et al., 1987, Ibáñez, 1990; De Miguel et al., 1992, Badi et al., 2004). Canas et al. (1989) mostraron que las soluciones del SRE presentan 


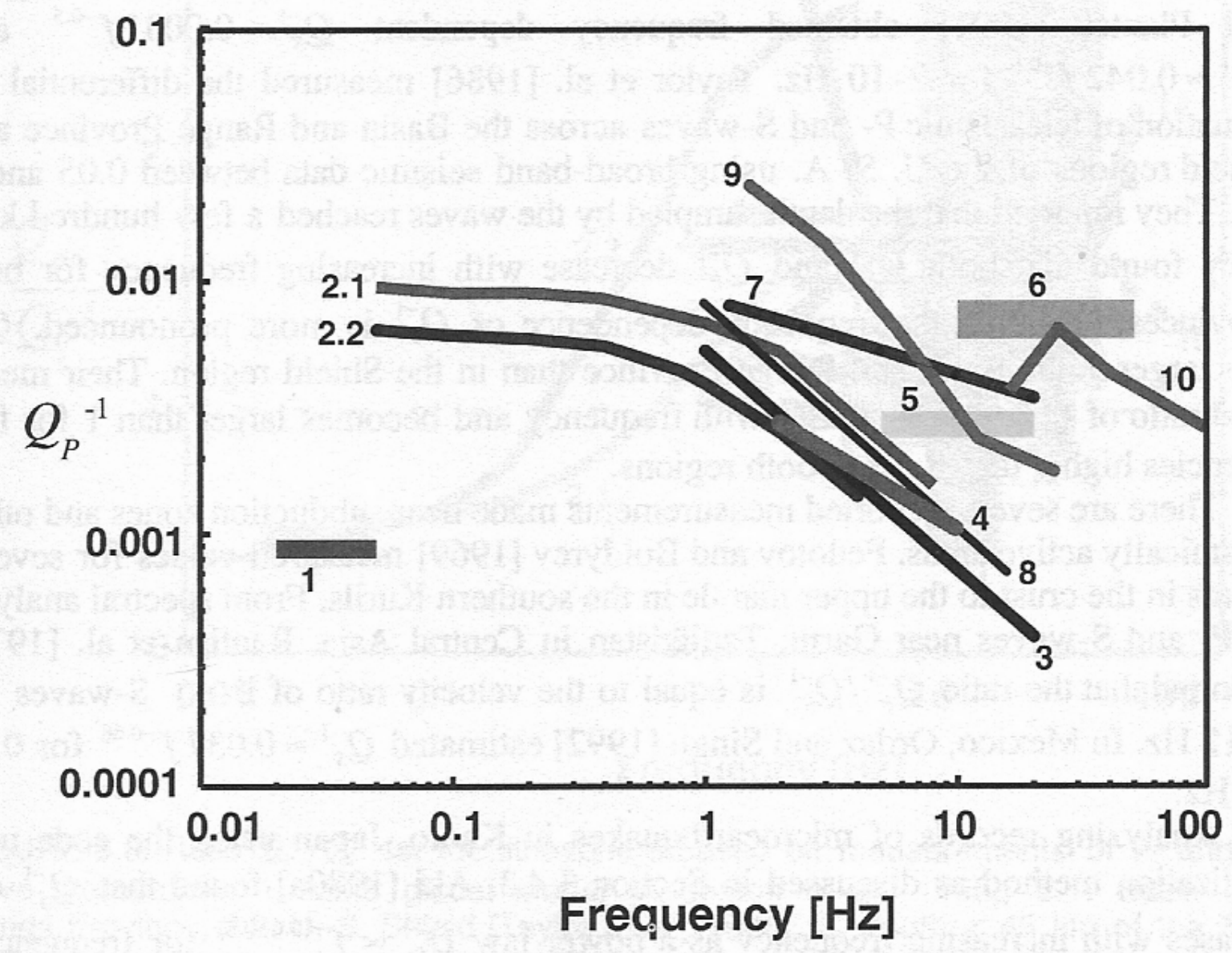

Fig. 2.22.-. Valores de $Q^{-1}$ de ondas $P$ para la litósfera estimados por diversos autores según análisis de ondas superficiales: 1, prof $<45 \mathrm{~km}$ del modelo global SL8 (Anderson y Hart, 1978). Análisis de decaimiento espectral: 2.1, provincia Basin and Range; 2.2, cratón EEUU (Taylor et al., 1986); 3, Pn, Canadá oriental (Zhu et al., 1991); 4, Pg, Francia (Campillo y Plantet, 1991); 5, prof. $<40 \mathrm{~km}$, Caribe norte (Frankel, 1982); 6, prof $<10 \mathrm{~km}$, Arette, Pirineos (Modiano y Hatzfeld, 1982); 7, prof. 5-25 km, Kuriles Sur (Fedotov y Boldyrev, 1969); 8, sur de Noruega (Kvamme y Havskov, 1989). Método de normalización de la coda extendido a ondas P (Sato y Fehler, 1998): 9, Kanto, Japón (Yoshimoto et al., 1993); 10, corteza superior en Nagano occidental, Japón (Yoshimoto et al., 1994). (Extractada de Sato y Fehler, 1998).

mayor estabilidad si las distancias entre estaciones son grandes. Cheng y Mitchell (1981) compararon la atenuación de ondas de corte en diferentes regiones de Estados Unidos estimando valores de la atenuación que tenían una relación directa con la actividad tectónica. Xie y Mitchell (1990) realizaron un exhaustivo análisis de la viabilidad del método SRE para la determinación de $Q$ de $L_{g}$ y codas de $L_{g}$, mediante aproximaciones estadísticas concluyendo que si se tiene en cuenta la geología atravesada por las ondas, el estudio conjunto de ambas ondas da buenos resultados aún en las regiones tectónicas más complicadas. Ibáñez et al. (1993) emplearon el método DRE de una forma alternativa para la determinación del factor de expansión geométrica de ondas $\mathrm{S}$ en el sur de España. Como se ha visto, las consideraciones geológicas que requiere el SRE se evitan en la aplicación del DRE, pero este ha tenido poca 
difusión debido a que la geometría requerida implica una menor disponibilidad de datos.

La mayoría de las determinaciones de $Q_{\mathrm{S}}{ }^{-1}$ han sido realizadas sobre datos de terremotos locales con registro en corto período y en todos los casos han mostrado que zonas tectónicamente activas tienen una variación de $Q_{\mathrm{S}}{ }^{-1}$ decreciente con la frecuencia y de forma

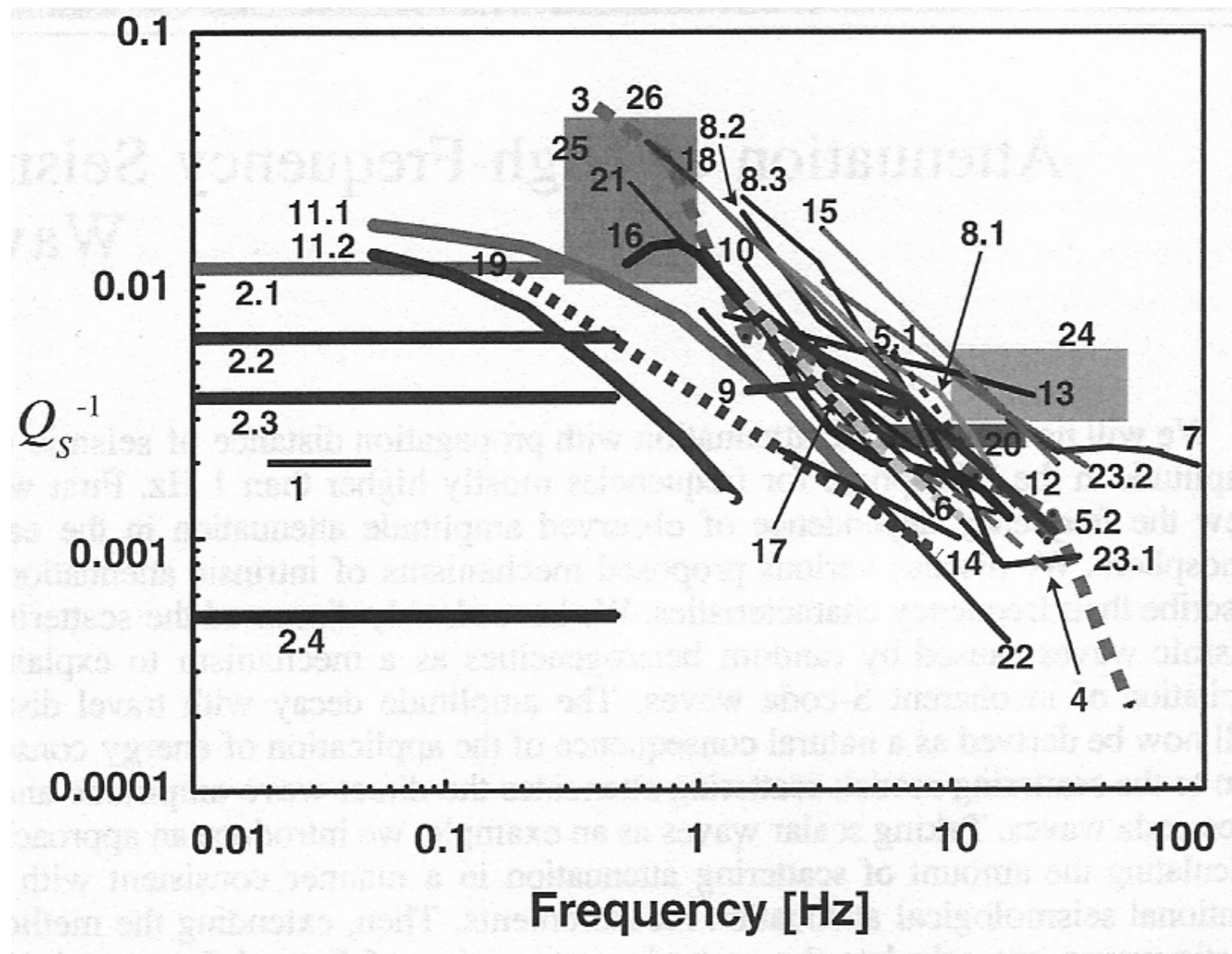

Fig. 2.23.- Valores calculados de $Q^{-1}$ de ondas $S$ para la litósfera a partir de: Análisis de ondas superficiales: 1,prof. $<45 \mathrm{~km}$, modelo global SL8 (Anderson y Hart, 1978); 2.1, corteza superior (prof. $<18 \mathrm{~km}$ ) en la provincia Basin and Range, EEUU ; 2.2, corteza superior en Colorado Plateau; 2.3, corteza superior en EEUU. oriental; 2.4, corteza inferior (prof $>18 \mathrm{~km}$ ) en EEUU. (Cheng y Mitchell, 1981). Método de NC: 3, Hindu-Kush (Roecker et al., 1982); 4, Kanto, Japón (Aki, 1980a); 5.1, Kanto oriental, Japón (Sato y Matsumura, 1980); 5.2, Kanto, Japón (Yoshimoto et al., 1993); 6, norte de Grecia (Hatzidimitriou, 1995); 7, corteza superficial en Nagano occidental, Japón (Yoshimoto et al., 1994). MLTWA (ver sección 2.4.2.1): 8.1, California Central; 8.2, Hawaii; 8.3, Long Valley en California, EEUU. (Mayeda et al., 1992); 9, KantoTokai, Japón (Fehler et al., 1992); 10, Kyushu, Japón (Hoshiba,1993). Decaimiento espectral: 11.1, Basin and Range, EEUU; 11.2, Cratón de EEUU (Taylor et al., 1986); 12, Sg y Lg, Utah, EEUU. (Brockman y Bollinger,1992); 13, prof. 5-25 km, sur de Kuriles (Fedotov y Boldyrev, 1969); 14, Lg, Francia (Campillo y Plantet, 1991); 15, falla Imperial, California (Singh et al., 1982); 16, prof. < $50 \mathrm{~km}$, sur de Kanto, Japón (Kinoshita, 1994); 17, costa Pacífica de Kanto, Japón (Takemura et al., 1991); 18, Montenegro, Yugoslavia (Rovelli, 1984); 19, México (Ordaz y Singh, 1992); 20, prof. $<40 \mathrm{~km}$, Caribe norte (Frankel, 1982); 21, norte de ltalia (Console y Rovelli, 1981); 22, sur de Noruega (Kvarnme y Havskov, 1989); 23.1, estado de New York, EEUU.; 23.2, sur de California, EEUU (Frankel et al., 1990); 24, prof. < $10 \mathrm{krn}$, Arette, Pirineos (Modiano y Hatzfeld, 1982); 25, falla de San Andrés, California (Kurita, 1975); 26, sur de ltalia (Rovelli, 1983). (Extractada de Sato y Fehler, 1998). 
bastante similar, con máximos entre 0.01 y 0.1 a frecuencias de $1 \mathrm{~Hz}$ y mínimos cercanos a 0.001 por encima de los $10 \mathrm{~Hz}$. Puede asumirse que la atenuación de las ondas $\mathrm{S}$ depende de la frecuencia según la relación $Q_{S}^{-1} \propto f^{-n}$ con $n$ entre 0.5 y 1 para frecuencias mayores que $1 \mathrm{~Hz}$. De la distribución de curvas de $Q_{\mathrm{s}}^{-1}$ en corto período puede verse que las regiones con mayor actividad tectónica muestran una mayor atenuación en todas las frecuencias como es el caso de California o el sur de Italia, mientras que Noruega o Francia muestran los menores valores relativamente.

Con datos de la región de Kanto, Japón, Aki (1980a, b) encontró que $Q_{S}^{-1}$ obtenido por $\mathrm{NC}$ es similar a $Q_{c}$. Sus resultados reforzaban la teoría del scattering simple de $\mathrm{S}$ a $\mathrm{S}$ para la generación de las codas de terremotos locales. Las conclusiones de Aki coincidían con las de Herrmann (1980) y Rautian y Khalturin (1978), excepto para el caso del volcán Kilauea, Hawai, donde las anomalías de $Q_{c}$ halladas, parecían indicar un mecanismo de atenuación más bien relacionado con un proceso de relajación activado termalmente. En cambio, Martínez-Arévalo et al. (2003) hallaron un comportamiento anómalo en la dependencia de $Q_{S}$ con la frecuencia en la Isla Decepción (Antártida) y que parecía indicar un scattering fuerte en las cercanías de la fuente como principal causa de la atenuación. Por otra parte, los valores de $Q_{S}$ hallados según el método de NC eran mayores a los obtenidos por métodos espectrales, interpretándose como un efecto del método que, al promediar las amplitudes, elimina la contribución de la atenuación más superficial y por lo tanto hace una sobrevaloración de $Q_{S}$.

Yoshimoto et al. (1993) extendieron el método NC para aplicarlo al análisis de las ondas P. Akinci et al. (1995b) lo aplicaron al análisis de ondas Lg y su coeficiente de expansión geométrica en Turquía y el sur de España. Los valores de $Q$ obtenidos mediante NC para ondas S o Lg resultan en general muy próximos a $Q_{c}$ (Aki, 1980a; Rautian y Khalturin, 1978; Herrmann, 1980). Hatzidimitriou (1995) y Kvamme y Havskov (1989) encontraron que para lapsos de tiempo largos, $Q_{c} \sim Q_{S}$, mientras que para lapsos de tiempo cortos $Q_{c}<Q_{S} \quad$ Estas diferencias pueden entenderse a partir del modelo de generación de ondas coda. La parte inicial de la coda contiene ondas que han sido dispersadas en la corteza superior más atenuativa, mientras que el $Q_{c}$ del final de coda se acerca al de las ondas $\mathrm{S}$ ya que muestrea un volumen de tierra mayor aproximándose al valor medio cortical. Fehler y Sato (2003) reivindicaron, a través de un análisis detallado, los resultados obtenidos con el método de NC sobre todo en el caso de medios aleatorios ricos en componentes de longitud de onda corta. Sin una teoría formal que explique la formación de la coda, el método de NC es una herramienta valiosa que a su vez ha sido aprovechada por otros métodos. 


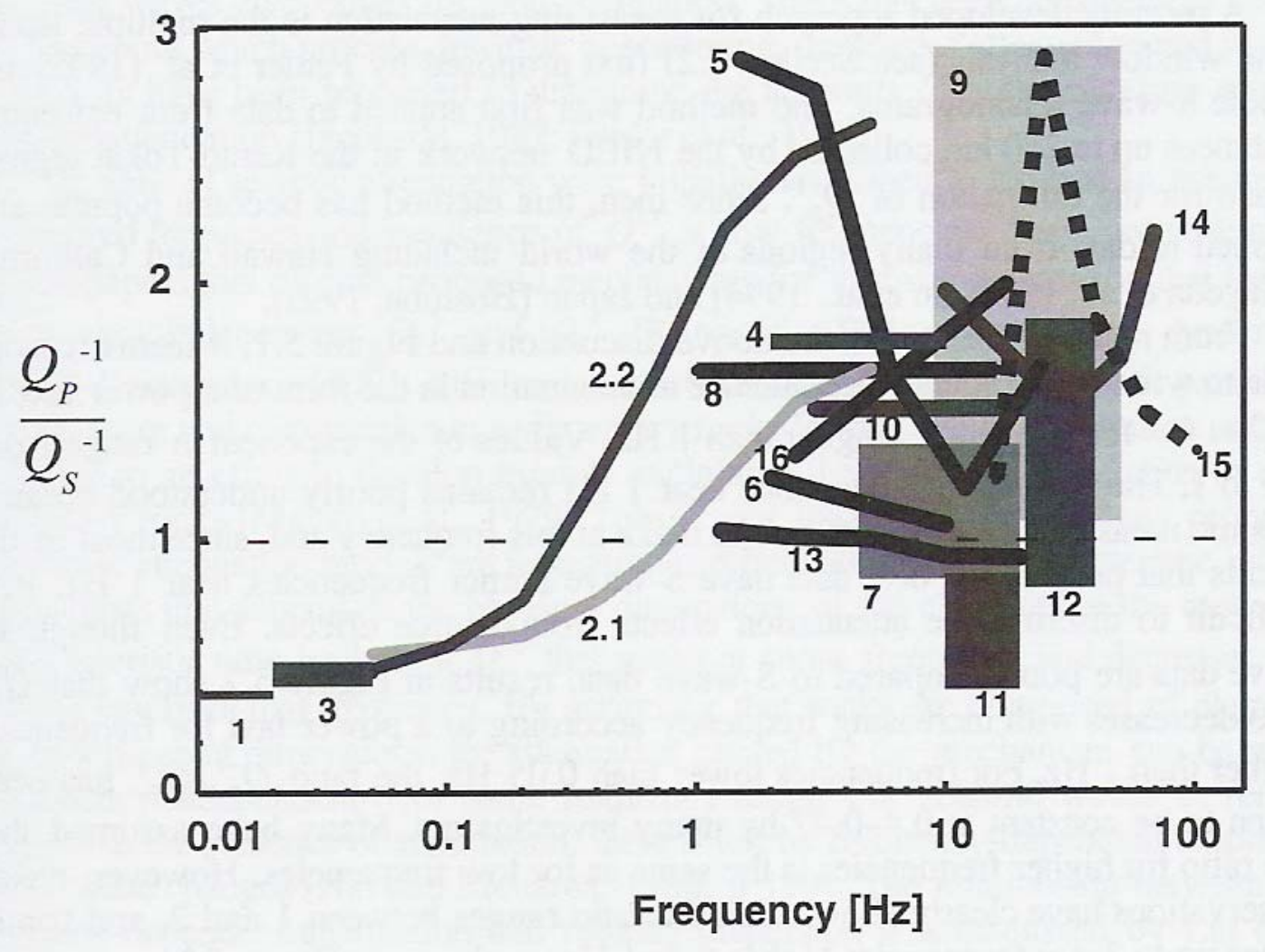

Fig. 2.24. Relación $Q_{P}^{-1} / Q_{S}^{-1}$. para la litósfera a partir de las estimaciones de atenuación para ondas $P$ y $S$ en diversas regiones del mundo: 1, modelo global MM8 (Anderson et al., 1965); 2.1, Basin and Range, EEUU; 2.2, Cratón EEUU Taylor et al., 1986); 3, prof. $<45 \mathrm{~km}$ del modelo global SL8 (Anderson y Hart, 1978); 4, Garm, Asia Central (Rautian et al., 1978); 5, Kanto, Japón (Yoshimoto et al., 1993); 6, Pg y Lg, Francia (Campillo y Plantet, 1991); 7, prof. $<40 \mathrm{~km}$, Caribe norte (Frankel, 1982); 8, Sur de Noruega (Kvamme y Havskov, 1989); 9, prof. $<10 \mathrm{~km}$, Arette, Pirineos (Modiano y Hatzfeld, 1982); 10, corteza superior del Rift Rio Grande, EEUU (Carpenter y Sanford, 1985); 11, prof. < 7 km, falla de San Andreas (Bakun et al., 1976); 12, prof. $<7$ km. Swabian Jura, Alemania (Hoang-Trong, 1983); 13, Kuriles sur (Fedotov y Boldyrev, 1969); 14, Anza, California (Hough et al., 1988); 15, corteza superior de Nagano occidental, Japón (Yoshimoto et al., 1994); 16, Pacífico occidental (Butler et al., 1987). (Extractada de Sato y Fehler, 1998)

A partir de las determinaciones de $Q_{P}^{-1}$ y $Q_{S}^{-1}$ suele establecerse la relación entre las mismas $Q_{P}^{-1} / Q_{S}^{-1}$. Dado que $Q_{P}^{-1}$ es función de $\left(Q_{\mu}^{-1}, Q_{K}^{-1}\right)$ y $Q_{S}^{-1}=Q_{\mu}^{-1}$, los valores de $Q_{P}^{-1} / Q_{S}^{-1}$ pueden ayudar a interpretar la importancia relativa de la atenuación debida a procesos compresivos y de corte y a través de eso conocer el estado de los materiales atravesados. A su vez, los valores de $Q_{P}^{-1} / Q_{S}^{-1}$ permiten comparar la atenuación general entre regiones. Una relación $Q_{P}^{-1} / Q_{S}^{-1}$ baja puede estar indicando una alta atenuación de ondas $\mathrm{S}$ o a su vez una baja atenuación de ondas P. El primer caso es más simple de entender, ya que la atenuación de ondas $\mathrm{S}$ se asocia directamente con la rigidez del medio y por lo tanto con la temperatura y el grado de fusión o la presencia de fluidos. Una baja atenuación de ondas P, 
suele asociarse a mayor consolidación y/o edad de las rocas del mismo modo que se asocia a la velocidad, pero también se asocia al coeficiente de rigidez. En la Fig. 2.24 se ve que los valores promedios globales de $Q_{P}^{-1} / Q_{S}^{-1}$ según los modelos de distribución de $Q$ en la Tierra MM8 y SL8 (Anderson et al., 1965, Anderson y Hart, 1978) se encuentran en el intervalo [0.4-0.7]. A frecuencias mayores que $1 \mathrm{~Hz}, Q_{P}^{-1} / Q_{S}^{-1} \approx 1-2$ o hasta 3 . En algunas regiones se observa una relación $Q_{P}^{-1} / Q_{S}^{-1}$ independiente de la frecuencia en el intervalo estudiado. Kanto, Japón (curva 5) muestra valores de $Q_{P}^{-1} / Q_{S}^{-1}$ decrecientes hasta los $10 \mathrm{~Hz}$ donde la relación muestra un brusco cambio de pendiente. En Nagano, Japón (curva 15) se observa también un comportamiento con brusco cambio a cierta frecuencia. Es curioso que en forma casi sistemática, los valores obtenidos en regiones diferentes resulten muy relacionados cuando se estudian con las mismas técnicas y criterios. Se ha visto que en muchos casos los métodos empleados ejercen una fuerte influencia sobre los resultados y es por eso que la utilización simultánea de distintas técnicas permite una mejor interpretación. Por otra parte la dispersión de los resultados obtenidos utilizando diferentes técnicas sobre las mismas ondas en una dada región muestra la necesidad del diseño de métodos más precisos.

\subsection{SEPARACIÓN DE LA ATENUACIÓN INTRÍNSECA y DE SCATTERING}

\subsubsection{EVOLUCIÓN DE LOS MODELOS DE SEPARACIÓN DE $Q_{\mathrm{i}}$ y $Q_{\mathrm{s}}$}

Como ya se ha mencionado, las variaciones regionales de la atenuación pueden entenderse estimando la contribución de los mecanismos de absorción y scattering a la atenuación de las ondas, lo que permitiría inferir importantes propiedades del medio de propagación relacionadas a su estado de actividad tectónica. La atenuación intrínseca ha sido atribuida a fracturas interconectadas y saturadas de fluidos pero también a temperaturas elevadas que pueden alcanzar el punto de fusión (Mitchell, 1995, Frankel et al. 1990, Zor et al., 2007, Xie et al., 2004, entre otros). El origen de la atenuación por scattering parece estar mejor definido a partir del scattering de las ondas debido a la topografía irregular, la geología superficial compleja y la estructura cortical heterogénea (Aki, 1969).

A partir de la expresión (2.49) de Dainty y Toksöz (1981) que manifestaba la linealidad de los efectos de la atenuación, varios autores han puesto sus esfuerzos en pos de individualizar los efectos de la atenuación dentro de la densidad de potencia espectral. Dainty (1981) hizo sus estimaciones asumiendo que la atenuación anelástica era independiente de la 
frecuencia y que el scattering era proporcional a ella. El modelo de scattering múltiple de Gao (Gao et al., 1983a, b y Gao 1984), a partir del análisis de la propagación de las ondas, ofreció una expresión de la densidad de potencia espectral que resultaba una primera aproximación no lineal para la separación de $Q_{\mathrm{i}}$ y $Q_{\mathrm{s}}$. Sus conclusiones fueron que según los lapsos de tiempo medidos variaba la influencia relativa del scattering y de la anelasticidad. Sería la teoría de la transferencia de energía (Wu, 1985) la que ofrecería una forma de sintetizar la envolvente completa del sismograma y daría un método viable para estimar la cantidad de atenuación que es causada por los mecanismos intrínsecos y de scattering (Wu y Aki, 1988). Sin embargo, la presencia de cierto nivel de ruido enmascara el final del registro provocando una subestimación de la energía total.

Frankel y Wennerberg (1987) en su modelo de flujo de energía hallaron un $Q_{c}^{-1}$ cercano al $Q_{i}^{-1}$ si se utilizaba el scattering simple, aunque no explicaron el mecanismo físico de su modelo de scattering. La insensibilidad de la coda al scattering fue justificada por la redistribución de energía de las ondas directas dispersada hacia la coda. Era esta la razón que justificaba las diferencias de comportamiento de $Q_{c}$ y $Q_{d}$ con la frecuencia y el tiempo (Frankel, 1991).

La simulación numérica de Hoshiba (1991) a la distribución espacio-temporal de energía sísmica afectada de scattering múltiple brindó una solución a las dificultades encontradas en la implementación del modelo de transferencia radiativa. Hoshiba et al. (1991) mejorarían la simulación de Hoshiba (1991) proponiendo el Método de las Ventanas Temporales Múltiples (MLTWA) que no requería la cuantificación de la energía total del sismograma.

Zeng et al. (1991) y Sato (1993) obtuvieron nuevas soluciones analíticas del scattering múltiple. Zeng (1991) dio una solución compacta de las expresiones de Zeng et al. (1991) que facilitaban su aplicación bajo una serie de condiciones y daba una alternativa para el cálculo de las curvas teóricas del MLTWA. Wennerberg (1993) describió la posibilidad de reinterpretar los valores de $Q_{c}$ obtenidos según modelos de scattering simple en términos del scattering múltiple. Observando las similitudes entre las aproximaciones de Abubakirov y Gusev (1990) y las de Zeng (1991), estableció relaciones simples para estimar $Q_{i}$ y $Q_{s}$ a partir de valores de $Q_{c}$ y $Q_{d}$. Sato et al. (1997) extendieron la solución analítica de Zeng (1991) al caso de un patrón de radiación no esférico, es decir en presencia de un mecanismo focal.

Trabajos posteriores han intentado incorporar modelos más reales en el cálculo de las curvas teóricas de energía integrada (Hoshiba, 1994, 1995, 1997, Hoshiba et al., 1997, 
Margerin et al., 1998, 1999, Yoshimoto, 2000), han acelerado los tiempos de cálculo (Ugalde et al., 1998a) o los han implementado en subrutinas bajo programas de cálculo de uso corriente (Bianco y Del Pezzo, 2002, 2010). En los últimos años, se ha difundido el uso de métodos estocásticos que incrementan la cantidad de información extractada de los sismogramas sobre todo en el caso de un conjunto de datos reducido y además mejoran significativamente la resolución de los modelos (Sato et al., 2002).

\subsubsection{MÉTODOS DE SEPARACIÓN DE $Q_{\mathrm{i}}$ y $Q_{\mathrm{s}}$}

En las siguientes secciones se desarrollarán dos de los métodos de mayor uso en la actualidad y que se implementan en esta tesis: el Método de las Ventanas Temporales Múltiples (Multiple Lapse Time Windows Analysis) (Hoshiba et al., 1991) y el Método de Wennerberg (1993).

\subsubsection{MÉTODO DE VENTANAS TEMPORALES MÚLTIPLES O MULTIPLE LAPSE TIME WINDOWS ANALYSIS (MLTWA)}

Este método brinda información acerca de los cambios temporales de la energía sísmica de las ondas, tanto directas como coda, durante su propagación considerando la distribución de la energía para varias ventanas temporales consecutivas del sismograma en función de la distancia hipocentral. Las bases numéricas del MLTWA fueron dadas por Hoshiba et al. (1991) a partir de la teoría de la transferencia radiativa (Sección 2.2.4.3), mostrando que la variación con la distancia hipocentral de la integral de la energía sísmica sobre ventanas temporales se relaciona directamente con el albedo (2.53) y la atenuación total, $Q_{T}^{-1}$.

Sobre la base del principio de conservación de la energía y utilizando el método de simulación Monte-Carlo, Hoshiba (1991) sintetizó la distribución espacio-temporal de la densidad de energía para el caso del scattering isótropo, sin absorción intrínseca y en un medio tridimensional. Presentó los coeficientes de expansión hasta el scattering de décimo orden para el caso en que la distancia recorrida por la onda dispersada era mucho más grande que la distancia hipocentral.

Hoshiba comparó su simulación con diversos modelos de scattering, hallando consistencia con la formulación propuesta por Wu (1985) para la teoría de transferencia radiativa en estado estacionario. La coincidencia se daba si se consideraban la onda directa y las partes inicial y final de la coda. Esta observación indicaba que debía ponerse atención en la selección de las ventanas temporales en aplicaciones prácticas.

Las bases establecidas por Hoshiba (1991) que resultaban similares a las de Sato (1977) 
y Gao et al. (1983b), eran:

- Las heterogeneidades puntuales con sección efectiva $\sigma_{0}$ se encuentran distribuidas aleatoriamente con una densidad $n_{0}$ y las ondas dispersadas se consideran incoherentes.

- Existe scattering isótropo cuya potencia por unidad de volumen está caracterizada por el coeficiente de scattering, $g \equiv \sigma_{0} n_{0}$.

- Las ondas S esféricas son radiadas desde una fuente puntual de muy corta duración.

- No hay conversión entre ondas P y S durante el scattering y la velocidad de las ondas $\mathrm{S}$ es constante e igual a $v$.

- No hay absorción intrínseca. En el mismo trabajo se vería la necesidad de modificar esta hipótesis.

El método en cuestión plantea la energía total como la suma de las contribuciones de las energías de los diferentes órdenes de scattering. Suponiendo que la propagación de la energía de la onda $\mathrm{S}$ está representada por el movimiento de muchas partículas que salen de la fuente con una unidad de energía, se calcula la energía que cada una de ellas acarrea luego de sufrir un scattering de orden $\mathrm{n}$ y se calcula la probabilidad de que dicha partícula atraviese el área unitaria que rodea al receptor.

Si la distancia fuente receptor está dada por $\left|R_{r}-R_{0}\right|$ y la distancia recorrida entre dos heterogeneidades consecutivas es $\left|r_{k}\right|=\left|R_{k}-R_{k-1}\right|$, entonces para el scattering de orden $n$, la distancia recorrida será $D=\left|r_{1}\right|+\left|r_{2}\right|+\left|r_{3}\right|+\ldots+\left|r_{n}\right|+\left|R_{r}-R_{n}\right|$

El número de partículas dividido por la velocidad de propagación y el intervalo de tiempo analizado dará la función de densidad de energía correspondiente al scattering de $n$ ésimo orden. La suma de las funciones de densidad de energía hasta un orden de scattering alto dará la densidad de energía para una dada distancia hipocentral.

Como la energía se conserva, deberá cumplirse que

$$
W_{0}=W_{0} e^{-g v t}+\int_{0}^{v t} E_{s}(r, t) 4 \pi r^{2} d r
$$

donde $W_{0}$ es la energía radiada por la fuente, $g$ es el coeficiente de scattering y $v$ la velocidad de las ondas. El primer término del segundo miembro representa la energía directa atenuada debido al scattering $\left(W_{d}(t)\right)$ y el segundo término es la integral volumétrica de la energía dispersada $\left(W_{s}(t)\right)$. El segundo miembro de (2.106) se calcula numéricamente comprobándose que su valor se mantiene casi igual a $W_{0}$ para todo $t$ cuanto mayor sea el número de 
dispersores. Si $E_{s n}(r, t)$ es la densidad de energía debida a la onda dispersada de orden $n$, entonces

$$
E_{s}(r, t)=E_{s 1}(r, t)+E_{s 2}(r, t)+\ldots
$$

es la densidad de energía total. La energía dispersada para el orden $n$ será

$$
W_{s n}(t)=\int_{0}^{v t} E_{s n}(r, t) 4 \pi r^{2} d r
$$

Hoshiba (1991) expresó el resultado de esta integral como el producto de una potencia y una exponencial

$$
W_{s n}(t)=W_{0} A_{n}(g v t)^{n} e^{-g v t}
$$

donde $\operatorname{los} A_{n}$ son coeficientes por determinar. Reemplazando (2.107) en (2.106) y considerando la (2.109), la expresión de la energía total queda de la forma

$$
W_{0}=W_{0} e^{-g v t} \sum_{i=0}^{n} A_{i}(g v t)^{i}
$$

por lo que deberá ser $A_{i}=1 / n$ !. La máxima contribución de la onda afectada por un scattering de orden $n$ a la energía total, se produce cuando $g v t=n$. El scattering simple es el dominante cuando gvt «1. El scattering múltiple se puede aproximar por $g v t \leq 3$ y el modelo de difusión por $g v t » 1$.

Al expresar el decaimiento temporal de la densidad de energía en la fuente, evaluando los coeficientes de expansión correspondientes hasta el décimo orden, Hoshiba (1991) observó discrepancias con los mismos coeficientes determinados por Gao et al. (1983b) (denominados $K_{i}$ en la Ecuación 2.47) para $n>2$. Dado que las hipótesis de ambos coincidían, Hoshiba (1991) halló la justificación en el hecho de que su método satisfacía la conservación de energía incluso para los órdenes superiores, mientras que el modelo de Gao et al. (1983b) no la respetaba. Los resultados obtenidos mostraban consistencia con las fórmulas del modelo de transferencia radiativa de $\mathrm{Wu}(1985)$.

Relacionando el decaimiento temporal de la energía en la fuente según el modelo de Hoshiba (1991) con el $Q_{c}^{-1}$, en el caso de la validez del scattering simple, se obtenía un $Q_{c}^{-1}$ idéntico a $Q_{\beta}^{-1}=g v / \omega$, $\left(Q^{-1}\right.$ de ondas $\mathrm{S}$ directas $)$ cuando no había absorción intrínseca. Considerando el scattering múltiple, Hoshiba (1991) observó que $Q_{c}^{-1}$ era afectado por las contribuciones de las ondas que sufrían scattering múltiple más que por las pérdidas del scattering simple. En este caso, $Q_{c}^{-1}$ daba negativo para todo valor de $g v t$, lo que significaba que la pendiente de la envolvente de coda corregida de la expansión geométrica, crecía con el 
tiempo. Pero como $Q_{\beta}^{-1}$ es positivo y crece según $g v t$, dicho resultado significaba que $Q_{c}^{-1}$ se volvía muy diferente de $Q_{\beta}^{-1}$ al incluir el scattering múltiple, contrariamente a lo observado por muchos autores. Estas discrepancias entre las predicciones teóricas y los valores observados fueron adjudicadas por Hoshiba (1991) a la consideración de ausencia de absorción intrínseca. Partiendo de las mismas hipótesis pero incorporando la atenuación intrínseca, Hoshiba et al. (1991) propusieron un método para la separación de ambos efectos de atenuación. La quinta hipótesis fue reemplazada por la siguiente:

- La absorción intrínseca es espacialmente uniforme y está dada por $Q_{\mathrm{i}}^{-1}$.

Teniendo en cuenta los cambios espaciales y temporales de la energía en la onda directa y en la coda, modelaron la energía en tres ventanas temporales sucesivas y de longitud finita. De ahí, el nombre que dieron al método: Multiple Lapse Time Window Analysis (MLTWA). La primera ventana contiene las ondas directas y la primera parte de la coda, mientras que las siguientes ventanas contienen sólo ondas dispersadas.

Sea $E(r, t \mid \omega)$ la densidad de energía para las ondas directa y dispersada a una distancia hipocentral $r$ y un lapso de tiempo $t$ para una banda de frecuencia angular centrada en $\omega$. Si no se admiten conversiones de onda entre frecuencias, entonces puede tratarse cada una independientemente y omitir $\omega$. Para cada una de las tres ventanas temporales, las integrales de energía se expresan

$$
\begin{aligned}
& E_{1}(r) \equiv v \int_{r / v}^{r / v+T_{1}} E(r, t) d t \\
& E_{2}(r) \equiv v \int_{r / v+T_{1}}^{r / v+T_{2}} E(r, t) d t \\
& E_{3}(r) \equiv v \int_{r / v+T_{2}}^{r / v+T_{3}} E(r, t) d t
\end{aligned}
$$

Los tiempos de inicio de las ventanas, $\mathrm{T}_{1}, \mathrm{~T}_{2}$ y $\mathrm{T}_{3}$ se miden a partir de la llegada de la onda $\mathrm{S}$ igual a $r / v$. Hoshiba et al. (1991) tomaron $\mathrm{T}_{1}=15 \mathrm{~s}, \mathrm{~T}_{2}=30 \mathrm{~s}, \mathrm{~T}_{3}=45 \mathrm{~s} \mathrm{y} v=4 \mathrm{~km} / \mathrm{s}$ como velocidad representativa de la corteza y manto superior. Las integrales de las expresiones (2.111) para el tiempo y la distancia se calculan mediante los parámetros $\bar{t}, \bar{r}$, para evitar la confusión con los valores observados. En este paso, Hoshiba et al. (1991) introdujeron la separación entre la atenuación intrínseca y de scattering por medio de la longitud de extinción, $L_{e}$, y el albedo, $B_{0},(2.19$ y 2.53$)$ pero expresados ahora en función de la atenuación total que es la suma de las componentes de scattering e intrínseca, de la forma 


$$
\begin{aligned}
& L_{e}^{-1} \equiv\left(Q_{s}^{-1}+Q_{i}^{-1}\right) \frac{\omega}{v}=Q_{T}^{-1} \frac{\omega}{v} \\
& B_{0} \equiv \frac{Q_{s}^{-1}}{Q_{T}^{-1}}
\end{aligned}
$$

Multiplicando en la (2.111) a la energía de la onda directa por el factor de atenuación total y a la energía de las ondas dispersadas por el factor de absorción intrínseca de la (2.112), se obtiene

$$
\begin{aligned}
& 4 \pi r^{2} E_{1}(r)=e^{-\bar{L}_{e}-1 \bar{r}}+4 \pi \bar{r}^{2} \frac{\bar{v}}{W_{0}} \int_{\bar{r} / \bar{v}}^{\bar{r} / \bar{v}+\bar{T}_{1}} E_{s}(\bar{r}, \bar{t}) e^{-\bar{h} \bar{v} t} d \bar{t} \\
& 4 \pi r^{2} E_{2}(r)=4 \pi \bar{r}^{2} \frac{\bar{v}}{W_{0}} \int_{\bar{r} / \bar{v}+\overline{T_{1}}}^{\bar{r} / \bar{v}+\bar{T}_{2}} E_{s}(\bar{r}, \bar{t}) e^{-\bar{h} \bar{v} \bar{t}} d \bar{t} \\
& 4 \pi r^{2} E_{3}(r)=4 \pi \bar{r}^{2} \frac{\bar{v}}{W_{0}} \int_{\bar{r} / \bar{v}+\overline{T_{2}}}^{\bar{r} / \bar{v}+\bar{T}_{3}} E_{s}(\bar{r}, \bar{t}) e^{-\bar{h} \bar{v} \bar{t}} d \bar{t}
\end{aligned}
$$

donde

$$
\begin{array}{ll}
\bar{L}_{e}^{-1}=\bar{g} / B_{0} ; & \bar{h}=\bar{L}_{e}^{-1}\left(1-B_{0}\right)=\bar{g}\left[\left(1-B_{0}\right) / B_{0}\right] \\
\bar{T}_{i}=T_{i}\left[B_{0} v /\left(L_{e} \bar{g} \bar{v}\right)\right] ; & \bar{r}=r\left[B_{0} /\left(L_{e} \bar{g}\right)\right]
\end{array}
$$

El primer término del lado derecho en la expresión de $E_{1}(\mathrm{r})$ es la energía de la onda directa afectada por la atenuación total. El factor $e^{-\bar{h} \bar{v}}$ introduce en cada expresión la absorción intrínseca. Las curvas de la Fig. 2.25 muestran la diferencia en el decaimiento de la densidad de energía entre la primera y la última ventana temporal debido al efecto de la atenuación total en la primera ventana.

Las distribuciones de energía fueron calculadas para distancias $r \leq 250 \mathrm{~km}$ y diferentes pares de $L_{e}$ y $B_{0}$, obteniendo curvas teóricas de $4 \pi r^{2} E_{i}(r)$ con $i=1,2,3$ (Fig. 2.26), que se utilizan para comparar con las observadas en datos reales. De la comparación resulta la selección del par $L_{e}$ y $B_{0}$ que mejor ajusta las curvas teóricas a los datos (Fig. 2.27). Las curvas originales provistas por Hoshiba et al. (1991) consideran que la energía radiada es igual a la unidad y que todos los efectos de sitio son idénticos, por lo que deben hacerse las correcciones correspondientes.

Hoshiba (1991) observó que la consideración de un $Q_{i}^{-1}$ espacialmente uniforme no bastaba para justificar la variación de $Q_{c}{ }^{-1}$ con el tiempo. Al aplicar el MLTWA a datos reales, Fehler et al. (1992) concluyeron que la hipótesis de scattering uniforme e isótropo y la suposición de que en todo el intervalo de tiempo analizado existían sólo ondas internas ponían limitaciones al ajuste sobre la tercera ventana (Mayeda et al., 1992, Hoshiba, 1993, Akinci et al., 1995). 

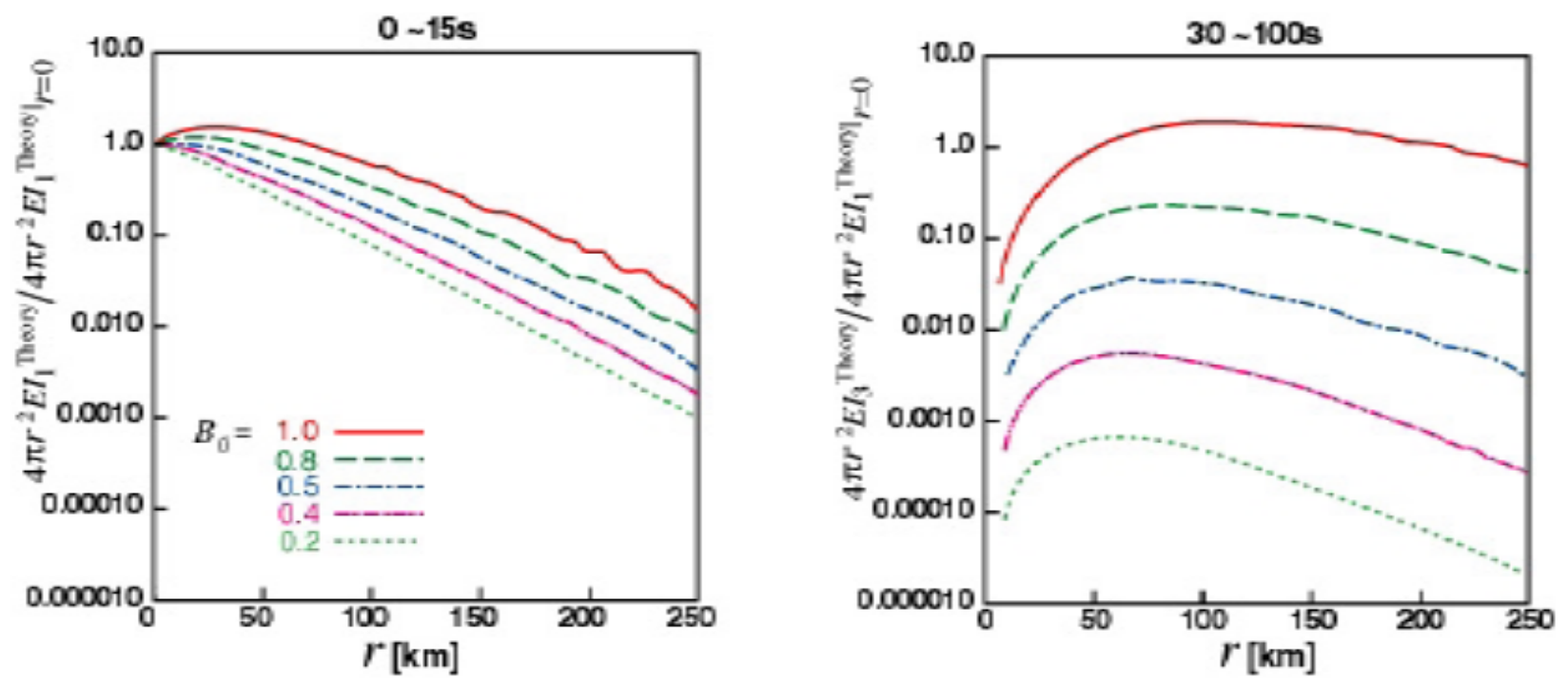

Fig. 2.25.-Energía integrada corregida por expansión geométrica para la primera ventana temporal (izquierda) y para la tercera ventana (derecha) evaluada en diferentes valores del albedo, $B_{0}$. Las curvas rojas corresponden al modelo sin atenuación intrínseca. (Extractada de Fehler et al., 1992).

Zeng et al. (1991) reunieron las teorías de scattering simple y múltiple, así como la del transporte de energía en una expresión teórica de la distribución de energía integrada. La versión 3D de la misma que sería dada por Zeng (1993) es

$$
E(\vec{r}, t)=E_{i n}\left(t-\frac{\left|\vec{r}-\vec{r}_{0}\right|}{v}\right) \frac{e^{-\eta\left|\vec{r}-\vec{r}_{0}\right|}}{4 \pi\left|\vec{r}-\vec{r}_{0}\right|}+\int_{V} \eta_{s} E\left(\vec{r}_{1}, t-\frac{\left|\vec{r}_{1}-\vec{r}\right|}{v}\right) \frac{e^{-\eta\left|\vec{r}_{1}-\vec{r}\right|}}{4 \pi\left|\vec{r}_{1}-\vec{r}\right|^{2}} d V_{1}
$$

donde $E(\vec{r}, t)$ es la densidad de energía dispersada, $E_{\text {in }}$ es la energía incidente, $\vec{r}_{0}$ es el vector posición de la fuente, $\vec{r}$ es el vector posición del receptor, $\vec{r}_{1}$ es el vector posición de todos los posibles dispersores en un diferencial de volumen $V_{1}$ dentro del volumen $V, \eta_{s}$ es el coeficiente de scattering, $\eta_{i}$ es el coeficiente de absorción, $\eta$ es el coeficiente de atenuación total $\left(\eta=\eta_{s}+\eta_{i}\right)$ y $v$ es la velocidad de las ondas $\mathrm{S}$.

La energía de la onda incidente proveniente de la fuente está representada por el primer término del segundo miembro, mientras que el segundo término es la superposición lineal de las energías de las ondas dispersadas en todas las heterogeneidades posibles en los puntos señalados por $\vec{r}_{1}$ y recibida en $\vec{r}$. 

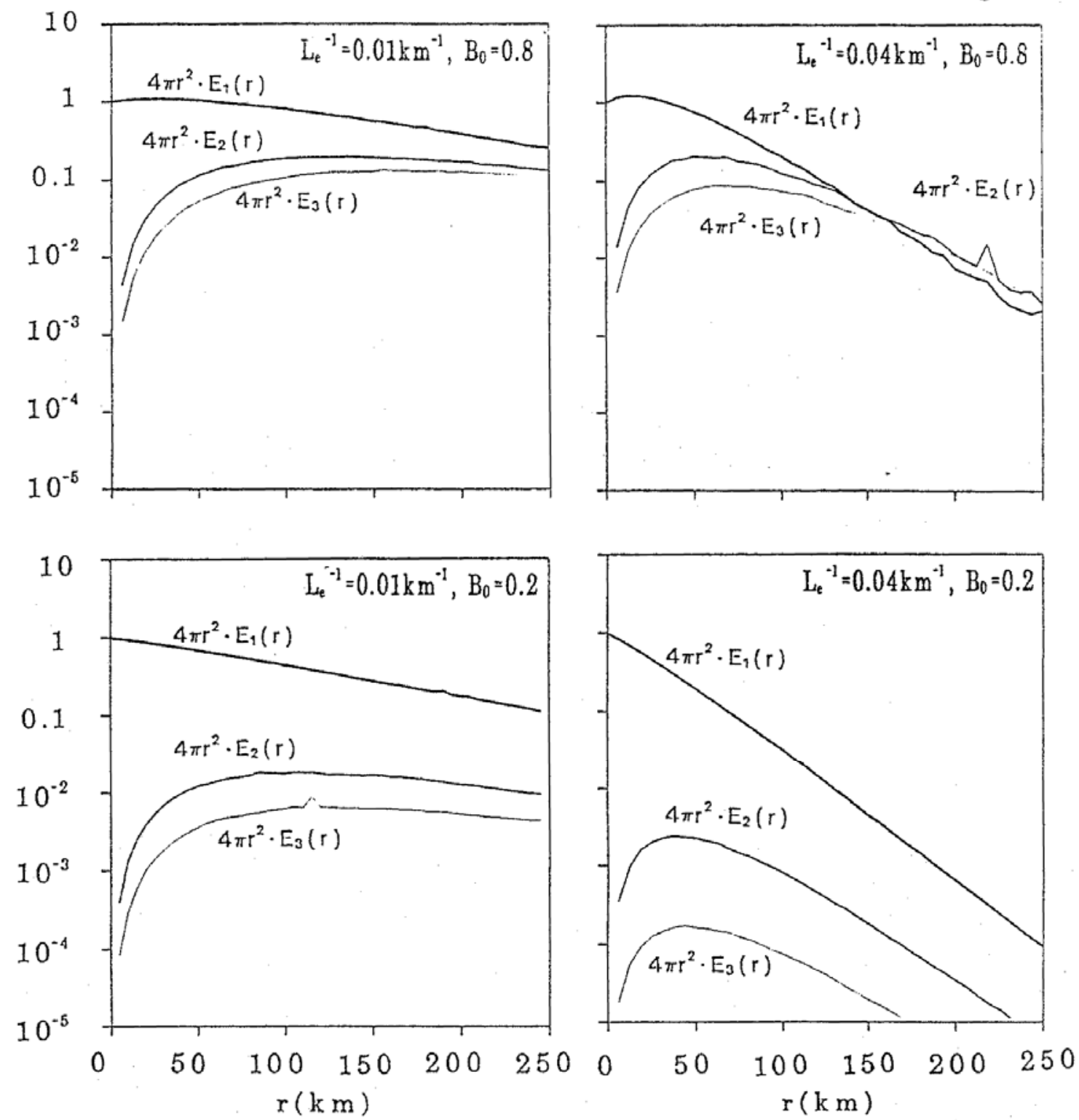

Fig. 2.26.-. Curvas de distribución de energía $4 \pi r^{2} E_{1}(r), 4 \pi r^{2} E_{2}(r)$ y $4 \pi r^{2} E_{3}(r)$ en función de la distancia epicentral $r(\mathrm{~km})$ para los casos donde $L_{e}^{-1}=0.01$ y $0.04 \mathrm{~km}^{-1}$ con $B_{0}=0.2$ y 0.8 . Los valores de $T_{1}, T_{2}$ y $T_{3}$ se fijaron a 15s, 30s y 45s. (Extractada de Hoshiba et al, 1991)

Zeng (1991) resolvió la (2.115) en el dominio de las transformadas integrales obteniendo una solución compacta que comparó con la simulación de Hoshiba (1991), con el modelo de scattering simple, con el de flujo de energía y el de difusión. A partir de sus resultados obtuvo una solución aproximada al scattering múltiple mediante un modelo híbrido de scattering simple y difusión

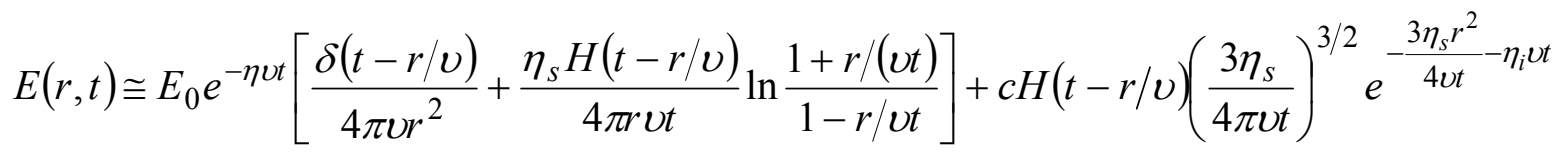


donde

$$
c=E_{0}\left[1-\left(1+\eta_{s} v t\right) e^{-\eta_{s} v t}\right] /\left(\frac{4}{\sqrt{\pi}} \int_{0}^{\sqrt{3 \eta_{s} v t} / 2} e^{-\alpha^{2}} d \alpha\right)
$$

$E_{0}$ es la energía total incidente a $t=0, t$ es el tiempo de viaje, $H$ es la función de Heaviside, $r$ es la distancia hipocentral y $\alpha=\frac{v t}{r}$. El coeficiente de atenuación intrínseca, $\eta_{i}=2 \pi f v^{-1} Q_{i}^{-1}$, y el coeficiente de atenuación por scattering, $\eta_{s}=2 \pi f v^{-1} Q_{s}^{-1}$, se relacionan a través de la atenuación total $\eta_{\mathrm{s}}+\eta_{i}=L_{e}^{-1}$ y el albedo sísmico $B_{0}=\eta_{s} /\left(\eta_{s}+\eta_{i}\right)$.

Para aplicar en datos reales la aproximación de Zeng (1991) al MLTWA, siguiendo a Bianco et al. (2002), se eligen tres ventanas sucesivas en el sismograma: la primera comienza en la llegada de la onda $\mathrm{S}$ y se extiende hasta el inicio de la coda, $\left[r / v, r / v+T_{1}\right]$, la segunda ventana comprende la parte media de la coda $\left[r / v+T_{1}, r / v+T_{2}\right]$ y la tercera contiene el final de la coda $\left[r / v+T_{2}, r / v+T_{3}\right]$. La longitud de las tres ventanas es idéntica y se elige de modo que la primera ventana contenga la mayor contribución de la energía de ondas directas mientras que en las restantes predominen las ondas dispersadas. Las densidades de energía
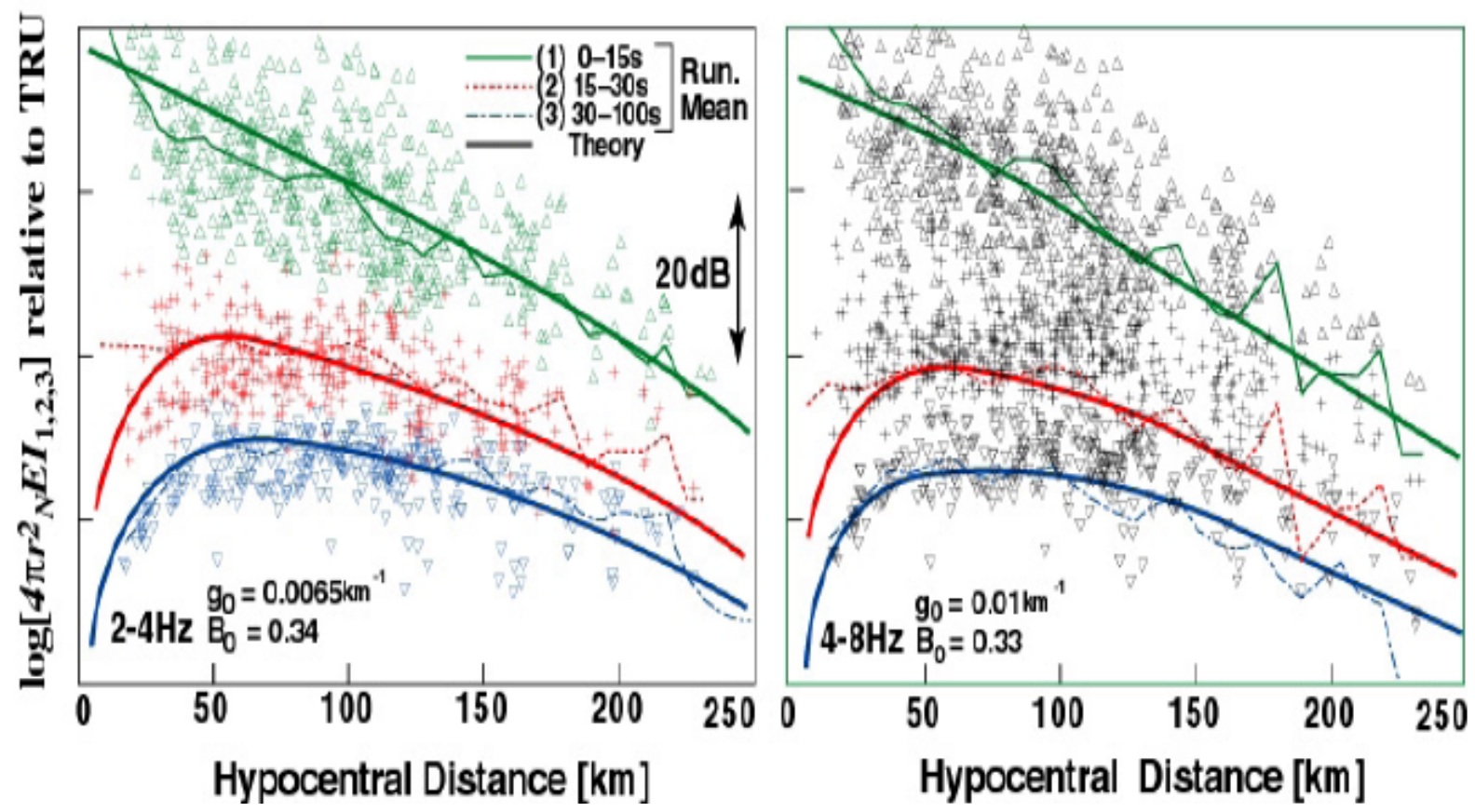

Fig. 2.27.- Primera aplicación del MLTWA a datos reales en Japón (extractada de Fehler et al., 1992). Los valores individuales de la energía integrada corregida de expansión geométrica vs. distancia. para las tres ventanas temporales en dos bandas de frecuencia se muestran como triángulos o cruces. Los promedios sobre ventanas móviles de $15 \mathrm{~km}$ de distancia se señalan con líneas punteadas. Las curvas teóricas seleccionadas como mejor ajuste a los datos se muestran con líneas sólidas. 
observadas en función de la distancia y la frecuencia, se calculan midiendo las amplitudes RMC de los sismogramas filtrados en una serie de bandas de paso elegidas de acuerdo a los datos. La energía de cada ventana debe ser corregida del efecto de sitio y del patrón de radiación de la fuente utilizando el método de NC, para lo cual se elige una ventana de coda a un tiempo $t_{r e f}>2 t_{S}$ para evitar la contaminación con las ondas $\mathrm{S}$ directas, quedando

$$
E_{i}\left(f, r_{m}\right)=\frac{e_{i}\left(f, r_{m}\right)}{E_{\text {coda }}\left(f, t_{\text {ref }}\right)} 4 \pi r_{m}{ }^{2} \quad i=1,2,3
$$

donde $e_{i}\left(f, r_{m}\right)$ es la amplitud RMC calculada en función de $f$ y $r_{m}$ integrada sobre cada una de las ventanas, $E_{\text {coda }}\left(f, t_{\text {ref }}\right)$ es la energía de la coda observada al tiempo de referencia $t_{\text {ref }}$ y $E_{i}\left(f, r_{m}\right)$ es la energía observada normalizada a la frecuencia $f$ en la ventana $i$. La corrección por expansión geométrica se realiza multiplicando cada energía integrada por $4 \pi r_{m}^{2}$.

Las curvas teóricas se obtienen integrando la (2.116) en cada intervalo de tiempo elegido $\left[\mathrm{t}_{\mathrm{i}}(r), \mathrm{t}_{\mathrm{i}+1}(r)\right]$

$$
E_{i}^{*}=\int_{t_{i}(r)}^{t_{i+1}(r)} E(r, t) d t \quad i=1,2,3
$$

En esta aproximación debe asumirse un valor de $v$, la velocidad promedio de las ondas S. Las energías de la (2.116) se calculan para varios valores de $L_{e}^{-1}$ y $B_{0}$ y se normalizan de modo que puedan compararse con diferentes espectros de fuente y efectos de sitio utilizando el método de NC.

$$
E c_{i}(r)=\log \left[\left(\frac{E_{i}^{*} /\left(t_{i+1}(r)-t_{i}(r)\right)}{E\left(r, t^{*}\right)}\right) 4 \pi r^{2}\right] \quad i=1,2,3
$$

Las energías observadas $E_{i}\left(f, r_{m}\right)$ se comparan entonces con las energías integradas teóricas corregidas $E c_{i}(r)$ para elegir la combinación más apropiada de $L_{e}$ y $B_{0}$ en cada frecuencia. La elección puede hacerse en forma automática (Bianco y Del Pezzo 2002) por mínimos cuadrados, utilizando la función de desajuste (misfit function) entre los datos observados y el modelo (Hoshiba et al., 1991)

$$
M\left(B_{0}, L_{e}^{-1}\right)=\sum_{k=1}^{N} \sum_{i=1}^{3}\left(E_{i}(f, r)-E c_{i}(r)\right)^{2}
$$

Los parámetros del modelo, $\left(\eta_{\mathrm{i}}, \eta_{\mathrm{s}}\right)$, que mejor ajusta a los datos son función de $L_{e}^{-1} \mathrm{y}$ $B_{0}$. Con ellos pueden calcularse los valores de $Q_{i}^{-1}$ y $Q_{s}^{-1}$ esperados despejando de la (2.112) las siguientes expresiones 


$$
\begin{aligned}
Q_{s}^{-1} & =v B_{0} /\left(L_{e} \omega\right) \\
Q_{i}^{-1} & =v\left(1-B_{0}\right) /\left(L_{e} \omega\right)
\end{aligned}
$$

Esta aproximación sería adoptada por numerosos autores como modelo de absorción y scattering en la corteza ya que se acerca suficientemente a la solución general de la (2.115) tanto para scattering débil como fuerte y para diferentes configuraciones de fuente y receptor (Pujades et al., 1997, Ugalde et al., 1998, Bianco et al., 2002, Giampiccolo et al, 2006).

\subsubsection{MÉTODO DE WENNERBERG (MWe)}

Sobre la base del balance entre la energía dispersada desde las ondas directas y la energía en la coda, Frankel y Wennerberg (1987) propusieron una formulación sencilla para el decaimiento de la coda que diferencia explícitamente entre atenuación intrínseca y de scattering (sección 2.2.4.4). Su teoría es válida tanto para el scattering débil como para el fuerte e incluye implícitamente el scattering múltiple. Demostraron que el modelo de scattering simple no ajusta el decaimiento de coda cuando el scattering es moderado a fuerte $(Q \leq 150)$; que el decaimiento de la coda es sensible al $Q_{i}$ del medio y que el cociente entre la amplitud de la coda y la energía de la onda directa da una medida de la atenuación por scattering.

$\mathrm{Wu}$ (1985), utilizando la teoría de transporte de energía o transferencia radiativa (Sección 2.2.4.3), había encontrado que la forma de la curva de densidad de energía en función de la distancia podía ser usada para separar $Q_{s}$ y $Q_{i}$. Zeng et al. (1991) demostraron que los modelos de scattering simple y múltiple y la teoría del transporte de energía podían unificarse en una única ecuación integral (2.115). Zeng (1991) desarrolló dos soluciones compactas de la (2.115) para el caso en que la fuente y el receptor coinciden en un punto. La primera solución considera scattering múltiple (SZ) y la segunda es una solución aproximada que combina el scattering simple y la difusión (SDZ) para obtener el scattering múltiple (2.116).

Los resultados para el scattering débil y fuerte mostraron que la solución híbrida (2.116) coincidía bastante bien con la solución exacta cuando la separación entre fuente y receptor era pequeña.

Varios autores (Phillips y Aki, 1986; del Pezzo et al., 1990; Abuvakirov y Gusev, 1990; Hoshiba, 1991; Mayeda et al., 1992; Fehler et al., 1992) habían observado una dependencia de $Q_{c}$ con el lapso de tiempo considerado. Esta variación no podía ser explicada sólo por el efecto del scattering múltiple, como sugirió Zeng (1991), sino que requería variaciones de la atenuación intrínseca y/o de scattering en el volumen involucrado en la generación de codas 
que se expandía con el lapso de tiempo.

Wennerberg (1993) postuló que, mientras la hipótesis de una distribución uniforme de heterogeneidades puntuales y la atenuación intrínseca no alcanzaban para justificar la dependencia temporal de $Q_{c}$, el modelo de scattering múltiple con esas hipótesis podía usarse para estimar las propiedades medias de un medio no uniforme. Dando una cuantificación de los papeles de la atenuación intrínseca y de scattering en la determinación de $Q_{c}^{-1}$, era posible separar, en principio, sus efectos a través de una combinación de medidas de $Q_{c}$ y $Q_{d}$. Un sistema de ecuaciones lineales sencillo relacionaba las cantidades mencionadas a los parámetros físicos de ambas atenuaciones y proveía una medida independiente que podía compararse con la propuesta por Abuvakirov y Gusev (1990) para $Q_{s}$. Presentó una simulación numérica que comparaba la forma de onda del modelo SBS con el modelo de Zeng (1991) para fuente y receptor espacialmente coincidentes y con el modelo de flujo de energía. A partir de la enorme similitud entre ellos para un cierto lapso de tiempo, analizando las contribuciones del scattering y la absorción a la coda, observó que la atenuación intrínseca controlaba al $Q_{c}$ y que ambos efectos combinados conducían a $Q_{c}^{-1}<Q_{i}^{-1}$. Este modelo implicaba que para que el scattering tuviera un efecto sobre la coda similar a la atenuación intrínseca, debía darse una relación $Q_{s}^{-1}>[5-15] Q_{i}^{-1}$ y a su vez, había predicho que $Q_{c}^{-1}$ debía ser menor que $Q_{d}^{-1}$. La igualdad entre estos valores
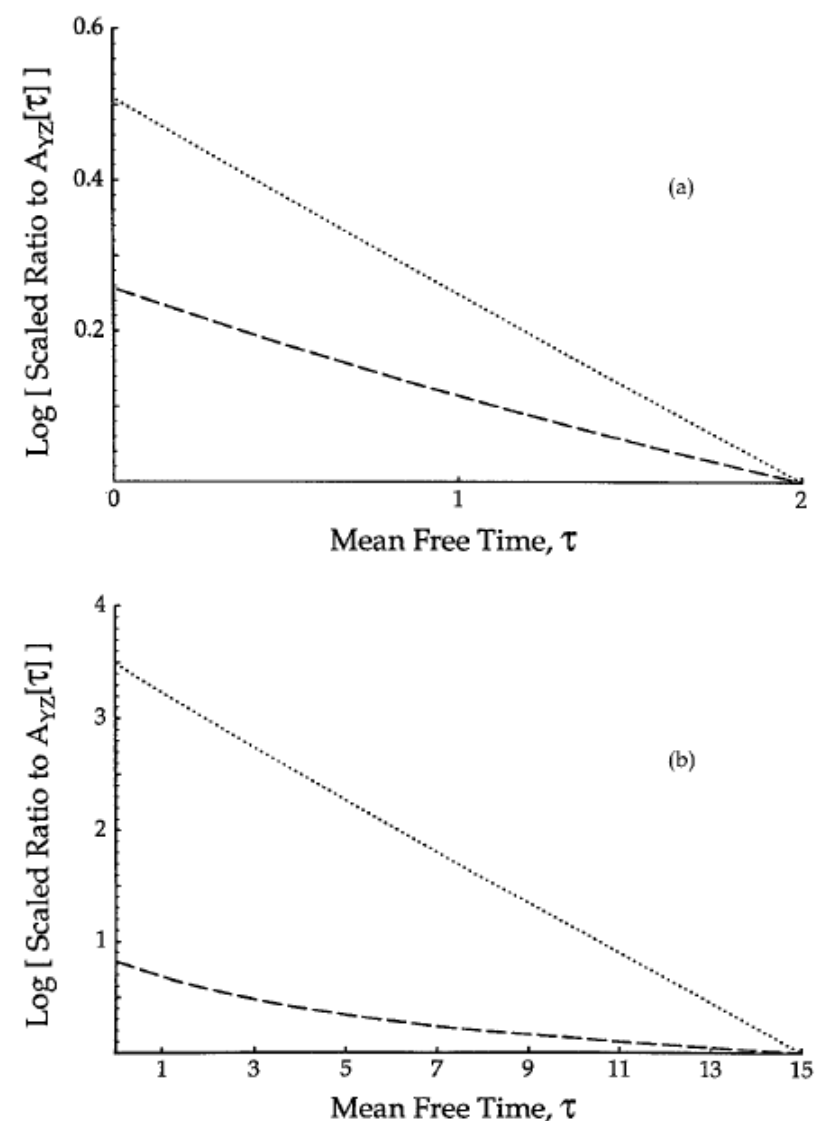

Fig. 2.28.- Logaritmos de los cocientes de las envolventes postuladas por el scattering simple (puntos) y el flujo de energía (segmentos) con los predichos por Zeng para scattering múltiple débil (a) y fuerte (b). Extractada de Wennerberg, 1993. observada por Aki (1980b) fue explicada por Wennerberg como debida a un fenómeno de scattering débil.

Observando las desviaciones de los cocientes entre las envolventes del SBS y del SZ con el FE, para scattering débil y fuerte en función del tiempo libre medio $\left(\tau=t \omega / Q_{s}=t \eta_{s} v\right)$, Wennerberg (1993) observó sus decaimientos exponenciales (Fig. 2.28). Si los cocientes de 
amplitudes pueden expresarse, genéricamente como $e^{-\delta \tau}$ entonces, la amplitud de la envolvente correspondiente a SBS se aproximaba por

$$
A_{S B S}(t) \propto \frac{e^{\frac{-\omega t}{2 Q_{c}}}}{t}
$$

y la correspondiente al SDZ, se simplificaba en la expresión

$$
A_{S D Z}(t) \propto \frac{e^{-\frac{\omega t}{2 Q_{s}}(1-2 \delta)}}{t}
$$

De allí resultaba que, si no había atenuación intrínseca, un valor de $Q_{c}$ observado podía relacionarse con el $Q_{s}$ de Zeng (1991) a través de

$$
\frac{1}{Q_{c}}=\frac{1-2 \delta}{Q_{s}}
$$

donde $\delta$ dependía del lapso de tiempo. La expresión de $\delta$ se estimaba mediante ajustes lineales de modo que como una buena aproximación numérica, Wennerberg (1993) estableció la relación

$$
\frac{1}{Q_{c}(\tau)}=\frac{-1}{(4.44+0.738 \tau) Q_{s}}
$$

Si se incluía la atenuación intrínseca, entonces

$$
\frac{1}{Q_{c}(\tau)}=\frac{1}{Q_{i}}+\frac{1-2 \delta(\tau)}{Q_{s}}
$$

donde seguía siendo $1-2 \delta(\tau)=-1 /(4.44+0.738 \tau)$ y a su vez, $\tau=2 \pi f t / Q_{s}$. Reuniendo la (2.127) con la (2.49) y suponiendo que la determinación de $Q_{\mathrm{d}}$ incluía ambos efectos de atenuación representando el efecto total de la atenuación, quedaba establecido un sistema de ecuaciones sencillo que permitía distinguir $Q_{i}$ y $Q_{s}$. Despejando se obtenía

$$
\begin{gathered}
\frac{1}{Q_{s}}=\frac{1}{2 \delta(\tau)}\left(\frac{1}{Q_{d}}-\frac{1}{Q_{c}(\tau)}\right) \\
\frac{1}{Q_{i}}=\frac{1}{2 \delta(\tau)}\left(\frac{1}{Q_{c}(\tau)}+\frac{2 \delta(\tau)-1}{Q_{d}}\right)
\end{gathered}
$$

Como $Q_{s}^{-1}$ debe ser positivo, la expresión (2.128) implica que $Q_{d}^{-1}$ debe ser mayor que $Q_{c}^{-1}$ y que si $Q_{c}^{-1}$ es cercano a $Q_{s}^{-1}$, entonces la atenuación por scattering debe ser muy pequeña (Wennerberg, 1993). 
La implementación del método se realiza seleccionando valores de $Q_{d}^{-1}$ y $Q_{c}{ }^{-1}$ estimados para un paquete de ondas directas y sus ondas coda a fin de analizar los efectos de la atenuación intrínseca y de scattering sobre las mismas ondas a lo largo de su propagación. Es así que deben seleccionarse lapsos de tiempo en la coda comparables a los tiempos de la propagación directa. Esto significa que si la onda directa demoró un tiempo $t_{d}=r / v$ en recorrer la trayectoria, las ondas coda habrán sido dispersadas en un elipsoide de semieje mayor $\tau=t_{c}=2 r / v$, para que se cumpla la validez del modelo SBS utilizado en la determinación de $Q_{\mathrm{c}}$. Si no se dispone de los valores de $Q_{\mathrm{c}}$ correspondientes a $t_{\mathrm{c}}$, estos pueden extrapolarse mediante la expresión del decaimiento de las amplitudes espectrales estimada previamente (Del Pezzo et al., 1995). En general los resultados de las aplicaciones del MWe que se encuentran en la bibliografía corresponden a tiempos de propagación de S o distancias hipocentrales muy cortos, como es en el caso de la sismología volcánica (Del Pezzo et al., 1995, Martínez Arévalo et al., 2003).

Reemplazando los valores de $Q_{d}^{-1}$ y $Q_{\mathrm{c}}$ en las (2.128) y (2.129) se resuelven las ecuaciones de segundo grado que resultan del despeje de las mismas, obteniéndose los valores de $Q_{\text {s }}$ y $Q_{\text {i }}$ por el MWe.

\subsubsection{ALGUNOS RESULTADOS DE LA SEPARACIÓN DE $\boldsymbol{Q}_{s}$ y $\boldsymbol{Q}_{\boldsymbol{i}}$}

La primera aplicación del método MLTWA, fue efectuada por Fehler et al. (1992) para la región de Kanto-Tokai, Japón (curva KTJ, Fig. 2.29). Para determinar los parámetros de atenuación utilizaron dos cocientes: 1) el cociente entre las energías integradas para la primera ventana (0-15 s) a $50 \mathrm{~km}$ y a $150 \mathrm{~km}$ de distancia hipocentral y 2) el cociente entre las energías integradas para $150 \mathrm{~km}$ y ventanas entre 0-15 s y 30-100 s. La corrección por efecto de sitio y función fuente fue realizada siguiendo el método de la onda coda indicado por Phillips y Aki (1986), quienes asumieron que la forma de la curva de decaimiento de la coda era la misma en todos los sitios y para todas las fuentes y que sólo difería su amplitud dependiendo del efecto de sitio y del factor fuente. Las bases físicas de la corrección, eran las mismas del método de FE (Frankel y Wennerberg, 1987). Para la banda de frecuencia entre 1 y $2 \mathrm{~Hz}$, Fehler et al. (1992) no pudieron ajustar completamente los datos y atribuyeron esta deficiencia a la hipótesis de scattering isótropo y uniforme. En la región de Kanto-Tokai el efecto del scattering (0.0011) resultó ser menor que la absorción intrínseca. Para las frecuencias altas, $Q_{c}^{-1}(0.0022)$ resultó aproximadamente igual a $Q_{i}^{-1}(0.0021)$. A partir de mediciones sobre registros en tres componentes, llegaron a la conclusión de que los parámetros de atenuación eran independientes del movimiento de partículas. 

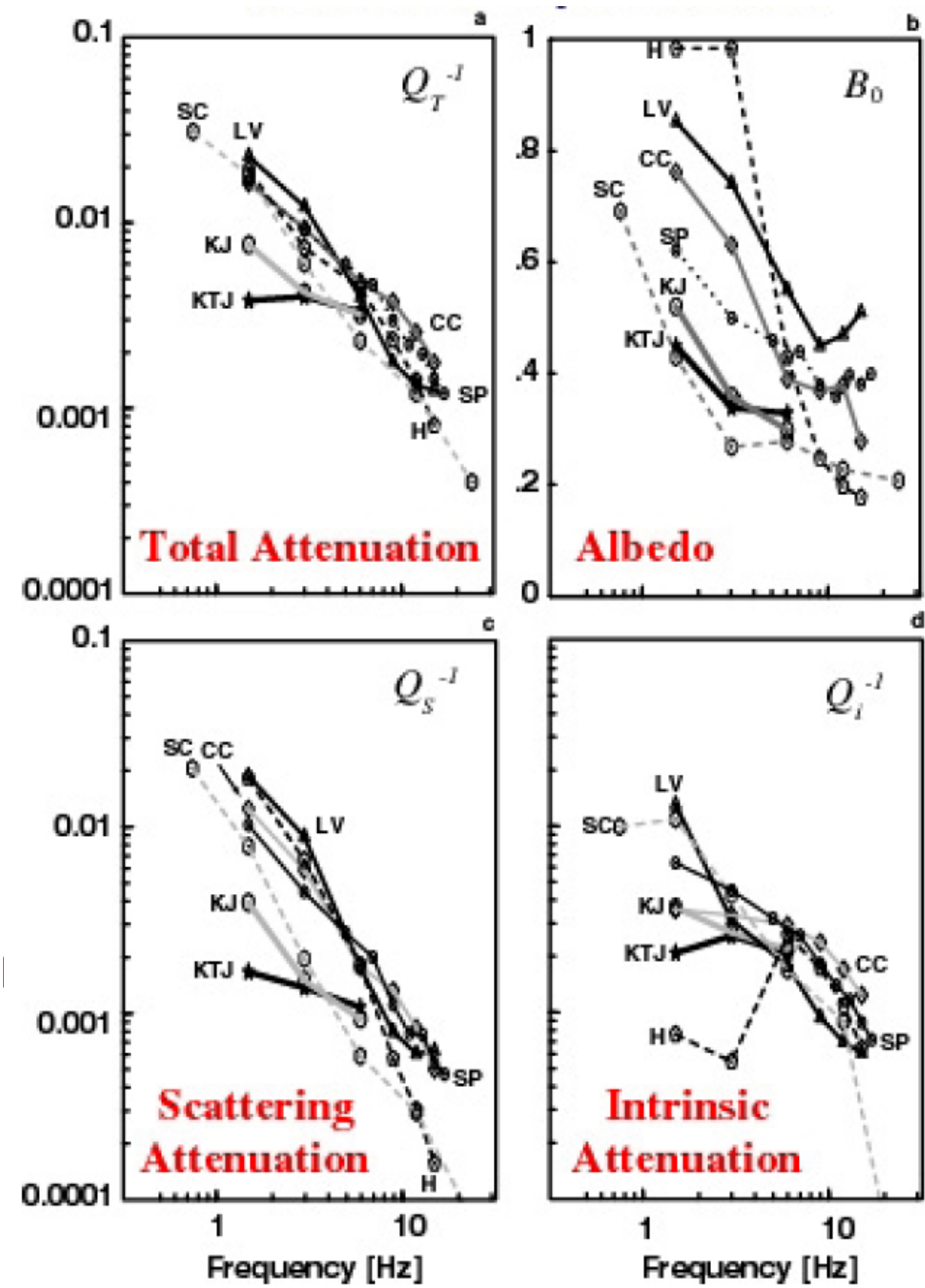

Fig. 2.29. Resumen de resultados obtenidos con el método MLTWA para varias regiones y frecuencias sobre ondas S: (a) atenuación total y (b) albedo sísmico; (c) atenuación por scattering y (d) atenuación intrínseca. KTJ: Kanto-Tokai, Japón (Fehler et al., 1992); KJ, Kanto, Japón (Hoshiba, 1993); LV, Long Valley, CC, Central California, H, Hawaii (Mayeda et al., 1992); SC, Southern California (Jin et al., 1994) y SP, datos de eventos entre 0-170km de profundidad en el sur de España (Akinci et al., 1995). Extractada de Sato y Fehler, 1998.

Hoshiba (1993) calculó los valores de la longitud de extinción y el albedo en Japón, aplicando el método de $\mathrm{NC}(\mathrm{Aki}, 1980)$ para la corrección de los sismogramas. Halló un $B_{0}$ igual a 0.2-0.7 para las frecuencias 1-2 Hz y 0.1-0.3 para el intervalo 4-8 Hz. El $Q_{T}^{-1}$ resultó cercano a $10^{-2}$ para $1 \mathrm{~Hz}$ y $10^{-3}$ para $10 \mathrm{~Hz}$. Tanto la atenuación intrínseca como la de scattering dependían de la frecuencia en forma de potencia con exponentes 0.7 para $Q_{i}^{-1}$ y 1.5 para $Q_{s}^{-1}$ (curva KJ, Fig. 2.29). El rango de distancias hipocentrales elegido afectaba a los 
resultados sobre todo en las frecuencias bajas, indicando la sobresimplificación al suponer isotropía y uniformidad espacial de la atenuación. Sobre la hipótesis del scattering anisótropo, Hoshiba(1995) estimó que en Japón occidental, la energía de la coda se concentraba justo atrás de la llegada de las ondas $\mathrm{S}$ mucho más de lo esperado para el modelo de scattering múltiple e isótropo. Explicó esta característica introduciendo una dependencia angular de tipo Gaussiana para el scattering.

Se han obtenido resultados del MLTWA en diversas regiones del mundo (Estados Unidos: Mayeda et al., 1992, Jin et al., 1994; España: Akinci et al., 1995; Pujades et al., 1997; Turquía: Akinci et al., 1995: Venezuela: Ugalde et al., 1998b; Grecia: Tselentis, 1998; México: Margerin et al., 1999; Norte de Chile: Hoshiba et al., 2001; Italia: Bianco et al., 2002, 2005, Giampiccolo et al., 2006; Francia: Lacombe et al., 2003; Japón: los ya mencionados y Matsunami y Nakamura, 2004; Holanda: Goutbeek et al., 2004; Alaska: Dutta et al., 2009, norte de la India: Padhy, 2009, Korea del Sur: Chung et al., 2010, entre otros). Sato y Fehler (1998) hicieron una síntesis de algunos de estos resultados como se muestra en la Fig. 2.29. Una amplia recopilación hecha por Del Pezzo y Bianco (2007) se presenta en el Capítulo V junto con los resultados de esta tesis (Fig. 5.4 a, b).

Mayeda et al. (1992) analizaron datos de numerosas estaciones en Hawaii, Long Valley y Central California entre 1.5 y $15 \mathrm{~Hz}$. Jin et al. (1994) concluyeron que en el sur de California, el albedo y el scattering mostraban dependencia espacial para frecuencias por debajo de $6 \mathrm{~Hz}$ pero eran similares por encima de $6 \mathrm{~Hz}$. La justificación hallada era que las frecuencias altas viajaban por la litósfera inferior más homogénea, resultando en una menor variación espacial de los parámetros. Así mismo, observaron muy poca variación en la atenuación intrínseca para las diferentes estaciones lo que mostraba cierta uniformidad del comportamiento anelástico en la región.

Akinci et al. (1995) aplicaron el MLTWA para comparar la atenuación en el sur de España y el oeste de Anatolia, Turquía, encontrando que el scattering dependía más de la frecuencia que la absorción intrínseca. En la Fig. 2.30, extractada de Akinci et al. (1995) puede verse que $Q_{c}^{-1}$ se aproxima al $Q_{i}^{-1}$ para ambas regiones y frecuencias intermedias, mientras que para las frecuencias altas se aproxima al $Q_{T}^{-1}$. En España, para frecuencias bajas, $Q_{c}^{-1}$ es similar al $Q_{s}^{-1}$ en las distancias cortas y permanece entre $Q_{i}^{-1}$ y $Q_{s}^{-1}$ para largas distancias. Pujades et al. (1997) extendieron el análisis hacia el sudeste de la península Ibérica, con resultados similares. De la regionalización surgió que la absorción intrínseca era más sensible a las características geológicas que el scattering (en cuencas Cuaternarias y 

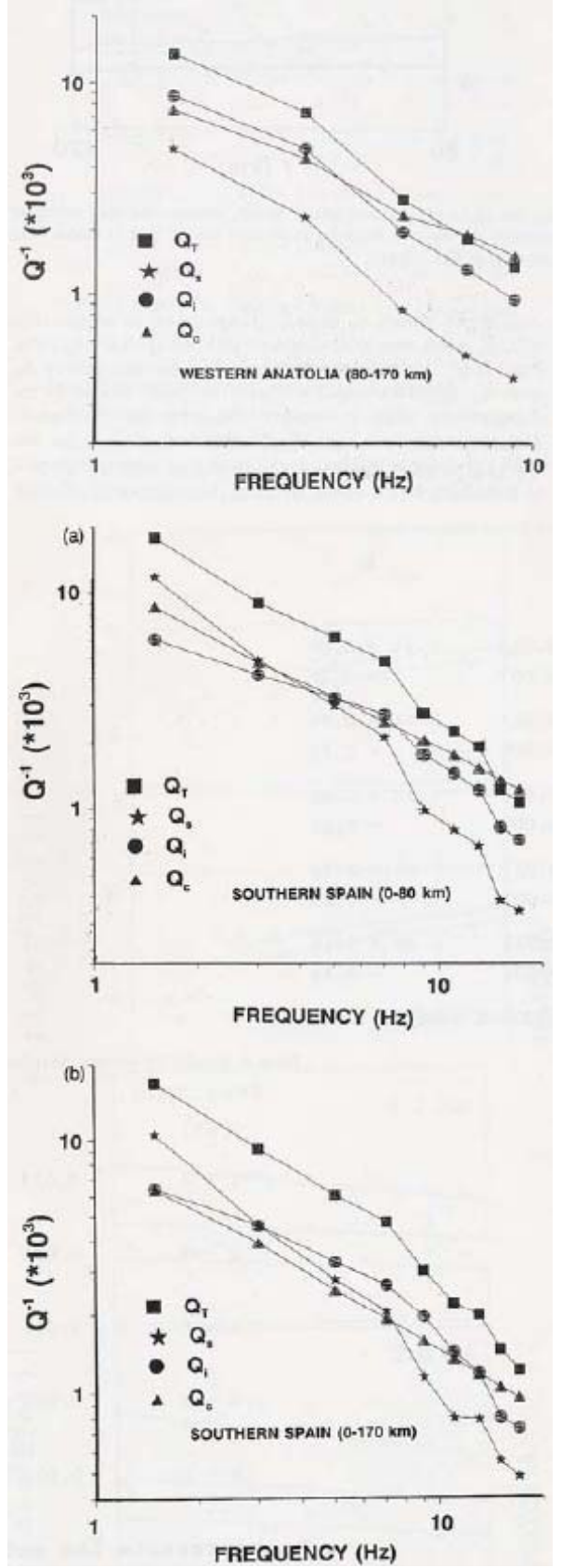

Fig. 2.30.- $Q^{-1}$ total (cuadrados), de scattering (estrellas), intrinseco (círculos) y coda (triángulos) en función de la frecuencia obtenidos en el oeste de Anatolia, Turquía (arriba) y el sur de España (medio: distancias cortas y abajo: distancias largas). Extractada de Akinci et al. (1995).
Neógenas, $Q_{s} \sim 80$ y $Q_{i} \sim 100$; en rocas Paleozoicas, $Q_{s} \sim 200$ y $\left.Q_{i} \sim 1000\right)$. Por otra parte, $Q_{\mathrm{s}}^{-1}$ dominaba la atenuación a bajas frecuencias mientras que $Q_{\mathrm{i}}^{-1}$ predominaba en las altas frecuencias. Una importante conclusión de este trabajo fue que el uso de modelos de scattering múltiple en la separación de $Q_{i}$ y $Q_{s}$, con la hipótesis de fuente $\mathrm{y}$ receptor coincidentes espacialmente, acarreaba dificultades debido a que ambos parámetros están fuertemente acoplados en el ajuste de los datos (Akinci et al., 1995a)

Hoshiba (1994) consideró atenuación variable con la profundidad y una fuente con profundidad no nula, observando que en ese caso la corrección por NC podía no ser la más adecuada y que el decrecimiento de $Q_{c}{ }^{-1}$ con el lapso de tiempo era explicado por la estratificación. Hoshiba $(1995,1997)$ y Hoshiba et al. (1997) comprobaron que los resultados del MLTWA se veían muy afectados por el modelo de velocidades asumido. Hoshiba (1995) incorporó el scattering no isótropo observando que en este caso aumentaba la concentración de energía en la primera ventana temporal.

Margerin et al. (1998, 1999) examinaron la atenuación en un medio con una corteza de velocidad y scattering variables con la profundidad y un manto transparente, prestando atención a la transición entre el régimen de scattering múltiple y la difusión. Observaron que la forma de la coda estaba controlada por la estratificación mientras que el valor de $Q_{\mathrm{c}}$ era determinado por las fugas de energía desde la corteza hacia el manto. Yoshimoto (2000) extendió la simulación para el caso de un gradiente positivo de velocidad con la profundidad, demostrando que la hipótesis de velocidad uniforme sobrevaloraba el coeficiente de scattering. Según los resultados de. Hoshiba et al. (2001) el mecanismo de la fuente no afectaba claramente a las ondas directas por encima de los $2 \mathrm{~Hz}$ y la atenuación por scattering 
era comparable o menor que la atenuación intrínseca para un modelo simple de corteza y manto con dos capas de velocidad uniforme. Estos resultados contradecían a Margerin et al. (1999). Sin embargo, Lacombe et al., (2003) atribuyeron las discrepancias entre los resultados de Margerin et al. (1999) y Hoshiba et al. (2001) a las diferentes ventanas temporales utilizadas en ambos trabajos, sosteniendo que en el comienzo de la coda, la atenuación de la energía dependía de la distancia mientras que al final de la coda este decaimiento era constante e independiente de aquella. Lacombe et al. (2003) obtuvieron en Francia un modelo con una longitud de camino libre medio de $250 \mathrm{~km}$ y una longitud de absorción de $150 \mathrm{~km}$. Sin embargo resultaba difícil establecer un predominio de alguno de ellos.

Ugalde et al. (1998a) introdujeron el cálculo paralelo para reducir el tiempo de estima de las envolventes por el método numérico, permitiendo de este modo introducir modificaciones a las hipótesis del modelo más sencillamente que en la forma analítica. Bianco y Del Pezzo $(2002,2010)$ elaboraron un programa que permite estimar las envolventes mediante la aproximación analítica de Zeng (1991). Aplicándolo sobre los datos de Akinci et al. (1995) compararon sus resultados con aquellos del método numérico de Hoshiba (1991) encontrando que las discrepancias se daban en las distancias muy cortas (menores que $10 \mathrm{~km}$ ) y principalmente en la primera ventana de energía.

Dutta et al. (2004) en Alaska obtuvieron una atenuación intrínseca similar a la de scattering en las bajas frecuencias $(\sim 1 \mathrm{~Hz})$, mientras que a mayores frecuencias la absorción anelástica domina el proceso de atenuación, además $Q_{\mathrm{c}}^{-1}$ a $1 \mathrm{~Hz}$ permanece entre $Q_{\mathrm{i}}^{-1}$ y $Q_{\mathrm{T}}^{-1}$ pero se acerca a $Q_{\mathrm{T}}^{-1}$ para frecuencias mayores. Padhy (2009) analizó datos del terremoto de Chamoli, India del año 1999 hallando una dependencia de ambos efectos de la atenuación con la frecuencia de la forma $Q_{s}^{-1}=0.006 \pm 0.004 f^{0.89 \pm 0.33}$ y $Q_{i}^{-1}=0.003 \pm 0.0005 f^{0.84 \pm 0.08}$ y atribuyendo el mayor scattering a la heterogeneidad lateral de la velocidad cortical.

Los resultados obtenidos usando el método de Wennerberg (1993) en la cuenca de Granada (Fig. 2.31, Del Pezzo et al., 1995) y en Grecia (Tselentis, 1998) mostraron un predominio de la atenuación intrínseca sobre la de scattering y valores de $Q_{\mathrm{c}}$ muy cercanos a $Q_{\mathrm{i}}$. En cambio, los resultados del mismo método en el Volcán Etna (Fig. 2.31, Del Pezzo et al., 1995), en el Monte Vesubio (Bianco et al., 1999) y en Isla decepción (Martínez Arévalo et al., 2003) mostraron un predominio del scattering en zonas volcánicas. Este predominio ha sido confirmado en varias regiones usando diferentes modelos, por ejemplo con un método de separación basado en el modelo del flujo de energía (Frankel, 1991) aplicado en zonas 

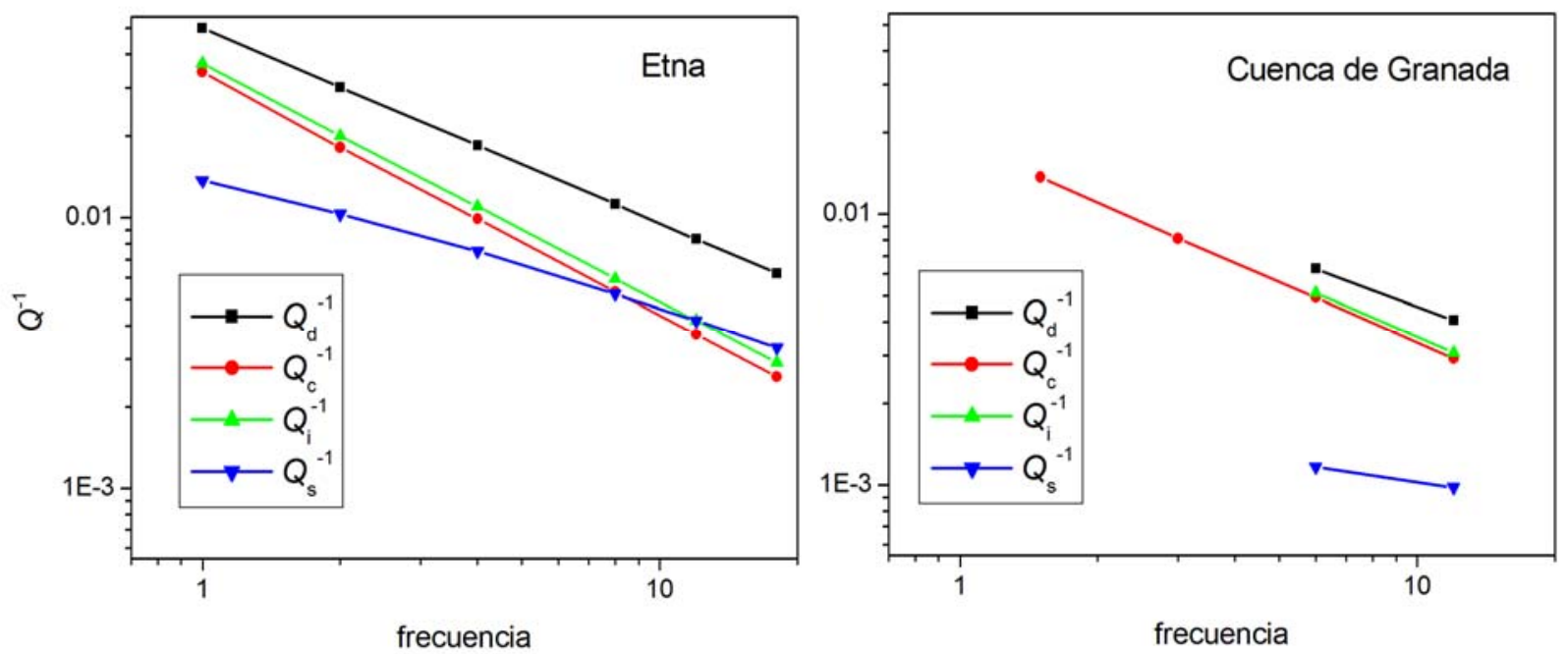

Fig. 2.31.- Valores de $Q_{d}^{-1}, Q_{c}^{-1}, Q_{i}^{-1}$ y $Q_{s}^{-1}$ Vs. frecuencia para el volcán Etna y la Cuenca de Granada obtenidos por Del Pezzo et al. (1995) con el método de Wennerberg (1983).

volcánicas de Italia (Del Pezzo et al., 1996), con el modelo de difusión aplicado en el Monte Vesubio (Del Pezzo et al., 2006) e incluso con el análisis de modelos 3D numéricos complejos en medios elásticos y viscoelásticos (OBrien y Bean, 2009).

El número de aplicaciones del método de Wennerberg que pueden hallarse en la bibliografía, es menor al de las aplicaciones del MLTWA seguramente debido a que las mismas expresiones que lo caracterizan eliminan a todos aquellos datos que resulten en un $Q_{\mathrm{d}}$ $>Q_{\mathrm{c}}$ y además debido a la exigencia del uso de un par de ventanas temporales relacionadas a través de $t_{c}=2 t_{s}$ que en algunos casos resulta en amplitudes de coda al nivel del ruido. Como señaló Wennerberg (1993), sería necesario incorporar modelos no uniformes de heterogeneidad y absorción a la envolvente de coda, sin embargo el scattering múltiple resulta útil para obtener valores promedio de los parámetros en el volumen definido por el lapso de tiempo de coda.

\subsection{MÉTODOS SELECCIONADOS PARA SU APLICACIÓN}

En el presente capítulo se ha recorrido la evolución de los modelos y métodos de determinación de la atenuación de ondas coda; se han presentado algunos métodos de determinación del factor de calidad de ondas directas para el caso de terremotos locales considerando dependencia o independencia de la atenuación con la frecuencia y se han analizado dos métodos de separación de la atenuación intrínseca y de scattering sobre la base de los modelos de generación de ondas coda antes analizados.

En el capítulo siguiente se describirá en detalle la geodinámica interna de la región en 
estudio y se presentarán los datos sismológicos para el análisis. Es por eso que en este punto deben elegirse los métodos a aplicar para así poder establecer los requisitos que deberán satisfacer los datos. Esta selección se efectúa de acuerdo a las observaciones realizadas en las secciones 2.2.5, 2.3.3 y 2.4.3 y teniendo en cuenta los trabajos previos de determinación de la atenuación sísmica en la Región de Nuevo Cuyo (RNC).

En el Capítulo IV y en el caso de la atenuación de ondas coda, se asumirá la validez del scattering simple utilizándose los métodos SBS (Sección 2.2.3.1) y SIS (Sección 2.2.3.2) en función de la frecuencia, el tiempo de coda y la profundidad focal para poder comparar a la RNC con otras regiones sísmicamente activas del mundo. La atenuación de ondas directas ( $\mathrm{P}$ y S) se analizará mediante diferentes técnicas sobre el mismo conjunto de datos. En primer lugar se utilizará el ME (Sección 2.3.2.2) en función de la frecuencia, luego se aplicarán el APP (2.3.2.1) para analizar la atenuación de las ondas P directas independientemente de la frecuencia, el NC (2.3.2.4) para estudiar la atenuación de las ondas S directas y el DRE (2.3.2.3) para el análisis de ondas $\mathrm{Lg}$ o Sg según el caso. Finalmente se implementarán el MLTWA (2.4.2.1) y el MWe (2.4.2.2) para la separación de los efectos de absorción y scattering. 


\section{CAPÍTULO III}

\section{Región de Nuevo Cuyo: Marco Sismotectónico y Datos Sismológicos}

El sábado 15 de enero de 1944, a las 20.52, un terremoto de 7.8 grados en la escala de Richter, destruyó la ciudad de San Juan y enlutó al país entero: la tragedia fue de tal magnitud que ninguna familia salió indemne de algún luto y prácticamente nadie salvó su casa. Nunca se supo exactamente la cantidad de muertos. Se estima que fueron entre 10 y 15 mil personas.

Desde la mañana los animales se habían comportado de manera extraña: los gallos no cantaron al amanecer sino al atardecer, los caballos relincharon inquietos y los perros, gimiendo de miedo, buscaron sin cesar algún lugar oscuro donde refugiarse.

Pero nadie se apercibió de lo que anunciaban y cuando las grietas se abrieron, ya fue muy tarde: en minutos todo se vino abajo, la Catedral incluida.

Y hasta los que habian logrado

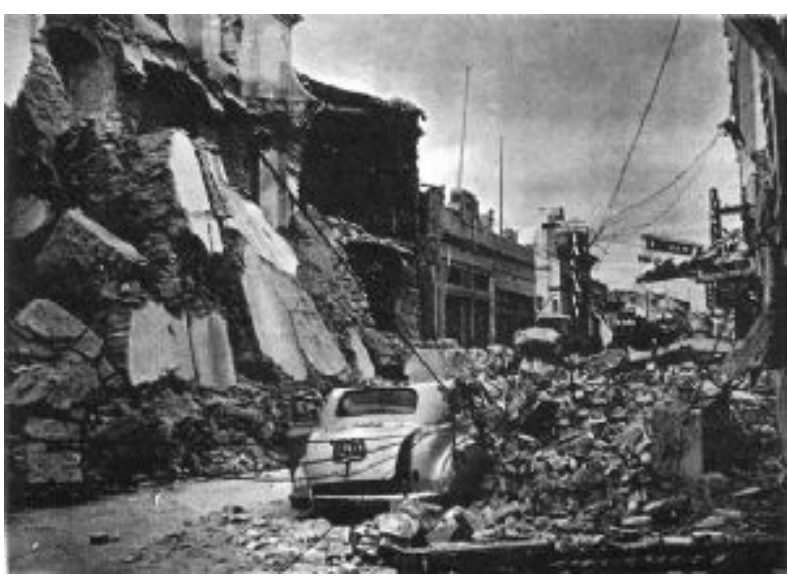
escapar hacia la calle, fueron aplastados por la mampostería de los viejos edificios, que se bambolearon y finalmente cayeron, incapaces de resistir, porque inexplicablemente -desde 1900 se sabia que San Juan estaba situada en zona altamente sísmica- no habian sido hechos para eso.

("A 64 años del terremoto que destruyó la ciudad”, Diario de Cuyo, San Juan, 15/01/2008. Fotografía extraida del libro "Historia Argentina", Diego Abad de Santillán. TEA, Tipográfica Editora Argentina. 1971, Buenos Aires, Argentina.). 


\subsection{LA REGIÓN DE NUEVO CUYO (RNC)}

El presente trabajo se desarrolla en el marco de una región calificada como la de más alta peligrosidad sísmica en la República Argentina (Giardini et al., 1999). Con una morfología y tectónica condicionadas por la subducción de la placa de Nazca bajo la Placa Sudamericana, la Región de Nuevo Cuyo se encuentra conformada por las provincias del Centro-Oeste argentino: San Juan, Mendoza, La Rioja y San Luis, teniendo como principal expresión topográfica a la Cordillera de los Andes.

En particular, la zona de estudio se extiende desde los $28^{\circ} \mathrm{S}$ hasta los $34^{\circ} \mathrm{S}$ y desde los

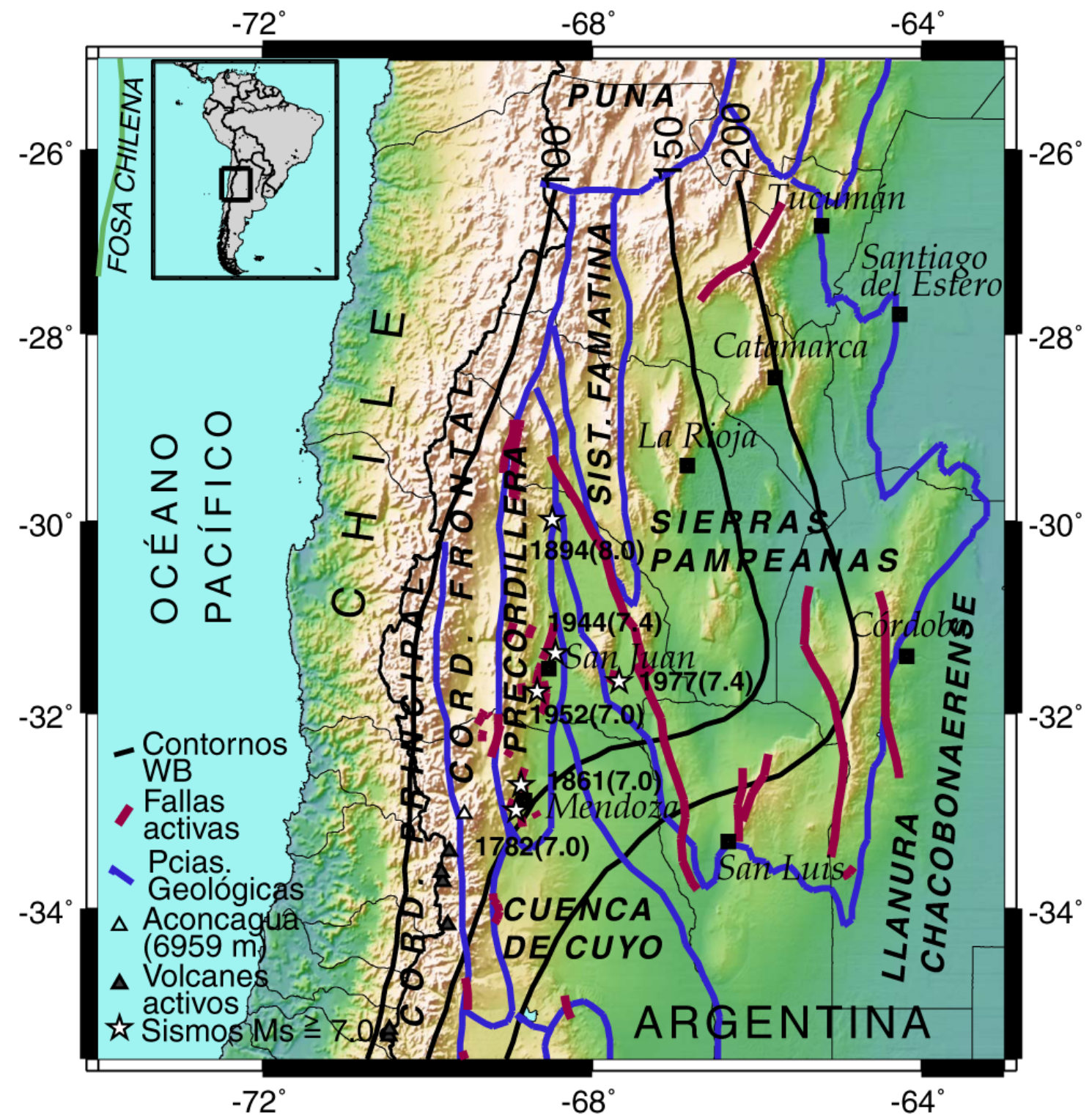

Fig. 3.1.- Región de Nuevo Cuyo y su marco tectónico. Provincias geológicas (Ramos, 1999b), principales fallas activas (Cortés et al., 1999; Costa, 1999), epicentros de sismos históricos con magnitud Ms $\geq 7.0$ (Alvarado et al., 2005; Costa, 1999) y contornos de Wadati-Benioff en km (Cahill y Isacks, 1992). Elaborado con GMT 4.0 (Wessel y Smith, 2004) 
$63^{\circ} \mathrm{O}$ hasta $\operatorname{los} 72^{\circ} \mathrm{O}$ (Fig. 3.1).

Esta zona, cuenta en su historia reciente con sismos destructivos como los que tuvieron epicentro en las ciudades de Mendoza (1861, Ms 7.0), San Juan (1944, Ms 7.4) y Caucete (1977, Ms 7.4) y frecuentemente se ve afectada por eventos sísmicos sentidos (Castano et al., 1999, INPRES, 2006).

La actividad sísmica intracontinental superficial se extiende hacia el este hasta unos 600 $\mathrm{km}$ de distancia de las mayores alturas andinas (Alvarado et al., 2005) ya que incluso Córdoba (1934) y San Luis (1936) han sido seriamente afectadas por terremotos superficiales cercanos.

\subsubsection{LA CORDILLERA DE LOS ANDES}

La Cadena Andina es un oróclino que al sur de la deflexión de Cochabamba o Codo de Arica $\left(20^{\circ} \mathrm{S}\right)$ adquiere un rumbo aproximadamente norte-sur (Fig. 3.3). Hacia el oeste queda flanqueada por la fosa Peruano-Chilena y hacia el este por una zona de retroarco caracterizada por el desarrollo de fajas plegadas y corridas. Los Andes son principalmente un cinturón montañoso no-colisional formado a lo largo de una subducción de larga data y aún activa (Jordan et al., 1983). Esta es la definición del margen tipo Andino, reconocido en diversos márgenes convergentes del registro geológico. La placa de Nazca, cuya corteza oceánica surge a la superficie en las Dorsales Pacífica Este y de Chile, subduce bajo la placa Sudamericana, continental y frágil, con un ángulo que varía de normal (unos $30^{\circ}$ ) a bajo (cerca de $5^{\circ}$ ) según las latitudes observadas.

La velocidad de convergencia total (C) entre las placas está dada por la suma de la velocidad de acortamiento (S) de la placa Sudamericana y la velocidad de subducción ( $\mathrm{Su}$ ) de la placa de Nazca. Según Doglioni et al. (2006), en la actualidad el cociente entre las velocidades de convergencia (alrededor de $7.3 \mathrm{~cm} / \mathrm{año}$ ) y acortamiento del orógeno (aproximadamente $4 \mathrm{~cm} / \mathrm{año}$ ) en los Andes Centrales es de 1.8. La diferencia entre ambas velocidades dará la velocidad con que la placa de Nazca subduce, $3.3 \mathrm{~cm} / \mathrm{año}$, un valor relativamente bajo que justifica el bajo ángulo de subducción. Fijada la velocidad de convergencia, a mayor viscosidad de la placa continental cabalgante, menor será el acortamiento en el orógeno y la subducción será más rápida y viceversa. Por lo tanto conocer la relación convergencia/acortamiento $(\mathrm{C} / \mathrm{S})$ puede ser un medio indirecto de evaluar la viscosidad. En la Fig. 3.2 extractada de Doglioni et al. (2006), la distribución de C/S para Sudamérica permite ver que los valores decrecen levemente hacia el sur, indicando menor viscosidad en la placa continental. 


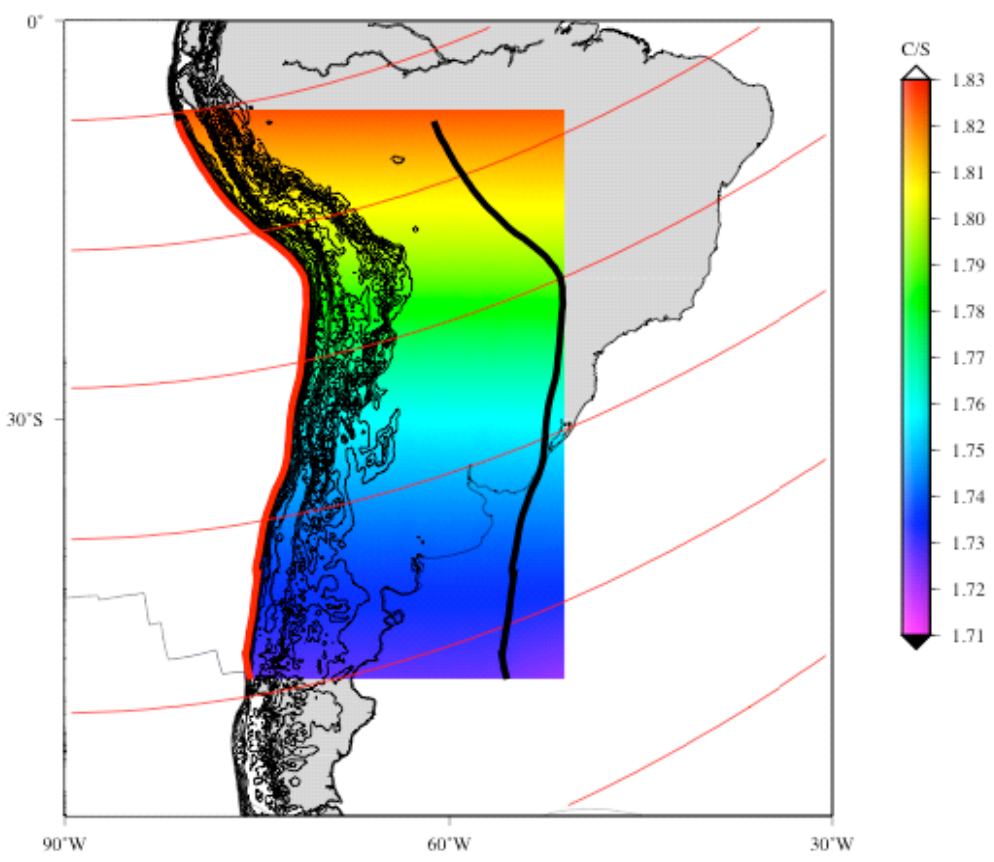

Fig 3.2.- Relación convergencialacortamiento (C/S) a lo largo de la Cordillera de los Andes. La línea gruesa negra se asume como referencia fija en la placa superior estable y la línea gruesa roja, como bisagra de la subducción, convergiendo relativamente pero a menor velocidad que la convergencia entre las placas Sudamericana y de Nazca. Las líneas rojas delgadas son círculos mínimos alrededor del polo de Euler correspondiente a dichas placas. Cuanto mayor sea C/S, mayor será la viscosidad de la litósfera sudamericana (extractada de Doglioni et al., 2006).

El acortamiento y el engrosamiento cortical en el segmento sur de los Andes Centrales, han sido estimados por diversos autores. Existe un gradiente del acortamiento de norte a sur, coincidente con la rotación del antearco (Isacks, 1988, Beck et al., 1996). El máximo acortamiento orogénico y el mayor engrosamiento cortical se dan en los Andes Centrales (Isacks, 1988, Allmendinger et al., 1997). A los $20^{\circ} \mathrm{S}$, los balances corticales a través del norte de Chile y Bolivia indican un acortamiento total de hasta $320 \mathrm{~km}$ durante el Cenozoico (Schmitz, 1994, Kley et al., 1999), mientras que entre los $30^{\circ}-32^{\circ} \mathrm{S}$ el acortamiento es de 160$140 \mathrm{~km}$ (Introcaso et al., 1992, Ramos et al., 1996) y entre los $37^{\circ}-39^{\circ} \mathrm{S}$ es de $44-20 \mathrm{~km}$ (Martínez et al., 1997). Las mediciones de velocidad de acortamiento internas actuales mediante GPS en la zona de subducción plana Pampeana $\left(27^{\circ} \mathrm{S}-33^{\circ} \mathrm{S}\right)$, resultan en valores de $12 \mathrm{~mm} / \mathrm{a}$ para Cordillera-Precordillera y de $5.4 \mathrm{~mm} / \mathrm{a}$ para Sierras Pampeanas (Norabuena et al., 1998, Kendrick et al., 1999).

Las diferentes inclinaciones de la placa subducida, manifestadas en los contornos de la zona de Wadati-Benioff, (Fig. 3.3) permiten entender los procesos que condujeron a la presente distribución de unidades y sus características morfoestructurales. Desarrollada coaxialmente con orogenias previas, la tectónica neógena (23-2.6 Ma) dio como resultado la 


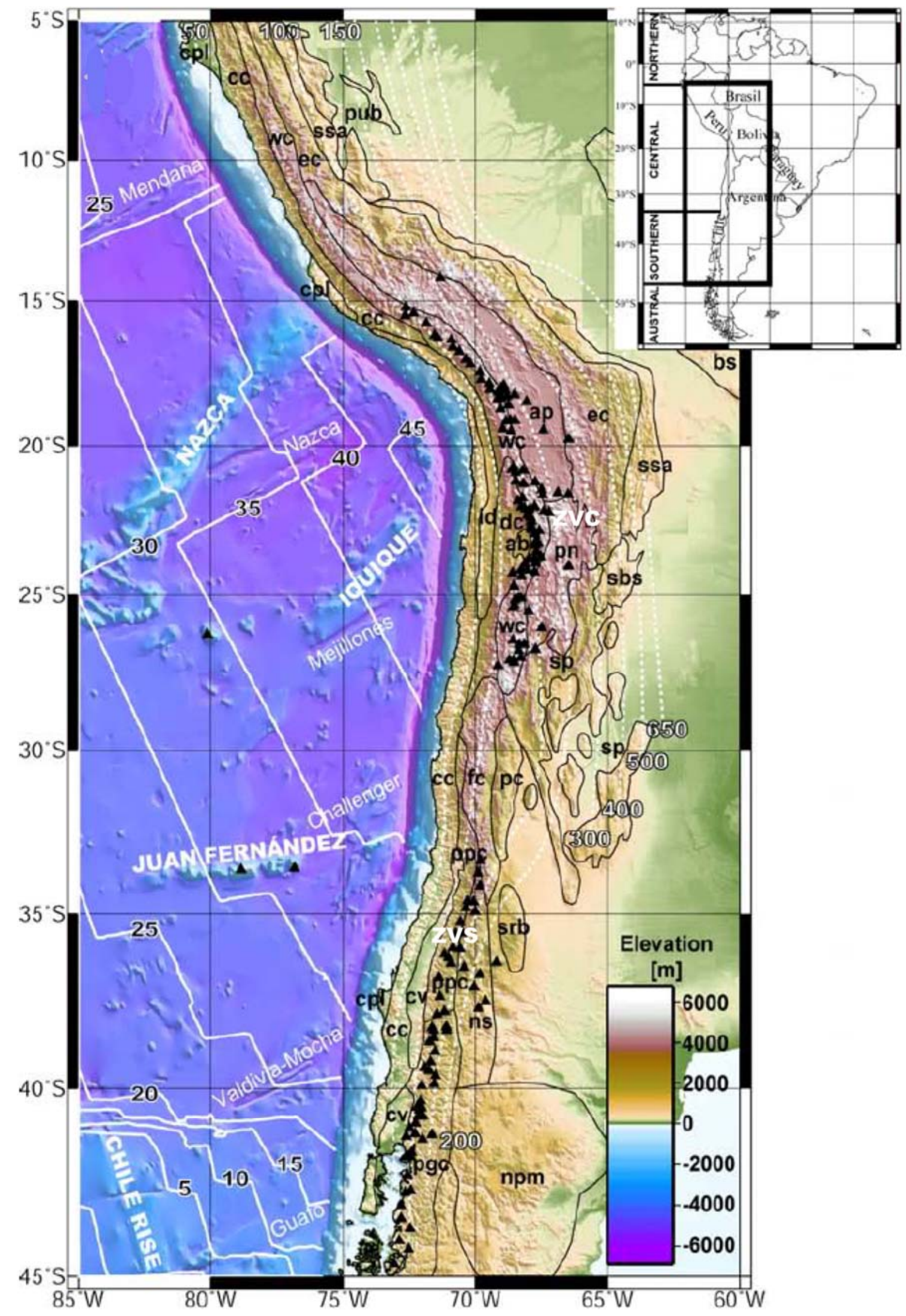


Fig 3.3.- (página anterior) Marco geotectónico de la subducción Nazca-Sudamérica recopilado por Tassara et al. (2006): el mapa inserto muestra el área considerada dentro de Sudamérica y la segmentación a escala continental del margen andino continental. El mapa de elevación digital ha sido tomado de http://www.ngdc.noaa.gov/mgg/gebco. Las líneas blancas costa afuera representan las isócronas de la placa de Nazca (Müller et al., 1997) con edades en millones de años (Ma), los nombres en letras mayúsculas corresponden a las dorsales asísmicas y a los centros activos de divergencia sobre la dorsal de Chile mientras que nombres en minúsculas corresponden a zonas de fractura. Sobre el continente, las líneas punteadas blancas son los contornos de Wadati-Benioff (en $\mathrm{km}$ ) provistos en la base de datos del paquete de programas GMT (Wessel y Smith, 1998), los triángulos son volcanes activos según la base de datos del Instituto Smithsoniano (http://www.volcano.si.edu/gvp/world), ZVC y ZVS (en letras blancas) son las zonas volcánica central y sur respectivamente, las líneas negras esquematizan los bordes de las unidades morfotectónicas según diferentes trabajos citados en Tassara et al. (2006): cpl, Planicies Costeras; pub, Bloques Elevados Peruanos; cc, Cordillera de la Costa; id, Depresión Intermedia; cv, Valle Central; dc, Cordillera de Domeyko; wc, Cordillera Occidental; fc, Cordillera Frontal; ppc, Cordillera Principal; ab, Cuenca de Atacama; ap, Altiplano; pn, Puna; pc, Precordillera; ec, Cordillera Oriental; sp, Sierras Pampeanas; ssa. Sierras Subandinas; sbs, Sistema Santa Barbara System; pgc, Cordillera Patagónica; srb, Bloque San Rafael; ns, Sistema de Neuquén; npm, Macizo Norpatagónico.

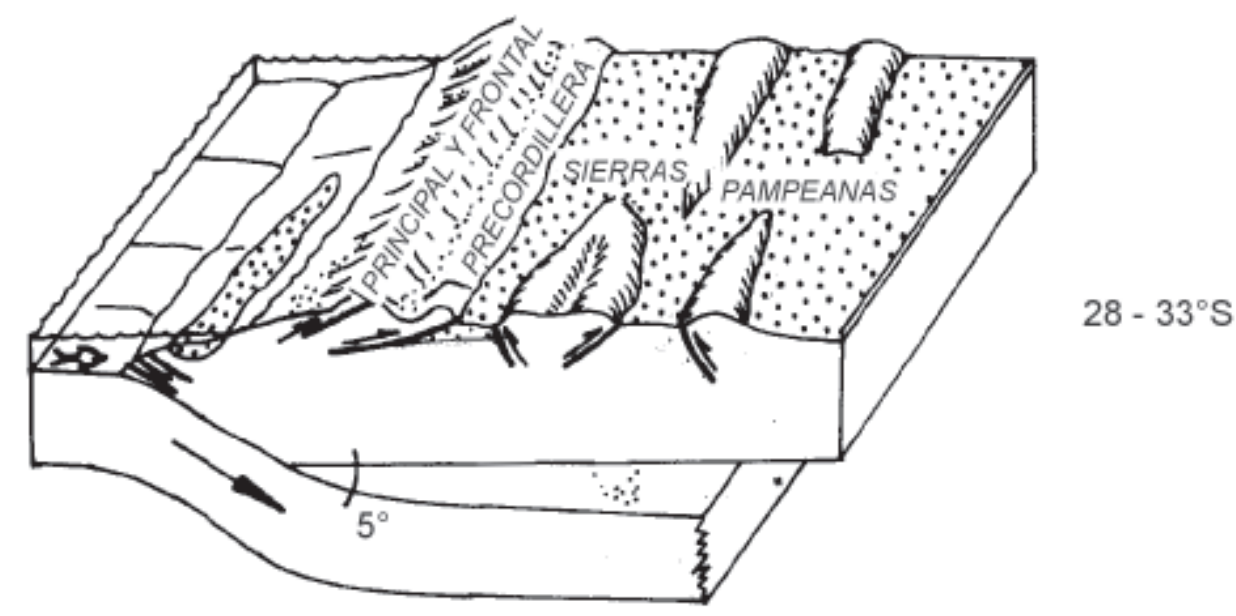

Fig. 3.4.- Diagrama de bloques para la configuración de la subducción subhorizontal entre los $27-28^{\circ} \mathrm{S}$ y los $33^{\circ} \mathrm{S}$ mostrando los rasgos tectónicos principales de la placa continental que resultan de esta configuración de placas. (Extractado de Ramos, 1999d, basado en Jordan et al., 1983).

característica segmentación paralela al eje de la cordillera de los rasgos principales en ambas placas. Dichos rasgos, además, se correlacionan espacial y temporalmente entre si.

En la zona de estudio (Fig. 3.4), entre los $27^{\circ} \mathrm{S}$ y $\operatorname{los} 33^{\circ} \mathrm{S}$, un segmento de subducción subhorizontal se corresponde en la placa superior, de oeste a este, con un aumento definido de la topografía desde la costa hasta las crestas de los Andes, un escasísimo magmatismo 
neógeno-cuaternario, una delgada faja de deformación epidérmica neógena a cuaternaria con migración oriental y una ancha zona de elevación plio-pleistocena (0.01-5.3 Ma) del basamento cristalino en fallas inversas (Jordan et al., 1983; Isacks, 1988; Smalley et al., 1993).

Al sur y al norte de dicho segmento se observa un ángulo de subducción normal, de aproximadamente $30^{\circ}$. El sector norte conocido como Zona Volcánica Central $\left(16^{\circ} \mathrm{S}\right.$ a $\left.26^{\circ} \mathrm{S}\right)$, representa el andinotipo por excelencia con un importante desarrollo del arco magmático, que durante el Neógeno migra en respuesta a los cambios de comportamiento de la placa subducida. Se extiende desde Arequipa hasta el norte de Chile a lo largo de la Cordillera Occidental que bordea al Altiplano-Puna. Sus volcanes se caracterizan por desarrollarse en una corteza engrosada en algunos casos hasta más de 70km de espesor, por lo que los magmas alcanzan un alto grado de diferenciación (Ramos, 1999a).

El sector sur denominado Zona Volcánica Sur, $\left(33.5^{\circ} \mathrm{S}\right.$ a $\left.46.5^{\circ} \mathrm{S}\right)$ incluye alrededor de sesenta volcanes activos que principalmente se desarrollan en la pendiente chilena de la cordillera. Entre ellos pueden citarse el Tupungatito $\left(33.40^{\circ} \mathrm{S}, 69.80^{\circ} \mathrm{O}\right)$, el San José $\left(33.78^{\circ} \mathrm{S}\right.$, $69.90^{\circ} \mathrm{O}$ ) y el Maipo $\left(34.16^{\circ} \mathrm{S}, 69.83^{\circ} \mathrm{O}\right)$ (Siebert y Simkin, 2002-2011) que comparten las características de hallarse en la región estudiada, en territorio limítrofe argentino-chileno y tener manifestaciones de actividad históricas. El sector norte de esta larga cadena volcánica muestra composición con mucha influencia cortical, mientras que al sur de los $37^{\circ} \mathrm{S}$ predominan los basaltos.

\subsubsection{PROVINCIAS GEOLÓGICAS EN LA REGIÓN DE NUEVO CUYO}

Según Rolleri (1976) una provincia geológica es una región caracterizada por una determinada sucesión estratigráfica, un estilo estructural propio y rasgos geomorfológicos peculiares, siendo el conjunto la expresión de una particular historia geológica.

Los datos sismológicos y geológicos muestran que la segmentación latitudinal de la litósfera oceánica bajo los Andes ejerce un control tectónico de primer orden sobre las provincias geológicas. Siguiendo la división del territorio argentino en provincias geológicas dada por Ramos (1999b), la Región de Nuevo Cuyo comprende en su totalidad a la Cordillera Frontal, la Sierra de Famatina y la Precordillera y en parte a la Cordillera Principal, las Sierras Pampeanas, la Llanura Chaco-Bonaerense, la Cuenca de Cuyo, el Bloque de San Rafael y Payenia (o Payunia) (Fig. 3.5). A continuación se describen las principales características de cada provincia o sector de provincia geológica incluido en la región, teniendo en cuenta 
aquellos aspectos de su estructura y evolución que pudieran relacionarse con su actual comportamiento tectónico (Figuras 3.5 y 3.1).

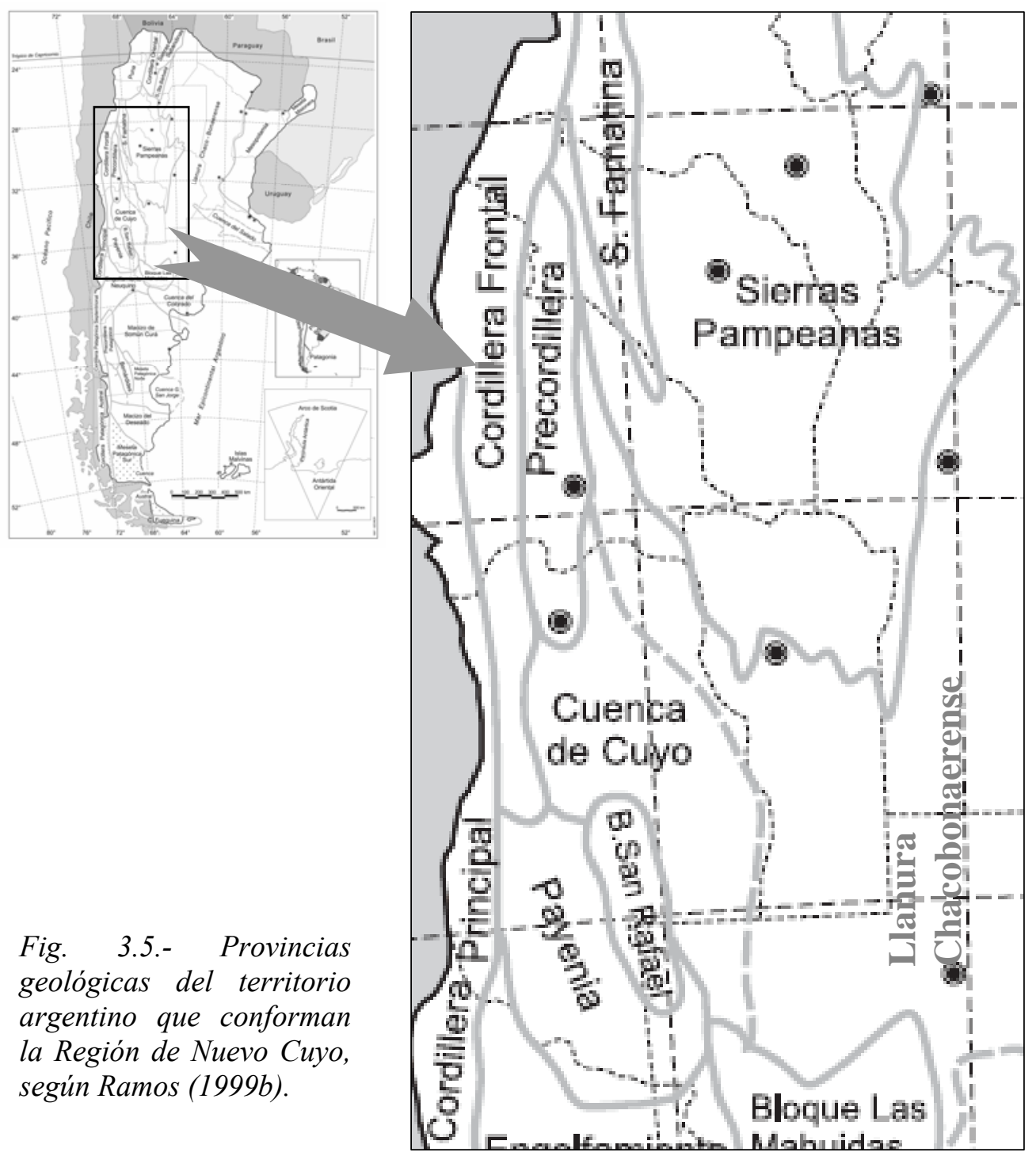

\subsubsection{LLANURA CHACOBONAERENSE O CHACOPAMPEANA}

La llanura Chacobonaerense es una extensa planicie desarrollada entre las Sierras Subandinas y el cinturón Sanrafaelino-Pampeano por el oeste y el río Paraná al este, rodeando Ventania y Tandilia hasta el litoral atlántico por el sudeste (Fig. 3.5). Chebli et al. (1999) establecen el límite superior en la cota $200 \mathrm{~m}$. Debido a la potente carpeta de loess cuaternario (de hasta $4000 \mathrm{~m}$ de espesor) que cubre los depocentros, los conocimientos de la geología se obtienen a través de datos de pozo. En sentido amplio es parte de un gran antepaís que recibió desde el Mioceno $(<23 \mathrm{Ma})$, sedimentos provenientes del levantamiento andino y que hoy constituye el lugar de tránsito de esos sedimentos hacia la plataforma y talud continental atlántico. Se caracteriza por su historia compleja en la que intervinieron diferentes procesos de subsidencia con levantamientos tectónicos leves y localizados, dados 
por la inversión andina sobre un basamento cristalino precámbrico.

La sucesión sedimentaria total de la llanura muestra a escala regional diferentes fosas que le dan al basamento la forma de grandes cubetas separadas por altos o dorsales que se desarrollaron desde el Paleozoico inferior hasta la actualidad. Se distinguen varias cuencas sedimentarias y en particular entre las provincias de La Pampa, Córdoba y San Luis se encuentra la llamada cuenca Chacoparanense. En el Mesozoico (251-65.5 Ma), estuvo sometida a un régimen extensional, con fallas directas que sirvieron de conductos para la introducción de material ígneo alternado con depósitos fluviales, fluviolacustres y eólicos y parece haber tenido conexión con las cuencas que se encuentran entre las Sierras Pampeanas. A partir de la tectónica andina las estructuras normales lístricas fueron invertidas. La intensidad de las reactivaciones se incrementa al acercarse a los Andes y se interrelaciona con los bloques de basamento del antepaís. En esta llanura la única manifestación de los acortamientos horizontales andinos es la inversión tectónica en la zona austral de la provincia de Córdoba, delimitada por fracturas extensionales correspondientes a zonas de debilidad cortical de escala regional.

\subsubsection{CORDILLERA PRINCIPAL}

Comprende el área cordillerana delimitada por las cuencas marinas que ingresaron desde el antiguo océano Pacífico al territorio argentino durante el Jurásico y el Cretácico, pudiéndose distinguir tres sectores diferentes: sanjuanino, mendocino y sur (Ramos, 1999b).

El sector sanjuanino (Fig. 3.6) está caracterizado por el desarrollo de la faja plegada y corrida de La Ramada que involucra el basamento exponiendo sustratos volcánicos y piroclastos paleozoico tardío-triásicos y las secuencias marinas mesozoicas a lo largo del límite argentino-chileno al sur de $\operatorname{los} 31^{\circ} \mathrm{S}$. En este sector de subducción horizontal no hay volcanismo de arco de edad plio-cuaternaria $(<5.3 \mathrm{Ma})$, los últimos rasgos de edificios volcánicos son de edad miocena media (13.8 Ma).

El sector mendocino, comprende la faja plegada y corrida del Aconcagua, de naturaleza epidérmica. En el borde oriental aflora el zócalo volcánico permotriásico como parte de la Cordillera Frontal. Se asientan depósitos marinos y volcánicos que difieren sólo en parte con el sector sanjuanino. En la parte norte del sector se asienta el macizo volcánico mioceno del Aconcagua, transportado tectónicamente a esta posición. 


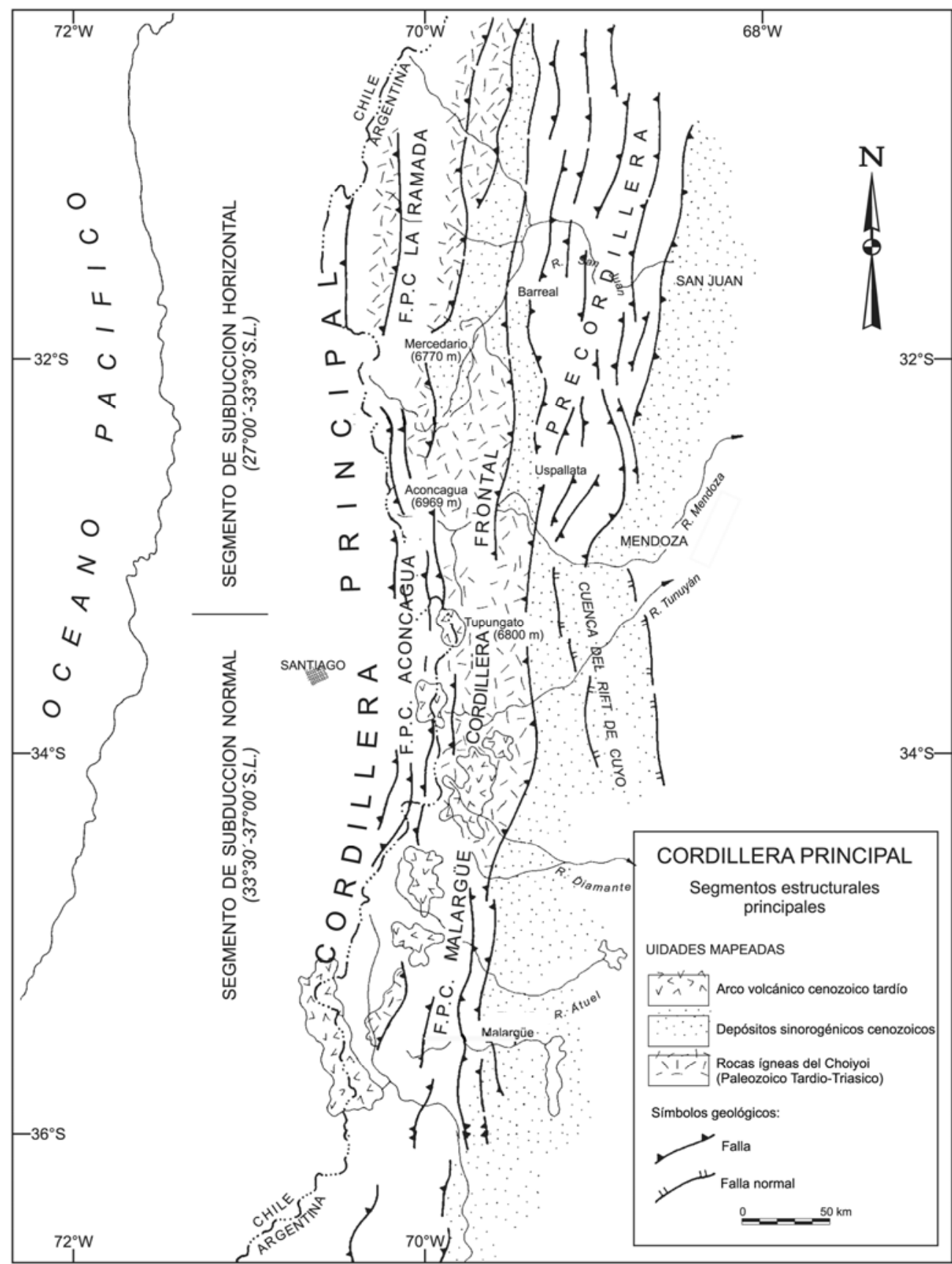

Fig. 3.6.- Cordillera Principal, Frontal y Precordillera (adaptada de Ramos, 1999b).

El sector sur, se extiende desde el río Diamante hasta el río Agrio en Neuquén. La estructura de la cordillera en este sector es compleja teniendo la particularidad de contar con los primeros depósitos marinos atlánticos en territorio argentino. El sustrato se halla fragmentado por fallamiento extensional. La deformación ándica originó la faja plegada y corrida de Malargüe dada por la inversión tectónica del sistema extensional. En este sector la subducción es normal y se diferencia además porque cuenta con una serie de volcanes pliopleistocenos entre los que se destacan grandes estratovolcanes cuaternarios, algunos de ellos activos, como el Tupungato, el San Juan, el Marmolejo, el San José y el Peteroa entre otros. 


\subsubsection{CORDILLERA FRONTAL}

Es la cadena montañosa que se extiende en el oeste de Catamarca, La Rioja y noroeste de San Juan (Fig. 3.6 y Fig. 3.7) y a lo largo de San Juan y Mendoza, se interpone entre la Precordillera y la Cordillera Principal extendiéndose hasta la altura del río Diamante (Ramos, 1999b). En ella se ha encontrado una sucesión de depósitos marinos carboníferos, intrusivos y volcánicos de subducción neopaleozoicos e intrusivos por extensión postectónica permotriásica. La fase orogénica San Rafael, interrumpió la evolución de las cuencas sedimentarias de la Cordillera Frontal y del Bloque de San Rafael (Sección 3.1.2.9) con una deformación frágil, sin metamorfismo. Como consecuencia de ello se produjo el ascenso de esta región que culminó con una superficie de erosión que abarcó casi toda la Cordillera Frontal (Llambías et al., 1993). Un rift triásico la segmentó al norte y al sur y de este modo participó como un bloque de basamento en la configuración de la Cordillera Principal cuando la deformación ándica la levantó a fines del Mioceno.

\subsubsection{PRECORDILLERA}

A pesar de que ha recibido diferentes nombres a lo largo de numerosos estudios, en todos ellos quedaba claro que era una unidad diferente de los Andes y con una antigüedad mayor. Dataciones hechas sobre circones en xenolitos de rocas volcánicas miocenas arrojan una edad mesoproterozoica (1118 $\pm 54 \mathrm{Ma}$ ) para el basamento de Precordillera (Kay et al., 1996).

La Precordillera se caracteriza por terrenos paleozoicos que se habrían levantado principalmente durante el Cenozoico Superior. Los diferentes ambientes (talud, plataforma, someros y continentales) de los que provienen los sedimentos que se alternan según la subunidad y el período analizado, son indicadores de sucesivos procesos de subducción y colisión en la conformación de esta provincia. Sobre la base de sus características estratigráficas y estructurales, esta unidad ha sido dividida en tres subunidades: la Precordillera Occidental, la Central y la Oriental (Ortiz y Zambrano, 1981).

Los sectores occidental y central están representados por una faja plegada y corrida epidérmica mientras que el oriental corresponde a un bloque de basamento con una estructura de igual vergencia que las contiguas Sierras Pampeanas (Ramos, 1999b). Allmendinger et al. (1990) y Smalley et al. (1991) sugieren tratar a la Precordillera Oriental como parte de las Sierras Pampeanas por sus similitudes en el estilo tectónico. En el sector oriental, al este del Valle de Matagusanos (Fig 3.7 y 3.12), se encuentran las secuencias cámbricas con la típica 


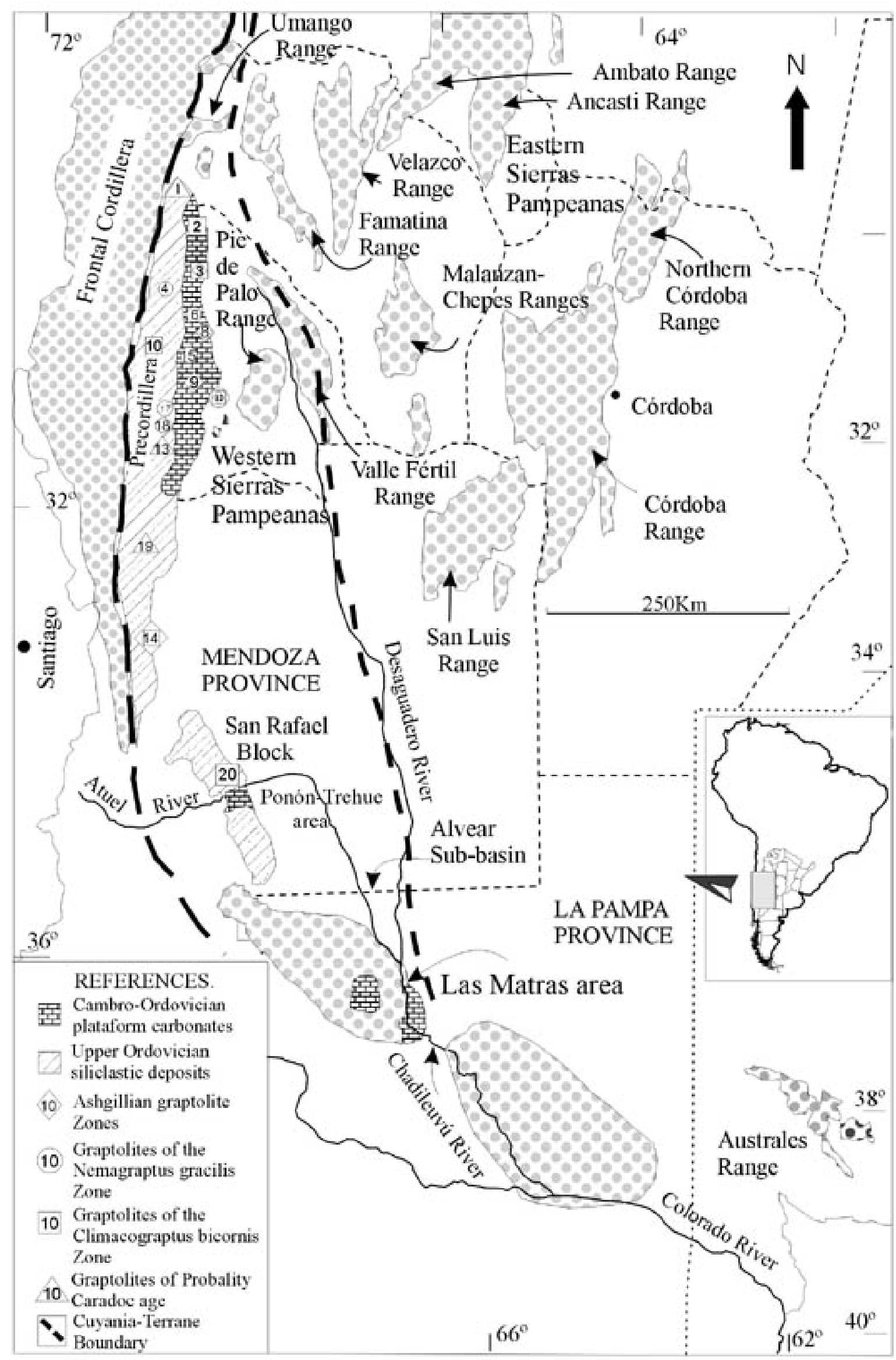

Fig. 3.7.- Ubicación de la Precordillera con relación a la Cordillera Frontal y las Sierras Pampeanas. Se distingue la Precordillera Oriental caracterizada por las plataformas carbonáticas. En las Sierras Pampeanas se señalan sus componentes principales. Las líneas segmentadas muestran los límites del Terreno de Cuyania (extractada de Sato et al., 2000). 
fauna de Olenellus que permitieron establecer los fuertes vínculos faunísticos con Laurentia.

Durante el Mioceno y el Plioceno, la Precordillera sufrió un paulatino levantamiento y apilamiento en forma sincrónica con la expansión del volcanismo de arco hacia el este (Jordan et al, 1983).

\subsubsection{SISTEMA DE FAMATINA}

Con la Precordillera y parte de las Sierras Pampeanas Occidentales al oeste y el resto de las Sierras Pampeanas al este, el Sistema de Famatina (Fig. 3.7 y 3.8) abarca un conjunto de bloques serranos que se reconoce como una unidad independiente de las típicas Sierras Pampeanas (Ramos, 1999b).porque presenta su máximo de actividad en el Ordovícico medio a superior (aprox. $450 \mathrm{Ma}$ ). Corresponde a un arco magmático desarrollado en un margen continental activo, seguido de granitos postorogénicos y una intensa deformación dúctil. Posteriormente se sucedieron intensos procesos extensionales que dieron origen a los depósitos de la cuenca de Paganzo (abarcando gran parte de la provincia de La Rioja y noroeste de San Juan) y a los del rift de Ischigualasto (al este del lineamiento Valle Fértil). En el Terciario se dio el levantamiento de la región occidental y se interdigitaron rocas volcánicas con depósitos sinorogénicos testimoniando la migración del arco volcánico hacia el antepaís entre los 10 y 4 Ma. La estructura del Sistema de Famatina es similar a la de las Sierras Pampeanas: bloques de basamento precámbrico-eopaleozoico levantados en el Cenozoico superior y basculados por fallas lístricas inversas.

\subsubsection{SIERRAS PAMPEANAS}

Como definiera González Bonorino (1950), las Sierras Pampeanas son un sistema de montañas en bloque limitadas por fallas inversas lístricas. Se dividen actualmente en dos provincias de características diferentes: las Sierras Pampeanas Occidentales y las Orientales. En la Fig. 3.7 se destacan las principales sierras que las componen y en la Fig. 3.8 las fallas activas según Costa (1999).

La estructura actual se originó principalmente a partir del Mioceno inferior. Antiguas fallas normales cretácicas del sector oriental, fueron reactivadas en el Eoceno (55.8-33.9 Ma) (Ramos, 1999b). Muchas de las fallas ándicas son inversión de fallas normales cretácicas (Sa. de Córdoba), fallas triásicas (Sa. La Huerta, al sur de la Sa. Valle Fértil), fallas neopaleozoicas (Sa. Los Llanos, sector norte de Sa. Chepes) o antiguas fajas miloníticas precámbricas o paleozoicas (Sa. de San Luis y Valle Fértil). 


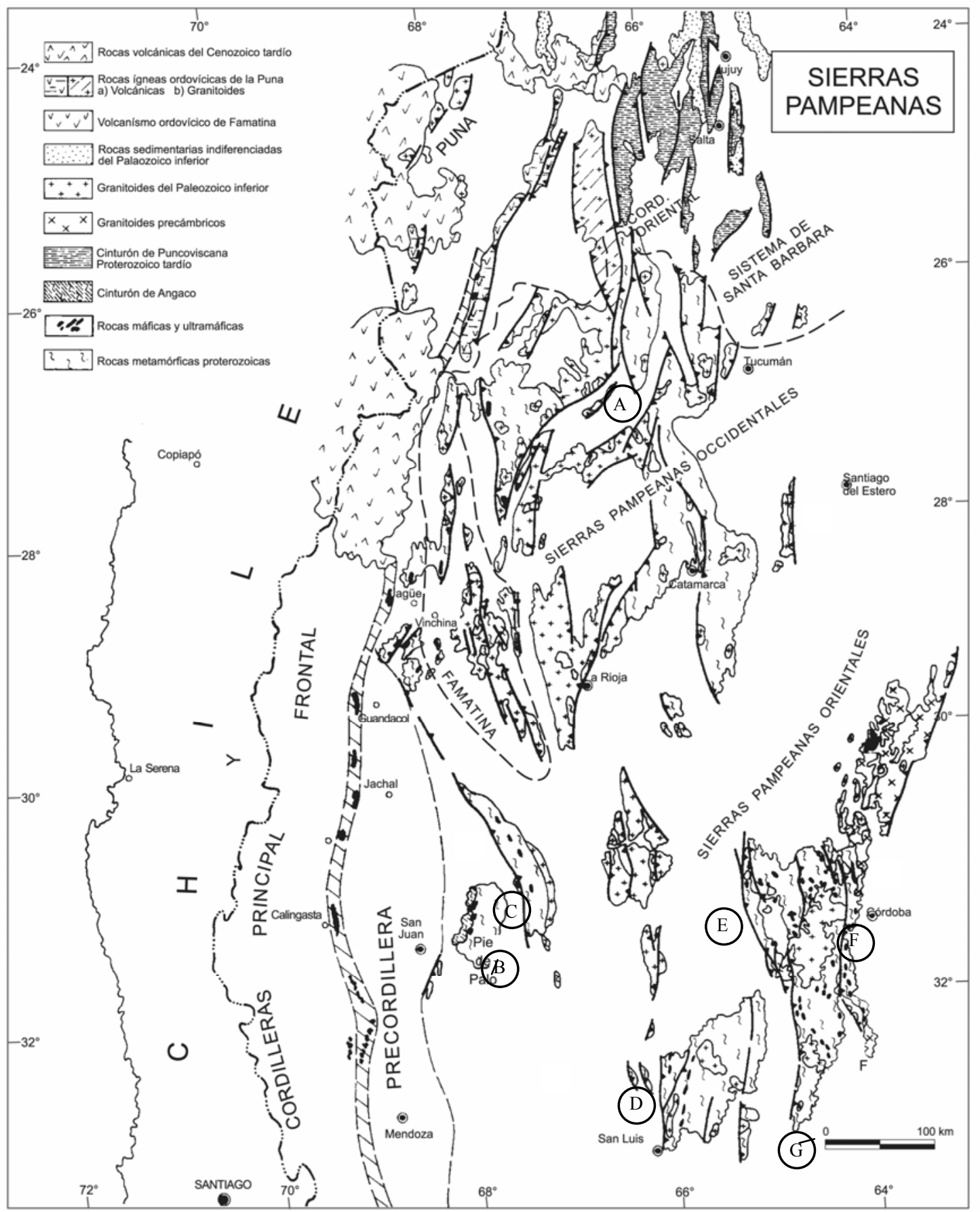

Fig. 3.8.- Sierras Pampeanas y Sistema de Famatina (Ramos, 1999b) y fallas activas según Costa (1999): A: Aconquija, B: Ampacama-Niquizanga, C: Las Chacras (parte del lineamiento Valle Fértil), D: San Luis, E: Comechingones, F: Sierra Chica, G: Las Lagunas (modificada de Ramos, 1999b).

Las Sierras Pampeanas Orientales corresponden a un orógeno con posterior colisión cerca del límite Precámbrico-Cámbrico mientras que las Sierras Pampeanas Occidentales corresponden a un orógeno con una subducción de cámbrica a ordovícica media y efectos post-colisionales a partir del Ordovícico superior. Luego se modelaron las depresiones que conforman la cuenca de Paganzo (Azcuy et al., 1987) y dieron origen al piso estructural donde 
se alojaron los depósitos neopaleozoicos con niveles piroclásticos procedentes de la Cordillera Frontal. En las proximidades de Río Cuarto, justo al sur de las Sierras Pampeanas Orientales, afloran pequeños aparatos volcánicos cretácicos. En Córdoba y San Luis, los volcanes de Pocho (4 Ma) y del Morro (1.9 Ma) de edad cenozoica superior son prueba de la migración del arco magmático hacia el este por la subhorizontalización de la placa de Nazca (Ramos, 1999d).

Por su historia tectónica (Ramos, 1988), este sustrato incluye varios terrenos (Chilenia, Cuyania y Pampia, Fig. 3.10), con distinta antigüedad y grado de cratonización, lo que sumado a la actividad derivada de grandes lineamientos estructurales como es el caso de la megafractura de Valle Fértil (Baldis et al., 1982, 1989), ha generado diferentes depocentros (Fernández Seveso et al., 1993). Así por ejemplo, la cuenca Saliniana, ubicada en las Salinas Grandes, entre las provincias de Córdoba, Santiago del Estero, Catamarca y La Rioja constituye un depocentro cretácico y terciario de $2500 \mathrm{~m}$ de espesor con orientación NE-SO, oblicuo a los bloques cristalinos de Sierras Pampeanas, separando las Occidentales de las Orientales (Fig. 3.8). Esta disposición puede acusar la acción de estructuras preexistentes y/o la sobreimpresión de la tectónica andina (Chebli et al., 1999). En la provincia de San Juan, modelos corticales obtenidos por datos gravimétricos, geológicos, sismológicos y sísmicos muestran la existencia de dos zonas de alta densidad que son interpretadas como paleosuturas (Gimenez et al., 2000).

El bloque de Pie de Palo, uno de los de mayor actividad sísmica de las Sierras Pampeanas, se encuentra entre Precordillera y la Sierra de Valle Fértil (Fig. 3.7). Está delimitado lateralmente por estructuras activas de tipo inverso y rumbo general NNE-SSO.

Fig. 3.9.- Imágen satelital donde se observan claramente los lineamientos NE-SO que dividen a la Sa. Pie de Palo y la importancia de la gran falla que la delimita por el este (Google earth (C) 2011 Google).

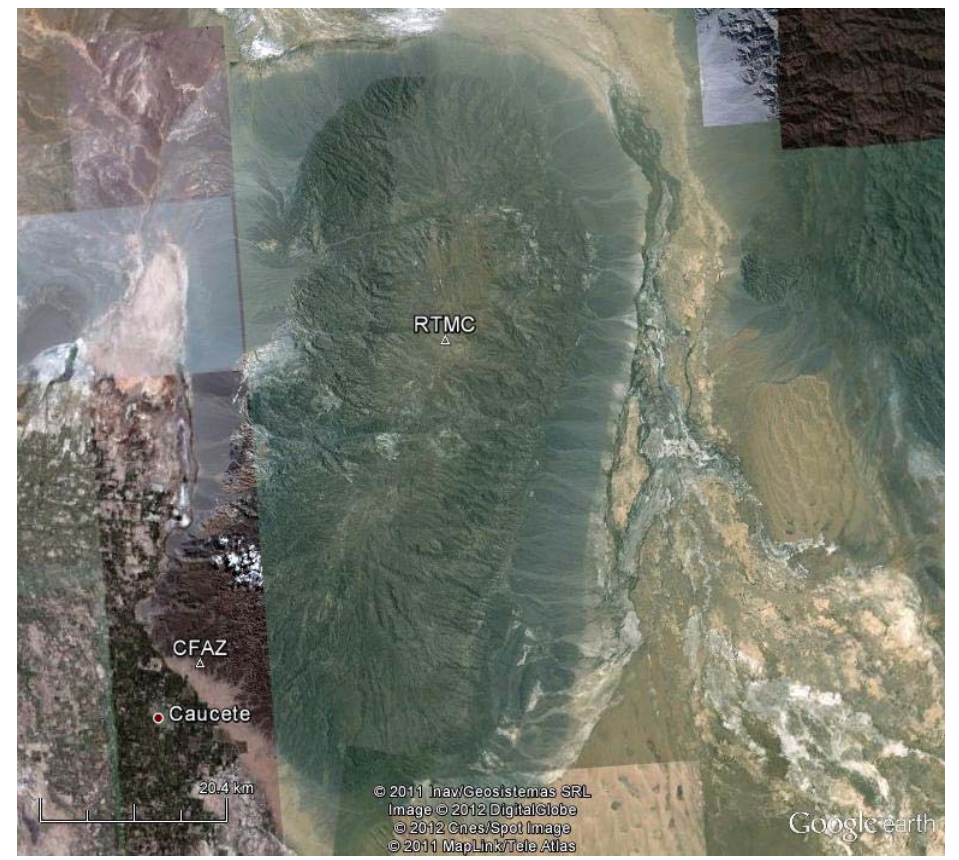


Por el este, fallas como las de Ampacama y Nikizanga marcan el inicio del Valle Bermejo que separa al bloque de la Sierra de Valle Fértil. Por el oeste, una fractura que bordea las localidades de Caucete, San Martín y Angaco marca el borde oriental del Valle de Tulum que separa a la Sierra de Pie de Palo de la Precordillera (Fig. 3.8). En el interior de la Sierra (Fig. 3.9) dos grandes fracturas de rumbo NE-SO, Guayaupa-Lima y El Molle-Potrerillos, la dividen en tres bloques (Perucca et al., 1979, Perucca et al., 1997, Castro et al., 2005). Al norte de Pie de Palo, la Megatraza de Salinas Grandes mantiene el mismo rumbo que las dos anteriores.

\subsubsection{CUENCA CUYANA}

La Cuenca de Cuyo se ubica principalmente en la provincia de Mendoza, al sur de la Precordillera, desde la zona de transición entre los segmentos de subducción horizontal y normal, bordeando el Bloque de San Rafael hasta Payenia y el Bloque de Las Mahuidas y extendiéndose hacia el este con un límite poco definido hasta la Llanura Chacobonaerense (Fig. 3.5).

Iniciada en el Triásico como una cuenca de rift con sucesiones clásticas y volcánicas (Fig. 3.10), la Cuenca Cuyana fue reactivada por la tectónica andina. Esta cuenca

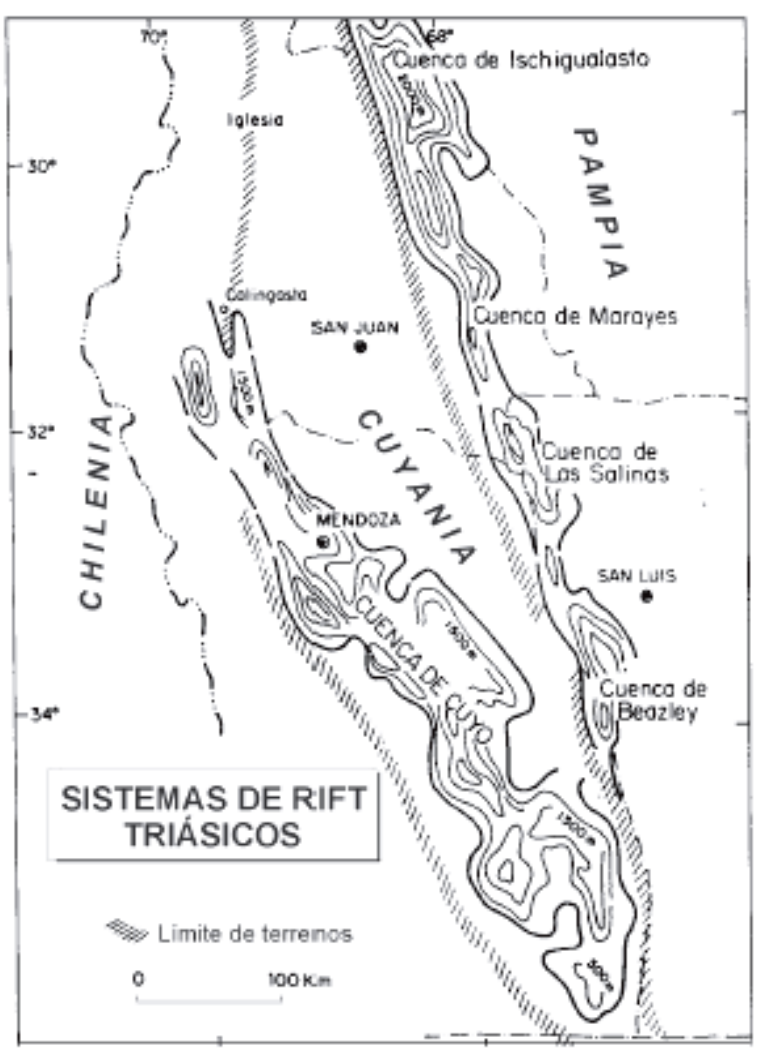

Fig. 3.10.- Terrenos, suturas que los delimitan y depocentros generados por la actividad de los grandes lineamientos (extractada de Ramos, 1999d). correspondía a la parte distal de la cuenca de antepaís generada en la Cordillera Principal por la faja plegada y corrida del Aconcagua entre los 20 y $8.6 \mathrm{Ma}$ (Mioceno) (Ramos et al., 1996). En particular, en la Precordillera Sur, la deformación neógena sobreimpuesta a las estructuras extensionales condujo a la inversión tectónica de los hemigrábenes y al ascenso y desmembramiento del relleno, configurando afloramientos aislados con diferentes grados de inversión y complejidad de deformación. A escala regional, tales variaciones en el estilo estructural andino están fuertemente influenciadas por la presencia de anisotropías oblicuas, como los bordes de la cuenca Cuyana o las extensas fajas de cizalla sanrafaélicas (Japas et al., 
2008)

Los sedimentos que constituyen la Cuenca de Cuyo actual tienen antigüedades que van de los 15.7 a 1 Ma, siendo la más joven la Formación Mogotes (Ramos, 1999c). Con posterioridad a la sedimentación de la Formación Mogotes, los depósitos sinorogénicos desarrollan una serie de estratos de crecimiento de edad cuaternaria en el flanco occidental del anticlinal Barrancas que se desarrolla entre los ríos Tunuyán y Mendoza (Fig. 3.6).

\subsubsection{PAYENIA}

Desarrollada principalmente al sur de la provincia de Mendoza pero extendiéndose hasta el noreste de Neuquén y noroeste de La Pampa, la Payenia se caracteriza por sus extensos campos volcánicos (Fig. 3.5). Recibe su nombre del volcán Payén, ubicado al oeste de la caldera del volcán Payún Matru $\left(36.42^{\circ} \mathrm{S}, 69.20^{\circ} \mathrm{O}, 3680\right.$ m), (Siebert y Simkin, 20022011).

Estos campos volcánicos son, en su mayoría, extensas coladas basálticas ubicadas al sur del cerro Diamante, sobre los márgenes del Bloque de San Rafael, sobre el frente de corrimientos más oriental de la Cordillera Principal y sobre el Engolfamiento Neuquino. Están representados por grandes estratovolcanes, calderas volcánicas, domos y volcanes monogénicos de composición básica alcalina que definen la provincia andino-cuyana de retroarco (Bermúdez et al., 1993). Tienen mayoritariamente una edad menor a 5 Ма у registran en algunos casos actividad histórica.

La estructura de esta región se caracteriza por un fallamiento extensional, detectado en parte por lineamientos de volcanes monogénicos de rumbo noroeste y por indicios de reactivación por fallamiento directo de estructuras más antiguas. (Ramos, 1999b).

\subsubsection{BLOQUE DE SAN RAFAEL}

El Bloque de San Rafael, ubicado entre Payenia y la Cuenca de Cuyo (Fig. 3.5), se caracteriza por la presencia de extensos afloramientos de rocas ígneas permo-triásicas correspondientes al ciclo magmático Choiyoi (Llambías et al., 1993) conformado por dos secciones que indican un cambio en el ambiente tectónico. La sección inferior (pérmica inferior) representa un magmatismo típico de arco continental en un margen convergente, mientras que la sección superior (pérmica superior-triásica inferior) indica la transición hacia un ambiente extensional de intraplaca continental (Llambías et al. 1993, Kleiman 1999, Kleiman 2002).

El Bloque de San Rafael tiene una historia geológica homogénea y una estructura y 
expresión geomorfológica distintiva. Comprende a un sistema orogénico eopaleozoico similar al de la Precordillera, al que se le sobreimpone el magmatismo neopaleozoico característico de la Cordillera Frontal (Rolleri y Criado Roqué, 1970). La región está representada por una antigua planicie de erosión regional, labrada durante el Jurásico y el Cretácico, que está siendo actualmente exhumada debido al levantamiento tectónico ándico. La estructura ándica de la región está caracterizada por una serie de corrimientos con vergencia hacia el este e importante actividad neotectónica en su frente oriental, indicando que este proceso está aún activo (Ramos, 1999b).

\subsubsection{RESEÑA GEOTECTÓNICA DE LA REGIÓN}

Yrigoyen (1999) hace un buen resumen de la situación de la Argentina en el marco geológico de América del Sur a la luz de la tectónica de placas. En los últimos años, un importante número de hipótesis e interpretaciones tratan de reconstruir los orígenes y las conexiones de las distintas partes que conforman el basamento sudamericano. Los modelos más aceptados plantean que algunos terrenos argentinos son el resultado de traslaciones y colisiones continente-continente muy antiguas.

Por ejemplo, la Precordillera es considerada como una microplaca fragmentada de Laurentia en el Proterozoico tardío o Cámbrico temprano (aprox. $500 \mathrm{Ma}$ ) que luego de un largo desplazamiento, colisionó con el margen gondwánico occidental o autóctono pampeano en el Ordovícico (aprox. $460 \mathrm{Ma}$ ). Ese terreno, denominado Cuyania (Fig. 3.10) u Occidentalia muestra fauna y secuencias carbonáticas poco frecuentes en el resto de Sudamérica pero que se asemejan a las de los Apalaches y a las del este de Laurentia. La presencia de bentonitas indica el fin del arco magmático en el momento de la colisión, las ofiolitas reflejan la obducción (Baldo et al., 1999) y por lo tanto la presencia de una corteza oceánica previamente subducida. Más tarde, en el Devónico (aprox. $380 \mathrm{Ma}$ ) y coaxialmente a la colisión previa, se acreciona el terreno de Chilenia (Fig. 3.10), aportando el basamento de Cordillera Frontal y Principal (Ramos, 1999d).

Iniciándose en el Ordovícico, pero con su mayor expresión durante el Silúrico y el Devónico se dieron una serie de episodios deposicionales que se manifiestan con mayor potencia hacia el norte del país. Es así que los terrenos de la Puna y Cordillera Oriental actuales funcionaron como área de aporte para las cuencas habilitadas hacia el este.

La integración del collage de los diferentes bloques fue mayormente completada durante el Paleozoico temprano y sufrió pocos reajustes durante el resto del Fanerozoico. Los 
terrenos basamentales del territorio argentino guardan cercana similitud composicional con las grandes áreas cratónicas de Amazonas, São Francisco y Guayanas, formando en conjunto el $44 \%$ de la superficie sudamericana. La conexión regional se hace más evidente a medida que avanzan los tiempos geológicos, al punto que en el Cretácico (145 a $65 \mathrm{Ma}$ ) ya las unidades geoestructurales son inseparables.

La apertura del Océano Atlántico activó la subducción a lo largo del margen pacífico, incrementando la convergencia entre la placa Pacífica y la placa Sudamericana. Según Ramos (1999d) el proceso de subducción que tuvo lugar en el margen occidental durante el Mesozoico y el Cenozoico presentó dos regímenes tectónicos distintivos. Desde el Triásico tardío al Cretácico temprano (230 a $135 \mathrm{Ma}$ ) el ritmo de convergencia parece haber sido lento a muy lento, por lo que la extensión dominó los esfuerzos actuantes en la corteza continental en las etapas de rift y apertura inicial atlántica. Esto reactivó fuertemente la actividad magmática en la faja andina. Al completarse la separación de África y Sudamérica, se inició el otro régimen, caracterizado por un incremento notorio de la deriva continental y por lo tanto de la subducción. El régimen de esfuerzos dominante fue compresivo durante el Cretácico medio (100 Ma), comenzando la deformación y sobreelevación de la Cordillera Andina. En el período Cenozoico, una serie de pliegues, retrocorrimientos, fallamientos y generación de anticlinales iniciados en el oeste, avanzó lentamente hacia el antepaís oriental provocando un apilamiento tectónico de sobrecorrimientos que aún siguen activos como lo muestra la sismicidad actual. Durante el Oligoceno (25 Ma) se produjo la ruptura de la placa Farallones en las placas de Cocos y Nazca. Entre los 28 y los 20 Ma la velocidad de subducción aumentó (de $6 \mathrm{~cm} /$ año a $15 \mathrm{~cm} / \mathrm{año}$ ), para luego reducirse moderadamente entre los 20 y los $10 \mathrm{Ma}$ (de $15 \mathrm{~cm} /$ año a $13 \mathrm{~cm} /$ año). La desaceleración de la convergencia ocurrida hace unos $20 \mathrm{Ma}$ pudo haber sido responsable del mayor acoplamiento entre las placas y la tendencia a la subhorizontalización (Wall y Lara, 2001). Si bien la relación entre el régimen de convergencia y la tectónica de la placa cabalgante es aún materia de debate, a partir de los $18 \mathrm{Ma}$ se habrían dado las condiciones para un régimen compresivo y el progresivo engrosamiento cortical que se observan a lo largo de los Andes.

En un modelo cinemático tridimensional de la evolución cenozoica tardía de los Andes centrales, Isacks (1988) combina levantamiento, geometría cambiante de la placa subducida y cambios en los lineamientos del frente orogénico. Los cambios en la placa Sudamericana afectaron la pendiente de la placa subducida y viceversa. Una concavidad inicial de Sudamérica hacia el mar, produjo cambios latitudinales en la pendiente de la subducción, afectando a los procesos convectivos en la cuña astenosférica y por lo tanto al ancho de la 
zona deformada en la placa superior. La curvatura del oroclino boliviano se vio incrementada y los segmentos peruanos y chilenos de los Andes se movieron relativamente hacia el mar, contribuyendo a la subhorizontalización de la placa subducida en el Mioceno tardío. El arco magmático migró hacia el este debido a la erosión en el borde continental por la subducción.

\subsubsection{SISMOTECTÓNICA DE LA REGIÓN DE NUEVO CUYO}

El orógeno andino es activo ya que manifiesta procesos neotectónicos significativos a lo largo del frente de corrimiento y variaciones de sismicidad cortical intraplaca correlacionadas con las variaciones del estilo tectónico (Fig. 3.11). El límite oriental de la sismicidad cortical coincide con el límite oriental de la zona de Wadati-Benioff (ZWB) tanto en la subducción subhorizontal como en la normal (Fig. 3.3). La profundidad de los sismos corticales también se correlaciona con el estilo tectónico. En regiones de subducción normal la sismicidad ocurre en corteza superior y media, (Cahill y Isacks, 1992) mientras que en regiones de subducción plana la sismicidad cortical se ubica principalmente en corteza media e inferior

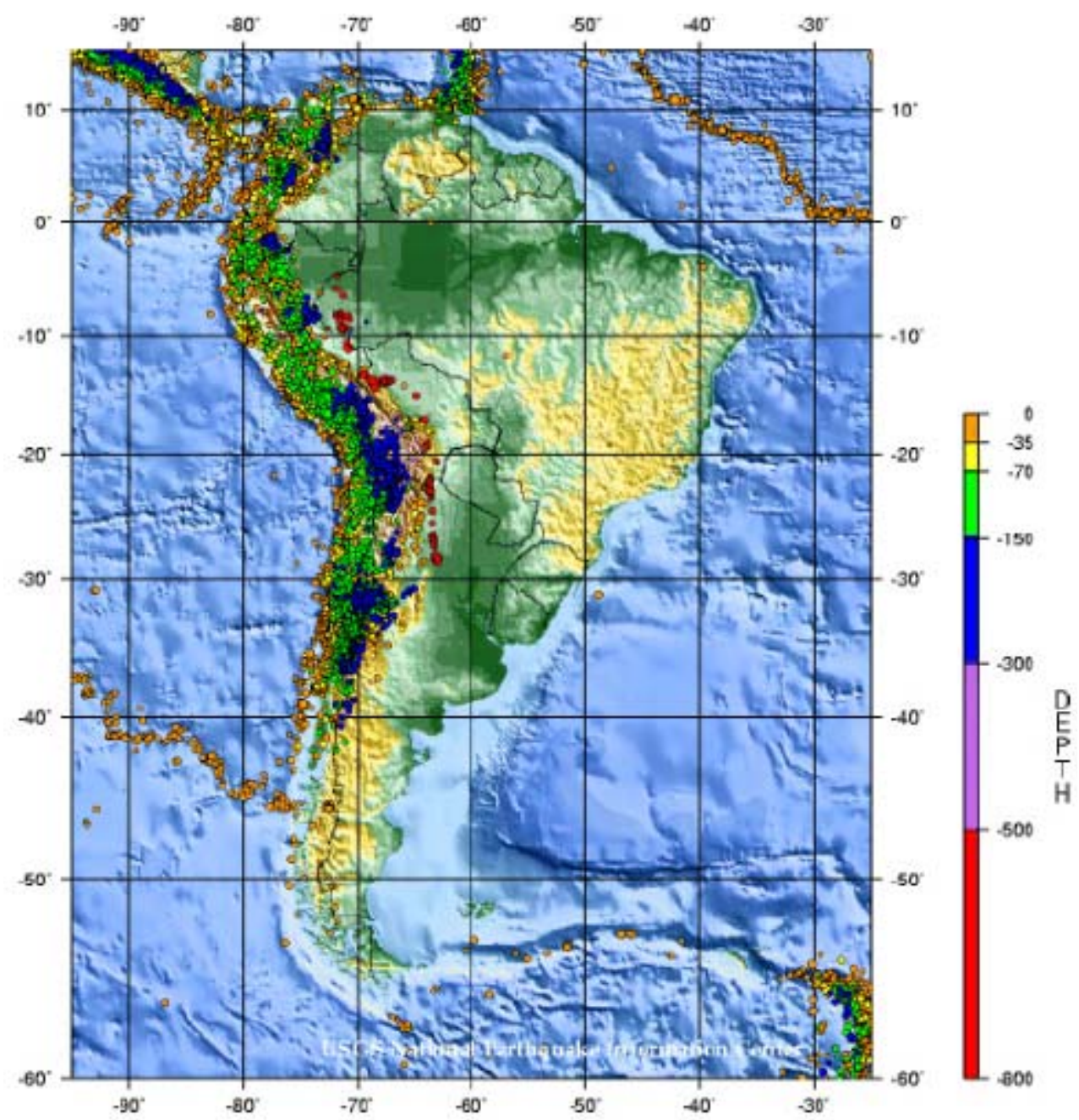

Fig. 3.11.- Mapa de sismicidad en Sudamérica entre los años 1990 y 2000. (fuente PDE USGS/NEIC) 
(Smalley y Isacks, 1990, Suárez et al, 1983). Los trabajos mencionados atribuyen dicha variación a diferencias regionales de las características termodinámicas y reológicas de la litósfera en direcciones transversal y longitudinal al orógeno.

Isacks (1988) propuso que la placa descendente revierte la orientación de su curvatura en profundidad en respuesta a los cambios de la curvatura lateral del margen occidental sudamericano. En un estudio con datos telesísmicos de eventos en la Placa de Nazca, Cahill y Isacks (1992) concluyeron que la transición entre la subducción normal y horizontal, entre los $27^{\circ} \mathrm{S}$ y $28^{\circ} \mathrm{S}$, es gradual y asociada a un cambio en la curvatura de la placa subducida, de convexa hacia arriba a cóncava hacia arriba y no debida a un desprendimiento de la placa como habían postulado Febrer et al. (1982) y Baldis et al. (1982) por estudios geotérmicos y magnetotelúricos. Sin embargo la idea de una ruptura en la placa sería retomada varios años más tarde algo más al sur (Booker et al., 2005a,b).

La distribución de la actividad sísmica en profundidad entre los $28^{\circ} \mathrm{S}$ y $32^{\circ} \mathrm{S}$ muestra un aumento en la inclinación inicial de la placa denotada por el acercamiento de los contornos de 50 y $75 \mathrm{~km}$ hacia la fosa y una horizontalización a una profundidad de 100-125 km que se extiende por centenares de kilómetros hacia el este antes de reiniciar su descenso (Cahill y Isacks, 1992) (Fig. 3.3). Este segmento coincide con la ausencia de volcanismo activo y el desarrollo de la Precordillera y las Sierras Pampeanas (Barazangi y Isacks, 1976, Jordan et al., 1983). Como resultado, en esta zona se encuentran las mayores alturas de los Andes

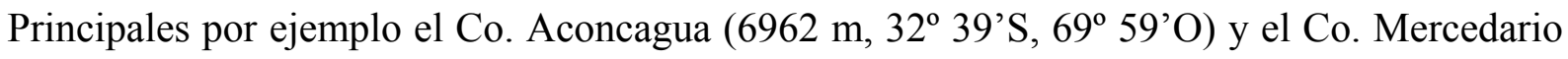
$\left(6770 \mathrm{~m}, 32^{\circ} \mathrm{S}, 70^{\circ} \mathrm{O}\right)$ (Siebert y Simkin, 2002-2011).

En particular, entre $\operatorname{los} 31^{\circ} \mathrm{S}$ y $32^{\circ} \mathrm{S}$ se desarrolla una región de alta sismicidad con un notable comportamiento bimodal en profundidad. Gutscher y Malavieille (1999) señalaron que la energía sísmica liberada en la región de subducción horizontal es en promedio 3 a 5 veces mayor que en las áreas de subducción normal adyacentes. Utilizando datos locales, Smalley et al. (1993) determinaron profundidades focales entre 5 y $35 \mathrm{~km}$ para los eventos corticales y una ZWB centrada aproximadamente en los $107 \mathrm{~km}$ con un espesor cercano a los $20 \mathrm{~km}$. La sismicidad cortical se concentra principalmente bajo Precordillera Oriental y Sierra Pie de Palo (Fig. 3.12) (Smalley et al., 1993, Regnier at al., 1992, Perucca y Bastias, 2006).

El frente de corrimiento del margen oriental de la Precordillera es un frente activo y está representado en la provincia de San Juan por una zona triangular de piel gruesa. El sistema de retrocorrimientos de la Precordillera Oriental es su principal fuente sismogénica, con directa 
incidencia en la ciudad de San Juan (Costa, 1999). El patrón de fracturas no muestra una relación simple con la deformación superficial (Smalley y Isacks, 1990). Según Smalley et al. (1993) esta sismicidad podría resultar de la reactivación neógena de una sutura devónica entre los terrenos de Precordillera y Sierras Pampeanas. Estudios de sismicidad, geología y

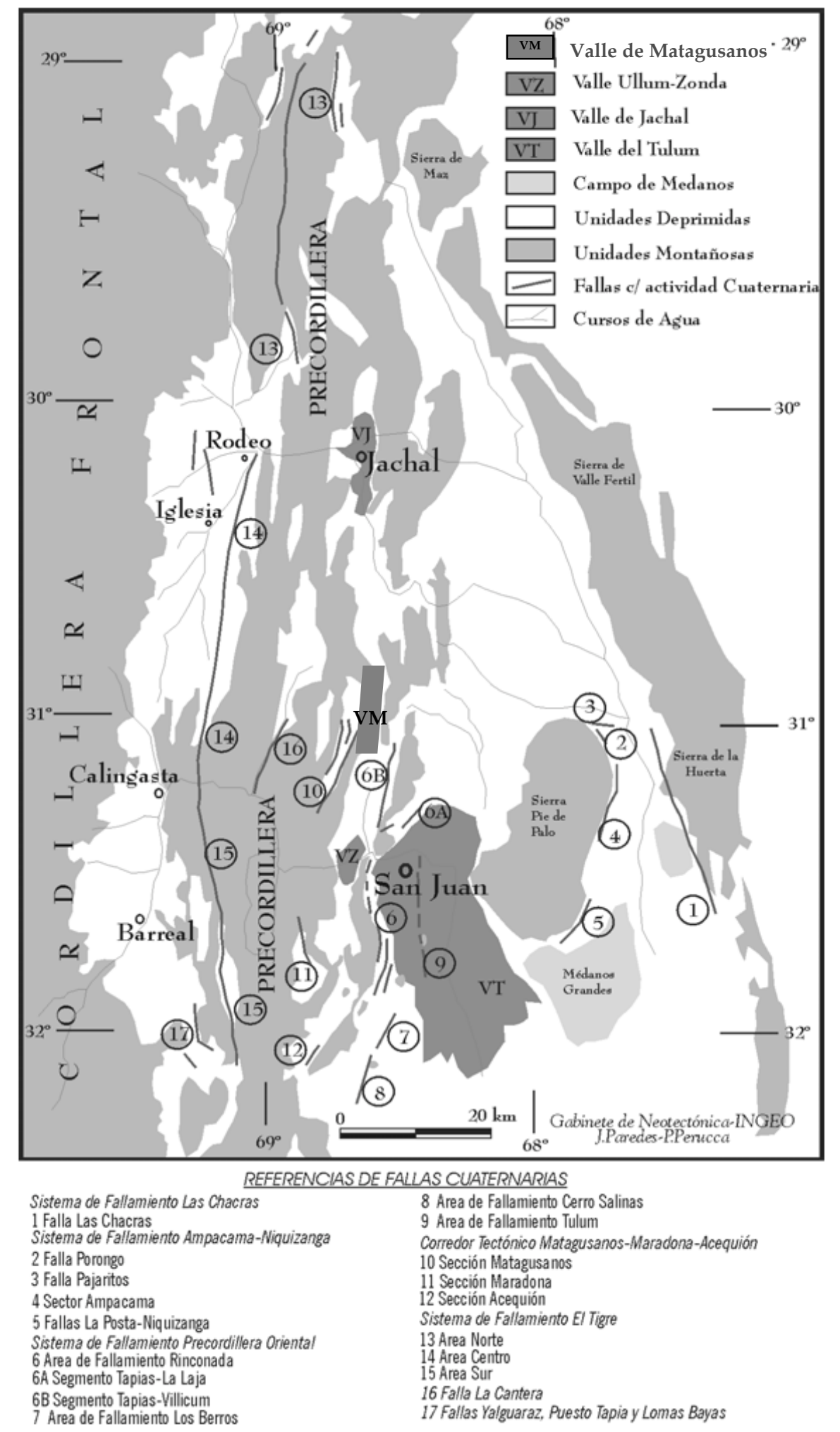

Fig. 3.12.- Geología de San Juan, unidades positivas, valles y fallas activas agrupadas en Sistemas de Fallamientos. Modificado de Perucca y Paredes (2004). 
reflexión sísmica mostraron a los corrimientos con inclinación hacia el este de la Precordillera Oriental como una prolongación hacia la superficie de fallamientos basales inferidos en las Sierras Pampeanas con la misma inclinación. En esta región tuvo lugar el mayor evento de la Precordillera, el terremoto Ms 7.4 de San Juan de 1944 (Fig. 3.1) localizado a 31.6 $\pm 0.4^{\circ} \mathrm{S}$, $68.5 \pm 0.6^{\circ} \mathrm{O}$ según Kadinsky-Cade (1985) y profundidad de $50 \mathrm{~km}$ (ISC). La incertidumbre de la localización impide su asociación a una falla específica. El mayor desplazamiento superficial fue observado en la falla de La Laja (Fig. 3.12) y por su profundidad y orientación, el evento se asocia a fallas basales de corrimiento con inclinación hacia el este. Debido a esto Smalley et al. (1993) sugirieron que el riesgo sísmico en San Juan debe ser mayor que el estimado únicamente a partir de estudios de geología superficial.

En la Sierra Pie de Palo, la sismicidad se concentra principalmente bajo los límites topográficos de la sierra, aunque se extiende hacia afuera en el SE de la misma. El patrón de sismicidad y los mecanismos focales corroboran la división de la sierra en bloques con zonas sismogénicas de inclinaciones diferentes debido a fallas de orientación NE-SO (Fig. 3.9) (Smalley et al., 1993). En esta sierra tuvo lugar otro gran evento con Ms 7.4, el terremoto de Caucete de $1977\left(31.03^{\circ} \mathrm{S}, 67.77^{\circ} \mathrm{O}, 13 \mathrm{~km}\right.$, PDE, USGS/NEIC o $17 \mathrm{~km}$, Chinn y Isacks, 1983). La ruptura que ocasionó el sismo principal no alcanzó la superficie aunque se midieron desplazamientos en la falla Ampacama-Niquizanga (Volponi et al., 1978) (Fig. 3.12). Se acepta que tuvo su origen en una falla inversa ciega y su mecanismo focal es de corrimiento puro con planos de orientación NS, pero la selección del plano de falla aún causa controversias. Kadisnky-Cade (1985) lo modeló como un evento doble con 20 s y 64 km de separación. Tanto la numerosa secuencia de réplicas como la actividad sísmica en años posteriores muestran una gran variedad de mecanismos focales indicando una geometría de fallas compleja (Regnier et al., 1992). Los patrones de sismicidad diferentes entre Pie de Palo y Precordillera Oriental, sugieren que dichas áreas tienen basamentos y reologías corticales diferentes.

La actividad sísmica cortical hacia el este tiene como ejemplo un evento de Ms 6.0 $\left(32.00^{\circ} \mathrm{S}, 66.00^{\circ} \mathrm{O}, 40 \mathrm{~km}\right.$, INPRES) que afectó en 1936 a las localidades de San Francisco del Monte de Oro y Gral. San Martín, ubicadas sobre el borde nor-occidental de la Sierra de San Luis (D en Fig. 3.8).

A profundidades intermedias la sismicidad se concentra principalmente bajo Precordillera, justo al oeste de Pie de Palo (Smalley et al., 1993, Badi et al., 2009) con una mayor intensidad bajo la falla de La Laja. Según Smalley et al. (1993), esta correlación 
espacial de los eventos superficiales e intermedios se relaciona con la interacción entre la dorsal Juan Fernández subducida y la placa Sudamericana a través de una estructura asísmica en la placa superior entre los 35 y $95 \mathrm{~km}$ de profundidad. Estudios de mecanismos focales intermedios (Stauder, 1973, Triep y de Cardinali, 1984) determinaron su naturaleza intraplaca correspondiendo a fallamiento normal por flexión en la litósfera oceánica subyacente y en respuesta a la convergencia de placas. Debido a esto, Smalley y Isacks (1987) estimaron un espesor litosférico continental de $80 \mathrm{~km}$ aproximadamente en la región de San Juan. En numerosos trabajos (Smalley y Isacks, 1987, 1990, Pujol et al., 1991, Regnier et al., 1994) se observa una aparente inclinación de la ZWB hacia el oeste bajo la Precordillera y Cordilleras Frontal y Principal. Este comportamiento fue interpretado por Regnier et al. (1994) como un efecto del modelo de estructura de velocidad en capas planas utilizado en la localización de eventos, que no permite visualizar el engrosamiento cortical hacia el arco denotado por estudios gravimétricos y análisis flexurales (Introcaso et al., 1992, Gimenez et al., 2000, Tassara y Yañez, 2003). Pujol et al. (1991), mediante localización conjunta de hipocentros, determinaron variaciones laterales de velocidad en forma cualitativa que permitieron eliminar la pendiente anómala hacia el oste de la ZWB.

Bajo las Sierras de Córdoba la actividad sísmica a profundidades intermedias desaparece sugiriendo que la placa de Nazca abandona la horizontalidad hundiéndose en el manto con una pendiente mayor. Una anomalía de resistividad en dirección este-oeste llevó a Booker et al. (2005a, b) a suponer una ruptura de la placa en esa zona de máxima flexión. La orientación de los ejes de máxima tensión en los mecanismos intraplaca estimados por Anderson et al. (2007) mostraron variaciones coincidentes con la anomalía magnetotelúrica.

A los $33^{\circ} \mathrm{S}$ una nueva transición entre los regímenes de subducción horizontal y normal, con un menor nivel de actividad sísmica, se alinea aproximadamente con la subducción de la dorsal asísmica Juan Fernández (Badi, 1991, Badi y Sabbione, 1994) y se caracteriza por ser una flexión cerrada (Smalley y Isacks, 1987, Cahill y Isacks, 1992) según indica la continuidad del contorno de la ZWB correspondiente a los $125 \mathrm{~km}$ de profundidad. A esta latitud, la Precordillera desaparece en coincidencia con el aumento de la inclinación de la ZWB. La deformación se localiza en el frente montañoso y en el extenso ambiente extracordillerano (Cortés et al., 1999, Smalley y Isacks, 1990) donde la tectónica holocena activó corrimientos ciegos o emergentes de vergencia oriental y pliegues asociados en las cerrilladas pedemontanas cercanas a la ciudad de Mendoza y reactivó el borde oriental del bloque de San Rafael. 
En el flanco oriental del anticlinal Barrancas (Fig. 3.6, Sección 3.1.2.7), un corrimiento ciego con inclinación oeste y una superficie de despegue a $6.1 \mathrm{~km}$ de profundidad, constituye la falla Lunlunta (Cortés et al., 1999), a la que el INPRES vinculó el terremoto con Ms 5.9 de Mendoza en $1985\left(33.12^{\circ} \mathrm{S}, 68.82^{\circ} \mathrm{O}\right)$ con una profundidad de $12 \mathrm{~km}$, mientras que Triep (1987) lo asoció a una falla contigua hacia el este y a una profundidad de $14 \pm 5 \mathrm{~km}$. En ambos casos la superficie de despegue está por encima de la profundidad focal estimada, por lo que podría vincularse a un nivel de despegue más profundo.

Hacia el este, ya en el ambiente de la llanura pampeana, en un juego de escarpas correspondientes al sistema de Fallas de Sampacho, tuvo lugar en 1934 un terremoto Ms 6.0 en el sudeste de la provincia de Córdoba $\left(33.5^{\circ} \mathrm{S}, 64.5^{\circ} \mathrm{O}, 30 \mathrm{~km}\right.$, fuente INPRES). Mingorance (1991) postuló, basado en rasgos morfológicos, que la falla Sampacho (Las Lagunas, Fig. 3.8) experimentó un desplazamiento vertical de $0.25 \mathrm{~m}$ durante el evento.

La subducción normal se extiende desde los $33^{\circ} \mathrm{S}$ hasta los $46^{\circ} 30^{\prime} \mathrm{S}$, donde se encuentra la unión triple de Chile entre las placas de Nazca, Sudamérica y Antártica. La pendiente de la ZWB es aproximadamente de $30^{\circ}$ hasta los $36^{\circ} \mathrm{S}$ y cercana a $40^{\circ}$ al sur de dicha latitud. (Badi y Sabbione, 1992, 1994, López et al., 1997).

Chen et al. (1999) compilaron los datos del catálogo de Harvard CMT relativos a la sismicidad intermedia y profunda para Sudamérica, determinando las variaciones de la subducción de la Placa de Nazca con la latitud y su correlación con estados de tensiones y volcanismo observado. Los autores concluyeron que: 1) el estado de tensiones en los terremotos intermedios y profundos para regiones de subducción normal $\left(20-30^{\circ}\right)$ es principalmente de extensión paralela a la inclinación mientras que en las regiones de subducción con bajo ángulo $\left(<10^{\circ}\right)$ muestran una dispersión mayor; 2$)$ en las regiones de subducción subhorizontal se observa una escasez o ausencia de volcanismo superficial que es explicada por los cambios en la reología de acuerdo a la geometría de la subducción; 3) los extremos norte y sur del margen de subducción muestran un volcanismo importante en regiones con muy poca actividad sísmica, sugiriendo que la fracturación no es condición necesaria para el volcanismo asociado a subducción.

Imágenes tomográficas de la estructura profunda de la subducción bajo Sudamérica mediante ondas $\mathrm{P}$ de telesismos han corroborado que la placa de Nazca es probablemente continua lateralmente y en profundidad prácticamente en toda su extensión presentando desgarros que sirven de transición entre los diferentes estilos de subducción (Rietbrock y Haberland, 2001). Esto queda además manifestado por los estados de tensión hallados en los 
mecanismos focales (Engdahl et al, 1995, Dorbath et al., 1996) y en la alteración en las profundidades de las discontinuidades del manto debido a la modificación de la estructura termal por la subducción de la placa oceánica (Collier y Helffrich, 2001, Ren et al., 2007).

\subsubsection{GEODINÁMICA DE LA REGIÓN DE NUEVO CUYO}

Desde un punto de vista geodinámico se han planteado tradicionalmente ciertos factores interrelacionados que pueden justificar la presencia de zonas de subducción horizontal en el margen Sudamérica-Nazca. Gutscher et al. (2000) examinaron dichos modelos analizándolos a partir de la comparación de varios ejemplos. Entre las hipótesis que pueden contribuir al mantenimiento de una subducción plana se destacaban: 1) una succión hidrostática entre las dos placas en contacto. Según esta hipótesis todas las subducciones tenderían a horizontalizarse; 2) la subducción de una placa oceánica joven, aún tibia, aumenta su flotabilidad. Esta hipótesis no alcanzaba para justificar la presencia de subducciones normales en lugares donde la misma dorsal que origina la placa está subduciendo, como es el caso del sur de Chile central $\left(40-42^{\circ} \mathrm{S}\right)$. Además la edad de la placa de Nazca que subduce entre los $28-33^{\circ} \mathrm{S}$ se estima entre 35 y $42 \mathrm{Ma}$ (Fig. 3.3); 3) la presencia de litósfera oceánica bajo la litósfera continental, reemplazando a la astenósfera, altera la estructura térmica del margen e incrementa la resistencia de la placa superior. Dicho enfriamiento del perfil geotérmico retrasa la transición basalto-eclogita permitiendo una mayor penetración hacia el este de la placa oceánica. Cuando dicha transición ocurre, el aumento de densidad provoca el hundimiento de la placa oceánica. Esta hipótesis debe reforzarse con la presencia de una dorsal asísmica en la subducción para justificar la exclusividad del proceso de horizontalización en algunas zonas; 4) la geometría del margen continental favorece la subducción horizontal. Esta hipótesis no parece surgir de la observación de los distintos casos existentes pues hay curvaturas de distintos tipos asociadas a subducciones de alto y bajo ángulo; 5) un movimiento absoluto del margen de subducción hacia la placa oceánica. Esto significa que la velocidad de la placa continental hacia el margen es mayor que la velocidad de hundimiento de la placa oceánica. Los cambios de estilo dentro del margen andino indican que este factor no puede ser la única causa de la horizontalización.

Anderson et al. (2004) agregaron un modelo basado en estudios de anisotropía en el manto superior: 6) existe un acople entre el flujo astenosférico y la geometría de la subducción. El flujo astenosférico hacia el este bajo la placa de Nazca es deflectado por el movimiento retrógado de la placa de Nazca, resultando un flujo hacia el sur que afecta la polarización de las ondas. La interacción de este flujo con la geometría local de la placa 
subducida produce un flujo neto en dirección E-O bajo la subducción horizontal.

Se han observado gaps sísmicos a profundidades intermedias en numerosos márgenes con zonas de subducción plana. Gutscher et al. (2000) observaron que en algunos casos esto coincide con la subducción de arcos magmáticos o cordilleras oceánicas, destacando que en Chile central existía una controversia entre las edades de los magmas asociados y la edad de la placa subducida. Kay y Mpodozis (2002) observaron que los cambios en los estilos magmáticos en la región de subducción plana a través del tiempo y su limitación espacial, revelan las variaciones en la convergencia Nazca-Sudamérica y los efectos de la subducción de la traza del punto caliente Juan Fernández. La llegada de la dorsal asísmica a la fosa marca la finalización del volcanismo de arco andesítico y la horizontalización extrema de la placa de Nazca en parte del margen de subducción. Como mencionaran Anderson et al. (2007) todos los análisis de sismicidad en la Región de Nuevo Cuyo muestran un gap a profundidades intermedias bajo las Sierras de Córdoba. Burd et al. (2010) elaboraron una estructura 3D de la conductividad del manto cuyos resultados iniciales les permitieron inferir la presencia de una cuña astenosférica al este del segmento de subducción horizontal con su techo en la base de la litósfera, limitada hacia el este por las raíces del Cratón del Río de la Plata y hacia el oeste por la placa de Nazca. Dicha cuña probablemente se extiende hasta la zona de transición en el manto sugiriendo que la placa de Nazca se ve obligada a hundirse cuando encuentra las raíces mecánicamente más fuertes del cratón. Una zona conductiva orientada este-oeste dentro de un manto resistivo sugiere un flujo astenosférico a través de la placa y por lo tanto una ruptura que justificaría la ausencia de sismicidad.

Tassara et al. (2006) elaboraron un modelo reológico de la subducción NazcaSudamérica cotejado con numerosos datos geofísicos. En su modelo analizaron las causas de la horizontalización en el contexto de las relaciones entre la estructura cortical de las dorsales en la placa de Nazca, la posición y la morfología de los segmentos de subducción horizontal y su clara correlación con la morfoestructura de la placa continental. Estas observaciones sugirieron que si bien el extremo sur del segmento de subducción plana peruano está claramente relacionado con la subducción de la gran dorsal de Nazca, la flotabilidad positiva de las dorsales subducidas con respecto al resto de la placa que las rodea (Gutscher et al., 2000; Yáñez et al., 2001) es una condición necesaria pero no suficiente para la horizontalización de la laja. En particular, Tassara et al. (2006) notaron que la subducción de la dorsal de Iquique (Fig. 3.3), que tiene raíces corticales más continuas que la dorsal Juan Fernández, está asociada a un segmento de subducción con mayor pendiente bajo el Altiplano. Los autores estimaron que otros dos factores pueden ser considerados como controles de la 
forma de la subducción: 7) ciertos aumentos locales de la flotabilidad de la placa oceánica por hidratación del manto superior al norte de la dorsal Juan Fernández, como fuera revelado por las velocidades sísmicas estimadas por Kopp et al. (2004) y 8) el control dinámico ejercido por el movimiento absoluto de la placa Sudamericana hacia el oeste y su composición, mostrado por los modelos termomecánicos de van Hunen et al. (2004). Las variaciones en la viscosidad de la placa Sudamericana pueden observarse a través de la disminución, de norte a sur, en la cantidad de convergencia que es absorbida por el acortamiento de la placa continental (Isacks, 1988, Doglioni et al., 2006).

El modelo del límite litósfera-astenosfera (lithosphere asthenosphere boundary, LAB) cotejado con datos independientes por Tassara et al. (2006) alcanza profundidades mayores que $120 \mathrm{~km}$ por debajo de las Sierras Pampeanas Orientales y a lo largo de una región orientada ENE. El límite noroeste de esta región se correlaciona con la supuesta ubicación de las suturas entre el cratón del Río de la Plata y el terreno Pampia y entre este último y Cuyania (Ramos, 1999d). La corteza bajo los Andes también presenta variaciones de espesor con la latitud. Bajo el Altiplano, se encuentra engrosada con espesores de más de $70 \mathrm{~km}$, mientras que en la Puna y en la cuenca de Atacama, justo al norte de los $25^{\circ} \mathrm{S}$, el Moho se halla por encima de los $60 \mathrm{~km}$ de profundidad. Al sur de $\operatorname{los} 26^{\circ} \mathrm{S}$, la cordillera presenta raíces corticales de más de $60 \mathrm{~km}$ y en la zona de interés de este estudio, entre la Cordillera Frontal y las Sierras Pampeanas Occidentales, la profundidad del Moho decrece de 60 a $40 \mathrm{~km}$. Esto sugiere la presencia de discontinuidades de escala litosférica entre los diferentes terrenos que conforman el continente (Tassara et al., 2006).

La discontinuidad intracortical (ICD) es definida como una discontinuidad de densidad (granítica-basaltica) en el modelo para ajustar las longitudes de onda corta de la anomalía de Bouguer. Una ICD profunda indica, una corteza félsica, mientras que una ICD somera indica una corteza máfica. Por otro lado, parece ser que una ICD profunda se relaciona con un mecanismo de deformación de piel gruesa mientras que una ICD somera se asocia a un mecanismo de piel fina, sugiriendo que la estructura composicional cortical ejerce un control importante en la deformación del margen continental. Las rocas de alta densidad máficas a ultramáficas aflorantes en algunos puntos de las Sierras Pampeanas Occidentales parecen ser la expresión superficial de un gran terreno caracterizado por una ICD con profundidades inferiores a $7.5 \mathrm{~km}$. La ubicación de este alto en la ICD coincide con la localización de Cuyania según Ramos (1999d), que sería un terreno alóctono máfico en comparación al basamento félsico más común en los Andes. En las Sierras Pampeanas se encuentran unas regiones con orientación ENE donde la ICD tiene profundidades menores que $5 \mathrm{~km}$ y que se 
correlacionan con las anomalías de velocidad en el manto halladas por Wagner et al. (2006). Estos datos sugieren que la forma de la ICD podría estar afectada por las variaciones de densidad en el manto causadas probablemente por la subducción de la dorsal Juan Fernández. Tassara y Yáñez (2003) encontraron los menores valores de espesor elástico efectivo máximo a $\operatorname{los} 33^{\circ} \mathrm{S}$, menores aún que los hallados en el Altiplano. El debilitamiento hallado en el antearco a esa latitud podría estar relacionado con un rejuvenecimiento de la placa de Nazca en su paso sobre el punto caliente de Juan Fernández (Fig. 3.3). Yáñez et al. (2001) estimaron una edad de 9-10 Ma para los edificios volcánicos en la placa de Nazca cerca de la fosa a los $33^{\circ} \mathrm{S}$. La horizontalización de la subducción en el segmento de la Cordillera Frontal podría estar asociada también a este hecho ya que coincide con un mayor acoplamiento mecánico en la zona de Wadati-Benioff, las mayores profundidades de la zona sismogénica cortical y la mayor energía liberada (Gutscher et al., 2000, Gutscher, 2002). Las anomalías térmicas asociadas a la dorsal Juan Fernández y el calor liberado por fricción en la subducción horizontal parecen ejercer juntos el mayor control termal en la rigidez del antearco.

En el Altiplano y Puna, la ICD se encuentra más profunda pero sin superar los 25-30 $\mathrm{km}$, sugiriendo que la composición cortical promedio es intermedia, coincidiendo con valores de velocidad de ondas $\mathrm{P}\left(V_{\mathrm{P}}\right)$ del orden de $6 \mathrm{Km} / \mathrm{s}$ a profundidades de corteza media-inferior (Beck y Zandt, 2002). Según Schmitz et al. (1997) la combinación de bajas $V_{\mathrm{P}} \mathrm{y}$ altas densidades a profundidades de corteza inferior requiere un grado importante de fusión parcial de un protolito máfico. En coincidencia, Haberland et al. (2003) han estimado una alta atenuación de ondas $\mathrm{P}\left(Q_{\mathrm{P}}=100\right)$ hasta unos $20 \mathrm{~km}$ de profundidad y Tassara y Yáñez (2003) han determinado bajas rigidez y viscosidad, las que permiten un flujo cortical que se piensa responsable de las deformaciones observadas. De este modo, Tassara et al. (2006) plantean a la ICD como una aproximación a las variaciones laterales de densidad causadas principalmente por cambios en la composición cortical pero además puede asociarse a variaciones de temperatura en la corteza inferior y a la posibilidad de un grado de hidratación y/o fusión parcial de las rocas corticales, concluyendo que las variaciones de composición cortical de intermedia a félsica en los Andes centrales y a máfica en los Andes del sur son probablemente la principal causa de la segmentación andina a escala continental.

\subsection{DATOS}

Los datos utilizados en este estudio han sido provistos por el Instituto Nacional de Prevención Sísmica (INPRES), dependiente de la Secretaría de Obras Públicas del Ministerio de Planificación Federal, Inversión Pública y Servicios. La Red Nacional de Estaciones 
Sismológicas del INPRES está compuesta por cincuenta (50) estaciones distribuidas en todo el territorio nacional divididas en tres redes zonales por razones topográficas y de interconectividad: Red Zonal Norte, Red Zonal Centro y Red Zonal Sur.

La Red Zonal Centro o Red Sismológica Zonal Nuevo Cuyo (RSZNC), cuenta actualmente con estaciones en las provincias de San Juan, Mendoza, La Rioja, San Luis, Catamarca y Córdoba. Los datos analizados han sido registrados por 23 estaciones de corto período y componente vertical con registro digital, pertenecientes a la RSZNC. La Fig. 3.13 muestra su distribución y los principales rasgos geotectónicos de la región.

En el Apéndice A se describen las características principales de las condiciones de

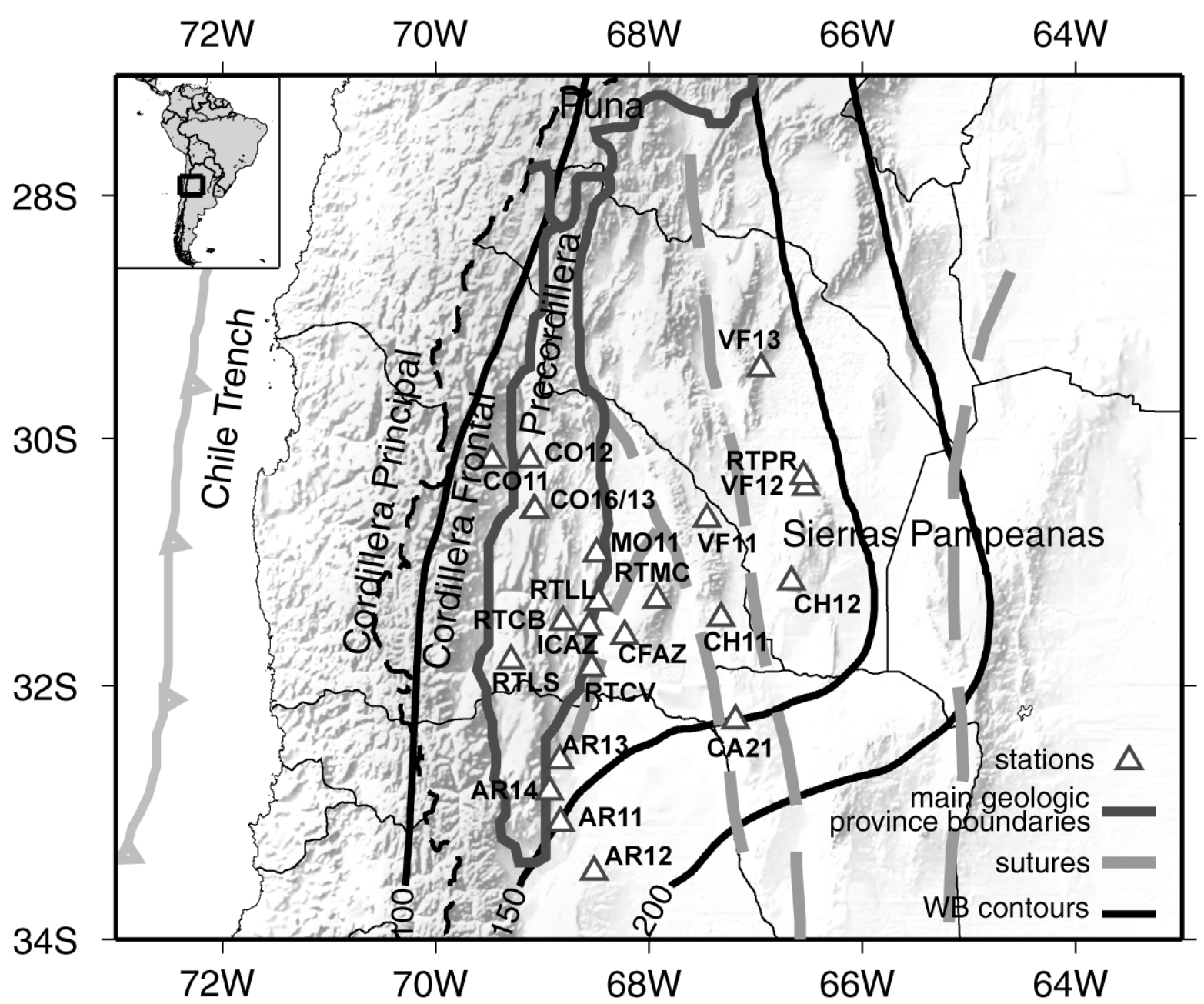

Fig. 3.13. Mapa de la Región de Nuevo Cuyo donde se incluyen la localización de las estaciones utilizadas (triángulos) de la Red Sismológica Zonal Nuevo Cuyo (RSZNC) perteneciente al INPRES cuyos códigos se explican en el anexo A y algunos rasgos tectónicos de importancia. Las líneas sólidas en gris oscuro definen las principales provincias geológicas, las líneas segmentadas en gris claro son suturas que coinciden con los mayores sistemas de fallas activos (Ramos et al., 2002). Las líneas sólidas en negro son los contornos de la zona de Wadati-Benioff (profundidad en $\mathrm{km}$ ) según Cahill y Isacks (1992).

instalación y de la registración de cada estación así como las constantes de las respuestas de 
instrumento construidas en este estudio a partir de las curvas de calibración provistas por el INPRES y utilizadas en el procesamiento de los datos. Cada estación, puede clasificarse como analógica con registro in situ, telemétrica analógica o telemétrica digital, en función del tipo de registro obtenido y de la forma en que se envían los datos al centro de recolección, para su tratamiento y análisis. Los datos son almacenados en formato PC-SUDS a un archivo por evento. Las frecuencias de muestreo utilizadas han sido de $100 \mathrm{mps}$ en la mayoría de las estaciones hasta agosto de 1998 cuando las estaciones fueron reconfiguradas a $80 \mathrm{mps}$ o 40 mps. En la configuración de la instalación, se han incluido filtros antialias analógicos con frecuencias de corte en 16,12 o $7.5 \mathrm{~Hz}$ dependiendo de la estación. Si bien estos circuitos reducen el contenido de frecuencias recuperables, las distancias epicentrales y las magnitudes analizadas permiten inferir frecuencias de corte en los espectros de ondas internas bastante por debajo de estos límites (Sección 1.2) y cercanas a 3-4 Hz.

A partir de la base de datos completa del INPRES para el período agosto 1995-marzo 1999 se han seleccionado en forma visual un conjunto de registros para el análisis de la atenuación de ondas internas y coda mediante los métodos descriptos en el Capítulo II. Dicho conjunto de datos verifica una serie de requisitos mínimos que son: 1) buena relación señalruido, 2) distancias estimadas a priori inferiores a 500-600 km, 3) ondas P y S no saturadas, 4) buen desarrollo de codas con más de $60 \mathrm{~s}$ de duración y decaimiento uniforme. Bajo estas condiciones se han seleccionado aproximadamente 500 eventos. Como ejemplo en la Fig. 3.14a se muestran los sismogramas de un evento de profundidad somera registrado por 9 estaciones de la RSZNC y en la Fig. 3. 14b, los correspondientes a un evento con foco en la placa de Nazca.

Se han efectuado las lecturas de los tiempos de llegada de las ondas $\mathrm{P}$ y S y las duraciones de los registros en forma manual utilizando los programas PCSuds y/o WinSuds (Banfill Software Engineering) dependiendo del sistema operativo de base. El proceso de localización inicial se ha efectuado mediante el programa Hypo71 (IASPEI Soft. Lib. Vol I, 1989) en forma individual para cada evento, utilizando tiempos de P y S, sin considerar elevación de estaciones y aplicando el modelo de velocidades que se muestra en la Tabla 3.1, provisto por el INPRES. Este es una modificación del que fuera obtenido por Herrin et al. (1968) como un modelo sobre-simplificado de los primeros $700 \mathrm{~km}$ del manto a fin de hacerlo útil en la localización mediante ondas $\mathrm{P}$ de eventos regionales superficiales. 


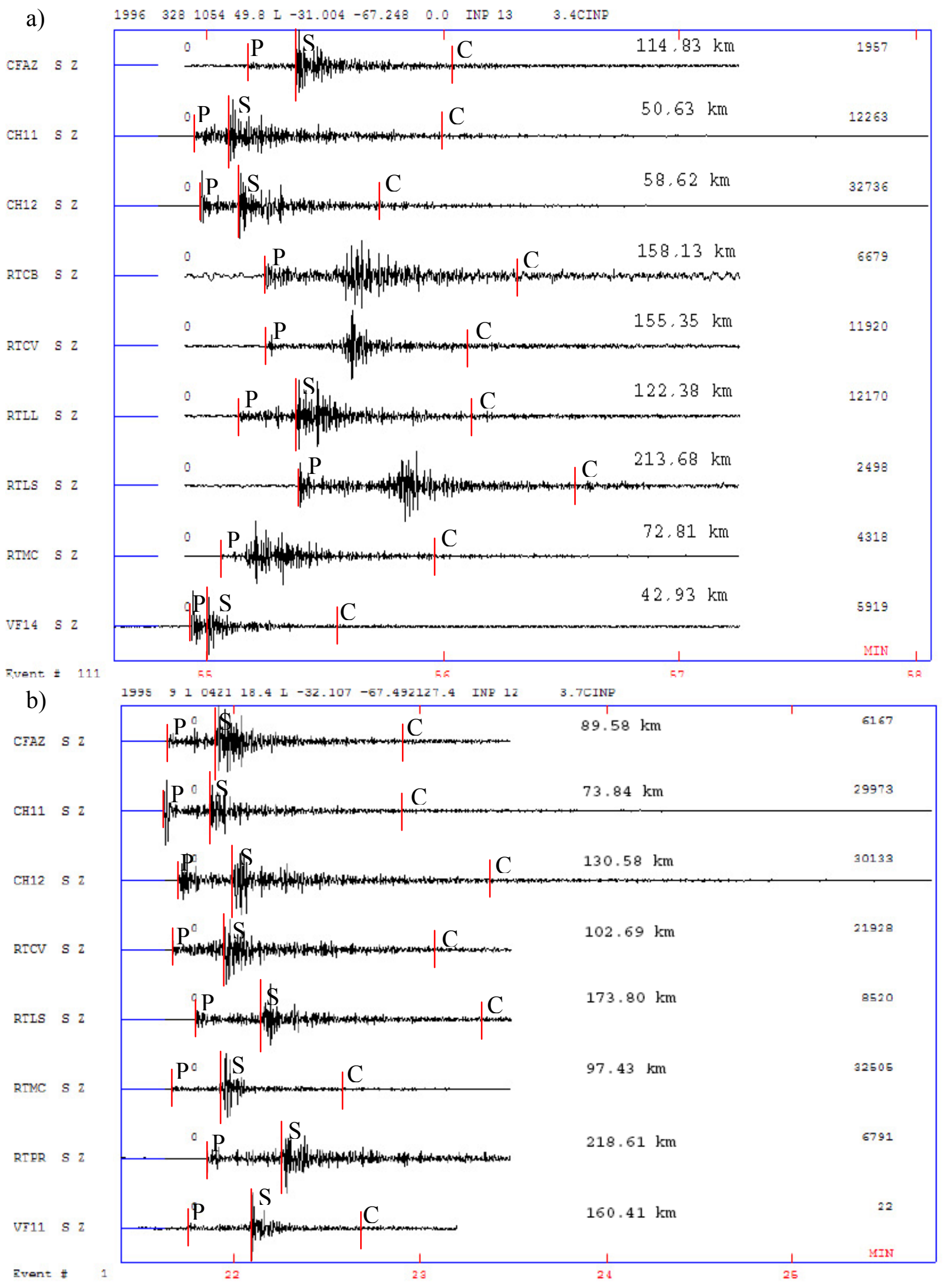

Fig. 3.14.- Ejemplo de sismogramas registrados por RSZNC en estaciones de componente vertical. Se observan una buena relación señal ruido en la mayoría de las estaciones y diferente polaridad y carácter del comienzo de la onda P según distancias y dirección de propagación. Se indican las distancias epicentrales en km. a) Sismo somero en la Sa. de Chepes, pcia. de La Rioja. La llegada de la onda S muestra diferente grado de definición entre estaciones. b) Sismo a 127.4 $\mathrm{km}$ de profundidad en la placa de Nazca bajo el NO de la pcia. de San Luis. Se observan tiempos de llegada de P y S claros. La mayor profundidad del hipocentro disminuye el número de reflexiones de $S$ en corteza haciendo más fácil la identificación de la llegada de la onda directa. 
El modelo propone una discontinuidad de Mohorovicic a profundidades entre 60 y $80 \mathrm{~km}$ Cada localización se ha efectuado mediante una sucesión de iteraciones manuales en las que se reasignan pesos a las lecturas de acuerdo a los residuos temporales de cada estación en la iteración previa. El objetivo es lograr la convergencia en la solución con un RMS mínimo, obteniendo una diferencia entre iteraciones consecutivas no significativa.

Los criterios de lectura y asignación de pesos utilizados fueron los indicados por el INPRES de acuerdo a las rutinas de localización empleadas. Durante el proceso de localización han debido utilizarse principalmente tiempos de llegada de ondas $\mathrm{P}$, en especial en el caso de eventos superficiales. Esto se debe a la

\begin{tabular}{ll}
\hline $\boldsymbol{V}_{P}[\mathrm{~km} / \mathrm{s}]$ & Prof. $[\mathrm{km}]$ \\
\hline 5.0 & 0 \\
6.2 & 10 \\
7.8 & 40 \\
7.85 & 60.3 \\
8.222 & 80.3 \\
8.273 & 100.8 \\
8.331 & 121.1 \\
8.398 & 141.6 \\
8.472 & 162.0 \\
8.554 & 182.6 \\
8.641 & 203.2 \\
8.735 & 223.9 \\
8.835 & 244.6 \\
\hline \multicolumn{2}{c}{$V_{P} / V_{S}=1.65$} \\
\hline Tabla 3.1. & Modelo de \\
velocidades de ondas $P\left(V_{P}\right)$ \\
su relación con la \\
velocidad de S $\left(V_{S}\right)$. Fuente \\
INPRES.
\end{tabular}
habitual presencia de múltiples comienzos de ondas $\mathrm{S}$ (por ej. Sg, S* y Sn) en el caso de eventos corticales registrados a distancias inferiores a 500 $\mathrm{km}$ y al hecho de contar con registros de una componente vertical donde la contaminación por la presencia de la coda de ondas $\mathrm{P}$ enmascara muchas veces el comienzo de las $\mathrm{S}$. De este modo se han ajustado las localizaciones al modelo de velocidades fijo resultando un total de 468 eventos localizados (Badi et al., 2007).

La distribución de sismicidad hallada (Fig. 3.15) muestra un comportamiento bimodal. Se aprecian los eventos corticales y los correspondientes a la zona de Wadati-Benioff, asociados a la Placa de Nazca que subduce bajo la Placa Sudamericana, aunque con cierta indefinición del espesor cortical y de la zona sismogénica en la Placa de Nazca, donde además aparece una leve pendiente hacia el oeste de la zona de Wadati Benioff (perfil inferior en la Fig. 3.15).

Se ha visto entonces la necesidad de obtener un modelo de velocidades ajustado a los datos de la RSZNC ya que los resultados obtenidos con el modelo de la Tabla 3.1 muestran una notable dispersión de los hipocentros respecto de las localizaciones esperadas según los procesos tectónicos conocidos en la región o en los peores casos, las localizaciones no convergen a posiciones razonables. La distribución de sismicidad obtenida en este apartado se ha tomado como la base original para la determinación del modelo unidimensional de ondas P mínimo como se verá en la siguiente sección. 


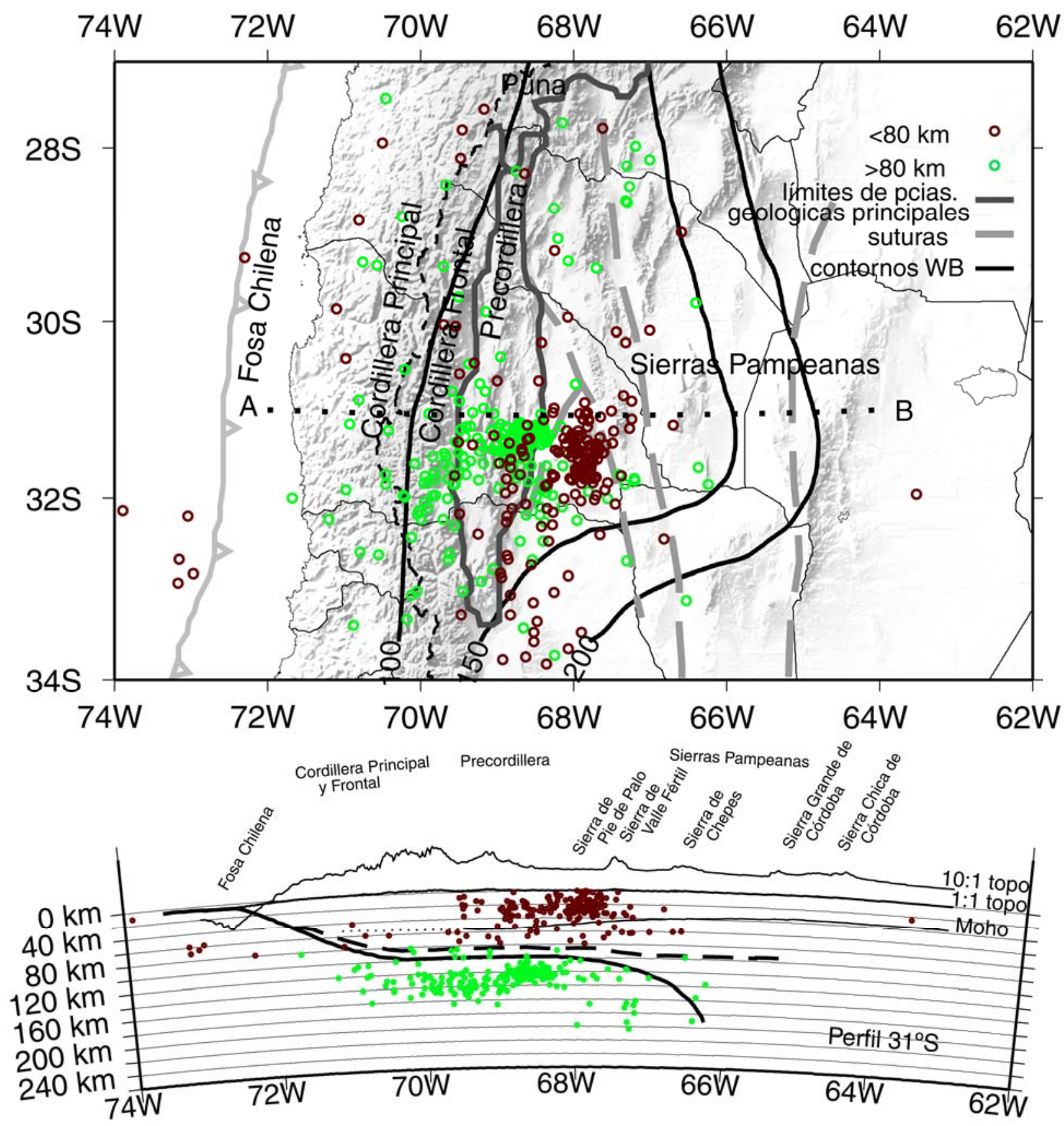

Fig.3.15.- Arriba: Distribución de epicentros obtenida con el modelo de velocidades inicial (Tabla 3.1) donde se diferencian en rojo, los eventos corticales $(<80 \mathrm{~km})$ y en verde, eventos intermedios (>80 km). La línea punteada AB marca el perfil sobre el que se proyectan los hipocentros en el gráfico inferior. Demás referencias coinciden con la Fig. 3.13. Abajo: proyección de hipocentros sobre el perfil $A B$ donde pueden diferenciarse los correspondientes a cada placa y la dispersión que presentan las soluciones. La línea gruesa sólida es la geometría inferida de la placa oceánica subductada y la línea gruesa segmentada es la base litosférica continental según Gutscher et al. (2000). La línea delgada sólida es la discontinuidad de Mohorovicic según Ramos et al. (2002) y una extrapolación hacia el oeste en línea de puntos. Realizado con GMT 4.0 (Wessel and Smith, 2004). 


\subsection{ESTRUCTURA UNIDIMENSIONAL DE VELOCIDAD DE ONDAS P}

\subsubsection{ANTECEDENTES Y METODOLOGÍA}

En la bibliografía pueden hallarse determinaciones de estructuras de velocidad para la Región de Nuevo Cuyo con técnicas diversas, utilizando datos de redes sismológicas portátiles desplegadas en Precordillera y/o Sierras Pampeanas, por ejemplo mediante análisis de funciones receptoras (Gilbert et al., 2006) o a través de estudios de Pn (Fromm et al., 2004). Uno de las primeras determinaciones de velocidad ha sido la de Pujol et al. (1991) quienes realizaron la determinación conjunta de hipocentros (Joint Hypocenter Determination, JHD, Douglas, 1967) para eventos superficiales e intermedios y obtuvieron correcciones de estación que asociaron a variaciones laterales cualitativas de la velocidad. En su análisis dejaron fijo el modelo de velocidad obtenido por Bollinger y Langer (1988).

En esta oportunidad se ha decidido utilizar el programa VELEST 3.1 (Kissling, 1995) a través de una implementación incluida en el paquete de programas SEISAN (Havskov y Ottemöller, 2005). El programa VELEST permite derivar un modelo unidimensional de velocidades apto para la localización de terremotos o como modelo de entrada para estudios de tomografía sísmica (Kissling, 1988, Kissling et al., 1994) resolviendo en forma iterativa el problema no lineal de la localización, trabajando en dos modos posibles: simple o simultáneo. En el modo simple realiza la localización de eventos en forma individual. En el modo simultáneo resuelve el problema del acople entre la localización de hipocentros y el modelo de velocidades realizando en forma conjunta la determinación de hipocentros (JHD), sugiriendo nuevas correcciones de estación y modelo de velocidad al final de cada iteración según numerosos parámetros que el usuario debe configurar. En ambos casos el problema directo se resuelve trazando el rayo sísmico a través de un modelo de capas planas desde la fuente hacia la estación. El problema inverso, no lineal, se resuelve por mínimos cuadrados. Una detallada explicación de la metodología y criterios de aplicación pueden hallarse en Kissling et al. (1994) y Kissling (1995). Utilizando el modo simultáneo mediante la técnica JHD se ha buscado la estructura de velocidades que optimizara la localización de todos los hipocentros en forma conjunta.

La implementación del programa VELEST dentro del paquete SEISAN está dada por el programa VELMENU que convierte automáticamente las lecturas de tiempos y las localizaciones preliminares del formato SEISAN (NÓRDICO) al propio del VELEST (CNV). Seguidamente genera el archivo de parámetros de entrada a partir de los archivos de la base de datos SEISAN, ejecuta el VELEST y convierte su salida a formato SEISAN. Otra ventaja 
de VELMENU es que permite la implementación de VELEST bajo Windows, dado que la versión original está diseñada para UNIX (Havskov y Ottemöller, 2008).

\subsubsection{DETERMINACIÓN DEL MODELO UNIDIMENSIONAL}

En primera instancia se han incorporado los datos a la base de datos SEISAN. El formato original utilizado por la RSZNC en el período 1995-1999 era SUDS. Para convertirlos a SEISAN sin pérdida de información, han debido convertirse primeramente a ASCII, incorporarles los datos del encabezamiento para cada estación, luego convertirlos a SEISAN y multiplexarlos para obtener nuevamente un archivo por evento. Este paso se ha automatizado mediante códigos en lenguaje FORTRAN.

Dentro del programa SEISAN, se han revisado todas las lecturas de tiempos de llegada de las ondas $\mathrm{P}$ y S seleccionando sólo aquellos comienzos claros para poder asignar a todas las lecturas el mismo peso en la localización. En este proceso se ha tomado a las localizaciones realizadas con HYPO71 (base original) como datos de partida. A la localización geográfica de las estaciones se les ha incorporado en esta etapa la elevación provista por el INPRES o en su defecto, estimada a partir de imágenes satelitales. Esta incorporación podía resultar significativa dado que la topografía de la región da lugar a desniveles de hasta $2000 \mathrm{~m}$ entre estaciones (Apéndice A).

Al contar con estaciones de una componente de registro vertical se ha optado por realizar el ajuste del modelo de velocidades para ondas P. Esta decisión se fundamenta en que los tiempos de llegada de las ondas $\mathrm{S}$ en componente vertical suelen estar menos definidos a causa de: 1) la polarización teóricamente transversal de las mismas que las hace mejor definidas en componentes horizontales cuando las ondas llegan a la estación en trayectorias no paralelas a la superficie; 2) la presencia de múltiples llegadas de ondas $\mathrm{S}\left(\mathrm{Sn}, \mathrm{Sg}, \mathrm{S}^{*}\right.$ y reflexiones) por efecto de la corteza heterogénea, que además varían su orden de llegada según la distancia y distribución de velocidades y 3) la habitual superposición del comienzo de las ondas S con la coda de $\mathrm{P}$ para las distancias epicentrales utilizadas.

A fin de proveer un modelo de $V_{\mathrm{S}}$ para la localización, debía darse una relación $V_{\mathrm{P}} / V_{\mathrm{S}}$ que ajustara a los datos. Una aproximación sencilla a dicha relación puede obtenerse mediante los diagramas de Wadati (Havskov y Ottemöller, 2005), donde se dibujan las diferencias de tiempos de llegada entre las ondas $\mathrm{S}$ y las ondas $\mathrm{P}$ en función del tiempo de llegada de $\mathrm{P}$ a partir de un origen arbitrario para todas las estaciones que lo hayan registrado (Fig. 3.16). El concepto de los diagramas de Wadati es muy simple. Si las ondas S y P 
demoran un tiempo $t_{\mathrm{s}}$ y $t_{\mathrm{p}}$ respectivamente en recorrer una distancia $\Delta$ viajando a velocidades medias $V_{\mathrm{S}}$ y $V_{\mathrm{P}}$, entonces la diferencia entre los tiempos de llegada de las ondas directas será igual a la diferencia de tiempos de viaje. Pueden establecerse las siguientes relaciones

$$
\begin{aligned}
& \Delta=V_{P} t_{P} \\
& \Delta=V_{S} t_{S}
\end{aligned}
$$

entonces despejando $t_{\mathrm{s}}-t_{\mathrm{p}}$ queda

$$
t_{S}-t_{P}=\frac{\Delta}{V_{P}}\left(\frac{V_{P}}{V_{S}}-1\right)=t_{P}\left(\frac{V_{P}}{V_{S}}-1\right)
$$

que permite obtener una relación promedio entre las velocidades de las ondas en su camino sísmico a partir del diagrama de Wadati. De la (3.2) se observa que la pendiente de la recta en la Fig. 3.16 es proporcional a $\left(V_{P} / V_{S}-1\right)$. Una vez calculada la relación de velocidades para cada terremoto, el promedio obtenido sobre todos los eventos de la base de datos permite establecer una relación $V_{\mathrm{P}} / V_{\mathrm{S}}$ para toda la región (Ojeda y Havskov, 2001, Havskov y Ottemöller, 2008). En la RNC se ha obtenido un valor medio de $V_{\mathrm{P}} / V_{\mathrm{S}}=1.76$ con una desviación standard de 0.05 (para todos aquellos datos registrados por más de 3 estaciones,

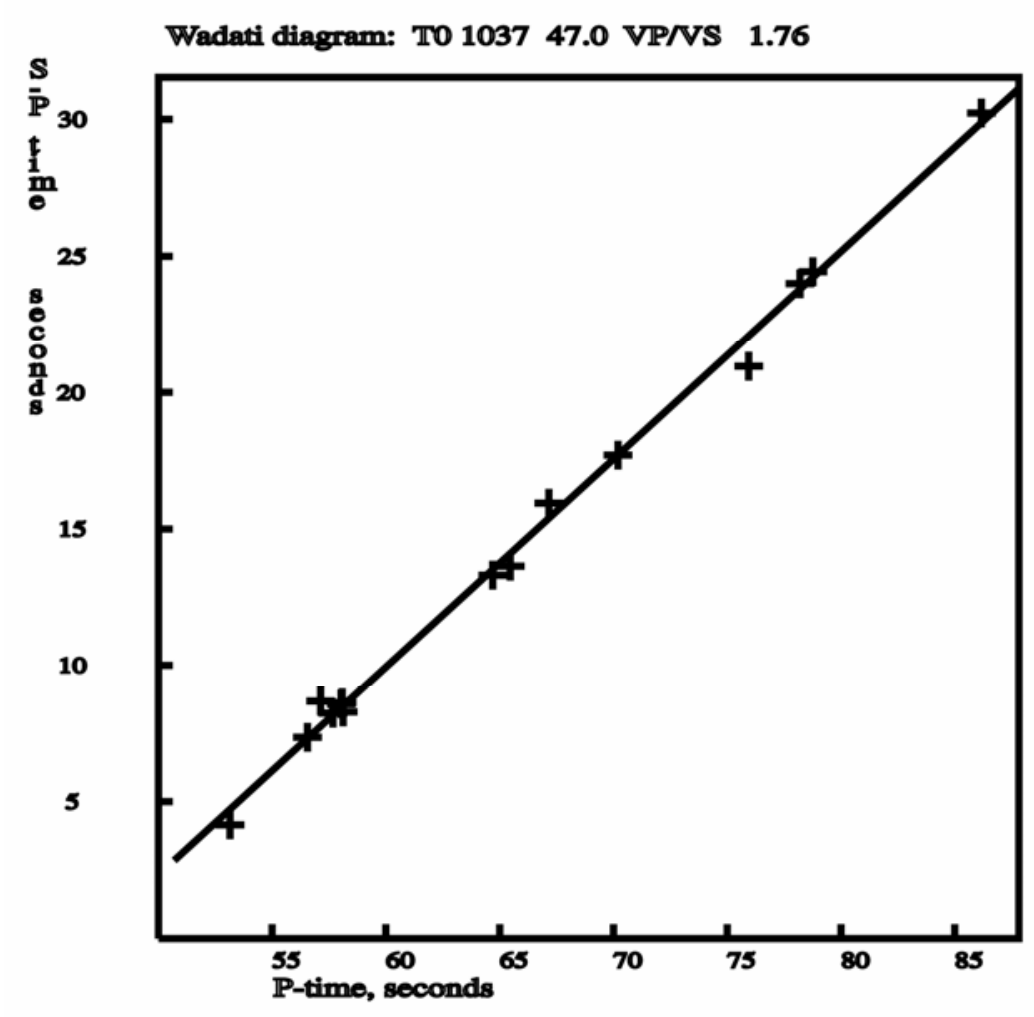

Fig. 3.16.- Ejemplo de Diagrama de Wadati para un sismo de la región. En el eje vertical se grafican diferencias de tiempos de llegada entre las ondas $S$ y $P$ (cruces) en las distintas estaciones, en las abscisas, tiempos de P desde un origen fijado a 10:37:47.0. El tiempo de origen del evento es 10:37:47.13UTC. La pendiente del diagrama indica un $V_{P} / V_{S}=1.76$. 
seleccionando los ajustes lineales de la (3.2) con un RMS $<1$ s y coeficientes de correlación mayores que 0.9).

La determinación del modelo de velocidad requiere la selección de una estación de referencia. Las correcciones de estación de las demás estaciones de la red se hacen relativas a la estación de referencia. Siguiendo los criterios establecidos por Kissling et al. (1994) y Ojeda y Havskov (2001) se ha seleccionado una estación de referencia localizada prácticamente en el centro geográfico de la red, con comienzos de ondas $\mathrm{P}$ y S generalmente claros y con el mayor número de lecturas. Resultando elegida la estación Coronel Fontana.

Utilizando el programa VELMENU se han procesado mediante JHD las lecturas de todos los eventos disponibles para obtener las correcciones de estación que minimizaran el RMS de las localizaciones. En esta etapa se han utilizado tiempos de $\mathrm{P}$ y de S, dejando fijo el modelo de velocidad original de $\mathrm{P}$ (Tabla 3.1) pero con una relación $V_{\mathrm{P}} / V_{\mathrm{S}}=1.76$.

Las correcciones de estación representan desviaciones del modelo de velocidad, de modo que correcciones positivas y negativas corresponden a anomalías negativas y positivas de velocidad en el área de la estación con respecto a la estación de referencia. Por lo tanto se relacionan con las condiciones del sitio y la topografía en la estación.

Una vez alcanzada una variación no significativa del RMS residual, se ha procedido a seleccionar entre los resultados, aquellos eventos que verificaran las siguientes condiciones a la distribución: un cubrimiento (GAP) menor a $200^{\circ}$, distancias epicentrales por debajo de 350 km y RMS menor a 1 s. Sobre esos datos se ha ajustado mediante JHD el modelo de velocidad preliminar de ondas P (Kissling, 1995).

Una vez alcanzado un mínimo de RMS, se ha obtenido el modelo unidimensional preliminar de velocidad de P. En estas iteraciones el programa elimina en cada iteración aquellos eventos cuya localización no converge. Para la determinación del modelo unidimensional mínimo se realiza una nueva selección de los datos. Esta surge de la localización en modo simple de todos los eventos de la base de datos original con el modelo preliminar de velocidad y las correcciones de estación correspondientes, seleccionando aquellos eventos que verifiquen las mismas condiciones a la distribución del paso anterior.

El modelo 1D mínimo se ha obtenido entonces procesando esta última selección subamortiguando los parámetros hipocentrales y el modelo de velocidad, sobreamortiguando las correcciones de estación e invirtiendo el modelo cada dos iteraciones (Kissling, 1995). Es decir que se deja variar la localización en cada iteración y al modelo, iteración por medio. En este paso, se han incorporado capas intermedias al modelo para poder ajustar a partir de ellas 


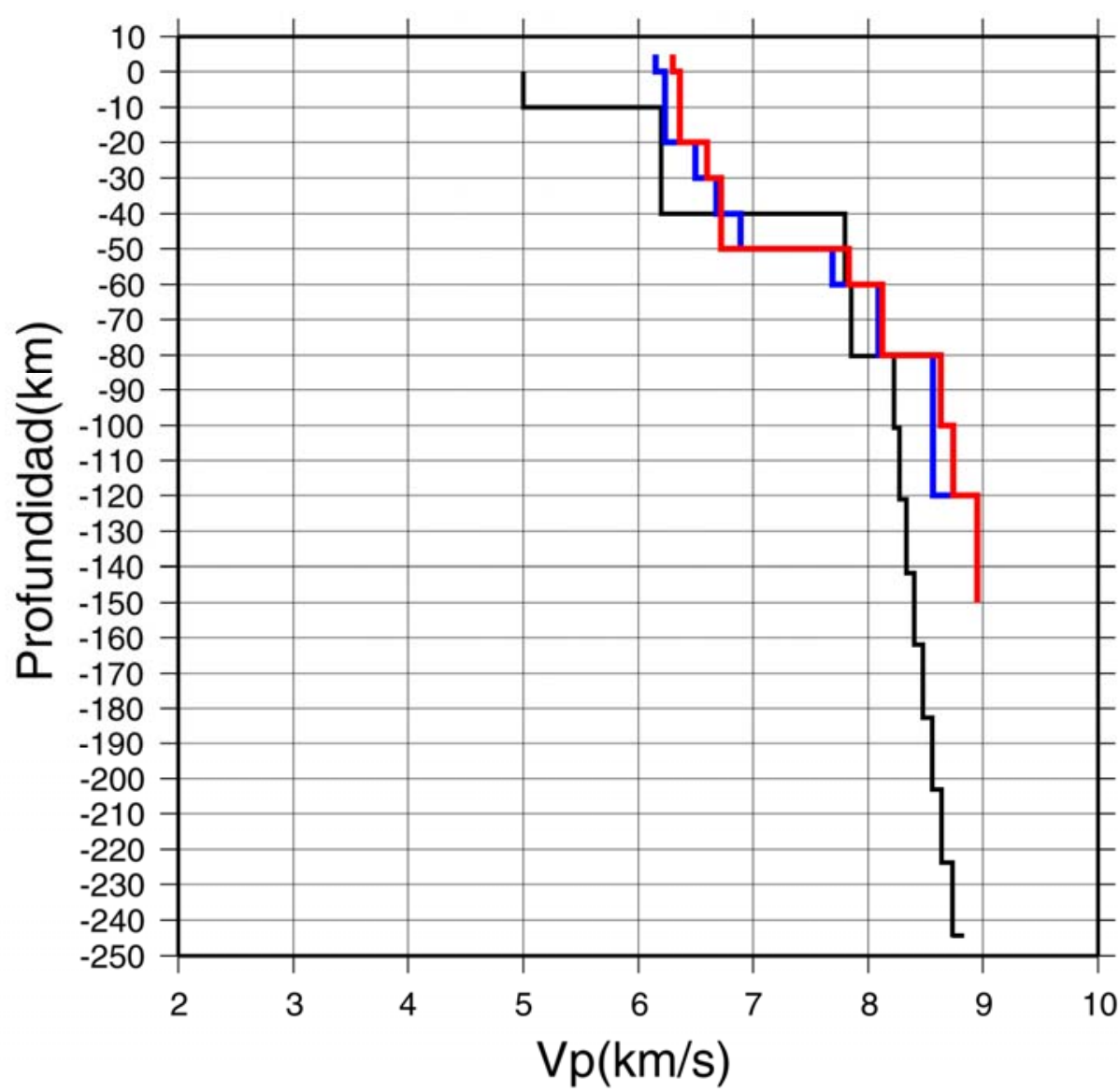

Fig. 3.17.- Perfiles de velocidad de ondas P. En línea negra delgada se muestra el modelo inicial, en azul el obtenido en este estudio con $V_{P} / V_{S}=1.80$ y en rojo el obtenido con $V_{P} / V_{S}=1.76 y$ seleccionado para el recálculo final de hipocentros.

el espesor cortical y un gradiente de velocidad. En cada paso a lo largo del proceso se han ido

\begin{tabular}{ll}
\hline $\boldsymbol{V}_{\boldsymbol{P}}[\mathrm{km} / \mathrm{s}]$ & Prof. $[\mathrm{km}]$ \\
\hline 6.30 & -5.00 \\
6.36 & 0.00 \\
6.60 & 20.00 \\
6.72 & 30.00 \\
7.83 & 50.00 \\
8.12 & 60.00 \\
8.63 & 80.00 \\
8.74 & 100.00 \\
8.95 & 120.00 \\
\hline \multicolumn{2}{c}{$\boldsymbol{V}_{\boldsymbol{P}} / \boldsymbol{V}_{S}=\mathbf{1 . 7 6}$} \\
\hline \multicolumn{3}{c}{}
\end{tabular}

Tabla 3.2. Modelo unidimensional de velocidad de ondas $P$ obtenido mediante JHD y relación de velocidades de $P$ y $S$ promedio, ajustada mediante diagramas de Wadati. combinando aquellas capas adyacentes que resultaran con velocidades semejantes. El objetivo de variar los coeficientes de amortiguamiento y los espesores de capa es hallar un modelo de velocidad estable que a su vez minimice los errores de las localizaciones.

Dado que los modelos obtenidos y/o utilizados por otros autores (Fromm et al., 2004, Alvarado et al., 2005, Anderson et al., 2007) consideran un $V_{P} / V_{S}=1.80$ para la región de las Sierras Pampeanas Occidentales, se repitió el proceso de ajuste con dicha relación e idéntica metodología, arribándose a resultados y valores de RMS mínimos muy similares.

En la Fig. 3.17 se compara el modelo inicial con los modelos obtenidos en este análisis para $V_{P} / V_{S}=1.80 \mathrm{y}$ 


\begin{tabular}{|c|c|c|c|c|c|}
\hline STN & Lat $\left[{ }^{\circ}\right]$ & Lon $\left[{ }^{\circ}\right]$ & Elev [m] & Ptcor [s] & Stcor [s] \\
\hline AR11 & $33.0857 \mathrm{~S}$ & $68.8275 \mathrm{~W}$ & 1159 & 1.20 & 1.23 \\
\hline AR12 & $33.4737 \mathrm{~S}$ & $68.5152 \mathrm{~W}$ & 1019 & 1.32 & 1.52 \\
\hline AR13 & $32.5917 \mathrm{~S}$ & $68.8340 \mathrm{~W}$ & 1000 & 0.80 & 0.47 \\
\hline AR14 & $32.8413 \mathrm{~S}$ & $68.9328 \mathrm{~W}$ & 1507 & 1.08 & 0.79 \\
\hline AR21 & $33.0857 \mathrm{~S}$ & $68.8275 \mathrm{~W}$ & 1159 & 1.31 & 2.59 \\
\hline AR22 & $33.4737 \mathrm{~S}$ & $68.5152 \mathrm{~W}$ & 1019 & 1.26 & 1.36 \\
\hline AR23 & $32.5917 \mathrm{~S}$ & $68.8340 \mathrm{~W}$ & 1000 & 0.89 & 5.02 \\
\hline CA11 & $32.2747 \mathrm{~S}$ & $67.1877 \mathrm{~W}$ & 634 & -0.02 & -0.13 \\
\hline CA21 & $32.2747 \mathrm{~S}$ & $67.1877 \mathrm{~W}$ & 634 & 0.16 & -0.74 \\
\hline CFAZ & $31.6032 \mathrm{~S}$ & $68.2322 \mathrm{~W}$ & 621 & 0.00 & -0.75 \\
\hline FO14 & $31.6032 \mathrm{~S}$ & $68.2322 \mathrm{~W}$ & 621 & 0.06 & -0.73 \\
\hline FO24 & $31.6032 \mathrm{~S}$ & $68.2322 \mathrm{~W}$ & 621 & -0.03 & -0.86 \\
\hline MO11 & $30.9397 \mathrm{~S}$ & $68.4923 \mathrm{~W}$ & 830 & 1.10 & 0.63 \\
\hline MO14 & $31.3093 \mathrm{~S}$ & $67.9240 \mathrm{~W}$ & 3100 & 0.47 & -0.15 \\
\hline VF12 & $30.3957 \mathrm{~S}$ & $66.5277 \mathrm{~W}$ & 725 & -0.09 & 0.42 \\
\hline VF13 & $29.4262 \mathrm{~S}$ & $66.9512 \mathrm{~W}$ & 1650 & 0.68 & 2.42 \\
\hline ICAZ & $31.5282 \mathrm{~S}$ & $68.5598 \mathrm{~W}$ & 660 & 0.50 & -0.15 \\
\hline RTCV & $31.8622 \mathrm{~S}$ & $68.5402 \mathrm{~W}$ & 670 & 0.19 & -0.83 \\
\hline RTCB & $31.4885 \mathrm{~S}$ & $68.8095 \mathrm{~W}$ & 1030 & 0.42 & -0.36 \\
\hline RTLL & $31.3292 \mathrm{~S}$ & $68.4748 \mathrm{~W}$ & 690 & 0.04 & -0.33 \\
\hline RTLS & $31.7995 \mathrm{~S}$ & $69.2950 \mathrm{~W}$ & 2560 & 0.67 & -0.44 \\
\hline RTPR & $30.3080 \mathrm{~S}$ & $66.5517 \mathrm{~W}$ & 460 & -0.07 & -0.45 \\
\hline RTMC & $31.3095 \mathrm{~S}$ & $67.9240 \mathrm{~W}$ & 3100 & 0.05 & -0.65 \\
\hline CO11 & $30.1670 \mathrm{~S}$ & $69.4733 \mathrm{~W}$ & 2745 & 0.81 & 0.78 \\
\hline CO12 & $30.1708 \mathrm{~S}$ & $69.1197 \mathrm{~W}$ & 1572 & 0.87 & 1.16 \\
\hline CO13 & $30.5885 \mathrm{~S}$ & $69.0810 \mathrm{~W}$ & 2792 & 0.65 & 0.76 \\
\hline $\mathrm{CO} 23$ & $30.5885 \mathrm{~S}$ & $69.0810 \mathrm{~W}$ & 3127 & 0.00 & -6.23 \\
\hline CO16 & $30.5877 \mathrm{~S}$ & $69.0662 \mathrm{~W}$ & 2792 & 0.80 & 0.00 \\
\hline RODE & $30.1670 \mathrm{~S}$ & $69.4733 \mathrm{~W}$ & 2745 & 0.86 & 0.93 \\
\hline CUES & $30.1708 \mathrm{~S}$ & $69.1197 \mathrm{~W}$ & 1572 & 0.61 & 0.77 \\
\hline CH12 & $31.1667 \mathrm{~S}$ & $66.6628 \mathrm{~W}$ & 1470 & -0.07 & -0.72 \\
\hline CH15 & $31.1667 \mathrm{~S}$ & $66.6628 \mathrm{~W}$ & 1470 & 0.03 & -1.19 \\
\hline CH11 & $31.4560 \mathrm{~S}$ & $67.3268 \mathrm{~W}$ & 615 & -0.09 & -1.01 \\
\hline VF11 & $30.6603 \mathrm{~S}$ & $67.4540 \mathrm{~W}$ & 910 & -0.01 & 0.04 \\
\hline VF14 & $30.6603 \mathrm{~S}$ & $67.4540 \mathrm{~W}$ & 910 & -0.01 & -0.33 \\
\hline
\end{tabular}

Tabla 3.3.- Correcciones de estación sugeridas por este estudio. En letras rojas se destaca la estación de referencia, Coronel Fontana. Deberá tenerse en cuenta que las correcciones para tiempos de $S$ han sido determinadas a partir de los tiempos leidos en componente vertical que luego han sido descartados en la etapa final de localización. 
$V_{P} / V_{S}=1.76$. Se observan diferencias mínimas en valores de velocidad y número de capas en ambas soluciones. En ambos casos la profundidad promedio de la discontinuidad de Mohorovicic para la RNC se estima en $50 \mathrm{~km}$.

La decisión de utilizar el valor $V_{P} / V_{S}=1.76$ se basa en que este es el valor obtenido a partir del ajuste de los diagramas de Wadati para los registros de la RSZNC, que no difiere prácticamente del 1.75 obtenido por Bollinger and Langer (1988) y utilizado por Pujol et al. (1991) y que a su vez coincide con el modelo inicial de Pardo et al. (2002). El modelo 1D resultante consta de 8 capas y un semiespacio cuyos valores de $V_{\mathrm{P}}$ se dan en la Tabla 3.2. La Tabla 3.3 proporciona las correcciones de estación finales para tiempos de $\mathrm{P}$ y S obtenidas en el ajuste.

Comparando el modelo 1D mínimo de la Tabla 3.2 con el modelo 3D obtenido por Pardo et al. (2002) para la misma zona con datos de estaciones en territorio chileno, se observa una buena correlación de la sismicidad y de los valores de velocidad en profundidad. Alvarado et al. (2009) mediante la integración de datos sismológicos y gravimétricos obtuvieron valores de $V_{\mathrm{P}}$ corticales cercanos a $6.4 \mathrm{~km} / \mathrm{s}$ en el terreno de Cuyania (zona central de la RSZNC) con relaciones $V_{\mathrm{P}} / V_{\mathrm{S}}=1.80-1.85$, espesores corticales entre 55-60 km y altas densidad y velocidad sísmica en corteza inferior. Por lo tanto el modelo obtenido en este estudio corrobora los resultados previos de otros autores a partir de bases de datos y metodologías diferentes, por lo cual se adopta como la estructura unidimensional de velocidades a utilizar en los análisis de atenuación en la RNC.

\subsection{LOCALIZACIÓN Y ANÁLISIS DE SISMICIDAD}

Con el objetivo de disponer de una distribución de sismicidad confiable para los estudios de atenuación se han vuelto a localizar todos los eventos de la base original (Sección 3.2) pero esta vez en forma conjunta y con el modelo de velocidad obtenido en la Sección 3.3.2. Para ello se ha preparado una base inicial del recálculo a partir de la base original de hipocentros. La base inicial del recálculo se ha conformado mediante las localizaciones efectuadas con un método simple (hypocenter, hypo71 o velest en modo simple) utilizando el modelo de velocidad y correcciones de estación propuestos en las Tablas 3.2 y 3.3, asignando a todas las lecturas de tiempos de $\mathrm{P}$ y S el mismo peso y efectuando una única iteración.

Partiendo de esta base inicial del recálculo se han localizado los eventos usando el método JHD, fijando el modelo de velocidad y las correcciones de estación y dejando variar los parámetros hipocentrales hasta lograr la estabilización en un mínimo del RMS. En este 
caso, al considerar tiempos de llegada de ondas $\mathrm{P}$ y S el método converge lentamente hasta un valor de RMS promedio igual a 0.36 para 7312 rayos sísmicos analizados. Dejando de lado los tiempos de $\mathrm{S}$ a partir de este punto, se ha continuado la localización sólo con tiempos de $\mathrm{P}$ por los motivos expuestos anteriormente y observándose una aceleración de la convergencia del método. El resultado final de la localización se presenta en el Apéndice B, habiéndose obtenido 452 eventos localizados con un RMS promedio de 0.16 para un total de 4205 rayos sísmicos analizados.

Una comparación de las distribuciones de RMS obtenidas en las etapas sucesivas de la localización, puede verse en la Fig. 3.18 donde se representan las mejores localizaciones en cada etapa $(\mathrm{RMS}<1)$. En primer lugar, en la base original que representa las localizaciones en modo simple, con Hypo71 y el modelo de velocidad de partida (Tabla 3.1), se ha obtenido una distribución de RMS (barras en negro) con una media de 0.199, una varianza igual a 0.765 y un coeficiente de asimetría (que caracteriza el grado de asimetría de una distribución con respecto a su media, siendo positivo cuando la distribución se extiende hacia valores mayores que la media y negativo en caso contrario) de 15.034. Volviendo a localizar los datos con el mismo modelo y método pero asignando igual peso a todas las lecturas se obtiene la distribución de RMS indicada en amarillo. Esta distribución presenta una varianza (4.523) notablemente mayor con una asimetría menor (7.359). La diferencia entre ambos resultados podría atribuirse a la subjetividad del operador al momento de asignar pesos en cada caso particular.

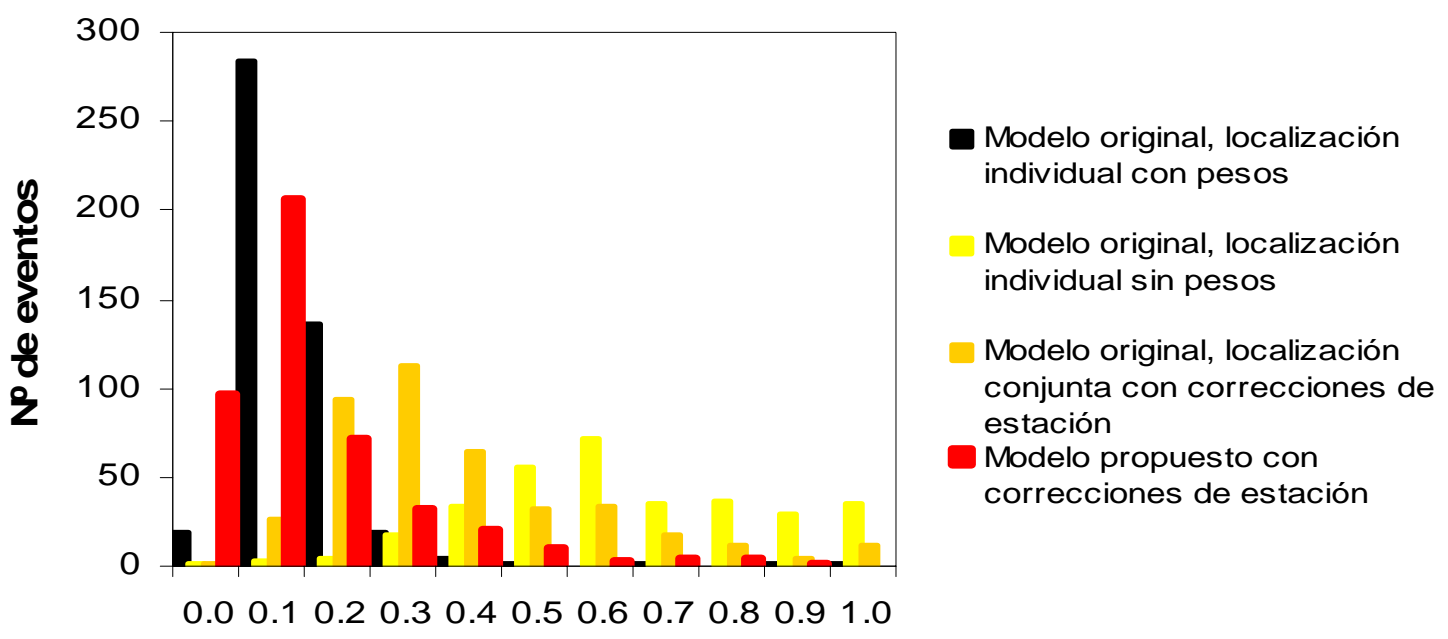

\section{RMS [s]}

Fig. 3.18.- Evolución de la distribución del número de eventos según RMS para RMS $<1$ con los modelos de velocidad utilizados. Como puede verse la localización con el modelo propuesto logra un RMS medio menor que las etapas previas de cálculo, aunque similar al original. Sin embargo se obtiene mediante un proceso más objetivo de selección de los datos, disminuyendo la dispersión de los parámetros estadísticos y ajustando mejor a la tectónica regional que la distribución original. 
La distribución obtenida mediante localización conjunta (JHD) utilizando el modelo original más las correcciones de estación que minimizan el RMS residual (naranja) tiene una varianza igual a 0.562 y una asimetría de 8.795 . La metodología utilizada en esta etapa es comparable a la de Pujol et al. (1991) ya que se ha dejado fijo el modelo permitiendo variar las correcciones de estación. El resultado final obtenido con el modelo de velocidad (Tabla 3.2) y las correcciones de estación (Tabla 3.3) propuestas en este estudio se presenta en rojo. La distribución de RMS muestra una media de 0.159 con una varianza de 0.025 y una asimetría igual a 2.548. Como puede verse, las distribuciones de RMS original y final presentan medias bastante próximas sin embargo la varianza y la asimetría son notablemente menores en la distribución final que se acerca de este modo a una distribución normal como ocurre en la mayoría de los procesos naturales. Más adelante se verá que la distribución de sismicidad asociada resulta mucho más ajustada a los rasgos tectónicos conocidos.

Las magnitudes de los eventos se calcularon mediante la fórmula de magnitud de coda $(M c)$ con la relación:

$$
M c=a \cdot \log (\operatorname{cod} a[s])+b \cdot \Delta[k m]+c
$$

que resulta válida para distancias $(\Delta)$ menores a $1500 \mathrm{~km}$ y donde coda es la duración del registro en segundos, medido desde el comienzo de P hasta donde la señal se confunde con el ruido de fondo para sismogramas con buena relación señal ruido (marcas $\mathrm{C}$ en la Fig. 3.14). Las constantes $a, b$ y $c$ han sido provistas por el INPRES para la RNC con valores: $a=1.175624, b=1.383656$ y $c=0.00062$. Las magnitudes $(M c)$ de los eventos han resultado

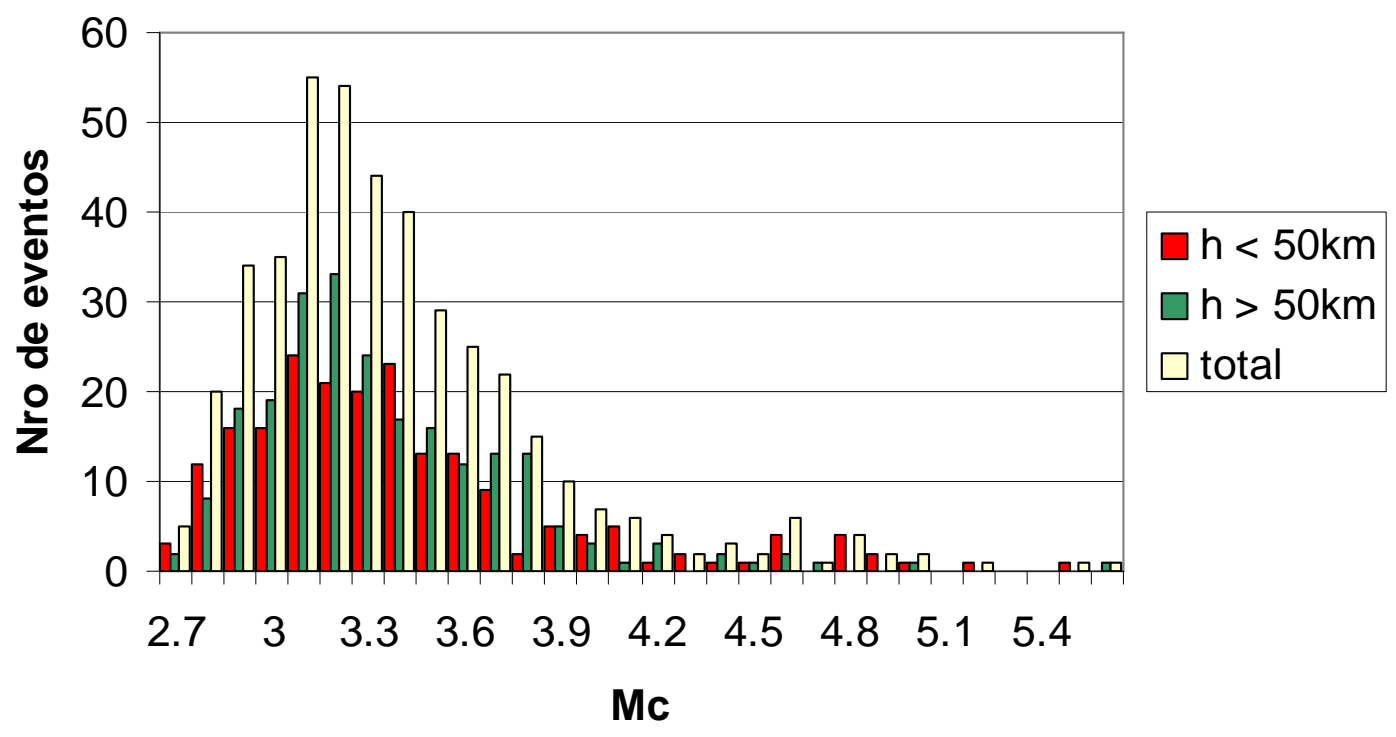

Fig. 3.19.- Distribución de magnitudes de coda (Mc) según la profundidad focal obtenida en la localización final considerando eventos corticales $(h<50 \mathrm{~km})$ y en el manto $(h>50 \mathrm{~km})$ de acuerdo a la profundidad estimada para el Moho en el modelo de velocidad de la Tabla 3.2. 


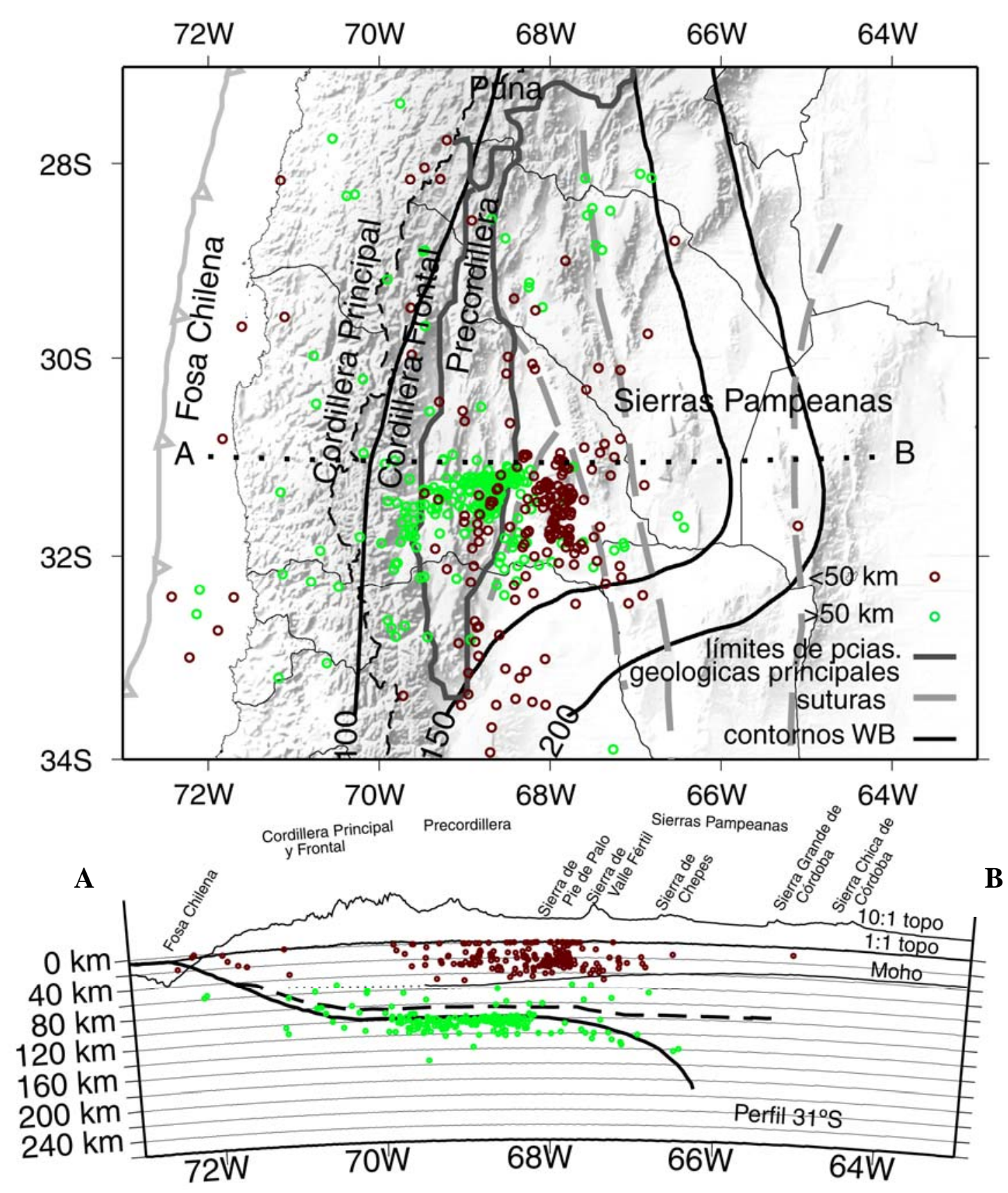

Fig. 3.20.- Arriba: Distribución de epicentros obtenida con el modelo de velocidades propuesto (Tabla 3.2) donde se diferencian en rojo, los eventos corticales $(h<50 \mathrm{~km})$ y en verde los eventos correspondientes a Placa de Nazca $(h>50 \mathrm{~km})$ La línea punteada AB marca el perfil sobre el que se proyectan los hipocentros en el gráfico inferior. Abajo: proyección de hipocentros sobre el perfil $A B$ donde puede observarse la menor dispersión de las localizaciones respecto de la base original (Fig. 3.15). Ver más observaciones en el texto. Las referencias coinciden con las de Fig. 3.13 y Fig. 3.15. 
hallarse dentro del intervalo [2.7-5.6] (Apéndice B) con una distribución del número de eventos por magnitud parametrizado según la profundidad focal como se muestra en la Fig. 3.19 .

En la Fig. 3.20 se presenta la distribución de hipocentros resultante en planta y en una proyección sobre una sección vertical. En el mapa se observa que los eventos corticales se concentran principalmente en Sierra Pie de Palo y en un claro alineamiento a lo largo de los valles de Matagusanos y Ullum-Zonda, al oeste del límite este de Precordillera Oriental, dado por el corrimiento Villicum-Pedernal que incluye las fallas de La Laja, Tapias -Villicum y Rinconada (Siame et al., 2006). En ambos casos las suturas planteadas por Ramos et al. (2002) delimitan la actividad sísmica cortical demostrando una vez más que las viejas discontinuidades corticales juegan un rol importante en la concepción y la geometría de las fallas principales.

Los sismos a profundidades intermedias mantienen su mayor concentración al sur de los $31^{\circ} \mathrm{S}$ pero se observa un mayor alineamiento de los mismos en dirección ENE que coincide con una zona somera de la ICD hallada por Tassara et al. (2006) y a su vez con las anomalías de velocidad en el manto determinadas por Wagner et al. (2006) (Sección 3.1.5). Esta direccionalidad de la zona de mayor fragilidad en la placa de Nazca coincide con la prolongación bajo el continente de la dorsal Juan Fernández subducida como ya había sido observado (Wagner et al., 2006, Anderson et al., 2007).

En la distribución de la sismicidad en profundidad de la Fig. 3.20, se observa una menor dispersión de los hipocentros acentuándose su comportamiento bimodal que se adapta perfectamente a los contornos estimados por Gutscher et al. (2000) y Ramos et al. (2002). Un grupo de eventos se concentra principalmente en corteza intermedia con sus profundidades máximas a $50 \mathrm{~km}$. El otro grupo se ubica en la parte superior de la placa de Nazca, a partir de los $100 \mathrm{~km}$ de profundidad. En el perfil, la pendiente aparente hacia el oeste de la zona de Wadati-Benioff obtenida anteriormente mediante la localización en modo simple parece haberse eliminado, tal como habían observado Pujol et al. (1991).

Anderson et al. (2007) estimaron la geometría de la subducción de la placa de Nazca a partir de la localización de eventos intermedios usando el algoritmo GMEL (Rodi et al., 2002a, b). Mediante el empleo de estructuras simples con velocidades promedio y la localización conjunta de eventos usando varios eventos maestros con localización controlada por otros estudios, observaron que la pendiente aparente hacia el oeste en la zona de subducción plana resultaba menor que con los métodos de localización simple, pero su 

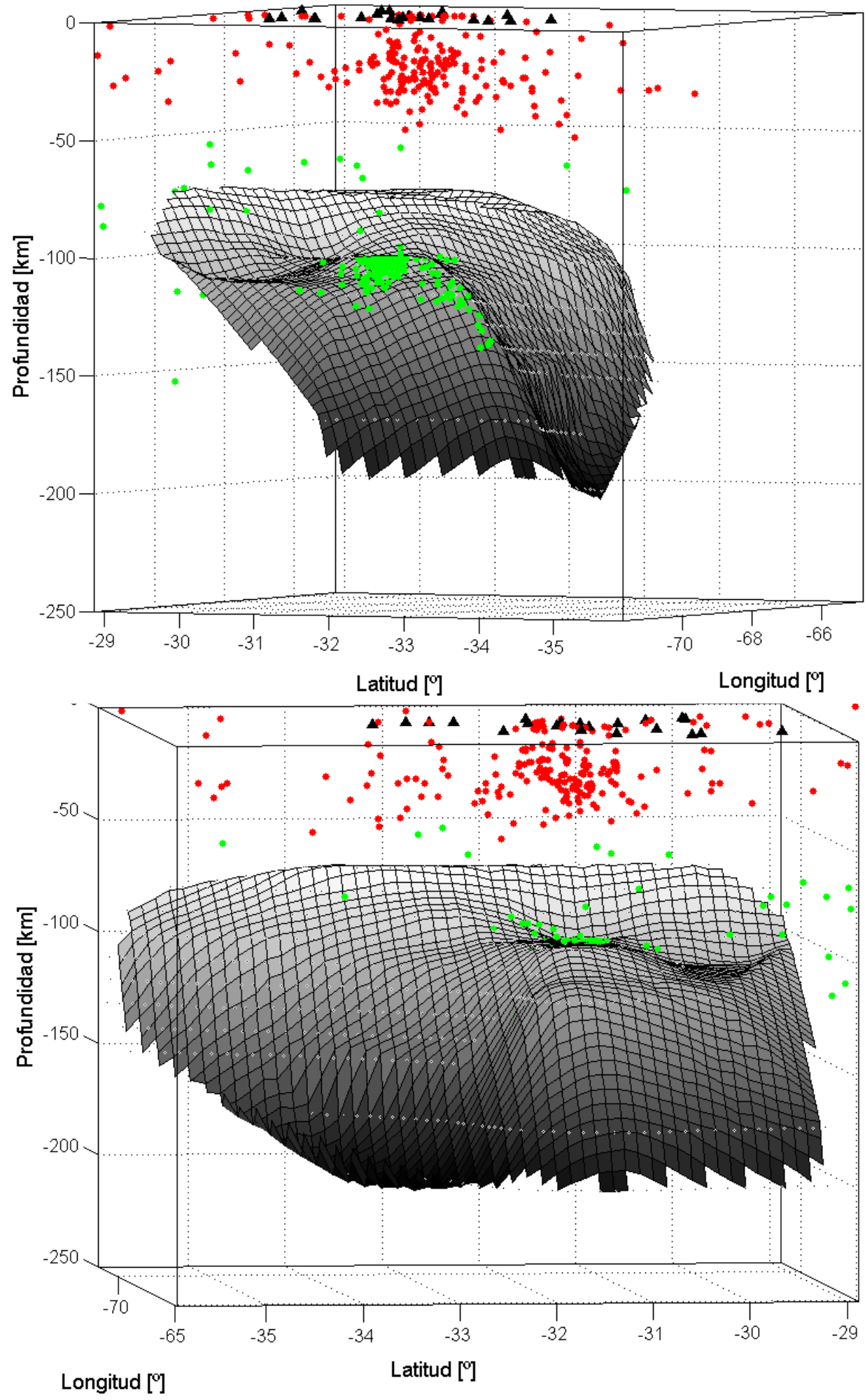

Fig. 3.21.- Gráfico 3D de la superficie de Wadatti-Benioff obtenida por interpolación cúbica de los contornos de la geometría de la placa de Nazca dados por Anderson et al. (2007) a la que se le han superpuesto los focos corticales (rojo) e intermedios (verde) así como las estaciones sismológicas en superficie (sin elevación topográfica). Arriba: vista en dirección SO-NE, abajo: vista ENE-OSO. Más explicaciones en el texto. 
existencia quedaba confirmada.

En la Fig. 3.21 puede compararse la distribución de hipocentros resultante con la geometría de la placa de Nazca construida a partir de la de los contornos de Wadati-Benioff cada $100 \mathrm{~km}$ dados por Anderson et al. (2007). En las dos vistas rotadas del mismo gráfico 3D se observa claramente la naturaleza intraplaca de los sismos intermedios y su mayor concentración en la elevación de la placa de Nazca asociada a la dorsal Juan Fernández. La carencia de una mayor resolución en las profundidades de los contornos de WB puede ser la causa de la localización de algunos focos justo por encima de la superficie. El resto de los eventos intermedios por encima de WB podrían ajustar mejor sus profundidades con un modelo 3D de velocidad.

En la Fig. 3.22 se presentan los perfiles de hipocentros en profundidad elaborados para intervalos de un grado de latitud con las localizaciones obtenidas en este trabajo para la sismicidad seleccionada. Las mayores concentraciones de eventos se obtienen entre $\operatorname{los} 30^{\circ} \mathrm{y}$ los $32^{\circ} \mathrm{S}$. A los $\left[31^{\circ}-32^{\circ} \mathrm{S}\right.$ ] aparece la pendiente de la placa subducida hacia el oeste tal como habían observado Anderson et al. (2007) usando datos, modelo y algoritmo diferentes. Las profundidades promedio de la zona frágil en la placa de Nazca resultan similares en ambos análisis con profundidades entre 100 y $120 \mathrm{~km}$ para el intervalo $\left[68^{\circ}-70^{\circ} \mathrm{O}\right]$ y $\left[30^{\circ}-32^{\circ} \mathrm{S}\right]$. La curvatura de la placa varía de norte a sur. A los $\left[30^{\circ}-31^{\circ} \mathrm{S}\right]$ la placa alcanza los $100 \mathrm{~km}$ de profundidad a $\operatorname{los} 70^{\circ} \mathrm{O}$ (Fig. 3.22d) y se mantiene estable a esa profundidad según la sismicidad registrada por Anderson et al. (2007). A los [31 $\left.{ }^{\circ}-32^{\circ} \mathrm{S}\right]$ (Fig. 3.22e) la placa desciende por debajo de los $100 \mathrm{~km}$ de profundidad a los $70^{\circ} \mathrm{O}$ para luego flexionar elevándose por encima de $\operatorname{los} 100 \mathrm{~km}$ a $\operatorname{los} 69^{\circ} \mathrm{O}$ antes de volver a descender a $\operatorname{los} 68^{\circ} \mathrm{O}$, donde aumenta la pendiente alcanzando los $140 \mathrm{~km}$ de profundidad a los $67^{\circ} \mathrm{O}$. Comparando la ubicación de los focos a profundidades intermedias bajo la Sierra de Chepes en las Figs. 3.15 y 3.20, se observa que la localización con el modelo propuesto define mejor la flexión de la placa corroborando la existencia del gap a profundidades intermedias al este de la Sierra. A los $32^{\circ} \mathrm{S}$, la placa desciende uniformemente hasta los $120 \mathrm{~km}$ de profundidad a los $70^{\circ} \mathrm{O}$ y en este caso (Fig. 3.22f) ya no se observa la pendiente hacia el oeste antes del descenso final que parece iniciarse hacia los $68^{\circ} \mathrm{O}$. 

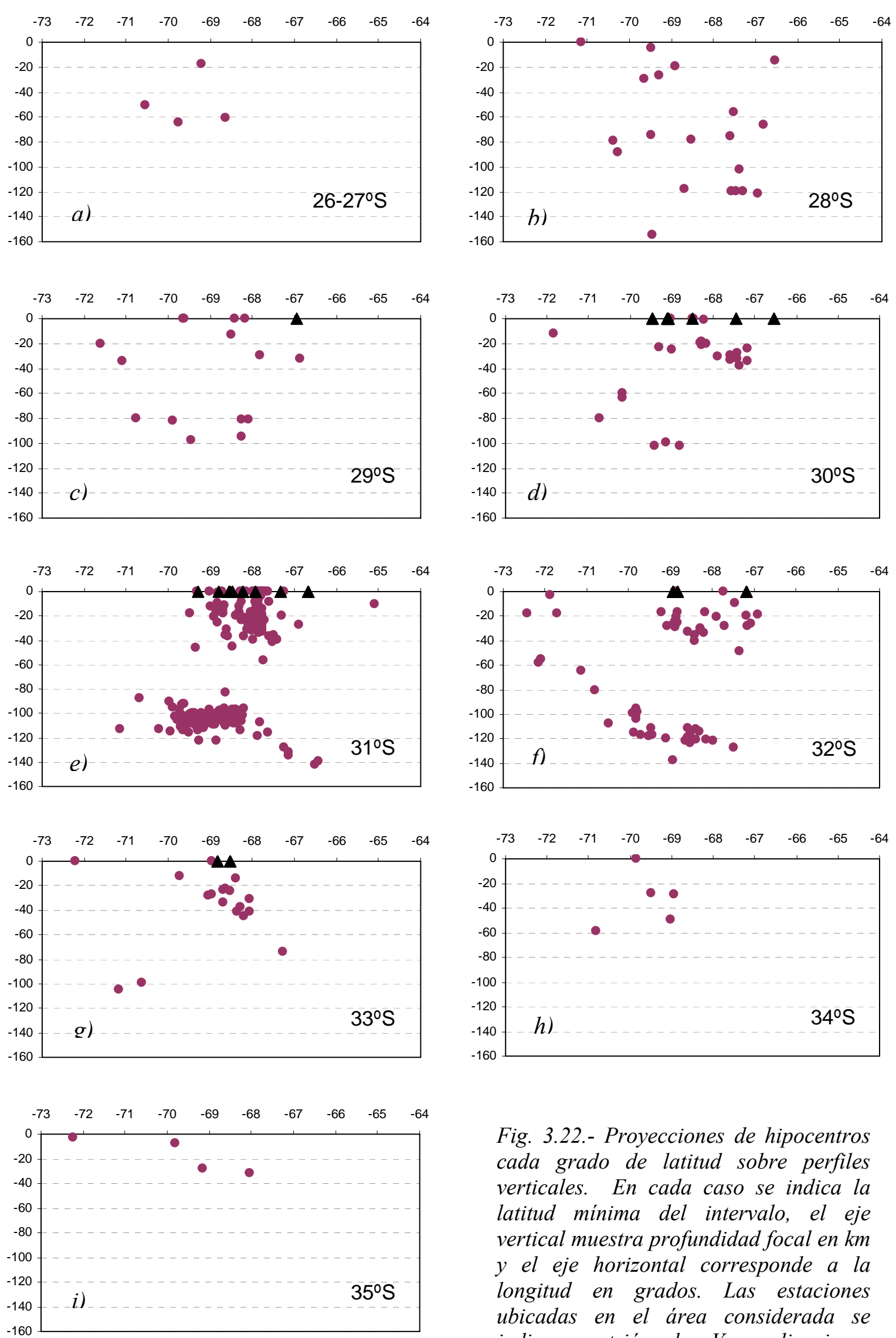

Fig. 3.22.- Proyecciones de hipocentros cada grado de latitud sobre perfiles verticales. En cada caso se indica la latitud mínima del intervalo, el eje vertical muestra profundidad focal en $\mathrm{km}$ $y$ el eje horizontal corresponde a la longitud en grados. Las estaciones ubicadas en el área considerada se indican con triángulos. Ver explicaciones en el texto. 

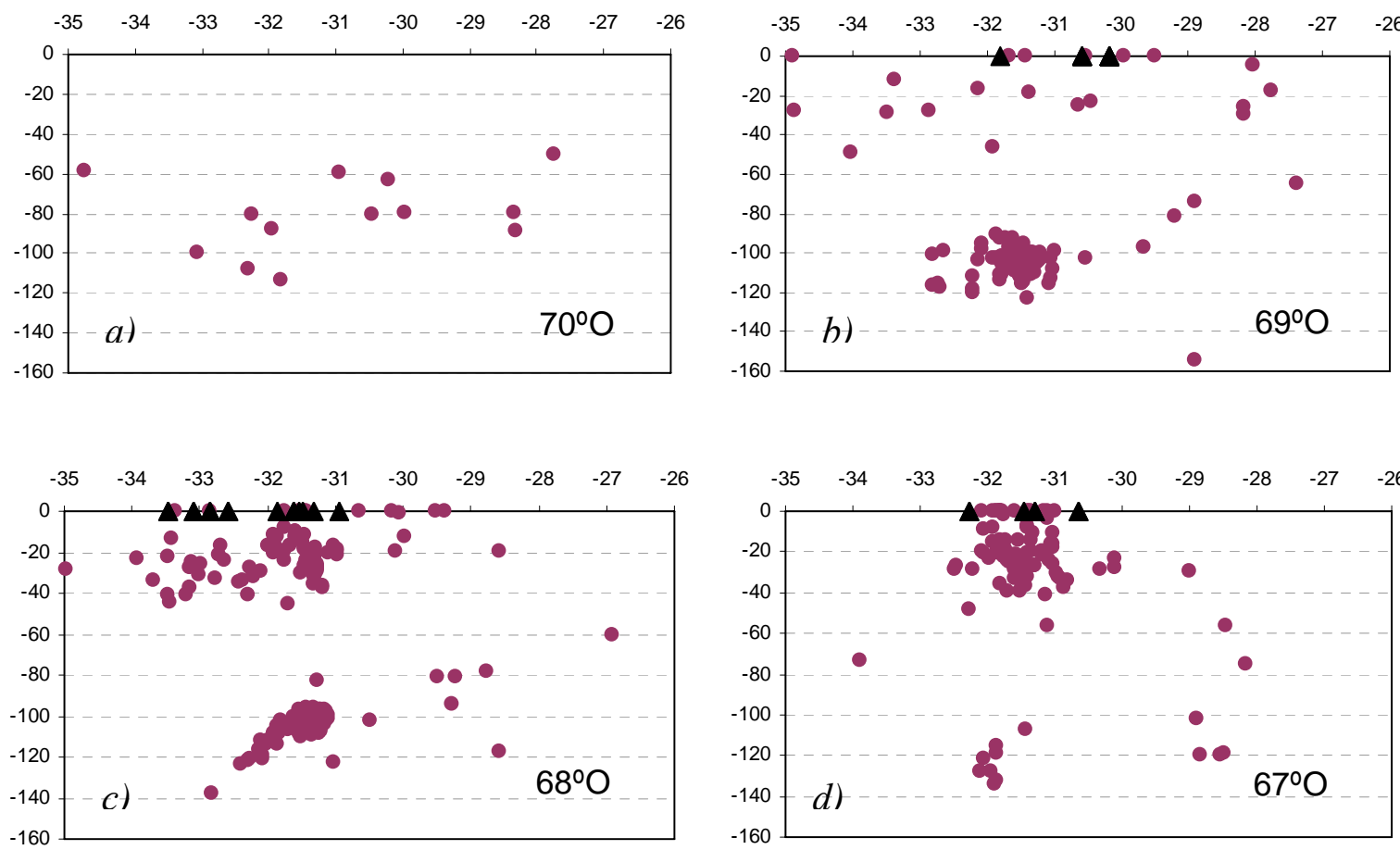

Fig. 3.23.- Proyecciones de hipocentros cada grado de longitud sobre perfiles verticales. En cada caso se indica la longitud mínima del intervalo, el eje vertical muestra profundidad focal en km y el eje horizontal corresponde a la latitud en grados. Las estaciones ubicadas en el área considerada se indican con triángulos. Ver explicaciones en el texto.

La Fig. 3.23 muestra las proyecciones de hipocentros sobre planos verticales para intervalos de un grado de longitud que permiten ver la profundización de la placa de Nazca variando con la latitud. Entre $\operatorname{los}\left[69^{\circ}-70^{\circ} \mathrm{O}\right]$ la selección de eventos no ha incluido focos intermedios al sur de los $33^{\circ} \mathrm{S}$. La sismicidad en el intervalo [30-33 $\left.{ }^{\circ} \mathrm{S}\right]$ se mantiene en una distribución plana horizontal con un techo a los $90 \mathrm{~km}$ de profundidad (Fig. 3.23b). En el intervalo $\left[68-69^{\circ} \mathrm{O}\right]$ se observa la flexión de la placa al sur de los $32^{\circ} \mathrm{S}$ (Fig. 3.23c) que a su vez parece ser correspondida con una mayor profundidad de la actividad sísmica cortical. A los $\left[67-68^{\circ} \mathrm{S}\right]$ la actividad sísmica intermedia disminuye notablemente y tiene profundidades que descienden levemente hacia el sur sin superar los $140 \mathrm{~km}$ de profundidad (Fig. 3.22d).

A fin de observar los efectos del nuevo modelo de velocidad en la localización, se analizan los cambios en la distribución de hipocentros en planta y perfil desde la base original (Fig. 3.15) hasta la localización final de este trabajo (Fig. 3.20). En general se observa (Fig. 3.24) que los focos intermedios disminuyen su profundidad concentrándose en una zona de Wadati-Benioff con profundidades cercanas a $100 \mathrm{~km}$. Se observa en promedio una tendencia general de los epicentros hacia la red y en particular, algunos eventos que habían sido descartados en la localización original, convergen en este proceso a una solución más real. 


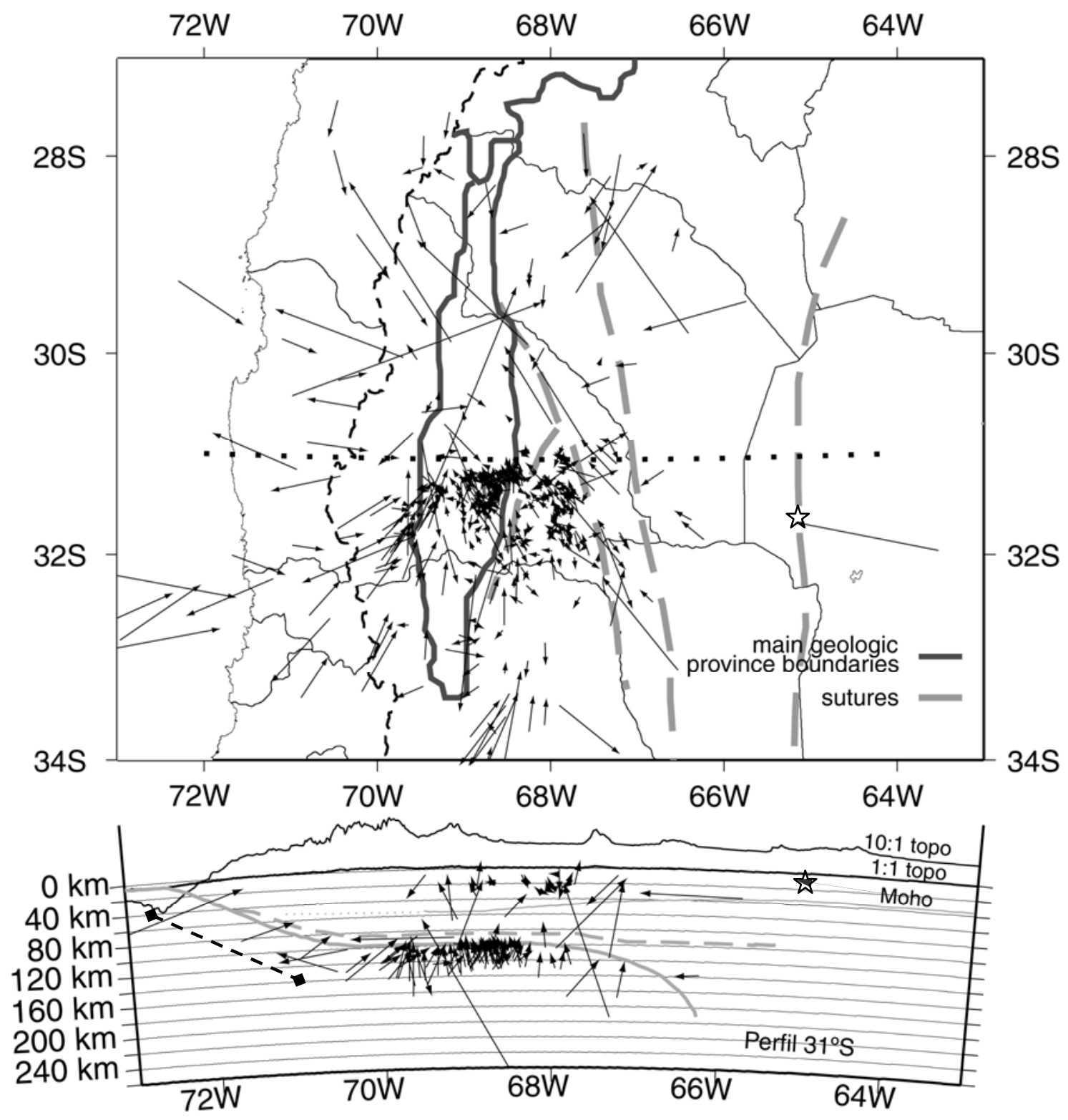

Fig. 3.24.- Arriba: desplazamiento de los epicentros nuevos respecto de los originales obtenidos con Hypo71 y el modelo de velocidades de Tabla 3.1. Abajo: Desplazamiento en longitud y profundidad para los mismos eventos. Las estrellas y la línea segmentada con rombos indican ejemplos citados en el texto.

El desplazamiento de la solución es mucho mayor para eventos alejados de la RSZNC y mínimo para eventos corticales bajo la red. Por una parte un alto porcentaje de trayectorias sísmicas atravesando la zona comprendida por la RSZNC permite obtener resultados más exactos para eventos en ese entorno y presupone menor exactitud para eventos alejados del mismo. Por otra parte, las nuevas localizaciones de epicentros, aún de aquellos a mayores distancias epicentrales, se vinculan más claramente a los rasgos tectónicos conocidos, dando crédito a la solución.

Como ejemplos de los resultados a grandes distancias de la red, pueden citarse: 1) el 
evento localizado bajo la Sierra Grande de Córdoba (señalado con una estrella en la Fig. 3.24), cuya nueva localización se ubica en la sutura Pampia-Cratón Río de La Plata a una profundidad de $10 \mathrm{~km}$ antes se ubicaba en medio de la provincia de Córdoba, al este de la Sierra Chica de Córdoba y a una profundidad de $25 \mathrm{~km}$; 2) los eventos intermedios y superficiales en territorio chileno (señalados en el perfil de la Fig. 3.24 por una línea segmentada con extremos de rombos) durante la relocalización migran sus posiciones redistribuyéndose a lo largo del contorno de la subducción de la placa de Nazca según Gutscher et al. (2000).

Los pequeños desplazamientos sufridos por los eventos corticales bajo el área central de RSZNC, permiten concluir que la metodología de localización sugerida originalmente por el INPRES da resultados muy precisos para eventos locales. Sin embargo el nuevo modelo permite aplicar una misma técnica a todos los eventos registrados por la red hasta distancias de al menos $500 \mathrm{~km}$, obteniéndose de manera menos subjetiva resultados más precisos y exactos.

La Fig. 3.25 muestra los desplazamientos de los hipocentros en planta y perfil desde la base inicial del recálculo hasta la localización final (Fig. 3.20). Los focos de eventos a profundidades intermedias se reacomodan distribuyéndose en la parte superior de la placa de Nazca con desplazamientos tanto hacia el este como hacia el oeste de sus posiciones iniciales. Bajo la Precordillera y las Sierras Pampeanas Occidentales se observan algunos desplazamientos de localización desde profundidades superficiales a intermedias y viceversa. Sin embargo la mayoría de los eventos corticales bajo Pie de Palo y Precordillera Oriental, como los de la placa de Nazca bajo Precordillera no cambian prácticamente su ubicación. Esta leves modificaciones explican la lenta convergencia del método cerca de la solución de RMS mínimo.

El desplazamiento promedio de los hipocentros durante la localización mediante JHD con modelo de velocidad fijo no muestra una dirección preferente ni una magnitud que pueda asociarse al emplazamiento de las estaciones de la RSZNC. Por lo tanto, el modelo propuesto resulta confiable para las rutinas de localización en la red y la nueva distribución de sismicidad de la base de datos seleccionada permite aplicar los métodos de determinación de la atenuación sísmica descriptos en el Capítulo II, efectuar regionalizaciones de los resultados e interpretarlos mediante su asociación a los procesos geodinámicos en el margen de subducción 


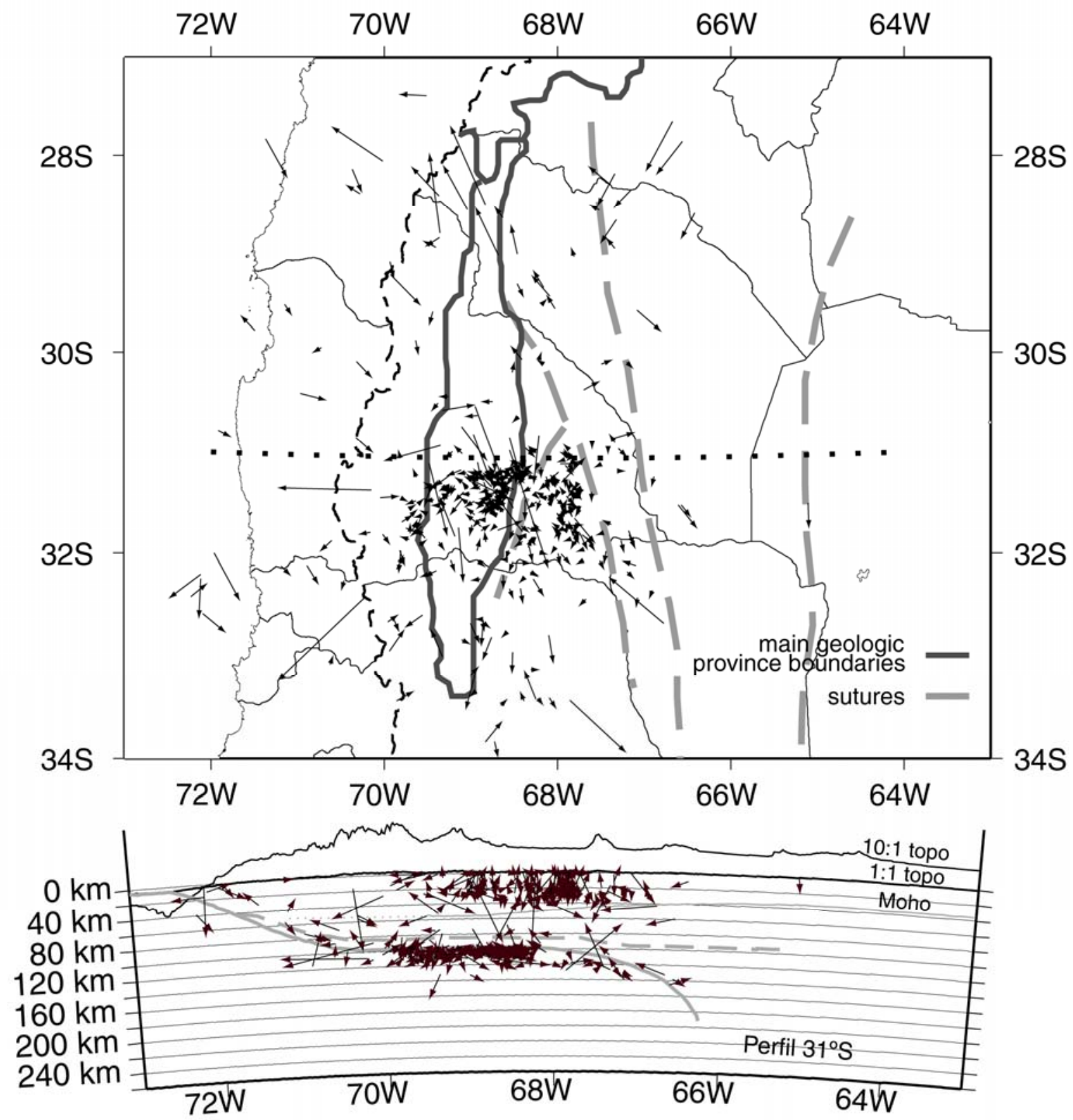

Fig. 3.25.- Arriba: desplazamiento de los epicentros nuevos respecto de los iniciales del recálculo. Abajo: Desplazamiento en longitud y profundidad para los mismos eventos. Más explicaciones en el texto. 


\section{Determinación de $Q$ en ondas coda, ondas $P$ y S: Aplicación, resultados e interpretación}

En este capítulo se presentan las estimaciones del factor de calidad para la Región de Nuevo Cuyo sobre los diferentes tipos de ondas registrados por la RSZNC, habiendo seleccionado aquellos métodos que mejor se adaptaran a los datos. En cada caso se plantean las consideraciones realizadas para la aplicación del método, los criterios utilizados y las herramientas de cálculo. Los resultados obtenidos se interpretan en planta y profundidad, comparando con los conocimientos actuales de la tectónica regional. Luego se separan los efectos de la absorción intrínseca y de scattering interpretándose su relación en función de los procesos geodinámicos que caracterizan a la región.

\subsection{ONDAS CODA}

\subsubsection{MÉTODOS DE BACK-SCATTERING SIMPLE (SBS) Y SCATTERING ISÓTROPO SIMPLE (SIS)}

\subsubsection{APLICACIÓN Y RESULTADOS}

El análisis de la atenuación de ondas coda se ha realizado simultáneamente por el método SBS (Aki y Chouet, 1975) y por el método SIS, incorporando al SBS la modificación propuesta por Sato (1977) en el caso en que la distancia fuente-receptor no fuese despreciable. Combinando las (2.28), (2.34) y (2.35), que aproximan la envolvente de la energía de la coda en función del tiempo y la frecuencia angular, puede expresarse la envolvente de la amplitud de la coda de un sismograma a una dada frecuencia (f) y lapso de tiempo ( $t$ ) medido desde el origen del evento

$$
A(f, t)=A_{0}(f) t^{-m} K(v)^{\frac{1}{2}} e^{-\frac{\pi f t}{Q_{c}}}
$$

donde $A_{0}(f)$ resume los efectos del patrón de radiación y el efecto de sitio; $t^{-m}$ representa la 
expansión geométrica de las ondas coda; $K(v)$ es el factor de corrección por expansión geométrica del SIS dado por la (2.35) en el que $v=v t / r=t / t_{s}$ con $t_{s}$, el tiempo de llegada de la onda $\mathrm{S} ; Q_{c}$ es el factor de calidad del medio que tiene en cuenta la atenuación total. En el caso del método SBS, el factor $K(v)$ se considera igual a la unidad.

El factor de divergencia geométrica, $m$, puede seleccionarse entre 1 y 0.5 según se considere a la coda como compuesta por ondas $\mathrm{S}$ dispersadas $\mathrm{u}$ ondas superficiales, respectivamente. Como ya se mencionara en el Cap. II, para un modelo cortical simplificado de dos capas, distancias inferiores a $500 \mathrm{~km}$ y focos en corteza superior, las ondas de corte en los sismogramas mostrarán fases $\mathrm{Sg}, \mathrm{S}^{*}$ y $\mathrm{Sn}$ en diferente orden según sea la distancia epicentral, mientras que para eventos en corteza inferior, no se espera hallar Sg (Kulhánek, 2002). Las ondas $\mathrm{S}$ de corto período pueden interferir en corteza y dar lugar a ondas superficiales, como las Rg, para el caso de focos con profundidades inferiores a $5 \mathrm{~km}$. Si bien se han observado ondas superficiales Lg a distancias apenas mayores que $100 \mathrm{~km}$ (Chun et al., 1987; De Miguel et al., 1992) lo habitual es que se produzcan a distancias de $5^{\circ}$ o más para trayectorias continentales (Kulhánek, 2002). En una corteza continental real, más compleja, pueden observarse un número mayor de comienzos de $\mathrm{P}$ y $\mathrm{S}$, pero en general la Sg muestra las mayores amplitudes y decae formando la coda. Aki (1980b) observó que la energía requerida para la generación de ondas coda mediante scattering simple coincidía con la atenuación de ondas $\mathrm{S}$, por lo que interpretó a la coda de terremotos locales como producto de la dispersión simple de S a S. Siguiendo a Aki (1980b), se ha asumido en este trabajo a las ondas coda como ondas $\mathrm{S}$ dispersadas tomando $m=1$.

Para el ajuste de los valores de $Q_{\mathrm{c}}$ a los sismogramas, se ha utilizado el programa QCODA, desarrollado por J. Ibáñez, con modificaciones realizadas para este trabajo (QCODA03) que incorporaron la entrada automática de las formas de onda (convertidas a formato ASCII de una columna) y de los parámetros de cálculo desde un fichero, grabando los resultados seleccionados en un archivo por estación. Los sismogramas han de tener codas no saturadas con más de $60 \mathrm{~s}$ de duración, buena relación señal ruido y debe contarse con la localización de hipocentros para la definición de los lapsos de tiempo.

Para cada estación, las trazas se han filtrado en cuatro bandas de frecuencia centradas en $f_{\mathrm{c}}=1.5,3,6$ y $12 \mathrm{~Hz}$ (a excepción de CFAZ y RTMC en las que la $f_{\mathrm{c}}$ máxima impuesta por las condiciones de registración ha sido $6 \mathrm{~Hz}$ ) y ancho de banda de una octava. Los filtros pasabanda aplicados son tipo Butterworth de orden cinco en el intervalo $\left[f_{\mathrm{c}}-f_{\mathrm{c}} / 2, f_{\mathrm{c}}+f_{\mathrm{c}} / 2\right]$. Las envolventes de la coda en cada banda de frecuencia, como las que se muestran en la Fig. 


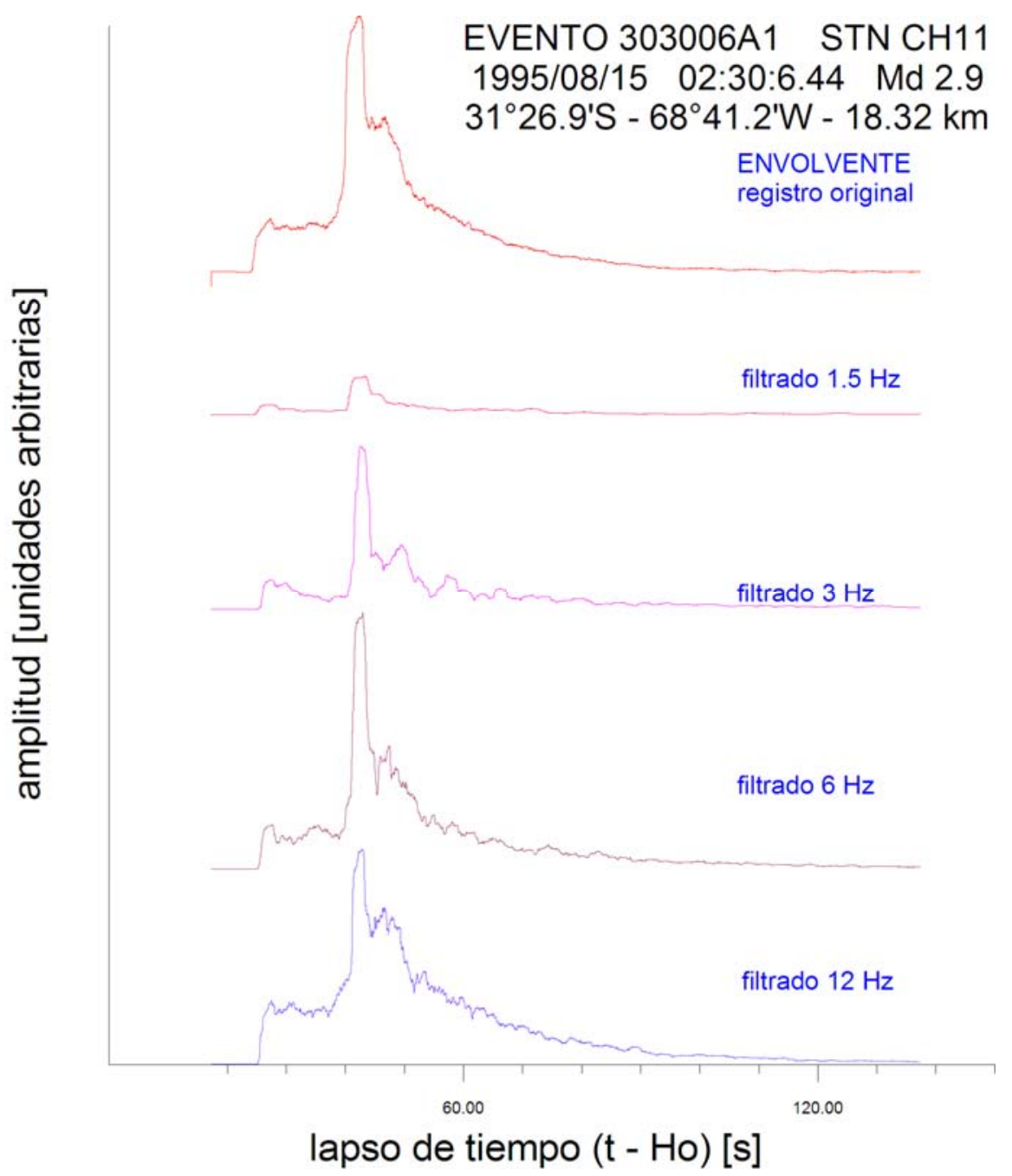

Fig. 4.1.- Ejemplo de envolventes calculadas sobre un registro de la estación Chepes. Arriba se observa la envolvente de la traza original y por debajo las envolventes de la señal filtrada en las frecuencias centrales elegidas.

4.1, han sido estimadas calculando la raíz cuadrada del valor cuadrático medio (RMC) de cada traza en el dominio temporal sobre unas ventanas móviles (Aki y Chouet, 1975) elegidas según la frecuencia $f_{\mathrm{c}}(3 \mathrm{~s}$ de duración para $1.5 \mathrm{~Hz}$ y $2 \mathrm{~s}$ para 3,6 y $12 \mathrm{~Hz})$ y con un solapamiento del $50 \%$.

Dado el amplio rango de distancias y magnitudes disponibles, la consideración $t_{\mathrm{c}}>2 t_{\mathrm{s}}$ establecida en el SBS no aseguraba una buena relación señal ruido en la coda de todos los registros. Siguiendo a Ibáñez et al. (1991) se ha definido su inicio en forma visual coincidiendo con el punto donde la envolvente corregida de divergencia geométrica comienza a decaer de forma regular (Fig. 4.2). El final de la coda suele establecerse a partir de la relación señal ruido, pero ésta puede variar de una estación a otra, por lo que se ha definido el final de la coda en el punto donde la envolvente de la coda corregida de divergencia geométrica comienza a crecer luego de haber alcanzado un mínimo (Ibáñez et al., 1991). A fin de estudiar la variación de $Q$ con el lapso de tiempo, se han calculado los ajustes en cada 


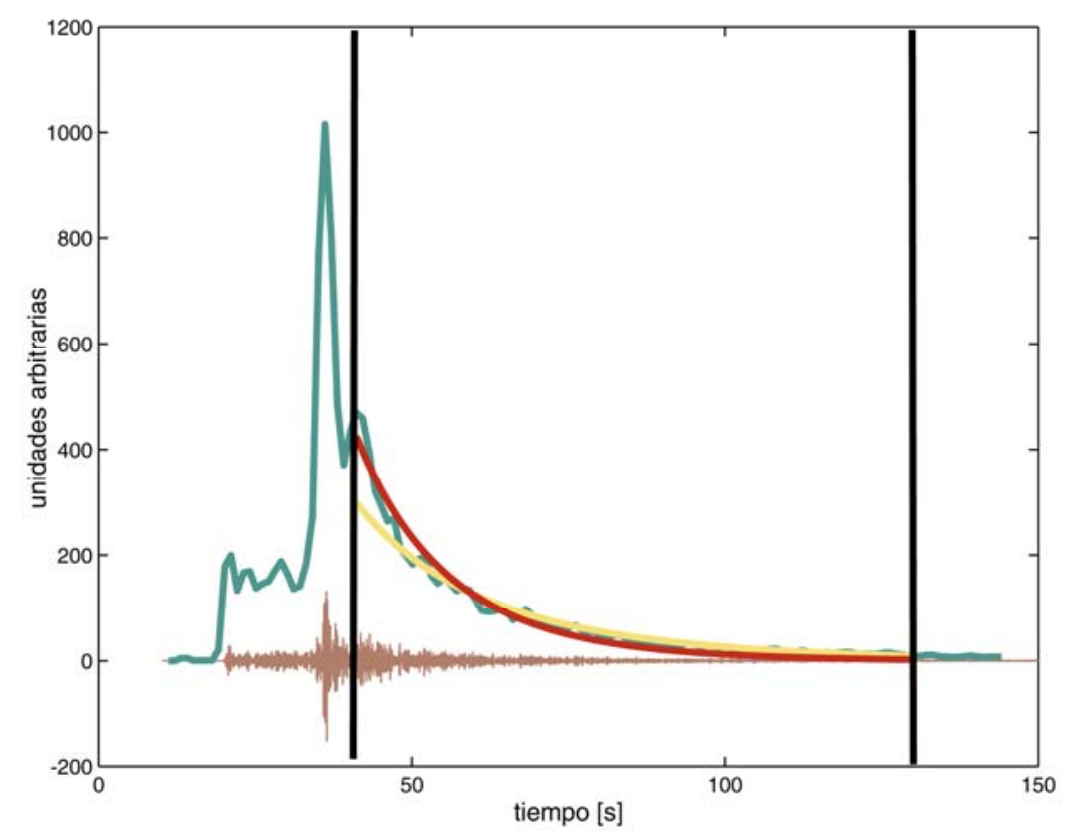

Fig. 4.2.- Ejemplo de registro filtrado a $6 \mathrm{~Hz}$, su envolvente $R M C$ (en verde) y las definiciones de inicio y fin de coda (en negro), según el criterio de Ibáñez et al (1991). En amarillo se muestra el ajuste lineal y en rojo, el ajuste no lineal.

envolvente para diferentes longitudes de coda, todas múltiplos de $10 \mathrm{~s}$. La máxima longitud analizada se extiende desde el inicio hasta el fin de la coda, definidos estos visualmente pero dentro de los límites objetivos (Ibáñez et al., 1991) y la mínima longitud es de $20 \mathrm{~s}$.

Para cada frecuencia y longitud de coda, el ajuste del decaimiento exponencial a la envolvente en el dominio del tiempo (Fig. 4.2), se puede realizar por transformación logarítmica de la (4.1) o por un ajuste no lineal (Gauss-Newton). Este último garantiza los requisitos del método de ajuste por mínimos cuadrados para la obtención del $Q$ (Ibáñez et al., 1993) supuesta una distribución gaussiana de errores en los datos. De este modo se han obtenido 60605 valores de $Q$ (para todas las frecuencias y lapsos de tiempo analizados) tanto con el ajuste lineal como con el no lineal.

En cada ajuste individual se ha observado que el método no lineal estima mejor el inicio de la ventana mientras que el método lineal lo hace sobre el final de la ventana. Esto ya había sido comprobado sobre datos sintéticos y reales por Ibáñez et al. (1990). Al comparar los resultados de los ajustes ha podido observarse que un $86 \%$ de los valores de $Q$ calculados con el método lineal son mayores que los correspondientes del método no lineal. (Fig. 4.2).

En un $99 \%$ de las soluciones, se ha logrado buena convergencia para ambos métodos, aunque los coeficientes de correlación del ajuste no lineal son en general, superiores al del método lineal. La Fig. 4.3 muestra las distribuciones de coeficientes de correlación en 

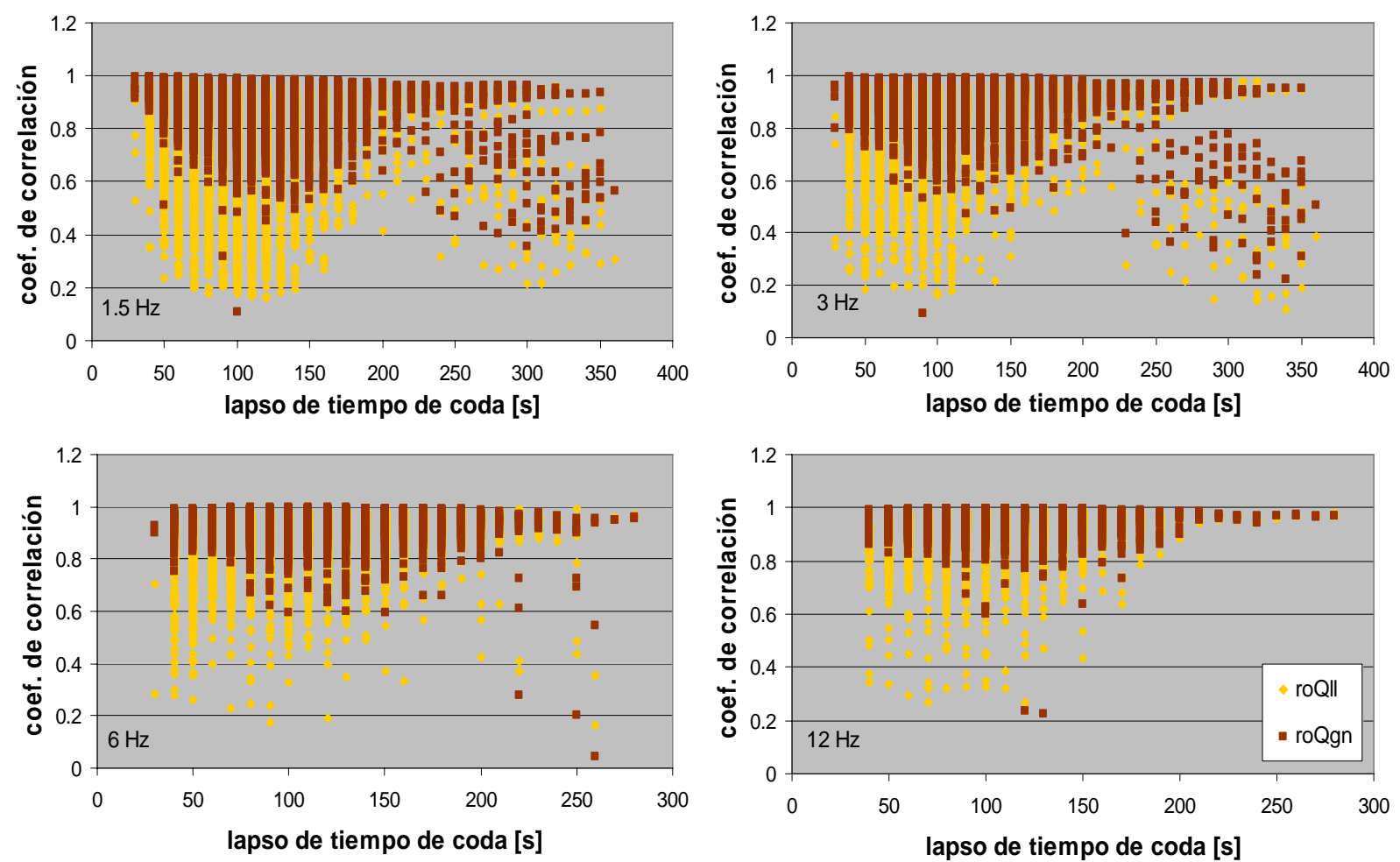

Fig. 4.3.- Coeficientes de correlación de los ajustes lineal (amarillo) y no lineal (rojo) de la ecuación 4.1 en función del lapso de tiempo de coda para las diferentes bandas de frecuencia consideradas. En cada caso se consideró todo el rango de profundidades focales disponibles.

función del lapso de tiempo para las frecuencias analizadas. Analizando los ajustes con coeficientes de correlación mayores que 0.7 en ambos métodos, se observa que los errores del método no lineal son menores que los del método lineal en el $61 \%$ de los casos, razón por la cual de aquí en más, los resultados de $Q_{\mathrm{c}}$ analizados son los del ajuste Gauss-Newton.

Observando el comportamiento de los valores de $Q_{\mathrm{c}}$ individuales obtenidos para toda la región, en función del lapso de tiempo y la profundidad, con la frecuencia como parámetro, se observa que $Q_{\mathrm{c}}$ crece con el lapso de tiempo para todas las frecuencias en ambos intervalos de profundidad, pero no muestra una clara dependencia con esta última.

La (2.21) puede aplicarse a la atenuación de ondas coda en la forma $Q_{c}=Q_{o}\left(f / f_{0}\right)^{n}$ permitiendo hallar valores de $Q_{o}$ y $n$ regionales, pero con el lapso de tiempo como parámetro. Para ellos, en primer lugar se han suavizado los valores de $Q_{c}$ con coeficientes de correlación superiores a 0.9 mediante un método de regresión polinómico ponderado (LOESS robusto) que asigna pesos locales a los datos con un ajuste por mínimos cuadrados lineal utilizando un polinomio de segundo grado y es resistente a los valores extremos. Seguidamente se ha ajustado la relación $Q(f)$ a los valores suavizados usando un método de mínimos cuadrados no lineal y robusto mediante pesos bicuadrados (Fig. 4.4). En este ajuste, los pesos se reasignan en las sucesivas iteraciones de acuerdo a los residuos obtenidos en cada una de ellas 

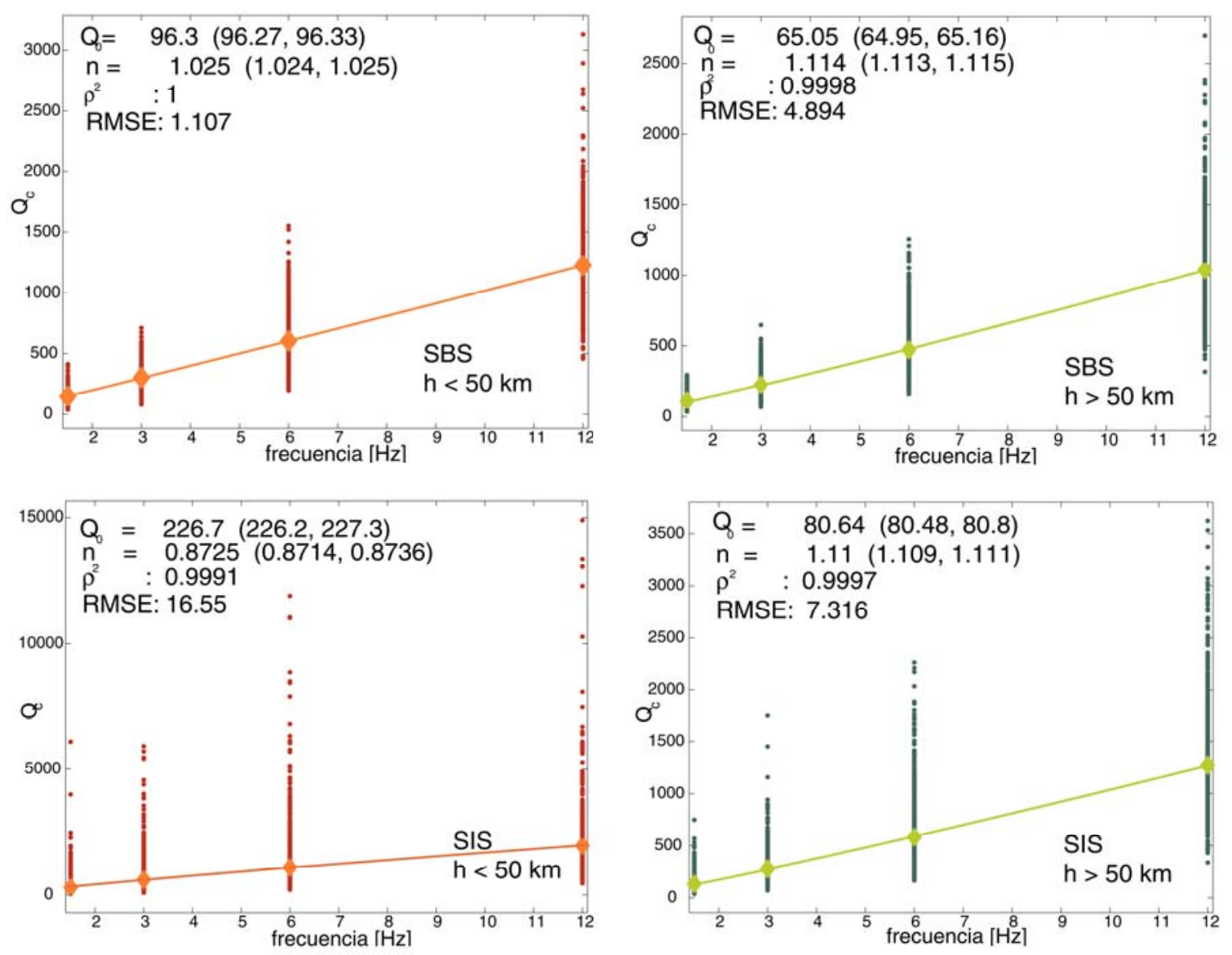

Fig. 4.4.- Distribución de $Q_{c}$ con la frecuencia a 90s de lapso de tiempo, para profundidades focales menores que $50 \mathrm{~km}$ (rojo) y mayores que $50 \mathrm{~km}$ (verde) según los métodos de back scattering simple (SBS) y scattering isótropo simple (SIS). Los rombos indican valores suavizados y las curvas corresponden al ajuste de la relación $Q(f)$ (ver texto). Se indican los coeficientes obtenidos en el ajuste con sus intervalos de confianza y bondad ( $\rho$ : correlación y RMSE: error medio cuadrático). No se incluyen los errores de $Q_{c}$.

asignando menor peso a los datos cuanto mayor sea su residuo $\left(\omega_{i}=\left(1-u_{i}^{2}\right)^{2}\right.$ para $\left|u_{i}\right|<1$ y $\omega_{i}=0$ para $\left|u_{i}\right| \geq 1$, donde $\omega_{i}$ son los pesos y $u_{i}$ son los residuos ajustados y estandarizados). En la Tabla 4.1 se muestran los valores medios regionales de $Q_{o}$ y $n$ obtenidos por el ajuste junto a sus errores admitidos, en los que se ha tenido en cuenta la propagación de errores en la determinación individual de cada $Q_{c}$. Se presentan también los coeficientes de correlación de los ajustes y su error medio cuadrático.

\begin{tabular}{|c|c|c|c|c|c|c|c|c|}
\hline Lapso & $\mathbf{Q}_{\mathbf{o}} \pm \mathbf{d} \mathbf{Q}_{\mathbf{o}}$ (SBS) & $\mathbf{n} \pm \mathbf{d n}(\mathbf{S B S})$ & $\mathbf{\rho}_{\mathbf{o}}{ }^{2}$ (SBS) & $\mathbf{R M S E ( S B S )}$ & $\mathbf{Q}_{\mathbf{o}} \pm \mathbf{d} \mathbf{Q}_{\mathbf{0}}(\mathbf{S I S})$ & $\mathbf{n} \pm \mathbf{d n}(\mathbf{S I S})$ & $\mathbf{\rho}_{\mathbf{o}}{ }^{2}$ (SIS) & $\mathbf{R M S E ( S I S )}$ \\
\hline 30 & $38 \pm 6$ & $0.71 \pm 0.1$ & 0.9700 & 5.12 & $104 \pm 5$ & $0.36 \pm 0.07$ & 0.9958 & 1.92 \\
\hline 40 & $40 \pm 5$ & $1.27 \pm 0.01$ & 0.9921 & 20.09 & $84 \pm 5$ & $1.31 \pm 0.03$ & 0.9674 & 96.00 \\
\hline 50 & $53 \pm 7$ & $1.26 \pm 0.01$ & 0.9998 & 5.75 & $80 \pm 7$ & $1.65 \pm 0.02$ & 0.9981 & 68.10 \\
\hline
\end{tabular}

Tabla 4.1.- (Página siguiente) $Q_{o}$ y n promedios regionales con errores, coeficiente de determinación $\left(\rho_{o}^{2}\right)$ y error medio cuadrático (RMSE) para eventos superficiales (tabla superior) e intermedios (tabla inferior). 


\begin{tabular}{|c|c|c|c|c|c|c|c|c|}
\hline 60 & $66 \pm 9$ & $1.16 \pm 0.02$ & 0.9068 & 135.50 & $173 \pm 9$ & $1.23 \pm 0.01$ & 1.0000 & 3.27 \\
\hline 70 & $80 \pm 11$ & $1.1 \pm 0.02$ & 1.0000 & 2.64 & $212 \pm 11$ & $0.96 \pm 0$ & 0.9980 & 31.97 \\
\hline 80 & $91 \pm 12$ & $1.04 \pm 0.02$ & 1.0000 & 0.50 & $256 \pm 12$ & $0.82 \pm 0$ & 0.9997 & 9.52 \\
\hline 90 & $96 \pm 12$ & $1.03 \pm 0.03$ & 1.0000 & 1.11 & $227 \pm 13$ & $0.87 \pm 0.01$ & 0.9991 & 16.55 \\
\hline 100 & $104 \pm 13$ & $1 \pm 0.03$ & 0.9999 & 4.10 & $255 \pm 13$ & $0.82 \pm 0.01$ & 0.9974 & 27.83 \\
\hline 110 & $113 \pm 13$ & $0.97 \pm 0.03$ & 0.9925 & 30.47 & $233 \pm 14$ & $0.84 \pm 0.01$ & 0.9995 & 11.16 \\
\hline 120 & $114 \pm 13$ & $0.97 \pm 0.03$ & 0.9959 & 21.84 & $229 \pm 14$ & $0.81 \pm 0.01$ & 0.9984 & 17.56 \\
\hline 130 & $117 \pm 13$ & $0.93 \pm 0.03$ & 0.8638 & 135.30 & $236 \pm 14$ & $0.78 \pm 0.01$ & 0.9961 & 24.73 \\
\hline 140 & $127 \pm 12$ & $0.9 \pm 0.02$ & 0.9971 & 15.41 & $224 \pm 13$ & $0.75 \pm 0.01$ & 0.9973 & 17.73 \\
\hline 150 & $130 \pm 11$ & $0.87 \pm 0.03$ & 0.8235 & 117.10 & $231 \pm 11$ & $0.72 \pm 0$ & 0.9908 & 24.60 \\
\hline 160 & $138 \pm 11$ & $0.86 \pm 0.01$ & 0.9994 & 6.22 & $245 \pm 11$ & $0.68 \pm 0.01$ & 0.9980 & 11.65 \\
\hline 170 & $136 \pm 11$ & $0.89 \pm 0.01$ & 0.9976 & 13.70 & $207 \pm 11$ & $0.76 \pm 0$ & 0.9997 & 4.85 \\
\hline 180 & $130 \pm 11$ & $0.91 \pm 0.01$ & 0.9992 & 7.73 & $163 \pm 11$ & $0.84 \pm 0.01$ & 0.9996 & 5.15 \\
\hline 190 & $118 \pm 10$ & $0.94 \pm 0.01$ & 0.9925 & 25.61 & $135 \pm 10$ & $0.93 \pm 0.01$ & 0.9951 & 21.59 \\
\hline 200 & $112 \pm 10$ & $1.01 \pm 0.02$ & 0.9994 & 7.96 & $130 \pm 10$ & $0.97 \pm 0.01$ & 0.9995 & 7.94 \\
\hline 210 & $127 \pm 21$ & $0.94 \pm 0.08$ & 0.9265 & 96.94 & $138 \pm 16$ & $0.94 \pm 0.05$ & 0.9608 & 76.51 \\
\hline 220 & $122 \pm 12$ & $0.98 \pm 0.02$ & 0.9998 & 1.00 & $141 \pm 12$ & $0.97 \pm 0.02$ & 0.9999 & 5.39 \\
\hline 230 & $119 \pm 10$ & $0.9 \pm 0.02$ & 0.9985 & 12.25 & $123 \pm 10$ & $0.93 \pm 0.04$ & 0.9945 & 25.67 \\
\hline 240 & $129 \pm 18$ & $0.89 \pm 0.06$ & 0.9981 & 18.75 & $160 \pm 118$ & $0.82 \pm 0.32$ & 0.9449 & 114.30 \\
\hline
\end{tabular}

\begin{tabular}{|c|c|c|c|c|c|c|c|c|}
\hline Lapso & $\mathrm{Q}_{0} \pm d Q_{0}(\mathrm{SBS})$ & $\mathrm{n} \pm \mathrm{dn}(\mathrm{SBS})$ & $\rho_{0}^{2}(\mathrm{SBS})$ & RMSE(SBS) & $\mathrm{Q}_{0} \pm d Q_{0}$ (SIS) & $\mathrm{n} \pm \mathrm{dn}$ (SIS) & $\rho_{0}^{2}$ (SIS) & RMSE(SIS) \\
\hline 40 & $37 \pm 15$ & $1.5 \pm 0.25$ & 0.9981 & 10.88 & $34 \pm 28$ & $1.86 \pm 0.48$ & 0.9963 & 29.02 \\
\hline 50 & $35 \pm 5$ & $1.01 \pm 0.02$ & 0.9950 & 10.37 & $40 \pm 5$ & $0.99 \pm 0.03$ & 0.9927 & 13.42 \\
\hline 60 & $32 \pm 5$ & $1.28 \pm 0$ & 0.9983 & 10.55 & $35 \pm 5$ & $1.3 \pm 0.03$ & 0.9983 & 12.20 \\
\hline 70 & $42 \pm 6$ & $1.23 \pm 0$ & 0.9993 & 7.79 & $46 \pm 6$ & $1.26 \pm 0.03$ & 0.9992 & 9.99 \\
\hline 80 & $53 \pm 7$ & $1.17 \pm 0$ & 0.9997 & 5.52 & $61 \pm 7$ & $1.19 \pm 0.02$ & 0.9996 & 7.89 \\
\hline 90 & $65 \pm 8$ & $1.11 \pm 0$ & 0.9998 & 4.89 & $81 \pm 8$ & $1.11 \pm 0.02$ & 0.9997 & 7.32 \\
\hline 100 & $75 \pm 9$ & $1.07 \pm 0$ & 0.9993 & 9.25 & $97 \pm 9$ & $1.06 \pm 0.02$ & 0.9987 & 16.00 \\
\hline 110 & $88 \pm 10$ & $1.03 \pm 0$ & 0.9991 & 10.96 & $117 \pm 10$ & $1 \pm 0.02$ & 0.9979 & 20.42 \\
\hline 120 & $102 \pm 12$ & $0.98 \pm 0$ & 0.9980 & 16.37 & $157 \pm 12$ & $0.89 \pm 0.02$ & 0.9930 & 36.08 \\
\hline 130 & $127 \pm 15$ & $0.9 \pm 0$ & 0.9961 & 23.24 & $172 \pm 15$ & $0.85 \pm 0.02$ & 0.9872 & 48.19 \\
\hline 140 & $129 \pm 16$ & $0.89 \pm 0$ & 0.9970 & 20.00 & $177 \pm 17$ & $0.82 \pm 0.02$ & 0.9699 & 69.78 \\
\hline 150 & $138 \pm 15$ & $0.87 \pm 0.01$ & 0.9953 & 25.41 & $165 \pm 16$ & $0.84 \pm 0.02$ & 0.9923 & 35.23 \\
\hline 160 & $144 \pm 16$ & $0.85 \pm 0$ & 1.0000 & 1.36 & $173 \pm 16$ & $0.81 \pm 0.02$ & 0.9985 & 14.62 \\
\hline 170 & $147 \pm 16$ & $0.86 \pm 0$ & 0.9997 & 6.74 & $173 \pm 16$ & $0.82 \pm 0.02$ & 0.9988 & 13.23 \\
\hline 180 & $156 \pm 16$ & $0.87 \pm 0.02$ & 0.9938 & 30.71 & $191 \pm 17$ & $0.81 \pm 0.02$ & 0.9913 & 37.09 \\
\hline 190 & $192 \pm 20$ & $0.82 \pm 0.04$ & 0.9850 & 50.39 & $243 \pm 24$ & $0.75 \pm 0.05$ & 0.9730 & 69.65 \\
\hline 200 & $197 \pm 21$ & $0.82 \pm 0.05$ & 0.9811 & 53.58 & $237 \pm 27$ & $0.78 \pm 0.05$ & 0.9756 & 64.37 \\
\hline 210 & $198 \pm 14$ & $0.83 \pm 0.01$ & 0.9995 & 6.38 & $260 \pm 14$ & $0.79 \pm 0.03$ & 0.9969 & 18.04 \\
\hline 220 & $175 \pm 14$ & $0.94 \pm 0.03$ & 0.9969 & 17.03 & $227 \pm 14$ & $0.9 \pm 0.02$ & 0.9989 & 12.02 \\
\hline 230 & $194 \pm 50$ & $0.61 \pm 0.13$ & 0.8066 & 94.65 & $216 \pm 18$ & $0.62 \pm 0.04$ & 0.9766 & 35.02 \\
\hline 240 & $199 \pm 37$ & $0.71 \pm 0.09$ & 0.9503 & 63.20 & $249 \pm 16$ & $0.64 \pm 0.01$ & 0.9994 & 7.31 \\
\hline 250 & $214 \pm 88$ & $0.69 \pm 0.19$ & 0.7871 & 152.50 & $266 \pm 33$ & $0.68 \pm 0.06$ & 0.9778 & 56.03 \\
\hline 260 & $196 \pm 15$ & $0.74 \pm 0$ & 0.9999 & 3.13 & $226 \pm 15$ & $0.75 \pm 0.01$ & 0.9997 & 6.54 \\
\hline 270 & $189 \pm 14$ & $0.79 \pm 0.01$ & 0.9997 & 5.95 & $229 \pm 14$ & $0.78 \pm 0.03$ & 0.9972 & 23.92 \\
\hline 280 & $200 \pm 15$ & $0.76 \pm 0.02$ & 0.9983 & 14.16 & $242 \pm 15$ & $0.76 \pm 0.01$ & 0.9992 & 11.06 \\
\hline 290 & $209 \pm 15$ & $0.72 \pm 0$ & 1.0000 & 0.00 & $263 \pm 15$ & $0.62 \pm 0$ & 1.0000 & 0.00 \\
\hline 300 & $204 \pm 14$ & $0.76 \pm 0$ & 1.0000 & 0.00 & $256 \pm 14$ & $0.67 \pm 0$ & 1.0000 & 0.00 \\
\hline
\end{tabular}

En la Fig. 4.5 pueden compararse las curvas de variación de los valores medios regionales de $Q_{o}$ y $n$ obtenidos en función del lapso de tiempo para eventos con focos a 
profundidades corticales y en la placa de Nazca. El factor de calidad regional crece con el lapso de tiempo analizado tal como se había observado en los valores individuales. Los valores del factor de calidad resultantes del SIS son siempre mayores que aquellos obtenidos por el SBS ya que el modelo de Sato, al considerar la distribución de energía en el frente de onda, atribuye un mayor efecto a la expansión geométrica. Con datos de eventos intermedios, ambos métodos dan valores de $Q_{0}$ con comportamientos crecientes con el lapso de tiempo de manera similar. La convergencia entre ambos métodos para lapsos de tiempo cortos, en incidencia casi vertical, parece indicar que las variaciones de la atenuación debidas a la trayectoria horizontal recorrida son más importantes que las debidas a la profundidad focal.

Para eventos superficiales, los valores de $Q_{\mathrm{c}}$ del método SIS se apartan notablemente de los correspondientes al método SBS para lapsos de tiempo por debajo de los 190s. De acuerdo a estudios comparativos entre ambos métodos, se esperaba hallar diferencias en el ajuste de la primera porción de la coda (Rautian y Khalturin, 1978; Herraiz y Espinosa, 1986; Novelo-Casanova y Lee,. 1991 e Ibáñez et al., 1991) que es más sensible a la asimetría del patrón de radiación y del scattering que la parte final. Sin embargo, a lapsos de tiempo entre 60 y $190 \mathrm{~s}$, la disparidad de resultados puede deberse a la disponibilidad de ese lapso de tiempo en un amplio rango de distancias hipocentrales. Para un dado lapso de tiempo, el efecto de la expansión geométrica corregida según el factor de Sato (Ec. 2.30) será mayor cuanto mayor sea la distancia fuente-receptor, aumentando más el valor del factor de calidad

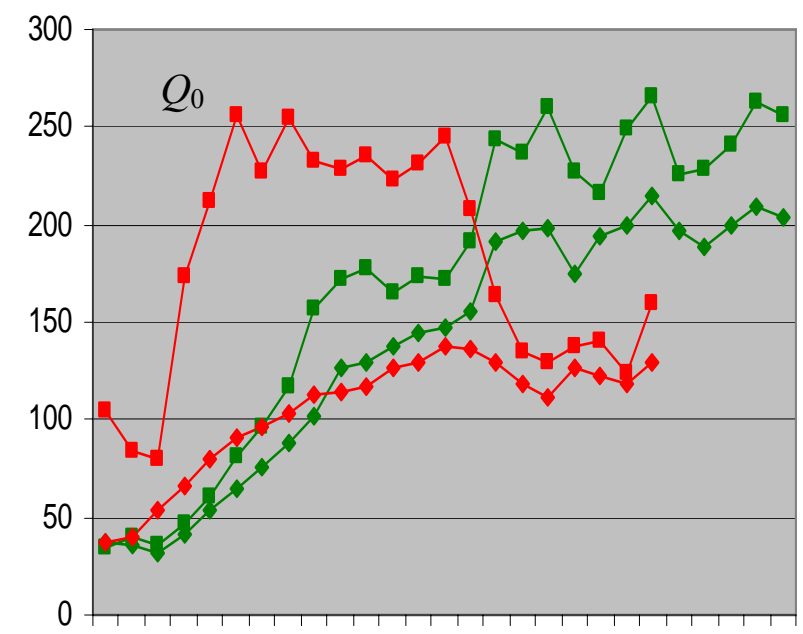

$3^{\circ} 6^{\circ} 9^{\circ} 2^{\circ} 2^{\circ} 2^{\circ} 2^{0}$ lapso de tiempo [s]

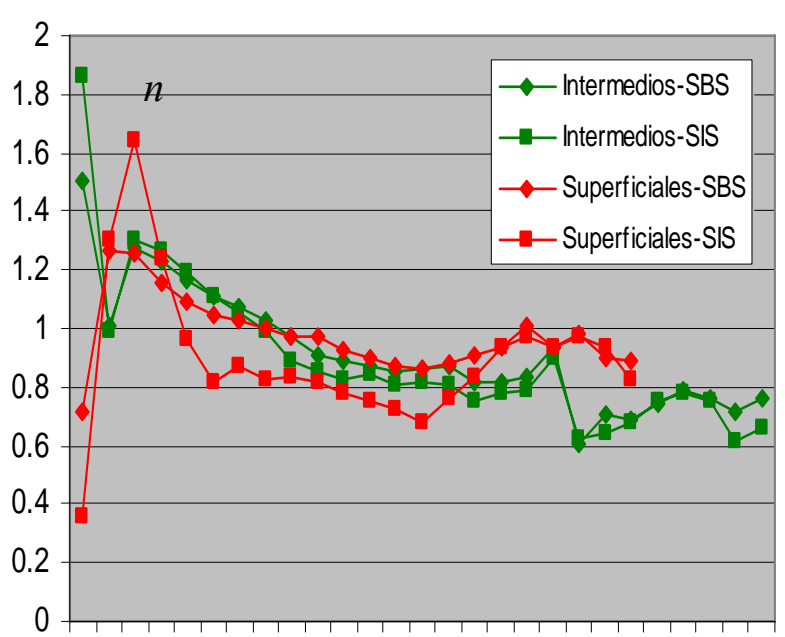

$3^{\circ} 6^{0} \quad 2^{\circ} 2^{0} \geq^{\circ} 2^{0} 2^{0}$

lapso de tiempo [s]

Fig. 4.5.- Valores medios regionales de $Q_{0}$ (izquierda) y $n$ (derecha) para eventos superficiales (rojo) e intermedios (verde) obtenidos del ajuste por mínimos cuadrados robusto en función de la frecuencia sobre todos los ajustes individuales de Qc por lapso de tiempo. Rombos indican resultados del método SBS y cuadrados corresponden al método SIS. 
ajustado en esos casos respecto de aquel obtenido a distancias menores. Por lo tanto, puede esperarse un apartamiento notable entre el método SBS y SIS si no se consideran longitudes de coda similares o rangos de distancia acotados. Por otra parte, los valores de la potencia $n$ se mantienen muy similares entre ambos métodos y a lo largo de toda la coda, mostrando un comportamiento levemente decreciente con el lapso de tiempo analizado conforme aumenta $Q_{0}$. La mayor diferencia se observa para lapsos de tiempo cortos, lo cual podría estar indicando una subestimación del $Q_{0}$ a profundidades intermedias pero sobre todo una sobrestimación a profundidades corticales, de acuerdo al cambio brusco de pendiente de las curvas de $n$.

\subsubsection{INTERPRETACIÓN}

Con el fin de detectar la existencia de variaciones en la atenuación dentro de la región, se han analizado los valores individuales de $Q_{\mathrm{c}}(f)$, para el método SIS, en función del backazimut para cada estación en los dos rangos de profundidades focales.

A profundidades intermedias (Fig. 4.6), las estaciones RTCB, RTLL, RTLS y RTPR muestran valores mayores de $Q_{\mathrm{c}} \mathrm{y}$ una mayor dispersión en función de la frecuencia para las trayectorias de dirección aproximadamente paralelas a los mayores lineamientos si se los compara con los valores de $Q_{\mathrm{c}}$ en las trayectorias transversales a los fallamientos principales o, lo que es lo mismo, paralelas a la dirección de convergencia de las placas tectónicas.

En el caso de la sismicidad superficial (Fig. 4.7), sólo las estaciones CFAZ, CA21 e ICAZ muestran alguna dependencia azimutal en los valores de $Q_{\mathrm{c}}$ para distintas frecuencias.

La dispersión de los valores de $Q_{\mathrm{c}}$ con la frecuencia es mayor para las trayectorias de eventos superficiales que para las trayectorias de eventos intermedios. En la teoría del scattering esto indicaría que cerca de la superficie la razón entre las densidades de heterogeneidades de grandes y pequeñas dimensiones es mayor que a profundidades intermedias. 


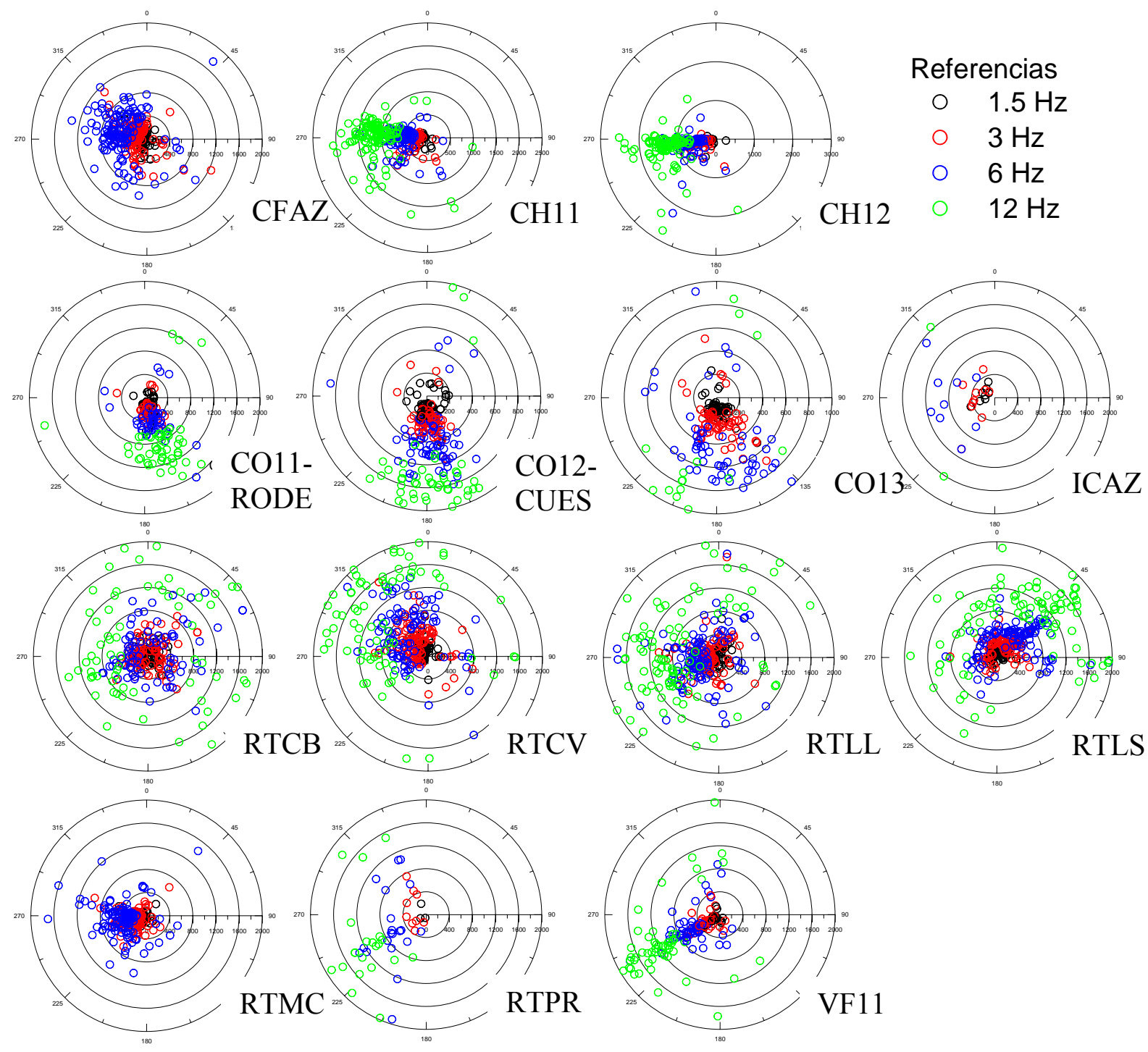

Fig.4.6.- Distribución de valores de $Q_{c}$ obtenidos correspondientes a $100 \mathrm{~s}$ de lapso de tiempo para eventos con profundidades mayores que $50 \mathrm{~km}$, por estación, en función del Backazimut y según la frecuencia central del filtro aplicado (circulos negros representan $Q_{c}$ a $1.5 \mathrm{~Hz}$; rojos, a $3 \mathrm{~Hz}$; azules, a $6 \mathrm{~Hz}$ y verdes, a $12 \mathrm{~Hz}$ ). 


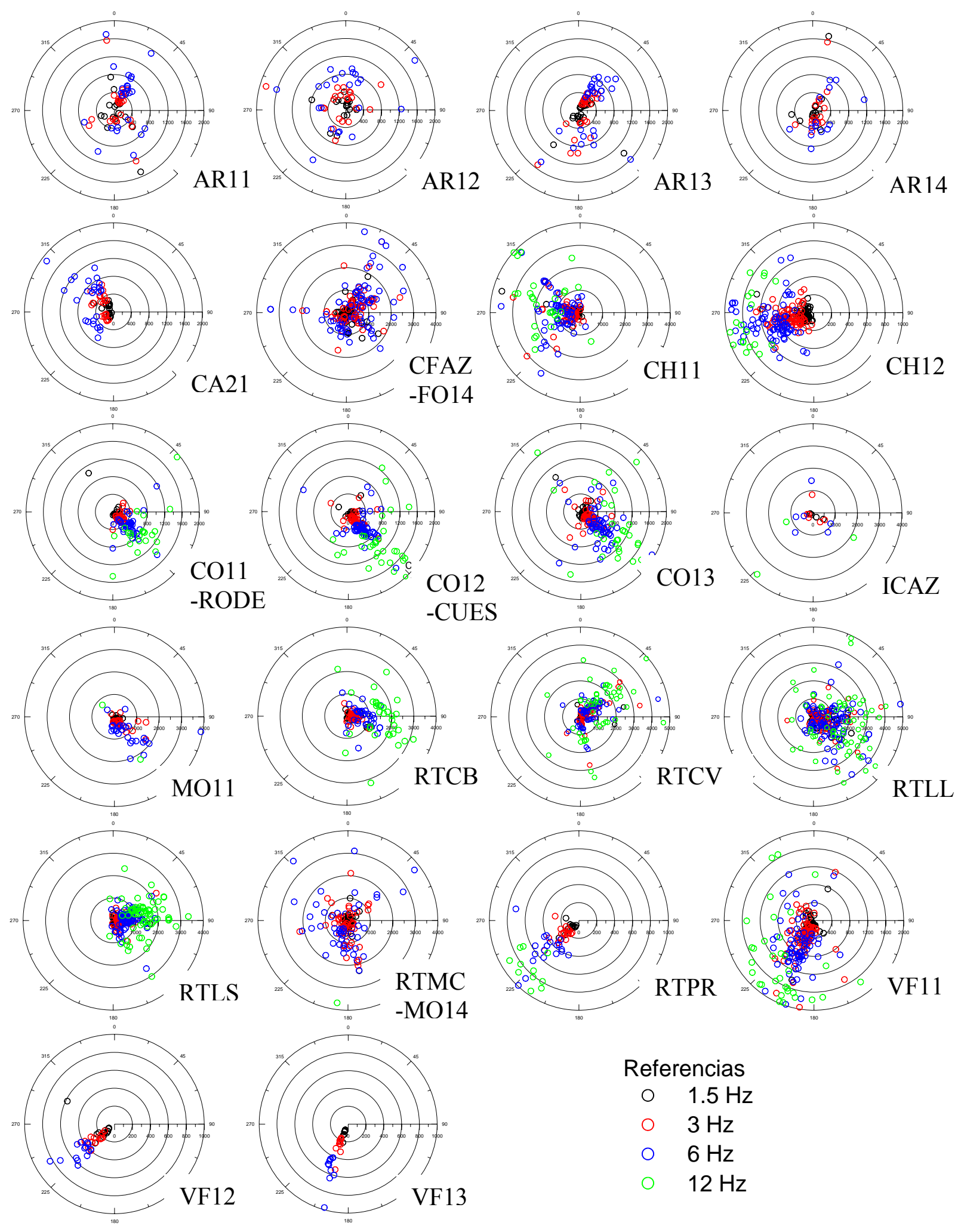

Fig. 4.7.- Distribución de valores de $Q_{c}$ para eventos con profundidades inferiores a $50 \mathrm{~km}$ obtenidos para cada estación en función del Backazimut y según la frecuencia central del filtro aplicado (circulos negros representan $Q_{c}$ a $1.5 \mathrm{~Hz}$; rojos, a $3 \mathrm{~Hz}$; azules, a $6 \mathrm{~Hz}$ y verdes, a $12 \mathrm{~Hz}$.). En todos los casos se graficaron los valores correspondientes a $90 \mathrm{~s}$ de lapso de tiempo, excepto para VF13 que se graficaron los valores correspondientes a $100 \mathrm{~s}$. 
Por otra parte, no se observan diferencias importantes de la atenuación de ondas coda en relación a la dirección de propagación de las ondas directas, por lo que se ha considerado la hipótesis de Pulli (1984) para la identificación de variaciones laterales de la atenuación. Según la misma, el $Q_{\mathrm{c}}$ estimado con el modelo de scattering simple para un lapso de tiempo dado, corresponde al decaimiento promedio de la amplitud de las ondas que han sido dispersadas en la superficie de un elipsoide que tiene a la estación y a la fuente como focos.

Puede considerarse como fuente para cada elipsoide, la localización promedio de un grupo de eventos asociados a una misma fuente sismogénica cuyos registros en una dada estación hayan arrojado valores de $Q_{\mathrm{c}}$ similares. En ese caso se obtiene un elipsoide promedio para los eventos mencionados y la estación (Kumar et al., 2005, Biescas et al., 2007). La proyección superficial del elipsoide tiene semieje mayor $a=v_{\text {med }} t / 2$ y semieje menor $b=\sqrt{\left(v_{\text {med }} t / 2\right)^{2}-\left(\Delta_{\text {med }} / 2\right)^{2}}$, donde $v_{\text {med }}$ es la velocidad promedio de las ondas coda para las trayectorias consideradas a partir del modelo (Tabla 3.2), $t$ es el lapso de tiempo al que se mide la amplitud de la coda y $\Delta_{\text {med }}$ es la distancia epicentral media en $\mathrm{km}$. La profundidad promedio a la que se asigna el valor de $Q_{c}$, está dada por $h=h_{\text {med }}+b$, donde $h_{\text {med }}$ es la profundidad media de los focos considerados.

Tabla 4.2.- Valores de $Q_{0}$ y $n$ ajustados a los datos de $Q_{c}$ en función de la frecuencia mediante la (2.21) para cada estación y grupo de epicentros seleccionados a partir de la figura 4.7. El epicentro promedio corresponde a las coordenadas del centro geográfico de cada grupo de epicentros, a es el semieje mayor de la elipse calculado según se indica en el texto y $h$ es la profundidad máxima que alcanza el elipsoide según la hipótesis de Pulli (1984).

\begin{tabular}{|c|c|c|c|c|c|c|c|c|c|}
\hline \multicolumn{2}{|c|}{ ESTACIÓN } & \multicolumn{2}{|c|}{ EPICENTRO promedio } & \multirow[t]{2}{*}{$a[\mathrm{~km}]$} & \multirow[t]{2}{*}{$h[\mathrm{~km}]$} & \multicolumn{2}{|c|}{ Single Back-Scattering } & \multicolumn{2}{|c|}{$\begin{array}{l}\text { Single Isotropic- } \\
\text { Scattering }\end{array}$} \\
\hline latitud $\left[^{\circ}\right]$ & longitud $\left[{ }^{\circ}\right]$ & latitud $\left[{ }^{\circ}\right]$ & longitud $\left[^{\circ}\right]$ & & & $Q_{0} \pm e Q_{0}$ & $n \pm e n$ & $Q_{0} \pm e Q_{0}$ & $n \pm e n$ \\
\hline \multicolumn{10}{|c|}{ lapso de tiempo $=40 \mathrm{~s}$} \\
\hline-31.603 & -68.232 & -31.528 & -68.095 & 74 & 84 & $25 \pm 0$ & $1.48 \pm 0.01$ & $32 \pm 1$ & $1.69 \pm 0.01$ \\
\hline-31.528 & -68.560 & -31.562 & -68.715 & 72 & 76 & $33 \pm 6$ & $1.31 \pm 0.08$ & $22 \pm 12$ & $1.94 \pm 0.21$ \\
\hline-31.862 & -68.540 & -31.731 & -68.661 & 73 & 78 & $37 \pm 4$ & $1.34 \pm 0.05$ & $57 \pm 21$ & $1.58 \pm 0.15$ \\
\hline-31.329 & -68.475 & -31.324 & -68.276 & 73 & 84 & $40 \pm 3$ & $1.31 \pm 0.03$ & $47 \pm 4$ & $1.6 \pm 0.03$ \\
\hline-31.310 & -67.924 & -31.369 & -67.852 & 74 & 83 & $17 \pm 1$ & $1.74 \pm 0.03$ & $14 \pm 2$ & $2.34 \pm 0.09$ \\
\hline-31.310 & -67.924 & -31.318 & -67.986 & 73 & 85 & $22 \pm 1$ & $1.64 \pm 0.03$ & $11 \pm 1$ & $2.78 \pm 0.04$ \\
\hline \multicolumn{10}{|c|}{ lapso de tiempo $=50 \mathrm{~s}$} \\
\hline-32.841 & -68.933 & -32.918 & -68.936 & 92 & 100 & $55 \pm 7$ & $1.18 \pm 0.08$ & $135 \pm 12$ & $1.47 \pm 0.05$ \\
\hline-31.603 & -68.232 & -31.491 & -68.078 & 91 & 100 & $43 \pm 0$ & $1.31 \pm 0$ & $70 \pm 2$ & $1.47 \pm 0.02$ \\
\hline-31.456 & -67.327 & -31.450 & -67.613 & 92 & 98 & $54 \pm 24$ & $1.58 \pm 0.19$ & $114 \pm 3$ & $1.23 \pm 0.01$ \\
\hline-31.528 & -68.560 & -31.584 & -68.725 & 90 & 92 & $48 \pm 3$ & $1.22 \pm 0.03$ & $112 \pm 18$ & $1.39 \pm 0.07$ \\
\hline-30.940 & -68.492 & -31.109 & -68.242 & 91 & 97 & $44 \pm 2$ & $1.37 \pm 0.02$ & $53 \pm 2$ & $1.48 \pm 0.02$ \\
\hline-31.489 & -68.810 & -31.327 & -68.487 & 92 & 95 & $25 \pm 2$ & $1.43 \pm 0.03$ & $24 \pm 3$ & $1.59 \pm 0.05$ \\
\hline-31.862 & -68.540 & -31.694 & -68.627 & 91 & 98 & $51 \pm 8$ & $1.23 \pm 0.07$ & $79 \pm 32$ & $1.42 \pm 0.17$ \\
\hline-31.862 & -68.540 & -31.954 & -68.635 & 91 & 100 & $58 \pm 29$ & $1.17 \pm 0.2$ & $35 \pm 3$ & $1.65 \pm 0.04$ \\
\hline
\end{tabular}




\begin{tabular}{|c|c|c|c|c|c|c|c|c|c|}
\hline \multicolumn{2}{|c|}{ ESTACIÓN } & \multicolumn{2}{|c|}{ EPICENTRO promedio } & $a[\mathrm{~km}]$ & $h[\mathrm{~km}]$ & Single $\mathrm{Bc}$ & -Scattering & \multicolumn{2}{|c|}{$\begin{array}{l}\text { Single Isotropic- } \\
\text { Scattering }\end{array}$} \\
\hline latitud $\left[^{\circ}\right]$ & longitud $\left[^{\circ}\right]$ & latitud $\left[{ }^{\circ}\right]$ & longitud $\left[{ }^{\circ}\right]$ & \multirow[b]{2}{*}{92} & \multirow[b]{2}{*}{96} & $Q_{0} \pm e Q_{0}$ & $n \pm e n$ & $Q_{0} \pm e Q_{0}$ & $n \pm e n$ \\
\hline-31.862 & -68.540 & -31.695 & -68.243 & & & $43 \pm 2$ & $1.31 \pm 0.02$ & $120 \pm 24$ & $1.25 \pm 0.08$ \\
\hline-31.329 & -68.475 & -31.385 & -68.205 & 91 & 97 & $70 \pm 3$ & $1.14 \pm 0.02$ & $123 \pm 9$ & $1.19 \pm 0.03$ \\
\hline-31.800 & -69.295 & -31.673 & -69.024 & 91 & 93 & $43 \pm 4$ & $1.34 \pm 0.04$ & $65 \pm 32$ & $1.46 \pm 0.2$ \\
\hline-31.310 & -67.924 & -31.162 & -67.908 & 91 & 98 & $33 \pm 10$ & $1.49 \pm 0.18$ & $42 \pm 16$ & $1.82 \pm 0.22$ \\
\hline-31.310 & -67.924 & -31.368 & -67.848 & 92 & 102 & $27 \pm 0$ & $1.51 \pm 0$ & $29 \pm 2$ & $1.99 \pm 0.03$ \\
\hline-31.310 & -67.924 & -31.318 & -67.987 & 92 & 103 & $30 \pm 0$ & $1.51 \pm 0.01$ & $21 \pm 1$ & $2.44 \pm 0.02$ \\
\hline-30.660 & -67.454 & -30.908 & -67.677 & 91 & 93 & $43 \pm 4$ & $1.33 \pm 0.03$ & $50 \pm 8$ & $1.49 \pm 0.06$ \\
\hline \multicolumn{10}{|c|}{ lapso de tiempo $=60 \mathrm{~s}$} \\
\hline-33.474 & -68.515 & -33.174 & -68.680 & 110 & 116 & $89 \pm 16$ & $0.83 \pm 0.11$ & $133 \pm 40$ & $0.72 \pm 0.19$ \\
\hline-32.841 & -68.933 & -33.062 & -68.907 & 110 & 116 & $68 \pm 6$ & $0.95 \pm 0.05$ & $106 \pm 23$ & $0.88 \pm 0.13$ \\
\hline-31.603 & -68.232 & -31.741 & -68.474 & 110 & 111 & $35 \pm 8$ & $1.47 \pm 0.11$ & $104 \pm 8$ & $1.44 \pm 0.04$ \\
\hline-31.603 & -68.232 & -31.481 & -68.076 & 110 & 118 & $56 \pm 11$ & $2.05 \pm 0.12$ & $42 \pm 12$ & $1.53 \pm 0.13$ \\
\hline-31.167 & -66.663 & -31.320 & -67.217 & 110 & 107 & $53 \pm 26$ & $1.01 \pm 0.18$ & $45 \pm 3$ & $1.22 \pm 0.03$ \\
\hline-31.528 & -68.560 & -31.438 & -68.308 & 109 & 119 & $81 \pm 45$ & $0.95 \pm 0.23$ & $56 \pm 12$ & $1.57 \pm 0.09$ \\
\hline-31.528 & -68.560 & -31.570 & -68.732 & 109 & 111 & $42 \pm 3$ & $1.25 \pm 0.03$ & $92 \pm 59$ & $1.25 \pm 0.29$ \\
\hline-31.489 & -68.810 & -31.433 & -68.416 & 110 & 112 & $59 \pm 1$ & $1.17 \pm 0.01$ & $74 \pm 3$ & $1.22 \pm 0.02$ \\
\hline-31.862 & -68.540 & -31.973 & -68.712 & 110 & 115 & $86 \pm 3$ & $1.01 \pm 0.02$ & $103 \pm 23$ & $1.43 \pm 0.09$ \\
\hline-31.862 & -68.540 & -31.692 & -68.627 & 109 & 117 & $79 \pm 32$ & $1.42 \pm 0.17$ & $79 \pm 3$ & $1.28 \pm 0.01$ \\
\hline-31.862 & -68.540 & -31.650 & -68.221 & 110 & 113 & $61 \pm 1$ & $1.2 \pm 0.01$ & $184 \pm 33$ & $0.91 \pm 0.08$ \\
\hline-31.329 & -68.475 & -31.396 & -68.180 & 109 & 115 & $87 \pm 2$ & $1.06 \pm 0.01$ & $184 \pm 8$ & $1.01 \pm 0.02$ \\
\hline-31.800 & -69.295 & -31.812 & -68.734 & 110 & 106 & $58 \pm 4$ & $1.17 \pm 0.03$ & $64 \pm 6$ & $1.22 \pm 0.04$ \\
\hline-31.310 & -67.924 & -31.489 & -67.935 & 109 & 117 & $64 \pm 7$ & $1.18 \pm 0.05$ & $59 \pm 5$ & $1.59 \pm 0.05$ \\
\hline-31.310 & -67.924 & -31.370 & -67.848 & 110 & 120 & $38 \pm 2$ & $1.35 \pm 0.03$ & $138 \pm 92$ & $1.16 \pm 0.26$ \\
\hline-30.660 & -67.454 & -30.985 & -67.703 & 110 & 112 & $70 \pm 2$ & $1.18 \pm 0.02$ & $119 \pm 12$ & $1.14 \pm 0.04$ \\
\hline \multicolumn{10}{|c|}{ lapso de tiempo $=70 \mathrm{~s}$} \\
\hline-33.086 & -68.828 & -33.340 & -68.634 & 129 & 139 & $87 \pm 4$ & $0.81 \pm 0.03$ & $73 \pm 33$ & $1.13 \pm 0.26$ \\
\hline-33.474 & -68.515 & -33.195 & -68.699 & 128 & 132 & $104 \pm 3$ & $0.87 \pm 0.02$ & $161 \pm 59$ & $0.88 \pm 0.23$ \\
\hline-32.592 & -68.834 & -32.083 & -68.433 & 128 & 119 & $36 \pm 12$ & $1.51 \pm 0.19$ & $34 \pm 13$ & $1.62 \pm 0.22$ \\
\hline-32.592 & -68.834 & -33.042 & -68.616 & 129 & 133 & $51 \pm 2$ & $1.16 \pm 0.02$ & $53 \pm 1$ & $1.23 \pm 0.01$ \\
\hline-32.841 & -68.933 & -33.061 & -68.825 & 128 & 134 & $61 \pm 0$ & $1.09 \pm 0$ & $140 \pm 16$ & $0.94 \pm 0.07$ \\
\hline-32.275 & -67.188 & -32.003 & -67.590 & 127 & 127 & $92 \pm 8$ & $1.04 \pm 0.05$ & $126 \pm 18$ & $1.03 \pm 0.08$ \\
\hline-31.603 & -68.232 & -31.825 & -68.498 & 128 & 127 & $41 \pm 7$ & $1.45 \pm 0.08$ & $90 \pm 2$ & $1.58 \pm 0.01$ \\
\hline-31.603 & -68.232 & -31.478 & -68.074 & 128 & 136 & $73 \pm 0$ & $1.16 \pm 0$ & $35 \pm 10$ & $1.71 \pm 0.13$ \\
\hline-30.589 & -69.081 & -30.934 & -68.534 & 128 & 119 & $75 \pm 6$ & $1.07 \pm 0.03$ & $82 \pm 7$ & $1.06 \pm 0.04$ \\
\hline-31.528 & -68.560 & -31.570 & -68.729 & 126 & 129 & $56 \pm 5$ & $1.14 \pm 0.04$ & $92 \pm 30$ & $1.37 \pm 0.14$ \\
\hline-30.940 & -68.492 & -31.219 & -68.223 & 128 & 132 & $35 \pm 3$ & $1.62 \pm 0.04$ & $21 \pm 5$ & $2.29 \pm 0.1$ \\
\hline-31.489 & -68.810 & -31.462 & -68.391 & 128 & 130 & $68 \pm 1$ & $1.14 \pm 0.01$ & $91 \pm 2$ & $1.17 \pm 0.01$ \\
\hline-31.862 & -68.540 & -31.710 & -68.639 & 127 & 135 & $71 \pm 5$ & $1.16 \pm 0.03$ & $149 \pm 35$ & $1.34 \pm 0.1$ \\
\hline-31.862 & -68.540 & -31.638 & -68.214 & 128 & 131 & $83 \pm 2$ & $1.1 \pm 0.01$ & $140 \pm 3$ & $1.08 \pm 0.01$ \\
\hline-31.862 & -68.540 & -32.054 & -68.687 & 128 & 133 & $92 \pm 1$ & $1.02 \pm 0$ & $170 \pm 3$ & $1.02 \pm 0.01$ \\
\hline-31.329 & -68.475 & -31.593 & -68.576 & 128 & 129 & $86 \pm 1$ & $1.01 \pm 0$ & $198 \pm 9$ & $0.85 \pm 0.02$ \\
\hline-31.800 & -69.295 & -31.658 & -68.997 & 128 & 135 & $57 \pm 2$ & $1.29 \pm 0.01$ & $118 \pm 23$ & $1.37 \pm 0.08$ \\
\hline-31.800 & -69.295 & -31.674 & -68.647 & 128 & 119 & $71 \pm 1$ & $1.13 \pm 0$ & $82 \pm 1$ & $1.14 \pm 0.01$ \\
\hline-31.310 & -67.924 & -31.371 & -67.841 & 129 & 138 & $35 \pm 1$ & $1.43 \pm 0.01$ & $34 \pm 2$ & $1.98 \pm 0.04$ \\
\hline-31.310 & -67.924 & -31.317 & -67.987 & 128 & 140 & $44 \pm 1$ & $1.36 \pm 0.01$ & $65 \pm 2$ & $1.85 \pm 0.02$ \\
\hline-30.660 & -67.454 & -31.039 & -67.712 & 128 & 128 & $77 \pm 1$ & $1.19 \pm 0$ & $156 \pm 6$ & $1.11 \pm 0.02$ \\
\hline \multicolumn{10}{|c|}{ lapso de tiempo $=80 \mathrm{~s}$} \\
\hline-33.086 & -68.828 & -33.368 & -68.672 & 148 & 159 & $84 \pm 12$ & $0.88 \pm 0.09$ & $125 \pm 25$ & $0.88 \pm 0.12$ \\
\hline-33.474 & -68.515 & -33.155 & -68.693 & 147 & 154 & $109 \pm 11$ & $0.86 \pm 0.06$ & $180 \pm 35$ & $0.75 \pm 0.12$ \\
\hline
\end{tabular}

Tabla 4.2. (continuación) 


\begin{tabular}{|c|c|c|c|c|c|c|c|c|c|}
\hline \multicolumn{2}{|c|}{ ESTACIÓN } & \multicolumn{2}{|c|}{ EPICENTRO promedio } & $a[\mathrm{~km}]$ & $\mathrm{h}[\mathrm{km}]$ & \multicolumn{2}{|c|}{ Single Back-Scattering } & \multicolumn{2}{|c|}{$\begin{array}{l}\text { Single Isotropic- } \\
\text { Scattering }\end{array}$} \\
\hline latitud $\left[^{\circ}\right]$ & longitud $\left[^{\circ}\right]$ & latitud $\left[^{\circ}\right]$ & longitud $\left[{ }^{\circ}\right]$ & \multirow[b]{2}{*}{148} & \multirow[b]{2}{*}{153} & $Q_{0} \pm e Q_{0}$ & $n \pm e n$ & $Q_{0} \pm e Q_{0}$ & $n \pm e n$ \\
\hline-32.592 & -68.834 & -33.041 & -68.602 & & & $71 \pm 3$ & $1.01 \pm 0.02$ & $57 \pm 4$ & $1.37 \pm 0.04$ \\
\hline-32.592 & -68.834 & -32.028 & -68.400 & 147 & 139 & $54 \pm 3$ & $1.34 \pm 0.03$ & $80 \pm 6$ & $1.07 \pm 0.04$ \\
\hline-32.841 & -68.933 & -33.057 & -68.836 & 147 & 152 & $80 \pm 6$ & $0.94 \pm 0.04$ & $152 \pm 1$ & $0.87 \pm 0$ \\
\hline-32.275 & -67.188 & -31.915 & -67.587 & 146 & 146 & $101 \pm 3$ & $1 \pm 0.02$ & $38 \pm 6$ & $1.41 \pm 0.1$ \\
\hline-32.275 & -67.188 & -32.491 & -67.980 & 147 & 137 & $37 \pm 6$ & $1.38 \pm 0.09$ & $137 \pm 5$ & $1 \pm 0.02$ \\
\hline-31.603 & -68.232 & -31.915 & -68.513 & 147 & 145 & $84 \pm 1$ & $1.03 \pm 0.01$ & $118 \pm 2$ & $0.98 \pm 0.01$ \\
\hline-31.167 & -66.663 & -31.333 & -67.301 & 146 & 140 & $72 \pm 1$ & $1.06 \pm 0.01$ & $86 \pm 1$ & $1.06 \pm 0.01$ \\
\hline-30.167 & -69.473 & -30.668 & -68.703 & 146 & 122 & $68 \pm 4$ & $1.04 \pm 0.03$ & $68 \pm 4$ & $1.04 \pm 0.03$ \\
\hline-30.171 & -69.120 & -30.730 & -68.524 & 146 & 128 & $77 \pm 4$ & $1.05 \pm 0.02$ & $82 \pm 5$ & $1.04 \pm 0.02$ \\
\hline-30.589 & -69.081 & -31.027 & -68.514 & 146 & 133 & $75 \pm 2$ & $1.08 \pm 0.01$ & $82 \pm 2$ & $1.08 \pm 0.01$ \\
\hline-31.528 & -68.560 & -31.565 & -68.731 & 144 & 147 & $49 \pm 7$ & $1.22 \pm 0.06$ & $68 \pm 22$ & $1.54 \pm 0.13$ \\
\hline-30.940 & -68.492 & -31.270 & -68.218 & 147 & 149 & $40 \pm 3$ & $1.58 \pm 0.03$ & $22 \pm 3$ & $2.34 \pm 0.07$ \\
\hline-31.489 & -68.810 & -31.475 & -68.376 & 146 & 149 & $82 \pm 0$ & $1.09 \pm 0$ & $123 \pm 0$ & $1.09 \pm 0$ \\
\hline-31.862 & -68.540 & -31.614 & -68.214 & 146 & 149 & $102 \pm 1$ & $1.04 \pm 0.01$ & $186 \pm 6$ & $1 \pm 0.01$ \\
\hline-31.862 & -68.540 & -32.206 & -68.626 & 147 & 150 & $107 \pm 5$ & $1 \pm 0.02$ & $194 \pm 7$ & $0.97 \pm 0.02$ \\
\hline-31.329 & -68.475 & -31.653 & -68.580 & 146 & 146 & $93 \pm 1$ & $1.03 \pm 0.01$ & $202 \pm 2$ & $0.88 \pm 0$ \\
\hline-31.800 & -69.295 & -31.670 & -68.617 & 146 & 137 & $79 \pm 1$ & $1.1 \pm 0$ & $98 \pm 0$ & $1.08 \pm 0$ \\
\hline-31.310 & -67.924 & -31.320 & -67.988 & 147 & 159 & $48 \pm 1$ & $1.34 \pm 0.01$ & $76 \pm 1$ & $1.19 \pm 0$ \\
\hline-31.310 & -67.924 & -31.375 & -67.827 & 147 & 157 & $48 \pm 2$ & $1.15 \pm 0.02$ & $68 \pm 6$ & $1.8 \pm 0.05$ \\
\hline-30.308 & -66.552 & -30.812 & -67.234 & 146 & 129 & $61 \pm 4$ & $1.14 \pm 0.02$ & $63 \pm 4$ & $1.14 \pm 0.03$ \\
\hline-30.396 & -66.528 & -30.843 & -67.213 & 146 & 132 & $101+2$ & $0.84+0.01$ & $110 \pm 3$ & $0.81 \pm 0.01$ \\
\hline \multicolumn{10}{|c|}{ lapso de tiempo $=90 \mathrm{~s}$} \\
\hline-33.474 & -68.515 & -33.155 & -68.693 & 165 & 173 & $115 \pm 7$ & $0.89 \pm 0.04$ & $87 \pm 3$ & $1.21 \pm 0.02$ \\
\hline-33.474 & -68.515 & -32.773 & -68.286 & 165 & 153 & $81 \pm 39$ & $1.2 \pm 0.29$ & $189 \pm 23$ & $0.8 \pm 0.08$ \\
\hline-33.086 & -68.828 & -32.485 & -68.426 & 165 & 156 & $110 \pm 3$ & $0.89 \pm 0.02$ & $128 \pm 5$ & $0.86 \pm 0.03$ \\
\hline-32.841 & -68.933 & -32.917 & -68.783 & 165 & 168 & $87 \pm 1$ & $0.98 \pm 0.01$ & $92 \pm 15$ & $1.74 \pm 0.09$ \\
\hline-32.592 & -68.834 & -33.064 & -68.625 & 166 & 172 & $80 \pm 8$ & $1.03 \pm 0.06$ & $85 \pm 0$ & $1.09 \pm 0$ \\
\hline-32.592 & -68.834 & -31.944 & -68.373 & 165 & 153 & $78 \pm 0$ & $1.09 \pm 0$ & $98 \pm 13$ & $1.05 \pm 0.08$ \\
\hline-32.275 & -67.188 & -32.674 & -67.907 & 166 & 157 & $58 \pm 3$ & $1.2 \pm 0.03$ & $60 \pm 3$ & $1.22 \pm 0.03$ \\
\hline-32.275 & -67.188 & -31.899 & -67.583 & 165 & 165 & $117 \pm 3$ & $0.92 \pm 0.01$ & $168 \pm 4$ & $0.9 \pm 0.01$ \\
\hline-31.862 & -68.540 & -32.383 & -68.521 & 166 & 166 & $107 \pm 1$ & $1 \pm 0$ & $150 \pm 10$ & $1.05 \pm 0.03$ \\
\hline-31.800 & -69.295 & -31.673 & -68.611 & 164 & 156 & $85 \pm 0$ & $1.1 \pm 0$ & $107 \pm 1$ & $1.11 \pm 0$ \\
\hline-31.603 & -68.232 & -32.035 & -68.507 & 165 & 162 & $94 \pm 1$ & $1.04 \pm 0.01$ & $138 \pm 5$ & $0.98 \pm 0.02$ \\
\hline-31.489 & -68.810 & -31.480 & -68.358 & 164 & 166 & $100 \pm 2$ & $1.04 \pm 0.01$ & $177 \pm 5$ & $0.99 \pm 0.01$ \\
\hline-31.329 & -68.475 & -31.649 & -68.578 & 164 & 160 & $100 \pm 0$ & $1 \pm 0$ & $186 \pm 5$ & $0.93 \pm 0.01$ \\
\hline-31.310 & -67.924 & -31.375 & -67.827 & 165 & 176 & $48 \pm 2$ & $1.21 \pm 0.02$ & $135 \pm 27$ & $1.8 \pm 0.12$ \\
\hline-31.310 & -67.924 & -31.325 & -67.985 & 165 & 177 & $58 \pm 1$ & $1.19 \pm 0.01$ & $70 \pm 5$ & $1.3 \pm 0.05$ \\
\hline-31.310 & -67.924 & -31.164 & -67.909 & 163 & 171 & $67 \pm 6$ & $1.34 \pm 0.05$ & $96 \pm 14$ & $1.46 \pm 0.09$ \\
\hline-31.167 & -66.663 & -31.345 & -67.312 & 165 & 160 & $79 \pm 3$ & $1.03 \pm 0.02$ & $101 \pm 1$ & $1.02 \pm 0.01$ \\
\hline-30.940 & -68.492 & -31.269 & -68.214 & 165 & 168 & $80 \pm 7$ & $1.25 \pm 0.04$ & $49 \pm 5$ & $2.03 \pm 0.04$ \\
\hline-30.589 & -69.081 & -31.021 & -68.502 & 164 & 154 & $90 \pm 3$ & $1.02 \pm 0.01$ & $102 \pm 2$ & $1.03 \pm 0.01$ \\
\hline-30.396 & -66.528 & -30.859 & -67.214 & 164 & 153 & $103 \pm 1$ & $0.87 \pm 0.01$ & $82 \pm 4$ & $1.06 \pm 0.02$ \\
\hline-30.308 & -66.552 & -30.846 & -67.228 & 164 & 149 & $77 \pm 4$ & $1.05 \pm 0.02$ & $88 \pm 4$ & $1.11 \pm 0.02$ \\
\hline-30.171 & -69.120 & -30.753 & -68.513 & 164 & 149 & $81 \pm 3$ & $1.1 \pm 0.02$ & $106 \pm 2$ & $0.96 \pm 0.01$ \\
\hline-30.167 & -69.473 & -30.696 & -68.716 & 164 & 143 & $100 \pm 2$ & $0.97 \pm 0.01$ & $118 \pm 1$ & $0.84 \pm 0.01$ \\
\hline
\end{tabular}

Tabla 4.2. (continuación) 

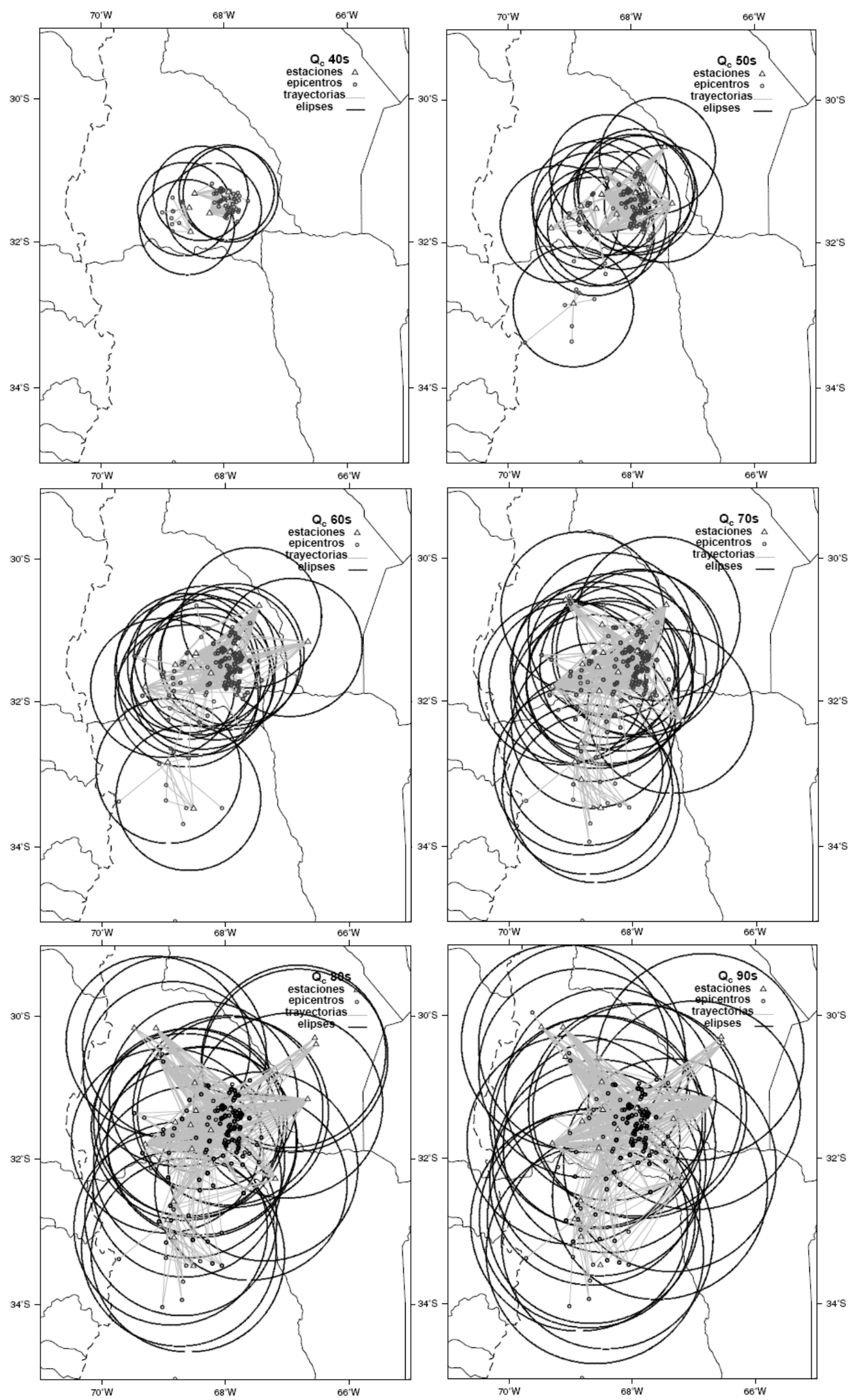

Fig. 4.8.- Contornos de las proyecciones superficiales de los elipsoides involucrados en la generación de ondas coda seleccionados. Para cada lapso de tiempo (entre 40 y 90 s) se graficaron los haces de trayectorias directas y las elipses con focos en la estación y en el centro del grupo de epicentros considerados. 
Para la construcción de las elipses se han seleccionado de la Fig. 4.7 grupos de eventos con profundidades menores que $50 \mathrm{~km}$, distancias epicentrales inferiores a $200 \mathrm{~km}$ y valores similares de $Q_{c}$. En la Tabla 4.2 se listan los valores de $Q_{0}$ y $n$ estimados para cada elipse, en lapsos de tiempo entre 40 y 90 s considerando los modelos SBS y SIS, sus errores y los parámetros que la definen (coordenadas de los focos: estación y epicentro promedio; semieje mayor, a y profundidad promedio, $h$ ). Los mapas de la Fig. 4.8 muestran los epicentros y estaciones seleccionados, las trayectorias consideradas en cada caso y las elipses calculadas.

Asumiendo un valor uniforme de $Q_{0}$ y $n$ dentro de cada elipse y promediando los valores de todas las elipses en una malla de 1' de espaciado, se han obtenido distribuciones de $Q_{0}$ y $n$ para los diferentes lapsos de tiempo analizados según el modelo de Sato. Se representan los contornos de $Q_{0}$ y $n$ (Figs. 4.9 a y b), ajustando una superficie cartesiana 2-D mediante una interpolación cúbica por trozos (splines) con funciones de Green, curvatura mínima y un espaciado de 3 '.

Los valores de $Q_{0}{ }^{-1}$, como medida de la atenuación se representan en escala de colores, observándose que al aumentar el volumen muestreado por las ondas, la atenuación se aproxima a un valor uniforme y cercano a 0.01 , para toda la región. Cerca de la superficie, entre 0 y $82 \mathrm{~km}$, la mayor atenuación se registra en la región oriental de la provincia de San Juan, hacia las Sas. Pie de Palo y Valle Fértil (ver Figs. 3.7 y 3.20), aunque aparece otro mínimo en el oeste, en Precordillera. Giroldi (1990) obtuvo para la provincia de San Juan un valor medio de $Q_{0}=100$ y Alvarado (1992) dedujo, para el norte de Mendoza, un valor promedio de $Q_{0}=122$, en ambos trabajos se consideró la longitud total de coda. En este trabajo puede verse que el valor de $Q_{0}$ varía con el lapso de tiempo utilizado. Sin embargo los resultados obtenidos no se apartan significativamente de los valores previamente mencionados para lapsos de tiempo del orden de $\operatorname{los} 100 \mathrm{~s}$, que es la longitud de coda preponderante. Por otra parte, se observa una fuerte dependencia de $Q_{\mathrm{c}}$ con la frecuencia, que se manifiesta en valores de $n$ en general superiores a 1 . En todos los casos, los mayores valores de $n$ se corresponden con los valores más bajos de $Q_{0 \text { c }}$, de modo que a mayor atenuación, mayor variación de ella con la frecuencia.

En la aproximación del scattering simple, la coda está compuesta por ondas S cuyo factor de calidad resume los efectos del scattering y la absorción intrínseca verificando la relación $Q_{c}^{-1}=Q_{s}^{-1}+Q_{i}^{-1}$ (Novelo Casanova y Martínez Bringas, 2005). 

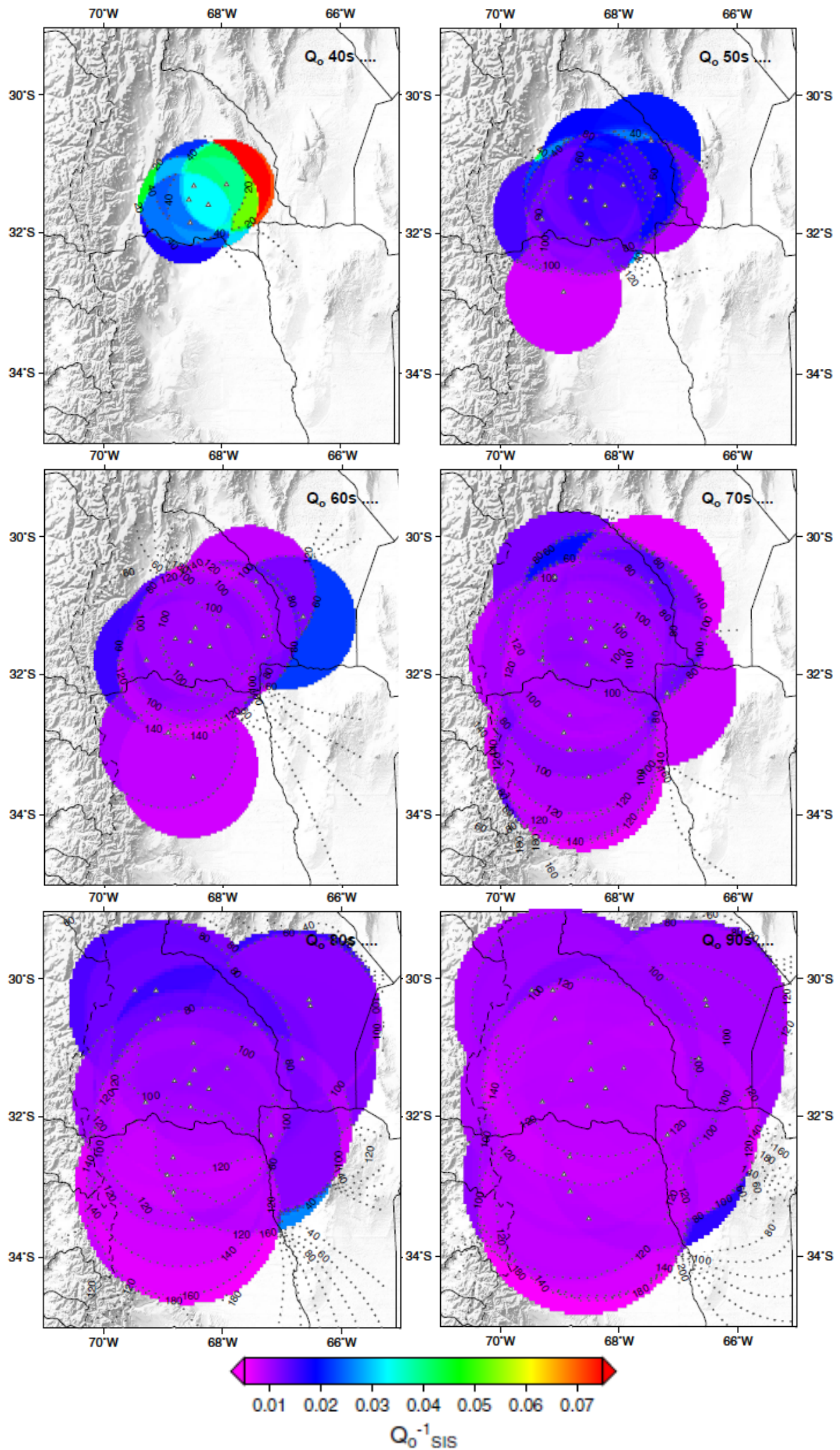

Fig. 4.9a.- Contornos de $Q_{0}$ según la interpretación de Pulli (1984) obtenidos promediando en una grilla los valores de $Q_{0}$ asignados a las elipses de la Fig. 4.8. En color se representa la función disipativa $\left(Q_{0}^{-1}\right)$ como medida de la atenuación. 

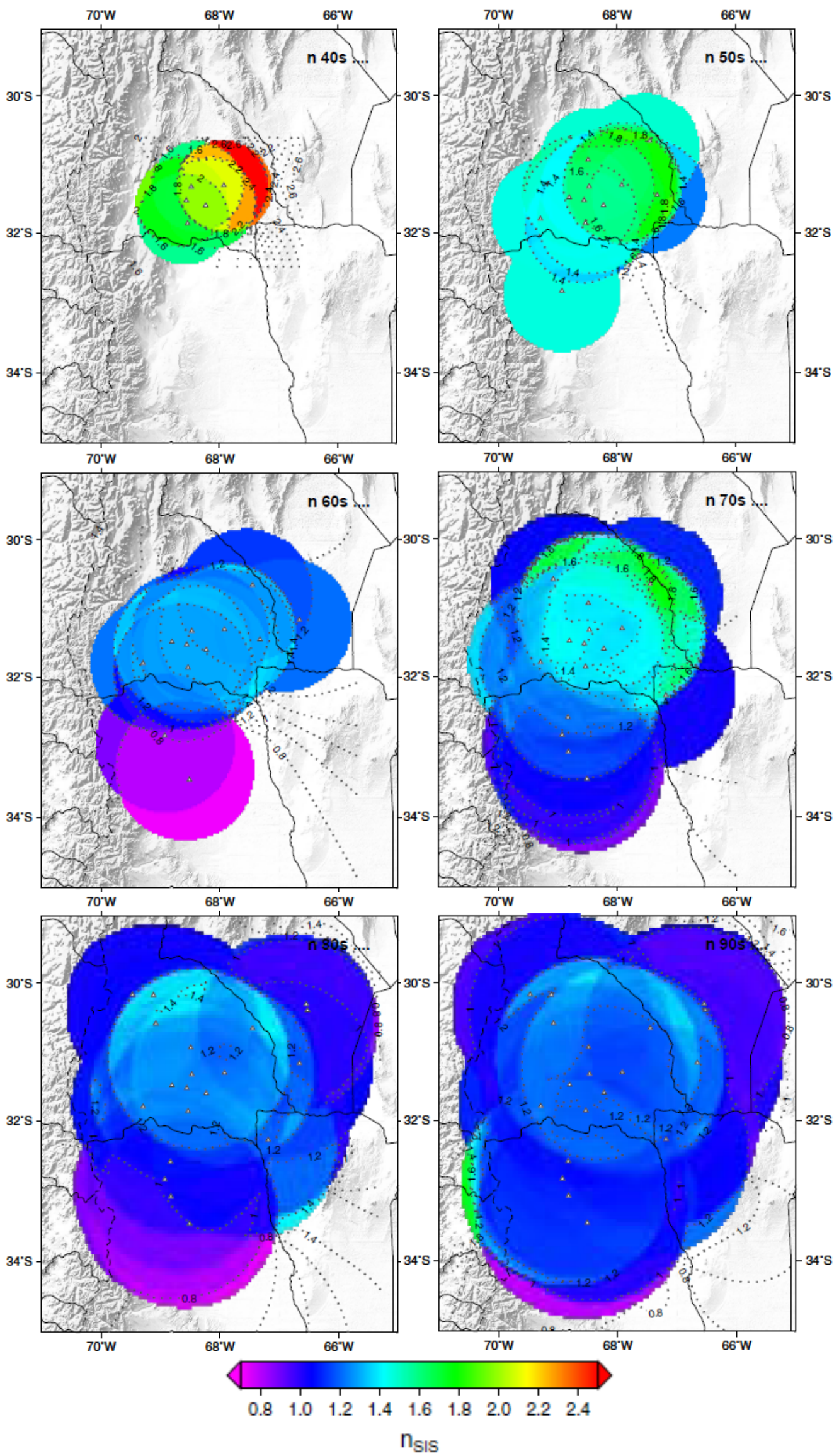

Fig. 4.9b.- Distribución del coeficiente n que ajusta la variación de $Q(f)=Q_{0} f^{n}$. Siguiendo la interpretación con volúmenes elipsoidales de Pulli (1984), se obtuvieron los valores de $n$ promediando en una grilla los valores asignados a las elipses de la Fig. 4.8. Comparando con la Fig. 4.9a, puede verse que los valores mayores de $n$ se dan en las áreas con mayor atenuación. 
Sobre la base de las conclusiones de otros autores citados en el Capítulo 2 y de los resultados de la separación de la atenuación intrínseca y de scattering de este trabajo que más adelante se presentarán, puede suponerse que los valores de $Q_{\mathrm{c}}$ son mayormente dominados por los mecanismos intrínsecos. De este modo, los valores de $Q_{\mathrm{c}}{ }^{-1}$ obtenidos para lapsos de tiempo de 40, 50, 60, 70, 80 y $90 \mathrm{~s}$, representan la atenuación promedio en volúmenes elipsoidales con profundidades máximas medias de 82, 97, 114, 132, 144 y $161 \mathrm{~km}$ respectivamente, según la interpretación de Pulli (1984). En cada lapso de tiempo se ha estimado la mediana de $Q_{0}$ obteniéndose los valores 30, 70, 100, 103, 100 y 112 respectivamente y se han calculado los residuos normalizados robustos de $Q_{0}{ }^{-1}$ que permiten analizar la variación de la atenuación en planta y profundidad. Dichos residuos se definen como $\left(Q_{0}^{-1}-\operatorname{MED}\left(Q_{0}^{-1}\right)\right) / \operatorname{MAD}\left(Q_{0}^{-1}\right)$ donde $\operatorname{MED}(x)$ representa la mediana de $x$ y $M A D(x)=\operatorname{MED}(\operatorname{abs}(x-M E D(x)))$ (Sachs, 1984). Asignando un plano a cada rango de profundidad como se muestra en la Fig. 4.10 se representan las desviaciones de la atenuación respecto de su mediana y se sobreimprimen los contornos de la ZWB (Anderson et al., 2007) correspondientes al rango de profundidad considerado. De acuerdo a los volúmenes implicados en la generación de las ondas coda, la atenuación para lapsos de tiempo mayores que o iguales a $60 \mathrm{~s}$ incluye el efecto de la placa de Nazca subyacente.

Se observa que en todos los rangos de profundidad el valor mediano de la atenuación se da principalmente en el área de mayor sismicidad que coincide con la horizontalización de la placa debido a la trayectoria de la dorsal Juan Fernández en su subducción bajo Sudamérica según estudios realizados por Gutscher et al. (2000) y Yañez et al. (2001) entre otros. Los máximos relativos de la atenuación en cada intervalo de profundidad se localizan en la dirección y sentido de subducción de la dorsal asísmica Juan Fernández. La mayor absorción de energía sísmica en dicha región coincidiría con un incremento del acoplamiento viscoso entre las placas continental y oceánica (Alvarado et al., 2010) debido a la morfología irregular de la placa de Nazca en la JFR, la escasez de material astenosférico entre placas, la consecuente alteración de la estructura geotérmica y por lo tanto el enfriamiento de la litósfera continental que resulta en un endurecimiento de su reología. 


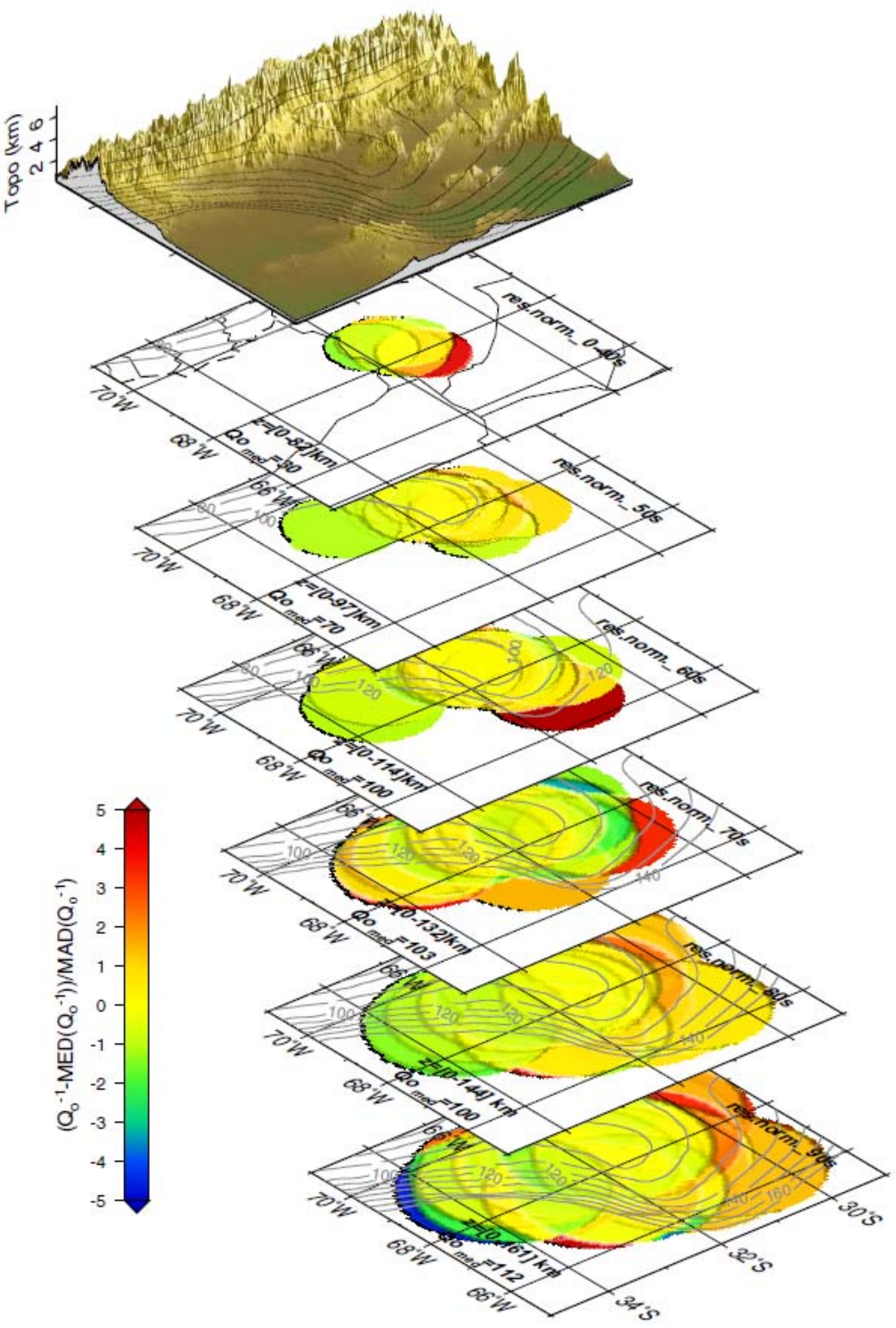

Fig. 4.10.- Los residuos normalizados de $Q_{0}^{-1}$ permiten ver las variaciones laterales de la atenuación para cada rango de profundidades muestreado en los intervalos de tiempo analizados (40,50, 60, 70, 80 y $90 \mathrm{~s})$. En la parte superior se muestra la topografía junto a los contornos de la ZWB. En cada mapa de $Q_{0}$ se expresa el valor de la mediana de $Q_{o}$ para dicho lapso de tiempo así como el intervalo de profundidades barrido por las ondas coda $v$ se sobreimprimen los contornos de la ZWB correspondientes. 
La mediana de $Q_{0}$ crece con el volumen muestreado, por lo que resulta de interés estudiar la variación de $Q_{0}$ con la profundidad.

Considerando a la atenuación como un efecto lineal puede estimarse la variación de $Q_{0}{ }^{-1}$ de ondas coda $\left(\Delta Q_{\mathrm{c}}{ }^{-1}\right)$ en un dado intervalo de profundidades $\left(\mathrm{z}_{2}-\mathrm{z}_{1}\right)$ substrayendo el efecto atenuativo desde la superficie hasta la menor profundidad $\left(\mathrm{z}_{1}\right)$ de aquel efecto hasta la mayor profundidad $\left(\mathrm{z}_{2}\right)$ (Novelo Casanova y Martínez Bringas, 2005):

$$
\left.t_{\left(z_{2}-z_{1}\right)} \Delta Q_{c}^{-1}\right|_{z_{1}} ^{z_{2}}=\left.t_{2} Q_{c}^{-1}\right|_{z_{2}}-\left.t_{1} Q_{c}^{-1}\right|_{z_{1}}
$$

donde $t_{(\mathrm{z} 2-\mathrm{z} 1)}$ es el tiempo que le lleva a las ondas propagarse por la capa entre $z_{2}$ y $z_{1}, t_{2}$ y $t_{1}$ son los lapsos de tiempo correspondientes a los elipsoides de profundidades máximas $z_{2}$ y $z_{1}$ respectivamente y $Q_{\mathrm{c}}{ }^{-1}$ es la mediana de la atenuación estimada en cada caso $\left(Q_{0}{ }^{-1}\right)$. Para poder comparar los valores de atenuación a diferentes profundidades y para asumir que las variaciones obtenidas son función sólo de la profundidad, se ha restringido el área estudiada a aquella muestreada por las codas de $40 \mathrm{~s}$, seleccionando para todos los lapsos de tiempo sólo datos de estaciones y eventos dentro de la misma área.

El significado estadístico de los cambios de $\Delta Q_{\mathrm{c}}{ }^{-1}$ se analizó mediante el test de suma de rangos conocido también como test de Mann-Whitnney o test de Wilcoxon (Hollander y Wolfe, 1999; Triola, 2004; Moore, 2005) donde el estadístico se relaciona con la suma de los rangos de cada muestra. Este test no paramétrico es indicado si la cantidad de datos es numerosa y su distribución no puede suponerse normal en un principio, ya que basa sus hipótesis en el rango completo de los datos. En todos los casos, un valor absoluto del estadístico superior a 1.96 permitió rechazar la hipótesis nula (igualdad de medianas en las muestras) con un nivel de significancia del 5\% (Mongay Fernández, 2005). Si bien a profundidades mayores que $130 \mathrm{~km}$ las medianas de $\Delta Q_{\mathrm{c}}^{-1}$ resultaron similares, el test mostró que los rangos y por lo tanto las muestras eran diferentes (UCLA: Academic Technology Services, Statistical Consulting Group, 2010).

Asumiendo entonces que las variaciones de la atenuación vistas en profundidad a través de la hipótesis de Pulli (1984) son significativas puede hacerse una interpretación de las variaciones de la atenuación con la profundidad relacionando, por ejemplo, su mediana con la distribución de sismicidad (Fig. 4.11).

De acuerdo a las magnitudes y distancias epicentrales de los datos, no pueden identificarse variaciones de atenuación (mediana de variación de $Q_{\mathrm{c}}{ }^{-1}$ en la Fig. 4.11 izquierda) dentro de los primeros $80 \mathrm{~km}$ de profundidad (lapsos de tiempo menores que $40 \mathrm{~s}$ ), 
que comprenden básicamente a la litósfera continental. Sin embargo, la condición frágil de la litósfera superior, marcada por la sismicidad hasta los $50 \mathrm{~km}$ de profundidad (Fig. 4.11 derecha), acompaña a una atenuación alta. Allí donde se da la transición litósfera continentallitósfera oceánica (Gutscher et al., 2000), a través de una delgada capa de material astenosférico, se da una disminución brusca de la atenuación y ausencia de terremotos. En la parte superior de la litósfera oceánica, a los $100 \mathrm{~km}$ de profundidad, se da la mayor concentración de eventos sísmicos, así como un aumento de la atenuación.

De acuerdo a estudios realizados en otras regiones de corteza continental tectónicamente activas, gran parte de los terremotos de mayor magnitud se localizan alrededor de la transición frágil-dúctil (TDF) (Sibson, 1982, Meissner y Strehlau, 1982, Chen y Molnar, 1983; Fernández, 2004). Relacionando la atenuación de ondas coda a un efecto anelástico, entonces el aumento de la atenuación entre los 100 y 114 km estaría indicando la posible ubicación de la TDF en la litósfera de Nazca subducida.
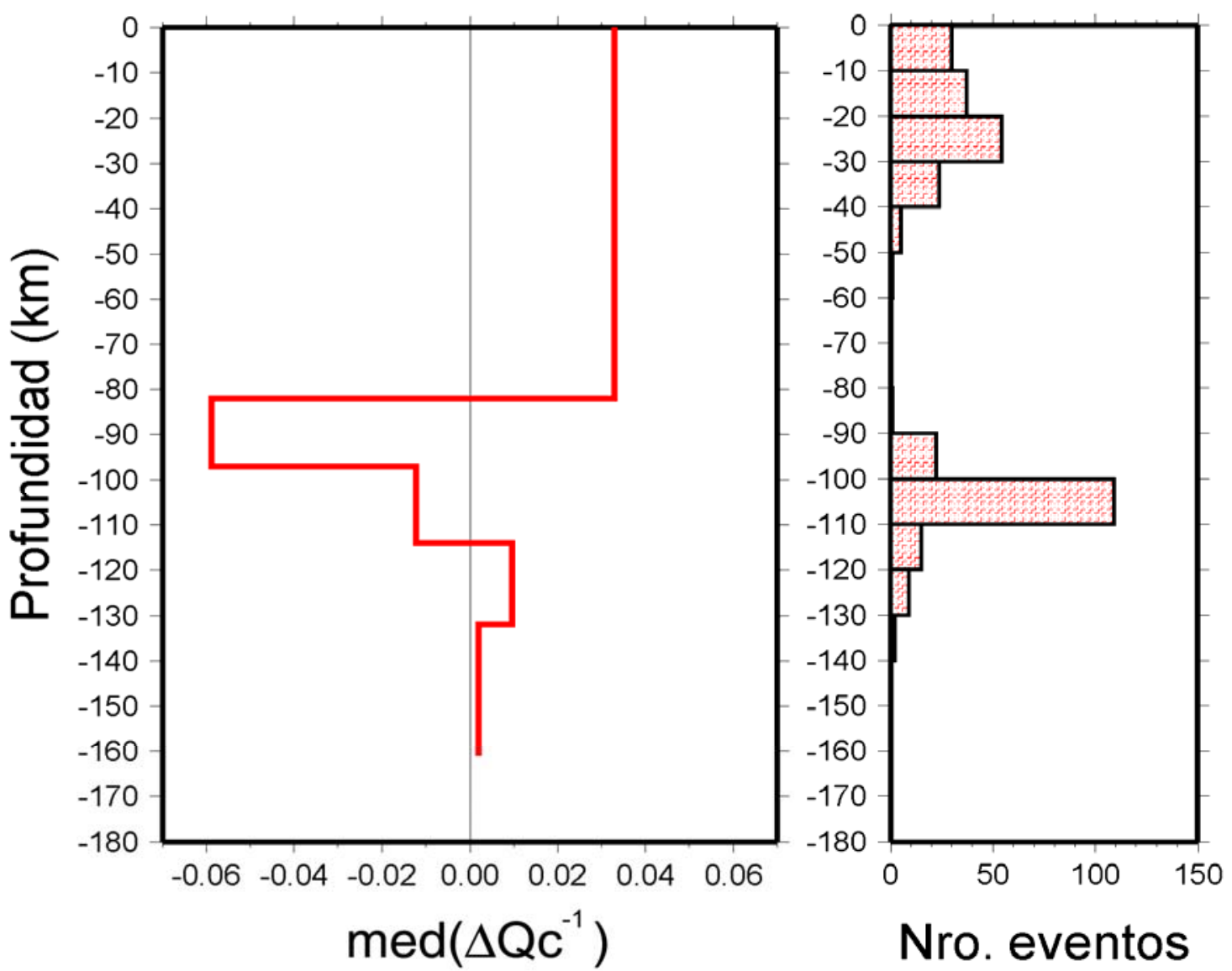

Fig.4.11.- Relación entre las medianas de la variación de la atenuación con la profundidad (izquierda) y el número de eventos en intervalos de $10 \mathrm{~km}$ de profundidad, seleccionados para este estudio dentro del área delimitada por $30.8^{\circ}-32.5^{\circ} \mathrm{S}$ y $69.4^{\circ}-67^{\circ} \mathrm{O}$ (derecha). 


\subsection{ONDAS P y $S$}

\subsubsection{MÉTODO ESPECTRAL (ME)}

\subsubsection{APLICACIÓN Y RESULTADOS}

Para la determinación del factor de calidad de ondas P y S $\left(Q_{\mathrm{P}}\right.$ y $\left.Q_{\mathrm{S}}\right)$ en función de la frecuencia a partir del decaimiento espectral, se ha aplicado la adaptación realizada por Giampiccolo et al. (2007) al método de las razones espectrales con una sola estación de Tsujiura (1966) y una nueva adaptación propuesta en este trabajo.

Utilizando la (2.81), la gráfica del $\ln \left[A\left(f_{i}\right) / A\left(f_{1}\right)\right]$ en función del tiempo tendrá una tendencia lineal que podrá ser ajustada con una recta de pendiente $m_{i}$ de la forma

$$
m_{i}=-\pi\left(f_{i} / Q_{i}-f_{1} / Q_{1}\right)
$$

donde se ha supuesto que los términos representados por los cocientes de las amplitudes espectrales en la fuente y de las funciones respuesta de sitio son constantes e independientes del tiempo de viaje para todos los registros analizados.

En la implementación de Giampiccolo et al. (2007) se supone que el factor de calidad es constante en el intervalo que va desde la frecuencia de referencia $f_{1}$ hasta cada $f_{i}$ analizada, mientras que en nuestra aproximación, se ajusta una variación del factor de calidad de la forma $Q_{i}(f)=Q_{o}\left(f_{i} / f_{0}\right)^{n}$ en cada intervalo de frecuencia considerado, con $Q(1 \mathrm{~Hz})=Q_{o}$.

Para aproximar los datos a la validez de las hipótesis del método, se han agrupado los eventos en aquellos con focos corticales $(\mathrm{h}<50 \mathrm{~km})$ y los que tienen su origen en la placa de Nazca $(\mathrm{h}>90 \mathrm{~km})$ restringiendo las magnitudes al rango [3.8 - 4.2].

Los espectros de desplazamiento han sido calculados utilizando la herramienta qspec (Havskov, 2005) del paquete SEISAN (SEISmic ANalysis System) (Ottemöller, Voss y Havskov, 2009). En primer lugar se convirtieron los datos a formato SEISAN y se elaboraron con el programa resp, las curvas de respuesta de instrumento en desplazamiento utilizando la información disponible (Tabla A1) para determinar las constantes correspondientes (Tabla A2).

En forma semiautomática se han calculado entonces los espectros de desplazamiento del suelo para eventos registrados por 14 estaciones, que tuvieran formas de onda no saturadas y 


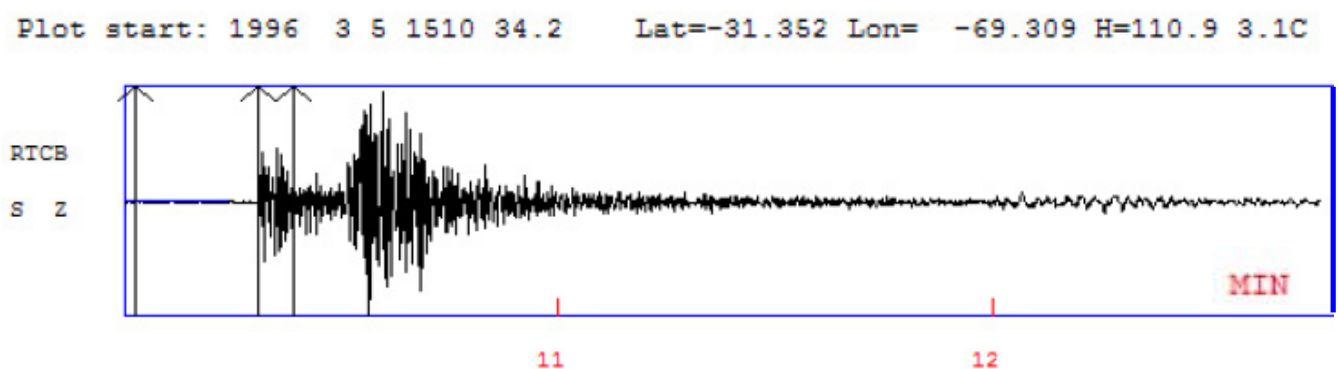

RTCB S $Z$ displacement

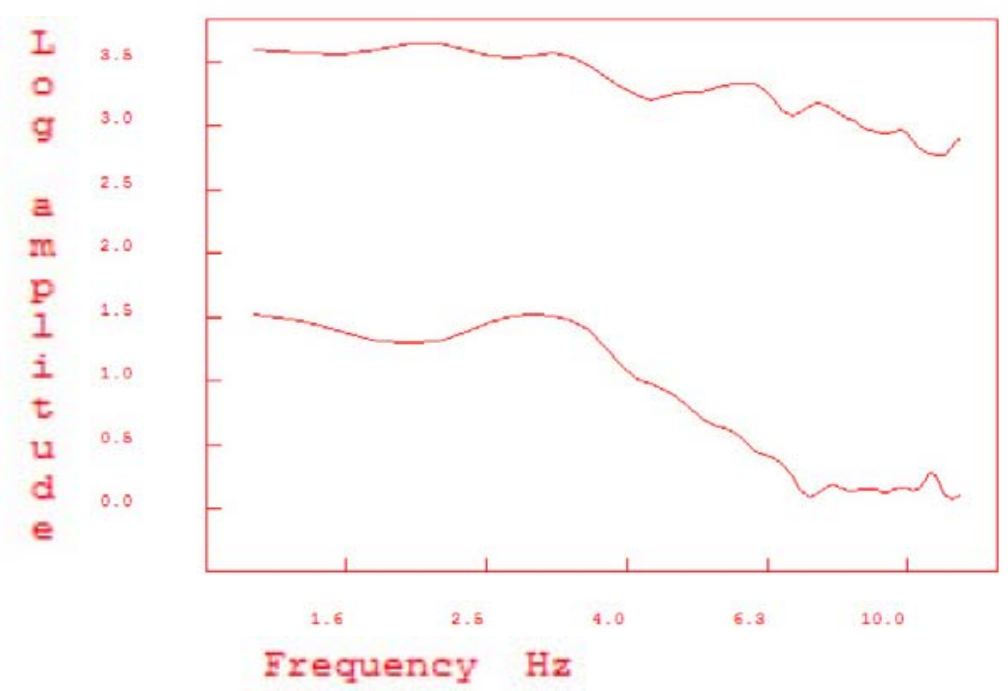

Fig. 4.12.- Ejemplos del cálculo de espectros de desplazamiento: Arriba registro de evento con profundidad focal intermedia donde se señala la ventana de señal de $P$ e inicio de la ventana de ruido para el cálculo de los espectros de amplitud suavizados que se muestran inmediatamente debajo. Abajo: ídem arriba pero para ondas $S$ de evento superficial.

Plot start: $1995112214350.0 \quad$ Lat $=-31.044$ Lon $=-68.245 \mathrm{H}=0.0$

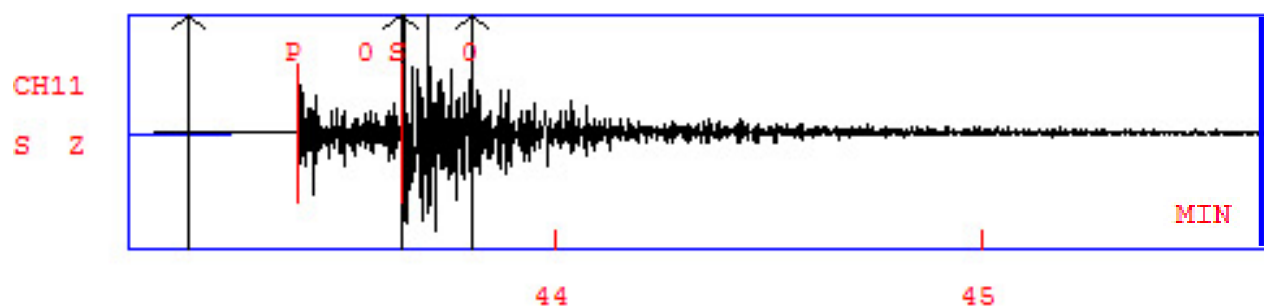

CH11 S Z displacement

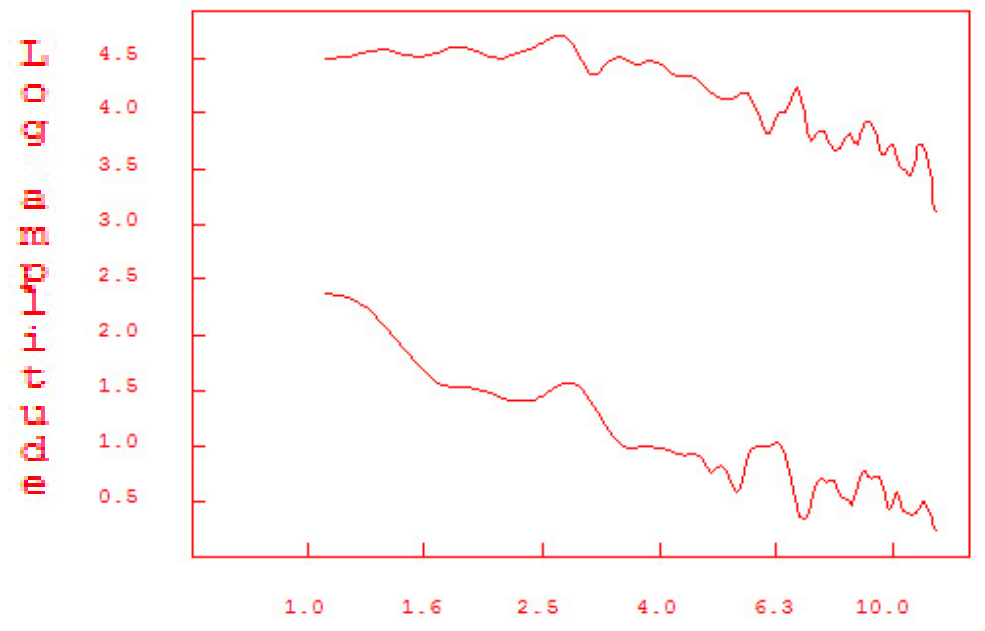

Frequency $\mathrm{Hz}$ 
localizaciones con bajo RMS, si bien el programa hace un control y rechazo de señales saturadas. Para ello se han seleccionado ventanas de 5 y $10 \mathrm{~s}$ de duración a partir del tiempo de llegada de las ondas $\mathrm{P}$ o $\mathrm{S}$ respectivamente, suavizando los bordes de las mismas en un $10 \%$ con una función coseno. En el caso de las ondas S, se ha optado por determinar el inicio de la ventana mediante el cálculo de tiempo de llegada con el modelo de velocidad y coordenadas de acuerdo a los resultados de la localización (Sección 3.4). Se quitaron la respuesta instrumental y el efecto de la expansión geométrica con la forma $r^{n} \operatorname{con} n=-1$ por ser ondas internas. Seguidamente se han calculado los espectros suavizados en 5 etapas, en bandas de frecuencia entre 1 y $12 \mathrm{~Hz}$ o entre 1 y $6 \mathrm{~Hz}$ de acuerdo a las respuestas instrumentales de cada estación, como puede verse en los ejemplos de la Fig. 4.12.

Las salidas del programa qspec incluyen entre otros, dos archivos principales en formato ASCII por estación. Estos archivos contienen, en un caso la información de los parámetros usados para el cálculo y los parámetros epicentrales de cada evento junto a la distancia epicentral y el tiempo de viaje de la onda analizada o los mensajes de error. En el

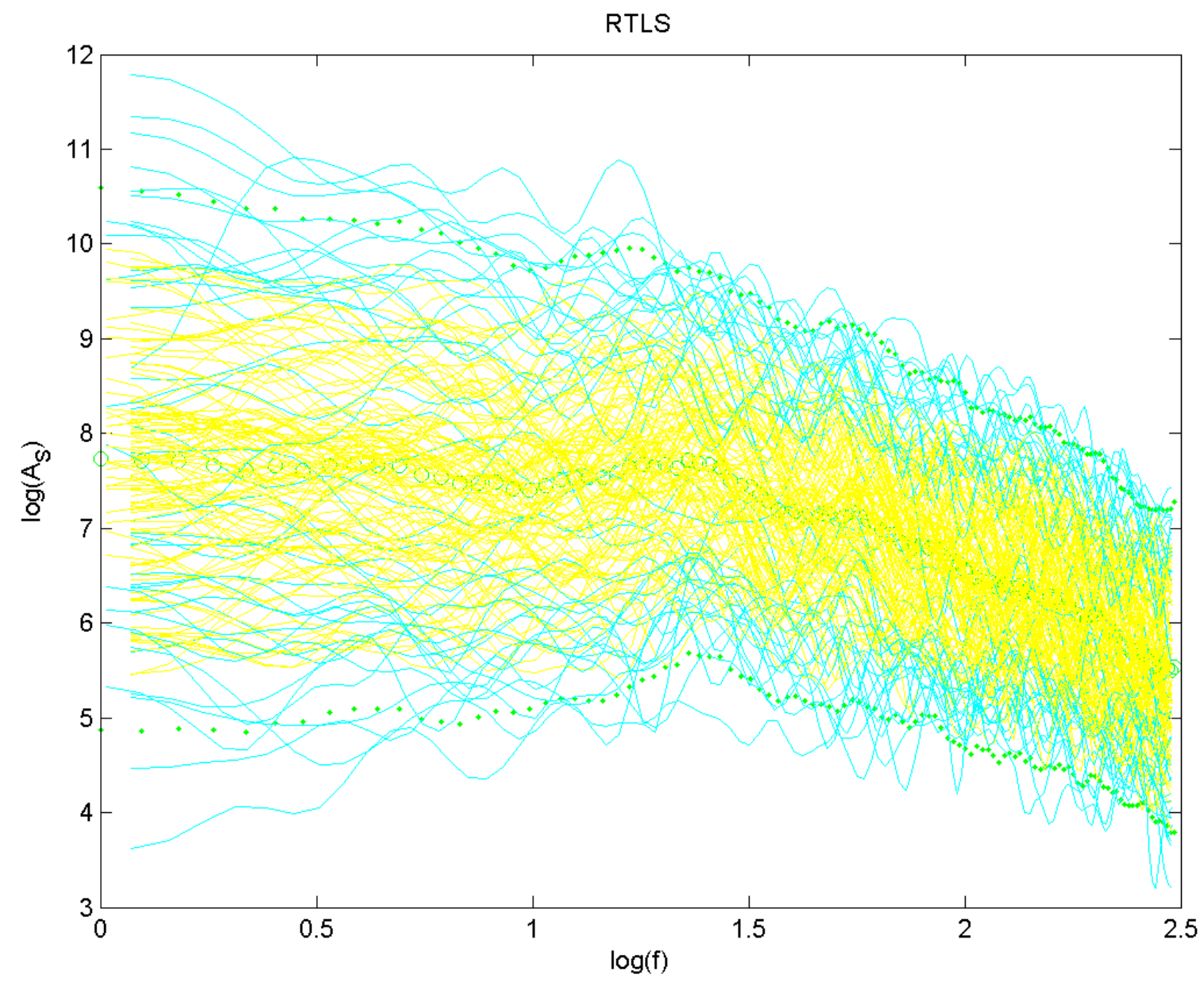

Fig. 4.13.- Selección de espectros por estación a partir de la dispersión del logaritmo natural de los espectros en función de la frecuencia. Círculos verdes indican la mediana; puntos verdes dan rango aceptado con un residuo máximo de $\pm 2 \sigma$, con $\sigma$ la desviación estándar; en cyan se presentan los espectros rechazados y en amarillo los considerados en el ajuste. 
otro archivo, se listan las amplitudes espectrales en formato secuencial a dos columnas (frecuencia y amplitud). De este modo se han calculado 1728 espectros de trayectorias desde la placa de Nazca y 1426 espectros para trayectorias corticales, tanto en ondas P como S.

Se ha desarrollado un código de programación que toma como entradas los listados de archivos de salida del programa qspec e implementa en forma simultánea los ajustes según la aproximación lineal, ME_L, (Giampiccolo et al., 2007) y no lineal, ME_NL, (este trabajo).

En primer lugar el programa compara los espectros por estación y rango de profundidad en función de la frecuencia, procediendo a restringir aún más la selección a fin de reducir su dispersión y lograr un mejor ajuste del método al buscar similitud de espectros en la fuente. Se ha adoptado $\pm 2 \sigma$ como residuo límite, con $\sigma$ la desviación estándar de los logaritmos naturales de los espectros respecto de la mediana de los mismos por estación (Fig. 4.13).

Calculadas las amplitudes espectrales promedios en ventanas de $1 \mathrm{~Hz}$ de ancho centradas en $f_{i}=1.5,2.5,3.5,4.5,5.5 \ldots 11.5 \mathrm{~Hz}$, de acuerdo a la banda de paso de cada estación, se ha
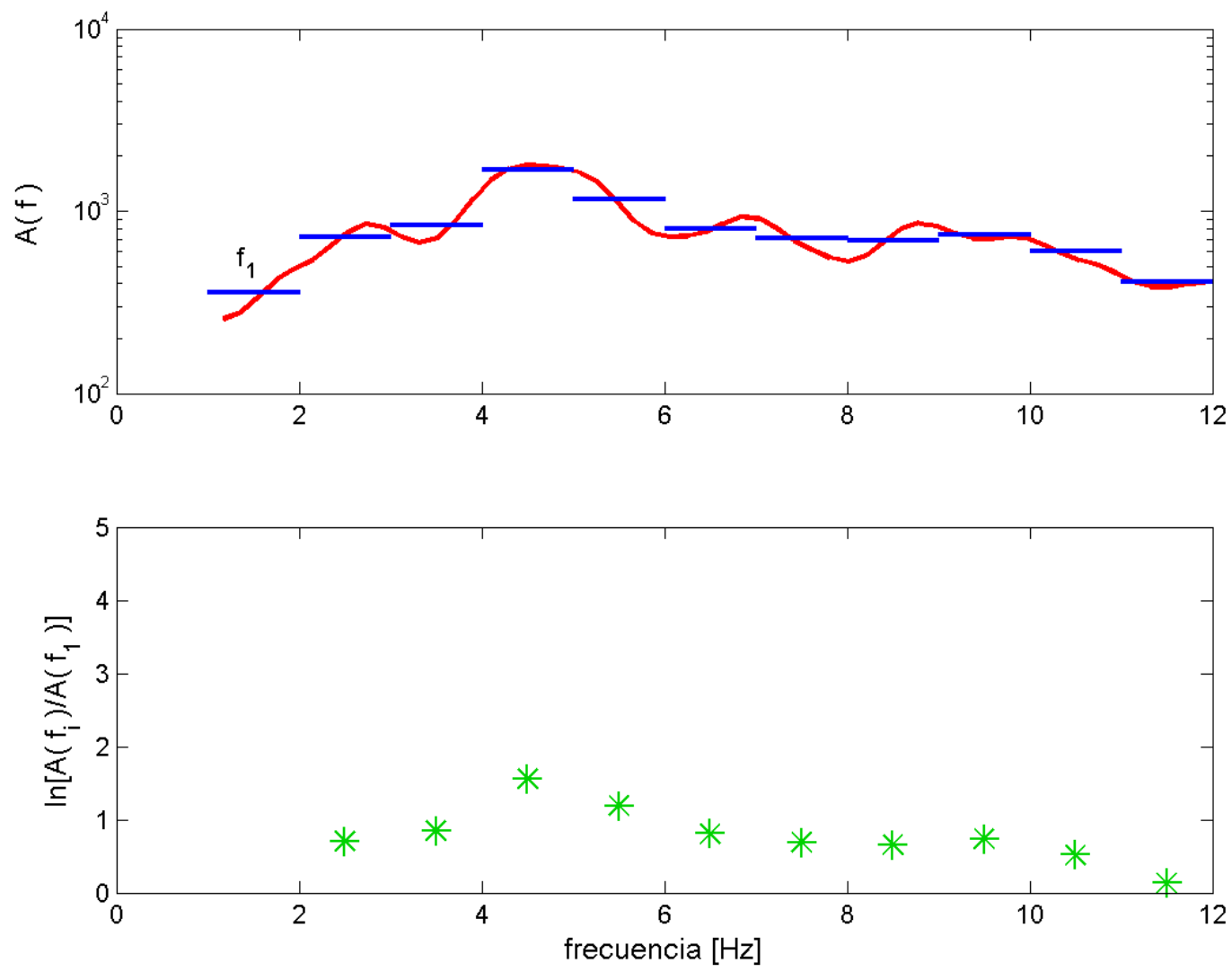

Fig. 4.14.- Ejemplo de aplicación del ME para calcular los cocientes de amplitudes espectrales medias en ventanas de frecuencia de $1 \mathrm{~Hz}$ de ancho. Arriba: espectro de amplitud de una onda P para un evento (rojo) y amplitudes medias (azul) en ventanas de frecuencia centradas en 1.5, 2.5, 3.5.., $11.5 \mathrm{~Hz}, f_{1}$ es la frecuencia de referencia para el cociente espectral. Abajo: logaritmo de los cocientes de amplitudes espectrales promedio en función de la frecuencia central de ventana. 
elegido $f_{1}=1.5$ como frecuencia de referencia para el cálculo de los cocientes $A\left(f_{i}\right) / A\left(f_{1}\right)$ en cada sismograma. En la Fig. 4.14 se ilustra el procedimiento para un espectro de ondas $\mathrm{P}$ de un evento con foco a $102.1 \mathrm{~km}$ de profundidad registrado a $183.1 \mathrm{~km}$ de distancia hipocentral. Los logaritmos de los cocientes entre las amplitudes medias de cada ventana y la frecuencia de referencia, $f_{1}$, se asignan a la frecuencia central de cada ventana como se muestra en la parte inferior de la figura.

Cada valor obtenido se asigna luego al tiempo de viaje de la onda $\mathrm{P}$, que en este caso es

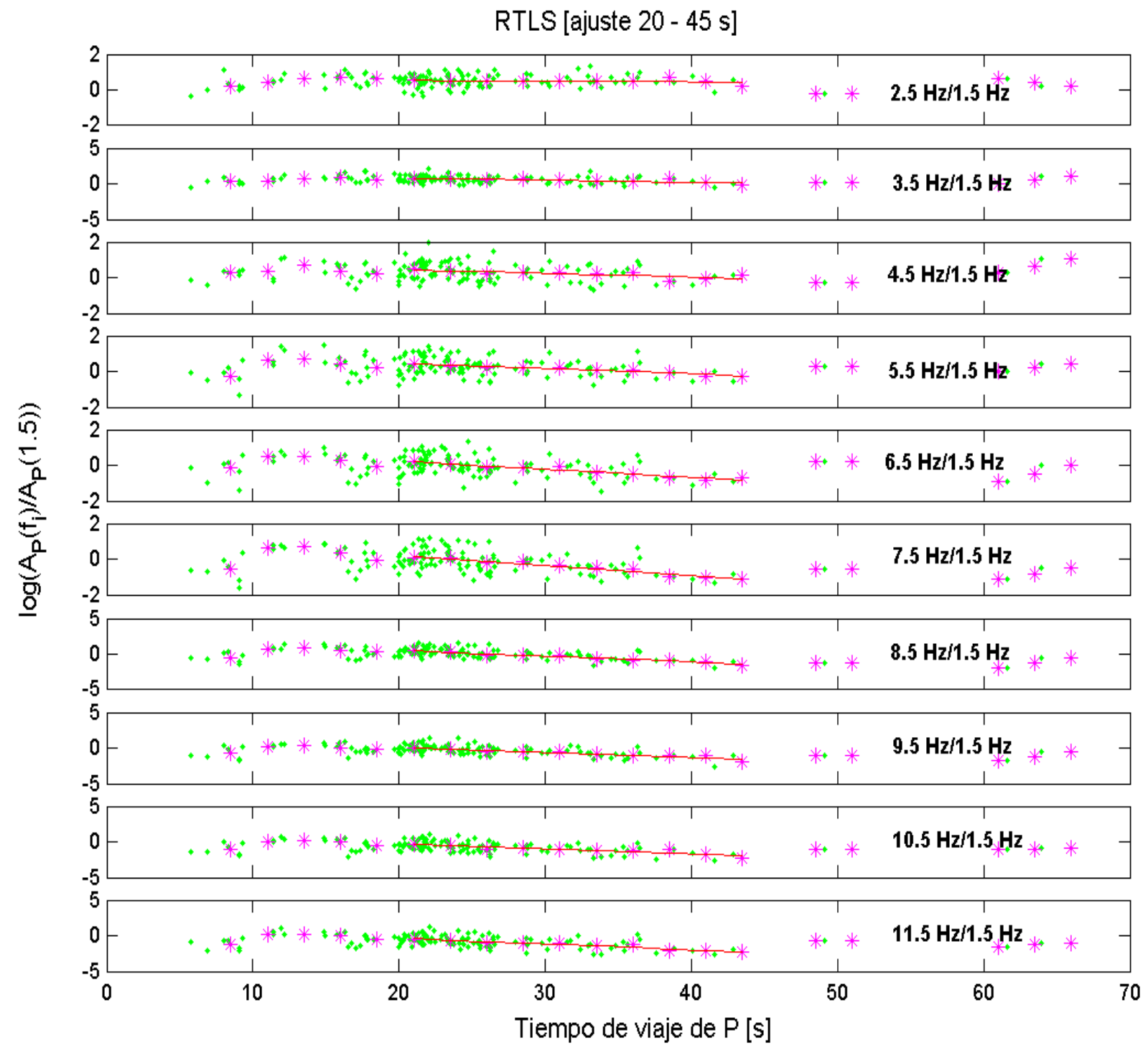

Fig. 4.15 a.- Ejemplo de aplicación del método espectral. Cocientes de amplitudes espectrales en función del tiempo de viaje y de la frecuencia (puntos verdes) para las ondas $P$ de eventos superficiales registrados en la estación RTLS. Los asteriscos fucsia indican promedios en ventanas temporales móviles y las líneas rojas indican ajustes lineales para el intervalo de tiempo seleccionado como se indica en el título de la figura (20-45s). 
CH11 [ajuste 12 - $70 \mathrm{~s}$ ]

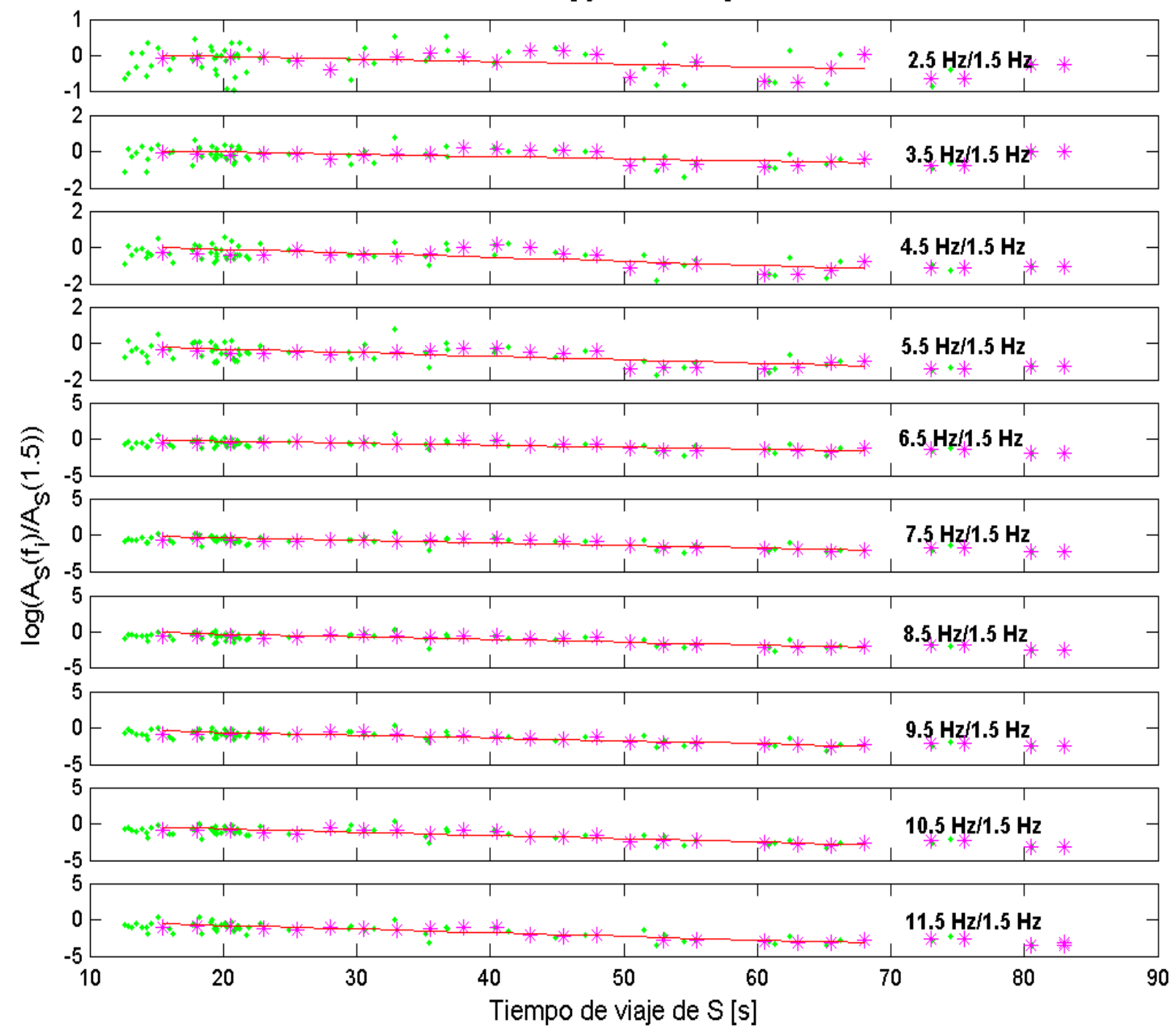

Fig. 4.15 b.- Ejemplo de aplicación del método espectral. Cocientes de amplitudes espectrales en función del tiempo de viaje y de la frecuencia (puntos verdes) para las ondas $S$ de eventos superficiales registrados por la estación CH11. Los asteriscos fucsia indican promedios en ventanas temporales móviles y las líneas rojas indican ajustes lineales para el intervalo de tiempo seleccionado como se indica en el titulo de la figura (12-70s)

de $25.7 \mathrm{~s}$. Seguidamente se construye un número de gráficos igual al número de pares de frecuencias $\left(f_{\mathrm{i}} / f_{1}\right)$ que puedan formarse para cada estación tal como aparece en una ventana emergente de la subrutina (Fig. 4.15 a, b). En cada gráfico se representa el $\ln \left[A\left(f_{i}\right) / A\left(f_{1}\right)\right]$ vs. $t$ para todos los sismogramas, donde $t$ es el tiempo de viaje de la onda analizada.

El programa permite ingresar por teclado varios intervalos de tiempo para el ajuste que se eligen por inspección visual de los gráficos, teniendo en cuenta que se espera observar una relación lineal, según la (2.81). Para cada intervalo de tiempo elegido, se suaviza la distribución de puntos calculando medianas en ventanas temporales móviles de $5 \mathrm{~s}$ con un $50 \%$ de solapamiento y se obtiene la pendiente, $m_{\mathrm{i}}$, de un ajuste lineal ortogonal a la 
distribución de medianas. Los resultados se muestran en el mismo gráfico (Figs. 4.15 a, b) y se guardan en un archivo de texto los intervalos de tiempo seleccionados, el valor de la pendiente ajustada y su coeficiente de correlación.

Según la aproximación no lineal (ME_NL), puede reescribirse la (4.3) suponiendo una variación de $Q$ con la frecuencia de la forma $Q(f)=Q_{0}\left(f / f_{0}\right)^{\alpha}$ para generar una función bivariada en $Q_{0}$ y $\alpha$ con $m_{i}, f_{\mathrm{i}}$ y $f_{1}$ como datos, considerando $f_{0}=1 \mathrm{~Hz}$

$$
\begin{aligned}
& m_{i}=-\pi\left(\frac{f_{i}}{Q_{0} f_{i}^{\alpha}}-\frac{f_{1}}{Q_{0} f_{1}^{\alpha}}\right) \\
& \Rightarrow 0=\frac{m_{i}}{\pi}+\frac{1}{Q_{0}}\left(f_{i}^{(1-\alpha)}-f_{1}^{(1-\alpha)}\right)
\end{aligned}
$$

El problema se reduce entonces a resolver un sistema de ecuaciones no lineales donde el número de ecuaciones varía con el número de ventanas de frecuencia consideradas. En el presente trabajo, dependiendo de la banda de paso de cada estación se ha obtenido un sistema de entre 4 y 10 ecuaciones que se puede resolver por mínimos cuadrados.

$$
F\left(Q_{0}, \alpha\right)=\sum_{i}\left\{\frac{m_{i}}{\pi}+\frac{1}{Q_{0}}\left(f_{i}^{(1-\alpha)}-f_{1}^{(1-\alpha)}\right)\right\}^{2}
$$

La solución puede hallarse por métodos iterativos tipo gradiente (Gauss-Newton) o tipo búsqueda (Búsqueda Exhaustiva). En este caso se ha optado por utilizar una Búsqueda Exhaustiva, evaluando la función $F\left(Q_{0}, \alpha\right)$ en valores de prueba de $Q_{0 \mathrm{j}} \mathrm{y} \alpha_{\mathrm{j}}$, dentro de un rango probable de variación para la región $\left(Q_{0 \mathrm{j}}=[0-500] \mathrm{y} \alpha_{\mathrm{j}}=[0-3]\right)$. Se construye entonces una superficie tridimensional $S\left(Q_{0 \mathrm{j}}, \alpha_{\mathrm{j}}, F\left(Q_{0 \mathrm{j}}, \alpha_{\mathrm{j}}\right)\right)$ cuyo mínimo global puede hallarse en forma aproximada por inspección visual en una gráfica de la superficie y contornos y a su vez determinando las coordenadas $Q_{0 j} *$ y $\alpha_{j} *$ que hacen mínima la tercera dimensión de la matriz $S$. Una vez mostradas todas las soluciones de una estación, el programa solicita al usuario la selección de un par de valores $Q_{0 j}{ }^{*}$ y $\alpha_{j} *$ para almacenar en memoria los valores correspondientes de las pendientes $m_{i}$. Una vez concluido el cálculo por estaciones, el programa estima un valor medio regional de la atenuación, utilizando el valor medio de las pendientes, $m_{i}$, en cada ventana de frecuencias para todas las estaciones y volviendo a minimizar la (4.5).

De esta manera se han obtenido valores de $Q_{\mathrm{o}} \mathrm{y} \alpha$ por estación para intervalos de tiempo seleccionados. En la Fig. 4.16 se presentan ejemplos de las superficies obtenidas con 

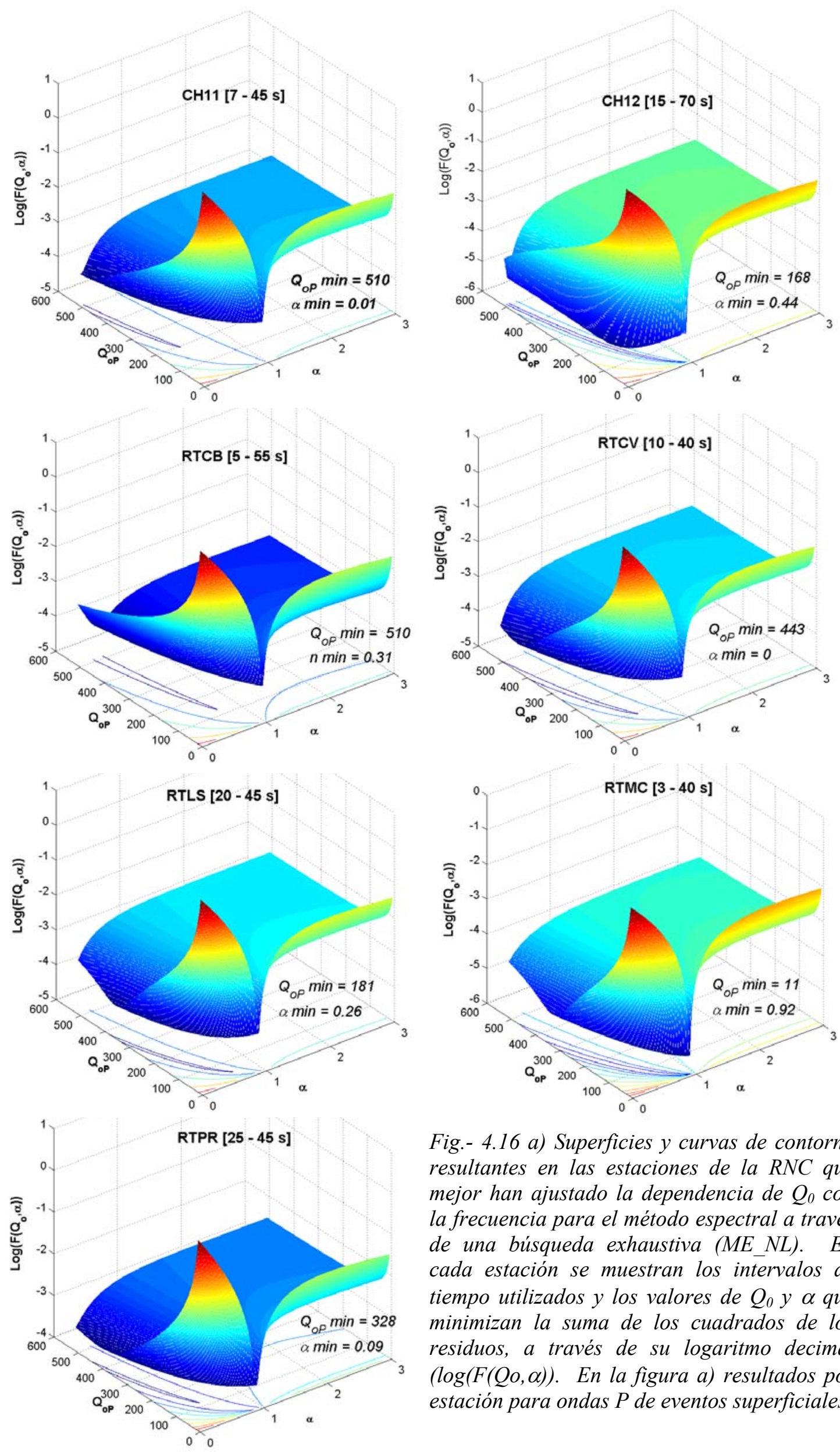

Fig.- 4.16 a) Superficies y curvas de contorno resultantes en las estaciones de la RNC que mejor han ajustado la dependencia de $Q_{0}$ con la frecuencia para el método espectral a través de una búsqueda exhaustiva (ME_NL). En cada estación se muestran los intervalos de tiempo utilizados y los valores de $Q_{0}$ y $\alpha$ que minimizan la suma de los cuadrados de los residuos, a través de su logaritmo decimal $(\log (F(Q o, \alpha))$. En la figura a) resultados por estación para ondas $P$ de eventos superficiales. 

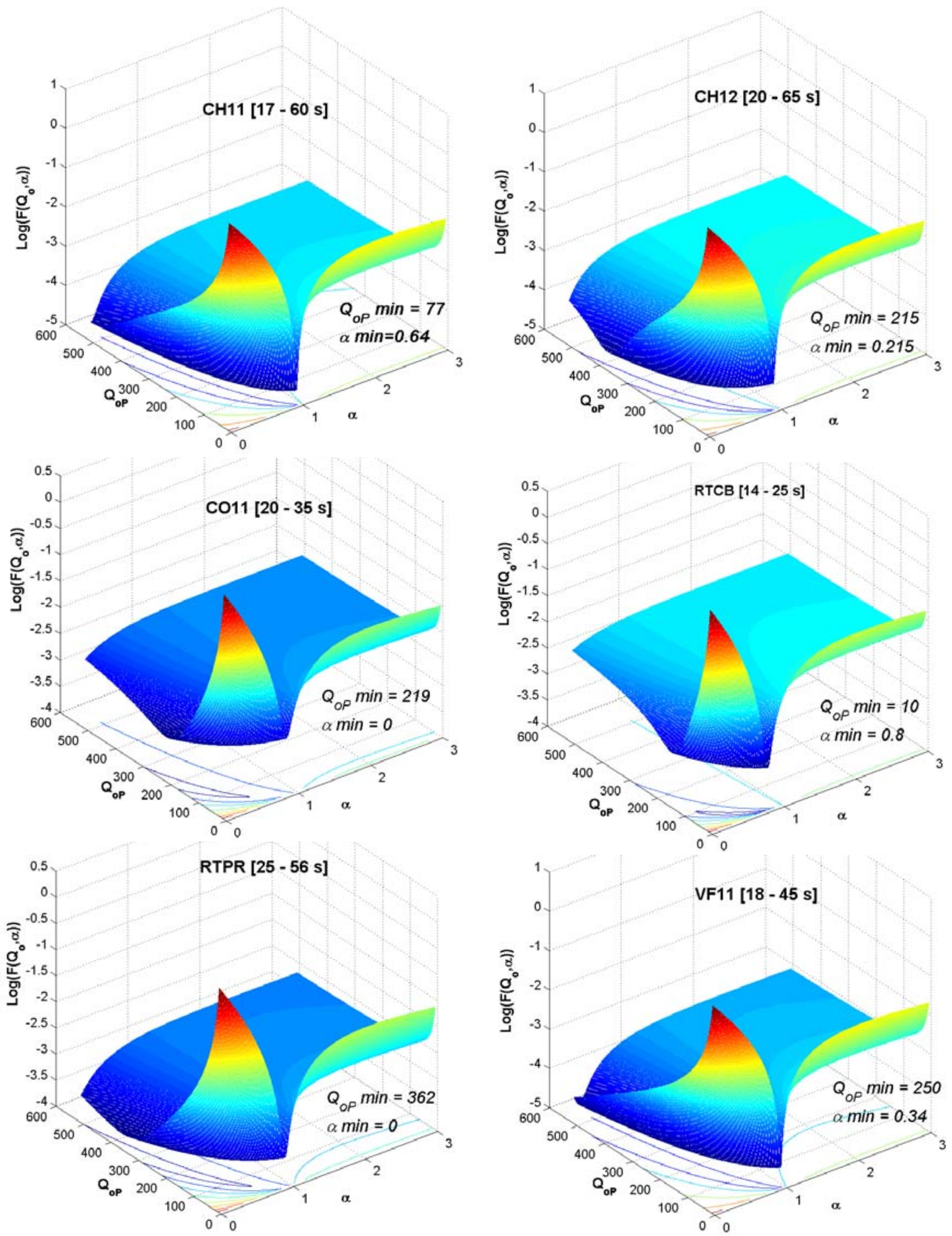

Fig 4.16.- b) resultados de la aplicación del ME_NL por estación para ondas $P$ de eventos intermedios. Ver más explicación en Fig. 4.16 a. 

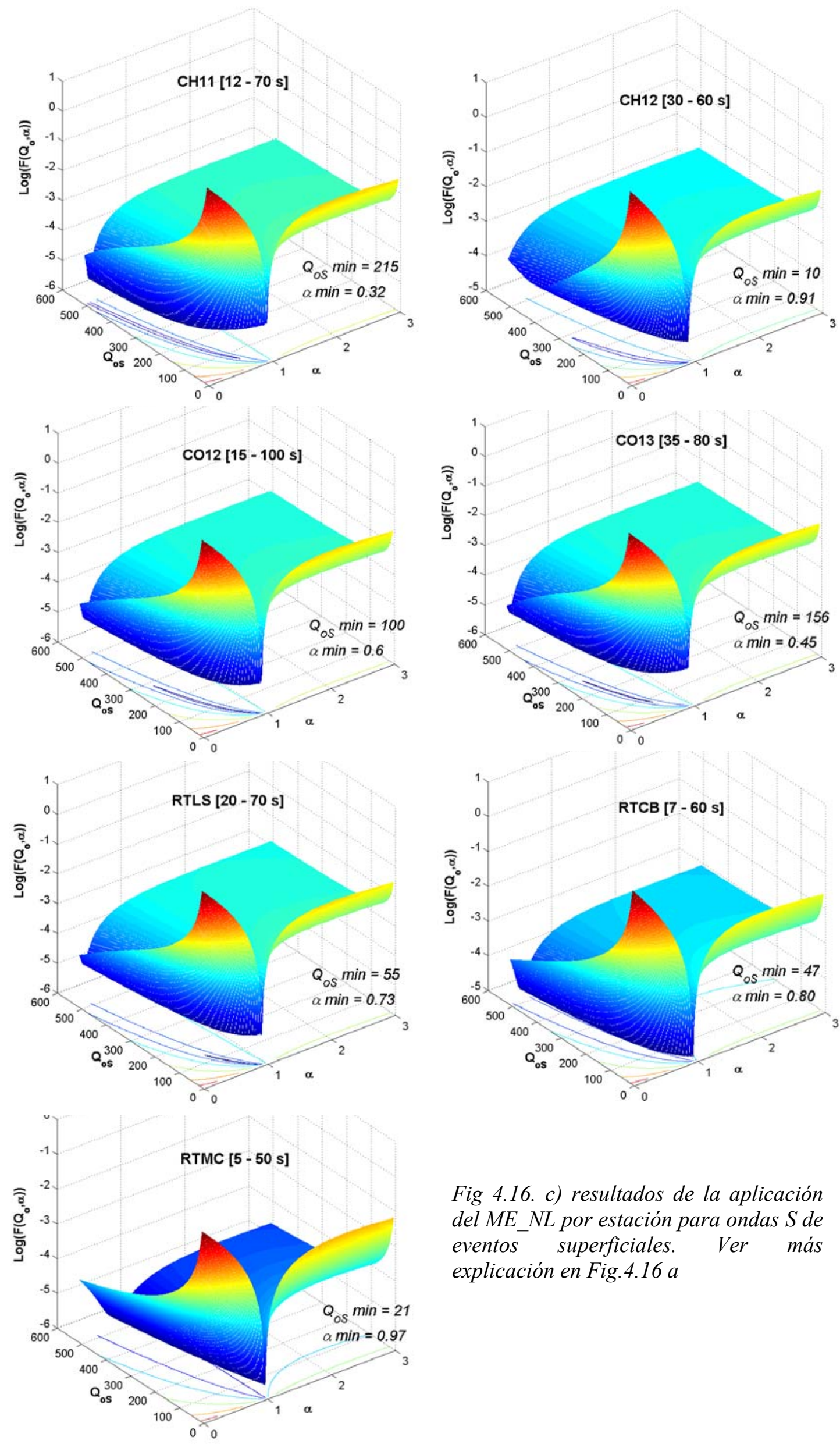

Fig 4.16. c) resultados de la aplicación del ME_NL por estación para ondas $S$ de eventos superficiales. Ver más explicación en Fig.4.16 a 

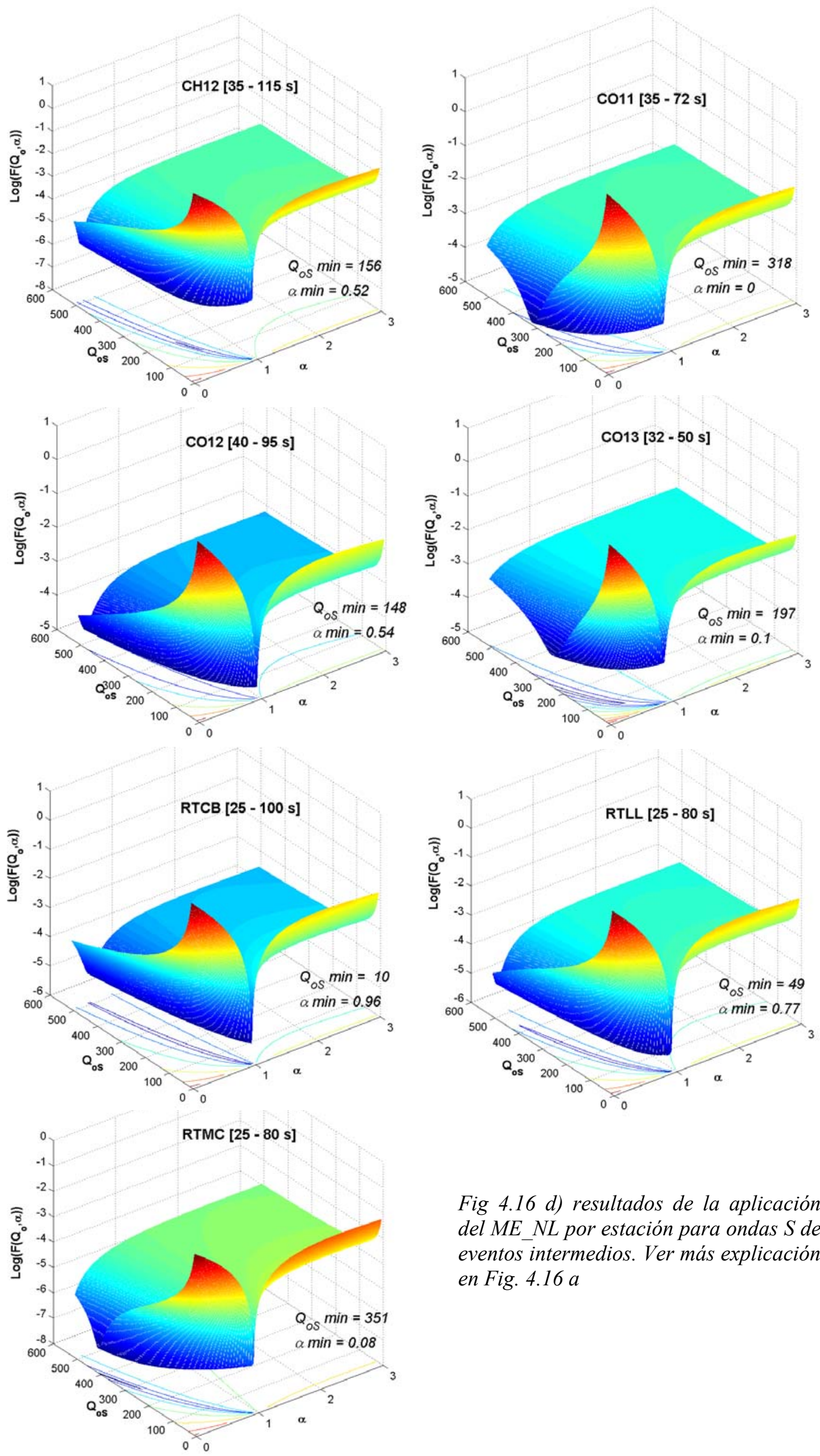

Fig 4.16 d) resultados de la aplicación del ME_NL por estación para ondas $S$ de eventos intermedios. Ver más explicación en Fig. $4.16 a$ 
los valores de prueba $Q_{0 \mathrm{j}}$ y $\alpha_{j}$, indicándose en cada caso el intervalo de tiempo de viaje en el que se ha efectuado el ajuste y los valores de $Q_{0}$ y $\alpha$ que minimizan la suma de los cuadrados de los residuos. Se presentan resultados para aquellos intervalos de tiempo en los que el ajuste lineal de $m_{i}$, ha logrado un coeficiente de correlación superior a 0.5. El rango de variación de $F\left(Q_{0}, \alpha\right)$ dentro del intervalo seleccionado para $Q_{0 \mathrm{j}}$ y $\alpha_{j}$, es muy amplio y por otra parte, el mínimo se encuentra sistemáticamente en un valle alargado de la función. A fin de facilitar la visualización de los mínimos se ha utilizado en este caso, una representación del $\log \left(F\left(Q_{0}, \alpha\right)\right)$ que permite una mayor resolución de la superficie en cercanías del mínimo.

En forma numérica el mínimo de la función $\log \left(F\left(Q_{0}, \alpha\right)\right)$ puede hallarse fácilmente y es único, pero la visualización de las superficies permite ver que la solución obtenida en el rango de prueba no está claramente definida. En todos los casos y dependiendo del margen de error aceptado, las soluciones matemáticamente posibles de $Q_{0}$ y $\alpha$ son numerosas pero mantienen una relación de tipo exponencial decreciente. Cuanto mayor sea $Q_{0}$ menor será el valor de $\alpha$, lo que ya se había observado también en la atenuación de ondas coda.

Por otra parte, comparando los resultados entre las distintas estaciones a intervalos de tiempo aproximadamente similares, para cada onda y rango de profundidad, se observan diferencias notables del valor de $Q_{0}$ obtenido, por lo que resultará interesante realizar más adelante una regionalización de dichos valores. Como era de esperar en este caso, la solución promedio regional calculada a partir de los valores individuales seleccionados no ha alcanzado una buena correlación de los ajustes y por lo tanto tampoco una clara definición de $Q_{0} \mathrm{y} \alpha$.

En segundo lugar, se ha procedido a aplicar el mismo método mediante la adaptación ME_L (Giampiccolo et al., 2007). Tomando nuevamente la (4.3) y suponiendo que $Q$ es constante entre $f_{\mathrm{i}}$ y $f_{1}$, puede escribirse $Q_{i}=Q_{1}=Q$ quedando entonces

$$
m_{i}=-\frac{\pi}{Q}\left(f_{i}-f_{1}\right)
$$

donde cada pendiente permite hallar un valor de $Q$ que se asocia a la frecuencia $f_{i}$ para modelar luego la dependencia de $Q \operatorname{con} f$.

Analizando todos los ajustes lineales del $\ln \left[A\left(f_{i}\right) / A\left(f_{1}\right)\right]$ vs. $t$ en cada estación con coeficiente de correlación mayor que 0.65 , se ha seleccionado el intervalo de tiempo de viaje que haya resultado con mayor correlación promedio para todas las ventanas de frecuencia en 


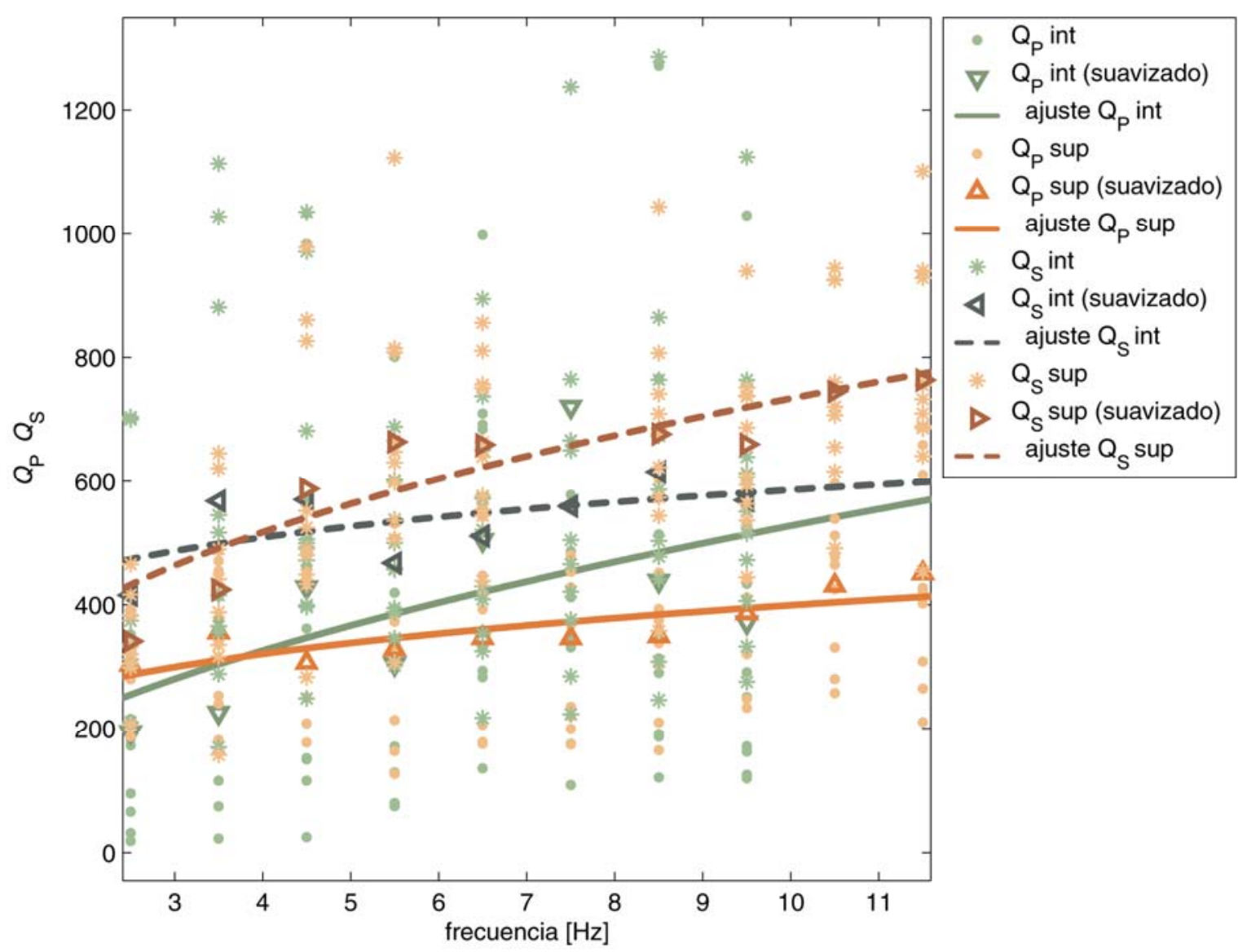

Fig. 4.17.- Ajustes regionales de los valores de $Q_{P}$ y $Q_{S}$ para la Región de Nuevo Cuyo, en función de la frecuencia obtenidos por el ME_L según la (4.6). Se indican valores individuales de $Q(f)$ con puntos (ondas $P, Q_{P}$ ) o asteriscos (ondas $S, Q_{S}$ ); valores suavizados con triángulos y ajustes de la función potencia en líneas sólidas para ondas $P$ y líneas segmentadas para ondas $S$. Se distinguen los resultados para datos de eventos superficiales en gama de rojos y los de eventos intermedios en gama de verdes.

el conjunto de pendientes. Esta condición se ha verificado para intervalos de tiempo promedio entre $16 \mathrm{~s}$ y $42 \mathrm{~s}$ para ondas $\mathrm{P}$ y entre $25 \mathrm{~s}$ y $83 \mathrm{~s}$ para ondas $\mathrm{S}$, teniendo en cuenta todas las estaciones y profundidades.

Calculados los factores de calidad en cada frecuencia para todas las estaciones, la gráfica de $Q$ vs. $f$, muestra una clara dependencia por lo que se ha ajustado la relación $Q_{i}=Q_{0}\left(f_{i} / f_{0}\right)^{\alpha}$ mediante un método de cuadrados mínimos robusto tal como se explicara en la Sección 4.1.1.1 (Fig. 4.17). De esta manera se han obtenido los valores de $Q_{0}$ y $\alpha$ que representan el efecto promedio regional de la atenuación sobre las ondas $\mathrm{P}$ y $\mathrm{S}$ en cada uno de los rangos de profundidad considerados ( $\mathrm{h} \leq 50 \mathrm{~km} \mathrm{y} \mathrm{h}>50 \mathrm{~km}$ ). La Tabla 4.3 contiene dichos valores de $Q_{0}$ y $\alpha$, resultantes del ajuste de la función potencia a los datos suavizados de cada grupo de eventos como se muestra en la Fig. 4.17. La bondad de los ajustes resulta mejor a profundidades corticales como puede comprobarse observando la dispersión de los valores suavizados. 


\begin{tabular}{|c|c|c|c|c|c|c|}
\hline ME_L & \multicolumn{3}{|c|}{ Ondas P } & \multicolumn{3}{c|}{ Ondas S } \\
\hline \multirow{2}{*}{ Profundidad } & $\boldsymbol{Q}_{\mathbf{0 P}} \pm \mathbf{e} \mathbf{Q}_{\mathbf{0 P}}$ & $\boldsymbol{\alpha}_{\mathbf{P}} \pm \mathbf{e} \boldsymbol{\alpha}_{\mathbf{P}}$ & $\rho_{\mathbf{p}}$ & $\begin{array}{c}\boldsymbol{Q}_{\mathbf{0}} \pm \\
\mathbf{e} Q_{\mathbf{0 S}}\end{array}$ & $\alpha_{\mathbf{S}} \pm \mathbf{e} \alpha_{\mathbf{S}}$ & $\rho_{\mathbf{S}}$ \\
\hline $\mathbf{0 - 5 0} \mathbf{~ k m}$ & $230 \pm 21$ & $0.24 \pm 0.04$ & 0.56 & $305 \pm 28$ & $0.38 \pm 0.04$ & 0.78 \\
\hline $\mathbf{5 0 - 1 5 0} \mathbf{~ k m}$ & $157 \pm 65$ & $0.52 \pm 0.21$ & 0.29 & $411 \pm 41$ & $0.15 \pm 0.06$ & 0.22 \\
\hline
\end{tabular}

Tabla 4.3.- Valores promedio regionales de $Q_{0} y \alpha$, sus errores y coeficientes de correlación del ajuste de la relación $Q(f)=Q_{0}\left(f / f_{0}\right)^{\alpha}$ para el método espectral (ME L) sobre ondas $P$ y $S$ según se muestra en la Fig. 4.17.

\subsubsection{INTERPRETACIÓN}

Como se ha visto anteriormente, las diferencias laterales halladas entre los valores de $Q$ por estación, amerita una interpretación en planta a fin de poder relacionarlas con la geodinámica de la región.

Utilizando los ajustes no lineales de $Q_{0}$ por estación, con coeficientes de correlación superiores a 0.5 , bien definidos y dentro de valores tectónicamente razonables (Fig. 4.16), se han confeccionado mapas de $Q_{0}$ espectral ajustando una superficie cartesiana 2-D mediante interpolación a trozos (splines) con funciones de Green, curvatura mínima y un espaciado de 5' (Fig. 4.18). En el caso de $Q_{0 \mathrm{P}}$ superficial (Fig. 4.18 a) se observa un mínimo alrededor de la Sierra Pie de Palo, hacia el este de San Juan, donde se concentra la mayor actividad sísmica superficial y dos máximos de $Q_{0 \mathrm{p}}$, uno de gran desarrollo superficial hacia el sur de Valle Fértil en el sudeste de la región, allí donde limitan San Luis, San Juan y Mendoza y otro de menor extensión hacia el centro-sur de San Juan. Si se observan las distribuciones de $Q_{0 \mathrm{~S}}$ superficial (Fig.4.18 c) se destaca un gran mínimo que se extiende de sudoeste a noreste de la región acompañando la trayectoria de la dorsal Juan Fernández subducida y en coincidencia con el desarrollo de la Sierra Pie de Palo. En el caso de las profundidades mayores que $50 \mathrm{~km}$ (Fig. 4.18 d) un mínimo muy definido de $Q_{0 \text { s }}$ coincide con la región de mayor concentración de sismicidad a profundidades intermedias, bajo la Precordillera y al oeste de la Sierra Pie de Palo, mientras que hacia el este se acentúa también el máximo de $Q_{0 \mathrm{~s}}$ que se había observado a menor profundidad. Por lo tanto, del análisis de la Fig. 4.18 puede concluirse que se observa la presencia de máximos de atenuación, tanto para ondas $\mathrm{P}$ como para ondas $\mathrm{S}$ en coincidencia con las regiones de mayor concentración de sismicidad tanto a profundidades superficiales como intermedias. Del mismo modo, coinciden para ambas ondas los mínimos de atenuación con una zona bien definida hacia el sudeste de la región. La distribución de $Q_{0 \text { P }}$ a profundidades intermedias (Fig. 4.18b), no muestra importantes variaciones que puedan 

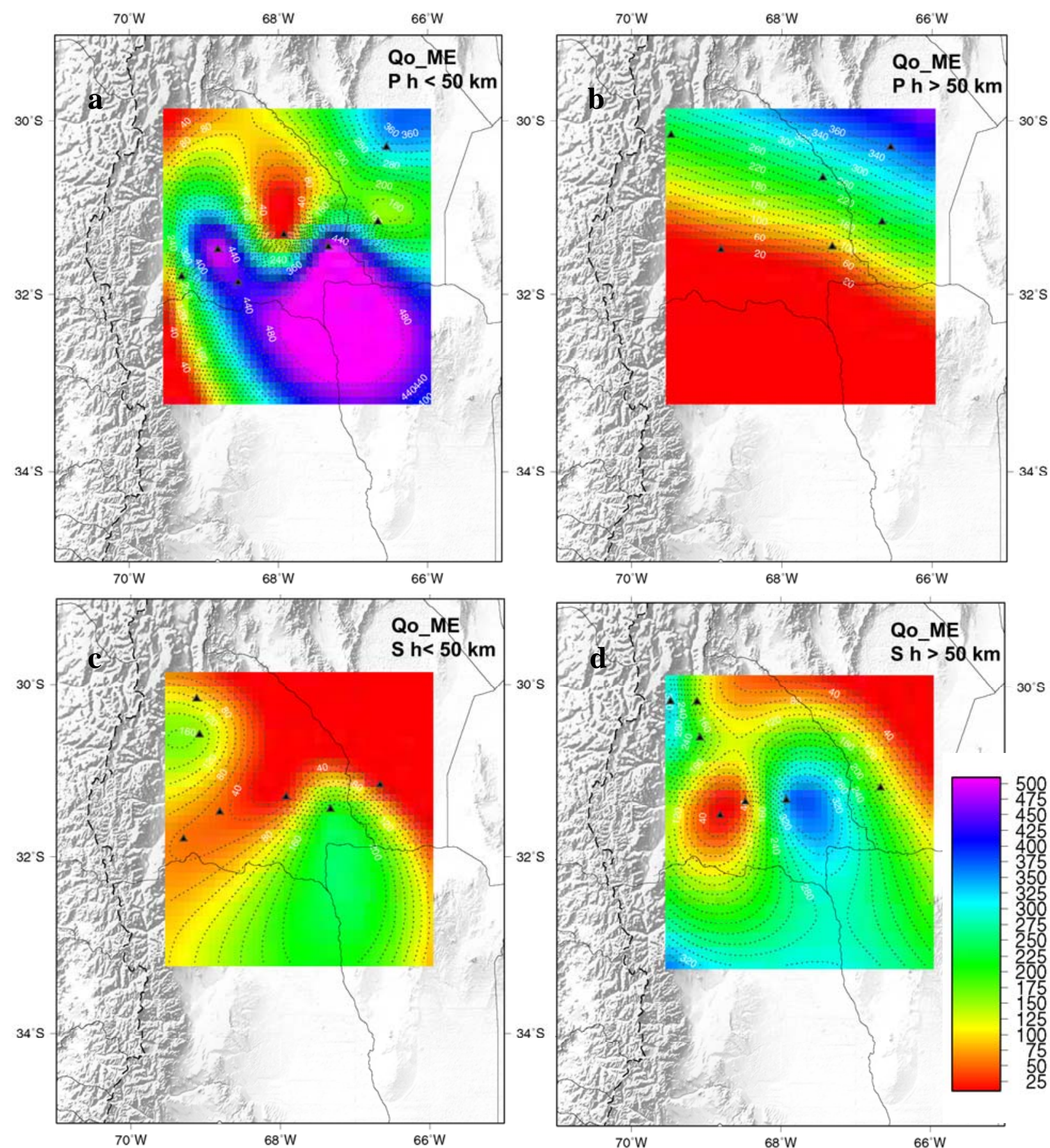

Fig. 4.18.- Mapas de distribución del factor $Q_{0}$ para el $M E \_N L$ según valores obtenidos por

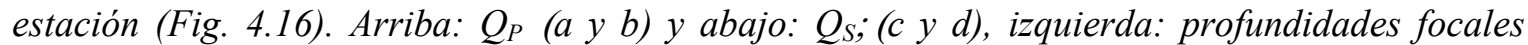
menores que $50 \mathrm{~km}$ y derecha: profundidades focales mayores que $50 \mathrm{~km}$. Los triángulos indican estaciones utilizadas a las que se asignaron los valores de $Q_{0}$ para ajustar una superficie cartesiana 2-D mediante interpolación a trozos con funciones de Green, curvatura mínima y un espaciado de 5'. Se muestran contornos en el rango correspondiente a cada grupo de datos.

relacionarse con la geodinámica de la región.

Los valores de $Q_{0}$ regionales obtenidos mediante el ajuste lineal son razonables para una región de gran actividad sísmica como puede verse comparando con los valores obtenidos por otro autores en diversas regiones del mundo (Fig. 2.21 y 2.22). Comparando la dependencia de $Q$ con la frecuencia para ondas P, S y coda (Fig. 4.5 y Tabla 4.3) a nivel regional, se 
observa que la atenuación de las ondas coda crece más rápidamente con la frecuencia que la de las ondas directas. Sin embargo, para valores obtenidos por estación con el ME_NL (Fig. 4.16), la variación de la atenuación de $\mathrm{S}$ en trayectorias superficiales, resulta mayor que la del resto y se aproxima al comportamiento de las ondas coda.

De la Fig. 4.17, se deduce que la atenuación de ondas $\mathrm{P}$ es mayor que la atenuación de ondas S en el intervalo de frecuencias analizadas en la región. En ambos tipos de ondas, los ajustes robustos regionales del $Q$ espectral no mostraron una clara variación de la atenuación con la profundidad. Existe sin embargo un detalle interesante y es que los valores de $Q_{\mathrm{P}}$ y $Q_{S}$ a profundidades mayores que $50 \mathrm{~km}$, convergen para frecuencias altas mientras que divergen a profundidades menores que $50 \mathrm{~km}$. Si se analiza la relación $Q_{\mathrm{P}}{ }^{-1} / \mathrm{Q}_{S}{ }^{-1}$, para ambos rangos de profundidad y en función de la frecuencia, se ve que $Q_{\mathrm{P}}{ }^{-1} / \mathrm{Q}_{S}{ }^{-1}>1$ para todo el intervalo (Fig. 4.19) y además ambos cocientes coinciden a una frecuencia entre 3.5 y $4 \mathrm{~Hz}$. Es decir, que a una dada frecuencia la atenuación superficial resulta igual a la atenuación intermedia en ambos tipos de ondas internas. Puede observarse una cierta estabilidad en función de la frecuencia para la atenuación de ondas $\mathrm{P}$ en trayectorias corticales $\mathrm{y}$ de ondas $\mathrm{S}$ en trayectorias que involucran el manto. La variedad de escalas de estructuras corticales así como las diferencias existentes en cuanto a la composición de los terrenos que la conforman causaría la mayor estabilidad de la atenuación de ondas $\mathrm{P}$ en corteza. La mayor homogeneidad a profundidades intermedias y la liberación de fluidos por la dinámica de la subducción sería el origen de los efectos de la atenuación en las ondas $\mathrm{S}$.

Al comparar las adaptaciones lineal y no lineal del método de Tsujiura (1966), puede decirse que el ME_NL resulta teóricamente más justificado ya que en ningún momento se plantea la independencia de $Q$ con la frecuencia. Las variaciones laterales observadas en los resultados obtenidos por estación con el ME_NL han permitido una regionalización de los valores de atenuación para ondas $\mathrm{P}$ y S, lo que no ha podido lograrse con el ME_L. Sin embargo, con el ME_L se han obtenido valores razonables de la atenuación promedio regional.

A partir de las consideraciones realizadas en ambas implementaciones y de los resultados obtenidos se observa que el ME_NL logra mejores ajustes por estación que el ME_L. Disponiendo de datos seleccionados específicamente para este método, en lo que se refiere a similitud de mecanismos de fuente e implementando algún otro método iterativo en la resolución del problema por mínimos cuadrados, podrían lograrse aún ajustes más definidos. Otro factor importante es el cálculo de los espectros de desplazamiento, pues en 
este trabajo se ha contado con datos de estaciones de corto período trabajando en rangos de frecuencia limitados por los filtros analógicos. El análisis visual de las frecuencias de corte espectrales, ha mostrado que en ciertos eventos las mismas podrían ser cercanas a las frecuencias de corte de los filtros analógicos, por lo que la recuperación de los espectros de desplazamiento podría no ser completa. Dado que se han encontrado algunos inconvenientes en el cálculo de espectros dentro de la versión del qspec utilizada y vista la dispersión de los ajustes del ME_NL, se observa que en futuros análisis sería conveniente incorporar el cálculo de los mismos al código de programación del método dando a la vez la posibildad de modificar parámetros en su determinación.

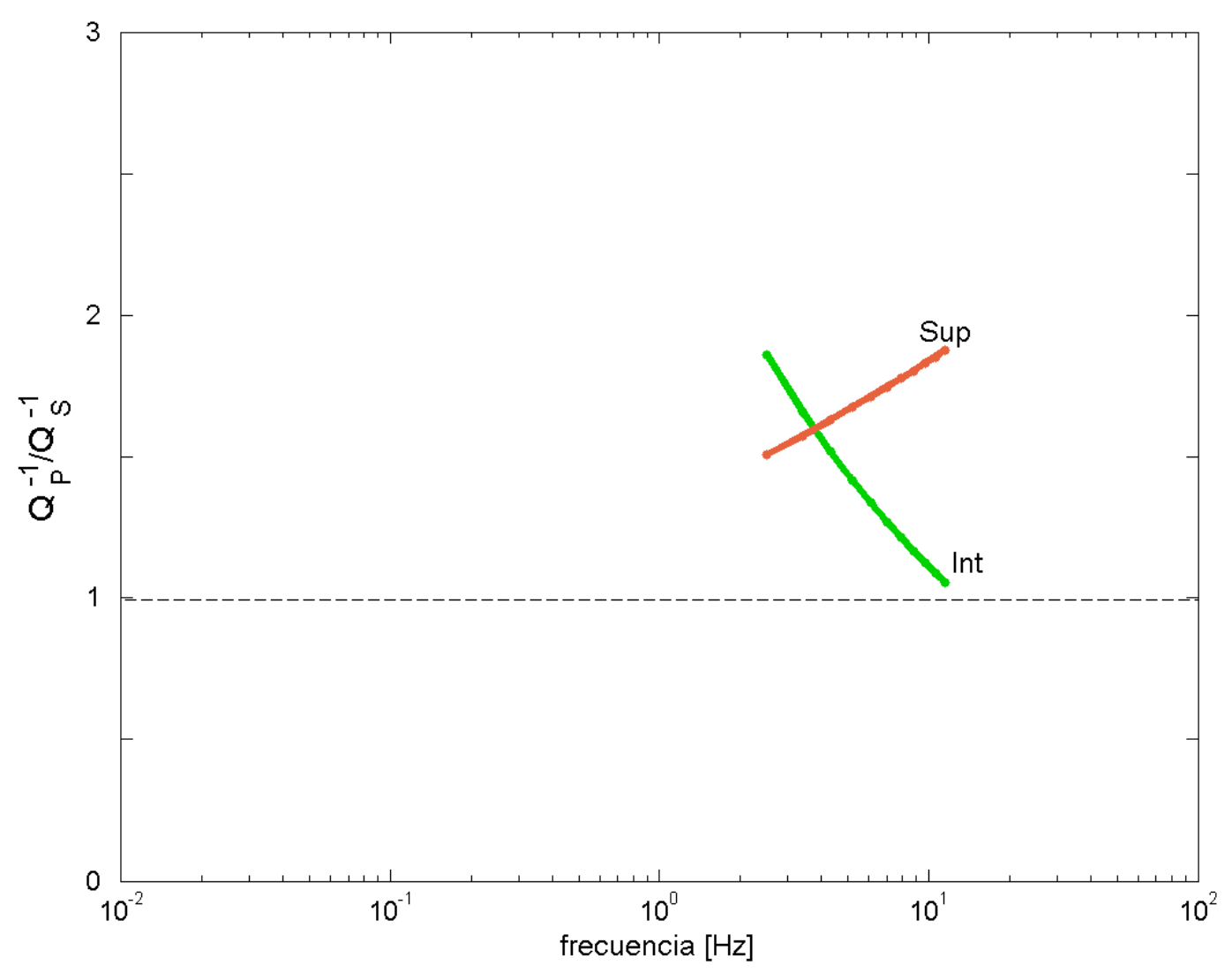

Fig. 4.19.- Relación $Q_{P}^{-1} / Q_{S}^{-1}$ para la región de Nuevo Cuyo obtenida en este estudio mediante el $M E \_L$. Resultados RNC a profundidades corticales se muestran en línea sólida roja y a profundidades de hasta $150 \mathrm{~km}$, en línea sólida verde. La escala del gráfico permite su comparación con la Fig. 2.24 que muestra los valores obtenidos en otras regiones del mundo. 


\subsubsection{MÉTODO DEL ANCHO DEL PULSO DE P (APP)}

\subsubsection{APLICACIÓN Y RESULTADOS}

La relación 2.71, establecida empíricamente por Gladwin y Stacey (1974b), permite determinar el factor de calidad de las ondas longitudinales a partir de la dependencia lineal entre el ancho del primer pulso de $\mathrm{P}$ en la estación y el tiempo de propagación. El valor de $Q_{P}$ obtenido a partir del ajuste por mínimos cuadrados de la (2.71) a los datos, se considera independiente de la frecuencia ya que se analiza la forma de onda tal cual es registrada y en el dominio del tiempo.

Para la medición del ancho del pulso de $\mathrm{P}$ se ha elaborado un código en lenguaje Basic (Ibáñez y Badi) que lee en forma automática el listado de sismogramas, así como los parámetros del hipocentro, los datos de registración y el tiempo de llegada de $\mathrm{P}$, necesarios para la identificación del primer pulso. Los sismogramas en velocidad se ingresan en formato ASCII de una columna, a razón de un archivo por evento y por estación. En primer lugar se corrige el sismograma por línea base, eliminando el valor medio de las amplitudes en la traza completa para establecer la línea de amplitud cero. Luego se muestra una ventana de $3 \mathrm{~s}$ centrada en el inicio de la onda $\mathrm{P}$, donde el usuario debe identificar el inicio y fin del primer pulso o rechazar el mismo si no se ajusta al criterio establecido para la selección (Fig. 2.18). Para medir la duración del pulso se prolonga linealmente la máxima pendiente positiva del primer pulso de $\mathrm{P}$ hasta intersectarla con la línea de amplitud cero marcando este punto como inicio. El ancho del pulso es el tiempo transcurrido desde dicha intersección hasta el primer cruce real de la traza con la línea de amplitud cero después de haber alcanzado su máximo. Sólo se deben seleccionar pulsos con pendientes y máximos claros. La duración del pulso en segundos y los parámetros del evento se graban en un archivo de salida.

El método requiere que el ancho del pulso en la fuente sea similar para todos los eventos, lo que es lo mismo que exigir ondas $\mathrm{P}$ con patrones de radiación similares. Para ello deben seleccionarse eventos con mecanismos focales similares. En caso de no contar con los mecanismos focales, el método puede aplicarse garantizando una misma zona sismogénica y un rango de magnitudes donde el ancho del pulso sea independiente de las mismas. Esta última condición permite suponer que las variaciones del ancho del pulso en la propagación, es decir su contenido en frecuencia, no guardan relación con el espectro de la fuente.

Habiendo calculado la magnitud coda (Mc) de los eventos mediante la (3.3), se han representado las medidas del ancho del pulso en función de Mc (Fig. 4.20) para eventos en la placa Sudamericana y en la placa de Nazca. Se observa que ambas variables no guardan una 

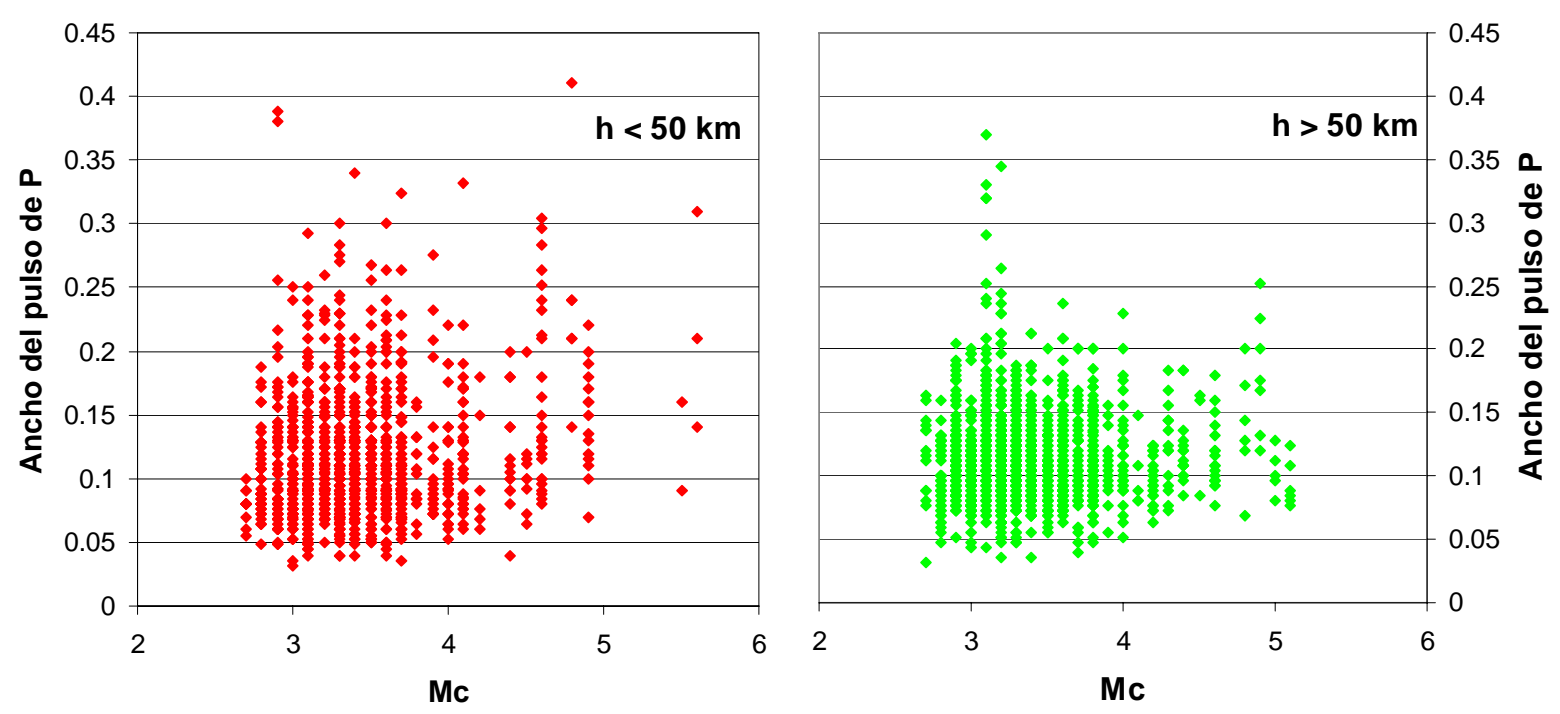

Fig. 4.20.- Distribución de duraciones del ancho del primer pulso de P en función de la Magnitud de coda (Mc) para eventos superficiales (izquierda: $0-50 \mathrm{~km}$ de profundidad) y eventos intermedios (derecha: $50-155 \mathrm{~km}$ de profundidad).

clara relación por debajo del valor $\mathrm{Mc}=4.2$ mientras que para valores de $\mathrm{Mc}$ mayores se puede ver una cierta dependencia directa. Por esta razón se ha decidido restringir el ajuste del factor de calidad a aquellos eventos con magnitudes en el rango 2.7-4.2.

A partir de la distribución de focos (Fig. 3.20) en planta y perfil, se observan dos nucleamientos bien definidos en los intervalos de profundidades 0-40 km y 90-120 km con proyecciones en superficie en la región definida por $31^{\circ}-32^{\circ} \mathrm{S}$ y $67^{\circ}-69^{\circ} \mathrm{O}$ para los eventos superficiales y en la región $31-32^{\circ} \mathrm{S}$ y $68-70^{\circ} \mathrm{O}$ para los eventos intermedios (rectángulos grises en Fig. 4.24). Se han definido entonces dos zonas sismogénicas, una superficial y otra intermedia, en cada una de las cuales los eventos se suponen con mecanismos focales similares entre sí.

En la Fig 4.21 se presentan los valores del ancho del pulso medidos en cada traza, en función del tiempo de viaje de la onda P. A fin de ajustar la (2.71) a los datos y dada la dispersión de los mismos se ha procedido a aplicar un ajuste por cuadrados mínimos robusto. Cabe aclarar que un ajuste por cuadrados mínimos convencional resulta en los mismos coeficientes de modo que este método solo mejora las bondades del ajuste. Para ello se han efectuado promedios de los valores individuales en ventanas temporales móviles de 4 segundos de duración y 50\% de solapamiento. Se han obtenido las pendientes y ordenadas al origen de las rectas que se muestran en la Fig. 4.21, mediante un ajuste lineal por mínimos cuadrados robusto sobre los valores promediados en función del tiempo de viaje. Utilizando 


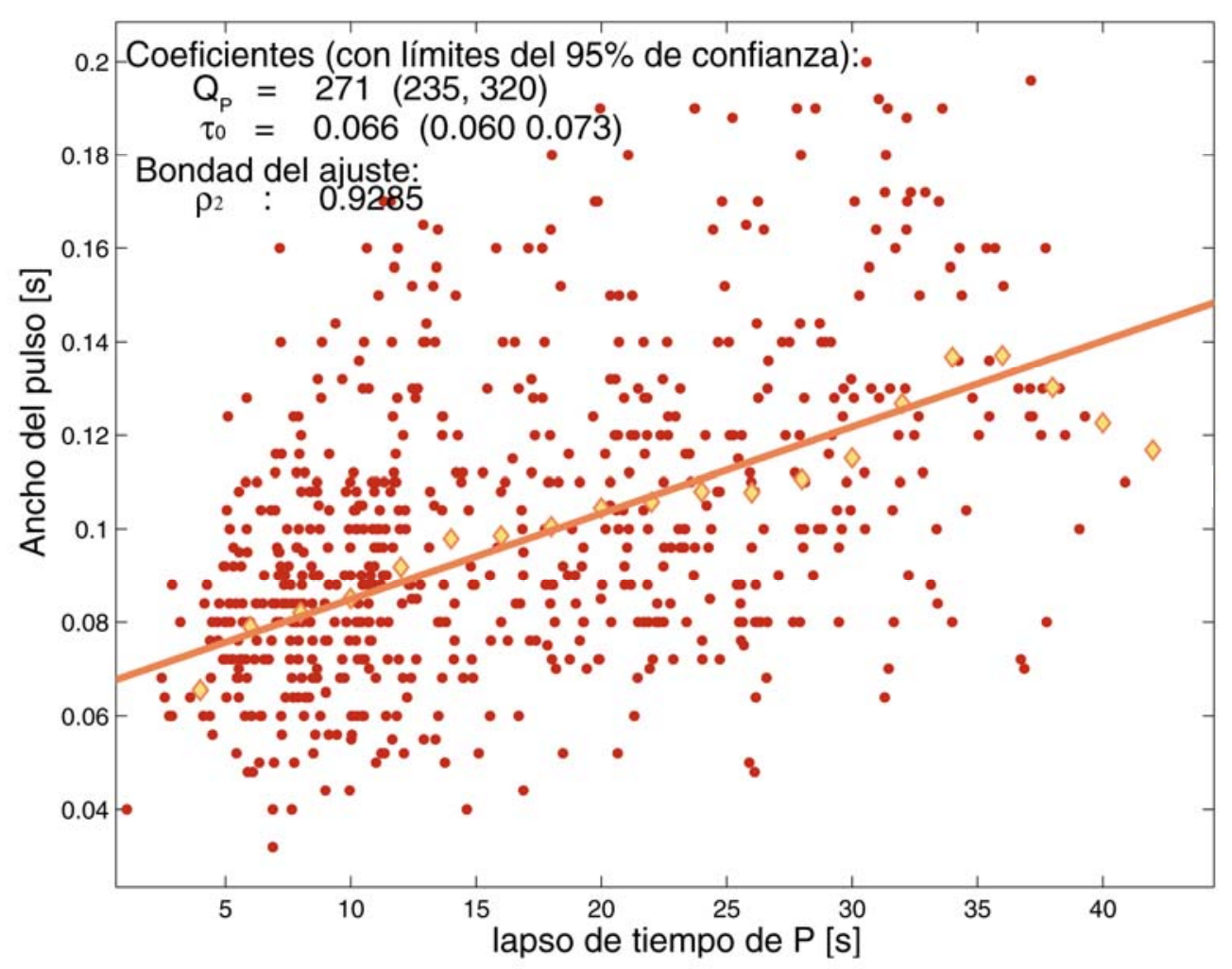

Fig. 4.21.- Distribución de la duración del primer pulso de P en función del lapso de tiempo para eventos con profundidades focales en el rango 0-40 km (arriba en puntos rojos) y 90-120 km (abajo en puntos verdes). Las líneas sólidas representan los ajustes por mínimos cuadrados de la (2.71) a los promedios temporales en ventanas móviles (rombos amarillos). En ambos gráficos se muestran los coeficientes del ajuste con límites de confianza del 95\% y el coeficiente de determinación.

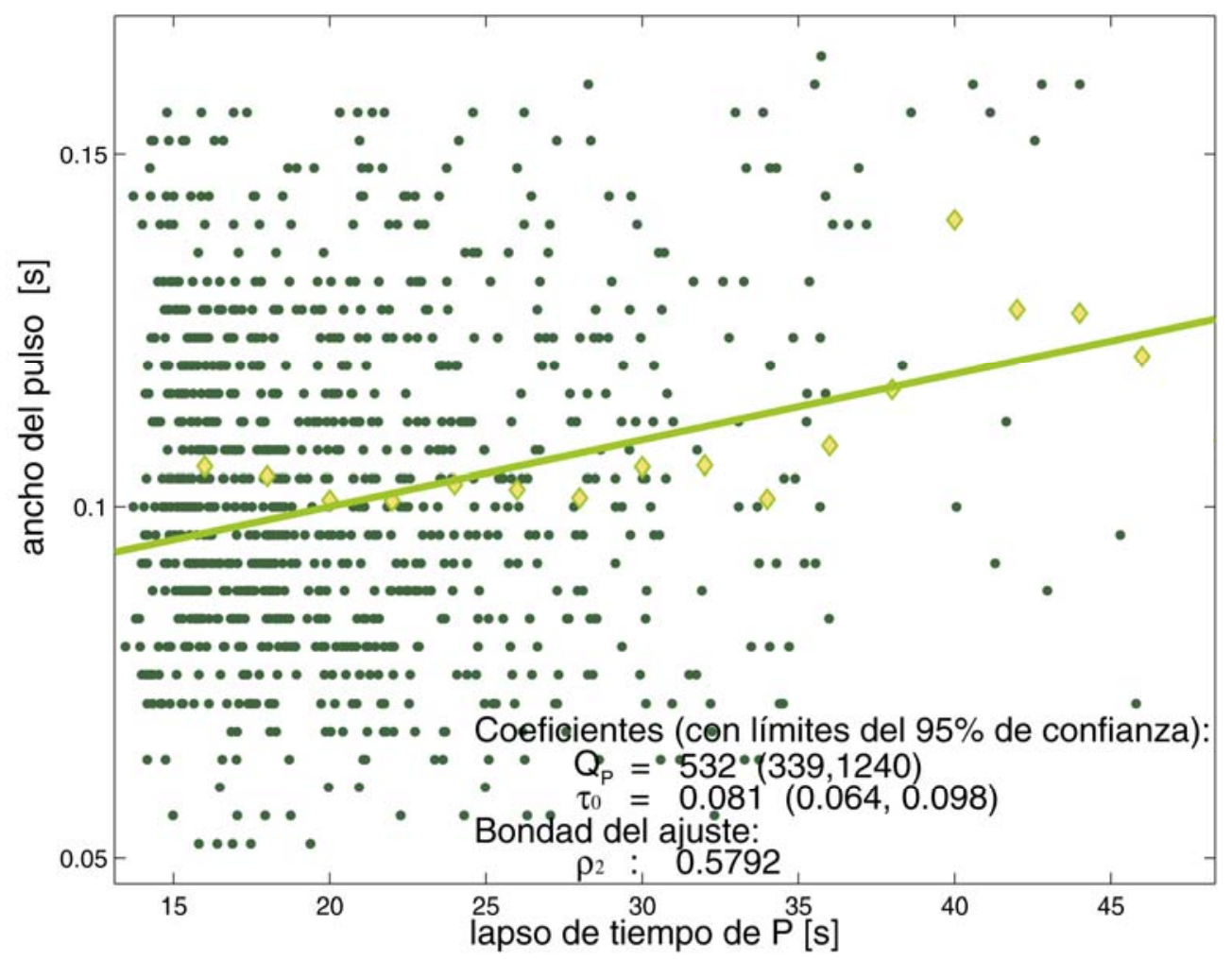


la (2.71) se han calculado los valores de $Q_{P}$ y $\tau_{0}$ asociados a la pendiente y la ordenada al origen del ajuste.

El valor de la constante $\mathrm{C}$, que relaciona el inverso de la pendiente con $Q_{P}$, ha sido experimentalmente estimado por diversos autores (Kjartansson, 1979, Blair y Spathis, 1982, Gladwin y Stacey, 1974b). Dado que se trata de registros en velocidad, en este trabajo se ha adoptado un valor de $C$ igual a 0.5 como fuera estimado por Gladwin y Stacey (1974b) y de acuerdo con el análisis realizado por Martínez Arévalo (2005). Jongmans (1991) recomendó el uso del método a distancias mayores que 1.2 veces las longitudes de onda analizadas.

Teniendo en cuenta la banda de paso de las estaciones utilizadas (en general entre 1 y 12 $\mathrm{Hz})$ y las velocidades medias de las ondas $\mathrm{P}$ en las trayectorias consideradas $(6.5 \mathrm{~km} / \mathrm{s}$ para el intervalo [0-40] $\mathrm{km}$ y $8.2 \mathrm{~km} / \mathrm{s}$ para [0-120] km), las distancias mínimas según lo recomendado son de $7.8 \mathrm{~km}$ y $9.8 \mathrm{~km}$ para eventos superficiales e intermedios respectivamente. Es así que los tiempos de propagación mínimos a considerar en los ajustes resultan del orden de 1.12 s. En la Fig. 4.21 puede verse que los tiempos de viaje de P considerados en los ajustes superan este límite en ambos intervalos de profundidad.

Junto a los coeficientes del ajuste se dan los rangos del 95\% de confianza y el coeficiente de determinación $\left(\rho^{2}\right)$ que es el cuadrado del coeficiente de correlación $(\rho)$. Se ha obtenido un valor de $Q_{\mathrm{P}}=271$ para trayectorias dentro de la corteza continental con lapsos de tiempo de hasta 38 s y un $Q_{\mathrm{P}}=532$ para aquellas ondas con trayectorias desde la placa de Nazca y lapsos de tiempo de hasta $45 \mathrm{~s}$. El valor de $Q_{\mathrm{P}}$ para trayectorias que se inician en la placa de Nazca debe considerarse además como un límite inferior ya que ajustes para lapsos de tiempo inferiores a $35 \mathrm{~s}$ (donde se concentra más del $87 \%$ de las muestras) dan valores del factor de calidad cercanos a 1000 si bien la dispersión de los valores resulta en un coeficiente de determinación por debajo del obtenido en el resultado presentado. La estimación de la atenuación de ondas $\mathrm{P}$ a partir de los promedios temporales del ancho del primer pulso para trayectorias superficiales logra una correlación $(\rho=0.97)$ más alta que a profundidades intermedias $(\rho=0.76)$, tal como puede apreciarse a simple vista en la distribución de mediciones en función del tiempo de la Fig. 4.21.

\subsubsection{INTERPRETACIÓN}

Considerando a la atenuación como un proceso lineal, independiente y directamente proporcional a la distancia recorrida por las ondas en medios de atenuación constante, podemos identificar el efecto atenuativo de cada uno de los mismos. Suponiendo un modelo con estratificación horizontal y ondas que viajan en trayectorias casi verticales, puede 
asumirse que

$$
\frac{d_{\text {total }}}{Q_{\text {total }}}=\sum_{i=1}^{n} \frac{d_{i}}{Q_{i}}
$$

donde el subíndice i se refiere a cada capa del modelo con espesor $d_{\mathrm{i}}$ y factor de calidad constante, $Q_{\mathrm{i}}$, mientras que los valores totales reflejan el efecto de toda la estructura atravesada.

De acuerdo a la distribución de sismicidad (Fig. 3.20), se plantea un modelo simple de dos capas y un semiespacio (Fig. 4.22) que representan dos zonas sismogénicas (Z.S.1 y Z.S.2) y una zona asísmica (Z.A.). La Z.S.1 se asocia a los sismos corticales hasta $40 \mathrm{~km}$ de profundidad, la Z.S.2 está representada por los sismos intraplaca (90-120 km de profundidad) en la placa de Nazca subducida y la Z.A. intermedia comprende los últimos kilómetros de corteza continental, el manto litosférico y quizás una zona delgada de material astenosférico interplaca. Si bien Tassara et al. (2006), plantean que la litósfera sudamericana debajo de los silencios volcánicos, en los segmentos de subducción horizontal, tiene un espesor que puede superar los $100 \mathrm{~km}$, el perfil obtenido por su modelo mantiene a los $31^{\circ} \mathrm{S}$ una zona de desacople entre litósfera continental y oceánica, como puede verse en el perfil de la Fig. 4.23.

Los valores de $Q_{P}$ obtenidos por este modelo se consideran válidos para la región comprendida en el recuadro negro de la Fig. 4.24, que comprende el área atravesada por las trayectorias de ondas $\mathrm{P}$ provenientes de la superposición de los conjuntos de focos a 0-40 km y $90-120 \mathrm{~km}$ de profundidad. De este modo el área analizada se extiende entre los $68-69^{\circ} \mathrm{O}$ y los $31-32^{\circ} \mathrm{S}$, hasta una profundidad de $120 \mathrm{~km}$.

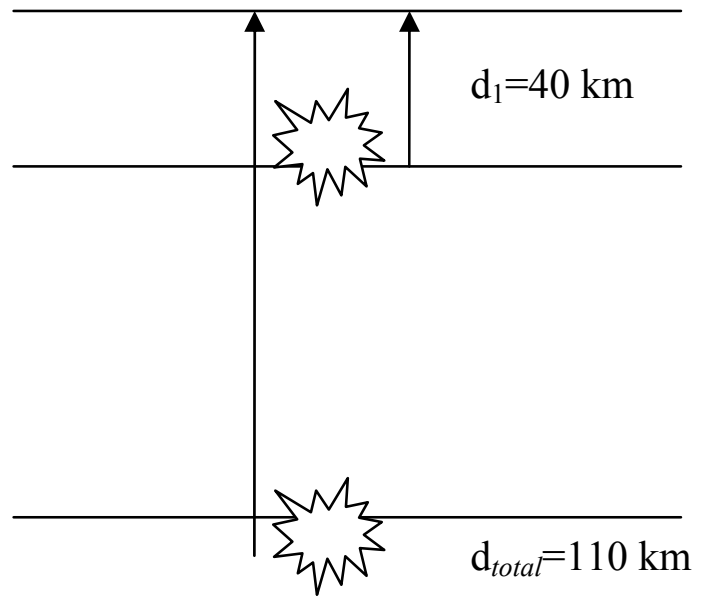
(Z.S.1) Zona Sismogénica
en Corteza Continental

(Z.A.) Zona Asísmica en Litósfera Continental Inferior y material astenosférico interplaca.

(Z.S.2) Zona Sismogénica en Litósfera Oceánica subducida

Fig. 4.22.- Modelo simplificado de estructura vertical para la región $31-32^{\circ} \mathrm{S}$ y $68-69^{\circ} \mathrm{O}$ utilizado en la interpretación de la distribución de valores de $Q_{P}$ obtenidos. $d_{1}$ es profundidad máxima de la zona sismogénica (S.Z.) cortical y $d_{\text {total }}$ es la profundidad promedio de la ZWB. Z.A. es la zona asísmica intermedia asociada a la litósfera inferior y al material astenosférico entre placas. 
Se asume una profundidad promedio de $110 \mathrm{~km}$ para la zona sismogénica en la placa de Nazca (Z.S.2) y un factor de calidad para las ondas allí originadas, considerado como el total, $Q_{\text {total }}=532$, obtenido para focos a profundidades entre 90 y $120 \mathrm{~km}$. Se supone que dichas ondas atraviesan una capa de $40 \mathrm{~km}$ de espesor dentro de la corteza con $Q_{1}=271$, según el resultado obtenido para la atenuación de focos superficiales (Z.S.1). Se trata de estimar la atenuación de la Zona Asísmica intermedia, entre 40 y $110 \mathrm{~km}$ de profundidad, a partir de los valores de $Q$ mencionados.

Expresando la (4.7) en términos del modelo queda

$$
\begin{aligned}
\frac{d_{\text {total }}}{Q_{\text {total }}} & =\frac{d_{Z . A .}}{Q_{Z . A .}}+\frac{d_{Z . S .1}}{Q_{Z . S .1}}, \\
\frac{110}{532} & =\frac{70}{Q_{Z . A .}}+\frac{40}{271}, \\
Q_{Z . A .} & =1183
\end{aligned}
$$

donde se ha obtenido un valor de $Q_{\mathrm{P}}=1183$ para la capa de $70 \mathrm{~km}$ de espesor que combina

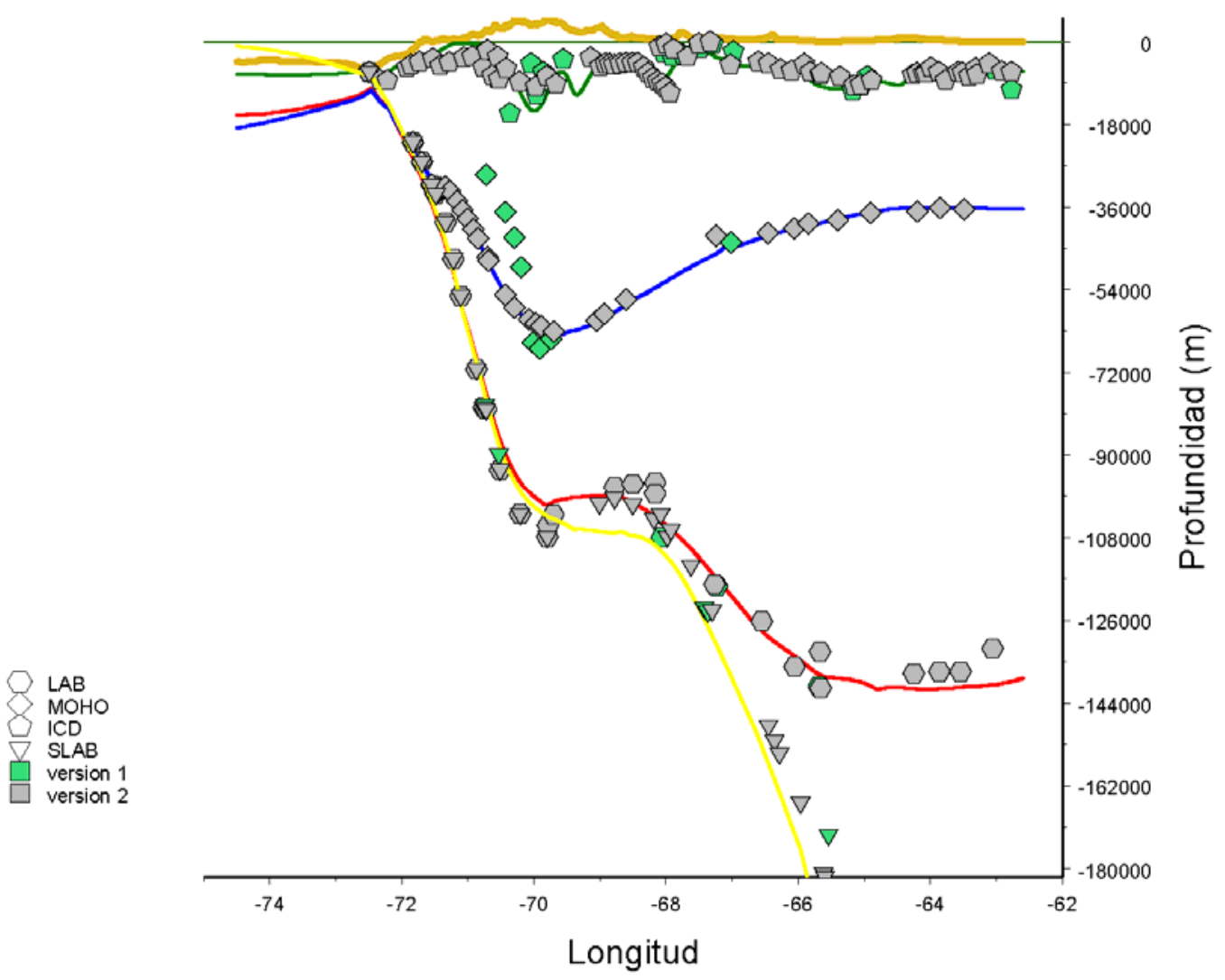

Fig. 4.23.- Perfil de la geometría 3D elaborado a los $31^{\circ} S$ por Tassara et al. (2011). En verde se dibuja la Discontinuidad de Densidad Intracortical (ICD); en azul, la Discontinuidad de Mohorovicic (MOHO); en rojo, el Límite Litósfera-Astenósfera (LAB) y en amarillo,el contorno de la placa subducida (SLAB). Se muestran también los valores individuales utilizados para elaborar el perfil según la versión 1 (Tassara et al., 2006) y la versión 2 (Tassara et al., 2011, inédita) 
litósfera continental inferior y material astenosférico interplaca.

Se observa entonces que la atenuación de las ondas $\mathrm{P}$ para eventos superficiales es netamente superior a la experimentada por aquellas ondas que provienen de focos en la placa de Nazca, ya que atraviesan una región de muy alto $Q$ en la litósfera inferior.

Al calcular los ajustes de la (2.71) a los datos de eventos intermedios, se han utilizado tiempos de viaje de hasta $42 \mathrm{~s}$. Para profundidades focales promedio iguales a $110 \mathrm{~km}$, en una

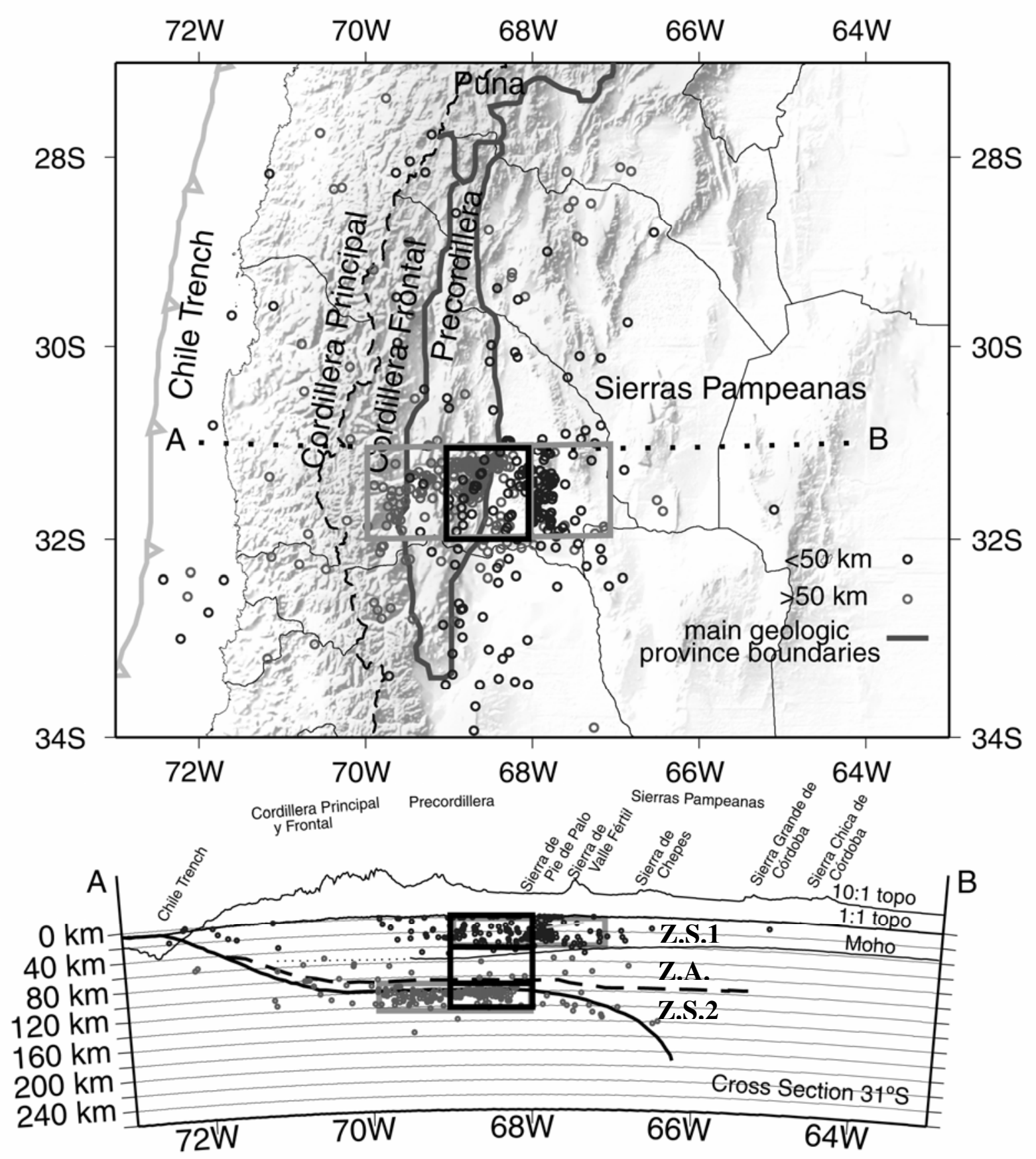

Fig. 4.24.- Sismicidad seleccionada para esta tesis en planta y perfil (Fig. 3.20) sobre la que se señala, con rectángulos grises, la selección de áreas de sismicidad considerada para la estimación de $Q_{P}$ a profundidades entre 0-40 km y 90-120km y con rectángulos negros, la zona de validez para la interpretación con la (4.8). Z.S.1 es la Zona Sismogénica en corteza, Z.A es la Zona Asísmica y Z.S.2 es la Zona Sismogénica. en la Placa de Nazca subducida. 
incidencia vertical como se plantea en este caso, se obtendría un tiempo de viaje para la onda $\mathrm{P}$ de unos $15 \mathrm{~s}$. Este tiempo se calcula suponiendo una velocidad promedio de $6.51 \mathrm{~km} / \mathrm{s}$ para los primeros $40 \mathrm{~km}$ de corteza y $7.94 \mathrm{~km} / \mathrm{s}$ para la Z.A según se deduce del modelo de velocidad (Tabla 3.2). De modo que para considerar incidencias verticales, deberían tenerse en cuenta tiempos de viaje significativamente menores que los disponibles. En este caso, al considerar un ajuste sobre tiempos de viaje por debajo de $\operatorname{los} 35 \mathrm{~s}$, se han logrado valores de $Q_{\mathrm{P}}$ del orden de 1000, pero con baja correlación. Por lo tanto, la distribución de valores de $Q_{\mathrm{P}}$ en profundidad obtenida, si bien se aparta considerablemente del modelo de incidencia vertical propuesto para su interpretación, vale como una primera aproximación a la espera de una colección de datos seleccionados a tal fin.

\subsubsection{MÉTODO DE NORMALIZACIÓN DE LA CODA (NC)}

\subsubsection{APLICACIÓN Y RESULTADOS}

Según el método de Normalización de la Coda propuesto por Aki (1980), el valor del factor de calidad de ondas de corte, $Q_{\mathrm{d}}$, puede obtenerse a partir del ajuste de la (2.100) a la distribución de amplitudes espectrales con la distancia hipocentral. Esto requiere el cálculo de las amplitudes espectrales sobre dos ventanas seleccionadas en el sismograma. La primera ventana, $A_{i}(\omega, D)$, incluye las máximas amplitudes de la onda directa y la segunda, $A_{i}^{c}\left(\omega, t_{c}\right)$, contiene las amplitudes de la coda a un tiempo de referencia fijo $t_{\mathrm{c}}$.

La implementación de este método se ha llevado a cabo mediante un código en lenguaje Basic (Ibáñez y Badi). El programa calcula amplitudes medias en una ventana de $\mathrm{S}$ elegida en pantalla por el usuario y en una ventana de coda a un tiempo de referencia pre-establecido sobre las trazas. Permite además calcular amplitudes y energía en otras ventanas de la onda $\mathrm{S}$, que son de utilidad para la separación de la atenuación intrínseca y de scattering, como se verá más adelante.

La entrada de datos se realiza a través de un par de archivos de texto que reúnen el listado de trazas y sus parámetros focales y de registración, así como los resultados previos obtenidos de la atenuación de ondas coda que se requieren posteriormente para el ajuste. El tiempo de viaje de $\mathrm{S}$ incorporado en la entrada de datos, permite presentar en pantalla directamente una ventana centrada en el mismo para agilizar el proceso manual de selección del comienzo de la ventana de S. 


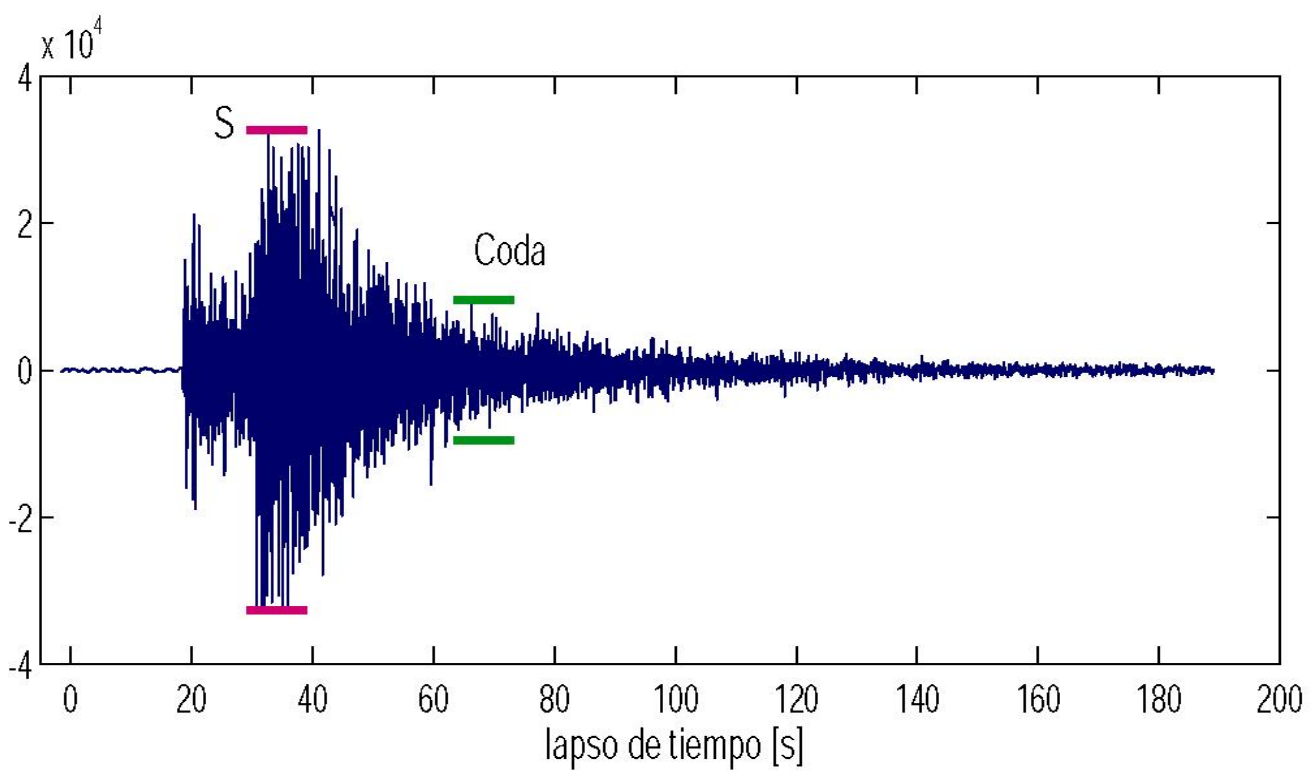

Fig.4.25.- Ejemplo de sismograma registrado a una distancia de $107 \mathrm{Km}$, donde se muestra la selección de la ventana de $S$ sobre su máxima amplitud y la ventana de coda al tiempo de referencia establecido en el texto para la aplicación del NC.

Cada traza ingresada es filtrada en bandas de frecuencia centradas en $f_{\mathrm{c}}: 1.5,3,6$ y 12 $\mathrm{Hz}$ y anchos de banda en el intervalo $\left[f_{\mathrm{c}}-f_{\mathrm{c}} / 2, f_{\mathrm{c}}+f_{\mathrm{c}} / 2\right]$. Sobre cada traza filtrada se miden las amplitudes espectrales de S y coda sobre el RMC calculado sobre las ventanas seleccionadas. Se han elegido las longitudes de las ventanas de $\mathrm{S}$ y de coda iguales entre sí y de $10 \mathrm{~s}$ de duración para hacerlas comparables en la normalización. De este modo se ha buscado garantizar la inclusión de todas las máximas amplitudes de la onda $\mathrm{S}$ directa evitando la contaminación con ondas $\mathrm{S}$ dispersadas. La ventana de $\mathrm{S}\left(t_{\mathrm{s}}\right)$ se ha ubicado visualmente en cada caso a partir del punto de máxima energía de la onda, habiéndose procesado 4074 sismogramas en total. En la Fig. 4.25 se muestra un ejemplo de sismograma con las ventanas de $\mathrm{S}$ y coda seleccionadas.

Debido al rango de distancias hipocentrales y magnitudes considerados, no ha sido posible fijar un único tiempo de referencia $\left(t_{\mathrm{c}}\right)$ mayor que $2 t_{\mathrm{s}}$ con una buena relación señal ruido donde centrar la ventana de coda en todos los sismogramas. La solución a este inconveniente fue sugerida e implementada en Aki (1980a) y consiste en tomar las amplitudes de coda en un conjunto de lapsos de tiempo $\left(t_{1}\right)$, seleccionados, para luego corregirlas a un tiempo de referencia fijo $\left(t_{\mathrm{c}}\right)$ mediante la expresión del decaimiento de coda $P(f, t)$. Para definir los tiempos $t_{1}$ se han contrastado los valores de $t_{\mathrm{s}}$, las distancias hipocentrales y los lapsos de tiempo máximos y mínimos seleccionados en cada caso para la estimación de $Q_{\mathrm{c}}$, donde ya se ha tenido en cuenta la relación señal ruido de las trazas. A partir de este análisis, los valores de $t_{1}$ elegidos han sido: $70 \mathrm{~s}$ para distancias inferiores a $150 \mathrm{~km}, 90 \mathrm{~s}$ para $150-250$ 
$\mathrm{km}$ de distancia, $110 \mathrm{~s}$ para $250-400 \mathrm{~km}$ de distancia y $130 \mathrm{~s}$ para distancias superiores a 400 $\mathrm{km}$.

En primer lugar se ha realizado una determinación de $Q_{d}$ sin normalizar las amplitudes de coda a un único tiempo de referencia (Badi et al., 2005), es decir realizando los ajustes de la (2.100) a los datos para cada $t_{1}$ en forma independiente, o lo que es lo mismo para los rangos de distancias asociados a esos $t_{1}$. De este modo, se eliminaba la influencia del modelo SBS o SIS sobre el método NC ya que no se requería una expresión del decaimiento de coda obtenida por otro ajuste. Modificando la (2.100), se han calculado los promedios del logaritmo natural de los cocientes de amplitudes mediante la relación

$$
\left\langle\ln \left\{D^{n} A_{i}(\omega, D) / A_{i}^{c}\left(\omega, t_{1}\right)\right\}\right\rangle_{D \pm \Delta D}=a-b D
$$

donde se ha elegido el coeficiente de expansión geométrica, $n=1$ o 0.5 según se considerara que las máximas amplitudes de la onda $\mathrm{S}$ fueran ondas internas o superficiales. En esta oportunidad se ha supuesto que para profundidades corticales y distancias grandes, podían tratarse de ondas superficiales atrapadas en corteza llegando inmediatamente luego de la $\mathrm{S}$, como se ha planteado en otros trabajos (Chun et al., 1987; De Miguel et al., 1992). De este modo se ha elegido $n=0.5$ para profundidades mayores que $65 \mathrm{~km}$ y distancias mayores que
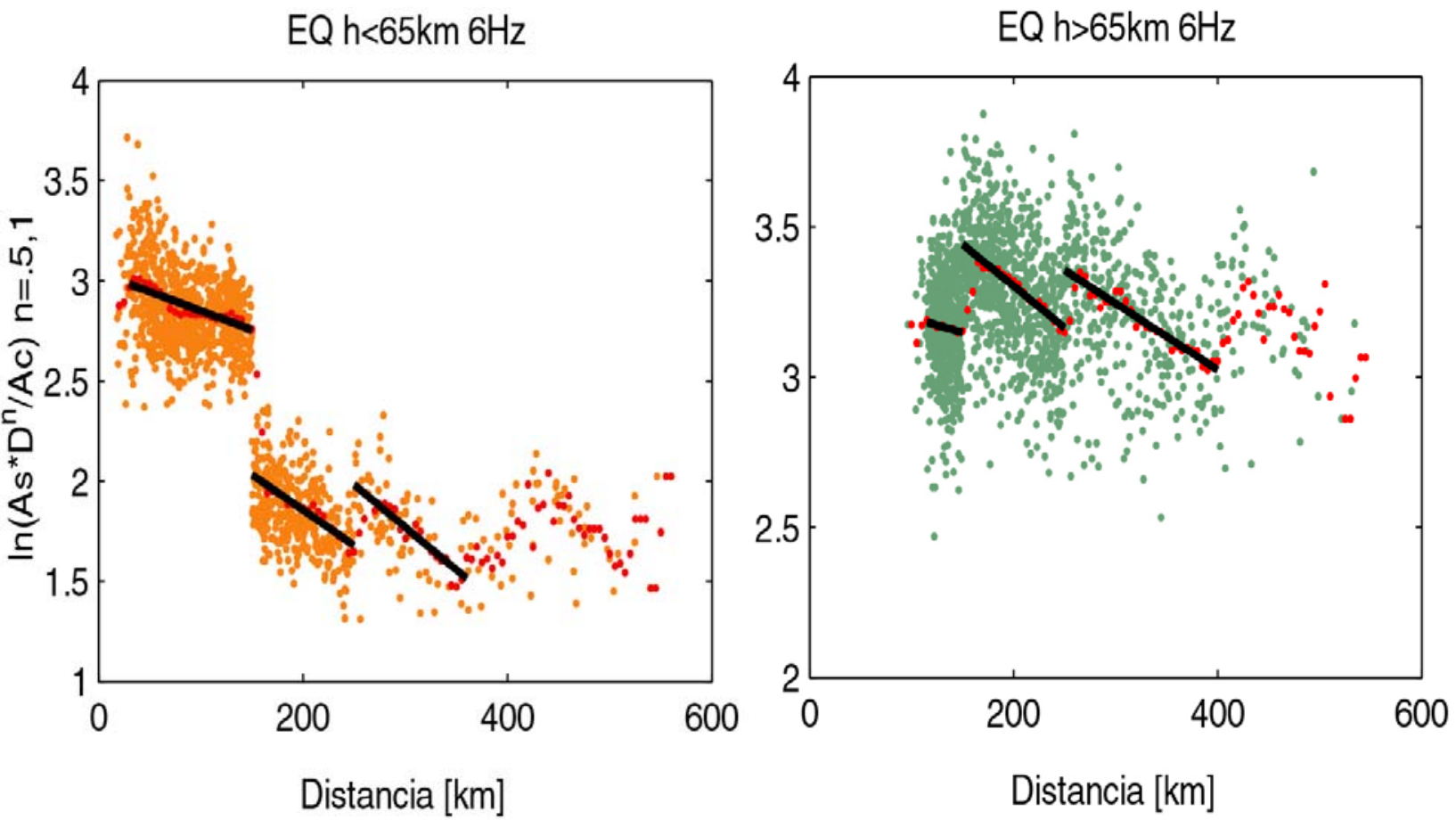

Fig. 4.26.- Ejemplos del ajuste del método NC para toda la región, sin corrección por decaimiento de coda para una frecuencia de $6 \mathrm{~Hz}$. A la izquierda se presentan los ajustes para eventos superficiales y a la derecha los eventos intermedios. Los datos individuales (puntos naranjas y verdes) se suavizaron (puntos rojos) para hacer el ajuste lineal (líneas sólidas)(Badi et al., 2005). 
Fig. 4.27.- $\quad$ Valores de Qd obtenidos para cada intervalo de tiempo ajustado con la (4.9) sin corrección a un tiempo de referencia único para la coda. En verde se muestran los correspondientes a trayectorias de eventos intermedios $(h>65$ km) y en naranja los $Q$ para trayectorias de eventos superficiales $(h<65 \mathrm{~km})$

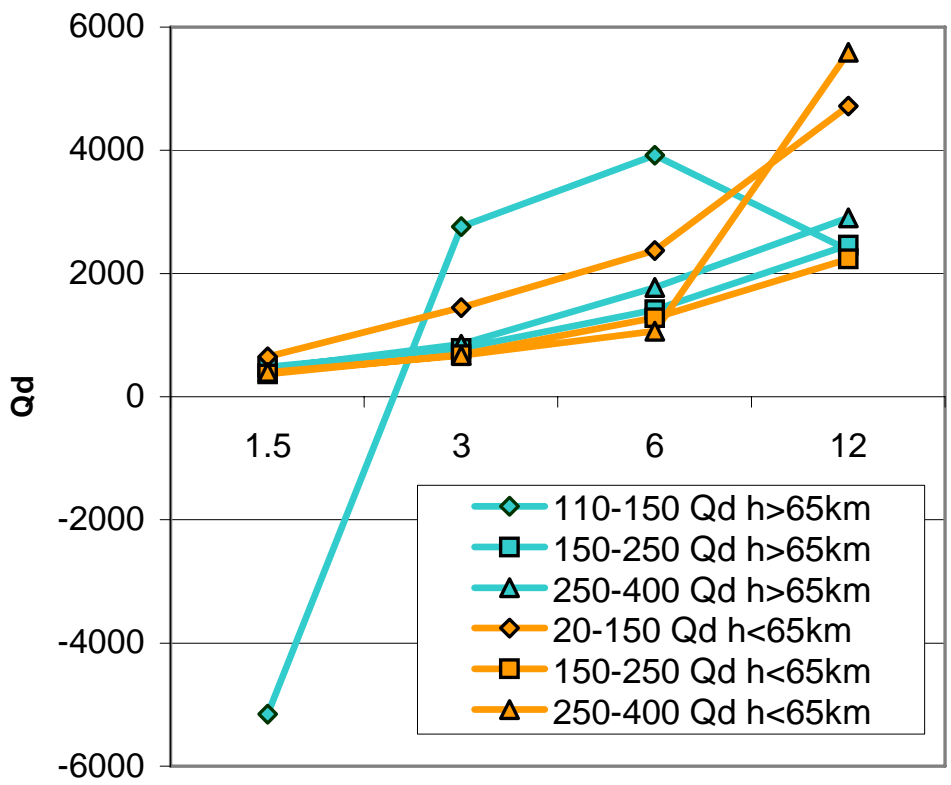

frecuencia $[\mathrm{Hz}]$

\begin{tabular}{|l|c|c|c|c|c|c|}
\hline & \multicolumn{3}{|c|}{ Intermedios } & \multicolumn{3}{c|}{ Superficiales } \\
\hline \multicolumn{1}{|c|}{ Distancias } & $\boldsymbol{Q}_{\boldsymbol{0}} \pm \boldsymbol{e} \boldsymbol{Q}_{\boldsymbol{0}}$ & $\boldsymbol{n} \pm \boldsymbol{e n}$ & $\boldsymbol{\rho}^{\mathbf{2}}$ & $\boldsymbol{Q}_{\boldsymbol{0}} \pm \boldsymbol{e} \boldsymbol{Q}_{\boldsymbol{0}}$ & $\boldsymbol{n} \pm \boldsymbol{e n}$ & $\boldsymbol{\rho}^{\mathbf{2}}$ \\
\hline$<\mathbf{1 5 0} \mathbf{~ k m}$ & $277.6 \pm 1455.5$ & $0.99 \pm 2.28$ & 0.04 & $475.1 \pm 87.9$ & $0.92 \pm 0.08$ & 0.98 \\
\hline $\mathbf{1 5 0}-\mathbf{2 5 0} \mathbf{~ k m}$ & $331.8 \pm 37.4$ & $0.81 \pm 0.05$ & 0.98 & $275.2 \pm 38.9$ & $0.84 \pm 0.06$ & 0.98 \\
\hline $\mathbf{2 5 0}-\mathbf{4 0 0}$ & $367.3 \pm 51.2$ & $0.84 \pm 0.06$ & 0.98 & $29.5 \pm 75.9$ & $2.11 \pm 1.05$ & 0.56 \\
\hline
\end{tabular}

Tabla 4.4.- Resultados del ajuste de la función $Q d=Q_{0}\left(f / f_{0}\right)^{n}$ a los valores de $Q d y$ sus coeficientes de determinación para toda la región como se muestran en la Fig. 4.27, a profundidades superficiales e intermedias

$100 \mathrm{~km}$. La profundidad de $65 \mathrm{~km}$ se ha elegido de acuerdo al modelo de velocidad originalmente utilizado en este trabajo (Tabla 3.1).

Los promedios en distancia, han sido estimados utilizando una ventana móvil de $15 \mathrm{~km}$ con un paso de $5 \mathrm{~km}$, a fin de visualizar la tendencia de los datos y estimar ajustes por mínimos cuadrados robustos. Así mismo se consideraron distancias hipocentrales entre $30 \mathrm{y}$ $400 \mathrm{~km}$ para eventos superficiales y entre 115 y $400 \mathrm{~km}$ para eventos intermedios dado que allí se concentraba la mayor población de datos y se maximizaba la correlación de los ajustes. Mediante un ajuste lineal por mínimos cuadrados en función de la distancia, se ha obtenido la pendiente, $b$ (Fig. 4.26). Conocida $b$, se ha calculado el valor de $Q_{d}$ para cada frecuencia y rango de distancia (Fig. 4.27), empleando un valor de $4.16 \mathrm{~km} / \mathrm{s}$ como velocidad de $\mathrm{S}$ promedio para eventos superficiales y $4.81 \mathrm{~km} / \mathrm{s}$ para los eventos intermedios de acuerdo al mismo modelo de velocidad. Los mejores ajustes regionales de $Q_{\mathrm{d}}$ en función de la 
frecuencia sin corrección a un único tiempo de referencia se han obtenido a distancias epicentrales entre 150 y $250 \mathrm{~km}$ en ambos rangos de profundidad (Badi et al., 2005), a distancias cortas en eventos superficiales y a distancias largas en eventos intermedios (Tabla 4.4).

Una vez obtenida la expresión del decaimiento de coda, se ha optimizado la aplicación del método efectuando la corrección de las amplitudes de la coda a un tiempo uniforme. Este procedimiento se ha realizado para dos valores de tiempo de referencia, $t_{\mathrm{c}}=0$ y $90 \mathrm{~s}$, considerando a las amplitudes de $\mathrm{S}$ como ondas internas por lo que el coeficiente de expansión geométrica se ha supuesto igual a la unidad, de acuerdo a lo establecido en la determinación de $Q_{\mathrm{c}}$ (Sección 4.1.1).

La elección de $t_{\mathrm{c}}=0$, implicaría llevar la amplitud de coda a su valor en el tiempo de origen del evento, es decir en la fuente. La corrección de amplitudes en este caso se ha efectuado mediante (2.104) con $K(t)=1$, es decir suponiendo la validez del modelo SBS y reemplazando en $Q_{\mathrm{c}}(\omega, t)$ los promedios regionales para las frecuencias y lapsos de tiempo correspondientes obtenidos por el modelo SBS. Los ajustes de la (2.100), para distancias de hasta $400 \mathrm{~km}$, han permitido nuevamente ajustar una relación $Q(f)$ tipo potencia y hallar valores regionales de $Q_{0 \mathrm{~d}}$ iguales a 90 y 160 para trayectorias superficiales e intermedias respectivamente y un exponente $n=0.7$ en ambos casos (Badi et al., 2007).

En la reducción de amplitudes de coda a $t_{\mathrm{c}}=90 \mathrm{~s}$, que es la longitud de coda predominante en la base de datos, se ha utilizado la (2.104) considerando el factor de corrección por expansión geométrica, $K(t)$, igual a la unidad en caso del modelo SBS (Badi et al., 2009) o calculando su valor en función de los tiempos de inicio de las ventanas de $\mathrm{S}$ y de coda en la consideración del modelo SIS (Badi et al., 2010). Así mismo, los valores de $Q_{\mathrm{c}}(\omega, t)$ se han reemplazado en la corrección a partir de:

1) valores individuales de $Q_{\mathrm{c}}$ para cada traza, intervalo de tiempo y frecuencia,

2) valores promedio regionales de $Q_{\mathrm{c}}$ por intervalo de tiempo, frecuencia y profundidad,

3) valores promedio de $Q_{\mathrm{c}}$ para cada lapso de tiempo y frecuencia en estaciones agrupadas de acuerdo a su entorno geotectónico,

4) la expresión de $Q(f)$ ajustada para cada lapso de tiempo en los mismos grupos de estaciones.

Las amplitudes así corregidas se han reemplazado entonces en el primer miembro de la (2.100) y se han representado en función de la distancia hipocentral. Se han estimado los 


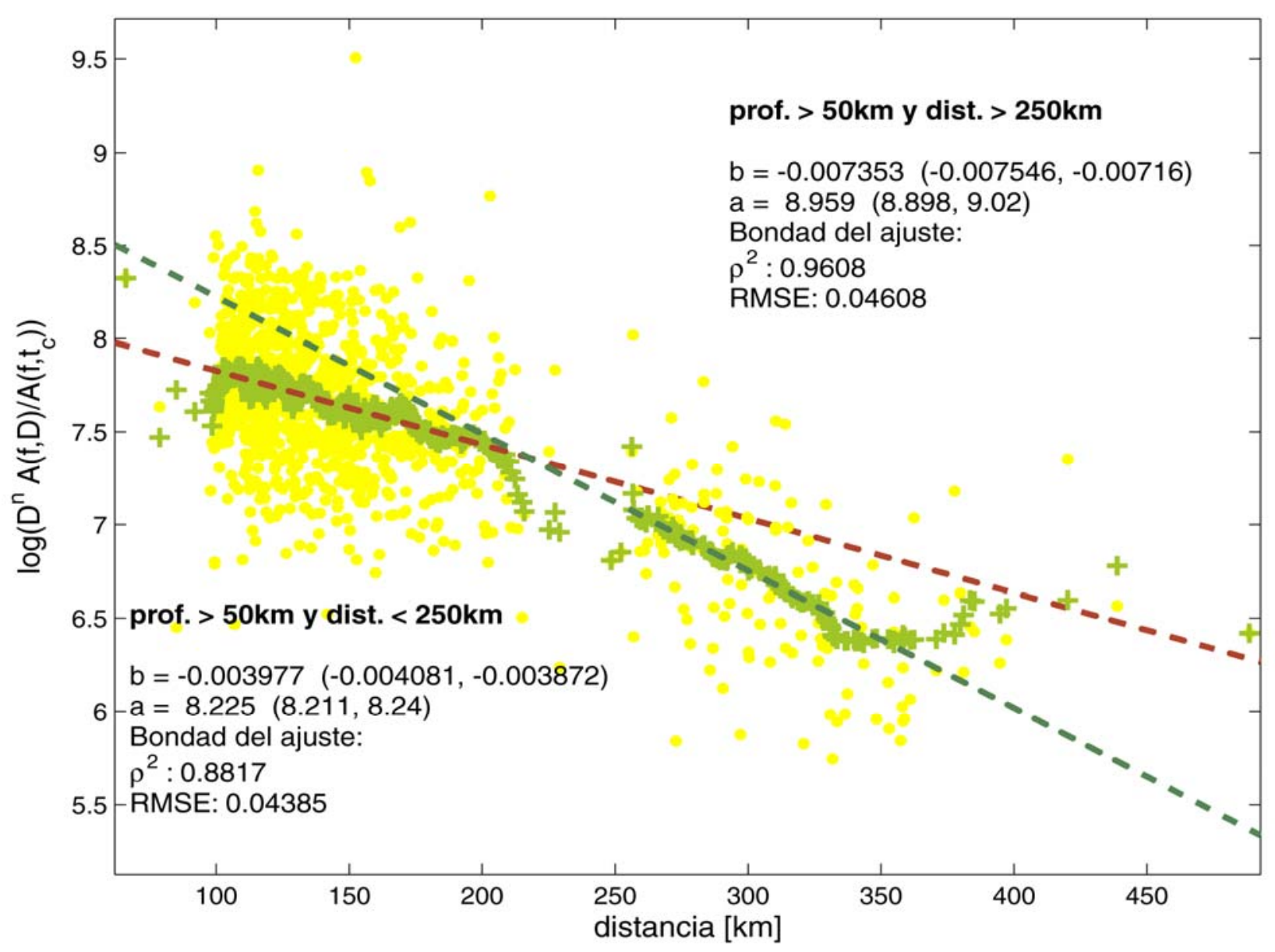

Fig. 4.28.- Ejemplo del ajuste lineal al logaritmo de la amplitud de $S$ normalizada por la amplitud de coda a $90 \mathrm{~s}$ para datos de eventos de profundidad intermedia (prof. $>50 \mathrm{~km}$ ) filtrados a $6 \mathrm{~Hz}$. A diferencia de los datos correspondientes a trayectorias corticales, en este caso se encontraron dos ajustes posibles según la distancia hipocentral, menor o mayor que $250 \mathrm{~km}$.

promedios del $\ln \left\{D A_{i}(\omega, D) / A_{i}^{c}\left(\omega, t_{c}\right)\right\}$ en ventanas móviles sobre $D$ con un ancho de $12 \mathrm{~km}$ y un solapamiento de $4 \mathrm{~km}$. Mediante un ajuste lineal ortogonal se han estimado los coeficientes $a$ y $b$ del segundo miembro de la (2.100) calculándose entonces el valor de $Q_{\mathrm{d}}$ para cada frecuencia mediante la relación $b=\omega / 2 Q_{d} v$. El valor de $v$, la velocidad promedio de $\mathrm{S}$ en la trayectoria, se ha estimado a partir de la localización definitiva y del modelo de velocidad obtenido en este trabajo (Apéndice B y Tabla 3.2). En el caso de focos superficiales $(h<50 \mathrm{~km}) \cup$ resulta igual a $3.71 \mathrm{~km} / \mathrm{s}$ e igual a $4.3 \mathrm{~km} / \mathrm{s}$ en el caso de focos intermedios $(\mathrm{h}>50 \mathrm{~km})$.

De la comparación de los resultados obtenidos según los diversos criterios de aplicación mencionados, se observa que los mejores ajustes de $Q_{\mathrm{d}}$ han surgido de emplear el modelo SIS reduciendo la amplitud de la coda a $t_{\mathrm{c}}=90 \mathrm{~s}$ por medio de valores promedios regionales de $Q_{\mathrm{c}}$ por frecuencia y lapso de tiempo para el caso de eventos intermedios. 
La Fig. 4.28 presenta un ejemplo del proceso de ajuste lineal sobre datos provenientes de focos a profundidades intermedias distribuidos en toda la región y para una frecuencia de 6 Hz. La tendencia observada en las amplitudes del $\ln \left\{D A_{i}(\omega, D) / A_{i}^{c}\left(\omega, t_{c}\right)\right\}$ en función de la distancia es diferente según se sea $\mathrm{D}>250 \mathrm{~km}$ o $\mathrm{D} \leq 250 \mathrm{~km}$ a dichas profundidades. Esta característica se repite en todas las bandas de frecuencia, por lo que se han efectuado dos ajustes lineales cada vez.

A profundidades corticales el ajuste regional de las amplitudes $\operatorname{del} \ln \left\{D A_{i}(\omega, D) / A_{i}^{c}\left(\omega, t_{c}\right)\right\}$ ha mostrado una gran dispersión por lo que se ha analizado la posibilidad de variaciones laterales en la atenuación superficial. Luego de considerar diferentes criterios de regionalización, se ha optado por la asociación de estaciones de la RSZNC de acuerdo a su entorno geológico.

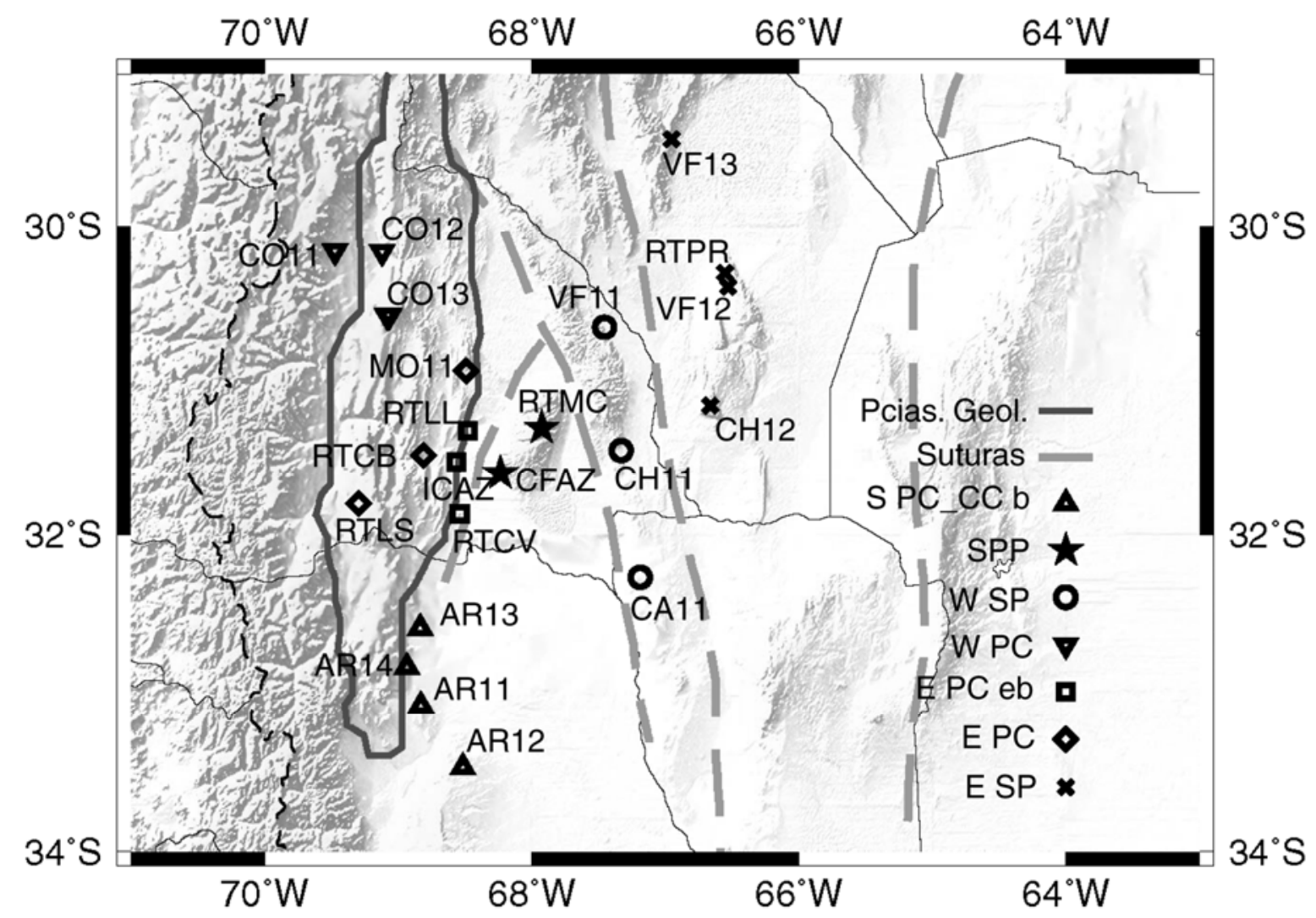

Fig. 4.29.- Grupos de estaciones elegidos para la regionalización del método NC. Se indica el nombre de cada estación de la RSZNC y se les asigna símbolo de acuerdo al grupo al que pertenecen. Ver más explicaciones en el texto.

La Fig. 4.29 muestra los diferentes grupos de estaciones considerados en relación a los principales rasgos tectónicos de la región, quedando definidos siete grupos:

W PC es la Precordillera occidental y está representada por las estaciones 
CO11/RODE, CO12/CUES, CO13 y CO16.

E PC es Precordillera oriental con las estaciones MO11, RTCB y RTLS.

E PC eb es el límite este de Precordillera oriental donde se sitúan RTLL, ICAZ y RTCV.

SPP es Sierra Pie de Palo con la presencia de las estaciones RTMC y CFAZ.

W SP son las Sierras Pampeanas occidentales donde están CA11, VF11 y CH11.

E SP son las Sierras Pampeanas orientales con VF12, RTPR, CH12 y VF13.

S PC_CC b es el límite entre Precordillera austral y Cuenca Cuyana donde se encuentarn AR11, AR12, AR13 y AR14.

Las tendencias de las amplitudes agrupadas de este modo han resultado más claramente definidas, disminuyendo su dispersión y mejorando los ajustes de $Q_{\mathrm{d}}$ con valores lógicos y

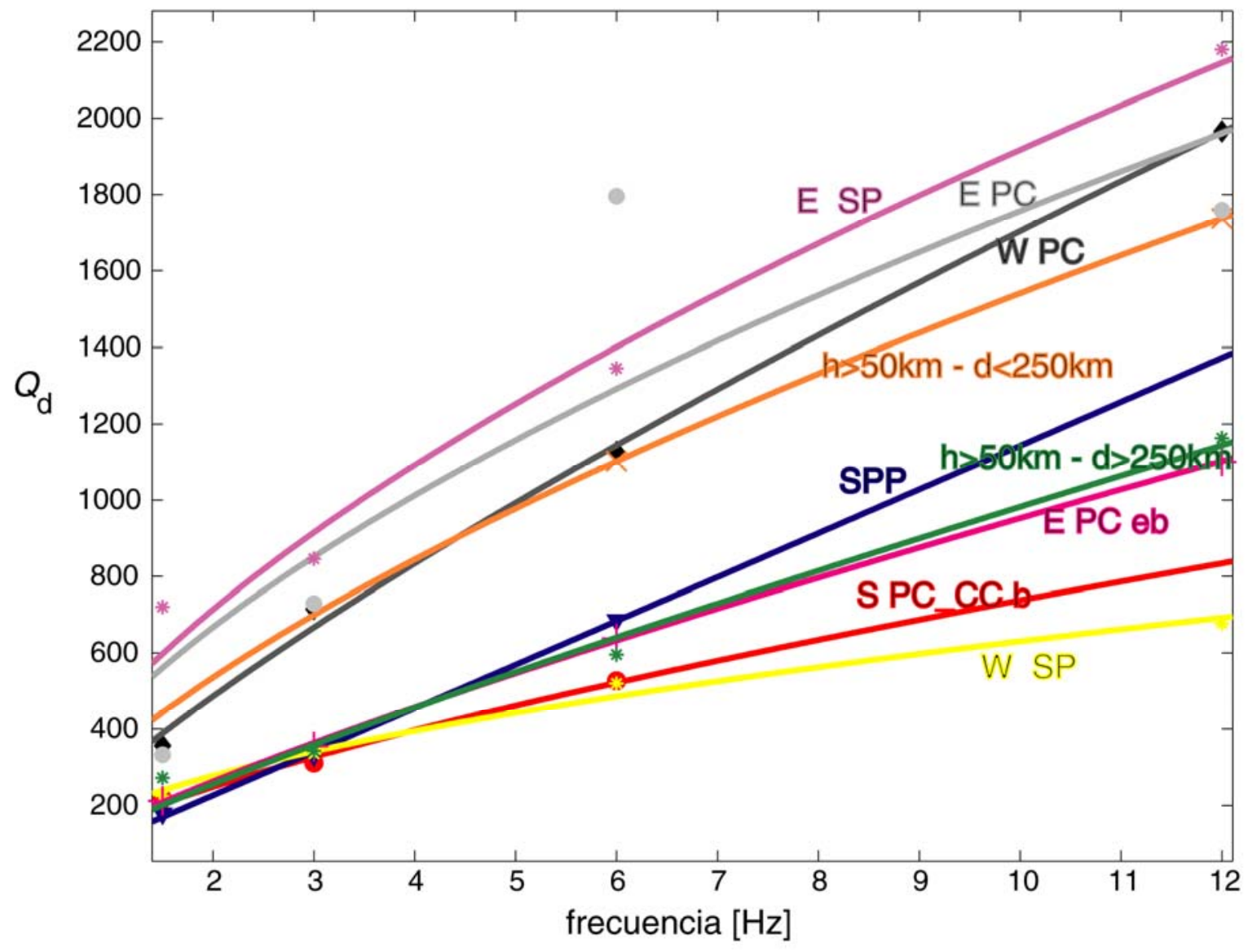

Fig. 4.30.- Distribución de $Q_{d}$ en función de la frecuencia para la región de Nuevo Cuyo obtenida con datos de estaciones agrupadas de acuerdo a los principales rasgos tectónicos de su emplazamiento. Las curvas que se indican con $h>50 \mathrm{~km}$ corresponden a ajustes para datos de focos en la placa de Nazca con distancias hipocentrales $d>250 \mathrm{~km} o$ $d<250 \mathrm{~km}$. Las restantes curvas corresponden a datos de focos en corteza continental según los grupos cuvas siglas se explican en el texto. 
buenos coeficientes de correlación. Por lo tanto, en el caso de eventos superficiales, se ha seleccionado la normalización de la coda por grupos de estaciones asumiendo nuevamente la validez del modelo SIS pero corrigiendo la amplitud de coda al tiempo de referencia, $t_{\mathrm{c}}=90 \mathrm{~s}$ por medio de los ajustes de $Q_{\mathrm{c}}(f)$ estimados sobre los datos de eventos superficiales registrados para cada grupo de estaciones.

En la Fig. 4.30 se presenta la distribución de los valores promedio de $Q_{\mathrm{d}}$ obtenida por ajuste ortogonal sobre valores promediados en ventanas de $12 \mathrm{~km}$ de ancho y $4 \mathrm{~km}$ de solapamiento. Para cada grupo se ha efectuado el ajuste en función de la frecuencia con una función potencia, para hallar los valores de $Q_{d}(1 \mathrm{~Hz})=Q_{0} \mathrm{y}$ el exponente $n$, que caracterizan su dependencia con la frecuencia. Se dibujan las curvas correspondientes a dichos ajustes en la misma figura y se muestran los coeficientes $Q_{0}$ y $n$ con sus errores y coeficientes de determinación correspondientes en la Tabla 4.5. Los errores obtenidos en las determinaciones del $Q_{d}$ medio para cada frecuencia han sido sensiblemente inferiores a los errores presentados en la tabla que surgen del ajuste de la (2.21).

\begin{tabular}{|l|c|c|c|}
\hline Zona & $\boldsymbol{Q}_{\mathbf{0}} \pm \boldsymbol{e} \boldsymbol{Q}_{\boldsymbol{0}}$ & $\boldsymbol{n} \pm \boldsymbol{e n}$ & $\boldsymbol{\rho}^{\mathbf{2}}$ \\
\hline W PC & $283 \pm 106$ & $0.78 \pm 0.17$ & 0.99 \\
\hline E PC & $440 \pm 1263$ & $0.60 \pm 1.34$ & 0.58 \\
\hline E PC eb & $151 \pm 17$ & $0.80 \pm 0.05$ & 0.99 \\
\hline SPP & $113 \pm 118$ & $1.00 \pm 0.64$ & 0.99 \\
\hline W SP & $195 \pm 93$ & $0.51 \pm 0.23$ & 0.97 \\
\hline E SP & $466 \pm 312$ & $0.61 \pm 0.31$ & 0.98 \\
\hline S PC_CC b & $155 \pm 233$ & $0.68 \pm 0.97$ & 0.98 \\
\hline $\mathbf{h}>\mathbf{5 0 k m}-\mathbf{d}<\mathbf{2 5 0 k m}$ & 340 & 0.66 & Obs.: sólo dos frec. \\
\hline $\mathbf{h}>\mathbf{5 0 k m}-\mathbf{d}>\mathbf{2 5 0 k m}$ & $143 \pm 136$ & $0.84 \pm 0.42$ & 0.97 \\
\hline
\end{tabular}

Tabla 4.5.- Estimaciones del factor de calidad de ondas $S$ a $1 \mathrm{~Hz}\left(Q_{0}\right)$ y potencia (n) que surgen del ajuste de los valores de $Q_{d}$ en función de la frecuencia, obtenidos por el método NC. Cada zona se define a partir de los rasgos tectónicos del emplazamiento de las estaciones (ver más explicación en el texto). Para eventos intermedios y distancias inferiores a $250 \mathrm{~km}$, el ajuste se realizó sobre valores para dos frecuencias, por lo que no pudo estimarse error en la determinación.

\subsubsection{INTERPRETACIÓN}

En el análisis de las curvas de la Fig. 4.30, se observan dos grupos de curvas con características diferentes. Por un lado se encuentra la atenuación estimada para las zonas $\mathrm{E}$ SP, W PC y E PC con valores de $Q_{0}$ altos y una variación con la frecuencia relativamente lenta, dada por exponentes menores que 0.8. Los resultados obtenidos para la Precordillera Oriental deben considerarse con cuidado debido al alto error en su determinación y por lo 
tanto su bajo coeficiente de correlación. La menor atenuación que caracteriza a las dos primeras zonas coincide con la menor sismicidad asociada a las mismas. Por otra parte se encuentran valores bajos de $Q_{0}$ y muy similares entre sí, para la SPP, E PC eb, W SP y S PC_CC b con una dependencia con la frecuencia no homogénea dentro del grupo. Vuelve a verificarse que a menor $Q_{0}$, más alto será el exponente de su relación potencia con la frecuencia. Analizando la relación entre los valores de $Q_{0}$ y la actividad sísmica, se ve la coincidencia entre los valores más altos de la atenuación y las áreas de mayor concentración de hipocentros. Por lo tanto, nuevamente se cumple que a mayor actividad tectónica mayor es la atenuación y a su vez esta crecerá más rápidamente con la frecuencia.

A partir de la distribución de los valores de $Q_{0}$, para profundidades corticales y por grupos de estaciones, se ha confeccionado un mapa de la atenuación de ondas $\mathrm{S}$ directas mediante el ajuste de una superficie cartesiana 2-D con interpolación spline y funciones de Green, curvatura mínima y un espaciado de 5', que se muestra en la Fig. 4.31.

Se destacan dos mínimos de $Q_{0}$, uno en la zona de la Sierra Pie de Palo y el otro en el NO de Mendoza. El primero de ellos se encuentra mejor definido por los valores de $Q_{0}$ ascendentes al este y al oeste, mientras que el otro surge de un único valor de $Q_{0}$ y del ajuste de la superficie cartesiana. A pesar del limitado número de valores disponibles para la interpolación, puede verse que ambos máximos de atenuación se sitúan en las proximidades de las principales fuentes sismogénicas de la Región de Nuevo Cuyo, donde tuvieron epicentro los sismos históricos de mayor magnitud de la región.

En el caso de las trayectorias de ondas $\mathrm{S}$ provenientes de focos intermedios se ha observado una misma tendencia de las amplitudes normalizadas en función de la distancia para todas las estaciones, por lo que se han interpretado en forma de promedio regional. A su vez, se ha comprobado en todas las frecuencias que el resultado del ajuste de la (2.100) dependía del intervalo de distancias seleccionado, obteniéndose dos pendientes bien diferentes (Fig. 4.28).

Para distancias inferiores a $250 \mathrm{~km}$, la atenuación a profundidades intermedias se caracteriza por un factor $Q_{0}=340$, mientras que para distancias mayores que $250 \mathrm{~km}$ resulta ser $Q_{0}=143$. Comparados estos ajustes con los de trayectorias superficiales, en la Fig. 4.30 puede verse que la atenuación intermedia a distancias menores, resulta similar a la hallada en Precordillera, mientras que para distancias mayores la atenuación a profundidades intermedias es menor y con un comportamiento muy similar al del límite este de Precordillera oriental. 


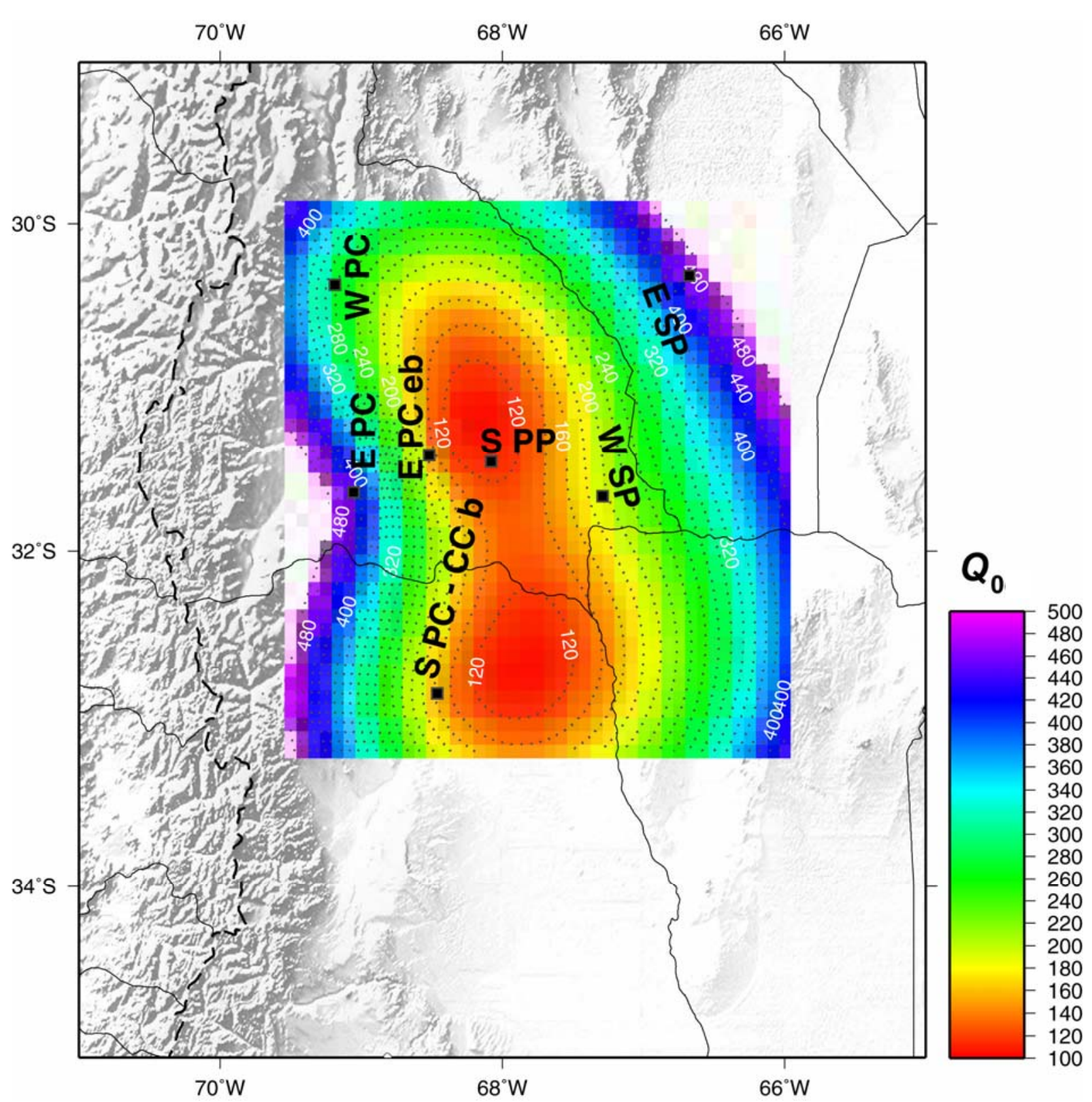

Fig. 4.31.- Mapa de distribución del factor de calidad de ondas $S$ a $1 \mathrm{~Hz}\left(Q_{0}\right)$, obtenido mediante el método NC para eventos corticales. Los cuadrados negros indican coordenadas medias de las estaciones ubicadas en cada una de las zonas delimitadas por los principales rasgos tectónicos. Las siglas se explican en el texto.

Al observar la distribución de hipocentros intermedios, ha podido verse que estos se concentran principalmente bajo Precordillera y Cordillera Frontal, por lo que las trayectorias correspondientes a distancias hipocentrales menores que $250 \mathrm{~km}$ han sido más afectadas seguramente por la atenuación correspondiente a dichas provincias geológicas.

Para distancias hipocentrales mayores que $250 \mathrm{~km}$, la atenuación de ondas $\mathrm{S}$ estimada a profundidades intermedias es menor y se aproxima a la de Sierra Pie de Palo, a la del límite este de Precordillera Oriental y a la del límite entre Precordillera austral y Cuenca de Cuyo. En este caso, dada la concentración de profundidades focales principalmente entre 90 y 120 $\mathrm{km}$, dichas trayectorias corresponderán a sismos bajo Precordillera y estaciones lejanas a la 
zona más sismogénica o a sismos intermedios más lejanos y estaciones en la zona más sismogénica, lo que significa, de cualquier modo, que dicha zona ha sido atravesada por las ondas afectando su amplitud.

En resumen, en el caso de sismos de profundidad intermedia, se puede asociar la atenuación de las ondas en las trayectorias largas a la mayor pérdida de energía en la zona más sismogénica de Nuevo Cuyo y la de las trayectorias cortas a una menor atenuación bajo Precordillera, en coincidencia con lo que ocurre a profundidades corticales.

La ausencia o acumulación relativa de sismos en una región puede darse por diferentes combinaciones de los parámetros geodinámicos: densidad, rigidez, temperatura, porosidad, presencia de fluidos, tensiones tectónicas, etc. El valor de $Q$ se relaciona a su vez con la densidad de heterogeneidades y la anelasticidad del medio, esta última vinculada a temperatura, presión, porcentaje de fusión y contenido de fluidos. Por lo tanto la separación de la atenuación de scattering e intrínseca en la región, puede aportar importante información para definir el grado de protagonismo de los diferentes parámetros geodinámicos en la distribución de la sismicidad.

\subsubsection{MÉTODO DE LAS DOBLES RAZONES ESPECTRALES (DRE)}

\subsubsection{APLICACIÓN Y DISCUSIÓN}

El método de las Dobles Razones Espectrales (DRE) fue diseñado originalmente por Chun et al. (1987) como una modificación del método de las Simples Razones Espectrales. Permite la determinación de la atenuación de ondas Lg eliminando por cocientes espectrales el efecto de sitio, el patrón de radiación y las respuestas instrumentales de las estaciones. Aunque diseñado originalmente para Lg, puede aplicarse también para determinar la atenuación de las ondas $\mathrm{S}$, ya que los efectos del patrón de radiación son eliminados por el método.

El DRE requiere, cada vez, cuatro sismogramas correspondientes a dos eventos registrados por dos estaciones. Cada cuarteto de dos estaciones y dos eventos debe permanecer en el mismo círculo máximo dentro de un margen de tolerancia, que dependerá de la onda considerada. En el caso de ondas Lg, esta condición es menos restrictiva que para otras fases (Xie et al., 2004) por lo que algunos autores han tomado tolerancias que van desde $\operatorname{los} 5^{\circ}$ hasta $\operatorname{los} 15^{\circ}$ (Chun et al.,1987, De Miguel et al., 1992, Zor et al., 2009). Otro detalle a tener en cuenta en la selección de los cuartetos es la observación efectuada por Chun et al. (1987) acerca de la mayor calidad de los ajustes para distancias entre estaciones más largas 
por sobre los ajustes para distancias cortas.

Se han encontrado en la bibliografía diferentes formas de seleccionar la ventana temporal para la estima del espectro. En el análisis de ondas Lg, Chun et al. (1987) comprobaron que estimando los espectros en ventanas definidas por intervalos de velocidad de grupo se obtenían resultados con menor dispersión que en la selección mediante tiempos fijos. En el caso de las ondas S, la forma más segura de determinar la ventana de tiempo es sin duda mediante la polarización transversal, por lo que debe contarse con registros en componentes horizontales.

Otro aspecto importante por el cual definir si las ondas registradas son ondas internas o superficiales, o existen ambas, es que, según la naturaleza de las ondas, se debe asumir el valor del coeficiente de expansión geométrica $(1,0.5)$, aunque también puede determinarse a través de una adaptación del mismo método (Ibáñez et al., 1993). En varias regiones se han observado ondas Lg a distancias algo mayores que 100 km (Chun et al., 1987, Ibáñez et al., 1991, Udías, 1999, Sec. 9.3). En la Región de Nuevo Cuyo, teniendo en cuenta un espesor cortical promedio de 50km (Fig. 3.20) y una discontinuidad intracortical de densidad somera (Tassara et al. 2006), la selección de distancias epicentrales mayores que $100 \mathrm{~km}$ y fuentes por encima de los $50 \mathrm{~km}$ de profundidad podrían permitir el desarrollo de ondas superficiales. Sin embargo, según Kulhánek (2002) las ondas Lg se desarrollan a distancias epicentrales a partir de $5^{\circ}$ y a profundidades corticales, mientras que las ondas $\mathrm{Rg}$ dominan las máximas amplitudes de los sismogramas a distancias entre 100 y $200 \mathrm{~km}$ y profundidades de 1 a $5 \mathrm{~km}$, pudiendo llegar a verse como máximo hasta los $600 \mathrm{~km}$ de distancia. En la sismicidad considerada en este trabajo, con distancias fundamentalmente inferiores a $500 \mathrm{~km} \mathrm{y}$ profundidades focales concentradas entre $0-50 \mathrm{~km}$ en la corteza y 100-150 km en la Placa de Nazca subducida, las ondas registradas se han considerado hasta ahora como Pn, Pg, Sn y Sg. Las Sg, suelen ser las ondas con mayor amplitud en los sismogramas de eventos en corteza superior cuyo decaimiento en amplitud forma la coda. Ahora bien, debido a la estructura interna de la corteza en la región, según sean la distancia epicentral y la profundidad focal, el inicio corresponderá a una onda $\mathrm{Sn}$ o $\mathrm{Sg}$. En un modelo sencillo de corteza con una capa granítica sobre otra capa basáltica, las ondas Sn no se observarán a distancias menores que una distancia crítica, que en corteza continental suele ser de $100 \mathrm{~km}$, mientras que las Sg podrán ser observadas a cualquier distancia. En realidad, la corteza real es bastante más compleja, por lo que se denomina Sg a las ondas refractadas en discontinuidades menores dentro de la corteza granítica (Kulhánek, 2002). Tassara et al. (2006) propusieron un modelo de la discontinuidad intracortical de densidad para la región andina con profundidades que 
varían entre los 5 y $15 \mathrm{~km}$ en la región de Nuevo Cuyo.

Con todas estas consideraciones en cuenta, se han efectuado búsquedas en la base de datos para implementar el método DRE en la región de Nuevo Cuyo hallándose muy pocas parejas de eventos y estaciones que permiten la aplicación del método y menos aún que den ajustes de calidad, ya sea considerando la existencia de ondas Lg o suponiendo ondas $\mathrm{Sg}$. Se hace necesario entonces contar con modelos corticales más definidos, con una mayor cantidad de datos a profundidades corticales $\mathrm{y}$, fundamentalmente, con sismogramas registrados en tres componentes. De esta manera se podrá esclarecer la naturaleza de las ondas de mayor amplitud en los sismogramas así como precisar su tiempo de inicio para luego estudiar su atenuación a través de este método.

\subsection{SEPARACIÓN DE LA ATENUACIÓN INTRÍNSECA y DE SCATTERING}

\subsubsection{MÉTODO DE LAS VENTANAS TEMPORALES MÚLTIPLES (MLTWA)}

\subsubsection{APLICACIÓN Y RESULTADOS}

La separación de la atenuación intrínseca y de scattering mediante el método de las Ventanas Temporales Múltiples (MLTWA) se basa en el análisis de la variación temporal de la energía en tres ventanas temporales sucesivas integradas en función de la distancia fuentereceptor y normalizadas. La energía en cada ventana se estima sobre la amplitud media cuadrática $(\mathrm{MC})$ de los sismogramas filtrados. Se efectúa la normalización mediante el método de $\mathrm{NC}$ a fin de eliminar el efecto de sitio y del patrón de radiación de la fuente y luego se corrige por expansión geométrica. Las densidades de energía en cada una de las tres ventanas y la coda han sido determinadas utilizando el mismo programa que en el método NC (Ibáñez y Badi)

Las curvas teóricas que describen el modelo de la distribución de energía integrada en función de la distancia hipocentral, han sido obtenidas a partir de la aproximación de Zeng et al. (1991) al scattering múltiple mediante un modelo híbrido de scattering simple y difusión (2.116) y (2.117). El cálculo de las curvas teóricas y su ajuste a los datos se ha efectuado mediante el programa Scat-Cad (MathCad ${ }^{2000}$ profesional) desarrollado por Bianco y Del Pezzo (2002) que ha sido actualizado en el MathLTWA (Mathematica 7) (Bianco y Del Pezzo, 2010). 
Cada traza se ha filtrado en cuatro bandas de frecuencia centradas en $f_{\mathrm{c}}=1.5,3,6,12$ $\mathrm{Hz}$ y con un ancho de una octava $\left(\left[f_{\mathrm{c}}-f_{\mathrm{c}} / 2, f_{\mathrm{c}}+f_{\mathrm{c}} / 2\right]\right)$. La duración de cada una de las tres ventanas temporales se ha tomado igual a 15 s (Hoshiba, et al., 1991) de modo de asegurar que las mayores amplitudes de la onda $\mathrm{S}$ quedasen dentro de la primera ventana. El inicio de la primera ventana se ha definido visualmente a partir de la llegada de la onda S. Seguidamente se ha estimado la envolvente de cada traza filtrada calculando su RMC en cada ventana. Para la normalización de la coda, se ha medido la amplitud de la envolvente en una ventana de $15 \mathrm{~s}$ de duración centrada en un lapso de tiempo de $70 \mathrm{~s}$. En la Fig. 4.32 se muestra un ejemplo de sismograma con las ventanas de $\mathrm{S}$ y de coda seleccionadas para el análisis. Para cada sismograma analizado el programa graba en un archivo ASCII los datos de las amplitudes espectrales, las energías y la frecuencia central del filtro, indicando además la distancia hipocentral, el tiempo de inicio de la primera ventana, el lapso de tiempo de coda elegido así como la información que permite identificar el evento y la estación correspondiente.

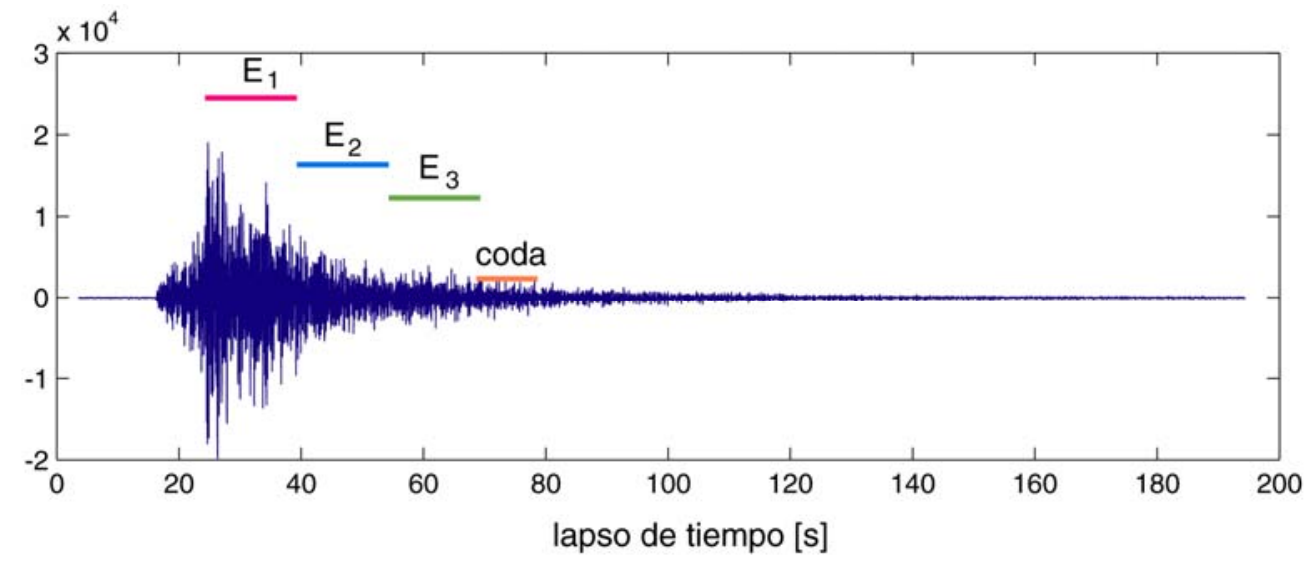

Fig. 4.32.- Ejemplo de sismograma regional. Los segmentos E1, E2 y E3 indican la posición y duración de las tres ventanas temporales sucesivas para el cálculo de la energía integrada en el MLTWA y "coda" marca la porción de la coda a $t>2$ ts utilizada para la normalización.

La (2.120) resume las correcciones realizadas sobre cada amplitud de energía integrada haciendo el cociente entre las amplitudes de la energía de cada ventana y la energía de una ventana en la coda a un lapso de tiempo fijo mayor que $2 t_{\mathrm{s}}$ y multiplicando por $4 \pi r^{2}$ para la corrección por expansión geométrica ya que se asume un frente de onda esférico para la onda S.

Se han analizado por separado los datos de sismos corticales $(0-40 \mathrm{~km})$ e intermedios $(40-150 \mathrm{~km})$. A fin de investigar la existencia de posibles variaciones laterales de la 


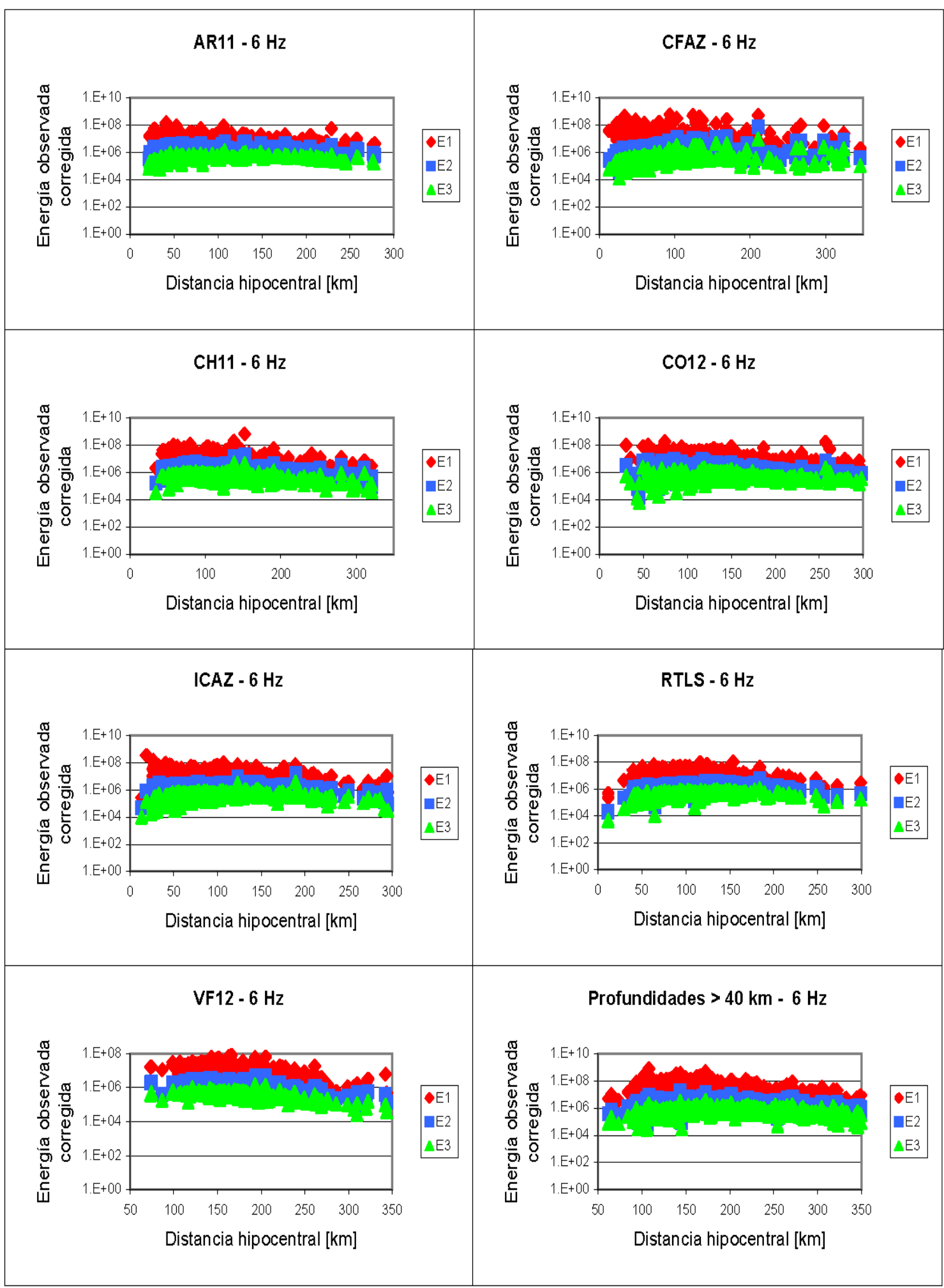

Fig. 4.33.- Ejemplos de energías integradas observadas en función de la distancia fuente receptor y para cada uno de los grupos de eventos seleccionados para el análisis en ventanas temporales múltiples. E1, E2 y E3 son las energías medidas sobre la RMC de cada sismograma filtrado en este caso a $6 \mathrm{~Hz}$, corregidas por normalización de la coda y expansión geométrica. 

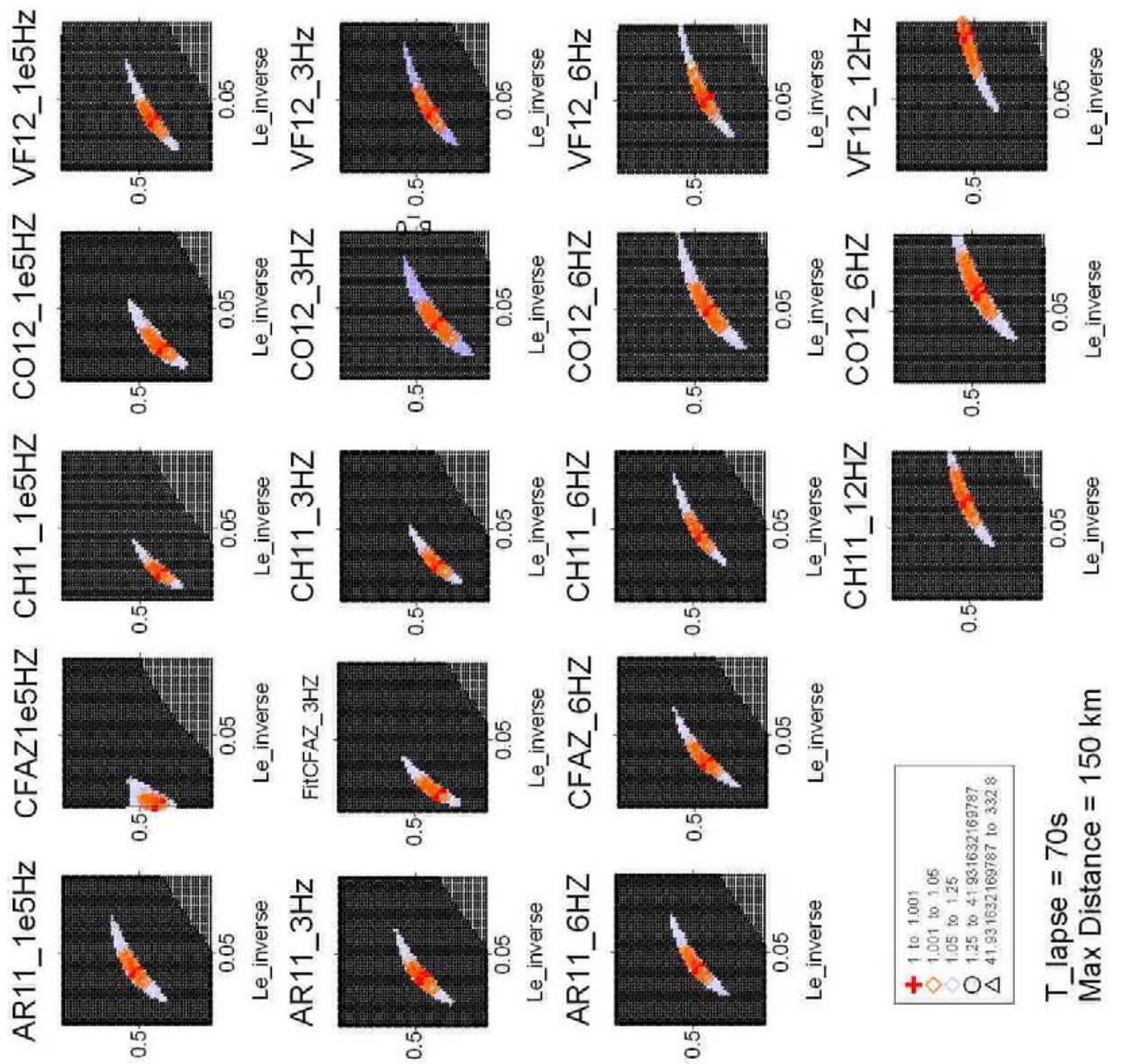

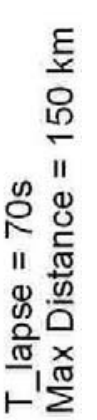

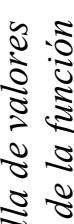

के ฏ

․․ำ

ปี है

ᄂ

\&.

के

:

is

홍

s.

¿

․ 2

苟这

i"

ป

\&

के ถ

ปิ

\&

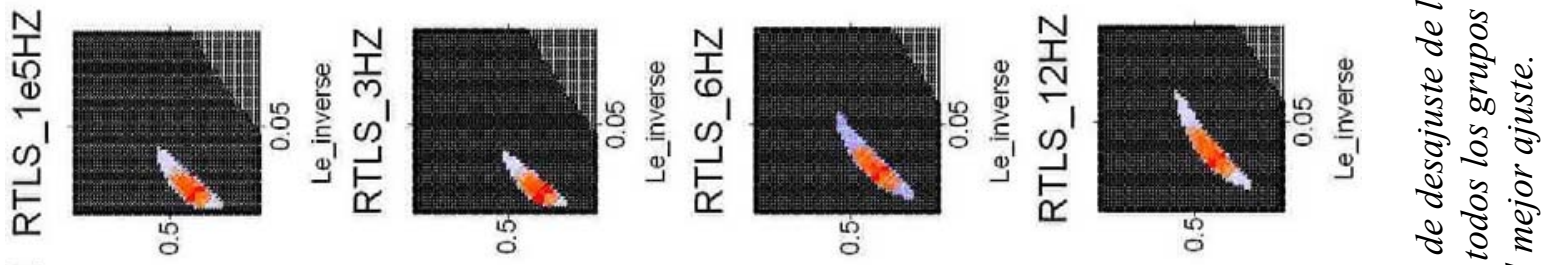

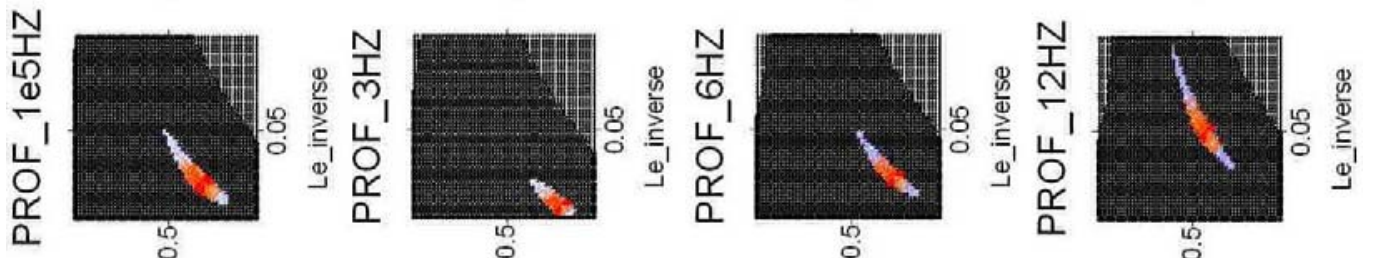

$2 \approx$

\&

$2 \pi$

$\approx 0$

ข $\approx$

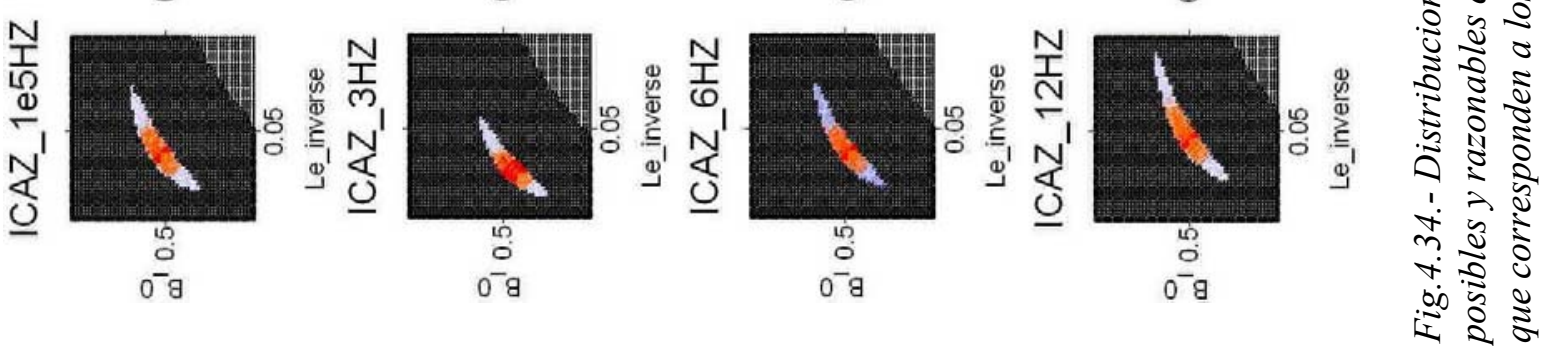


atenuación en la corteza, se han agrupado las 23 estaciones sismológicas en 7 grupos de acuerdo a criterios de homogeneidad tectónica, de modo tal que los grupos considerados son los mismos que se han presentado en el método de Normalización de la Coda (Sección 4.2.3.1), es decir: W PC, E PC, E PC eb, SPP, S PC-CC b, W SP y E SP. En el caso de los sismos a profundidades intermedias se han considerado todos los datos reunidos en un único grupo dado que los focos se concentran en un volumen relativamente pequeño.

En la Fig. 4.33 se muestran los resultados de la distribución de energías integradas corregidas en función de la distancia para las trazas filtradas a $6 \mathrm{~Hz}$ de todas las estaciones para el caso de sismos intermedios y para cada grupo en los sismos corticales. Las energías integradas para sismos intermedios muestran menor dispersión en su distribución que las de sismos corticales. La mayor dispersión se observa en la primera ventana temporal debido a que el método no tiene en cuenta los efectos del patrón de radiación (Bianco et al., 2005) y también en las distancias mayores que $200 \mathrm{~km}$ en el caso de sismos corticales. La distribución de energía se ha ajustado sobre cada grupo de datos correspondientes a sismos en corteza hasta distancias hipocentrales de $90 \mathrm{~km}$ como máximo, para evitar la influencia de la atenuación en trayectorias que atravesaran áreas vecinas.

Una vez evaluadas las energías observadas para las tres ventanas en función de la distancia, el programa Scat-Cad (Bianco y Del Pezzo, 2002) ajusta el modelo de Zeng (1991) a los datos del mismo modo que en Bianco et al. (2005). Mediante una función de desajuste norma L2 (2.121), es decir por mínimos cuadrados, se aplica un método de búsqueda exhaustiva en una grilla de valores razonables y posibles de $L_{e}^{-1}$ y $B_{0}$. Las amplitudes teóricas son calculadas en cada punto de la grilla con la (2.116), integradas en cada ventana (2.119) y corregidas también por normalización de la coda y expansión geométrica del mismo modo que los datos reales (2.120). Sus valores se parametrizan en términos de los coeficientes de la atenuación intrínseca y de scattering $\left(\eta_{i}, \eta_{s}\right)$, expresados a su vez en función de $Q_{\mathrm{i}}$ y $Q_{s}$ mediante $\eta_{i, s}=2 \pi f / v Q_{i, s}$, donde $v$ es la velocidad media de las ondas $\mathrm{S}$ y $f$ es la frecuencia. En este análisis se ha asumido una velocidad de $\mathrm{S}$ igual a $4.36 \mathrm{~km} / \mathrm{s}$, estimada como promedio pesado dentro del volumen atravesado por las ondas en sus trayectorias.

Siguiendo a Bianco y Del Pezzo (2002), el mínimo de la función de desajuste se busca gráficamente sobre la grilla de valores calculados normalizados por su mínimo (Fig. 4.34). La significación de cada estima y sus intervalos de error se han calculado mediante una distribución F o de Fisher-Snedecor (Vargas Sabadías, 1995) a un nivel de confianza del 70\%, 


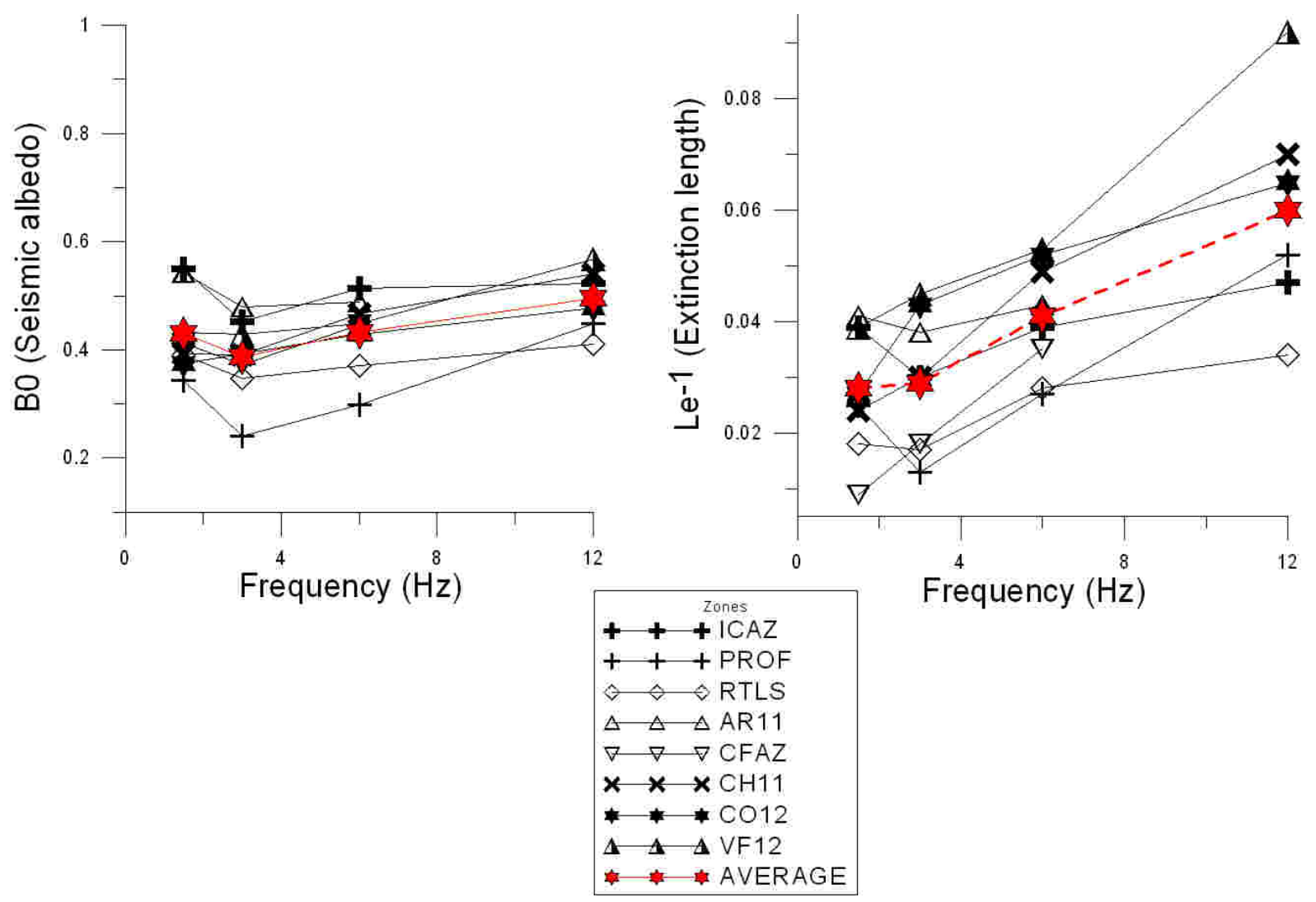

Fig. 4.35.- Valores de $L_{e}^{-1}$ y $B_{0}$ obtenidos para todos los grupos analizados. Las estrellas rojas indican valores medios para todos los eventos y profundidades. A partir de estos resultados se ha decidido reunir a los eventos corticales en un único grupo considerando el valor medio de sus parámetros como representativo de corteza para toda la RNC.

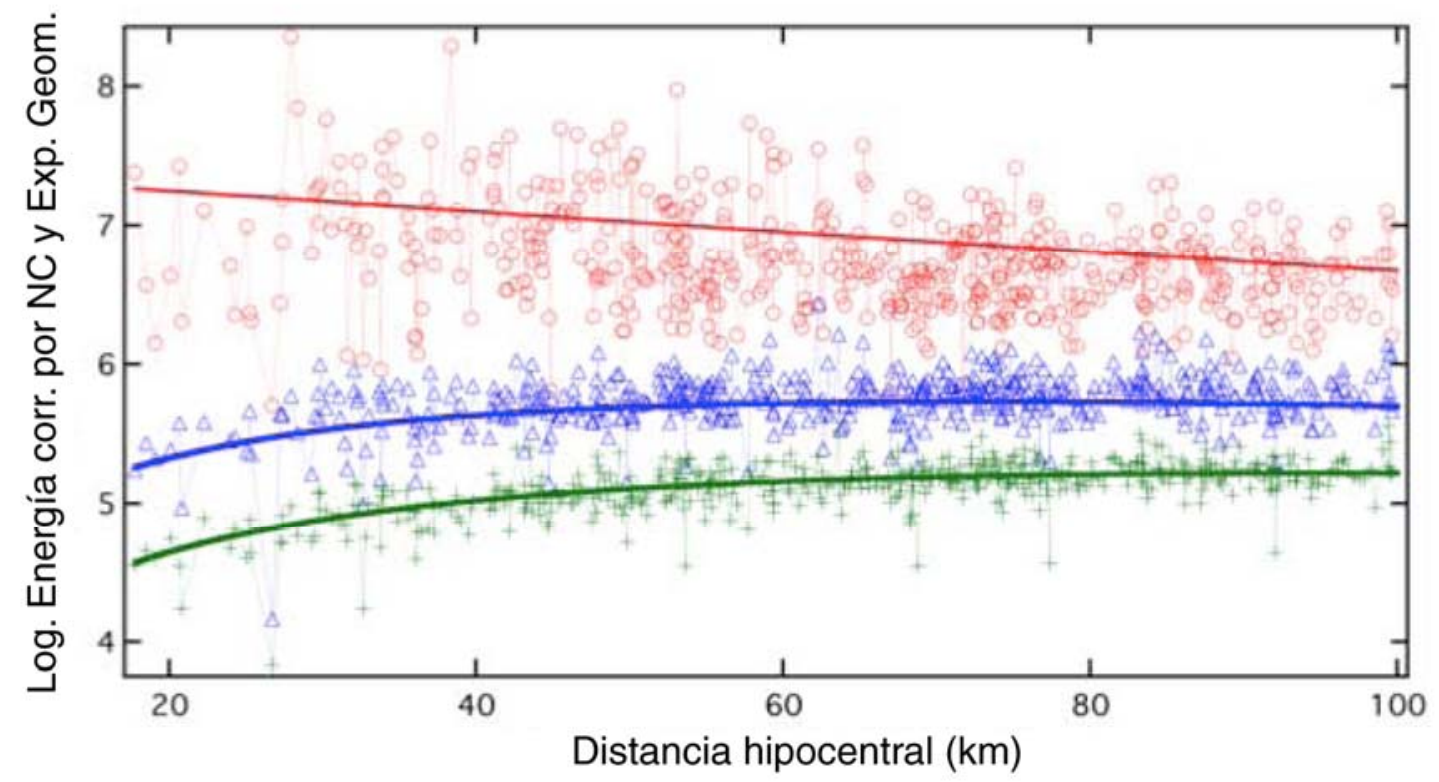

Fig. 4.36.- Ejemplo de la distribución del Log. de la energía corregida por normalización de la coda y expansión geométrica en función de la distancia hipocentral para eventos superficiales a $6 \mathrm{~Hz}$. Los círculos rojos corresponden a la ventana $E_{1}$, triángulos azules representan $E_{2}$ y cruces verdes a $E_{3}$. Las líneas sólidas muestran los mejores ajustes teóricos del MLTWA. 
utilizando para ello el cociente entre las dos variables aleatorias cada una de ellas con un número de grados de libertad $N_{d f}=N_{\text {data }}-2$, donde $N_{\text {data }}$ es el número de datos y 2 es el número de variables independientes. En este caso todas las soluciones con residuos normalizados más grandes que 1.1 son estadísticamente diferentes (Badi et al., 2009).

Al analizar los resultados correspondientes a todos los grupos de estaciones para eventos corticales se ha visto que, dentro de los rangos de error, estos presentan valores similares de $L_{e}^{-1}$ y $B_{0}$ (Fig. 4.35). Por esta razón se han considerado los promedios de los resultados para todos los grupos de eventos superficiales como parámetros característicos de corteza, extendiéndose la distancia del ajuste hasta $100 \mathrm{~km}$.

La Fig. 4.36 muestra un ejemplo de las energías integradas corregidas en función de la distancia a frecuencias centradas en $6 \mathrm{~Hz}$ para eventos superficiales junto a las curvas teóricas que mejor ajustan la distribución en cada ventana temporal. En la Tabla 4.6 se presentan los valores de $L_{e}^{-1}$ y $B_{0}$ (y los valores asociados de $\eta_{i}$ y $\eta_{s}, Q_{i}$ y $Q_{s}$ ) seleccionados indicándose el

\begin{tabular}{|c|c|r|r|r|r|r|}
\hline $\begin{array}{c}\text { Frequency } \\
\text { (Hz) }\end{array}$ & \multicolumn{1}{l|}{ B0 } & \multicolumn{1}{c|}{ Le $^{-1}$} & \multicolumn{1}{c|}{$\boldsymbol{\eta}_{\mathbf{i}}$} & \multicolumn{1}{c|}{$\boldsymbol{\eta}_{\mathbf{s}}$} & \multicolumn{1}{c|}{$\mathbf{Q}^{-1}$} & $\mathbf{Q s}^{-1}$ \\
\hline 1,5 & 0,46 & 0,039 & 0,021 & 0,018 & 0,008 & 0,007 \\
\hline 3 & 0,42 & 0,042 & 0,024 & 0,018 & 0,005 & 0,003 \\
\hline 6 & 0,45 & 0,055 & 0,03 & 0,025 & 0,003 & 0,002 \\
\hline 12 & 0,52 & 0,078 & 0,037 & 0,04 & 0,002 & 0,001 \\
\hline
\end{tabular}

Tabla 4.6.- Arriba: Ajustes del MLTWA para eventos corticales. $B_{0}$ (albedo sísmico) y $L_{e}^{-1}$ (inverso de la longitud de extinción) están afectados por un error inferior al $4 \%$ y $20 \%$ respectivamente para un nivel de dispersión aceptado de $1 \sigma$ (desviación estándar), 7\% y 35\% para 2 бy $10 \%$ y $50 \%$ a 3 б.

Abajo: Ajustes del MLTWA para eventos a profundidades intermedias. $B_{0}$ y $L_{e}^{-1}$ están afectados por un error inferior al $8 \%$ y 40\% respectivamente para un nivel de dispersión aceptado de $1 \sigma$.

\begin{tabular}{|c|r|r|r|r|r|r|}
\hline $\begin{array}{c}\text { Frequency } \\
(\mathrm{Hz})\end{array}$ & \multicolumn{1}{c|}{$\mathrm{B} 0$} & \multicolumn{1}{c|}{$\mathrm{Le}^{-1}$} & \multicolumn{1}{c|}{$\boldsymbol{\eta}_{\mathbf{i}}$} & \multicolumn{1}{c|}{$\boldsymbol{\eta}_{\mathbf{s}}$} & \multicolumn{1}{c|}{$\mathbf{Q i}^{-1}$} & $\mathbf{Q s}^{-1}$ \\
\hline 1,5 & 0,34 & 0,031 & 0,02 & 0,01 & 0,007 & 0,004 \\
\hline 3 & 0,24 & 0,016 & 0,012 & 0,004 & 0,002 & 0,0007 \\
\hline 6 & 0,3 & 0,034 & 0,023 & 0,01 & 0,002 & 0,0009 \\
\hline 12 & 0,45 & 0,065 & 0,036 & 0,03 & 0,002 & 0,001 \\
\hline
\end{tabular}


porcentaje de error de acuerdo al nivel de dispersión aceptado para los datos de eventos corticales e intermedios (Badi et al., 2009). Los resultados se dan en forma gráfica en la Fig. 4.37a y $4.37 \mathrm{~b}$, donde se ve el comportamiento de $\eta_{i}$ y $\eta_{s}$ con la frecuencia así como la de sus asociados $Q_{i}^{-1}$ y $Q_{s}^{-1}$

\subsubsection{INTERPRETACIÓN}

Como se observa de la Fig. 4.37a, $Q_{i}^{-1}$ para eventos corticales es mayor que $Q_{i}^{-1}$ para eventos intermedios a las frecuencias de 3 y $6 \mathrm{~Hz}$, mostrando que la atenuación intrínseca aumenta del manto a la corteza. Aún cuando el MLTWA incluye implícitamente las fugas de energía hacia el interior terrestre además de las pérdidas reales por absorción, se asume que los valores estimados de $Q_{i}^{-1}$ están principalmente asociados a las propiedades anelásticas de las estructuras geológicas complejas de la región. Por otra parte, el $Q_{\mathrm{s}}^{-1}$ obtenido de eventos intermedios es mucho menor que el $Q_{\mathrm{s}}{ }^{-1}$ de eventos corticales (Fig. 4.37b), mostrando una disminución del scattering al pasar de corteza a manto, probablemente debido a la mayor homogeneidad estructural del manto litosférico atravesado por las ondas sísmicas. A profundidades corticales, $Q_{i}^{-1}$ es similar a $Q_{\mathrm{s}}^{-1}$, indicando una contribución similar de ambos procesos de atenuación. En cambio, $Q_{\mathrm{s}}^{-1}$ es mucho menor que $Q_{\mathrm{i}}^{-1}$ en el manto litosférico, lo que puede interpretarse como una mayor contribución de la absorción anelástica por sobre la atenuación de scattering.

Es interesante comparar los valores de $Q_{\mathrm{i}}^{-1}$ y $Q_{\mathrm{s}}^{-1}$ obtenidos con los valores promedios regionales de la atenuación de ondas coda y ondas $\mathrm{S}, Q_{\mathrm{c}}^{-1}$ y $Q_{\mathrm{d}}^{-1}$, obtenidos sobre el mismo conjunto de datos e intervalos de profundidad 0-40 km y más de $40 \mathrm{~km}$. El promedio regional de $Q_{\mathrm{c}}{ }^{-1}$ se ha calculado a partir de los ajustes de envolventes de coda con coeficiente de correlación mayor que 0.9 y un lapso de tiempo de $90 \mathrm{~s}$. Este lapso de tiempo corresponde a un elipsoide de scattering con un semieje mayor de longitud cercana a $120 \mathrm{~km}$ según el modelo de velocidad utilizado (Pulli, 1984). Esta distancia es próxima a los $100 \mathrm{~km}$ considerados como distancia máxima en el ajuste del MLTWA. Para esta comparación se han elegido los resultados promedios regionales del método SBS por dos razones. En primer lugar a fin de evitar la dispersión observada en los resultados regionales del SIS (Fig. 4.5) a un lapso de 90s para la base de datos completa. En segundo lugar, por el hecho de que el ajuste regional obtenido por el SBS para distancias de hasta $100 \mathrm{~km}$ se aproxima al promedio de los valores obtenidos por el SIS sobre grupos de estaciones con distancias máximas de $200 \mathrm{~km}$ coincidiendo con lo observado por Ibáñez et al. (1991). 

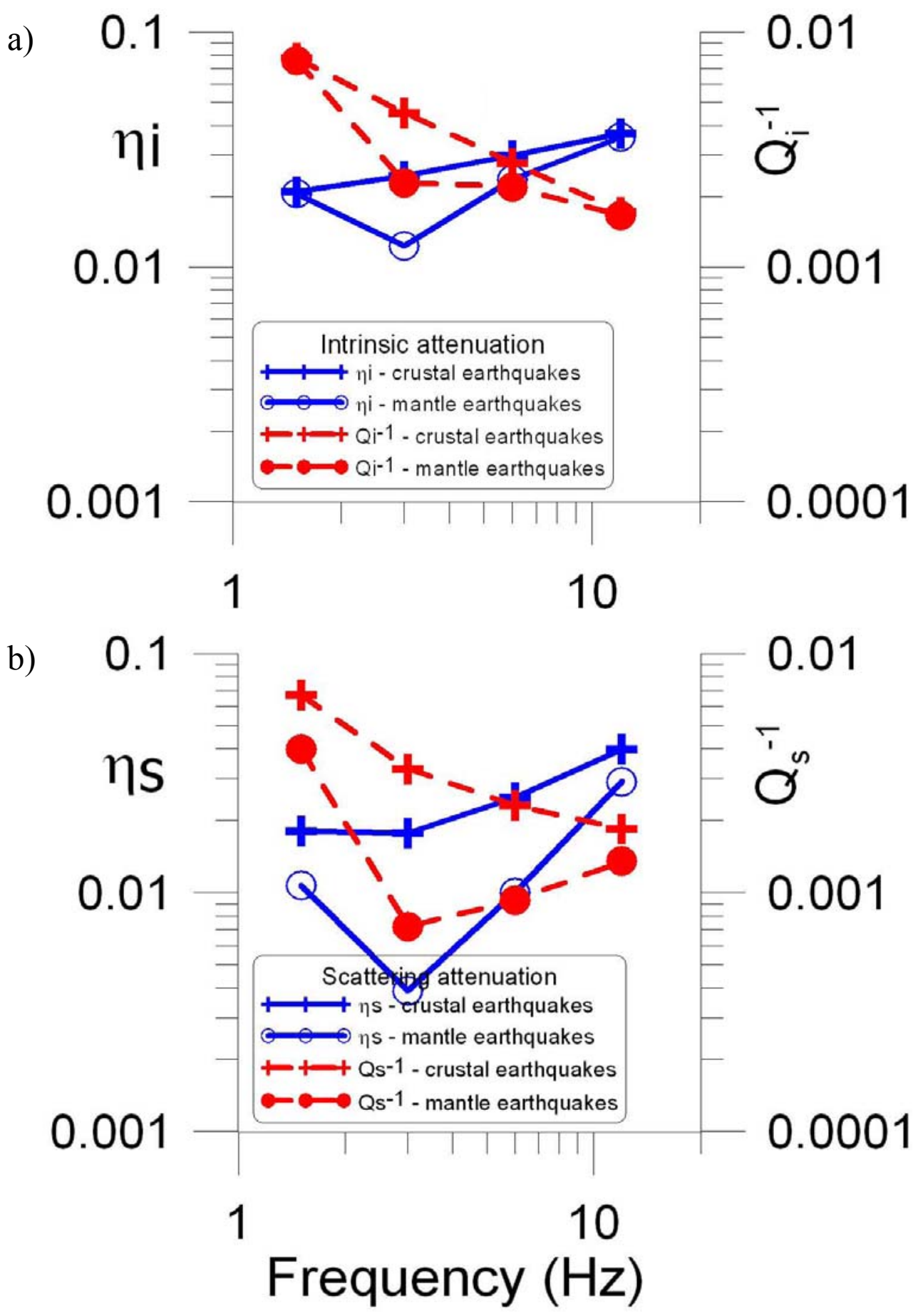

Fig. 4.37.- Resultados de la atenuación intrínseca y de scattering para eventos superficiales e intermedios obtenidos por el MLTWA en función de la frecuencia. En azul se muestran los valores de $\eta_{i}$ y $\eta_{s}$, los coeficientes de atenuación y en rojo los valores de $Q_{i}^{-1}$ y $Q_{s}^{-1}$. Los círculos indican valores en el manto y las cruces señalan los resultados en corteza. a) Valores de $\eta_{i} y Q_{i}^{-1}$ y b) valores de $\eta_{s}$ y $Q_{s}^{-1}$ (Badi et al., 2009).

El valor de $Q_{\mathrm{d}}{ }^{-1}$ se ha estimado aplicando el método de Normalización de la Coda del modo descrito en la Sección 4.2.3.1. Se ha seleccionado una ventana de $10 \mathrm{~s}$ de duración ubicada en el comienzo de la onda $\mathrm{S}$ y una ventana de coda de $10 \mathrm{~s}$ de duración, centrada a un tiempo de referencia de 90s. La corrección de la amplitud de la coda al tiempo de referencia se ha hecho, cuando fue necesario, mediante los valores de $Q_{\mathrm{c}}$ obtenidos por SBS. Los 
valores de $Q_{\mathrm{d}}^{-1}$ se han estimado mediante un ajuste lineal ortogonal en el intervalo de 40 a 250 $\mathrm{km}$ de distancia hipocentral.

En las Fig. 4.38a y 4.38b se comparan los valores medios de $Q_{\mathrm{i}}^{-1}, Q_{\mathrm{s}}^{-1}$ y $Q_{\mathrm{T}}^{-1}$ estimados por el MLTWA con $Q_{\mathrm{c}}^{-1}, Q_{\mathrm{d}}^{-1}$. Como ya se había visto en la determinación de la atenuación de ondas coda, en los promedios regionales no se observan variaciones importantes de $Q_{\mathrm{c}}{ }^{-1}$ con la profundidad y en ambos intervalos de profundidades focales sus valores se aproximan al $Q_{\mathrm{i}}^{-1}$ estimado por el MLTWA. Este resultado coincide con las observaciones realizadas en muchos trabajos (e. g. Sato y Fehler, 1998, Secciones 5.1 a 7.2). Como era de esperarse, los valores de $Q_{\mathrm{d}}^{-1}$ son similares a los de $Q_{\mathrm{T}}^{-1}$ obtenidos sumando $Q_{\mathrm{i}}^{-1}$ y $Q_{\mathrm{s}}^{-1}$ en manto y corteza. Las importantes diferencias en los valores de $Q_{\mathrm{s}}^{-1}$ para manto y corteza indican que el scattering se concentra principalmente en corteza. A $3 \mathrm{~Hz}$, se observa una interesante anomalía en el comportamiento de $Q_{\mathrm{s}}^{-1}$ con la frecuencia que podría estar indicando, de acuerdo con la (2.21), cierta relación entre el tamaño predominante de los dispersores y la longitud de onda.

Las claras diferencias observadas en la región de Nuevo Cuyo entre el scattering a profundidades corticales e intermedias, pueden interpretarse en términos de los diferentes volúmenes de tierra donde las ondas son dispersadas. Las ondas dispersadas generadas a profundidades intermedias involucran un volumen que comprende el manto litosférico por encima de la placa de Nazca en la zona de subducción plana, mientras que las ondas generadas por los terremotos superficiales se encuentran principalmente atrapadas en una corteza altamente heterogénea. El MLTWA da estimaciones de valores medios de los parámetros de atenuación intrínseca y de scattering en dichos volúmenes por lo que sus diferencias se refieren a diferencias en los valores medios de las propiedades de scattering y anelasticidad de los medios.

El predominio de la atenuación intrínseca en el manto litosférico puede ser explicado por la presencia de agua u otros fluidos liberados en la litósfera continental por la dinámica del proceso de subducción. Esto concuerda con la relación establecida por Booker et al. (2005a) entre las variaciones de la resistividad eléctrica observadas y la distribución de sismicidad intermedia y cortical en la región. La gran heterogeneidad que se deduce de los resultados de la atenuación en corteza, coincide con la complejidad tectónica de la placa continental en la región de Nuevo Cuyo donde se encuentran tanto las mayores alturas de la Cordillera de los Andes (6959 m), como la presencia de valles, una tectónica de piel fina y fallas inversas reactivadas sobre un basamento cristalino. 

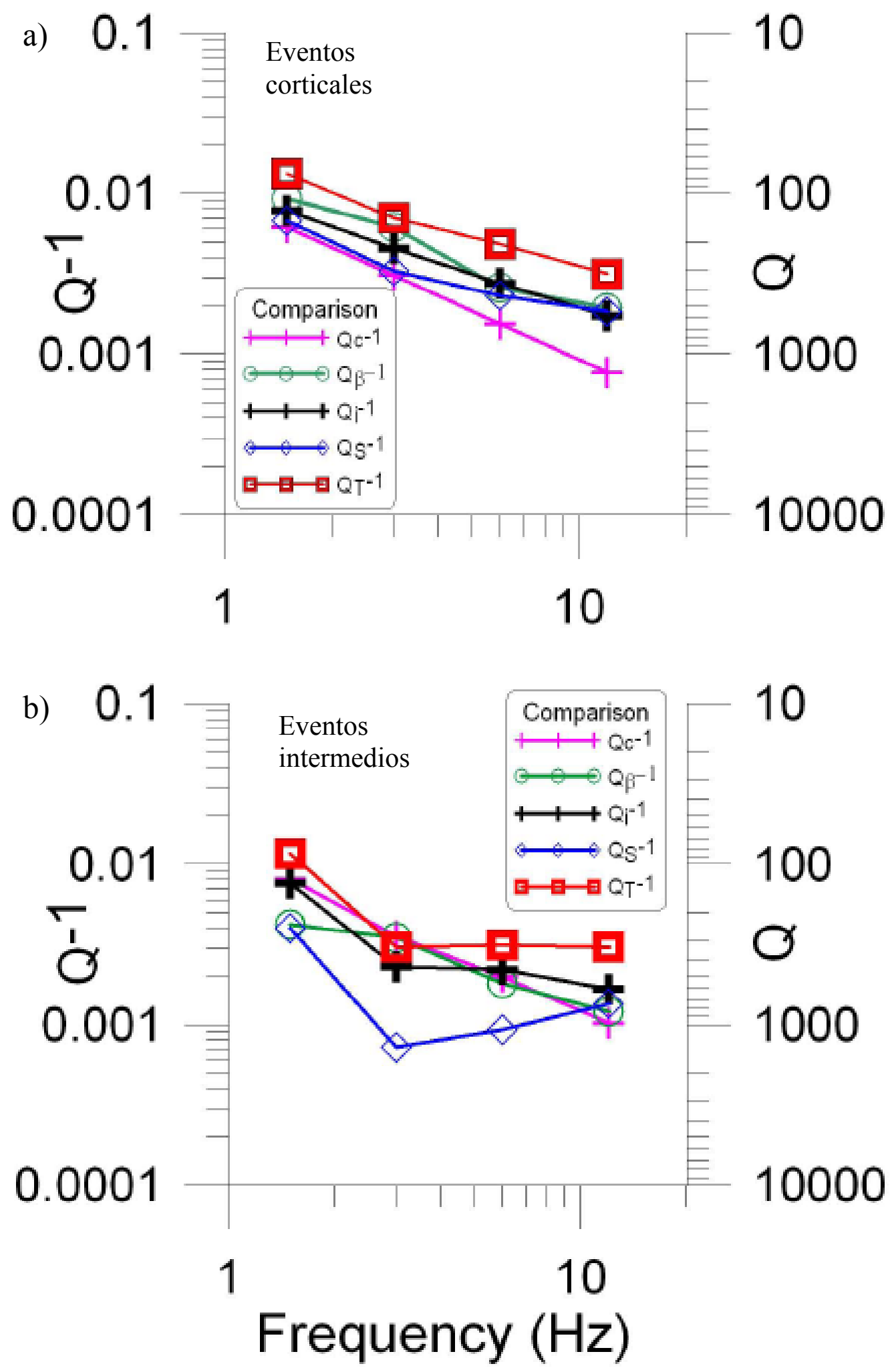

Fig. 4.38. Comparación entre los valores medios de $Q_{i}^{-1}, Q_{s}^{-1}$ y $Q_{T}^{-1}$ estimados por el MLTWA con $Q_{c}^{-1}, Q_{\beta}^{-1}=Q_{d}^{-1}$ obtenidos por SBS y NC respectivamente, para (a) terremotos corticales y (b) terremotos intermedios (Badi et al., 2009). 


\subsubsection{MÉTODO DE WENNERBERG (MWe)}

\subsubsection{APLICACIÓN Y RESULTADOS}

El método de Wennerberg (1993), MWe, permite realizar de manera sencilla la separación de la atenuación intrínseca y de scattering a partir del conocimiento de la atenuación de la onda directa y de su coda, utilizando las (2.128) y (2.129). En dichas ecuaciones $Q_{\mathrm{d}}$ hace referencia a la atenuación total calculada sobre la onda directa, mientras que $Q_{\mathrm{c}}$ es la atenuación de las ondas dispersadas en la coda. En este caso, habiéndose analizado la atenuación de codas de $\mathrm{S}$, debe entenderse a $Q_{\mathrm{d}}$ como el correspondiente a las ondas S directas.

La atenuación de ondas coda se ha estimado en función de la frecuencia y del lapso de tiempo según los métodos de Back-Scattering Simple y Scattering Isótropo Simple. La atenuación de ondas S se ha analizado por el método Espectral y por el de Normalización de la Coda, en ambos casos en función de la frecuencia y de la distancia hipocentral.

Para poder calcular la variación de $Q_{\mathrm{i}}$ y $Q_{\mathrm{s}}$ con el lapso de tiempo, se necesita estimar $Q_{\mathrm{d}}$ para tiempos de viaje comparables a los lapsos de tiempo medidos en la coda. Esto significa que si $t_{\mathrm{c}}$ es el lapso de tiempo de la coda, el tiempo de propagación de la onda directa deberá ser $t_{\mathrm{s}} \leq t_{\mathrm{c}} / 2$ para que se cumpla la validez de la comparación de Wennerberg (1993) entre el SBS y la aproximación híbrida de Zeng (1991) al scattering múltiple (SDZ). La base de datos utilizada en este trabajo cuenta con una gran variedad de distancias hipocentrales que llegan hasta los $500 \mathrm{~km}$ aproximadamente y con profundidades de hasta $150 \mathrm{~km}$ por lo que se dispone de un amplio rango de tiempos de propagación de la onda directa. Por otro lado dado que los factores de calidad son positivos por definición, la (2.128) exige que $Q_{\mathrm{d}}<Q_{\mathrm{c}}$ para obtener soluciones razonablemente posibles de $Q_{\text {s. }}$.

Utilizando el método Espectral se han aplicado dos tipos de ajustes, uno por estaciones para intervalos de tiempo de propagación seleccionados a partir de los datos y otro promediando el efecto de la atenuación a nivel regional (Giampiccolo et al., 2007). En la primera aproximación, se han calculado valores de $Q_{\mathrm{d}}$ para tiempos de viaje que van de los 20 s a 100 s como máximo, obteniéndose resultados para las estaciones $\mathrm{CH} 11, \mathrm{CH} 12, \mathrm{CO} 12$, CO13, RTLS, RTCB y RTMC en el caso de los eventos superficiales y para CH12, CO11, CO12, CO13, RTCB, RTLL y RTMC en el caso de eventos intermedios. En el ajuste regional, se han utilizado los mismos intervalos de tiempo que en el ajuste por estaciones pero estimando un único valor de $Q_{\mathrm{d}}$ promediando los ajustes del decaimiento espectral para todas las estaciones. 
En el método de Normalización de la Coda, en el caso de eventos superficiales, se han hecho los ajustes del factor de calidad para distancias de hasta $350 \mathrm{~km}$, agrupando las estaciones en 7 grupos de acuerdo a criterios de homogeneidad tectónica (Fig. 4.29). Los datos de eventos intermedios han sido reunidos en un solo grupo para obtener una distribución promedio para toda la región, evaluándose ajustes del factor de calidad en dos intervalos de distancia hipocentral, por encima y por debajo de los $250 \mathrm{~km}$ de acuerdo a las tendencias observadas.

De este modo, en el caso del ME, se dispone de tiempos de propagación de $\mathrm{S}$ por debajo de los $100 \mathrm{~s}$ en eventos superficiales e intermedios, lo que implica tiempos de coda del orden de 190-200 s. En el caso del método de NC, para los eventos superficiales, las distancias máximas de $350 \mathrm{~km}$ equivalen a un tiempo promedio de propagación de la onda directa de unos $95 \mathrm{~s}$ a una velocidad media de $\mathrm{S}$ de $3.7 \mathrm{~km} / \mathrm{s}$. Para eventos intermedios, se puede suponer un tiempo de propagación de $58 \mathrm{~s}$ aproximadamente, para distancias máximas de 250 $\mathrm{km}$ y velocidad promedio de $4.3 \mathrm{~km} / \mathrm{s}$.

Los ajustes regionales del SBS y SIS se han podido efectuar en lapsos de tiempo a partir de $40 \mathrm{~s}$. A profundidades intermedias han dado resultados con comportamientos similares en todos los lapsos de tiempo. En cambio a profundidades corticales los valores del factor de calidad del SIS se apartan notablemente del SBS por debajo de los $180 \mathrm{~s}$ y ambos métodos, muestran un comportamiento anómalo del exponente que describe su variación con la frecuencia para lapsos de tiempo cortos, cercanos a $40 \mathrm{~s}$. Por lo tanto, dada la estabilidad de las soluciones para lapsos de tiempo largos puede suponerse la validez del método utilizando longitudes de coda largas, de 120 s o de 190 s según sea el tiempo de propagación de la onda S directa. Los ajustes de $Q_{\mathrm{c}}$ seleccionados para este análisis, son los efectuados según el método de SBS para los mismos grupos de estaciones del método de Normalización de la Coda.

En primer lugar se ha hecho la separación de la atenuación intrínseca y de scattering tomando los valores de $Q_{\mathrm{d}}$ obtenidos con el ME para cada estación, asignando sus valores o el promedio de ellos, al grupo al que pertenecen. Los valores de $Q_{\mathrm{c}}$ se eligieron para un lapso de tiempo de coda de $190 \mathrm{~s}$ de acuerdo a los tiempos de propagación de la onda $\mathrm{S}$ empleados en el ajuste del método espectral. Reemplazando en las (2.128) y (2.129) se resolvieron las ecuaciones de segundo grado que resultan del despeje de las mismas, obteniéndose los valores de $Q_{\mathrm{s}}$ y $Q_{\mathrm{i}}$ por el MWe. En la Fig. 4.39 se presentan los valores posibles de $Q_{\mathrm{s}}^{-1}, Q_{\mathrm{i}}^{-1}$ y $Q_{T}^{-1}$ 


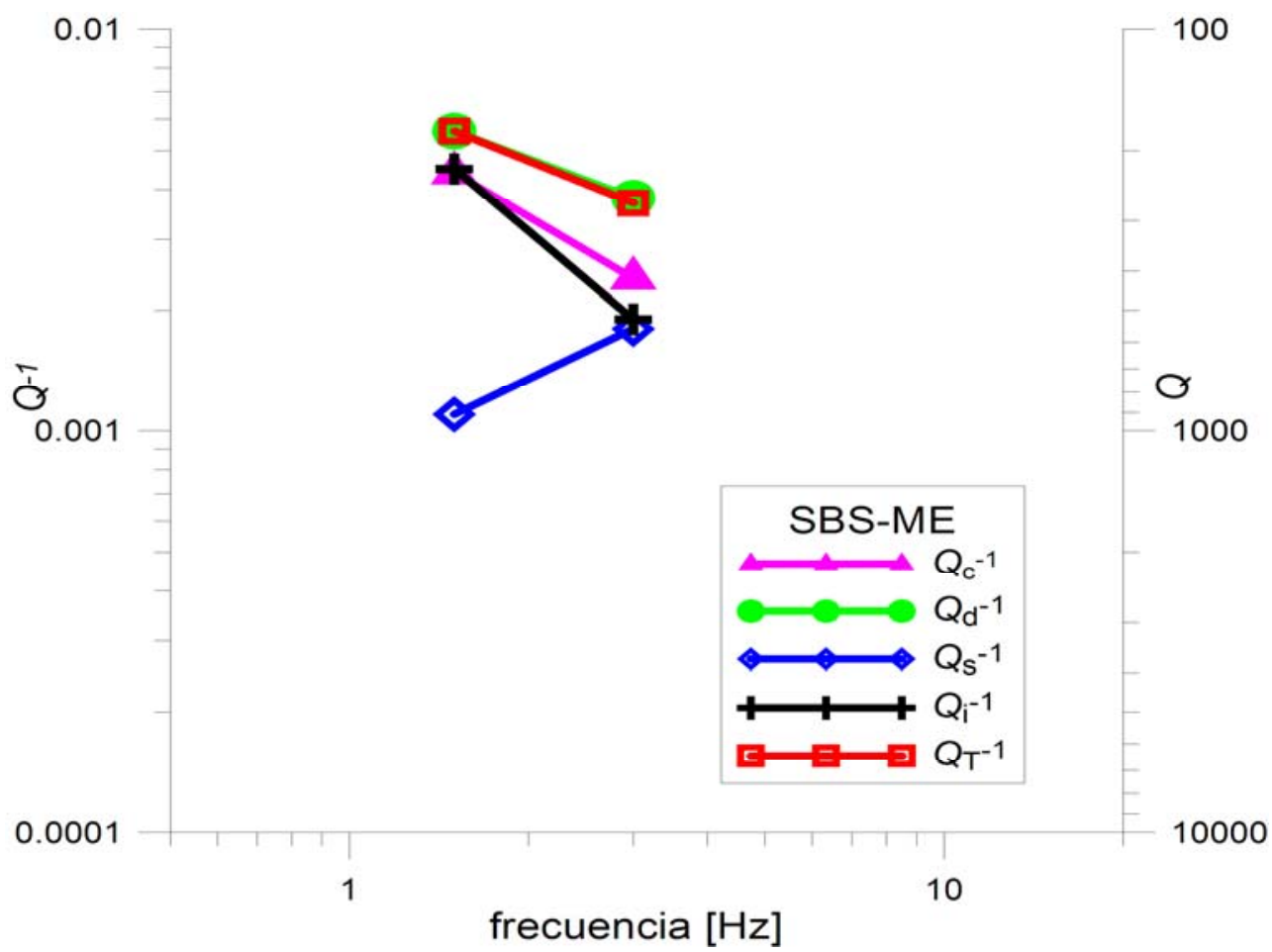

Fig. 4.39.- Valores promedio regionales de la separación de la atenuación intrínseca, $Q_{i}^{-1}, y$ de scattering, $Q_{s}^{-1}$, en función de la frecuencia mediante la aproximación de Wennerberg (1993) al scattering múltiple de Zeng (1991), utilizando los ajustes de $Q_{c}$ del método de Back- Scattering Simple y de $Q_{d}=Q_{S}$ mediante el Método Espectral.

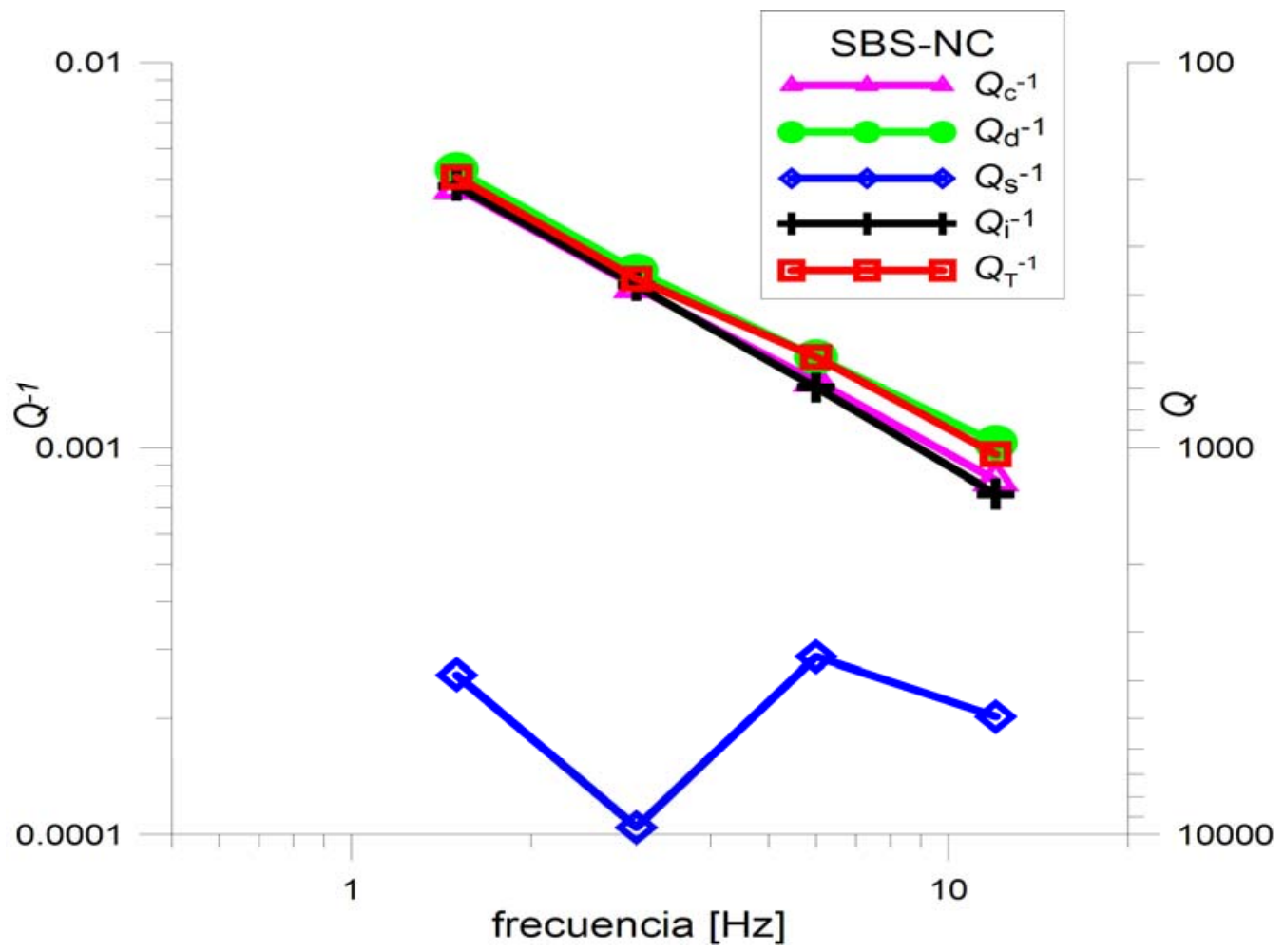

Fig. 4.40.- Valores promedio regionales de la separación de la atenuación intrínseca, $Q_{i}^{-1}$, y de scattering, $Q_{s}^{-1}$, en función de la frecuencia mediante la aproximación de Wennerberg (1993) al scattering múltiple de Zeng (1991), utilizando los ajustes de $Q_{c}$ del método de Back- Scattering Simple y de $Q_{d}$ mediante el Método de Normalización de la Coda. 
medios obtenidos para el caso de eventos superficiales. Comparándolos con los valores empleados de $Q_{\mathrm{c}}^{-1}$ y $Q_{\mathrm{d}}^{-1}$, se observa que $Q_{\mathrm{c}}^{-1}, Q_{\mathrm{d}}^{-1}$ y $Q_{\mathrm{i}}^{-1}$ presentan la misma tendencia mientras que $Q_{\mathrm{s}}^{-1}$ crece con la frecuencia. La atenuación por scattering resulta mucho menor que la de origen intrínseco a $1.5 \mathrm{~Hz}$, dado que $Q_{\mathrm{d}}^{-1}$ y $Q_{\mathrm{c}}{ }^{-1}$ toman valores próximos entre sí, como se deduce de la (2.128) (Wennerberg, 1993). Sin embargo, para $3 \mathrm{~Hz}$, la influencia de la atenuación por scattering es similar a la absorción intrínseca. Por otra parte $Q_{\mathrm{i}}^{-1}$ se mantiene próximo a $Q_{\mathrm{c}}^{-1}$ y la atenuación total estimada como suma de $Q_{\mathrm{s}}^{-1}$ y $Q_{\mathrm{i}}^{-1}$, es próxima a $Q_{\mathrm{d}}^{-1}$. Se ha considerado también la separación de $Q_{\mathrm{s}}$ y $Q_{\mathrm{i}}$ empleando los ajustes medios regionales del $\mathrm{ME}$ en ambos rangos de profundidad, pero en este caso, los valores de $Q_{\mathrm{d}}$ estimados superan ampliamente a los valores de $Q_{c}$, por lo que no puede aplicarse la (2.128).

En segundo lugar, se ha realizado la separación de $Q_{\mathrm{s}}$ y $Q_{\mathrm{i}}$ mediante la combinación de los resultados del SBS y del NC. En este caso, se han tomado los ajustes de $Q_{\mathrm{c}}$ para lapsos de tiempo de $190 \mathrm{~s}$ en eventos superficiales y de $120 \mathrm{~s}$ en el caso de eventos intermedios, dado que los ajustes de $Q_{\mathrm{d}}$ se han efectuado hasta distancias máximas de $350 \mathrm{~km}$ y $250 \mathrm{~km}$ respectivamente. Resolviendo el sistema de ecuaciones con las nuevas variables y seleccionando los valores razonables y posibles de $Q_{\mathrm{s}}$ y $Q_{\mathrm{i}}$ se ha elaborado la Fig. 4.40. A frecuencias entre 1 y $12 \mathrm{~Hz}, Q_{\mathrm{i}}^{-1}$ permanece cercano a $Q_{\mathrm{c}}^{-1}$ mientras que $Q_{\mathrm{T}}^{-1}$ prácticamente coincide con $Q_{\mathrm{d}}{ }^{-1}$. La atenuación intrínseca y la total disminuyen con la frecuencia como ya se había observado anteriormente. Nuevamente la atenuación por scattering resulta ser muy débil y esto se debe a que los valores de $Q_{\mathrm{d}}^{-1}$ se encuentran próximos a los de $Q_{\mathrm{c}}{ }^{-1}$. Los resultados de la atenuación por scattering no permiten establecer una dependencia clara con la frecuencia. Relacionando al scattering con la densidad de heterogeneidades, a la longitud de onda con la dimensión de las mismas y a la variación de la atenuación con la frecuencia con la

\begin{tabular}{|c|c|c|c|c|c|}
\hline Ajuste Wennerberg & Frecuencia [Hz] & $\boldsymbol{Q}_{\mathbf{c}}^{-1}$ & $\boldsymbol{Q}_{\mathbf{d}}{ }^{-1}$ & $\boldsymbol{Q}_{\mathbf{s}}^{-1}$ & $\boldsymbol{Q}_{\mathbf{i}}^{-1}$ \\
\hline \multirow{2}{*}{ SBS-ME } & 1.5 & 0.0044 & 0.0056 & 0.0011 & 0.0045 \\
\cline { 2 - 6 } & 3 & 0.0024 & 0.0038 & 0.0018 & 0.0019 \\
\hline \multirow{3}{*}{ SBS-NC } & 1.5 & 0.0047 & 0.0053 & 0.0003 & 0.0048 \\
\cline { 2 - 6 } & 3 & 0.0026 & 0.0029 & 0.0001 & 0.0026 \\
\cline { 2 - 6 } & 6 & 0.0015 & 0.0017 & 0.0003 & 0.0014 \\
\cline { 2 - 6 } & 12 & 0.0008 & 0.0011 & 0.0002 & 0.0019 \\
\hline
\end{tabular}

Tabla 4.7.- Comparación de valores promedio de la atenuación de ondas coda $\left(Q_{c}^{-1}\right)$, ondas transversales directas $\left(Q_{d}^{-1}\right)$, scattering $\left(Q_{s}^{-1}\right)$ y absorción intínseca $\left(Q_{i}^{-1}\right)$ para la Región de Nuevo Cuyo según la aproximación de Wennerberg al scattering múltiple de Zeng. SBS-ME indica que la resolución del método se hizo con ajustes del Método Espectral para ondas directas y del BackScattering Simple para codas, mientras que SBS-NC indica que se utilizaron ajustes del método de Normalización de la Coda para ondas directas v del SBS para codas. 
distribución de dichas dimensiones (Sección 2.1.2) puede decirse que, según esta aproximación, la densidad de los dispersores no guarda clara relación con sus dimensiones.

La Tabla 4.7 presenta los valores promedio regionales de $Q_{\mathrm{c}}^{-1}, Q_{\mathrm{d}}^{-1}, Q_{\mathrm{s}}^{-1}$ y $Q_{\mathrm{i}}^{-1}$ obtenidos por la aproximación de Wennerberg mediante el método espectral y el método de normalización de la coda.

\subsubsection{INTERPRETACIÓN}

El método de Wennerberg para la separación del scattering y la absorción intrínseca resulta ser de muy sencilla aplicación, pero son numerosas las consideraciones que deben hacerse sobre los datos, tal como se ha visto en la sección anterior. Además sus soluciones se ven afectadas por la propagación de los errores en la determinación de la atenuación de ondas directas (ME, NC) y de ondas coda (SBS) por lo que sus resultados deben considerarse como una aproximación, sobre todo para aquellos métodos en que la implementación debe aún ser optimizada.

En la mayoría de las aplicaciones del MWe a datos reales que se encuentran en la bibliografía, los tiempos de propagación de $\mathrm{S}$ empleados son muy cortos y los rangos de distancia, pequeños, como es en el caso de la sismología volcánica (Del Pezzo et al., 1995, Martínez Arévalo, 2005). En este trabajo se ha contado con lapsos de tiempo entre 40 y $200 \mathrm{~s}$ para las ondas coda y distancias de hasta $350 \mathrm{~km}$ o tiempos de viaje de hasta $100 \mathrm{~s}$, para el análisis de ondas $\mathrm{S}$ directas. Viendo que en la atenuación de ondas directas los mejores ajustes se daban a distancias grandes y que en la atenuación de ondas coda los dos métodos de ajuste convergen a lapsos de tiempo largos, involucrando volúmenes de scattering de escala regional, se ha realizado la separación de $Q_{\mathrm{s}}$ y $Q_{\mathrm{i}}$ mediante el método de Wennerberg (1993) en lapsos de tiempo largos. Los resultados obtenidos se interpretan como valores medios regionales tanto en superficie como en profundidad, ya que lapsos de tiempo de $190 \mathrm{~s} \mathrm{y}$ distancias hipocentrales máximas del orden de $300 \mathrm{~km}$ implican profundidades máximas de los elipsoides cercanas a los $400 \mathrm{~km}$, de acuerdo a la hipótesis de Pulli (1984). Por lo tanto, pueden asignarse los valores de $Q_{\mathrm{i}}^{-1}$ y $Q_{\mathrm{s}}^{-1}$ a los efectos de la atenuación intrínseca y de scattering en una capa que abarca el espesor del sistema de placas Sudamericana-Nazca yuxtapuestas e inclusive el manto astenosférico por debajo de ellas hasta profundidades apenas por encima del inicio de la zona de transición en el manto según los modelos globales (Dziewonski, 2005).

Del análisis de los valores $Q_{\mathrm{s}}^{-1}$ y $Q_{\mathrm{i}}^{-1}$ obtenidos, puede estimarse que la atenuación intrínseca juega un rol preponderante en el proceso de subducción y es muy próxima a la 
atenuación de ondas coda. Este resultado puede compararse con los valores obtenidos en el MLTWA a profundidades intermedias. Según la aproximación de Wennerberg y de acuerdo a los valores de la atenuación total observados en la región, la atenuación por scattering resulta ser muy débil. En el caso de la región de Nuevo Cuyo, donde los procesos geodinámicos establecidos en la subducción incluyen modificaciones de geotermas, deshidratación de placa oceánica, anisotropías en el flujo convectivo del manto, fusión parcial y tensiones tectónicas de gran magnitud, entre otros, todos ellos interrelacionados, siendo causa y efecto a la vez, es lógico pensar que el proceso de atenuación dominante por encima de los $400 \mathrm{~km}$ de profundidad sea la absorción intrínseca. 


\section{CAPÍTULO V}

\section{Análisis comparativo de los resultados}

En este capítulo se comparan los valores de la atenuación obtenidos para las ondas $\mathrm{P}, \mathrm{S}$ y coda según los diferentes métodos empleados con los valores de la atenuación intrínseca y de scattering. Seguidamente se cotejan con algunos de los muchos resultados que pueden hallarse en la bibliografía y que han sido obtenidos por otros autores en diversas regiones sísmicamente activas del mundo.

\subsection{LA REGIÓN DE NUEVO CUYO}

A fin de tener una imagen completa de la atenuación en la región de Nuevo Cuyo, se presentan algunas figuras que permiten comparar los resultados obtenidos en este estudio para las distintas ondas analizadas y según los métodos. En la Fig. 5.1a se presentan los valores de $Q$ obtenidos para ondas directas en los dos intervalos de profundidad considerados. En la Fig. $5.1 \mathrm{~b}$ se muestran los valores de $Q$ obtenidos para ondas directas que recorren trayectorias corticales y los valores de $Q_{\mathrm{c}}$ para lapsos de tiempo de coda de 40, 90 y 190 s obtenidos de los ajustes para eventos superficiales. En la Fig. 5.1c se observan los valores $Q$ obtenidos para ondas directas que inician sus trayectorias en la placa de Nazca subducida junto a los valores de $Q_{\text {c }}$ para lapsos de tiempo de coda de 40, 90 y 190 s obtenidos de los ajustes para eventos superficiales. De los resultados del ME, denotados en este caso como $Q_{\alpha}$ (que representa al $Q$ de ondas P) y $Q_{\beta}$ (que representa al $Q$ de ondas S), se ha visto que $Q_{\alpha}$ y $Q_{\beta}$ convergen hacia las altas frecuencias a profundidades intermedias, mientras que divergen a profundidades corticales. De las Fig. 5 b y c puede verse además que a profundidades corticales $Q_{\alpha}$ y $Q_{\beta}$ divergen desde un valor cercano al valor de $Q_{\mathrm{P}}$ obtenido por el APP y a profundidades intermedias convergen a un valor cercano al $Q_{\mathrm{P}}$ correspondiente a esa profundidad. A profundidades intermedias, el ME da resultados para ondas $\mathrm{S}$ similares al $\mathrm{NC}\left(Q_{\mathrm{d}}\right)$ a bajas frecuencias, mientras que los resultados del ME para ondas P se aproximan al APP para altas frecuencias. A profundidades corticales, los resultados del ME y el APP se asemejan a bajas 


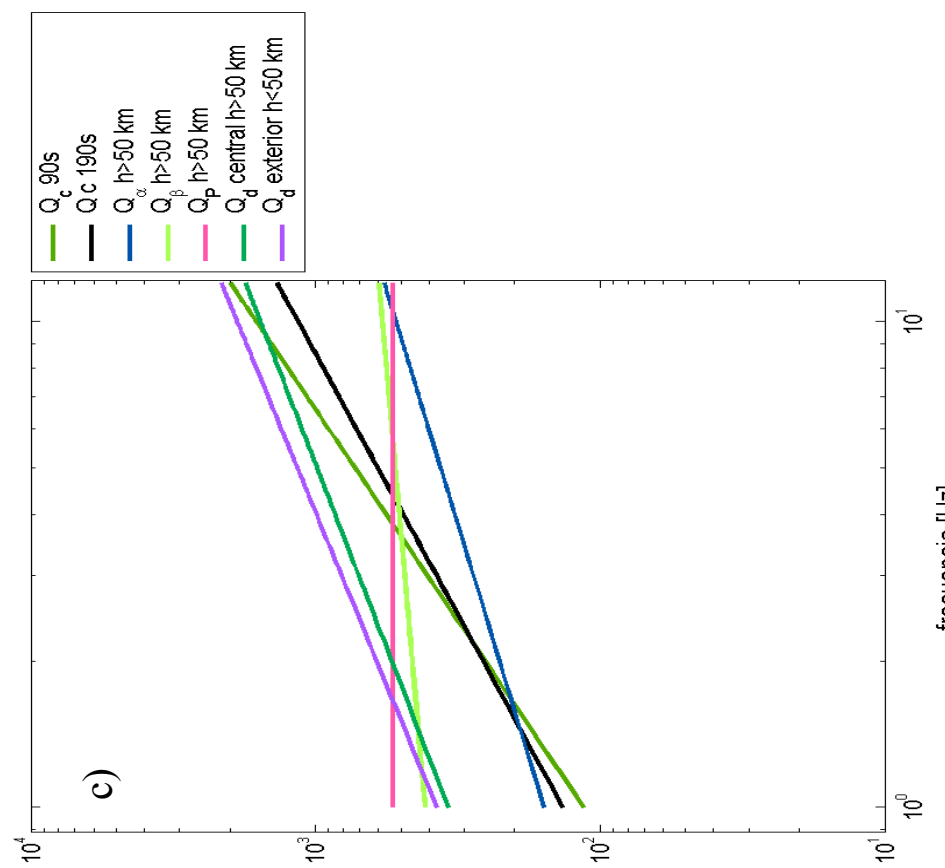

过

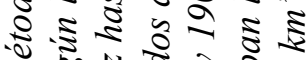
इ

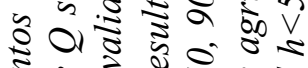

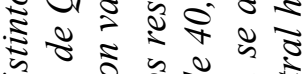
ป ป

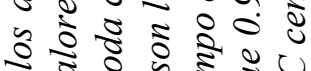

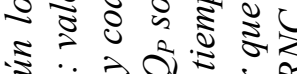

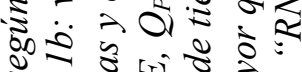

T्र का डू

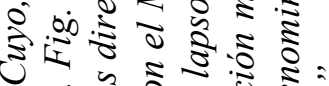

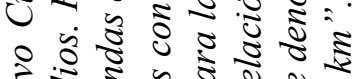

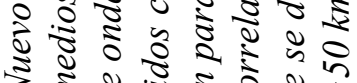

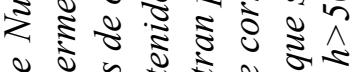

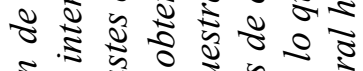

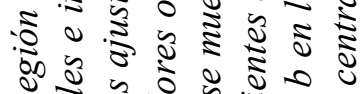

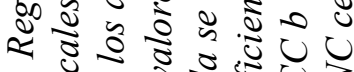

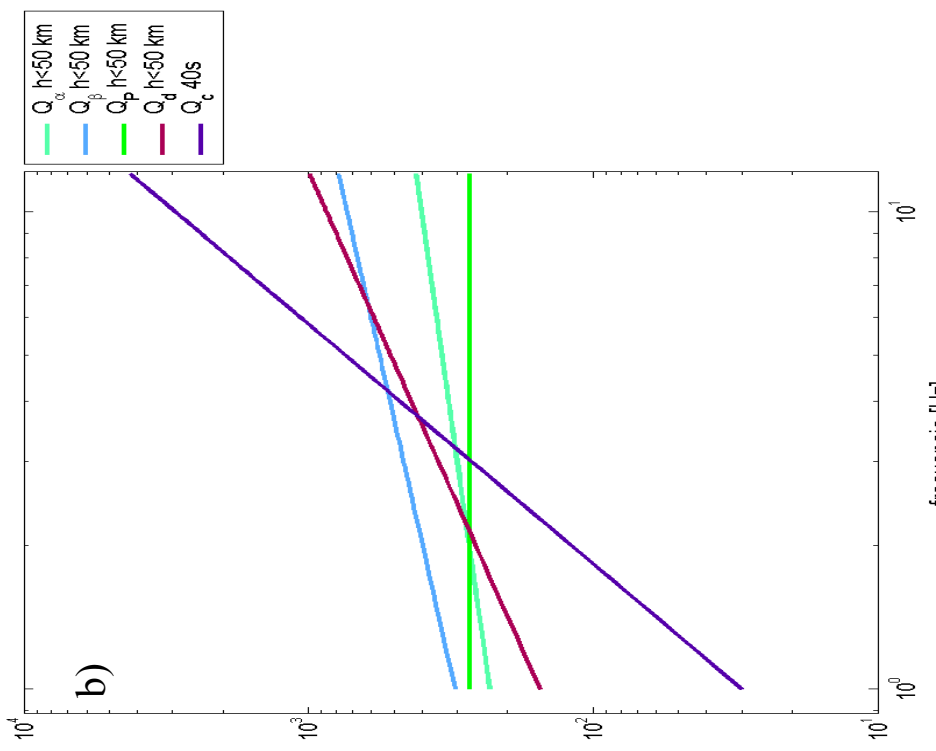

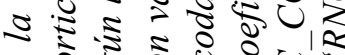

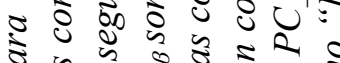
¿. : ปे चे

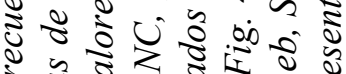

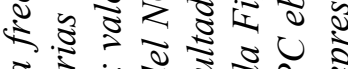

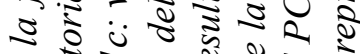

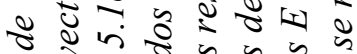

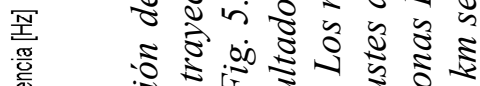

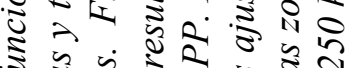

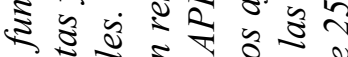
จ ช ซ 人)

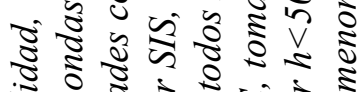

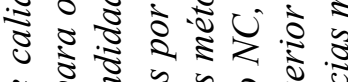

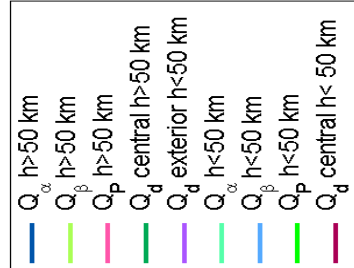

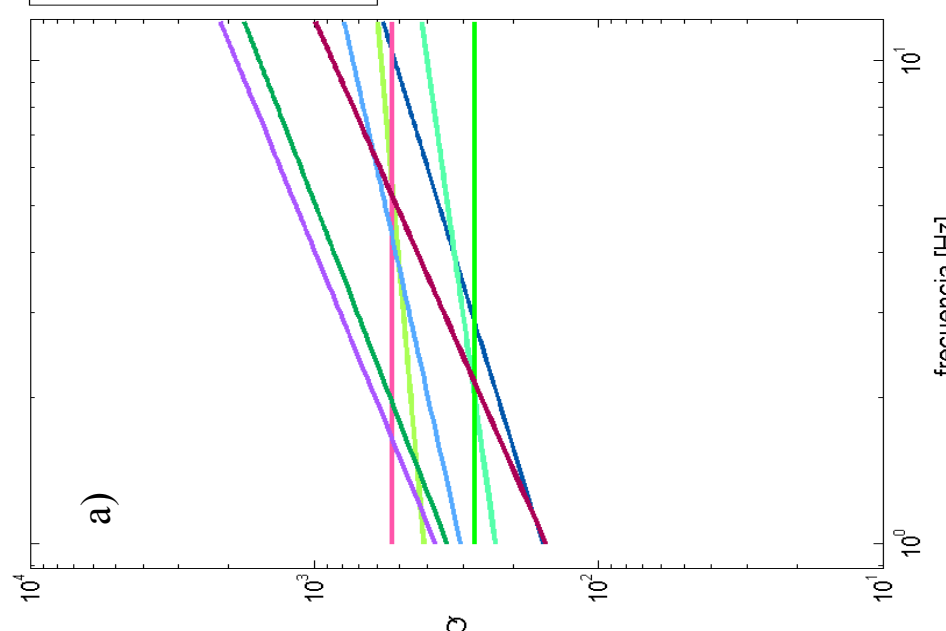

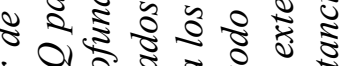

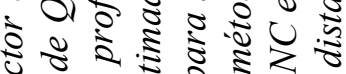

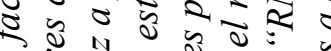

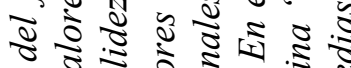
2.

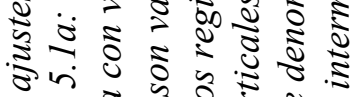
施

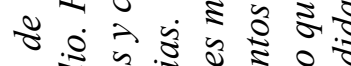

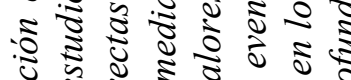

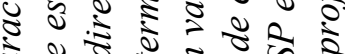

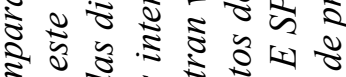

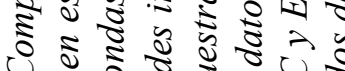

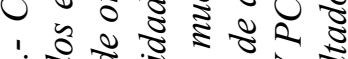
- ₹

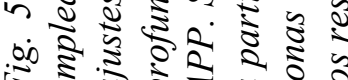

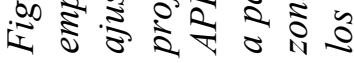


frecuencias, mientras que el ME y el NC lo hacen hacia las altas frecuencias. En todos los casos la mayor dependencia con la frecuencia se observa en el $Q_{\mathrm{c}}$.

Deshayes et al. (2008) han presentado una tomografía de atenuación de ondas P y S utilizando datos de estaciones temporales (proyectos OVA99 y CHARSME) y estaciones permanentes chilenas con el modelo de velocidad 3D de Pardo et al. (2004). En sus resultados (Fig. 5.2) hallaron una atenuación altamente heterogénea por encima de los $20 \mathrm{~km}$ de profundidad a $\operatorname{los} 31.5^{\circ} \mathrm{S}$, con anomalías de signos opuestos para ondas $\mathrm{P}$ y S que podrían indicar circulación de fluidos y/o una distribución compleja de los mismos. Dichas anomalías no se mostraban correlacionadas con las anomalías de velocidad. A mayores profundidades la atenuación resultaba más homogénea y menor que la supuesta en el modelo inicial. Al comparar sus resultados con las anomalías halladas en este estudio, se ha visto que a profundidades corticales, los máximos y mínimos de $Q$ de ondas $\mathrm{S}$ obtenidos por los métodos ME y NC coinciden aproximadamente con los de Deshayes et al.(2008) en corteza superior. En corteza inferior y manto, Deshayes et al.(2008) no muestran anomalías importantes, mientras que los resultados del ME mantienen las anomalías que se observan a menores profundidades. En cuanto a las anomalías de la atenuación de ondas $\mathrm{P}$ a profundidades corticales, los signos de las anomalías encontrados por Deshayes et al. (2008) a $31.5^{\circ} \mathrm{S}$, coinciden con las anomalías observadas en este estudio mediante ME algo más al sur. Los resultados de Deshayes et al. (2008) no definen el importante mínimo hallado por el ME en Sierra Pie de Palo.
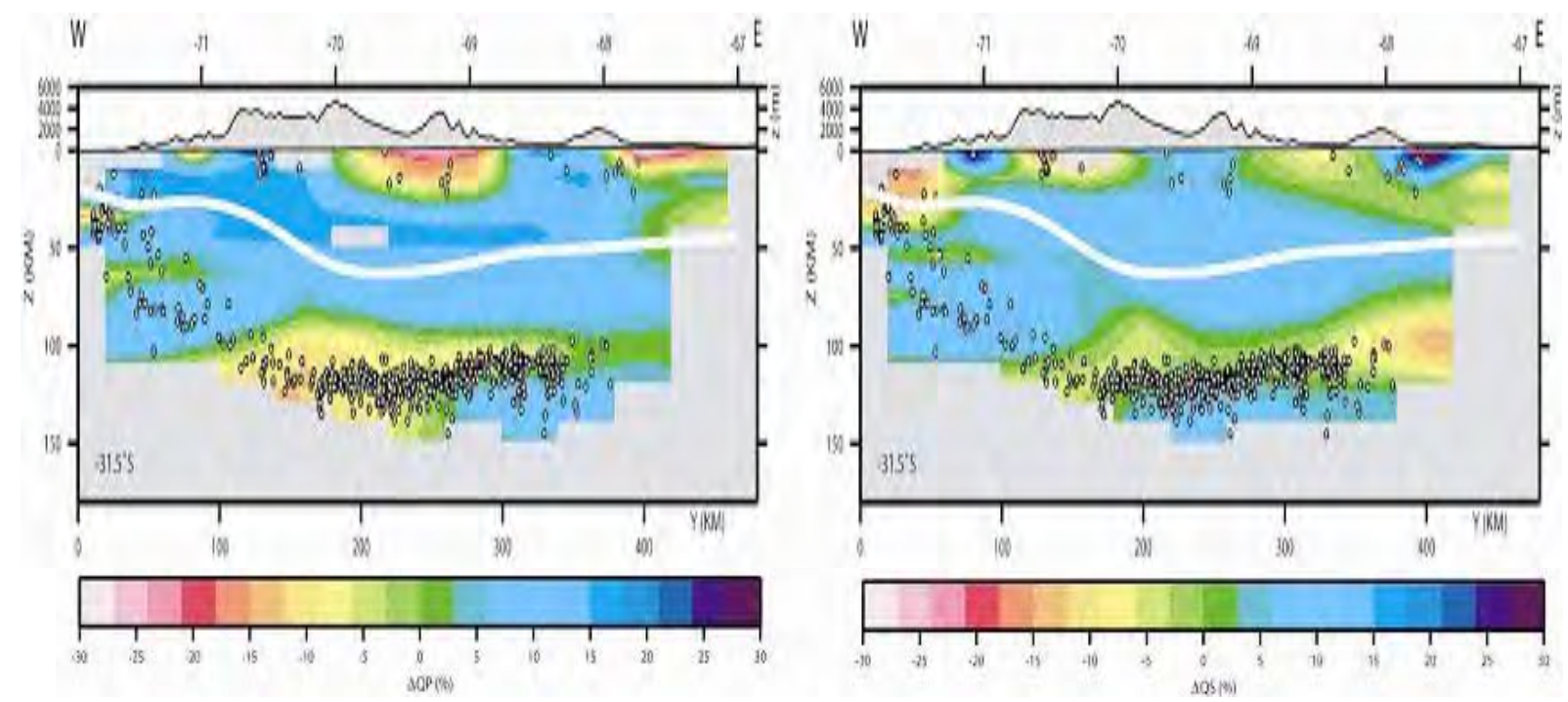

Fig. 5.2.- Secciones verticales de atenuación de ondas $P$ (izquierda) y $S$ (derecha) a los $31.5^{\circ} S$ obtenidas por Deshayes et al. (2008). La escala de colores indica porcentajes de de desviación. Las zonas con pobre resolución se indican en gris. Las profundidades del Moho son de Tassara (2005) y los puntos blancos indican los sismos utilizados. Extracatada de Deshayes et al. (2008) 


\subsection{LA REGIÓN DE NUEVO CUYO Vs. OTRAS REGIONES}

\subsubsection{ONDAS CODA}

La Tabla 5.1 resume valores de la atenuación de ondas coda a través de los ajustes de $Q_{\mathrm{c}}$ en función de la frecuencia para diversas regiones utilizando los métodos SBS o SIS. Los ejemplos se presentan en orden decreciente de $Q_{0}$, indicándose en algunos casos el lapso de tiempo utilizado para el ajuste. Los resultados de este estudio estimados para trayectorias de eventos superficiales y lapsos de tiempo de 40, 90 y 190 s se presentan como RNC. La RNC presenta una atenuación que dependiendo del lapso de tiempo elegido puede compararse al sur de Noruega, el Sur de España o la región de Abruzzo en Italia. Para los lapsos de tiempo más cortos, la dependencia con la frecuencia en la RNC resulta mayor que en las otras regiones, lo que podría relacionarse con diferencias en las escalas de las heterogeneidades.

\begin{tabular}{|l|l|l|}
\hline Región & $Q_{c}=Q_{0} f^{\alpha}$ & Cita \\
\hline Centro de EEUU & $1000 f^{0.2}$ & Singh y Herrmann, 1983 \\
\hline New England, EEUU & $660 f^{0.4}$ lapso $>100 \mathrm{~s}$ & Pulli, 1984 \\
\hline Quebec, Canadá & $305 \pm 15 f^{0.0 .8 \pm 0.02}$ lapso $=80 \mathrm{~s}$ & Woodgold, 1994 \\
\hline Francia & $278 \pm 5 f^{0.67 \pm 0.05}$ & Chevrot y Cansi, 1996 \\
\hline NO Himalaya & $158 f^{1.05}$ & Kumar et al., 2005 \\
\hline NE de Brasil & $151 \pm 99 f^{0.98 \pm 0.05}$ & Pereira Dias y de Souza, 2004 \\
\hline Oeste de EEUU & $150 f^{0.45}$ & Singh y Herrmann, 1983 \\
\hline New England, EEUU & $140 f^{0.95}$ lapso $<100 \mathrm{~s}$ & Pulli, 1984 \\
\hline RNC, Argentina & $135 \pm 10 f^{0.93 \pm 0.01}$ lapso $=190 \mathrm{~s}$ & este estudio \\
\hline Sur de Noruega & $120 f^{1.09}$ & Kvamme y Havskov, 1989 \\
\hline Granada, España & $118 \pm 8.9 f^{0.86 \pm 0.02}$ lapso $=70 \mathrm{~s}$ & Ibáñez et al., 1990 \\
\hline RNC, Argentina & $114 \pm 7 f^{1.15 \pm 0.03}$ lapso $=90 \mathrm{~s}$ & este estudio \\
\hline Costa Rica & $91 \pm 8.4 f^{0.72 \pm 0.071}$ & González y Persson, 1997 \\
\hline Venezuela & $75 \pm 1.09 f^{0.96 \pm 0.05}$ & García et al., 2004 \\
\hline Valle del Irno, Italia & $67.3 f^{0.74}$ & Del Pezzo et al., 1991 \\
\hline Antequera, Sur de España & $65 f^{0.89}$ lapso $=60 \mathrm{~s}$ & Ibáñez et al., 1993 \\
\hline Cuba & $62 \pm 2 f^{0.84 \pm 0.01}$ & Biescas et al., 2007 \\
\hline Abruzzo, Italia & $34 f^{0.71}$ lapso $=50 \mathrm{~s}$ & Ibáñez et al., 1993 \\
\hline RNC, Argentina & $30 \pm 7 f^{1.99 \pm 0.09}$ lapso $=40 \mathrm{~s}$ & este estudio \\
\hline & & \\
\hline
\end{tabular}

Tabla 5.1.- Valores del factor de calidad de ondas coda obtenidos mediante SBS o SIS por otros autores en diferentes regiones y en la región de Nuevo Cuyo (RNC) por este estudio. Se presentan valores en la RNC para lapsos de tiempo de 40, 90 y $190 \mathrm{~s}$ ajustados sobre datos de eventos superficiales. Las regiones se listan en orden de Qc decreciente. 


\subsubsection{ONDAS P y S}

En la Tabla 5.2 se presentan factores de calidad de ondas $\mathrm{P}$ y S obtenidos en diferentes regiones del mundo por otros autores utilizando métodos dependientes o independientes de la frecuencia y en algunos casos, tomografía de atenuación. Como valores de la región de

\begin{tabular}{|c|c|c|c|}
\hline Región & $Q_{\alpha}=Q_{0} f^{\alpha}$ Ondas $\mathbf{P}$ & $Q_{\beta}=Q_{0} f^{\alpha}$ Ondas $\mathbf{S}$ & Cita \\
\hline $\begin{array}{l}\text { RNC, Argentina } \\
\text { zona central* }\end{array}$ & - & $\begin{array}{l}153 \pm 115 f^{0.75 \pm 0.47} \mathrm{~h}<50 \mathrm{~km} \\
340 f^{0.66} \mathrm{~h}>50 \mathrm{~km}\end{array}$ & $\begin{array}{l}\text { este estudio } \\
\text { método } \mathrm{NC}\end{array}$ \\
\hline $\begin{array}{l}\text { RNC, Argentina } \\
\text { zona exterior** }\end{array}$ & - & $374 \pm 209 f^{0.70 \pm 0.24} \mathrm{~h}<50 \mathrm{~km}$ & $\begin{array}{l}\text { este estudio } \\
\text { método NC }\end{array}$ \\
\hline RNC, Argentina & $\begin{array}{l}230 \pm 21 f^{0.24 \pm 0.04} \mathrm{~h}<50 \mathrm{~km} \\
157 \pm 65 f^{0.52 \pm 0.21} \mathrm{~h}>50 \mathrm{~km}\end{array}$ & $\begin{array}{l}305 \pm 28 f^{0.38 \pm 0.04} \mathrm{~h}<50 \mathrm{~km} \\
411 \pm 41 f^{0.15 \pm 0.06} \mathrm{~h}>50 \mathrm{~km}\end{array}$ & $\begin{array}{l}\text { este estudio } \\
\text { método ME }\end{array}$ \\
\hline RNC, Argentina & $\begin{array}{l}271 \mathrm{~h}<40 \mathrm{~km} \\
532 \mathrm{~h}>40 \mathrm{~km}\end{array}$ & - & $\begin{array}{l}\text { este estudio } \\
\text { método APP }\end{array}$ \\
\hline Kuriles, Pacífico O. ${ }^{\&}$ & $140 f^{0.8}$ & $172 f^{0.9}$ & Butler, 1987 \\
\hline Kuriles Sur. & - & $\begin{array}{l}39 f^{1.0} \mathrm{~h}<50 \mathrm{~km} \\
500 f^{1.0} \mathrm{~h}>50 \mathrm{~km}\end{array}$ & Maeda y Sasatami, 2006 \\
\hline Honshu N, Japón & $150-180$ & - & Tsumura et al., 2000 \\
\hline Honshu S., Japón & 150 & - & Tsumura et al., 2000 \\
\hline Kanto, Japón & $32.26 f^{0.95}$ & $83.3 f^{0.73}$ & Yoshimoto et al., 1993 \\
\hline NE de Japón & - & $70-120$ & Takanami et al., 2000 \\
\hline Jujuy, Argentina \&\& & $\begin{array}{l}>500 \\
<350\end{array}$ & $\begin{array}{l}>350 \\
<200\end{array}$ & Whitman et al., 1992 \\
\hline $\begin{array}{l}\text { Andes Centrales } \\
\text { (lim. Arg.-Ch.-Bol.) }\end{array}$ & $\begin{array}{l}80-150 \text { en corteza } \\
600-2000 \text { corteza }+ \text { manto }\end{array}$ & - & Schurr et al., 2003 \\
\hline Norte de Marianas & $\begin{array}{l}56-70 f^{0.27}(\text { arco }) \\
43-60 f^{0.27} \text { (retroarco) }\end{array}$ & $\begin{array}{l}45-58 f^{0.27} \\
33-42 f^{0.27}\end{array}$ & Pozgay et al., 2009 \\
\hline Tonga - Lau & $47-60$ & - & Wiens et al., 2008 \\
\hline Alaska continental & $266 f^{0.27}$ & $138 f^{0.27}$ & Stachnik et al., 2004 \\
\hline Costa Rica & - & $84-88 f^{0.27}$ & Rychert et al., 2008 \\
\hline Nicaragua & - & $76-78 f^{0.27}$ & Rychert et al., 2008 \\
\hline
\end{tabular}

Nuevo Cuyo (RNC) se presentan los ajustes promedios regionales del NC, del APP y del ME

Tabla 5.2.- Valores del factor de calidad de ondas $P$ y $S$ obtenidos por otros autores en diferentes regiones y en la región de Nuevo Cuyo (RNC) por este estudio.

* Valores promedio obtenidos con el método NC. RNC central representan a la zona de mayor actividad sísmica a profundidades corticales (E PC eb, SPP, W SP, S PC_CC b) y en el caso $h>$ $50 \mathrm{~km}$ a las distancias menores que $250 \mathrm{~km}$.

** Valores promedio obtenidos con el método NC RNC exterior representa el valor medio de $W$ PC y E SP a profundidades corticales.

\& Butler (1992) presenta sus resultados como $Q_{P}=300$ a $2.5 \mathrm{~Hz}$ y 1500 a $17.5 \mathrm{~Hz}$ con una relación $f^{0.7}$ y $Q_{S}=400$ a $2.5 \mathrm{~Hz}$ y 3000 a $22.5 \mathrm{~Hz}$ con una relación $f^{1.1}$.

\&\& Whitman et al. (1992) evalúan $Q_{S}$ y $Q_{P}$ en trayectorias de alta y baja atenuación. 
en la RNC en este estudio. En particular, los resultados del NC se han separado según dos zonas, una zona central donde se concentra la mayor actividad sísmica y los valores de atenuación son mayores y otra zona exterior, donde la actividad sísmica es menor y que comprende a las zonas occidental y oriental de la RNC.

Comparando las curvas de $Q_{\alpha}{ }^{-1} / \mathrm{Q}_{\beta}{ }^{-1}$ de la RNC obtenidas mediante el ME (Fig. 4.17) con aquellas recopiladas por Sato y Fehler (1998) (Fig. 2.22) puede verse (Fig. 5.3) que la RNC muestra una relación $Q_{\alpha}{ }^{-1} / \mathrm{Q}_{\beta}{ }^{-1}$ hasta profundidades de $150 \mathrm{~km}$, con un comportamiento similar al obtenido por Yoshimoto et al. (1993) para Kanto, Japón, mientras que a profundidades corticales, la RNC tiene valores muy cercanos a los obtenidos por Butler et al. (1987) para las Islas Kuriles en la región Pacífico Occidental.

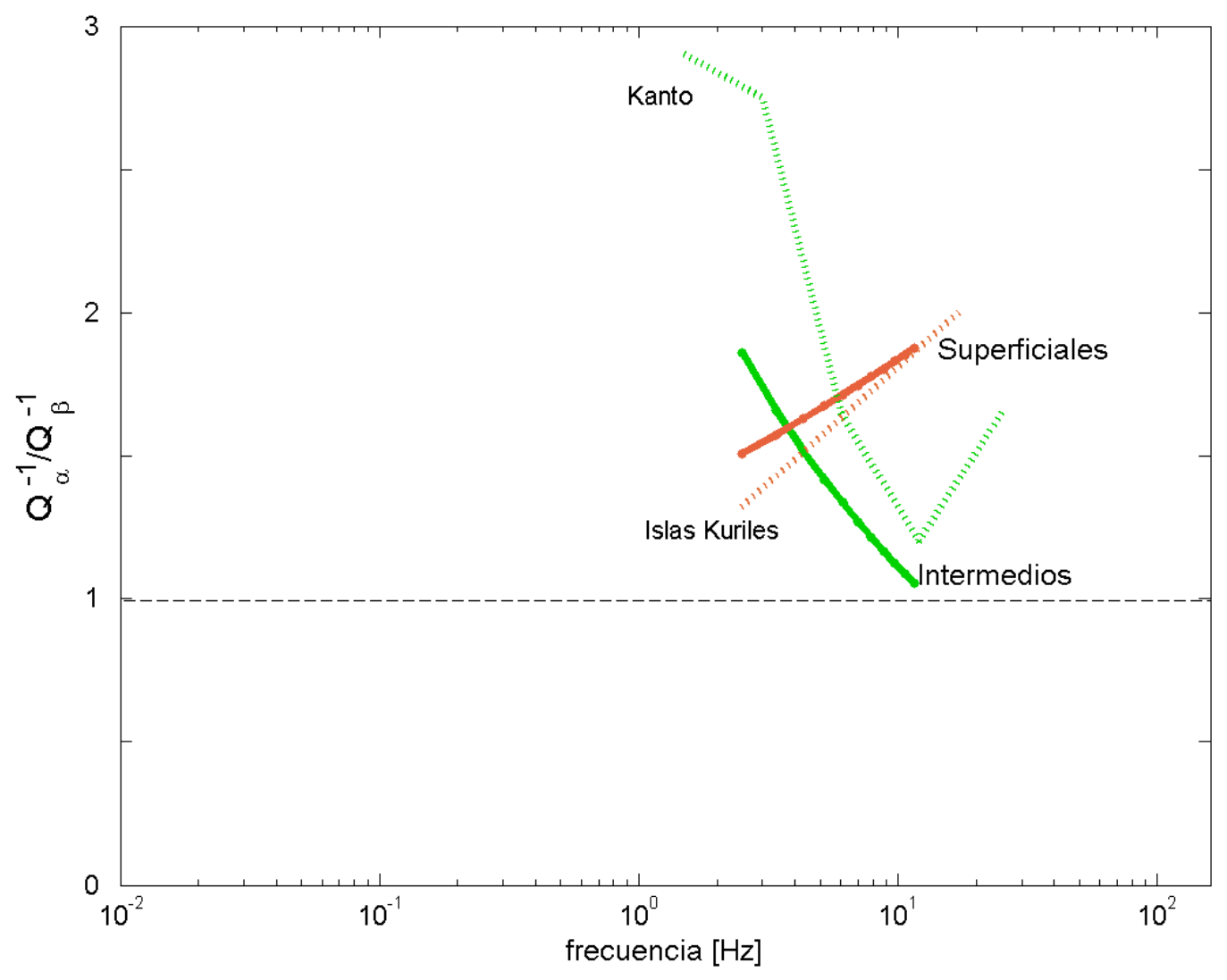

Fig.5.3- Relación $Q_{\alpha}^{-1} / Q_{\beta}^{-1}$ para la región de Nuevo Cuyo obtenida en este estudio y su comparación con otras regiones del mundo. Región de Nuevo Cuyo a profundidades corticales (línea sólida roja); Islas Kuriles según Butler et al., 1987 (línea punteada roja); Región de Nuevo Cuyo a profundidades hasta $150 \mathrm{~km}$ (línea sólida verde); Kanto, Japón según Yoshimoto et al., 1993 (linea punteada verde) 


\subsubsection{ATENUACIÓN INTRÍNSECA y DE SCATTERING}

Las Fig. 5.4a y 5.4b permiten comparar los resultados de la separación de $Q_{\mathrm{s}}^{-1}$ y $Q_{\mathrm{i}}^{-1}$ mediante el MLTWA en la región de Nuevo Cuyo con los resultados obtenidos en otras regiones del mundo basados en la misma aproximación. A profundidades corticales, la región de Nuevo Cuyo se encuentra entre las zonas caracterizadas por una alta atenuación como por ejemplo California Central, Estados Unidos y Andalucía, España. En cambio para eventos a profundidades intermedias, la atenuación en el manto para la región de Nuevo Cuyo es similar a la de otras zonas de baja atenuación de scattering como son los Apeninos Australes o el Friuli en Italia (Badi et al., 2009). Los valores de la atenuación intrínseca y de scattering según la aproximación de Wennerberg en la región de Nuevo Cuyo son válidos hasta profundidades de $400 \mathrm{~km}$, por lo que se comparan con los valores del MLTWA a profundidades intermedias. De acuerdo a los valores de la atenuación de ondas directas observados en la región, la atenuación por scattering resulta ser muy débil si se la compara

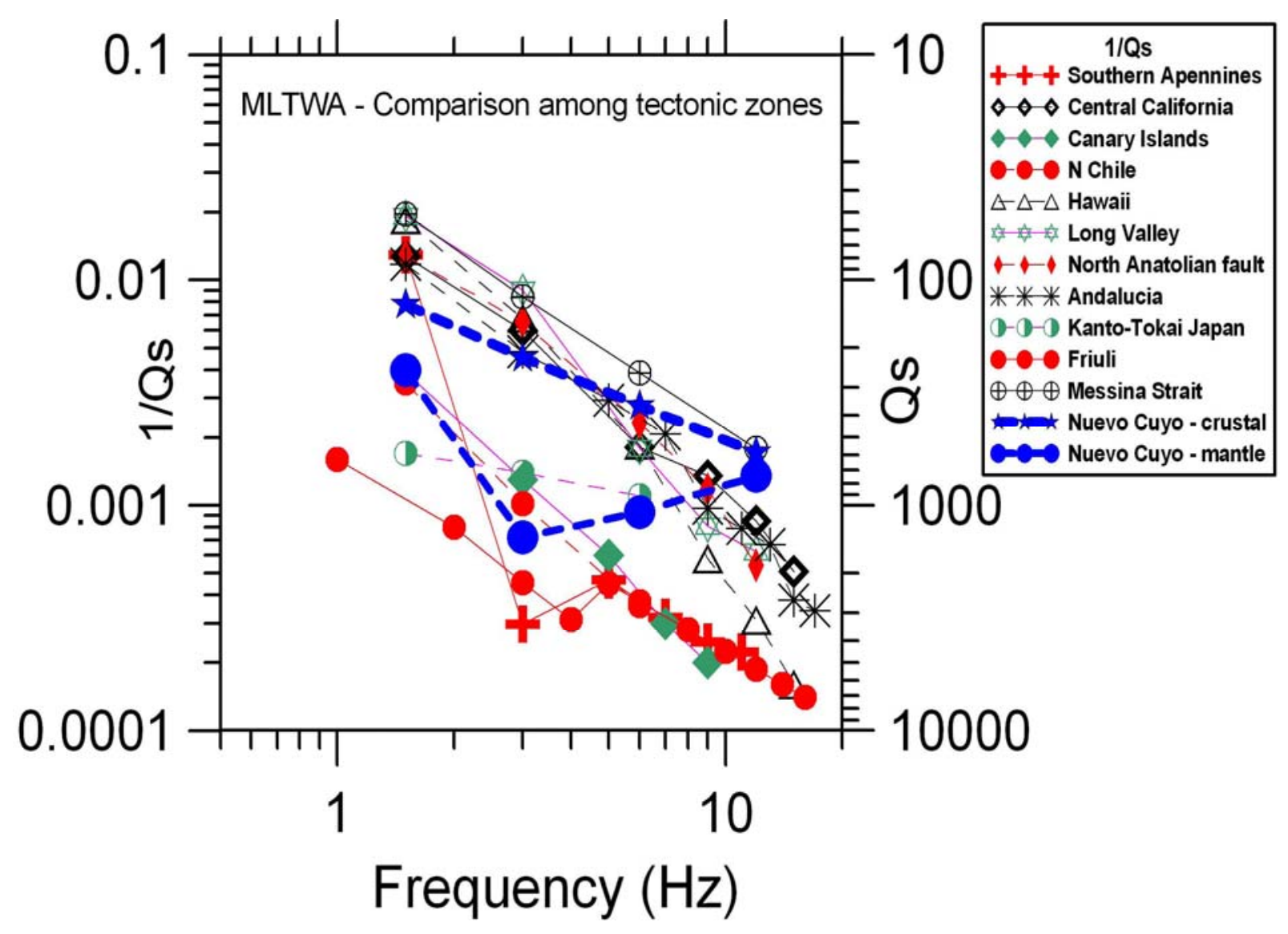

Fig. 5.4a.- Comparación entre los valores de $Q_{s}^{-1}$ obtenidos mediante el MLTWA en diversas zonas tectónicamente activas del mundo (valores extractados de Bianco et al., 2005 y Del Pezzo and F. Bianco, Earth Print Repository, 2007, http://www.earth-prints.org/) y los obtenidos para la región de Nuevo Cuyo a profundidades superficiales e intermedias. (Badi et al., 2009). 
con la atenuación intrínseca. Esta situación es similar a la observada por Xie y Mitchell (1990) en la región de las Basin and Range, USA, al separar los efectos anelásticos y de scattering sobre ondas Lg y codas de Lg tomadas 200 s después de la llegada de las Lg.

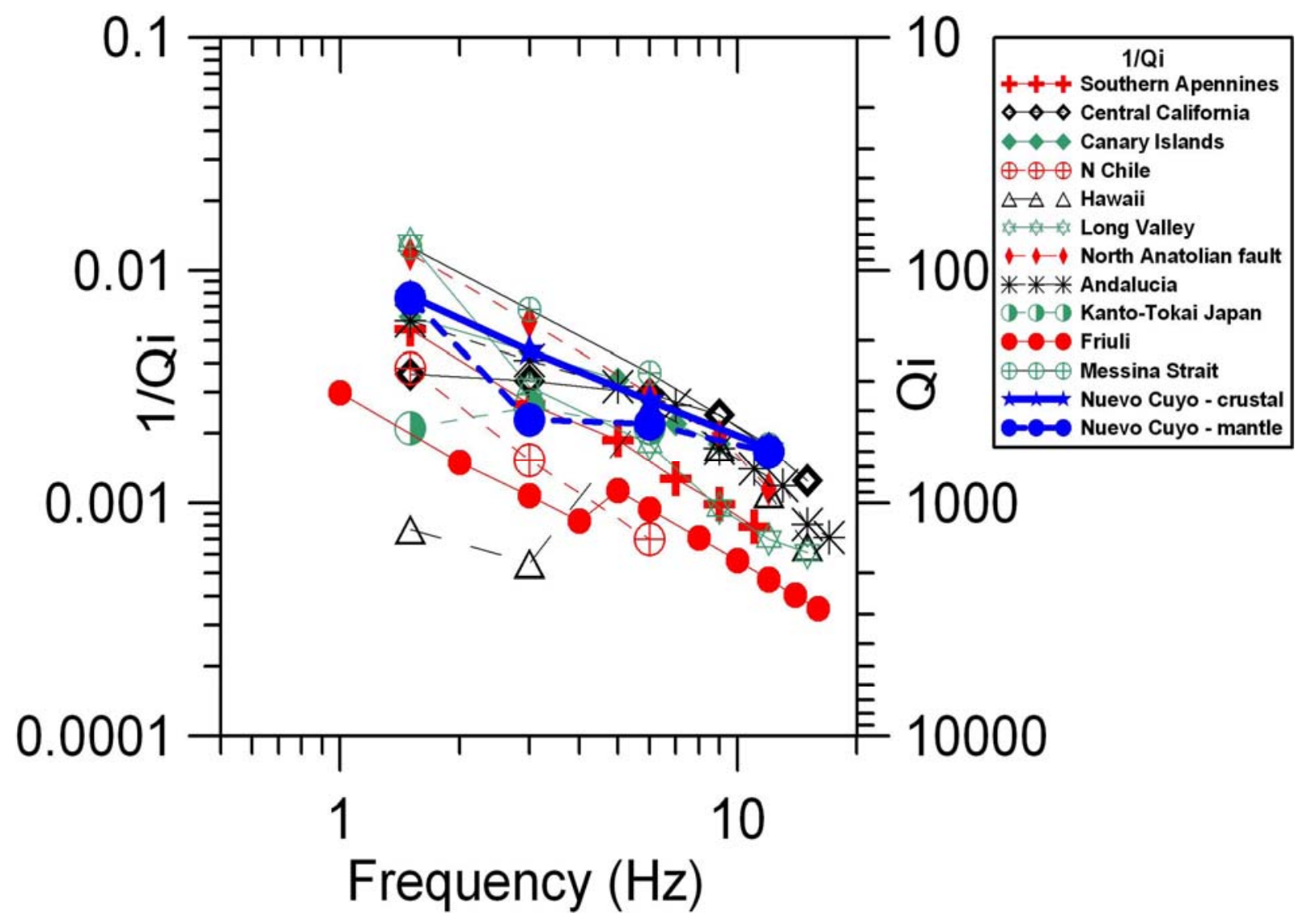

Fig. 5.4b.- Comparación entre los valores de $Q_{i}^{-1}$ obtenidos mediante el MLTWA en diversas zonas tectónicamente activas del mundo (valores extractados de Bianco et al., 2005 y Del Pezzo and F. Bianco, Earth Print Repository, 2007, http://www.earth-prints.org/) y los obtenidos para la región de Nuevo Cuyo a profundidades superficiales e intermedias. (Badi et al., 2009). 


\section{CAPÍTULO VI}

\section{Resumen y Conclusiones}

En esta tesis se ha realizado el primer trabajo de atenuación sísmica utilizando y comparando distintos métodos con datos digitales de ondas $\mathrm{P}, \mathrm{S}$ y coda registradas por estaciones permanentes en la región de Nuevo Cuyo Argentina, $\left(28^{\circ}-34^{\circ} \mathrm{S}\right.$ y $\left.65^{\circ}-72^{\circ} \mathrm{O}\right)$, una región calificada como de alta peligrosidad sísmica que se asocia a un segmento de subducción subhorizontal de la placa de Nazca bajo la Placa Sudamericana. En primer lugar se ha obtenido un modelo de velocidad que define con claridad la geometría de la subducción en la región. Se ha encontrado que la subducción de la dorsal Juan Fernández se asocia a valores bajos de atenuación tanto de ondas coda como de ondas directas. Las variaciones de la atenuación en profundidad han permitido inferir la profundidad de la transición frágil dúctil en la placa subducida que a su vez coincide con la distribución de sismicidad. Se ha observado que las zonas de mayor sismicidad cortical se corresponden también con las zonas de mayor absorción de energía sísmica lo que estaría indicando un mayor acoplamiento entre las placas. En todas las determinaciones se ha encontrado una disminución de la atenuación con la frecuencia que se interpreta como un aumento del camino libre medio o del tiempo de relajación dependiendo de si se considera el efecto del scattering o de la absorción anelástica. Se ha obtenido la separación de dichos efectos, que ha mostrado un predominio de la anelasticidad que se vincula fuertemente a los parámetros reológicos. La comparación con valores de dichos parámetros obtenidos independientemente por otros métodos geofísicos o geológicos en análisis de variaciones de densidad, resistividad, temperatura o velocidad valida los resultados de esta tesis, lo que permite integrarlos para obtener un modelo más acertado de la región, resultando entonces un aporte concreto para futuras aplicaciones.

\subsection{LOS DATOS y LOS MÉTODOS}

En este trabajo se han utilizado datos seleccionados entre los registros de 23 estaciones permanentes, de corto período y componente vertical, pertenecientes a la Red Sismológica Zonal Nuevo Cuyo, que depende del INPRES, en el período agosto de 1995 a marzo de 1999. 
Para estudiar la atenuación del medio se hace indispensable contar con una distribución de sismicidad que tenga localizaciones precisas y con una estructura de velocidad que permita plantear modelos para la interpretación de los resultados. Para ello se ha determinado una estructura unidimesional de velocidad de ondas $\mathrm{P}$, utilizando un método de localización conjunta de eventos. El modelo resultante consta de 8 capas y un semiespacio. Mediante diagramas de Wadati se ha establecido una relación $V_{P} / V_{S}=1.76$. La aplicación del nuevo modelo y correcciones de estación a la localización ha resultado en una mejor convergencia de las soluciones, una mayor definición de la zona de Wadati-Benioff y una mejor alineación de los epicentros superficiales con los principales sistemas de fallas activas. La comparación de la distribución de hipocentros resultante con las profundidades de las principales discontinuidades y las distribuciones de sismicidad obtenidas en otros trabajos a partir de bases de datos y metodologías diferentes corrobora la confiabilidad del modelo para los análisis de atenuación en la RNC.

De esta manera la zona de estudio ha quedado definida finalmente entre los $27^{\circ}$ y los $34^{\circ}$ de latitud sur y desde los $65^{\circ}$ hasta los $72^{\circ}$ de longitud oeste, de acuerdo a la distribución de los 452 sismos localizados. Las profundidades focales superficiales han resultado estar concentradas principalmente en corteza intermedia, hasta los $50 \mathrm{~km}$ de profundidad, bajo la Sierra Pie de Palo y también al oeste a lo largo del corrimiento Villicum-Pedernal. A profundidades intermedias los eventos se distribuyen en los primeros $20 \mathrm{~km}$ de la placa de Nazca subducida, es decir entre los 100 y $120 \mathrm{~km}$ de profundidad con una clara concentración bajo la Precordillera.

Se ha determinado la atenuación de ondas directas y ondas coda y se ha hecho la separación de los efectos anelásticos y de scattering en el intervalo de frecuencias entre 1 y 12 $\mathrm{Hz}$ de acuerdo a las respuestas instrumentales de las estaciones. Los métodos de estudio han sido seleccionados en primer lugar según las características de los datos (como por ejemplo: distribución de sismicidad, magnitudes, definición de los tiempos de llegada, naturaleza de las ondas, relación señal ruido, respuesta instrumental y distribución de estaciones) y en segundo lugar según la difusión de dichos métodos en la literatura para poder realizar la comparación de los resultados en la región de Nuevo Cuyo con otras regiones del mundo. En algunos casos, la implementación de los métodos se efectuó mediante programas existentes desarrollados a tal fin, en otros se adaptaron los programas para optimizar su aplicación a los datos y también, se desarrollaron nuevas implementaciones de algunos métodos de acuerdo a los criterios adoptados en este estudio. Es sobre todo en estas nuevas aplicaciones que pueden sacarse conclusiones. En primer lugar, los resultados han sido comparables a aquellos 
obtenidos por otros programas. En algunos casos han mostrado una mejor definición permitiendo extraer mayor información de los mismos y en otros se ha visto que pueden aún ser optimizados. Los criterios utilizados en dichas aplicaciones han sido más rigurosos con las hipótesis del modelo empleado que las implementaciones ya existentes disponibles.

\subsection{LAS ONDAS CODA}

La determinación del factor de calidad $Q_{\mathrm{c}}$ se ha efectuado con dos métodos en forma simultánea y usando un ajuste no lineal. Se ha supuesto la validez del Back-Scattering Simple (Aki-Chouet, 1975) en la generación de las codas y también se ha aplicado la adaptación de Sato (1977) para el Scattering Isótropo Simple (Sato, 1977). En valores medios regionales, tanto a profundidades corticales como en el manto, ambos métodos demuestran una fuerte dependencia del $Q_{\mathrm{c}}$ con la frecuencia pudiendo ajustarse una relación de tipo potencia $\left(Q_{c}=Q_{o}\left(f / f_{0}\right)^{n}\right)$. A profundidades intermedias la atenuación disminuye con el lapso de tiempo independientemente del método usado. A profundidades corticales, la atenuación de ondas coda según el SBS muestra también un comportamiento decreciente hasta los $180 \mathrm{~s}$ donde comienza a estabilizarse. En cambio, los resultados del SIS se apartan notablemente del SBS para lapsos entre los 60 y 170 s, donde comienza a converger hacia el SBS al disminuir la sensibilidad a la asimetría del patrón de radiación y del scattering. Los valores de la potencia $n$ se mantienen muy similares entre ambos métodos y a lo largo de toda la coda, mostrando un comportamiento levemente decreciente con el lapso de tiempo analizado conforme aumenta $Q_{0}$.

Analizando valores de atenuación obtenidos por estación, no se han observado variaciones importantes de la atenuación superficial de ondas coda con la dirección de propagación de las ondas directas. Por lo tanto se ha considerado la hipótesis de Pulli (1984) asignando los valores de $Q_{0}$ obtenidos al volumen de scattering que da origen a las ondas coda. Cerca de la superficie, entre 0 y $82 \mathrm{~km}$, la mayor atenuación se registra en la región oriental de la provincia de San Juan, hacia las Sas. Pie de Palo y Valle Fértil; otro mínimo se da en el oeste, en Precordillera. Hacia los 90 s de lapso de tiempo, se homogeiniza la distribución de $Q_{0}$ tomando un valor medio cercano a 100. Por otra parte, se observa una fuerte dependencia de $Q_{\mathrm{c}}$ con la frecuencia, que se manifiesta en valores de $n$ en general superiores a 1. En todos los lapsos de tiempo, los mayores valores de $n$ se corresponden con los valores más bajos de $Q_{0}$, de modo que a mayor atenuación, mayor variación de ella con la frecuencia. Según este análisis puede asumirse un valor homogéneo de $Q_{0}$ igual a 30 hasta los $82 \mathrm{~km}$ de profundidad, 70 hasta los $97 \mathrm{~km}, 100$ hasta los $114 \mathrm{~km}, 103$ hasta los $132 \mathrm{~km}, 100$ 
hasta los $144 \mathrm{~km}$ y 112 hasta los $161 \mathrm{~km}$ (en todos los casos se trata de valores desde la superficie hasta las profundidades indicadas). Estos valores medios se asignan al área donde la placa de Nazca permanece horizontal, disminuyendo en la dirección de avance de la dorsal Juan Fernández subducida. La mayor absorción de energía sísmica indicada por esos mínimos coincidiría con un incremento del acoplamiento viscoso entre las placas continental y oceánica (Alvarado et al., 2010) debido a la morfología irregular de la placa de Nazca en la JFR, la escasez de material astenosférico entre placas, la consecuente alteración de la estructura geotérmica y por lo tanto el enfriamiento de la litósfera continental que resulta en un endurecimiento de su reología.

Del análisis comparativo entre la variación de $Q_{0}$ y la distribución de sismicidad con la profundidad se observa que la condición frágil de la litósfera superior hasta los $50 \mathrm{~km}$ de profundidad coincide con una atenuación alta. Donde se da la transición litósfera continentallitósfera oceánica se da una disminución brusca de la atenuación y ausencia de terremotos. En la parte superior de la litósfera oceánica, a los $100 \mathrm{~km}$ de profundidad, se da la mayor concentración de eventos sísmicos, así como un aumento de la atenuación. Relacionando la atenuación de ondas coda a un efecto anelástico, el aumento de la atenuación entre los $100 \mathrm{y}$ 114 km estaría indicando la posible ubicación de la Transición Frágil Dúctil en la litósfera de Nazca subducida.

\subsection{LAS ONDAS DIRECTAS}

La determinación de la atenuación de la onda directa $\mathrm{P}$ se ha realizado en función de la frecuencia mediante el Método Espectral, ME, e independientemente de ella, mediante el método del Ancho del Primer Pulso, APP. Para las ondas S, se han empleado el Método Espectral, y la Normalización de la Coda, NC.

El ME se ha implementado mediante el método de Tsujiura (1966) con una sola estación, utilizando el criterio de Giampiccolo et al. (2007) con un ajuste lineal (ME_L) y una nueva adaptación propuesta en este trabajo que considera un ajuste no lineal de los factores de calidad (ME_NL). Los factores de calidad medios regionales, obtenidos con el ME_L resultaron: $230 \pm 21 \cdot f^{0.24 \pm 0.04}$ para ondas $\mathrm{P}$ a profundidades corticales; $157 \pm 65 \cdot f^{0.52 \pm 0.21}$ para ondas $\mathrm{P}$ a profundidades intermedias; $305 \pm 28 \cdot f^{0.38 \pm 0.04}$ para ondas $\mathrm{S}$ a profundidades corticales y $411 \pm 41 \cdot f^{0.15 \pm 0.06}$ para ondas $\mathrm{S}$ a profundidades intermedias. Las variaciones de los ajustes del ME_NL interpretadas en planta, muestran máximos de la atenuación de ondas P y S directas a profundidades corticales bajo la Sierra Pie de Palo y en la proyección superficial de la dorsal Juan Fernández subducida. La mayor atenuación de ondas S directas a 
profundidades intermedias se encuentra bajo Precordillera y al oeste de la Sa. Pie de Palo, coincidiendo con la concentración de sismicidad en esas profundidades. Del mismo modo, coinciden para ondas $\mathrm{P}$ y S los mínimos de atenuación en una zona bien definida hacia el sudeste de la región. De la comparación entre el ME_NL y el ME_L puede decirse que el ME_NL resulta tener una mejor justificación teórica y sus resultados han permitido una regionalización de los valores de atenuación para ondas $\mathrm{P}$ y S.

Analizando la relación entre la atenuación de ondas $\mathrm{P}$ y la de ondas $\mathrm{S}$ en función de la frecuencia, se ve que $Q_{\alpha}{ }^{-1} / \mathrm{Q}_{\beta}{ }^{-1}>1$ y además que es creciente en la corteza, mientras que a profundidades intermedias, $Q_{\alpha}{ }^{-1} / \mathrm{Q}_{\beta}^{-1}>1$ pero decreciente con la frecuencia indicando que la atenuación de las ondas $\mathrm{S}$ tiende a estabilizarse a profundidades intermedias mientras que la atenuación de ondas P lo hace a profundidades corticales. La variedad de escalas en las estructuras corticales así como las diferentes composiciones de los terrenos que la conforman pueden ser la causa de la estabilidad de la atenuación de ondas P en corteza. La mayor homogeneidad a profundidades intermedias hace que la presencia de fluidos en la dinámica de la subducción afecte principalmente a la atenuación de las ondas $\mathrm{S}$.

La atenuación de ondas $\mathrm{P}$ mediante el ensanchamiento del primer pulso de $\mathrm{P}$ con el tiempo de viaje, se ha determinado mediante la relación establecida por Gladwin y Stacey (1974b). Definiendo dos zonas sismogénicas a profundidades 0-40 km y 90-120 km en la zona central de la $\mathrm{RNC}$, se ha obtenido un valor de $Q_{\mathrm{P}}=271$ en corteza y un $Q_{\mathrm{P}}=532$ para trayectorias que se inician en la placa de Nazca. Mediante un modelo simple de estructura en capas paralelas homogéneas se ha obtenido un valor de $Q_{\mathrm{P}}=1183$ para la capa asísmica intermedia de $70 \mathrm{~km}$ de espesor que combina litósfera continental inferior y el escaso material astenosférico interplaca.

El método de Normalización de la Coda propuesto por Aki (1980), ha sido aplicado en numerosas regiones, por lo que resultaba interesante aplicarlo también en la RNC para estudiar la atenuación de las ondas $\mathrm{S}$ directas. Los mejores ajustes de $Q_{\mathrm{d}}$ a profundidades intermedias resultaron al normalizar mediante valores medios regionales de $Q_{\mathrm{c}}$ por frecuencia y lapso de tiempo obtenidos del modelo SIS y a un tiempo de referencia $t_{\mathrm{c}}=90 \mathrm{~s}$. A profundidades corticales se ha analizado la variación lateral de la atenuación de ondas directas mediante ajustes sobre grupos de estaciones asociadas de acuerdo a su entorno geotectónico. En este caso la normalización se ha hecho con ajustes del SIS obtenidos en los mismos grupos de estaciones. Los resultados muestran dos niveles de atenuación diferentes en la región. Por un lado se encuentra la atenuación estimada para las zonas E SP, W PC y E PC con valores de 
$Q_{0}$ altos y una variación con la frecuencia relativamente lenta, dada por exponentes menores que 0.8. Por otra parte se encuentran valores bajos de $Q_{0}$ y muy similares entre sí, para la SPP, E PC eb, W SP y S PC_CC b con una dependencia con la frecuencia no homogénea dentro del grupo. Relacionando los valores de la atenuación con la distribución de sismicidad, se observa nuevamente que a mayor actividad tectónica mayor es la atenuación y a su vez mayor es su dependencia con la frecuencia. Sobre un mapa de la atenuación de ondas S directas, se destacan dos mínimos de $Q_{0}$, uno en la zona de la Sierra Pie de Palo y el otro en el NO de Mendoza, donde tuvieron epicentro los sismos históricos de mayor magnitud de la región. Los resultados a profundidades intermedias permiten una interpretación que corrobora lo observado a profundidades corticales.

\subsection{LA ATENUACIÓN INTRÍNSECA y DE SCATTERING}

La separación de la atenuación intrínseca y de scattering mediante el método de las Ventanas Temporales Múltiples, MLTWA, (Hoshiba et al., 1991) se ha efectuado utilizando el programa desarrollado por Bianco y Del Pezzo (2002). Del análisis de los resultados a profundidades corticales se ha visto que, dentro de los rangos de error, toda la región presenta valores similares de $L_{e}^{-1}$ y $B_{0}$. Comparando valores promedio regionales en profundidades corticales y del manto se observa que la atenuación intrínseca aumenta del manto a la corteza, lo que se puede asociar a las propiedades anelásticas de las estructuras geológicas complejas de la región. El scattering disminuye al pasar de corteza a manto, probablemente debido a la mayor homogeneidad estructural del manto litosférico. A profundidades corticales, $Q_{i}^{-1}$ es similar a $Q_{\mathrm{s}}^{-1}$, indicando una contribución similar de ambos procesos de atenuación, mientras que en el manto litosférico, $Q_{\mathrm{s}}^{-1}$ es mucho menor que $Q_{\mathrm{i}}{ }^{-1}$, interpretándose como una mayor contribución de la absorción anelástica por sobre la atenuación de scattering.

La separación de la atenuación intrínseca y de scattering mediante el método de Wennerberg (1993), MWe, se ha efectuado a partir de los valores de la atenuación de las ondas S directas y de su coda para longitudes de coda largas. Puede decirse que los resultados del MWe han sido congruentes con el MLTWA, utilizando indistintamente los valores de atenuación de ondas S dados por el Método Espectral o por el método de Normalización de la Coda y los resultados del método de Back-Scattering Simple para la coda. Los valores de $Q_{\mathrm{i}}^{-1}$ y $Q_{\mathrm{s}}^{-1}$ estimados por el MWe en este estudio pueden asignarse a los efectos de la atenuación intrínseca y de scattering en el espesor total del sistema de placas Sudamericana-Nazca yuxtapuestas en la zona de subducción plana e inclusive al manto astenosférico por debajo de ellas hasta la zona de transición. La atenuación intrínseca juega un rol preponderante en el 
proceso de subducción y es muy próxima a la atenuación de ondas coda, mientras que la atenuación total prácticamente coincide con la atenuación de las ondas directas. La atenuación intrínseca y la total disminuyen con la frecuencia como ya se había observado anteriormente. La atenuación por scattering resulta ser muy débil y no muestra una dependencia clara con la frecuencia pudiendo asociarse a una densidad de dispersores constante en función de su tamaño.

De los dos métodos puede concluirse que la absorción anelástica domina la atenuación total por sobre los efectos de scattering. La gran heterogeneidad que se deduce de los resultados del MLTWA en corteza, coincide con la complejidad tectónica de la placa continental en la región de Nuevo Cuyo con la presencia de valles, una tectónica de piel fina y fallas inversas reactivadas sobre un basamento cristalino. El predominio de la atenuación intrínseca en el manto litosférico puede ser explicado por la presencia de agua u otros fluidos liberados en la litósfera continental desde la placa oceánica subducida.

\subsection{LA REGIÓN}

Los valores de atenuación encontrados en la región de Nuevo Cuyo son una clara manifestación de su estado de actividad tectónica. La RNC se caracteriza por una alta atenuación de ondas coda a profundidades corticales estabilizándose su valor cuando los volúmenes de scattering alcanzan una profundidad más allá de la zona sismogénica a profundidades intermedias. Las determinaciones de la atenuación de ondas directas, realizadas por los distintos métodos convergen entre sí para algunos métodos, frecuencias y profundidades. Los valores medios de atenuación de ondas $\mathrm{P}$ y S permiten inferir el rol de las diferencias composicionales superficiales en la atenuación de las ondas P para trayectorias corticales y el efecto de la presencia de fluidos en la litósfera debido a la subducción dados los efectos observados sobre las ondas $\mathrm{S}$ en trayectorias que abarcan profundidades intermedias. La separación de la atenuación intrínseca y de scattering ha mostrado que la absorción anelástica es el efecto predominante, más aún cuanto mayor sea la profundidad de validez de los resultados. No son las heterogeneidades las que dominan la atenuación a gran escala sino los parámetros anelásticos del medio. La subducción subhorizontal océano-continente y todos los procesos geodinámicos asociados a ella son los protagonistas.

\subsection{LA TESIS y EL FUTURO}

Este es el momento de pararse y mirar hacia atrás, ver qué se ha hecho, cuál es el aporte, qué se puede mejorar y entonces decidir los próximos pasos. Bien dicen que la tesis más que 
cerrar una etapa, abre varias. Fundamentalmente, esta tesis ha servido para integrar los conocimientos y el trabajo de un grupo de personas e instituciones que han hecho sus aportes a lo largo de estos años. Con la energía de estos aportes se han podido interpretar los fríos resultados que surgen del análisis de las ondas, elevando la temperatura de los mismos hasta fundirlos con suficiente coherencia como para enunciarlos.

La implementación de las metodologías que se han hecho ya sea siendo fieles a su diseño original, replanteando los criterios de aplicación o mejorando las técnicas de cálculo, forman un conjunto de herramientas que serán de utilidad en otras áreas tectónicas en las que se está colaborando y que en algunos casos tienen como protagonista a la actividad volcánica, lo que sin duda representará un nuevo desafío. Sin embargo también abre las puertas a la colaboración en otras regiones y a la incorporación de nuevos datos de la Región de Nuevo Cuyo que permitan densificar la información obtenida. Seguramente de este modo podrán satisfacerse aquellas inquietudes que han surgido a partir de los resultados. En particular se cree que podrían lograrse mejores resultados incorporando rutinas que efectúen algunas etapas de procesamiento previas de modo de eliminar posibles fuentes de error. Ya se han dado los primeros pasos a fin de extender los análisis de la atenuación de una a dos dimensiones. En colaboración con investigadores del Istituto Nazionale di Geofisica e Vulcanologia, Sezione di Catania, Catania, Italy se está trabajando en la elaboración de la tomografía de velocidad de ondas P que luego permitirá elaborar una tomografía de atenuación.

A lo largo de esta tesis se ha visto que el análisis de los efectos de la atenuación permite tener otro punto de vista de los parámetros que definen el medio por donde se propagan las ondas y que se relacionan con los procesos geodinámicos activos en la región. Se espera que estos resultados puedan ser incorporados y con éxito a la elaboración de modelos reológicos más completos de la región de subducción plana argentina.

Existe otra finalidad de gran importancia y aplicación y es que los valores de los factores de atenuación, permiten establecer fórmulas que describen el decaimiento de las amplitudes con la distancia. El correcto planteo de estas fórmulas es fundamental para la realización de estudios de peligrosidad y riesgo sísmico y por lo tanto para la elaboración de normas de construcción sismorresistente. En este sentido se espera que esta tesis pueda aportar a los trabajos que al respecto viene haciendo el Instituto Nacional de Prevención Sísmica (INPRES) ya sea a partir de los resultados obtenidos en la región de Nuevo Cuyo o en otras regiones sísmicamente activas del país donde sea de interés la aplicación de las técnicas aquí implementadas. 
Apéndice A

\section{Detalle de condiciones y parámetros de registración de las estaciones utilizadas de la Red Sismológica Zonal Nuevo Cuyo (RSZNC - INPRES)}

En este apartado se presentan dos tablas que contienen las condiciones de registración de las estaciones sismológicas utilizadas para este estudio y los parámetros de las curvas de respuesta instrumental.

En la tabla A1 se recopila la información brindada por el INPRES acerca de la localización geográfica de las estaciones, sus períodos de funcionamiento, el tipo de roca en el emplazamiento de la instalación para consideraciones del efecto de sitio y la modalidad de envío de datos (analógico o digital) hasta la sede del INPRES en San Juan. Se incluyen además el modelo de sensor empleado, que en estas estaciones puede ser S-13, un modelo corto período de Geotech-Teledyne o GURALP, un sensor banda ancha marca Guralp pero sin especificación del modelo. En algunos casos se indica el modelo de la tarjeta de conversión analógico-digital (CAD). En todas las estaciones, se informan la resolución del digitizador, la frecuencia de muestreo utilizada y el filtro analógico antialias implementado que en general define la banda de paso de la estación dada la frecuencia de muestreo elegida.

En la tabla A2 se presentan los parámetros necesarios para la construcción de las curvas de respuesta de las estaciones utilizando, por ejemplo, el programa resp.exe que se incluye en el paquete SEISAN (Havskov y Ottemöller, 2008). A partir de los resultados de calibraciones instrumentales efectuadas por el INPRES, dichos parámetros fueron calculados mediante una subrutina MATHCAD implementada por G. Alguacil (Havskov y Alguacil, 2004). Las curvas de respuesta han sido construidas con el programa resp para su empleo en el procesamiento de los datos cada vez que ha sido necesario remover la respuesta instrumental. 
Tabla A.1.- Características de emplazamiento y registración. En cada fila se muestra: código de estación en cuatro letras, nombre de estación, códigos alternativos correspondientes a diferentes intervalos de tiempo o curvas e respuesta, fechas de instalación y desinstalación de equipos si las hubiere, coordenadas, elevación y tipo de rocas en el emplazamiento, modelo de sensor (S-13 es modelo corto período de Geotech-Teledyne y GURALP es marca de sensor e indica banda ancha), formato de telemetría hasta la Central del INPRES, tipo de convertidor analógico digital de acuerdo a marca-diseño de plaqueta de adquisición, resolución en bits y frecuencia del filtro antialiasing. Las frecuencias de muestreo fueron en todos los casos de 100 mps para eventos anteriores al 06/08/1998. Para los datos de eventos posteriores a esa fecha, la frecuencia cambió a 40 mps a excepción de las estaciones RTLL, RTLS, RTCV, ICAZ, CFAZ (no asi FO24) que cambiaron a 80 mps. Debido a la existencia de más de un canal de datos y denominación para algunas estaciones, se adoptó una sigla por emplazamiento de estación, manteniéndose los nombres originales de los canales en las extensiones de los archivos ASCII a través de una selección de tres letras de las cuatro que conforman la sigla. En la tabla, los guiones separan nombres o configuraciones de diferentes canales a veces coetáneos, las barras separan configuraciones sucesivas en el tiempo.

* Se sabe que dejó de funcionar con la configuración original el 01-Nov-88. Luego se ha vuelto a instalar con la configuración mencionada en la tabla pero dejó de funcionar entre abril y julio de 1996.

** La fecha de inicio corresponde a la instalación de la estación con denominación MO13 (m13), MO14 (m14) se sabe que funcionó además como RTMC hasta abril de 1997.

\begin{tabular}{|c|c|c|c|c|c|c|c|c|c|c|c|c|c|}
\hline SIGLA & UBICACIÓN & Extensión & Inicio & Cierre & Latitud & Longitud & Elev. & $\begin{array}{l}\text { Roca de } \\
\text { sitio }\end{array}$ & Sensor & Telemetría & CAD & $\begin{array}{l}\text { Resol. } \\
\text { (bits). }\end{array}$ & AAF \\
\hline AR11 & Agrelo & $\operatorname{ar} 11-\operatorname{ar} 21$ & $12 / 06 / 97$ & & -33.08567 & -68.8275 & 1159 & Sedimentaria & S-13 & Analógica & & 24 & $16 \mathrm{~Hz}$ \\
\hline AR12 & Vizcacheras & $\operatorname{ar} 12-\operatorname{ar} 22$ & $01 / 10 / 96$ & & -33.47367 & -68.51517 & 1019 & Sedimentaria & S-13 & Analógica & & 24 & $16 \mathrm{~Hz}$ \\
\hline AR13 & Salagasta & $\operatorname{ar} 13-\operatorname{ar} 23$ & $09 / 06 / 97$ & & -32.59167 & -68.834 & $1000 ?$ & Sedimentaria & S-13 & Analógica & & 24 & $16 \mathrm{~Hz}$ \\
\hline AR14 & Co. Arco & $\operatorname{ar} 14$ & No disp. & & -32.84133 & -68.93283 & - & - & - & - & & 24 & $16 \mathrm{~Hz}$ \\
\hline CA21 & Catantal & ca21-ca11 & $28 / 09 / 96$ & & -32.27467 & -67.18767 & 634 & Sedimentaria & GURALP & Digital & & 24 & $16 \mathrm{~Hz}$ \\
\hline CFAZ & Coronel Fontana & $\begin{array}{l}\text { cfaz-fo24- } \\
\text { fo14 }\end{array}$ & $\begin{array}{l}01 / 01 / 85 \\
03 / 02 / 95\end{array}$ & & -31.60317 & -68.23217 & 621 & Metamórfica & $\begin{array}{l}\text { S-13/ } \\
\text { GURALP }\end{array}$ & $\begin{array}{l}\text { Analógica/ } \\
\text { Digital }\end{array}$ & IASPEI & $16-24$ & $7.5 \mathrm{~Hz}-16 \mathrm{~Hz}$ \\
\hline CH11 & Marayes & $\operatorname{ch} 11$ & $03 / 02 / 95$ & & -31.456 & -67.32683 & 615 & Metamórfica & S-13 & $\begin{array}{l}\text { Digital (96) } \\
\text { Analógica (98) }\end{array}$ & REF-TEK & 16 & $\begin{array}{l}25 \mathrm{~Hz} \mathrm{AAF} / 40 \mathrm{mps} \\
\text { y } 16 \mathrm{~Hz} \mathrm{AAF} /\end{array}$ \\
\hline
\end{tabular}




\begin{tabular}{|c|c|c|c|c|c|c|c|c|c|c|c|c|c|}
\hline SIGLA & UBICACIÓN & Extensión & Inicio & Cierre & Latitud & Longitud & Elev. & $\begin{array}{l}\text { Roca de } \\
\text { sitio }\end{array}$ & Sensor & Telemetría & CAD & $\begin{array}{l}\text { Resol. } \\
\text { (bits). }\end{array}$ & AAF \\
\hline CH12 & Chepes & $\operatorname{ch} 15-\operatorname{ch} 12$ & $03 / 02 / 95$ & & -31.16667 & -66.66283 & 1470 & Metamórfica & S-13 & Digital & REF-TEK & 16 & $\begin{array}{l}\text { sin AAF/ idem } \\
\text { CH11 }\end{array}$ \\
\hline CO11 & Rodeo & co11-rode & No disp. & & -30.167 & -69.47333 & 2745 & Ígnea & S-13 & Analógica & REF-TEK & 16 & idem $\mathrm{CH} 11$ \\
\hline CO12 & $\begin{array}{l}\text { Cuesta } \\
\text { del Viento }\end{array}$ & co12-cues & $03 / 02 / 95$ & & -30.17083 & -69.11967 & 1572 & Sedimentaria & S-13 & Analógica & REF-TEK & 16 & idem CH11 \\
\hline CO13 & Co. Coronel & co13-co23 & $03 / 02 / 95$ & & -30.5885 & -69.081 & 2792 & Metamórfica & S-13 & Digital & REF-TEK & 16 & idem $\mathrm{CH} 12$ \\
\hline CO16 & $\begin{array}{l}\text { cerca de Co. } \\
\text { Coronel }\end{array}$ & co16 & No disp. & & -30.58769 & -69.06611 & - & - & - & Digital & REF-TEK & 16 & idem $\mathrm{CH} 12$ \\
\hline ICAZ & INPRES Central & icaz & $01 / 01 / 80$ & & -31.52817 & -68.55983 & 660 & Sedimentaria & S-13 & Analógica & IASPEI & 16 & -- \\
\hline MO11 & Mogna & mo11 & $25 / 09 / 96$ & & -30.93967 & -68.49233 & 830 & Sedimentaria & S-13 & Analógica & & 24 & $40 \mathrm{mps}$ y $16 \mathrm{~Hz} \mathrm{AAF}$ \\
\hline RTCB & Co. Blanco & $\mathrm{rtcb}$ & $01 / 09 / 75$ & $10 / 11 / 96$ & -31.4885 & -68.8095 & 1030 & Ígnea & S-13 & Analógica & IASPEI & 16 & $12.5 \mathrm{~Hz}$ \\
\hline RTCV & Co. Valdivia & rtcv & $01 / 09 / 75$ & & -31.86217 & -68.54017 & 670 & Metamórfica & S-13 & Analógica & IASPEI & 16 & $12.5 \mathrm{~Hz}$ \\
\hline RTLL & La Laja & rtll & $01 / 09 / 75$ & & -31.32917 & -68.47483 & 690 & Sedimentaria & S-13 & Analógica & IASPEI & 16 & $12.5 \mathrm{~Hz}$ \\
\hline RTLS & El Leoncito & rtls & $02 / 02 / 94$ & & -31.7995 & -69.295 & 2560 & Sedimentaria & S-13 & Analógica & IASPEI & 16 & $12.5 \mathrm{~Hz}$ \\
\hline RTMC & $\begin{array}{l}\text { Mogote } \\
\text { Los Corralitos }\end{array}$ & $\begin{array}{l}\text { rtmc-mo13- } \\
\text { mo14 }\end{array}$ & $01 / 10 / 96^{* *}$ & & -31.3095 & -67.924 & 3100 & Metamórfica & S-13 & Digital & IASPEI & $16-24-24$ & $\begin{array}{l}7.5 \mathrm{~Hz} / 16 \mathrm{~Hz} \text { AAF } \\
\text { a 40mps }\end{array}$ \\
\hline RTPR & Patquia & rtpr & 01/05/83* & $*$ & -30.308 & -66.55167 & 460 & Metamórfica & S-13 & Analógica & IASPEI & 16 & $12.5 \mathrm{~Hz}$ \\
\hline VFabla & Aalle Certilacterí & ticldsudetemp & platariphento & y registra & iôn. $6(603$ tin & 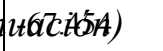 & 910 & Metamórfica & S-13 & Digital & REF-TEK & $16 / 24$ & $16 \mathrm{~Hz}$ \\
\hline VF12 & Punta de los & vf12 & $20 / 06 / 97$ & & -30.39567 & -66.52767 & 725 & Sedimentaria & S-13 & Analógica & & 24 & $16 \mathrm{~Hz}$ \\
\hline
\end{tabular}




\begin{tabular}{|c|c|c|c|c|c|c|c|c|c|c|c|c|c|}
\hline SIGLA & UBICACIÓN & Extensión & Inicio & Cierre & Latitud & Longitud & Elev. & $\begin{array}{l}\text { Roca de } \\
\text { sitio }\end{array}$ & Sensor & Telemetría & CAD & $\begin{array}{l}\text { Resol. } \\
\text { (bits). }\end{array}$ & AAF \\
\hline & Llanos & & & & & & & & & & & & \\
\hline VF13 & Co. La Cruz & vf13 & 19/06/97 & & -29.42617 & -66.95117 & 1650 & Ígnea & S-13 & Analógica & & 24 & $16 \mathrm{~Hz}$ \\
\hline
\end{tabular}

Tabla A.1.- Características de emplazamiento y registración (continuación) 


\begin{tabular}{|c|c|c|c|c|c|c|c|c|c|c|c|c|}
\hline STAT & COMP & PERIOD & DAMP & $\begin{array}{l}\text { GENCON } \\
{[\text { V.s.m }} \\
-1\end{array}$ & $\begin{array}{c}\text { AMP_GAIN } \\
\text { [dB] }\end{array}$ & $\begin{array}{c}\text { REC_MEDIA_GAIN } \\
\text { [counts. }{ }^{-1} \text { ] }\end{array}$ & $\begin{array}{c}\text { FLOW } \\
{[\mathrm{Hz}]}\end{array}$ & $\mathbf{P}$ & \begin{tabular}{|c|} 
FHIG \\
{$[\mathrm{Hz}]$}
\end{tabular} & $\mathbf{P}$ & $\begin{array}{c}\text { G@1_HZ } \\
{\left[\times 10^{09} \text { counts. } \eta \mathrm{m}^{-1}\right]}\end{array}$ & $\begin{array}{c}\text { G@5_HZ } \\
{\left[\times 10^{09} \text { counts. } \eta \mathrm{m}^{-1}\right]}\end{array}$ \\
\hline CFAZ & S_Z & 1 & 0.85 & 331.1 & 53.6 & 3280 & 0.5 & 2 & 7.5 & 2 & 1.87 & 14.655 \\
\hline CH11 & S_Z & 1 & 0.81 & 345 & 54.2 & 8740 & 0.01 & 2 & 25 & 2 & 6 & 47.914 \\
\hline CH12 & S_Z & 1 & 0.7 & 382.9 & 54.2 & 8740 & & & & & 7.65 & 53.893 \\
\hline CH15 & S_Z & 1 & 0.7 & 382.9 & 18.1 & 8740 & & & & & 0.12 & 0.844 \\
\hline CO11 & S_Z & 1 & 0.69 & 388.1 & 54.2 & 8740 & 0.01 & 2 & 25 & 2 & 7.92 & 54.67 \\
\hline CO12 & S_Z & 1 & 0.81 & 345 & 54.2 & 8740 & 0.01 & 2 & 25 & 2 & 6 & 47.914 \\
\hline CO16 & S_Z & 1 & 0.7 & 382.9 & 18.1 & 8740 & & & & & 0.12 & 0.844 \\
\hline CUES & S_Z & 1 & 0.81 & 345.1 & 54.2 & 8740 & 0.5 & 2 & 7.5 & 2 & 5.82 & 43.833 \\
\hline ICAZ & S_Z & 1 & 0.71 & 380.2 & 0 & 3280 & & & & & 0.0055 & 0.039 \\
\hline RODE & S_Z & 1 & 0.69 & 388.1 & 54.2 & 8740 & 0.5 & 2 & 7.5 & 2 & 7.69 & 50 \\
\hline RTCB & S_Z & 1 & 0.69 & 388.1 & 78.1 & 3280 & 0.2 & 2 & 12.5 & 2 & 46.5 & 317.659 \\
\hline RTCV & S_Z & 1 & 0.69 & 388.1 & 67 & 3280 & 0.2 & 2 & 12.5 & 2 & 13 & 88.506 \\
\hline RTLL & S_Z & 1 & 0.69 & 388.1 & 69.6 & 3280 & 0.2 & 2 & 12.5 & 2 & 17.5 & 119.388 \\
\hline RTMC & S_Z & 1 & 0.85 & 331 & 52 & 3280 & 0.5 & 2 & 7.5 & 2 & 1.55 & 12.185 \\
\hline RTPR & S_Z & 1 & 0.69 & 388.1 & 66 & 3280 & 0.2 & 2 & 12.5 & 2 & 11.5 & 78.88 \\
\hline VF11 & S_Z & 1 & 0.7 & 382.9 & 54.2 & 8740 & & & & & 7.65 & 53.893 \\
\hline VF14 & S_Z & 1 & 0.7 & 382.9 & 18.1 & 8740 & & & & & 0.12 & 0.844 \\
\hline
\end{tabular}

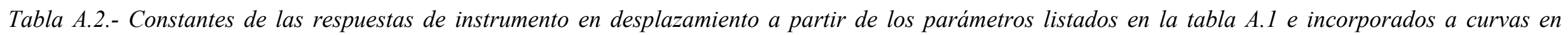

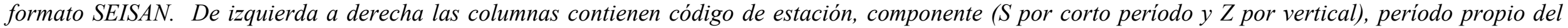

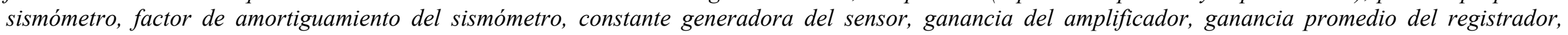

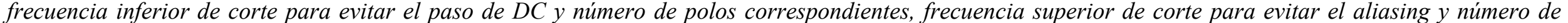
polos correspondientes, ganancia total en desplazamiento a $1 \mathrm{~Hz}$ y a $5 \mathrm{~Hz}$. 


\section{Apéndice B}

\section{Catálogo de eventos seleccionados}

El catálogo original de sismos está compuesto por eventos con profundidades estimadas a priori tanto superficiales como intermedias y registrados digitalmente por las estaciones de la Red Sismológica Zonal Nuevo Cuyo, INPRES, entre agosto de 1995 a marzo de 1999. Las condiciones impuestas en la selección han sido: una buena relación señal-ruido, amplitudes no saturadas y codas con duraciones cercanas o superiores a 60s. Estas resultan ser las condiciones básicas para asegurar una base de datos apta para la implementación de las técnicas de análisis de atenuación propuestas. La localización preliminar se ha efectuado en forma individual y con el programa HYPO71. La estructura de velocidad unidimensional de ondas $\mathrm{P}$ fue ajustada a los datos mediante la técnica de determinación conjunta de hipocentros y velocidad (JHD) implementada en el programa VELEST (Kissling et al., 1994, Kissling, 1995). Con el modelo de velocidades (Tabla 3.2) y las correcciones de estación (Tabla 3.3) obtenidos se han relocalizado todos los eventos del catálogo original. En la siguiente tabla se presentan los resultados que consisten en 452 eventos localizados con un RMS promedio de 0.16 para un total de 4205 rayos sísmicos analizados.

\begin{tabular}{|r|r|r|r|r|r|c|c|r|r|r|}
\hline año & mes & dia & hora & min & \multicolumn{1}{c|}{ seg } & lat & \multicolumn{1}{c|}{ long } & prof & Mc & RMS \\
\hline 1995 & 8 & 1 & 1 & 48 & 33.98 & -31.8398 & -67.7832 & 15.11 & 3.6 & 0.273 \\
\hline 1995 & 8 & 1 & 13 & 29 & 44.85 & -31.1264 & -67.5113 & 41.39 & & 0.546 \\
\hline 1995 & 8 & 2 & 1 & 17 & 26.44 & -31.8021 & -69.5859 & 110.98 & 3.2 & 0.074 \\
\hline 1995 & 8 & 3 & 17 & 59 & 6.34 & -32.4092 & -71.7042 & 18.17 & 3.7 & 0.33 \\
\hline 1995 & 8 & 4 & 1 & 21 & 7.72 & -31.3402 & -69.2672 & 110.12 & 3.4 & 0.171 \\
\hline 1995 & 8 & 4 & 17 & 9 & 3.19 & -33.2029 & -71.1807 & 104.63 & 3.1 & 0.409 \\
\hline 1995 & 8 & 5 & 9 & 59 & 36.28 & -31.2222 & -69.6400 & 104.81 & 3.3 & 0.074 \\
\hline 1995 & 8 & 6 & 2 & 53 & 4.5 & -31.5542 & -68.6943 & 102.25 & 2.8 & 0.045 \\
\hline 1995 & 8 & 6 & 4 & 49 & 26.07 & -31.5333 & -68.7527 & 104.12 & 2.9 & 0.046 \\
\hline 1995 & 8 & 8 & 4 & 24 & 9.08 & -30.9618 & -67.8839 & 30.62 & 2.9 & 0.038 \\
\hline 1995 & 8 & 8 & 18 & 26 & 37.12 & -28.9079 & -69.4873 & 74.09 & 3.3 & 0.104 \\
\hline 1995 & 8 & 8 & 22 & 54 & 39.08 & -31.4650 & -68.8323 & 100.56 & 2.7 & 0.048 \\
\hline 1995 & 8 & 9 & 5 & 4 & 26.41 & -32.0846 & -68.1316 & 120.53 & 2.8 & 0.045 \\
\hline 1995 & 8 & 9 & 14 & 22 & 20.98 & -31.1329 & -68.7258 & 101.17 & 2.9 & 0.066 \\
\hline 1995 & 8 & 9 & 23 & 56 & 39.23 & -29.4869 & -69.6323 & 0.1 & 3.2 & 0.179 \\
\hline 1995 & 8 & 11 & 17 & 18 & 44.03 & -31.1881 & -68.7633 & 103.36 & 3.2 & 0.038 \\
\hline
\end{tabular}




\begin{tabular}{|c|c|c|c|c|c|c|c|c|c|c|}
\hline año & $1 e s$ & dia & hora & $\min$ & seg & lat & long & prof & Mc & RMS \\
\hline 1995 & 8 & 13 & 12 & 19 & 16.13 & -32.9883 & -68.8371 & 25.45 & 3.2 & 0.251 \\
\hline 1995 & 8 & 13 & 12 & 50 & 22.38 & -28.1639 & -69.6396 & 29.28 & 3.3 & 0.09 \\
\hline 1995 & 8 & 14 & 13 & 22 & 23.52 & -31.8649 & -67.8638 & 118.52 & 2.8 & 0.056 \\
\hline 1995 & 8 & 15 & 2 & 30 & 6.44 & -31.4477 & -68.6870 & 18.32 & 2.9 & 0.17 \\
\hline 1995 & 8 & 15 & 3 & 40 & 29.52 & -32.2209 & -69.0939 & 119.77 & 2.9 & 0.166 \\
\hline 1995 & 8 & 17 & 19 & 19 & 34.47 & -31.2557 & -68.5317 & 103.76 & 2.8 & .075 \\
\hline 1995 & 8 & 17 & 19 & 33 & 9.78 & -31.5742 & -68.7819 & 103.9 & 2.9 & 0.055 \\
\hline 1995 & 8 & 18 & 1 & 42 & 39.09 & -27.7558 & -69.2086 & 17.26 & & 0.091 \\
\hline 1995 & 8 & 19 & 9 & 5 & 6.55 & -31.9444 & -70.6890 & 87.6 & 3.4 & 0.05 \\
\hline 1995 & 8 & 19 & 9 & 16 & 59.21 & -31.3572 & -71.1548 & 112.75 & 3.3 & 0.4 \\
\hline 1995 & 8 & 19 & 13 & 9 & 7.69 & -31.6988 & -68.9259 & 106.67 & 3.3 & .061 \\
\hline 1995 & 8 & 20 & 3 & 4 & 18.38 & -31.9172 & -68.9118 & 20.32 & 3.0 & 0.162 \\
\hline 1995 & 8 & 20 & 15 & 17 & 10.81 & -31.0706 & 369 & 115.18 & 3.1 & .605 \\
\hline 1995 & 8 & 20 & 21 & 28 & 44.73 & -31.7723 & -69.7026 & 111.12 & 3.6 & 0.171 \\
\hline 1995 & 8 & 21 & 23 & 47 & 39.25 & -31.4929 & 627 & 07.96 & 4.0 & .072 \\
\hline 1995 & 8 & 23 & 5 & 56 & 5.45 & -31.7705 & -69 & 101.57 & 3.3 & 0.241 \\
\hline 1995 & 8 & 23 & 13 & 15 & 13 & -31 & -68 & 106.67 & 3.3 & 044 \\
\hline 1995 & 8 & 23 & 14 & 18 & 4.28 & -31 & 435 & 82.41 & 3.3 & 0.388 \\
\hline 1995 & 8 & 24 & 14 & 14 & 25.47 & -31.4473 & -68 & 17.94 & 2.9 & 0.157 \\
\hline 1995 & 8 & 25 & 0 & 32 & 5.04 & -32.1781 & 317 & 64.94 & 3.5 & 0.229 \\
\hline 1995 & 8 & 25 & 1 & 51 & 34.02 & -32.7130 & 587 & 115.32 & 5.0 & 0.198 \\
\hline 1995 & 8 & 25 & 2 & 31 & 7.61 & -31 & 995 & 02.62 & 3.6 & 0.08 \\
\hline 1995 & 8 & 25 & 13 & 23 & 11.07 & -32.0018 & 748 & 111.79 & 3.2 & 0.009 \\
\hline 1995 & 8 & 25 & 16 & 45 & 43.33 & -31.7721 & 13 & 105.5 & 3.0 & 101 \\
\hline 1995 & 8 & 25 & 20 & 39 & 11.24 & 18 & 281 & 104.38 & 3.6 & 0.054 \\
\hline 1 & 8 & 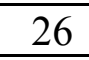 & 3 & 59 & & & & & 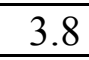 & \\
\hline 1995 & 8 & 26 & 23 & 56 & 0.94 & - & 69 & 101.54 & 2.9 & 0.033 \\
\hline 1995 & 8 & 27 & 21 & 6 & 49.33 & -31 & & 96.71 & 3.5 & .068 \\
\hline 1995 & 8 & 27 & 21 & 46 & 5.73 & -31 & 969 & 95.35 & 3.8 & 0.147 \\
\hline 1995 & 8 & 29 & 3 & 33 & 28.61 & -31.5292 & 841 & 109.17 & 3.7 & 0.146 \\
\hline 1995 & 9 & 1 & 4 & 21 & 18.37 & -32 & 921 & 27.39 & 3.2 & 0.108 \\
\hline 1995 & 9 & 2 & 14 & 9 & 52.49 & -31 & & 102.15 & 2.9 & 0.027 \\
\hline 1995 & 9 & 4 & 1 & 41 & 39.46 & -29.0023 & 222 & 29.18 & 3.6 & 0.304 \\
\hline 1995 & 9 & 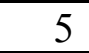 & 0 & 32 & 12.7 & & & 11.94 & 3.8 & 0.323 \\
\hline 1995 & 9 & & 0 & 26 & 48 & & & & 2.9 & 45 \\
\hline 1995 & 9 & 0 & 4 & 43 & 22.49 & -32 . & 256 & 121.78 & 3.3 & 0.059 \\
\hline 1995 & 9 & 9 & 23 & 45 & 58.06 & -31.8582 & 484 & 102.21 & 3.2 & 0.072 \\
\hline 1995 & 9 & 11 & 10 & 27 & 6.31 & -26.9218 & 229 & 60.27 & & 0.161 \\
\hline 1995 & 9 & 11 & 12 & 19 & 11.69 & -31.4668 & 194 & 99.87 & 3.1 & 0.059 \\
\hline 1995 & 9 & 12 & 19 & 38 & 58.32 & -31.2649 & 7886 & 102.24 & 3.2 & 0.022 \\
\hline 1995 & 9 & 14 & 9 & 30 & 57.33 & -28 & 863 & 26.32 & 3.7 & 0.107 \\
\hline 1995 & 9 & 15 & 23 & 21 & 17.94 & -28.3231 & -70.2850 & 88.46 & 4.1 & 0.099 \\
\hline 1995 & 9 & $1^{\prime}$ & 3 & 31 & 47.92 & -31.3035 & 930 & 100.08 & 3.0 & 0.035 \\
\hline 1995 & 9 & 17 & 3 & 38 & 10.87 & -30.9769 & -68.2784 & 18.25 & 3.4 & 0.092 \\
\hline 1995 & 9 & 17 & 4 & 13 & 52.09 & -31.2111 & -69.0879 & 103.47 & 3.5 & 0.406 \\
\hline 1995 & 9 & 18 & 5 & 32 & 3.59 & -31.6091 & -69.5212 & 106.94 & 3.1 & 0.072 \\
\hline 1995 & 9 & 19 & 0 & 51 & 22.7 & -32.2565 & -68.9256 & 27.49 & 3.1 & 0.095 \\
\hline
\end{tabular}




\begin{tabular}{|c|c|c|c|c|c|c|c|c|c|c|}
\hline año & mes & dia & hora & $\min$ & seg & lat & long & prof & Mc & RMS \\
\hline 1995 & 9 & 19 & 9 & 5 & 12.18 & -29.7481 & -66.8573 & 32.2 & 3.7 & 0.512 \\
\hline 1995 & 9 & 20 & 6 & 28 & 34.27 & -31.6270 & -67.9573 & 26.22 & 3.1 & 0.048 \\
\hline 1995 & 9 & 22 & 21 & 58 & 35.27 & -35.6278 & -72.2466 & 2.65 & & 0.159 \\
\hline 1995 & 9 & 24 & 8 & 34 & 34.65 & -31.3154 & -68.6619 & 95.57 & 3.2 & 0.062 \\
\hline 1995 & 9 & 24 & 11 & 59 & 4.44 & -31.7859 & -67.7986 & 20.66 & 3.4 & 0.018 \\
\hline 1995 & 9 & 25 & 6 & 24 & 43.7 & -31.2747 & -68.0421 & 28.34 & 3.2 & 0.099 \\
\hline 1995 & 9 & 28 & 0 & 52 & 35.37 & -31.4257 & -68.6590 & 102.3 & 3.1 & 0.038 \\
\hline 1995 & 9 & 30 & 5 & 13 & 20.21 & -31.3963 & -69.2594 & 122.67 & 3.0 & 0.554 \\
\hline 1995 & 9 & 30 & 18 & 57 & 28.44 & -31.1508 & -68.4581 & 98.56 & 3.1 & 0.071 \\
\hline 1995 & 10 & 1 & 5 & 22 & 59.94 & -31.2533 & -68.4788 & 100.97 & 3.1 & 0.055 \\
\hline 1995 & 10 & 1 & 22 & 3 & 4.72 & -28.8989 & -69.4656 & 154.87 & 3.8 & 0.38 \\
\hline 1995 & 10 & 3 & 15 & 11 & 50.55 & -31.8074 & -70.2275 & 113.23 & 3.3 & 0.139 \\
\hline 1995 & 10 & 4 & 2 & 26 & 15.21 & -31.3981 & -67.9337 & 8.36 & 2.9 & 0.233 \\
\hline 1995 & 10 & 5 & 4 & 1 & 11.09 & -30.4943 & -68.8090 & 102.08 & 2.9 & 0.031 \\
\hline 1995 & 10 & 6 & 16 & 58 & 33.37 & -31.1 & -68.4107 & 102.38 & 3.0 & 0.189 \\
\hline 1995 & 10 & 7 & 17 & 54 & 0.16 & -31.2852 & -68.0077 & 21.76 & 2.8 & 0.093 \\
\hline 1995 & 10 & 10 & 9 & 37 & 47.01 & -31.8 & -67 & 132 & 2.8 & 0.14 \\
\hline 1995 & 10 & 10 & 11 & 53 & 2.9 & -31.2762 & -68.4084 & 99.16 & 2.8 & 0.074 \\
\hline 1995 & 10 & 10 & 18 & 54 & 58.62 & -31. & -68 & 104.16 & 3.0 & 18 \\
\hline 1995 & 10 & 18 & 17 & 13 & 8.66 & -31.7935 & -69.6411 & 113.64 & 3.2 & 0.102 \\
\hline 1995 & 10 & 19 & 8 & 21 & 42.12 & -31.3564 & -68.6169 & 98.69 & 2.9 & 0.049 \\
\hline 1995 & 10 & 19 & 13 & 12 & 55.27 & -31.7079 & -66.4343 & 139.39 & 3.2 & 0.084 \\
\hline 1995 & 10 & 19 & 18 & 18 & 19.27 & -32.0631 & -67.9003 & 20.8 & 3.4 & 0.084 \\
\hline 1995 & 10 & 20 & 16 & 48 & 57.84 & -31.8289 & -68.3793 & 108.61 & 3.1 & 0.056 \\
\hline 1995 & 10 & 21 & 9 & 18 & 55.96 & -31.2850 & -68.5547 & 101.48 & 3.1 & 0.07 \\
\hline 1995 & 10 & 21 & 20 & 42 & 44.44 & -32.0300 & -68 & 113.69 & 3.1 & 0.063 \\
\hline 1995 & 10 & 22 & 10 & 53 & 44.51 & -31.5454 & -68.7623 & 104.32 & 3.2 & 0.847 \\
\hline 1995 & 10 & 25 & 15 & 52 & 40.62 & -31.5153 & -68. & 100.95 & 3.1 & 048 \\
\hline 1995 & 10 & 25 & 17 & 30 & 2.96 & -31.4281 & -67. & 106.97 & 3.0 & 0.091 \\
\hline 1995 & 10 & 27 & 23 & 42 & 36.96 & -31.5072 & -68 & 109.8 & 3.1 & 0.047 \\
\hline 1995 & 10 & 29 & 15 & 8 & 52.1 & -32.6905 & -69.7015 & 117.21 & 3.6 & 0.042 \\
\hline 1995 & 10 & 31 & 19 & 0 & 2.89 & -31.2901 & -68.1126 & 31.11 & 3.1 & 0.048 \\
\hline 1995 & 11 & 1 & 13 & 42 & 42.33 & -31.3928 & -68.5874 & 102.26 & 3.1 & 0.052 \\
\hline 1995 & 11 & 4 & 22 & 20 & 27.19 & -31.3986 & -68.6684 & 99.72 & 3.1 & 0.023 \\
\hline 1995 & 11 & 2 & 3 & 21 & 21.13 & -31.5788 & -69.3465 & 108.9 & 3.2 & 0.119 \\
\hline 1995 & 11 & 2 & 21 & 43 & 54.39 & -31.6173 & -68.3771 & 102.42 & 3.9 & 0.097 \\
\hline 1995 & 11 & 3 & 15 & 1 & 17.64 & -31.3032 & -68.7518 & 103.2 & 3.1 & 0.026 \\
\hline 1995 & 11 & 3 & 15 & 40 & 31.08 & -32.1289 & -69.8295 & 103.59 & 3.4 & 0.103 \\
\hline 1995 & 11 & 4 & 15 & 55 & 10.72 & -31.2044 & -68.9017 & 100.9 & 3.2 & 0.028 \\
\hline 1995 & 11 & 5 & 11 & 11 & 26.7 & -33.2035 & -68.3576 & 41.25 & 3.7 & 0.104 \\
\hline 1995 & 11 & 6 & 5 & 15 & 0.96 & -31.5750 & -69.1991 & 107.42 & 3.3 & 0.274 \\
\hline 1995 & 11 & 7 & 3 & 6 & 2.23 & -31.2036 & -68.4220 & 101.01 & 3.1 & 0.091 \\
\hline 1995 & 11 & 7 & 14 & 5 & 9.67 & -31.0114 & -68.3083 & 17.11 & 4.1 & 0.06 \\
\hline 1995 & 11 & 10 & 17 & 31 & 13.99 & -31.5568 & -69.6963 & 100.43 & 3.3 & 0.049 \\
\hline 1995 & 11 & 11 & 11 & 41 & 20.49 & -31.3321 & -68.1245 & 31.49 & 3.0 & 0.114 \\
\hline 1995 & 11 & 11 & 12 & 3 & 2.92 & -32.0528 & -67.9841 & 121.36 & 3.2 & 0.043 \\
\hline 1995 & 11 & 12 & 5 & 31 & 16.25 & -31.4861 & -69.5157 & 115.84 & 3.3 & 0.115 \\
\hline
\end{tabular}




\begin{tabular}{|c|c|c|c|c|c|c|c|c|c|c|}
\hline año & 1es & dia & hora & $\min$ & seg & lat & long & prof & Mc & RMS \\
\hline 1995 & 11 & 12 & 16 & 57 & 14.46 & -31.9112 & -67.6828 & 0.01 & 3.3 & 0.171 \\
\hline 1995 & 11 & 14 & 3 & 56 & 34.97 & -32.0937 & -67.1822 & 19.99 & 3.3 & 0.547 \\
\hline 1995 & 11 & 15 & 5 & 14 & 1.23 & -31.4901 & -69.0666 & 100.83 & 3.0 & .055 \\
\hline 1995 & 11 & 15 & 14 & 57 & 42.28 & -31.4984 & -69.6849 & 111.55 & 3.4 & .042 \\
\hline 1995 & 11 & 16 & 1 & 42 & 2.49 & -29.4800 & -68.0913 & 80.96 & 3.2 & 0.067 \\
\hline 1995 & 11 & 18 & 5 & 40 & 10.94 & -31.4192 & -69.0414 & 101.3 & 3.2 & 0.01 \\
\hline 1995 & 11 & 21 & 22 & 53 & 33.67 & -31.7568 & -68.8383 & 0.85 & 3.1 & .208 \\
\hline 1995 & 11 & 22 & 4 & 53 & 22.99 & -33.0007 & -72.2217 & 0.1 & & 0.4 \\
\hline 1995 & 11 & 24 & 9 & 32 & 2.98 & -32.1241 & -68.5359 & 16.81 & & .037 \\
\hline 1995 & 11 & 25 & 0 & 24 & 2.71 & -31.2713 & -68.0403 & 25.5 & 2.8 & 0.043 \\
\hline 1995 & 11 & 25 & 22 & 32 & 27.72 & -32.2 & -67 & 28.28 & 3.1 & .097 \\
\hline 1995 & 11 & 26 & 7 & 40 & 53.04 & -31.2055 & -68 & 102.4 & 2.9 & 0.024 \\
\hline 1995 & 11 & 26 & 10 & 47 & 17.1 & -31 & & 106.81 & 3.0 & 058 \\
\hline 1995 & 11 & 27 & 0 & 40 & 33.57 & -31.7 & -67 & 0 & 2.7 & 0.349 \\
\hline 1995 & 11 & 27 & 5 & 7 & 27.81 & & & 07.16 & 3.2 & 046 \\
\hline 1995 & 11 & 28 & 23 & 5 & 8.12 & -31.8 & 299 & 104.91 & 3.1 & 0.047 \\
\hline 1995 & 11 & 29 & 14 & 2 & 24.32 & -31 & 264 & 3.51 & 3.1 & 027 \\
\hline 1995 & 11 & 29 & 16 & 55 & 8.69 & -31 & 569 & 04.64 & 3.1 & .042 \\
\hline 1995 & 11 & 29 & 19 & 6 & 32.1 & -31 & 87 & 102.06 & 3.4 & 084 \\
\hline 1995 & 11 & 29 & 20 & 16 & 5.26 & -31 & 69 & 110.57 & & 0.047 \\
\hline 1995 & 12 & 1 & 7 & 8 & 45.36 & -31. & 97 & 100.89 & 3.1 & 0.143 \\
\hline 1995 & 12 & 1 & 14 & 24 & 4.37 & & & 27.18 & 3.2 & 059 \\
\hline 1995 & 12 & 2 & 5 & 50 & 10.55 & -28 . & & 19.66 & 3.6 & 0.083 \\
\hline 1995 & 12 & 2 & 14 & 23 & 20.26 & -32 & & 137.46 & 3.3 & 059 \\
\hline 1995 & 12 & 3 & 10 & 21 & 0.48 & -31 & & 28.86 & 3.2 & 0.085 \\
\hline 1995 & 12 & 5 & 8 & 16 & 19.16 & -31 & & 100.78 & 2.9 & 81 \\
\hline 1995 & 12 & 5 & 9 & 50 & 41.02 & 99 & & 97.29 & 3.0 & 111 \\
\hline 1995 & 12 & 6 & 0 & 44 & 2.41 & -30 & & 37.39 & 3.4 & 13 \\
\hline 1995 & 12 & 6 & 7 & 17 & 25.04 & -3 & 972 & 80 & 3.6 & 0.205 \\
\hline 1995 & 12 & 6 & 14 & 54 & 3.37 & -31 & & 100.62 & 3.1 & 0.148 \\
\hline 1995 & 12 & 7 & 8 & 26 & 12.41 & -31 & 91 & 26.77 & 3.0 & 0.147 \\
\hline 1995 & 12 & 13 & 7 & 47 & 45.64 & -31 & & 100.16 & 3.1 & 0.028 \\
\hline 1995 & 12 & 14 & 15 & 6 & 20.86 & -31 & 87 & 20.01 & 2.9 & .076 \\
\hline 1995 & 12 & 16 & 14 & 37 & 25.64 & -31 & & 103.36 & 3.2 & 0.008 \\
\hline 1995 & 12 & 17 & 9 & 11 & 8.11 & -31 & 26 & 105.59 & 3.0 & 0.018 \\
\hline 1995 & 12 & 18 & 2 & 36 & 7.87 & & & 109 & 2.9 & 059 \\
\hline 1995 & 12 & 20 & 11 & 14 & 52.65 & -31 & & 108.45 & 3.1 & 0.041 \\
\hline 1995 & 12 & 20 & 22 & 12 & 20.11 & -30.8179 & 300 & 33.95 & 3.2 & 0.134 \\
\hline 1995 & 12 & 21 & 8 & 16 & 46.85 & -30.9805 & & 99.02 & 3.5 & 0.212 \\
\hline 1995 & 12 & 21 & 19 & 48 & 0.48 & -31.3442 & 732 & 101.08 & 3.0 & 0.066 \\
\hline 1995 & 12 & 23 & 9 & 42 & 59.32 & -31 & 87 & 12.29 & 3.3 & 0.024 \\
\hline 1995 & 12 & 23 & 17 & 59 & 35.15 & -31.3900 & -68.8308 & 25.67 & 2.9 & 0.064 \\
\hline 1995 & 12 & 25 & 8 & 18 & 2.4 & 70 & & 28.13 & 2.9 & 0.056 \\
\hline 1995 & 12 & 27 & 4 & 2 & 48.8 & -31.2676 & -68.6769 & 102.28 & 3.3 & 0.041 \\
\hline 1995 & 12 & 27 & 23 & 52 & 54.94 & -31.1029 & -67.7335 & 56.25 & 3.1 & 0.501 \\
\hline 1995 & 12 & 28 & 22 & 28 & 32.63 & -32.2714 & -67.3499 & 48.51 & 3.5 & 0.417 \\
\hline 1995 & 12 & 30 & 8 & 10 & 25.11 & -31.8523 & -67.7833 & 0.07 & & 0.092 \\
\hline
\end{tabular}




\begin{tabular}{|c|c|c|c|c|c|c|c|c|c|c|}
\hline año & mes & dia & hora & $\min$ & seg & lat & long & prof & Mc & RMS \\
\hline 1995 & 12 & 30 & 15 & 3 & 0.12 & -31.5166 & -68.8979 & 108.8 & 3.1 & 0.039 \\
\hline 1995 & 12 & 31 & 8 & 32 & 42.42 & -31.3711 & -68.5755 & 101.19 & 3.2 & 0.084 \\
\hline 1995 & 12 & 31 & 15 & 18 & 42.76 & -31.2172 & -68.3752 & 99.88 & & 0.11 \\
\hline 1996 & 1 & 1 & 15 & 30 & 19.37 & -32.2103 & -69.5202 & 118.18 & 3.2 & 0.061 \\
\hline 1996 & 1 & 1 & 19 & 45 & 43.34 & -31.2170 & -68.7016 & 105.49 & 3.1 & 0.043 \\
\hline 1996 & 1 & 5 & 7 & 29 & 42.65 & -32.5771 & -72.1359 & 58.39 & & 0.293 \\
\hline 1996 & 1 & 6 & 17 & 10 & 5.91 & -31.5735 & -68.6482 & 107.1 & 3.2 & 0.047 \\
\hline 1996 & 1 & 9 & 19 & 57 & 50.99 & -32.3308 & -72.1012 & 55.29 & 3.7 & 0.243 \\
\hline 1996 & 1 & 10 & 3 & 26 & 33.3 & -31.6948 & -65.0996 & 9.97 & 3.6 & 0.169 \\
\hline 1996 & 1 & 10 & 10 & 8 & 3.74 & -28.1503 & -66.8153 & 65.86 & 3.4 & 0.356 \\
\hline 1996 & 1 & 11 & 19 & 50 & 22.27 & -32.0943 & -68.3809 & 112.16 & 3.2 & 0.045 \\
\hline 1996 & 1 & 12 & 19 & 26 & 49.78 & -31.5809 & -68.8466 & 104.02 & 3.9 & 0.089 \\
\hline 1996 & 1 & 13 & 1 & 44 & 56.58 & -31.7650 & -67.9448 & 1.86 & 2.9 & 0.162 \\
\hline 1996 & 1 & 13 & 9 & 34 & 54.45 & -31.2250 & -68.4279 & 100.07 & 3.3 & 0.108 \\
\hline 1996 & 1 & 14 & 15 & 45 & 1.5 & -31.2 & -68.7704 & 98.33 & 3.4 & 0.075 \\
\hline 1996 & 1 & 15 & 0 & 46 & 9.62 & -31.4334 & -68.7770 & 97.71 & 3.1 & 0.093 \\
\hline 1996 & 1 & 15 & 10 & 23 & 7.05 & -30.9 & -68 & 21.12 & 2.8 & 0.111 \\
\hline 1996 & 1 & 16 & 3 & 35 & 12.91 & -31.1132 & -68.4841 & 100.9 & 2.8 & 0.131 \\
\hline 1996 & 1 & 16 & 21 & 57 & 30.35 & -31.2698 & -68 . & 103.67 & 3.5 & 0.037 \\
\hline 1996 & 1 & 17 & 4 & 53 & 15.62 & -31.2679 & -68.0445 & 27.97 & 2.9 & 0.131 \\
\hline 1996 & 1 & 17 & 5 & 38 & 28.25 & -32.3903 & -68.5370 & 123.3 & 3.9 & 0.053 \\
\hline 1996 & 1 & 18 & 6 & 58 & 30.34 & -31.7581 & -68.3828 & 105.91 & 3.3 & 0.073 \\
\hline 1996 & 1 & 18 & 23 & 23 & 37.56 & -31.0248 & -69.2524 & 108.18 & 3.7 & 0.085 \\
\hline 1996 & 1 & 20 & 21 & 59 & 39.01 & -31.7223 & -67.7741 & 18.96 & 3.0 & 0.004 \\
\hline 1996 & 1 & 21 & 4 & 16 & 5.73 & -31.2134 & -69.2141 & 100.05 & 3.7 & 0.098 \\
\hline 1996 & 1 & 21 & 10 & 31 & 43.49 & -31.4820 & -69.4648 & 103.1 & 3.2 & 0.101 \\
\hline 1996 & 1 & 30 & 11 & 59 & 39.51 & -31.5092 & -68.7941 & 103.42 & 3.6 & 0.044 \\
\hline 1996 & 1 & 31 & 10 & 32 & 13.64 & -31.5054 & -69.5946 & 111.74 & 3.2 & 0.113 \\
\hline 1996 & 2 & 1 & 10 & 42 & 51.59 & -30.6407 & -69.0026 & 25.16 & 3.2 & 0.137 \\
\hline 1996 & 2 & 1 & 18 & 49 & 4.97 & -33.0592 & -70.6147 & 99.6 & & 0.148 \\
\hline 1996 & 2 & 2 & 0 & 2 & 8.09 & -31.4793 & -67.8532 & 27.44 & 3.3 & 0.08 \\
\hline 1996 & 2 & 3 & 10 & 26 & 33.88 & -31.2187 & -68.7854 & 98.26 & 3.5 & 0.013 \\
\hline 1996 & 2 & 4 & 11 & 38 & 28.26 & -31.2564 & -68.0456 & 26.81 & 3.0 & 0.084 \\
\hline 1996 & 2 & 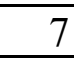 & 7 & 31 & 35.25 & -31.9094 & -69.5678 & 102.34 & 3.5 & 0.077 \\
\hline 1996 & 0 & 7 & 15 & 54 & 8.26 & -31.6235 & -68.7185 & 100.35 & 3.6 & 0.071 \\
\hline 1996 & 2 & 7 & 18 & 46 & 20.72 & -31.1644 & -68.3504 & 100.31 & 3.4 & 0.141 \\
\hline 1996 & 2 & 8 & 18 & 1 & 12.51 & -32.3020 & -70.4761 & 107.65 & 3.4 & 0.112 \\
\hline 1996 & 2 & 9 & 14 & 29 & 20.51 & -31.1340 & -68.3996 & 97.39 & 3.0 & 0.086 \\
\hline 1996 & 2 & 15 & 5 & 30 & 41.9 & -31.5906 & -68.7581 & 102.61 & 3.6 & 0.092 \\
\hline 1996 & 2 & 15 & 6 & 36 & 32.54 & -31.2002 & -68.5190 & 101.92 & 3.1 & 0.067 \\
\hline 1996 & 2 & 16 & 5 & 46 & 54.32 & -31.7555 & -68.2563 & 23.66 & 3.2 & 0.032 \\
\hline 1996 & 2 & 18 & 21 & 42 & 28.31 & -29.1965 & -69.9031 & 81.8 & 4.0 & 0.112 \\
\hline 1996 & 2 & 23 & 18 & 53 & 2.26 & -31.3778 & -69.2365 & 103.08 & & 0.079 \\
\hline 1996 & 3 & 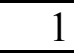 & 0 & 25 & 11.03 & -32.8467 & -68.8772 & 0.1 & 3.5 & 0.171 \\
\hline 1996 & 3 & 4 & 3 & 58 & 36 & -31.3643 & -67.7455 & 0.1 & 3.3 & 0.215 \\
\hline 1996 & 3 & 4 & 4 & 2 & 1.9 & -31.3834 & -67.7301 & 0.01 & 3.2 & 0.261 \\
\hline 1996 & 3 & 4 & 11 & 3 & 5.96 & -31.4507 & -68.6046 & 104.65 & 2.7 & 0.042 \\
\hline
\end{tabular}




\begin{tabular}{|c|c|c|c|c|c|c|c|c|c|c|}
\hline año & $1 e s$ & dia & hora & $\min$ & seg & lat & long & prof & Mc & RMS \\
\hline 1996 & 3 & 4 & 12 & 54 & 55.87 & -31.2192 & -68.4900 & 107.67 & 3.5 & 0.048 \\
\hline 1996 & 3 & 4 & 5 & 43 & 49.62 & -31.2128 & -68.3816 & 100.58 & 3.1 & .117 \\
\hline 1996 & 3 & 4 & 20 & 40 & 42.65 & & -71.8326 & 11.85 & 4.6 & 324 \\
\hline 1996 & 3 & 4 & 22 & 18 & 4.9 & -32.0627 & -69.7858 & 98.27 & 3.4 & .132 \\
\hline 1996 & 3 & 5 & 6 & 45 & 59.5 & -33.1154 & -68.5245 & 24.64 & 3.4 & .161 \\
\hline 1996 & 3 & 5 & 7 & 39 & 49.98 & -31.8681 & -69.9755 & 90.34 & 3.3 & .228 \\
\hline 1996 & 3 & 5 & 9 & 21 & 58.86 & -31.3384 & -69.4143 & 99.68 & 3.1 & .095 \\
\hline 1996 & 3 & 5 & 15 & 10 & 35.77 & -31.3516 & -69.3094 & 110.89 & 3.8 & .097 \\
\hline 1996 & 3 & 5 & 16 & 50 & 31.65 & -31.3 & -68.6132 & 100.72 & 2.9 & .042 \\
\hline 1996 & 3 & 5 & 16 & 57 & 49.26 & -31.2848 & -69.1252 & 109.84 & 3.7 & .732 \\
\hline 1996 & 3 & 5 & 21 & 47 & 11.52 & -31.2087 & -68.9676 & 102.81 & 3.1 & 034 \\
\hline 1996 & 3 & 6 & 1 & 15 & 28.07 & -31.7502 & -67.7906 & 20.64 & 2.8 & 56 \\
\hline 1996 & 3 & 6 & 8 & 48 & 16.65 & -28.3 & -70.3781 & 79.33 & & 224 \\
\hline 1996 & 3 & 6 & 9 & 42 & 47.62 & -30.5361 & -69.0228 & 0.1 & 4.3 & .625 \\
\hline 1996 & 3 & 6 & 11 & 41 & 40.13 & -29.5 & & 0.1 & 4.8 & .066 \\
\hline 1996 & 3 & 6 & 11 & 51 & 477.91 & -31.1699 & -68.4688 & 97.11 & 3.0 & 0.069 \\
\hline 1996 & 3 & 6 & 12 & 32 & 43.86 & & 102 & 0.1 & 3.4 & 258 \\
\hline 1996 & 3 & 6 & 14 & 50 & 38.9 & -32 . & 103 & 35.1 & 3.0 & 0.07 \\
\hline 1996 & 3 & 6 & 16 & 10 & 54.16 & -29 & 476 & 94.33 & 3.2 & \\
\hline 1996 & 3 & 6 & 17 & 31 & 15.36 & -32 & 946 & 121.16 & 4.0 & 0.082 \\
\hline 1996 & 3 & 7 & 9 & 52 & 8.71 & -31.6 & -69.5916 & 105.74 & 4.4 & 11 \\
\hline 1996 & 3 & 7 & 12 & 14 & 43.73 & -31.5 & -67.7961 & 30.83 & 2.7 & 092 \\
\hline 1996 & 3 & 7 & 22 & 22 & 25.22 & -31.2 & -69.0077 & 105.23 & 2.8 & .062 \\
\hline 1996 & 3 & 8 & $F$ & 15 & 10.27 & -30 & -69.4086 & .25 & 3.7 & 103 \\
\hline 1996 & 3 & 8 & 5 & 59 & 15.25 & -31 & 018 & 105.36 & 3.0 & .064 \\
\hline 1996 & 3 & 8 & 6 & 17 & 35.69 & -31 & 252 & 0 & 3.0 & 123 \\
\hline 1996 & 3 & 8 & 9 & 0 & 10.58 & -28 & 960 & 119.11 & & 0.197 \\
\hline 1996 & 3 & 8 & 12 & 47 & 59.96 & -31 & 918 & 96.44 & 3.0 & 0.11 \\
\hline 1996 & 3 & 8 & 17 & 31 & 53.85 & & & 104.02 & 3.5 & 063 \\
\hline 1996 & 3 & 8 & 18 & 38 & 4.23 & -31 & 505 & 112.96 & 3.7 & 07 \\
\hline 1996 & 3 & 8 & 20 & 19 & 31.14 & & 484 & 127.78 & 3.3 & 069 \\
\hline 1996 & 3 & 8 & 22 & 8 & 25.88 & & 810 & 104.15 & 3.8 & 0.079 \\
\hline 1996 & 3 & & 18 & 50 & 38.48 & -30 & 994 & 32.97 & 3.2 & .075 \\
\hline 1996 & 3 & & 23 & 19 & 18.03 & & 883 & 56.43 & 3.5 & 0.191 \\
\hline 1996 & 3 & 1 & 0 & 23 & 58.6 & -31 & 641 & 11.37 & & 55 \\
\hline 1996 & 3 & & & 25 & 2.96 & & 764 & 113.45 & 3.3 & .033 \\
\hline 1996 & 3 & - & 5 & 32 & 51.19 & & 097 & .4 & 3.3 & 91 \\
\hline 1996 & 3 & & & 15 & 25.68 & & -68.8106 & 98.6 & 3.2 & .052 \\
\hline 1996 & 3 & 12 & 11 & 6 & 54.34 & -31.2454 & -68.7402 & 102.91 & 3.2 & 0.075 \\
\hline 1996 & 3 & 12 & 23 & 31 & 30.01 & -31.3944 & -68.7028 & 105.93 & 3.0 & 0.028 \\
\hline 1996 & 3 & & 2 & 1 & 20.08 & & 660 & 97.29 & 3.7 & 0.145 \\
\hline 1996 & 3 & 13 & 8 & 24 & 45.97 & -31.9013 & -67.1325 & 134.37 & 2.9 & 0.146 \\
\hline 1996 & 3 & & & 55 & 48.41 & & 5744 & 92.87 & 3.2 & 0.029 \\
\hline 1996 & 3 & 14 & & 2 & 48.68 & -30.2112 & -70.1925 & 63.47 & & 0.044 \\
\hline 1996 & 3 & 12 & 10 & 8 & 6.92 & -32.7384 & -71.8864 & 2.67 & 3.8 & 0.239 \\
\hline 1996 & 3 & 14 & 18 & 37 & 46.71 & -31.4464 & -67.7934 & 28.17 & 3.0 & 0.137 \\
\hline 1996 & 3 & 15 & 18 & 22 & 21.65 & -30.9561 & -67.4150 & 32.19 & 3.5 & 0.098 \\
\hline
\end{tabular}




\begin{tabular}{|c|c|c|c|c|c|c|c|c|c|c|}
\hline año & mes & dia & hora & $\min$ & seg & lat & long & prof & Mc & RMS \\
\hline 1996 & 3 & 16 & 16 & 7 & 21.69 & -32.7997 & -69.4362 & 116.53 & 3.2 & 0.114 \\
\hline 1996 & 3 & 17 & 4 & 32 & 12.19 & -31.8136 & -68.5320 & 101.83 & 3.8 & 0.074 \\
\hline 1996 & 3 & 17 & 4 & 39 & 24.4 & -31.1656 & -68.4700 & 104.36 & 3.4 & 0.098 \\
\hline 1996 & 3 & 17 & 10 & 42 & 44.75 & -31.3334 & -67.8437 & 10.85 & 3.1 & 0.302 \\
\hline 1996 & 3 & 17 & 11 & 41 & 58.16 & -31.2647 & -68.4229 & 102.6 & 3.2 & 0.09 \\
\hline 1996 & 3 & 17 & 15 & 26 & 50.7 & -31.2616 & -68.9623 & 103.88 & 3.5 & 0.082 \\
\hline 1996 & 3 & 17 & 15 & 42 & 10.18 & -31.3527 & -68.9412 & 106.66 & 3.5 & 0.06 \\
\hline 1996 & 3 & 17 & 20 & 55 & 7.09 & -28.1726 & -71.1551 & 0.1 & 5.0 & 0.151 \\
\hline 1996 & 3 & 17 & 22 & 43 & 55.45 & -31.2516 & -68.5985 & 108.52 & 3.5 & 0.052 \\
\hline 1996 & 3 & 17 & 22 & 55 & 10.88 & -31.4656 & -69.7917 & 105.14 & 3.4 & 0.156 \\
\hline 1996 & 3 & 18 & 7 & 9 & 17.66 & -32.4044 & -72.4285 & 17.84 & 4.6 & 0.407 \\
\hline 1996 & 3 & 18 & 9 & 21 & 59.87 & -28.1063 & -66.9446 & 121.19 & 3.8 & 0.212 \\
\hline 1996 & 3 & 18 & 14 & 10 & 43.55 & -31.5182 & -68.6377 & 102.21 & 2.9 & 0.052 \\
\hline 1996 & 3 & 18 & 15 & 19 & 24.59 & -31.5401 & -68.7622 & 101.29 & 3.0 & 0.046 \\
\hline 1996 & 3 & 18 & 16 & 23 & 30.7 & -31.5027 & -69.3378 & 104.85 & 3.5 & 0.042 \\
\hline 1996 & 3 & 18 & 20 & 41 & 7.28 & -31.5216 & -69.3188 & 102.47 & 3.5 & 0.119 \\
\hline 1996 & 3 & 18 & 22 & 0 & 50.92 & -31.4907 & -68 & 33 & 3.0 & 0.04 \\
\hline 1996 & 3 & 18 & 23 & 17 & 6.42 & -31.0335 & -68.8565 & 122.26 & 3.7 & 0.931 \\
\hline 1996 & 3 & 19 & 0 & 9 & 38.82 & -31.2323 & -68.7161 & 99.29 & 3.6 & .076 \\
\hline 1996 & 3 & 19 & 5 & 48 & 16.73 & -31.3764 & -68.6073 & 103.87 & 3.6 & 0.082 \\
\hline 1996 & 3 & 19 & 8 & 11 & 11.53 & -31.4211 & -67.7560 & 28.75 & 3.3 & 0.156 \\
\hline 1996 & 3 & 19 & 14 & 39 & 22.06 & -32.4678 & -67.0821 & 26.65 & 3.1 & 0.236 \\
\hline 1996 & 3 & 19 & 15 & 18 & 0.17 & -30.1571 & -68.5090 & 0.02 & 4.0 & 0.217 \\
\hline 1996 & 3 & 19 & 20 & 5 & 7.61 & -31.2187 & -68.4191 & 100.31 & 2.9 & .135 \\
\hline 1996 & 3 & 20 & 1 & 35 & 12.73 & -31.3719 & -69.2087 & 108.55 & 3.8 & 0.088 \\
\hline 1996 & 3 & 20 & 2 & 7 & 20.31 & -29.3929 & -68 . & 0.1 & 4.8 & 0.06 \\
\hline 1996 & 3 & 20 & 3 & 18 & 33.49 & -31.6568 & -69.7295 & 97.18 & 3.8 & 0.213 \\
\hline 1996 & 3 & 20 & 7 & 12 & 26.36 & -28.5728 & -68.6 & 117.5 & 4.5 & 375 \\
\hline 1996 & 3 & 20 & 10 & 3 & 50.18 & -31.7213 & -69.6081 & 102.77 & 4.4 & 0.153 \\
\hline 1996 & 3 & 20 & 19 & 24 & 13.14 & -32.6346 & -69.8990 & 99.2 & 3.8 & 0.136 \\
\hline 1996 & 3 & 20 & 22 & 38 & 25.23 & -31.2203 & -68.6783 & 104.7 & 3.4 & 0.061 \\
\hline 1996 & 3 & 20 & 23 & 29 & 16.9 & -31.5957 & -66.5071 & 142.25 & 2.9 & 0.133 \\
\hline 1996 & 3 & 21 & 10 & 8 & 57.06 & -32.1281 & -69.2054 & 16.49 & 3.9 & 0.173 \\
\hline 1996 & 3 & 21 & 11 & 48 & 19.96 & -31.8554 & -67.6152 & 115.52 & 3.6 & 0.05 \\
\hline 1996 & 3 & 21 & 20 & 2 & 27.66 & -29.9730 & -70.7705 & 79.87 & 4.6 & 0.209 \\
\hline 1996 & 3 & 21 & 21 & 11 & 36.49 & -31.4509 & -69.2949 & 114.25 & 3.7 & 0.131 \\
\hline 1996 & 3 & 22 & 13 & 17 & 7.21 & -31.3802 & -68.6325 & 100.54 & 3.0 & 0.033 \\
\hline 1996 & 3 & 22 & 13 & 19 & 53.08 & -28.0440 & -69.4698 & 4.35 & 4.3 & 0.068 \\
\hline 1996 & 3 & 22 & 15 & 28 & 0.59 & -31.3196 & -67.7718 & 27.04 & 3.0 & 0.137 \\
\hline 1996 & 3 & 22 & 16 & 54 & 2.92 & -32.0638 & -68.5925 & 118.72 & 3.4 & 0.102 \\
\hline 1996 & 3 & 22 & 17 & 49 & 49.26 & -31.3334 & -69.4798 & 108.47 & 3.3 & 0.055 \\
\hline 1996 & 3 & 23 & 0 & 55 & 27.96 & -31.7795 & -68.0138 & 20.15 & 3.2 & 0.238 \\
\hline 1996 & 3 & 23 & 15 & 44 & 8.74 & -28.1541 & -67.5898 & 75.39 & 3.8 & 0.168 \\
\hline 1996 & 3 & 23 & 18 & 39 & 8.13 & -29.6793 & -71.6068 & 20.12 & 4.9 & 0.354 \\
\hline 1996 & 3 & 24 & 2 & 0 & 15.62 & -28.5386 & -67.5660 & 119.4 & 4.2 & 0.182 \\
\hline 1996 & 3 & 24 & 2 & 41 & 43.66 & -32.0763 & -69.8088 & 95.24 & 3.7 & 0.108 \\
\hline 1996 & 3 & 24 & 10 & 21 & 10.58 & -30.9612 & -70.1824 & 59.7 & 3.2 & 0.127 \\
\hline
\end{tabular}




\begin{tabular}{|c|c|c|c|c|c|c|c|c|c|c|}
\hline año & $1 e s$ & dia & hora & $\min$ & seg & lat & long & prof & Mc & RMS \\
\hline 1996 & 3 & 24 & 20 & 1 & 39.74 & -28.7765 & -68.5239 & 78.43 & 4.2 & 0.262 \\
\hline 1996 & 3 & 25 & 3 & 4 & 47.54 & -31.2296 & -68.3999 & 96.88 & 3.3 & 0.051 \\
\hline 1996 & 3 & 25 & 20 & 31 & 52.08 & -31.1699 & -68.5423 & 100.11 & 3.3 & .066 \\
\hline 1996 & 3 & 26 & 2 & 19 & 56.05 & -27.7388 & -70.5478 & 50.61 & 3.9 & .171 \\
\hline 1996 & 3 & 26 & 5 & 57 & 39.76 & -31.3453 & -69.0569 & 104.51 & 3.2 & 0.09 \\
\hline 1996 & 3 & 26 & 6 & 54 & 48.42 & -31.1346 & -68.2301 & 101.52 & 2.9 & 0.129 \\
\hline 1996 & 3 & 26 & 10 & 1 & 32.16 & -28.8952 & -67.3905 & 102.31 & 3.4 & .153 \\
\hline 1996 & 3 & 26 & 19 & 55 & 40.7 & -31.0974 & -68.5046 & 99.53 & & 077 \\
\hline 1996 & 3 & 27 & 6 & 16 & 2.06 & -31.7310 & -69.6459 & 92.37 & 3.7 & .099 \\
\hline 1996 & 3 & 27 & 7 & 3 & 50.76 & -31.5236 & -68.8820 & 108.83 & 3.5 & 0.071 \\
\hline 1996 & 3 & 27 & 8 & 56 & 7.75 & -31.7067 & -68.4709 & 45.31 & 3.0 & .768 \\
\hline 1996 & 3 & 27 & 12 & 25 & 25.33 & -31.4343 & -68.6309 & 101.67 & 3.3 & 0.043 \\
\hline 1996 & 3 & 27 & 15 & 31 & 4.51 & -31.7941 & 275 & 92.26 & 2.9 & 099 \\
\hline 1996 & 3 & 28 & 8 & 56 & 16.15 & -32.2025 & -69.4707 & 111.75 & 3.5 & 0.136 \\
\hline 1996 & 3 & 28 & 10 & 54 & 49.82 & 043 & 477 & 0 & 3.4 & 806 \\
\hline 1996 & 3 & 28 & 11 & 58 & 42.14 & -31.2449 & -68.8667 & 104.5 & 3.8 & 0.026 \\
\hline 1996 & 3 & 28 & 14 & 27 & 26.78 & -29.2317 & 435 & 81.12 & 3.6 & 095 \\
\hline 1996 & 3 & 28 & 17 & 49 & 22.1 & -29.9826 & 958 & 12.65 & 3.6 & 149 \\
\hline 1996 & 3 & 29 & 12 & 8 & 44.81 & -31.2096 & 255 & 01.31 & 3.3 & 074 \\
\hline 1996 & 3 & 29 & 21 & 0 & 18.41 & -31.0416 & 232 & 02.86 & 3.7 & 0.197 \\
\hline 1996 & 3 & 30 & 6 & 6 & 42.21 & -32.7946 & -69 & 100.99 & 3.8 & 0.124 \\
\hline 1996 & 3 & 30 & 15 & 4 & 40.27 & -31.1985 & & 97.88 & 3.9 & 889 \\
\hline 1996 & 3 & 31 & 17 & 42 & 3.45 & -30.4624 & 406 & 80.05 & 4.2 & 0.349 \\
\hline 1996 & 3 & 31 & 19 & 48 & 50.67 & 256 & 217 & 96.55 & 3.4 & 087 \\
\hline 1996 & 7 & 10 & 16 & 16 & 50.82 & -31.9082 & 53 & 15.51 & 3.9 & 0.104 \\
\hline 1996 & 7 & 11 & 22 & 24 & .25 & $\underline{z}$ & 369 & & 1 & 11 \\
\hline 1996 & 7 & 15 & 19 & 27 & 52.69 & 02 & & 30.09 & 3.9 & 089 \\
\hline 1996 & 7 & 15 & 22 & 54 & 12.6 & -31 & & 16.61 & 3.1 & 11 \\
\hline 1996 & 7 & 18 & 12 & 20 & 52.31 & -30.9781 & 094 & 19.26 & 4.0 & .086 \\
\hline 1996 & 7 & 18 & 13 & 6 & 56.73 & -31.4547 & & & 3.2 & 0.282 \\
\hline 1996 & 7 & 24 & 1 & 20 & 12.97 & 351 & 622 & 21.79 & 3.3 & 0.126 \\
\hline 1996 & 7 & 7 & 3 & 17 & 31.87 & -31.5597 & & 21.36 & 2.9 & 0.004 \\
\hline 1996 & 7 & 25 & 20 & 52 & 35.02 & -31.4144 & 96 & 0.01 & 3.2 & .143 \\
\hline 1996 & 7 & 27 & 9 & 42 & 44.36 & -31.4502 & 309 & 26.87 & 3.4 & 0.002 \\
\hline 1996 & 7 & 27 & 20 & 56 & 18.5 & -31.1496 & 479 & 0.04 & 3.4 & 0.046 \\
\hline 1996 & 8 & 4 & $\tau$ & 2 & 34.13 & 07 & & 28.36 & 3.4 & 0.021 \\
\hline 1996 & 8 & 4 & 12 & 24 & 2.47 & -31 & 37 & 0.01 & 3.4 & 0.079 \\
\hline 1996 & 8 & 5 & 10 & 56 & 26.1 & -31.1147 & 3000 & 0.03 & 3.2 & 0.085 \\
\hline 1996 & 8 & 9 & 16 & 22 & 29.23 & -31.7046 & 423 & 24 & 2.8 & 0.017 \\
\hline 1996 & 8 & 9 & 18 & 22 & 27.94 & -31.8766 & -67.6385 & 0.42 & 3.0 & 0.071 \\
\hline 1996 & 8 & 14 & 15 & 58 & 49.15 & -31.3996 & 7653 & 20.41 & 3.7 & 0.022 \\
\hline 1996 & 8 & 16 & 7 & 18 & 51.86 & -31.8236 & -67.9886 & 0.01 & 2.8 & 0.136 \\
\hline 1996 & 8 & 18 & 11 & 36 & 36.17 & -31.5279 & 25 & 14.68 & 3.4 & 0.118 \\
\hline 1996 & 8 & 21 & 21 & 21 & 23.59 & -31.3095 & -68.0433 & 27.4 & 3.2 & 0.085 \\
\hline 1996 & 8 & 21 & 22 & 11 & 23.34 & -30.3226 & -67.5760 & 29.05 & 3.9 & 0.16 \\
\hline 1996 & 8 & 22 & 16 & 39 & 9.89 & -31.5957 & -67.9185 & 21.87 & 3.1 & 0.053 \\
\hline 1996 & 8 & 25 & 7 & 24 & 13.34 & -31.7721 & -68.2848 & 19.34 & 2.9 & 0.037 \\
\hline
\end{tabular}




\begin{tabular}{|c|c|c|c|c|c|c|c|c|c|c|}
\hline año & mes & dia & hora & $\min$ & seg & lat & long & prof & Mc & RMS \\
\hline 1996 & 8 & 25 & 12 & 25 & 24.24 & -31.1931 & -68.1920 & 36.92 & 3.1 & 0.1 \\
\hline 1996 & 8 & 26 & 1 & 45 & 3.57 & -32.7082 & -68.8547 & 21.05 & 3.9 & 0.063 \\
\hline 1996 & 8 & 26 & 22 & 41 & 34.96 & -31.9212 & -69.3443 & 46.56 & 3.7 & 0.309 \\
\hline 1996 & 9 & 1 & 0 & 59 & 22.19 & -31.5855 & -67.7632 & 32.65 & 3.1 & 0.032 \\
\hline 1996 & 9 & 3 & 12 & 44 & 16.62 & -27.3735 & -69.7573 & 64.54 & & 0.233 \\
\hline 1996 & 9 & 3 & 20 & 7 & 22.28 & -32.3692 & -68.1970 & 33.67 & 3.4 & 0.071 \\
\hline 1996 & 9 & 3 & 22 & 26 & 7.46 & -31.5204 & -67.8322 & 34.09 & 2.8 & 0.052 \\
\hline 1996 & 9 & 4 & 23 & 28 & 46.35 & -31.3319 & -68.6246 & 35.6 & 3.6 & 0.067 \\
\hline 1996 & 9 & 8 & 18 & 46 & 26.12 & -30.1058 & -68.1781 & 19.97 & 3.4 & 0.348 \\
\hline 1996 & 9 & 8 & 23 & 24 & 20.75 & -30.0560 & -68.2111 & 0.69 & 3.7 & 0.115 \\
\hline 1997 & 1 & 2 & 13 & 26 & 17.37 & -31.0795 & -67.9332 & 24.3 & 2.9 & 0.077 \\
\hline 1997 & 1 & 5 & 22 & 21 & 5.17 & -31.4846 & -68.7032 & 14.99 & 2.9 & 0.177 \\
\hline 1997 & 1 & 6 & 16 & 11 & 37.13 & -31.3982 & -67.9987 & 26.11 & 3.0 & 0.105 \\
\hline 1997 & 1 & 6 & 16 & 31 & 46.57 & -31.5011 & -67.9704 & 39.76 & 2.8 & 0.111 \\
\hline 1997 & 1 & 8 & 23 & 46 & 9.33 & -31.3165 & -68 & 31.38 & 2.8 & 0.1 \\
\hline 1997 & 1 & 9 & 19 & 38 & 49.46 & -31.8533 & -68.8318 & 12.34 & 2.9 & 0.001 \\
\hline 1997 & 1 & 11 & 8 & 32 & 16.94 & -31.4050 & -67 & 6.82 & 3.1 & 0.144 \\
\hline 1997 & 1 & 12 & 20 & 1 & 57.53 & -30.4445 & -69.2963 & 23.21 & 3.5 & 0.109 \\
\hline 1997 & 1 & 16 & 2 & 19 & 53.18 & -31.8758 & -68 & 16.89 & 3.1 & 0.103 \\
\hline 1997 & 1 & 18 & 9 & 31 & 52.63 & -31.1165 & -67.7828 & 3.76 & 2.8 & 0.198 \\
\hline 1997 & 1 & 18 & 14 & 52 & 6.62 & -31.8559 & -67.7880 & 20.16 & 2.8 & 0.116 \\
\hline 1997 & 1 & 18 & 17 & 23 & 0.95 & -31.4248 & -68.2643 & 0.25 & 3.1 & 0.173 \\
\hline 1997 & 1 & 18 & 22 & 37 & 41.54 & -31.6558 & -69.0078 & 0.33 & 3.5 & 0.253 \\
\hline 1997 & 1 & 21 & 2 & 50 & 13.16 & -31.7991 & -67.9917 & 0 & 3.6 & 0.206 \\
\hline 1997 & 1 & 21 & 4 & 3 & 21.9 & -31.7459 & -67.8521 & 22.39 & 2.9 & 0.045 \\
\hline 1997 & 1 & 22 & 7 & 40 & 38.57 & -31.8007 & -67 & 0.05 & 3.1 & 0.155 \\
\hline 1997 & 1 & 24 & 0 & 45 & 47.54 & -31.2890 & -68.0113 & 17.72 & 3.0 & 0.051 \\
\hline 1997 & 1 & 24 & 5 & 8 & 25.39 & -31.7474 & -68 & 8.42 & 3.1 & 0.088 \\
\hline 1997 & 1 & 24 & 7 & 29 & 1.38 & -31.5795 & -68.8208 & 12.03 & 3.3 & 0.122 \\
\hline 1997 & 1 & 24 & 9 & 24 & 45.8 & -31.5747 & -68 & 9.86 & 3.2 & 0.139 \\
\hline 1997 & 2 & 7 & 19 & 19 & 28.46 & -33.0171 & -68.0576 & 31.31 & 3.4 & 0.353 \\
\hline 1997 & 2 & 9 & 14 & 54 & 15.21 & -31.1882 & -67.2899 & 19.74 & 3.4 & 0.099 \\
\hline 1997 & 2 & 14 & 21 & 37 & 22.09 & -31.3654 & -69.4723 & 18.17 & 3.7 & 0.189 \\
\hline 1997 & 2 & 26 & 7 & 32 & 12.43 & -31.6567 & -67.9769 & 24.76 & 2.8 & 0.131 \\
\hline 1997 & 2 & 27 & 21 & 55 & 58.24 & -30.0990 & -67.4369 & 27.86 & 4.1 & 0.281 \\
\hline 1997 & 3 & 3 & 17 & 42 & 52.84 & -31.9079 & -68.3196 & 11.89 & 3.1 & 0.156 \\
\hline 1997 & 3 & 5 & 22 & 51 & 36.69 & -31.2874 & -66.8992 & 27.25 & 3.4 & 0.372 \\
\hline 1997 & 3 & 0 & 2 & 24 & 10.26 & -33.4699 & -69.0446 & 28.47 & & 0.697 \\
\hline 1997 & 3 & 8 & 4 & 36 & 2.57 & -32.0453 & -67.4523 & 8.91 & 3.3 & 0.738 \\
\hline 1997 & 3 & 9 & 12 & 43 & 49.92 & -32.3864 & -66.9142 & 18.53 & 3.3 & 0.122 \\
\hline 1997 & 3 & 9 & 17 & 42 & 7.08 & -29.5782 & -71.1045 & 33.69 & 4.6 & 0.094 \\
\hline 1997 & 3 & 10 & 9 & 25 & 37.86 & -31.7040 & -67.4156 & 39.44 & 3.1 & 0.065 \\
\hline 1997 & 3 & 11 & 9 & 23 & 28.41 & -33.1404 & -68.2825 & 37.12 & 3.2 & 0.125 \\
\hline 1997 & 3 & 11 & 18 & 29 & 24.38 & -32.2880 & -68.4234 & 40.56 & 3.5 & 0.139 \\
\hline 1997 & 3 & 13 & 11 & 1 & 17.89 & -31.3489 & -67.8701 & 19.33 & 4.0 & 0.108 \\
\hline 1997 & 3 & 15 & 16 & 9 & 40.64 & -30.6608 & -68.4682 & 0.01 & 4.1 & 0.138 \\
\hline 1997 & 3 & 22 & 16 & 57 & 51.92 & -31.6121 & -67.9398 & 24.28 & 3.4 & 0.085 \\
\hline
\end{tabular}




\begin{tabular}{|c|c|c|c|c|c|c|c|c|c|c|}
\hline año & nes & dia & hora & $\min$ & seg & lat & long & prof & Mc & RMS \\
\hline 1997 & 3 & 24 & 6 & 6 & 46.48 & -29.9625 & -69.6220 & 0.1 & 4.5 & 0.414 \\
\hline 1997 & 3 & 26 & 2 & 25 & 17.89 & -31.1842 & -67.8654 & 21.01 & 3.4 & 085 \\
\hline 1997 & 3 & 30 & 10 & 12 & 22.88 & -31.5661 & & 24.39 & 3.1 & 073 \\
\hline 1997 & 4 & 6 & 12 & 16 & 3.97 & -28.8001 & 401 & 14.77 & 4.9 & 333 \\
\hline 1997 & 4 & 6 & 16 & 32 & 20.51 & -31.0958 & -67.8284 & 19.8 & 3.0 & .041 \\
\hline 1997 & 4 & 14 & 3 & 46 & 35.14 & -31.0470 & -67.8467 & 17.66 & 3.5 & .094 \\
\hline 1997 & 4 & 22 & 7 & 9 & 6.22 & -31.0496 & -67.8462 & 16.8 & 3.6 & 0.15 \\
\hline 1997 & 4 & 23 & 11 & 10 & 45.07 & -31.0461 & 67.8491 & 16.1 & 3.4 & .107 \\
\hline 1997 & 4 & 29 & 8 & 4 & 22.25 & -32.0952 & -68.8952 & 29.11 & 3.5 & .277 \\
\hline 1997 & 4 & 29 & 21 & 29 & 7.76 & -31.2705 & -68.0498 & 26.72 & 3.2 & 093 \\
\hline 1998 & 8 & 6 & 15 & 4 & 20.6 & -32.1952 & 860 & 31.92 & 4.1 & 235 \\
\hline 1998 & 8 & 6 & 18 & 19 & 49.06 & -32.0819 & -67.7184 & 0.1 & 3.3 & 249 \\
\hline 1998 & 8 & 8 & 5 & 24 & 12.86 & -31.9664 & 130 & 23.62 & 3.3 & 187 \\
\hline 1998 & 8 & 15 & 11 & 6 & 46.32 & -31.4401 & -68.0626 & 23.91 & 3.2 & .231 \\
\hline 1998 & 8 & 16 & 23 & 22 & 4 & -31.5 & 581 & 31.22 & 3.6 & 108 \\
\hline 1998 & 8 & 19 & 1 & 38 & 38.95 & -31.8 & 767 & 14.21 & 3.1 & .411 \\
\hline 1998 & 8 & 25 & 16 & 59 & 51.14 & & & 28.2 & 3.6 & 246 \\
\hline 1998 & 8 & 29 & 9 & 4 & 54.32 & -32.1 & 880 & 29.95 & 3.3 & .266 \\
\hline 1998 & 9 & 11 & 4 & 26 & 22.4 & -33 & & 76 & 3.5 & 774 \\
\hline 1998 & 9 & 12 & 14 & 18 & 13.31 & -31 & 92 & 25.75 & 3.2 & 65 \\
\hline 1998 & 1 & 4 & 15 & 37 & 41.48 & -31.5 & 900 & 0.1 & 2.7 & 75 \\
\hline 1998 & 1 & 7 & 3 & 56 & 50.05 & -31 & 991 & 31.8 & 3.6 & 345 \\
\hline 1998 & 1 & 7 & 18 & 46 & 28.69 & -31 & 042 & 25.9 & 3.6 & .309 \\
\hline 1998 & 1 & 7 & 23 & 31 & 11.25 & -31 & 017 & 18.27 & 3.3 & 198 \\
\hline 1998 & 1 & 9 & 10 & 40 & \begin{tabular}{|l|}
46.67 \\
\end{tabular} & -31 & 620 & 26.47 & 3.7 & 0.136 \\
\hline 1998 & 11 & 10 & 6 & 16 & 34.5 & -32 & 945 & 32.81 & 3.3 & 296 \\
\hline 1998 & 11 & 13 & 10 & 37 & 46.79 & -31 & & 10.94 & 3.4 & 889 \\
\hline 1998 & 11 & 18 & 18 & 51 & 53.58 & -3 & 42 & 24 & 2.9 & \\
\hline 1998 & 11 & 20 & 10 & 12 & 34.9 & & & 13.64 & 3.4 & 459 \\
\hline 1998 & 11 & 23 & 17 & 35 & 20.6 & -31 & & & 3.0 & 15 \\
\hline 1998 & 11 & 27 & 21 & 7 & 18.43 & -31 & & 16.14 & 3.8 & 294 \\
\hline 1998 & 11 & 30 & T & 17 & 38.09 & -32 & & 27.87 & 3.0 & 0.385 \\
\hline 1999 & 4 & 2 & 16 & 30 & 28.87 & -31 & 98 & 36.44 & 4.0 & 0.296 \\
\hline 1999 & 4 & 5 & 19 & 37 & 46.5 & & & 16.91 & 3.1 & 0.19 \\
\hline 1999 & 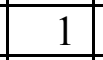 & 6 & 17 & 9 & 52.47 & -33 & 93 & 0.1 & 3.1 & .433 \\
\hline 1999 & 1 & 19 & 19 & 18 & 41.33 & & & 5 & 3.4 & 513 \\
\hline 1999 & 1 & 19 & 21 & 45 & 1 & & & & 3.6 & 64 \\
\hline 1999 & 1 & 22 & 4 & 34 & 31.41 & -28 . & 638 & 119.82 & 4.6 & 371 \\
\hline 1999 & 1 & 24 & 2 & 5 & \begin{tabular}{|l|}
31.29 \\
\end{tabular} & -31 . & 56 & 36.95 & 3.1 & 0.327 \\
\hline 1999 & 1 & 29 & 23 & 6 & 6.17 & -31.7334 & 848 & 14.62 & 4.1 & 0.158 \\
\hline 1999 & 1 & 29 & 23 & 49 & 16.67 & -33 & & 22.32 & & 0.939 \\
\hline 1999 & 2 & 1 & 7 & 57 & 58.31 & -32.0004 & -68.1836 & 16.99 & 4.2 & 0.515 \\
\hline 1999 & 2 & 1 & 13 & 29 & 4.46 & & & 23.44 & 4.4 & 0.414 \\
\hline 1999 & 2 & 2 & 20 & 2 & 1.14 & -30.1184 & -67.1797 & 23.61 & 3.5 & 0.466 \\
\hline 1999 & 2 & 4 & 5 & 38 & 37.69 & & -68.9574 & 27.4 & 3.4 & 0.473 \\
\hline 1999 & 2 & 5 & 16 & 7 & 12.56 & -34.7508 & -70.8096 & 58.55 & 5.6 & 0.176 \\
\hline 1999 & 2 & 12 & 2 & 58 & 1.06 & -34.9807 & -68.9532 & 28.44 & 4.8 & 0.158 \\
\hline
\end{tabular}




\begin{tabular}{|c|r|r|r|r|r|c|c|c|c|c|}
\hline año & mes & dia & hora & min & seg & lat & long & prof & Mc & RMS \\
\hline 1999 & 2 & 21 & 10 & 32 & 52.32 & -31.2692 & -68.0573 & 28.95 & 3.5 & 0.217 \\
\hline 1999 & 2 & 26 & 4 & 4 & 53.67 & -35.1695 & -68.0298 & 31.23 & 5.5 & 0.034 \\
\hline 1999 & 3 & 6 & 9 & 19 & 22.21 & -31.9560 & -68.0365 & 16.95 & 3.3 & 0.227 \\
\hline 1999 & 3 & 7 & 3 & 20 & 26.12 & -31.3615 & -67.8043 & 14.48 & 3.5 & 0.293 \\
\hline 1999 & 3 & 9 & 9 & 14 & 12.41 & -34.8843 & -69.8500 & 0.15 & & 0.446 \\
\hline 1999 & 3 & 9 & 19 & 41 & 58.3 & -34.8584 & -69.4837 & 28.13 & 4.6 & 0.252 \\
\hline 1999 & 3 & 10 & 5 & 55 & 8.87 & -34.0311 & -69.0177 & 49.05 & 4.8 & 0.228 \\
\hline 1999 & 3 & 10 & 12 & 0 & 38.23 & -31.4259 & -67.7550 & 24.23 & 3.3 & 0.326 \\
\hline 1999 & 3 & 17 & 5 & 25 & 26.65 & -35.0436 & -69.8223 & 7.58 & & 0.288 \\
\hline 1999 & 3 & 26 & 4 & 6 & 10.79 & -32.6473 & -68.8837 & 24.11 & 3.3 & 0.167 \\
\hline 1999 & 3 & 26 & 11 & 52 & 9.96 & -33.4677 & -68.0519 & 41.1 & 3.5 & 0.403 \\
\hline 1999 & 3 & 27 & 6 & 38 & 30.94 & -35.2002 & -69.1415 & 27.53 & 5.2 & 0.806 \\
\hline 1999 & 3 & 29 & 20 & 39 & 14.42 & -33.9028 & -67.2616 & 73.69 & 4.7 & 0.677 \\
\hline
\end{tabular}




\section{BIBLIOGRAFÍA}

Abers, G. A., P. E. van Keken, E. A. Kneller, A. Ferris, and J. C. Stachnik, 2006. The thermal structure of subduction zones constrained by seismic imaging: Implications for slab dehydration and wedge flow. Earth Planet. Sci. Lett. 241, 387- 397.

Abubakirov, I. R. and A. A. Gusev, 1990. Estimation of scattering properties of lithosphere of Kamchatka based on Monte-Carlo simulation of record envelope of near earthquake. Phys. Earth Planet. Interiors 64, 52-67.

Aki, K., 1956. Correlogram analyses of seismograms by means of a simple automatic computer. J. Phys. Earth 4, 71-79.

Aki, K., 1967. Scaling law of the seismic spectrum. J. Geophys. Res. 72, 1217-1231.

Aki K., 1969. Analysis of the seismic coda of local earthquakes as scattered waves. J. Geophys. Res. 74, pp. 615-631.

Aki, K., 1980a. Attenuation of shear-waves in the lithosphere for frequencies from 0.05 to $25 \mathrm{~Hz}$. Phys. Earth Planet. Interiors 21, 50-60.

Aki, K., 1980b. Scattering and attenuation of shear waves in the lithosphere. J. Geophys. Res. 85, B11, 6496-6504.

Aki, K., 1982. Scattering and attenuation. Bull. Seism. Soc. Am. 72, 319-330.

Aki K., M. Bouchon, B. Chouet and S. Das, 1977. Quantitative prediction of strong motion for a potential earthquake fault. Annali di Geofisica 30, 3-4 (Annals of Geophysics 53, 1, .81-91, 2010)

Aki K. and B. Chouet, 1975. Origin of Coda Waves: Source, Attenuation and Scattering Effects. J. Geophys. Res. 80, 23, 3322-3342.

Aki, K. and P. Richards, 1980, 2002. Quantitative Seismology. 1st. Ed, 1980, 2nd Ed., 2002. University Science Books, 700 pp.

Aki, K and M. Tsujiura, 1959. Correlation study of near earthquake waves. Bull. Earthq. Inst. Univ Tokyo 37, 207-232.

Akinci, A., E. del Pezzo and J. M. Ibáñez, 1995a. Separation of scattering and intrinsic attenuation in southern Spain and western Anatolia (Turkey). Geophys. J. Int. 121, 337-353.

Akinci, A., J. M. Ibáñez, E. del Pezzo and J. Morales, 1995b. Geometrical spreading and attenuation of Lg waves: a comparison between western Anatolia (turkey) and southern Spain. Tectonophysics 250, 47-60.

Allmendinger, R. W., T. E. Jordan, S. M. Kay and B. L. Isacks, 1997. The evolution of the AltiplanoPuna Plateau of the Central Andes. Annual Reviews of Earth and Planetary Sciences 25, 139174.

Alvarado, P. M., 1992. Atenuación sísmica en el norte de Mendoza. Trabajo final de Licenciatura en Geofísica. Facultad de Cs. Exactas, Físicas y Naturales. Universidad Nacional de San Juan.

Alvarado, P.M., S. Beck, G. Zandt, M. Araujo and E. Triep, 2005. Crustal deformation in the southcentral Andes backarc terranes as viewed from regional broad-band seismic waveform modelling. Geophys. J. Int., doi: 10.1111/j.1365-246X.2005.02759.x

Alvarado P., M. Pardo, H. Gilbert, S. Miranda, M. Anderson, M. Saez and S. Beck, 2009. Flat-slab subduction and crustal models for the seismically active Sierras Pampeanas region of Argentina. GSA Memoirs 2009, 204, 261-278. doi: 10.1130/2009.1204(12)

Alvarado, P., G. Sánchez, M. Saez, B. Castro de Machuca, 2010. Nuevas evidencias de la actividad sísmica del terreno Cuyania en la región de subducción de placa horizontal de Argentina: Revista Mexicana de Ciencias Geológicas 27, 2, 278-291.

Anderson, A. L., A. Ben-Menahem and C. B. Archambeau, 1965. Attenuation of seismic energy in the upper mantle. J. Geophys. Res. 70, 1441-1448.

Anderson, A. L. and R. S. Hart, 1978. $Q$ of the Earth. J. Geophys. Res. 83, 5869-5882. 
Anderson, D. L., 2000. Thermal state of the upper mantle: No role for mantle plumes. Geophys. Res. Letters 27, 3623-3626, doi:10.1029/2000GL011533.

Anderson, J. G. and S. Hough, 1984. A model for the shape of the Fourier amplitude spectrum of acceleration at high frequencies. Bull. Seismol. Soc. Am. 74, 1969-1993.

Anderson, M., P. Alvarado, G. Zandt, S.L. Beck, 2007, Geometry and brittle deformation of the subducting Nazca plate, central Chile and Argentina, Geophys. J. Int., doi:10.1111/j.1365246X.2007.03483.x, 2007.

Anderson, M., G. Zandt, E. Triep, M. Fouch and S. Beck, 2004. Anisotropy and mantle flow in the Chile-Argentina subduction zone from shear wave splitting analysis. Geophys. Res. Lett. 31, L23608, doi:10.1029/2004GL020906

Azcuy, C.L., R.R. Andreis, A. Cuerda, M. A. Hünicken, M. V. Pensa, D. A. Valencio y J. F. Vilas, 1987. Cuenca Paganzo. En: S. Archangelsky (Ed.), El Sistema Carbonífero en la República Argentina, Academia Nacional de Ciencias, Cap. III: 41-100. Córdoba.

Azimi, Sh. A., A. Y. Kalinin, V. B. Kalinin and B. L. Pivovarov, 1968. Impulse and transient characteristics of media with linear and quadratic absorption laws. Phys. Solid Earth 2, 88-93.

Badi, G. A., 1991. Relación de la sismicidad y tectónica en América del Sur entre los $22^{\circ}$ y $32^{\circ} \mathrm{S}$ con la subducción de la Placa de Nazca. Práctica de la Especialidad. Facultad de Cs. Astronómicas y Geofísicas. Universidad Nacional de La Plata.

Badi, G., E. Del Pezzo, J. M. Ibanez, F. Bianco, N. Sabbione and M. Araujo, 2009. Depth dependent seismic scattering attenuation in the Nuevo Cuyo region (southern central Andes). Geophys. Res. Lett. 36, 24, L24307. http://dx.doi.org/10.1029/2009GL041081

Badi, G., J. Ibáñez, , E. Del Pezzo, N. Sabbione, F. Bianco, 2010. Depth Dependent Seismic Attenuation in the Nuevo Cuyo Region, Argentina. EOS Trans. AGU, 91(26), Meet. Am. Suppl S23B-07.

Badi G., J. Ibáñez Godoy y N. Sabbione, 2000. Determinación de Q coda en la región centro-oeste de la Rep. Argentina con datos digitales. Actas de la 20va. Reunión Científica de la Asociación Argentina de Geofísicos y Geodestas. En CD, 85-90.

Badi G., J. Ibáñez Godoy y N. Sabbione, 2005. Atenuación de ondas de corte en la Región de Nuevo Cuyo. Actas de la XXII Reunión de la Asociación de Geofísicos y Geodestas, Buenos Aires, 6 al 10 de octubre de 2004. pp. 1-5, en CD.

Badi G., J. Ibáñez Godoy y N. Sabbione, 2007. Atenuación sísmica de corto período en la Región de Nuevo Cuyo. GEOACTA 32, 193-205.

Badi G., M. L. Rosa, N. C. Sabbione y M. Plasencia, 2004. Atenuación de Ondas Rayleigh en el extremo sur del Continente Sudamericano. 27-52. En: E. Triep, A. Introcaso (ed.). Tópicos de Geociencias. Un Volumen de Estudios Sismológicos, Geodésicos y Geológicos en homenaje al Ing. Fernando Séptimo Volponi. Editorial: EFU Fundación Universidad Nacional de San Juan., 334 pp.

Badi G. y N. Sabbione, 1992. Relación de la Sismicidad y Tectónica en América del Sur entre los $22^{\circ}$ y $32^{\circ} \mathrm{S}$ con la subducción de la Placa de Nazca. Exposición Oral en la XVII Reunión de la Asociación de Geofísicos y Geodestas, Buenos Aires, Argentina, 27 de octubre, Res. MaMB2.

Badi G. y N. Sabbione, 1994. Sismicidad en la zona de convergencia de las placas Sudamericana y de Nazca. Exposición Oral en la Asamblea Regional de Sismología en América del Sur. IASPEI - CERESIS - ISOP, Brasilia, Brasil, 22 al 26 de agosto, Res. SSR. 20.

Baldis, B.A., M. S. Beresi, O. Bordonaro y A. Vaca, 1982. Síntesis evolutiva de la Precordillera Argentina. $5^{\circ}$ Congreso Latinoamericano de Geología, 4:399-445.

Baldis, B., O. Bordonaro, C. Armella, M. Beresi, N. Cabaleri, S. Peralta y H. Bastías, 1989. La Cuenca Paleozoica inferior de la Precordillera Argentina. En: G.A. Chebli y L.A. Spalleti (Eds.), Cuencas Sedimentarias Argentinas, Serie Correlación Geológica No.6, 101-122. S. M. Tucumán.

Baldis, B. A., J. Febrer y A. Vaca, 1982. Transducción: un nuevo fenómeno asociado a los procesos de la tectónica global. Quinto Congreso Latinoamericano de Geología, Argentina. Actas III, 705718.

Baldo E. G., J. Saavedra, C. W. Rapela, R. J. Pankhurst, C. Casquet y C. Galindo, 1999. Síntesis geocronológica de la evolución paleozoica inferior del borde sur occidental de Gondwana en las Sierras Pampeanas, Argentina. Acta Geológica Hispánica 32, no 1-2, 17-2. 
Barazangi, M. and B. Isacks, 1976. Spatial distribution of earthquakes and subduction of the Nazca plate beneath South America. Geology 4, 686-692.

Beck, S. and G. Zandt, 2002. The nature of orogenic crust in the central Andes. J. Geophys. Res. 107, B10, 2230, doi:10.1029/2000JB000124.

Beck S. L., G. Zandt, S. C. Myers, T. C. Wallace, P. G. Silver and L. Drake, 1996. Crustal-thickness variations in the central Andes. Geology 24, 407-410.

Ben-Menahem, A., 1965. Observed attenuation and $Q$ values of seismic surface waves in the upper mantle. J. Geophys. Res. 70, 4641-4651.

Ben-Menahem, A. and S. Singh, 1998. Seismic waves and sources. Sec. Ed. Courier Dover Pub., 1136 pp.

Benz, H. M., A. Franke and D. M. Boore, 1997. Regional Lg attenuation for the Continental United States. Bull. Seism. Soc. Am. 87, 3, 606-619.

Bermúdez, A., D. Delpino, F. Frey y Saal, A., 1993. Los basaltos de retroarco extraandinos. En: Ramos, V.A. (Ed.) Geología y Recursos Naturales de Mendoza.12. Congreso Geológico Argentino y $2^{\circ}$ Congreso de Exploración de Hidrocarburos, Relatorio I - 13: 161-172.

Bianco, F., M. Castellano, E. Del Pezzo and J. M. Ibañez, 1999. Attenuation of short-period seismic waves at Mt Vesuvius, Italy. Geophys. J. Int. 138, 67-76.

Bianco, F. and E. Del Pezzo, 2002. Scat-Cad: a Mathcad 2000 professional package to model the energy decay due to seismic attenuation. Computers \& Geosciences, 28, 851-855.

Bianco, F. and E. Del Pezzo, 2010. MathLTWA: Multiple lapse time window analysis using Wolfram Mathematica 7. Computers \& Geosciences, 36, 10, 1388-1392.

Bianco F., E. Del Pezzo, M. Castellano, J. Ibanez and F. Di Luccio, 2002. Separation of intrinsic and scattering seismic attenuation in the Southern Apennine zone, Italy. Geophys. J. Int., 150, 1022.

Bianco F., E. Del Pezzo, L. Malagnini, F. Di Luccio and A. Akinci, 2005. Separation of depth dependent intrinsic and scattering seismic attenuation in the northeastern sector of the Italian Peninsula. Geophys. J. Int. 161, 130-142, doi: 10.1111/j.1365-246X.2005.02555.x

Biescas, B., Z. Rivera, J.A. Zapata, 2007. Seismic attenuation of coda waves in the eastern region of Cuba. Tectonophysics 429, 99-109.

Biswas, N. N. and K. Aki, 1984. Characteristics of coda waves: central and southcentral Alaska. Bull. Seism. Soc. Am. 74, 493-507.

Bisztricsany, E. A., 1958. A new method for determination of the magnitude of earthquakes. Geofiz. Kozlemen., 7, 2.

Blair, D.P. and A.T. Spathis, 1982. Attenuation of explosion generated pulse in rock masses. J. Geophys. Res. 87, 3885-3892.

Bloch, S., A. L. Hales and M. Landisman, 1969. Velocities in the crust and upper mantle of southern Africa from multi-mode surface-wave dispersion. Bull. Seism. Soc. Am. 59, 1599-1629.

Bollinger, G. and C. Langer, 1988. Development of a velocity model for locating aftershocks in the Sierra Pie de Palo region of western Argentina. U. S. Geol. Surv. Bull. 1795, 1-16.

Booker, J. R., A. Favetto, C. Pomposiello and F. Xuan, 2005a. The role of fluids in the Nazca flat slab near $31^{\circ} \mathrm{s}$ revealed by the electrical resistivity structure. 6th Int. Symposium on Andean Geodynamics (ISAG 2005, Barcelona), Extended Abstracts: 119-122.

Booker, J. R., C. Pomposiello, A. Favetto and A. Burd, 2005b. Implications of Electrical Conductivity Structure Associated With the Nazca Slab Beneath Argentina. Am. Geophys. Union, Fall Meeting 2005, abstract \#GP34A-01.

Brune, J. N., 1962. Attenuation of dispersed wave trains. Bull. Seism. Soc. Am. 52, 109-112.

Brune, J. N., 1970. Tectonic stress and the spectra of seismic shear waves from earthquakes. J. Geophys. Res. 75, 4997-5009.

Burd, A.I., J.R. Booker, R.L. Mackie, C.Pomposiello, A. Favetto and J.C. Larsen, 2010. Three and two-dimensional electrical conductivity of the mantle near the Chile-Argentina Nazca Flat Slab: insights into slab behavior. Am. Geophys. Union, Fall Meeting 2010, abstract \#GP21B02.

Butler, R., C. McCreery, L. Frazer, and D. Walker, 1987. High-Frequency Seismic Attenuation of Oceanic P and S Waves in the Western Pacific, J. Geophys. Res. 92, B2, 1383-1396.

Byerlee, J.D., 1978. Friction of rocks. Pure Appl. Geophys. 116, 616-626. 
Cahill, T. and B. L. Isacks, 1992. Seismicity and shape of the subducted Nazca Plate. J. Geophys. Res. 97, 17503-17529.

Campillo, M. and A. Paul, 1992. Influence of the lower crustal structure on the early coda of regional seismograms. . J. Geophys. Res. 97, 3405-3416.

Canas, J. A. and B. Mitchell, 1978. Lateral variation of surface-wave anelastic attenuation across the Pacific. Bull. Seism. Soc. Am. 68, 1637-1650.

Canas, J. A. and B. Mitchell, 1981. Rayleigh wave attenuation and its variation across the Atlantic Ocean. Geophys. J. R. Astr. Soc. 67, 159-176.

Canas, J. A., L. Pujades, J. Badal, G. Payo, F. de Miguel, F. Vidal, G. Alguacil, J. Ibáñez and J. Morales, 1991. Lateral variation and frequency dependence of coda- $Q$ in the southern part of Iberia. Geophys. J. Int. 107, 57-66.

Castano, J. C., M. A. Araujo, M. H. Millán, C. A. Navarro, M. V. Castano, 1999. Epicentros de los terremotos destructivos ocurridos en la República Argentina. INPRES, Secretaría de Minería e Indutria. Subsecretaría de Minería. Mapa.

Castro, C. E., J. C. Perucca, W. Simon y H. Sosa, 2005. Las Lajas de Nikizanga. Flanco Oriental de la Sierra de Pie de Palo. San Juan - Argentina. Actas de la XIII Reunión Anual Iberoamericana de Enseñanza Superior de la Minería, Lima, Perú, 13pp.

Chebli, G.A., M. E. Mozetic, E.A. Rossello y M. Bühler, 1999. Cuencas sedimentarias de la Llanura Chacopampeana. En: Geología Argentina. Instituto de Geología y Recursos Minerales. Buenos Aires. Anales 29(20), 627-644.

Chen, P., C. R. Bina, and E. A. Okal, Slab stress and volcanism in Nazca plate subduction: variations with dip angle and intermediate-depth seismicity, Eos, Transactions of the American Geophysical Union, 80, Fall Supplement, S12F-02, 1999.

Chen, W. P. y Molnar, P. 1983. Focal depth of intracontinental and intraplate earthquakes and their implications for the thermal and mechanical properties of the lithosphere. J. Geophys. Res. 88, 4183- 4214.

Cheng, C. C. and B. J. Mitchell, 1981. Crustal $Q$ structure in the United States from multi-mode surface waves. Bull. Seism. Soc. Am. 71, 161-181.

Chernov, L. A., 1960. Wave propagation in a random medium. McGraw-Hill. New York, 168 pp.

Chevrot, S. and Y. Cansi, 1996. Source spectra and site response estimates using the coda of Lg waves in western Europe. Geophys. Res. Lett. 23, 13, 1605-1608.

Chinn, D. S. and B. L. Isacks, 1983. Accurate source depths and focal mechanisms of shallow earthquakes in western South America and in the New Hebrides Island Arc. Tectonics 2, 529563

Chouet, B., 1979. Temporal variation in the attenuation of earthquake coda near Stone Canyon, California. Geophys. Res. Lett. 6, 143-146.

Chun, K., G. F. West, R. J. Kokoski, and C. Samson, 1987. A Novel Technique for Measuring Lg Attenuation - Results from Eastern Canada Between 1 to $10 \mathrm{~Hz}$. Bull. Seism. Soc. Am. 77, 398-419.

Chung, T. W., K. Yoshimoto and S. Yun, 2010. The Separation of Intrinsic and Scattering Seismic Attenuation in South Korea. Bull. Seism. Soc. Am. 100, 6; 3183-3193; doi: $10.1785 / 0120100054$

Collier, J. D. and G. R. Helffrich, 2001. The thermal influence of the subducting slab beneath South America from 410 and $660 \mathrm{~km}$ discontinuity observations. Geophys. J. Int. 147, 319-329.

Comte, D., M. Pardo, L. Dorbath, C. Dorbath, H. Haessler, L. Rivera, A. Cisternas and L. Ponce, 1994. Determination of seismogenic interplate contact zone and crustal seismicity around Antofagasta, northern Chile, using local data. Geophys. J. Int. 116, 553-561.

Cormier, V. F., 1982. The effect of attenuation on seismic body waves. Bull. Seism. Soc. Am. 72, 6, S169-S200.

Correig, A. M. and B. J. Mitchell, 1989. Attenuative body wave dispersion at La Cerdanya, eastern Pyrenees. Phys. Earth Planet. Inter. 57, 304-310.

Correig, A. M. B. J. Mitchell and R. Ortiz, 1990. Seismicity and coda $Q$ values in the eastern Pyrenees: first results from the La Cerdanya Seismic Network. Pure Appl. Geophys. 132, 311- 329. 
Cortés, J. M., P. Vinciguerra, M. Yamín and M. M. Pasini, 1999. Tectónica cuaternaria de la región andina del Nuevo Cuyo (28 - $38^{\circ}$ LS). En: Geología Argentina. Instituto de Geología y Recursos Minerales. Buenos Aires. Anales 29 (24) , 760-778.

Dainty, A. M., 1981. A scattering model to explain seismic $Q$ observations in the lithosphere between 1 and $30 \mathrm{~Hz}$. Geophys. Res. Lett., 8, 1126-1128.

Dainty, A. M., 1984. High-frequency acoustic backscattering and seismic attenuation. J. Geophys. Res. 89, B5, 3172-3176.

Dainty, A. M. and M. N. Toksöz, 1981. Seismic codas on the earth and the moon: A comparison. Phys. Earth Planet. Inter. 26, 250-260.

Dainty, A. M. and M. N. Toksöz, 1990. Array analysis of seismic scattering. . Bull. Seism. Soc. Am. $80,2242-2260$.

Deshayes, P., T. Monfret, M. Pardo and E. Vera. Three-dimensional P- and S-wave seismic attenuation models in central Chile - western Argentina $\left(30^{\circ}-34^{\circ} \mathrm{S}\right)$ from local recorded earthquakes. 7th International Symposium on Andean Geodynamics (ISAG 2008, Nice), Extended Abstracts: 184-187

De Miguel, F., J. M. Ibáñez, G. Alguacil, J. A. Canas, F. Vidal, J. Morales, J. A. Peña, A. M. Posadas and F. Luzón, 1992. 1-18Hz L Lattenuation in the Granada Basin (Southern Spain). Geophys. J. Int. 111, 270-280.

De Souza J. L. and B. J. Mitchell, 1998. Lg Coda Q Variations across South America and their Relation to Crustal Evolution. Pure Appl. Geophys. 153, 587-612.

Del Pezzo, E., 2008. Seismic wave scattering in volcanoes. http://www.earthprints.org/bitstream/2122/3845/1/SeismicWaveScatteringinVolcanoes_DelPezzo.pdf

Del Pezzo, E., R. Allota and D. Patane, 1990. Dependence of Qc (coda Q) on coda duration time interval: model or depth effect? Bull. Seism. Soc. Am. 80, 1028-1034.

Del Pezzo, E. and F. Bianco, 2007. 30 years of coda observations: Qc, Qi and Qs. A summary of the main results obtained worlwide, in memory of Keiiti Aki. Earth Print Repository, http://www.earth-prints.org/.

Del Pezzo, E., Bianco, F., Zaccarelli, L., 2006. Separation of $\mathrm{Q}_{\mathrm{i}}$ and $\mathrm{Q}_{\mathrm{s}}$ from passive data at Mt. Vesuvius: a reappraisal of the seismic attenuation estimates. Phys. Earth Planet. Int. 159, 202212.

Del Pezzo, E., S. de Martino, F. de Miguel, J. Ibáñez and A. Sorgente, 1990. Characteristics of the Seismic attenuation in two tectonically active zones of southern Europe. Pure Appl. Geophys. 135, 91-106.

Del Pezzo, E., F. Ferulano, A. Giarrusso and M. Martini, 1983a. Seismic coda $Q$ and scaling law of the source spectra at the Aeolian Island, Southern Italy. Bull. Seism. Soc. Am. 73, 97-108.

Del Pezzo, E., G. Iannaccone, G. Martini and R. Scarpa, 1983b. The November 1980 southern Italy earthquake. Bull. Seism. Soc. Am. 73, 187-200.

Del Pezzo, E., J. Ibáñez, J. Morales, A. Akinci and R. Maresca, 1995. Measurements of intrinsic and scattering seismic attenuation in the crust, Bull. Seism. Soc. Am. 85, 1373-1380.

Del Pezzo, E., M. Simini and J.M. Ibáñez, 1996. Separation of intrinsic and scattering $Q$ for volcanic areas: comparison between Etna and Campi Flegrei. J. Volc. Geotherm. Res. 70, 213-219.

Doglioni, C., E. Carminati and M. Cuffaro, 2006. Simple kinematics of subduction zones. International Geology Review. 4, 479-493.

Dorbath, C. and the Lithoscope Andean Group, 1996. Mapping the continuity of the Nazca plate through its aseismic part in the Arica elbow by teleseismic tomography. Third ISAG, St Malo (France)

Douglas, A., 1967. Joint epicentre determination. Nature 215, 47-48.

Dutta, U., N. N. Biswas, D. A. Adams, and A. Papageorgiou, 2004. Analysis of S-Wave Attenuation in South-Central Alaska. Bull. Seism. Soc. Am. 94, 1, 16-28.

Dziewonski, A. M., 2005. The robust aspects of global seismic tomography. In Foulger, G.R., Natland, J. H., Presnall, D. C. and Anderson D. L., eds, Plate, plumes and paradigms: Geol. Soc. Am. Special Paper 388, 147-154. doi:11.1130/2005.2388(12)

Dziewonski, A. M., S. Bloch and M. Landisman, 1969. A technique for the analysis of transient seismic signals. Bull. Seism. Soc. Am. 59, 427-444.

Engdahl, E. R., R. D. van der Hilst and J. Berrocal, 1995. Imaging of subducted lithosphere beneath South America. Geophys. Res. Lett. 22, 16, 2317-2320. 
Ewing, W. M., W. S. Jardetzky and F. Press, 1957. Elastic waves in layered media. McGraw Hill, New York.

Ewing, W. M. and F. Press, 1954. Mantle Rayleigh waves from the Kamchatka earthquake of November 4, 1952. Bull. Seism. Soc. Am. 44, 471-479.

Febrer, J, B. Baldis, J. Gasco, M. Mamani y C. Pomposielo, 1982. La anomalía geotérmica Calchaquí en el noroeste argentino: un nuevo proceso geodinámico asociado a la subducción de la Placa de Nazca. Quinto Congreso Latinoamericano de Geología, Argentina. Actas III, 705-718.

Fedotov, S. A. and S. A. Boldyrev, 1969. Frequency dependence of body-wave absoption in the crust and the upper mantle of the Kuril-island chain. Izv. Akad. Sci. USSR, Phys. Solid Earth 9, 1733.

Fehler, M., M. Hoshiba, H. Sato and K. Obara, 1992. Separation of scattering and intrinsic attenuation for the Kanto-Tokai region, Japan, using measurements of S-wave energy versus hypocentral distance. Geophys. J. Int. 108, 787-800.

Fehler, M. and H. Sato, 2003. Coda. Pure Appl. Geophys. 160, 541-554.

Fernández Ibáñez, Fermín, 2004. Condicionantes Reológicos de la Sismicidad Cortical en Béticas-Rif y Alborán Trabajo de Investigación Tutelada Bienio 2002/2004. Departamento de Geodinámica, Universidad de Granada.

Fernández Seveso, F., M. Pérez, I. Brisson y L. Álvarez, 1993. Análisis de cuenca: Técnicas aplicadas en la serie carbónica-pérmica del Paganzo. Boletín de Informaciones Petroleras, YPF, 33:77107.

Feruglio, E., 1946. Sistemas orográficos de la Argentina. Geografía de la República Argentina. Sociedad Argentina de Estudios Geográficos, GAEA 4, 536pp.

Foulger, G.R., B.R. Julian, D.P. Hill, A.M. Pitt, P.E. Malin and E. Shalev, 2004. Non-double-couple microearthquakes at Long Valley caldera, California, provide evidence for hydraulic fracturing. J. Volcanol. Geothern. Res. 132, 45-71.

Frankel, A., 1991. Mechanisms of seismic attenuation in the crust: scattering and anelasticity in New York State, South Africa, and Southern California. J. Geophys. Res. 96, B4, 6269-6289.

Frankel, A. and R. Clayton, 1984. A finite-difference simulation of wave propagation in twodimensional random media. Bull. Seism. Soc. Am. 74, 2167-2186.

Frankel, A. and R. Clayton, 1986. Finite difference simulations of seismic scattering: Implications for the propagation of short-period seismic waves in the crust and models of the crustal heterogeneity. J. Geophys. Res. 91, 6465-6489.

Frankel, A., A. McGarr, J. Bicknell, J. Mori, L. Seeber and E. Cranswick, 1990. Attenuation of highfrequency shear waves in the crust: Measurements from New York State, South Africa and Southern California. J. Geophys. Res. 95, 17441-17457.

Frankel A. and L. Wennerberg, 1987. Energy-flux model of seismic coda: separation of scattering and intrinsic attenuation. Bull. Seism. Soc. Am. 77, 1223-1251.

Fromm, R., Zandt, G. \& Beck, S.L., 2004. Crustal thickness beneath the Andes and Sierras Pampeanas at $30^{\circ} \mathrm{S}$ inferred from Pn apparent phase velocities. Geophys. Res. Lett. 31, L006625, doi:10.1029/2003GL019231.

Gao, L. S. 1984. Coda waves analysis for distinguishing attenuation due to isotropic scattering from attenuation due absorption. Pure Appl. Geophys. 122, 1-9.

Gao, L. S., L. C. Lee, N. N. Biswas and K. Aki. 1983a. Comparison of the effects between single and multiple scattering on coda waves for local earthquakes. Bull. Seism. Soc. Am. 73, 377-389.

Gao, L. S., N. N. Biswas, L. C. Lee and K. Aki. 1983b. Effects of multiple scattering on coda waves in three-dimensional medium. Pure Appl. Geophys. 121, 1, 3-15.

García, S. y J. Mendoza, 2004. Determinación del factor de calidad Q para Venezuela. Congreso SOVG.

Giampiccolo, E., S. D’Amico, D. Patanè and S. Gresta, 2007. Attenuation and Source Parameters of Shallow Microearthquakes at Mt. Etna Volcano, Italy. Bull. Seismol. Soc. Am. 97, 184-197.

Giampiccolo, E., T. Tuvè, S. Gresta and D. Patanè, 2006. S-waves attenuation and separation of scattering and intrinsic absorption of seismic energy in southeastern Sicily (Italy). Geophys. J. Int. $165,211-222$

Giardini, D., G. Grünthal, K. Shedlock and P. Zhang, 1999. Global Seismic Hazard Map in The global seismic hazard assessment program (GSHAP) 1992-1999. D. Giardini, ed. Annali di Geofisica $42,6,1230 \mathrm{pp}$. 
Gilbert, H., Beck, S. \& Zandt, G., 2006. Lithosperic and upper mantle structure of Central Chile and Argentina, Geophys. J. Int. 165, 383-398.

Gimenez, M., P. Martínez and A. Introcaso, 2000. A crustal model based mainly on gravity data in the area between the Bermejo Basin and the Sierras de Valle Fértil, Argentina. J. South American Earth Sciences 13, 275-286.

Gir, R., S. Mohan, G. Subhash and M. Choudhury, 1978. Investigation of crustal structure by the analysis of reverberation periodicities. Bull. Seism. Soc. Am. 68; 5; 387-1397.

Giroldi, L. G., 1990. Atenuación sísmica en San Juan. Trabajo final de Licenciatura en Geofísica. Facultad de Cs. Exactas y Naturales. Universidad Nacional de San Juan.

Gladwin, M. T. and F. D. Stacey, 1974a. Ultrasonic pulse velocity as a rock stress sensor. Tectonophysics 21, 39-45. doi:10.1016/0040-1951(74)90060-2

Gladwin, M. T. and F. D. Stacey, 1974b. Anelastic degradation of acoustic pulses in rock. Phys. Earth Planet. Inter., 8, 332-336.

Gonzalez, V. and L. Persson, 1997. Regional coda Q in Costa Rica, Central America. J. Seismology 1, 269-287

González Bonorino, F., 1950. Algunos problemas geológicos de las Sierras Pampeanas.Asociación Geológica Argentina, Revista 5(3): 81-110.

Goutbeek, F. H., B. Dost and T. van Eck, 2004. Intrinsic absorption and scattering attenuation in the southern part of the Netherlands. J. Seism. 8, 1, 11-23.

Groeber, P., 1938. Mineralogía y Geología. Espasa-Calpe Argentina, 1-492, Buenos Aires.

Gutscher, M.A., 2002. Andean subduction styles and their effect on thermal structure and interplate coupling. J. South Am. Earth Sci. 15, 3-10.

Gutscher, M. and J. Malavieille, 1999. Style of upper plate deformation linked to interplate coupling over flat slab segments: evidence from the Andean Margin. En: McKlay, K., ed., Thrust Tectonics 99, London, abstracts 214-217.

Gutscher, M., W. Spakman, H. Bijwaard and E.R. Engdahl, 2000. Geodynamic of flat subduction: Seismicity and tomographic constraints from the Andean margin. Tectonics 19, 5, 814-833.

Guzev, A. A. and V. K. Lemzikov, 1985. Properties of scattered elastic waves in the lithosphere of Kamchatka: Parameters an temporal variations. Tectonophysics 112, 137-153.

Haberland C. and A. Rietbrock, 2001. Attenuation tomography in the western central Andes: A detailed insight into the structure of a magmatic arc. J. Geophys. Res. 106, 11151-11167.

Haberland, C., A. Rietbrock, B. Schurr and H. Brasse, 2003. Coincident anomalies of seismic attenuation and electrical resistivity beneath the southern Bolivian Altiplano plateau. Geophys. Res. Lett. 30(18), 1923, doi:10.1029/2003GL017492.

Hadley, K., 1976. Comparison of calculated and observed crack densities and seismic velocities in Westerley granite. J. Geophys. Res. 81, 3484-3494.

Hanks, T. C., 1979. b-values and $\omega^{-\gamma}$ seismic source models: implications for tectonic stress variations along active crustal fault zones and the estimation of high-frequency strong ground motion. J. Geophys. Res. 84, 2235-2242.

Hartse, H., W. S. Phillips, M. C. Fehler and L. S. House, 1995. single-station spectral discrimination using coda waves. Bull. Seism. Soc. Am. 85, 1464-1474.

Hatzidimitriou, P. M., 1995. S-wave attenuation in the crust in northern Greece. Bull. Seism. Soc. Am. 85, 1381-1387.

Havskov J, 2005. Q and spectral analysis in SEISAN. University of Bergen, Norway

Havskov, J. and G. Alguacil, 2004. Instrumentation in earthquake seismology 22, Modern approaches in geophysics. Springer, $358 \mathrm{pp}$.

Havskov J., S. Malone, D. McClurg and R. Crosson, 1989. Coda $Q$ for the state of Washington. Bull. Seism. Soc. Am. 79, 1024-1038.

Havskov J. and L. Ottemöller, 2008. SEISAN: the earthquake analysis software for windows, solaris, linux and macosx. Version 8.2.1. University of Bergen, Norway, British Geological Survey, UK.

Havskov J., J. A. Peña, J. M Ibáñez, L. Ottemöller and C. Martínez-Arévalo, 2003. Magnitude scales for very local earthquakes. Application for Deception Island volcano (Antarctica). J. Volcanol. Geotherm. Res. 128, 115-133.

Herrmann, R. B., 1980. $Q$ estimates using the coda of local earthquakes. Bull. Seism. Soc. Am. 70, 447-468. 
Herraiz, M. and A. F. Espinosa, 1986. Scattering and attenuation of high-frequency seismic waves: development of the theory of coda waves. U.S.G.S. Open File Report 86-455.

Herrin, E., W. Tucker, J. Taggart, D. W. Gordon and J. L. Lobdell, 1968. Estimation of surface focus P travel times. Bull. Seism. Soc. Am. 58, 1273-1291.

Hollander, M., and D. A. Wolfe, 1999. Nonparametric Statistical Methods. Hoboken, NJ: John Wiley $\&$ Sons, Inc.

Hong, T., B. Kennett and R. Wu, 2004. Effects of the density perturbation in scattering. Geophys. Res. Lett. 31, LI3602, doi:10.1029/2004GL019933.

Hoshiba, M., 1991 Simulation of multiple-scattered coda wave excitation based on the energy conservation law, Phys. Earth Planet. Int.. 67, 123-136.

Hoshiba, M., 1993. Separation of scattering attenuation and intrinsic absorption in Japa, using the Multiple Lapse Time Window Analysis of full seismogram envelope. J. Geophys. Res. 98, 15809-15824.

Hoshiba, M. 1994. Simulation of coda wave envelope in depth dependent scattering and absorption structure. Geophys. Res. Lett. 21, 2853-2856.

Hoshiba, M., 1995. Estimation of nonisotropic scattering in western Japan using coda wave envelopes: Application of a multiple nonisotropic scattering model. J. Geophys. Res. 100, 645-657.

Hoshiba, M., A. Rietbrock, F. Scherbaum, H. Nakahara \& C. Haberland, 2001. Scattering attenuation and intrinsic absorption using uniform and depth dependent model - Application to full seismogram envelope recorded in Northern Chile. J. of Seismology 5, 157-179.

Hoshiba, M., H. Sato and M. Fehler, 1991. Numerical basis of the separation of scattering and intrinsic absorption from full seismogram envelope - a Monte-Carlo simulation of Multiple Isotropic Scattering. Papers in Met. and Geophys. 42, 65-91

Hudson, J. and J. R. Heritage, 1981. The use of the Born approximation in seismic scattering problems. Geophys. J. Roy. Astr. Soc. 66, 221-240.

Hwang, H. and B. Mitchell, 1987. Shear velocities, $Q_{\beta}$, and the frequency dependence of $Q_{\beta}$ in stable and tectonically active regions from surface wave observations. Geophys. J. R. Astr. Soc. 90, 575-613.

Ibáñez, J. M., 1990. Atenuación de ondas coda y Lg en el sur de España y de Italia a partir de sismogramas digitales. Tesis Doctoral. Facultad de Ciencias. Universidad de Granada, España.

Ibáñez, J. M., E. del Pezzo, F. De Miguel, M. Herraiz, G. Alguacil and J. Morales, 1990. Depthdependent seismic attenuation in the Granada zone (southern Spain). Bull. Seism. Soc. Am. 80, 1232-1244.

Ibáñez, J. M. , E. del Pezzo, M. Martín, D. Patané, F. de Miguel, F. Vidal and J. Morales, 1993. Estimates of Coda- $Q$ using a non-linear regression. J. Phys. Earth., 41, 203-219.

Ibáñez, J. M., F. de Miguel, G. Alguacil, J. Morales, F. Vidal, E. Del Pezzo y A. M. Posadas, 1991. Análisis de Q coda en las Béticas Centrales con datos digitales. Rev. de Geofísica 47, 59-74.

Ibáñez, J. M., Carmona, E., Almendros, J., Saccorotti, G., Del Pezzo, E., Abril, M., Ortiz, R., 2003. The 1998-1999 seismic series at Deception Island volcano, Antarctica. J. Volcanol. Geothern. Res. 128, 65-88.

Introcaso, A., M. C. Pacino and H. Fraga, 1992. Gravity, isostasy and Andean crustal shortening between latitudes $30^{\circ}$ and $35^{\circ} \mathrm{S}$. Tectonophysics 205 (1-3): 31-48.

INPRES, 2006. Instituto Nacional de Prevención Sísmica, Secretaría de Obras Públicas, Ministerio de Planificación Federal, Inversión Pública y http://www.inpres.gov.ar/seismology/linkppal.htm.

INPRES-CIRSOC 103, 1983. Reglamento: "Normas Argentinas para las construcciones sismorresistentes", Parte I. Construcciones en general. Ed. Nov.1983. INTI, Bs.As., Argentina, $109 \mathrm{pp}$.

Isacks, B. L., 1988. Uplift of the Central Andean Plateau and Bending of the Bolivian Orocline. J. Geophys. Res. 93, 3211-3231.

Japas, M. S., J. M. Cortés y M. Pasini, 2008. Tectónica extensional triásica en el sector norte de la Cuenca Cuyana: primeros datos cinemáticos. Revista de la Asociación Geológica Argentina 63 (2): $213-222$ 
Jeffreys, H., 1929, 1959. The Earth, its origin, history and physical constitution, Cambridge University Press, 2nd. Edition, 1929, 4th edition, 1959.

Jin, A. and K. Aki, 1986. Temporal change in coda $Q$ before the Tangshan earthquake of 1976 and the Haicheng earthquake of 1975. J. Geophys. Res. 91, 665-673.

Jongmans, D., (1991). Near-source pulse propagation: application to Q-determination. Geophysical Prospecting 39, 943-952.

Jordan, T. E. , B. L. Isacks, R. W. Allmendinger, J. A Brewer, V. A. Ramos and C. J. Ando, 1983. Andean tectonics related to geometry of subducted Nazca plate. Geol. Soc. of America Bulletin 94, 341-361.

Kay, S.M., S. Orrell and J.M. Abbruzzi, 1996. Zircon and whole rock Nd-Pb isotopic evidence for a Grenville age and a Laurentian origin for the Precordillera terrane in Argentina. J. Geology. 104, 637-648.

Kay, S. M. and C Mpodozis, 2002. Magmatism as a probe of the Neogene shallowing of the Nazca plate beneath the modern Chilean flat-slab. J. South Am. Earth Scs. 15, 39-57.

Kadinsky-Cade, K., 1985. Seismotectonics of the Chile margin and the 1977 Caucete earthquake of western Argentina, Ph. D Thesis, Cornell University, Ithaca, New York.

Kendrick, E. C., M. Bevis, R. F. Smalley Jr., O. Cifuentes, F. Galván, 1999. Current rates of convergence across the central Andes: Estimates from continuous GPS observations. Geophys. Res. Lett., 26(5), 541-544.

Kissling, E., 1995. Velest User's guide - Short Introduction to Velest Version 3.1 by E. Kissling, U. Kradolfer and H. Maurer. Institute of Geophysics and Swiss Seismological Service, Switzerland.

Kissling, E., 1988, Geotomography with local earthquake data, Rev. Geophys. 26, 659-698.

Kissling, E., W. L. Ellsworth, D. Eberhart-Phillips, U. Kradolfer, 1994. Initial reference models in seismic tomography. J. Geophys. Res. 99, B10, 19635 - 19646

Kjartansson, E., 1979. Constant Q-wave propagation and attenuation. J. Geophys. Res., 84, 47374748.

Kleiman, L. E. 1999. Mineralogía y petrología del volcanismo permo-triásico y triásico del Bloque de San Rafael en el área de Sierra Pintada, provincia de Mendoza, y su relación con las mineralizaciones de uranio. Tesis Doctoral. Facultad de Ciencias Exactas y Naturales, Universidad de Buenos Aires, $286 \mathrm{pp}$.

Kleiman, L. E. 2002. Magmatism and tectonic evolution of the Choiyoi and Puesto Viejo volcanics (Late Paleozoic-Early Mesozoic) at $34-35^{\circ}$ S Latitude, San Rafael, Mendoza Argentina. $15^{\circ}$ Congreso Geológico Argentino (Santa Cruz), Actas 1: 15-16.

Kley, J., Monaldi, C. R. and Salfity, J. A., 1999. Along-strike segmentation of the Andean foreland: causes and consequences. Tectonophysics, 301, 75-94.

Knopoff, L. and J. A. Hudson, 1964. Scattering of elastic waves by small inhomogeneities. J. Acoust. Soc. Am. 36, 338-343.

Knopoff, L. and J. A. Hudson, 1967. Frequency dependence of amplitude of scattered elastic waves. J. Acoust. Soc. Am. 42, 18-20.

Kopnichev, Y. F., 1977. The role of multiple scattering in the formation of a seismogram's tail (English Transl.), Izv. Akad. Nauk SSSR, Fiz. Zemli, 13, 394-398.

Kopp, H., E. R. Flueh, C. Papenberg and D. Klaeschen, 2004. Seismic investigations of the O'Higgins Seamount Group and Juan Fernández Ridge: Aseismic ridge emplacement and lithosphere hydration. Tectonics, 23, TC2009, doi:10.1029/2003TC001590.

Kulhánek O., 1990. Anatomy of Seismograms. Develop. Solid Earth Geophysics. 18 Elsevier, 178 pp.

Kulhánek O., 2002. The Structure and Interpretation of Seismograms. In: International handbook of earthquake and engineering seismology. Lee, Kanamori, Jennings and Kisslinger Ed., Academic Press, 933 pp.

Kumar, N., I. A. Parvez and H. S. Virk, 2005. Estimation of coda wave attenuation for NW Himalayan region using local earthquakes. Phys. Earth Planet. Inter. 151, 243-258.

Kuwahara, Y., H. Ito, H. Kawakatsu, T. Ohminato and T. Kiguchi, 1997. Crustal heterogeneity as inferred from seismic coda wave decomposition by small-aperture array observation. Phys. Earth Planet. Inter. 104, 247-256.

Kvamme, L. and J. Havskov, 1989. Q in southern Norway. Bull. Seism. Soc. Am. 79, 1575-1588. 
Lacombe C., M. Campillo, A. Paul and L. Margerin, 2003. Separation of intrinsic absorption and scattering attenuation from Lg coda decay in central France using acoustic radiative transfer theory. Geophys. J. Int. 154, 417-425.

Lay, T., 1987. Analysis of near-source contributions to early P-wave coda for underground explosions. II. Frequency dependence. Bull. Seism. Soc. Am. 77, 4, 1252-1273.

Lay, T., 2005. The deep mantle thermo-chemical boundary layer: The putative mantle plume source. In Foulger, G.R., Natland, J. H., Presnall, D. C. and Anderson D. L., eds, Plate, plumes and paradigms: Geol. Soc. Am. Special Paper 388, 193-205. doi:11.1130/2005.2388(12).

Lifshitz, E. M., L. D. Landau and L. P. Pitaevskii, 1986. Física Estadística. Vol. 9, Parte 2. Ed. Reverté, 472 pp.

Liu, H. P., D. L. Anderson and H. Kanamori, 1976. Velocity dispersion due to anelasticity: implications for seismology and mantle composition. Geophys. J. R. Astr. Soc. 47, 41-58.

López, G., D. Hatzfeld, R. Madariaga, S. Barrientos, J. Campos, H. Lyon-Caen, A. Zollo, G. Giannacone y E. Kausel, 1997. Microsismicidad en la zona centro-sur de Chile: $8^{\circ}$ Congreso Geológico de Chile, Antofagasta, Actas 3, 1771-1774.

Llambías, E .J., L. E. Kleiman y J. A. Salvarredi, 1993. El magmatismo gondwánico. En: Geología y Recursos Naturales de Mendoza. $12^{\circ}$ Congreso Geológico Argentino y $2^{\circ}$ Congreso de Exploración de Hidrocarburos (Mendoza), Relatorio 53-64.

Maeda, T. and T. Sasatani, 2006. Two-layer Qs structure of the slab near the southern Kurile trench. Earth Planets Space 58, 543-553.

Mai, P. M., and G. C. Beroza, 2002. A spatial random field model to characterize complexity in earthquake slip. J. Geophys. Res. 107(B11), 2308, doi:10.1029/2001JB000588.

Mancilla, F., R.B. Herrmann, J. Morales and D. Stich, 2008. Vertical Ground Motion in Southern Spain. Bull. Seism. Soc. Am. 98, 733-745.

Margerin, L., M. Campillo, and B. van Tiggelen, 1998. Radiative transfer and diffusion of waves in a layered medium: new insight into coda Q. Geophys. J. Int. 134, 596-612.

Margerin, L., M. Campillo, N. M. Shapiro N.M. and B. van Tiggelen, 1999. Residence time of diffuse waves in the crust as a physical interpretation of coda Q: application to seismograms recorded in Mexico. Geophys. J. Int. 138, 343-352.

Margheriti, L., L. Wennerberg and J. Boatwright, 1994. A Comparison of Coda and S-Wave Spectral Ratios as Estimates of Site Response in the Southern San Francisco Bay Area. Bull. Seism. Soc. Am. 84, 1815-1830.

Martínez, M.P., M.E. Giménez, A. Introcaso y J. A. Robles, 1997. Excesos de espesores corticales y acortamientos andinos en tres secciones ubicadas en $36^{\circ}, 37^{\circ}$ y $39^{\circ}$ de latitud sur. $7^{\circ}$ Congreso Geológico Chileno. Actas 1: 101-105.

Martínez Arévalo C., 2005. Estructura superficial de atenuación para ondas sísmicas directas, P y S, en ambientes volcánicos. Aplicación al volcán Isla Decepción (Antártida) y volcán Etna (Italia). Tesis de doctorado en Cs. Físicas, Univ. de Granada, España.

Martínez-Arévalo, C., F. Bianco, J. Ibáñez and E. del Pezzo, 2003. Shallow seismic attenuation and shear-wave splitting in the short period range of Deception Island volcano (Antarctica). J. Volcanol. Geotherm. Res. 128, 89-113.

Martínez Catalán, J. R., 2002. Geología Estructural y Dinámica Global. 4.-Deformación a escala cristalina. http://web.usal.es/ gabi/APUNTES/TEMA4.PDF

Masuda, T., 1988. Corner Frequencies and Q Values of P and S Waves by Simultaneous Inversion Technique. Tôhoku Geophys. Journ. (Sci. Rep. Tôhoku Univ., Ser. 5) 31, 101-125.

Matsunami, K. and M. Nakamura, 2004. Seismic attenuation in a nonvolcanic swarm region beneath Wakayama, southwest Japan. J. Geophys. Res. 109, B09302, doi:10.1029/2003JB002758.

Mayeda, K., S. Koynagi,.M. Hoshiba, K. Aki and Y. Zeng, 1992. A comparative study of scattering, intrinsic and coda $Q^{-1}$ for Hawaii, Long Valley and Central California between 1.5 and $15 \mathrm{~Hz}$. J. Geophys. Res. 97, 6643-6659.

McCall, K. and R. Guyer, 1994. Equation of state and wave propagation in hysteretic nonlinear elastic materials. J. Geophys. Res. 99, 23887-23897.

McNutt, S. R., 2005. Volcanic Seismology. Annu. Rev. Earth Planet. Sci. 32: 461-491, doi:10.1146/annurev.earth.33.092203.122459.

Meissner, R. y Strehlau, J. 1982. Limits of stresses in continental crusts and their relation to the depthfrequency distribution of shallow earthquakes. Tectonics 1, 73-89. 
Mitchell, B., 1995. Anelastic structure and evolution of the continental crust and upper mantle from seismic surface wave attenuation. Rev. Geophys. 33, 441-462.

Mohan, S., G. Subhash and M. A. Choudhury, 1979. Coda power and modulation characteristics of a complex P signal from underground nuclear explosions. Tectonophysics 53, 1-2, T33-T39, doi:10.1016/0040-1951(79)90350-0

Mongay Fernández, C., 2005. Quimiometría. Universitat de València, 423pp.

Moore, D. S., 2005. Estadística Básica Aplicada. Antoni Bosch Ed., 876pp.

Morales, J., J. Ibáñez, F. Vidal, F. de Miguel, G. Alguacil and A. Posadas, 1991. $Q_{c}$ site dependence in the Granada basin (southern Spain). Bull. Seism. Soc. Am. 81, 2486-2492.

Morelli, A., A.M. Dziewonski and J.H. Woodhouse, 1986. Global upper mantle tomography of seismic velocities and anisotropies. J. Geophys. Res. 96, 20337-20351.

Morse, P. M. and K. U. Ingard, 1986. Theoretical acoustics. Princeton University Press, 927 pp.

Myers, S. C., S. Beck, G. Zandt, and T. Wallace, 1998. Lithospheric-scale structure across the Bolivian Andes from tomographic images of velocity and attenuation for $\mathrm{P}$ and $\mathrm{S}$ waves, J. Geophys. Res. 103, $21233-21252$.

Nakamura, Y., 1977. Seismic energy transmission in an intensively scattering environment. J. Geophys. 43, 389-399.

Nakanishi, I. and D. L. Anderson, 1983. Measurements of mantle wave velocities and inversion for lateral heterogeneity and anisotropy. Part I: analysis of great circle phase velocities. J. Geophys. Res. 88, 10267-10283.

Nishizawa, O. T., T. Satoh, X. Lei and Y. Kuwahara, 1997. Laboratory studies of seismic waves propagation in inhomogeneous media using a laser Doppler vibrometer. Bull. Seism. Soc. Am. 87, 809-823.

Novelo-Casanova, D. A. and W. H. K. Lee, 1991. Comparison of techniques that use the Single Scattering Model to compute de quality factor $Q$ from coda waves. Pure Appl. Geophys. 135, $1,77-89$.

Novelo Casanova, D. A y A. Martínez Bringas, 2005. A seismic attenuation zone below Popocapépetl Volcano inferred from coda waves of local earthquakes. Geofísica Internacional 44, 2, 177 186.

Nur, A., 1971. Viscous phase in rocks and the low-velocity zone. J. Geophys. Res. 76, 1270-1277.

O'Brien, G.S. and C.J Bean, 2009. Volcano topography, structure and intrinsic attenuation: Their relative influences on a simulated 3D visco-elastic wavefield, J. Volcanol. Geotherm. Res., doi:10.1016/j.jvolgeores.2009.03.004

Ojeda, A. and J. Havskov, 2001. Crustal structure and local seismicity in Colombia. J. Seismology 5, 575-593.

Ortiz, A. y J.J. Zambrano, 1981. La provincia geológica Precordillera oriental. $8^{\circ}$ Congreso Geológico Argentino, Actas 3: 59-74.

Osagie, E., 1986. Anelasticity of the crust and upper mantle of South America from the inversion of observed surface wave attenuation. Geophys. J. R. Astr. Soc. 86, 1-17.

Padhy, S., 2009. Inversion of Seismogram Envelopes Using a Multiple Isotropic Scattering Model in Garhwal Himalaya. Bull. Seism. Soc. Am. 99, 2A, 726-740, doi: 10.1785/0120080076.

Pardo, M.; T. Monfret, E. Vera, A. Eisenberg, S. Gaffet, E. Lorca and A. Perez, 2002. Seismotectonic and Body-wave Tomography of Central Chile-NW Argentina Flat-slab Subduction Zone Using Local Earthquakes. Proceedings EGS XXVII General Assembly, Nice, 469-472.

Pardo, M., T. Monfret, E. Vera, G. Yanez, and A. Eisenberg, 2004. Flat-Slab to Steep Subduction Transition Zone in Central Chile-Western Argentina: Body Waves Tomography and State of Stress. AGU Fall Meeting Abstracts, B164.

Pereira Dias, A. and J. L. de Souza, 2004. Estimates of coda Q attenuation in the João Câmara area (Northeastern Brazil). J. Seismology 8: 235-246.

Perucca, J. C., M. C. Puertas, E. R. Uliarte y J. J. Zambrano, 1979. Carta Geotectónica de Cuyo. Boletín No 4, Fac. de Cs. Exactas Físicas y Naturales. Universidad Nacional de San Juan.

Perucca, J. C., G. Salinas de Salmuni, M. C Puertas y J. Zambrano, 1997. Aporte de las imágenes ERS-1 a la carta geotectónica de Cuyo. Sector centro-oeste de la provincia de San Juan, Argentina. Proceedings of an International Seminar on the Use and Applications of ERS in Latin America, Viña del Mar, Chile. ESA SP-405. 
Perucca, L. y H. Bastias, 2006. Regiones Sismotectónicas en el centro-oeste argentino. Provincias de La Rioja, San Juan y Mendoza. Temas de Geología Argentina I (2). INSUGEO, Serie Correlación Geológica, 21:209-222. Tucumán. ISSN 1514-4186. ISSN 1666-9479 en línea.

Perucca, L. y J. Paredes, 2004. Descripción del Fallamiento Activo en la Provincia de San Juan. Tópicos de Geociencias. Un Volumen de Estudios Sismológicos, Geodésicos y Geológicos en Homenaje al Ing. Fernando Séptimo Volponi. Editorial: EFU Fundación Universidad Nacional de San Juan. E. Triep, A. Introcaso (ed.). 269-309.

Phillips, W. S. and K. Aki, 1986. Site amplification of coda waves from local earthquakes in central California. Bull. Seism. Soc. Am. 76, 627-648.

Pozgay, S. H., D. A. Wiens, J. A. Conder, H. Shiobara, and H. Sugioka, 2009. Seismic attenuation tomography of the Mariana subduction system: Implications for thermal structure, volatile distribution, and slow spreading dynamics, Geochem. Geophys. Geosyst., 10, Q04X05, doi:10.1029/2008GC002313.

Pujades, L. G., A. Ugalde, J. A. Canas, M. Navarro, F. J. Badal and V. Corchete, 1997. Intrinsic and scattering attenuation from observed seismic codas in the Almeria Basin (southeastern Iberian Peninsula). Geophys. J. Int. 129, 281-291.

Pujol, J., J. M. Chiu, R. Smalley Jr., M. Regnier, B. Isacks, J.L. Chatelain, J. Vlasity, J. Castano and N. Puebla, 1991. Lateral velocity variations in the andean foreland in Argentina determined with the JHD method. Bull. Seism. Soc. Am. 81, 2441-2457.

Pulli, J., 1984. Attenuation of coda waves in New England. Bull. Seism. Soc. Am. 74, 1149-1166.

Raffaele, R., L. Scarfi, G. Badi, J. Ibáñez, S. Imposa, 2010. Sub-horizontal Subduction in Western Argentina, between $29^{\circ} \mathrm{S}$ and $34^{\circ} \mathrm{S}$, as viewed from a Local Seismic Network. Póster presentado en Cities on Volcanoes 6th, Puerto de la cruz, Tenerife, Islas Canarias, España, 31 de mayo al 4 de junio de 2010.

Ramos, V.A., 1988. Late Paleozoic-early Paleozoic of South America, a collisional history. Episodes, 2:168-173.

Ramos, V.A., 1999a. Plate tectonic setting of the Andean Cordillera. Episodes 22, 183-190.

Ramos, V.A., 1999b. Las provincias geológicas del territorio argentino. En: Geología Argentina. Instituto de Geología y Recursos Minerales. Buenos Aires. Anales 29(3), 41-96.

Ramos, V.A., 1999c. Los depósitos sinorogénicos terciarios de la Región Andina. En: Geología Argentina. Instituto de Geología y Recursos Minerales. Buenos Aires Anales 29 (22): 651682.

Ramos, V.A., 1999d. Evolución Tectónica de la Argentina. En: Geología Argentina. Instituto de Geología y Recursos Minerales.Buenos Aires. Anales 29 (24), 714-759.

Ramos, V.A., Cegarra, M. and Cristallini, E., 1996. Cenozoic tectonics of the High Andes of westcentral Argentina, $\left(30^{\circ}-36^{\circ} \mathrm{S}\right.$ latitude). Tectonophysics 259: 185-200.

Ramos, V. A., E. O. Cristallini and D. J. Pérez, 2002. The Pampean flat-slab of the Central Andes. J. South Am. Earth Sciences 15, 59-78.

Rautian, T. G. and V. I. Khalturin, 1978. The use of the coda for determination of the earthquake source spectrum. Bull. Seism. Soc. Am. 68, 923-948.

Ren, Y., E. Stutzmann, R. D. van der Hilst, and J. Besse, 2007. Understanding seismic heterogeneities in the lower mantle beneath the Americas from seismic tomography and plate tectonic history, J. Geophys. Res. 112, B01302, doi:10.1029/2005JB004154.

Regnier, M. J., C. Chatelain, R. Smalley Jr., J.M. Chiu, B. L. Isacks and M. Araujo, 1992. Seismotectonics of Sierra Pie de Palo, a basement block uplift in the Andean foreland of Argentina. Bull. Seism. Soc. Am. 82, 6, 2549-2571.

Regnier, M. J., J.M. Chiu, R. Smalley Jr., B. L. Isacks and M. Araujo, 1994. Crustal Thickness Variation in the Andean Foreland, Argentina, from Converted Waves. Bull. Seism. Soc. Am. $84,4,1097-1111$.

Rietbrock A., 2001. P wave attenuation structure in the fault area of the 1995 Kobe earthquake. J. Geophys. Res., 106, 4141-4154.

Rietbrock, A. and C. Haberland, 2001. A Tear in the subducting Nazca Slab: Evidence from Local Earthquake Tomography and High Precision Hypocenters. American Geophysical Union, Fall Meeting 2001, abstract \#T31A-0822 
Rodi, W., C. A Schultz, W. G. Hanley, S. Sarkar and H. S. Kuleli, 2002a. Grid-search location methods for ground-truth collection from local and regional seismic networks, in The 24th Seismic Research Review, 394-402.

Rodi, W., E. R. Engdahl, E. A. Bergman, F. Waldhauser, G. L. Pavlis, H. Israelsson, J. Dewey and M. N. Toksoz, 2002b. A new grid-search multiple-event location algorithm and a comparison of methods, in The24th Seismic Research Review, 403-411.

Rolleri, E.O., 1976. Sistema de San Bárbara. $6^{\circ}$ Congreso Geológico Argentino, Actas 1: 240-255.

Rolleri, E.O. y P. Criado Roqué, 1970. Geología de la provincia de Mendoza. 4a Jornadas Geológicas Argentinas, Actas 2: 1-60.

Romanowicz, B., 2003. Global mantle tomography: progress status in the last 10 years, Annu. Rev. Geophys. Space Phys. $31,1,303$.

Roth, E. G., D. A. Wiens, L. M. Dorman, J. Hildebrand, and S. C. Webb, 1999. Seismic attenuation tomography of the Tonga-Fiji region using phase pair methods, J. Geophys. Res. 104, B3, 4795-4809, doi:10.1029/1998JB900052.

Rychert, C. A., K. M. Fischer, D. G. A. Abers, T. Plank, E. Syracuse, J. M. Protti, V. Gonzalez and W. Strauch, 2008. Strong along-arc variations in attenuation in the mantle wedge beneath Costa Rica and Nicaragua. Geochem. Geophys. Geosyst. 9, Q10S10, doi:10.1029/2008GC002040.

Sachs, L., 1984. Applied Statistics: A Handbook of Techniques. Springer-Verlag, New York, 253pp.

Saito, T., H. Sato and M. Ohtake, 2002. Envelope broadening of spherically outgoing waves in threedimensional random media having power law spectra. J. Geophys. Res. 107, B5, 2089, doi: 10.1029/2001JB000264

Saito, T., H. Sato, M. Ohtake and K. Obara, 2005. Unified explanation of envelope broadening and maximum amplitude decay of high-frequency seismograms based on the envelope simulation using the Markov approximation: Forearc side of the volcanic front in northeastern Honshu, Japan. J. Geophys. Res., 110, B01304, doi:10.1029/2004JB003225.

Sato, A. M., Tickyj, H., Llambías, E. J. \& Sato, K., 2000. The Las Matras Tonalitic-trondhjemitic pluton, central Argentina: Grenvillian-age constraints, geochemical characteristics, and regional implications. J. South Am. Earth Sciences 13, 587-610.

Sato, H., 1977a. Energy propagation including scattering effects-single isotropic scattering approximation. J. Phys. Earth 25, 27-41.

Sato, H., 1977b. Single Isotropic Scattering model including wave conversions. Simple theoretical model of the short period body wave propagation. J. Phys. Earth 25, 163-176.

Sato, H. 1979. Wave propagation in one dimensional inhomogeneous elastic media. J. Phys. Earth 27, 455-466.

Sato, H., 1984. Attenuation and envelope formation of three-component seismograms of small local earthquakes in randomly inhomogeneous lithosphere. J. Geophys. Res. 89, 1221-1241.

Sato, H., 1988. Is the single scattering model invalid for the coda excitation at long lapse time? Pure Appl. Geophys. 128, 43-47.

Sato, H., 1993. Energy transportation in one- and two-dimensional scattering media: Analytic solutions of the multiple isotropic scattering model. Geophys. J. Int. 112, 141-146.

Sato, H., 1994. Multiple isotropic scattering model including P-S conversions for the seismogram envelope formation. Geophys. J. Int. 117, 487-494.

Sato, H., R. Dmowska and M. Fehler, 2009. Advances in geophysics, Vol. 50. Academic Press, 476 pp.

Sato, H. and M. C. Fehler, 1998. Seismic wave propagation and scattering in the heterogeneous earth. Springer-Verlag, New York, 308pp.

Sato, H., M. C. Fehler and R. S. Wu, 2002. Scattering and Attenuation of Seismic Waves in the Lithosphere. In: International handbook of earthquake and engineering seismology. Lee, Kanamori, Jennings and Kisslinger Ed., Academic Press, 933 pp.

Sato, H., H. Nakahara and M. Ohtake, 1997. Synthesis of scattered energy density for the nonspherical radiation from a point shear dislocation source based on the radiative transfer theory. Phys. Earth Planet. Int. 104, 1-13.

Savage, J. C., 1966. Thermoelastic attenuation of elastic waves by cracks. J. Geophys. Res. 71, 39293938.

Scherbaum F., 1990. Combined inversion for the three-dimensional Q structure and source parameters using microearthquake spectra, J. Geophys. Res. 95, 12423-12438. 
Scherbaum, F., D. Gillard and N. Deichmann, 1991. Slowness power spectrum analysis of the coda composition of two microearthquake clusters in northern Switzerland. Phys. Earth Planet. Inter. 67, 137-161.

Schmitz, M., 1994. A balanced model of the southern Central Andes. Tectonics 13, 2, 484-492.

Schmitz, M., W. Heinsohn and F. Schilling, 1997. Seismic, gravity and petrological indications for partial melting beneath the thickened central Andean crust $21^{\circ}-23^{\circ} \mathrm{S}$. Tectonophysics 270 , 313-326.

Schurr, B., G. Asch, A. Rietbrock, R. Trumbull and C. Haberland, 2003. Complex patterns of fluid and melt transport in the central Andean subduction zone. Earth Planet. Sc. Lett. 215, 105-119.

Siame, L., O. Bellier and M. Sebrier, 2006. Active tectonics in the Argentine Precordillera and Western Sierras Pampeanas. Rev. Asoc. Geol. Arg. 61 (4), 604-619.

Sibson, R. H. 1982. Fault zone models, heat flow, and the depth distribution of earthquakes in the continental crust of the United States. Bull. Seism. Soc. Am. 72(1), 151-163.

Siebert L. and T. Simkin, 2002-2011. Volcanoes of the World: an Illustrated Catalog of Holocene Volcanoes and their Eruptions. Smithsonian Institution, Global Volcanism Program Digital Information Series, GVP-3, (http://www.volcano.si.edu/world/).

Singh, S. and R. Herrmann, 1983. Regionalization of Crustal Coda $Q$ in the continental United States. J. Geophys. Res. 88, 527-538.

Smalley, R. F. Jr. and B. L. Isacks, 1987. A high-resolution local network study of the Nazca Plate Wadati-Benioff Zone under western Argentina. J. Geophys. Res. 92, 13903-13912.

Smalley, R. F. Jr. and B. L. Isacks, 1990. Seismotectonics of Thin-and Thick-Skinned Deformation in the Andean Foreland from Local Network Data: Evidence for a Seismogenic Lower Crust. J. Geophys. Res. 95, 12487-12498.

Smalley, R. F. Jr., J. Pujol, M. Regnier, J. Chiu, J. Chatelain, B. Isacks, M. Araujo and N. Puebla, 1993. Basement seismicity beneath the Andean Precordillera thin-skinned thrust belt and implications for crustal and lithospheric behaviour. Tectonics 12, 63-76.

Stachnik, J. C., G. A. Abers, and D. H. Christensen, 2004. Seismic attenuation and mantle wedge temperatures in the Alaska subduction zone. J. Geophys. Res. 109, B10304, doi:10.1029/2004JB003018.

Solomon, S. C., 1972a. On $Q$ and seismic discrimination. Geophys. J. of the R.A.S. 31, 163-177.

Solomon, S. C., 1972b. Seismic-Wave attenuation and partial melting in the upper mantle of North America. J. Geophys. Res. 77, 8, 1483-1502.

Stacey, F. D., M. T. Gladwin, B. McKavanagh, A. T. Linde and L. M. Hastic, 1975. Anelastic damping of acoustic and seismic pulses. Geophys. Surv. 2, 133-157.

Stauder, W., 1973. Mechanism and spatial distribution of Chilean earthquakes with relation to subduction of oceanic plate. J. Geophys. Res.78, 23, 5033-5061.

Stein, S. and M. Wysession, 2003. An Introduction to Seismology, Earthquakes and Earth Structure. Blackwell Pub., 498 pp.

$\mathrm{Su}, \mathrm{F}$., K. Aki and N. Biswas, 1991. Discriminating quarry blasts from earthquakes using coda waves. Bull. Seism. Soc. Am. 81, 162-178.

Suárez, G., P. Molnar and B. C. Burchfiel, 1983. Seismicity, fault plane solutions, depth of faulting and active tectonics of the Andes of Peru, Ecuador and southern Colombia. J. Geophys. Res. 88, B12, 10403-10428.

Takanami, T., I. Selwyn Sacks and A. Hasegawa, 2000. Attenuation structure beneath the volcanic front in northeastern Japan from broad-band seismograms. Phys. Earth Planet. Inter. 121, 339357, doi:10.1016/S0031-9201(00)00169-2.

Tassara, A., 2005. Interaction between the Nazca and South American plates and formation of the Altiplano-Puna plateau: Review of a flexural analysis along the Andean margin $\left(15^{\circ}-34^{\circ} \mathrm{S}\right)$, Tectonophysics 399, 39-57.

Tassara, A., H. J. Götze, S. Schmidt and R. Hackney, 2006. Three-dimensional density model of the Nazca plate and the Andean continental margin. J. Geophys. Res. 111, B09404, doi:10.1029/2005JB003976.

Tassara, A. y G. Yañez, 2003. Relación entre el espesor elástico de la litosfera y la segmentación tectónica del margen andino $\left(15-47^{\circ} \mathrm{S}\right)$. Rev. Geol. Chile 30, 2, 159-186. doi:10.4067/S071602082003000200002 . 
Triep, E. y C. B. de Cardinali, 1984. Mecanismos de sismos en las Sierras Pampeanas Occidentales. Actas del Noveno Congreso Geológico Argentino, III, 61-80.

Triola, M. F., 2004. Estadística. Pearson Education, 838 pp.

Tripathi, J. N., 2002. Scattering coefficient for $\mathrm{S}$ wave incident in a random medium characterized by exponential correlation function. Geophys. J. Int. 150 (2), 415-421 doi:10.1046/j.1365246X.2002.01693.X

Tripathi, J. N., A. Ram, 1997. Elastic-wave scattering in a random medium characterized by the von Kàrmán correlation function and small-scale inhomogeneities in the lithosphere. Geophys. J. Int. 131, 682-698.

Tsai, Yi-Ben and K. Aki, 1969. Simultaneous determination of the seismic moment and attenuation of seismic surface waves. Bull. Seism. Soc. Am. 59, 275-287.

Tselentis, G-A., 1998. Intrinsic and Scattering Seismic Attenuation in W. Greece. Pure and Applied Geophysics 153, 703-712, doi: 10.1007/s000240050215.

Tsujiura, M., 1966. Frequency analysis of seismic waves: 1. Bull. Earthquake Res. Inst., Tokyo Univ. 44, 873-891.

Tsujiura, M., 1978. Spectral analysis of the coda waves from local earthquakes. Bull. Earthquake Res. Inst., Tokyo Univ. 53, 1-48.

Tsumura, N., S. Matsumoto, S. Horiuchi, and A. Hasegawa, 2000. Three-dimensional attenuation structure beneath the northeastern Japan arc estimated from spectra of small earthquakes. Tectonophysics 319, 241-260, doi:10.1016/S0040-1951(99)00297-8.

Turcotte, D. L. and G. Schubert, 2002. Geodynamics. Cambridge University Press, 456 pp.

UCLA: Academic Technology Services, Statistical Consulting Group, 2010. Statistical Computing. from http://www.ats.ucla.edu/stat/mult_pkg/faq/general/mann-whitney.htm.

Udías Vallina, A., 1999. Principles of Seimology. Cambridge University Press, 475 pp.

Ugalde, A., L.G Pujades y J.A Canas, 1998a. Aplicación del cálculo paralelo al análisis de energía sísmica en la coda de terremotos regionales. Rev. Int. Mét. Num. Cálculo y Diseño en Ingeniería, 14, 4, 539-555.

Ugalde, A., L.G Pujades, J.A Canas and A, Villasenor, 1998b. Estimation of the intrinsic absorption and scattering attenuation in northeastern Venezuela (southeastern Carribean) using coda. Pure Appl. Geophys. 105, 685-702.

van Hunen, J., A. van den Berg and N. Vlaar, 2004. Various mechanisms to induce present-day shallow flat subduction and implications for the younger Earth: A numerical parameter study. Phys. Earth Planet. Inter. 146, 179-194.

Vargas Sabadías, A., 1995. Estadística descriptiva e inferencial. Colección Ciencia y Técnica, Univ. de Castilla La Mancha, 576pp.

Volponi. F., M. Quiroga y A. Robles, 1978. El terremoto de Caucete del 23 de noviembre de 1977. Instituto Sismológico Zonda, Universidad Nacional de San Juan, 81 pp, San Juan.

Wagner, L. S., S. Beck, G. Zandt and M. N. Ducea, 2006. Depleted lithosphere, cold, trapped asthenosphere, and frozen melt puddles above the flat slab in central Chile and Argentina. Earth and Planet. Sc. Lett. 245, 289-301

Wall, R. M. and L. E. Lara, 2001. Lavas Las Pataguas: alkaline volcanism in the Early Miocene Andean forearc of central Chile. Rev. Geol. Chile. [online]. Dec. 2001, vol.28, no.2, p.243258. Available from WWW: $<\mathrm{http}: / / \mathrm{www}$.scielo.cl/scielo.php?script=sci_arttext\&pid=S071602082001000200006\&lng=en\&nrm=iso >. ISSN 0716-0208.

Walsh, J. B., 1966. Seismic wave attenuation in rock due to friction. J. Geophys. Res. 71, 2591-2599.

Walsh, J. B., 1969. New analysis of attenuation in partially melted rock. J. Geophys. Res. 74, 43334337.

Wegler, U., 2004. Diffusion of seismic waves in a thick layer: Theory and application to Vesuvius volcano. J. Geophys. Res. 109, B07303, doi:10.1029/2004JB003048.

Wennerberg, L., 1993. Multiple-scattering interpretations of coda- $Q$ measurements. Bull. Seism. Soc. Am. 83, 279-290.

Wessel, P., and W.H.F. Smith, 1998. New, improved version of the Generic Mapping Tools released, EOS Trans. AGU, 79, 579.

Wessel, P., and W.H.F. Smith, 2004. The Generic Mapping Tools Technical Reference and Cookbook, Version 4.0, pp. 132. 
Wiens, D A., J.A. Conder and U.H. Faul, 2008. The seismic structure and dynamics of the mantle wedge. Annu. Rev. Earth Planet. Sciences 36, 421-455, doi:10.1146/annurev.earth.33.092203.122633.

Whitman, D., B. L. Isacks, J.-L. Chatelain, J.-M. Chiu, and A. Perez, 1992. Attenuation of highfrequency seismic waves beneath the central Andean plateau. J. Geophys. Res. 97, 19 929-19 947.

Woodgold, C., 1990. Estimation of Q in eastern Canada using Coda Waves. Bull. Seism. Soc. Am. $80,411-429$.

Woodgold, C., 1994. Coda Q in the Charlevoix, Quebec, Region: Lapse-Time dependence and spatial and temporal comparisons. Bull. Seism. Soc. Am., 84, 1123-1131.

Woodhouse, J. and A. M. Dziewonski, 1984. Mapping the upper mantle: three dimensional modeling of earth structure by inversion of seismic waveforms. J. Geophys. Res., 89, B7, 5953-5986.

$\mathrm{Wu}, \mathrm{H} .$, 1985. Multiple scattering and energy transfer of seismic waves - Separation of scattering effect from intrinsic attenuation - I. Theoretical modeling. Geophys. J. R. Astron. Soc. 82, 57-80

$\mathrm{Wu}, \mathrm{H}$. and K. Aki, 1985a. Scattering characteristics of the elastic waves by an elastic heterogeneity. Geophysics 50, 582-595.

$\mathrm{Wu}, \mathrm{H}$. and $\mathrm{K}$. Aki, 1985b. Elastic waves scattering by a random medium and the small-scale inhomogeneities in the lithosphere. J. Geophys. Res. 90, 10261-10273.

$\mathrm{Wu}, \mathrm{H}$. and K. Aki, 1988. Multiple scattering and energy transfer of seismic waves - Separation of scattering effect from intrinsic attenuation - II. Application of the theory to the Indu-Kush region. Pure Appl. Geophys. 128, 49-80.

$\mathrm{Wu}, \mathrm{H}$. and J. M. Lees, 1996. Attenuation structure of Coso geothermal area, California, from wave pulse widths. Bull. Seism. Soc. Am. 86, 1574-1590.

Xie, J., R. Gok, J. Ni and Y. Aoki, 2004. Lateral variations of crustal seismic attenuation along the INDEPTH profiles in Tibet from Lg Q inversion, J. Geophys. Res. 109, B10308, doi 10.1029/2004JB002988.

Xie, J. and B. Mitchell, 1990. Attenuation of multiphase surface waves in the Basin and Range province, part I: Lg and Lg coda. Geophys. J. Int. 102, 121-137.

Yáñez, G., C. R. Ranero, R. von Huene and J. Díaz, 2001. Magnetic anomaly interpretation across the southern central Andes $\left(32^{\circ}-34^{\circ}\right)$ : The role of the Juan Fernández Ridge in the late Tertiary evolution of the margin. J. Geophys. Res. 106, 6325-6345.

Yoshimoto, K., 2000. Monte Carlo simulation of seismogram envelopes in scattering media. J. Geophys. Res. 105, 6153-6161.

Yoshimoto, K., Sato, H. and Ohtake, M. (1993), Frequency-Dependent Attenuation of P and S Waves In the Kanto Area, Japan, Based On the Coda-Normalization Method. Geophys. J. Int. 114: 165-174. doi: 10.1111/j.1365-246X.1993.tb01476.x

Yoshimoto, K., U. Wegler and M. Korn, 2006. Volcanic Front as a Boundary of Seismic-Attenuation Structures in Northeastern Honshu, Japan. Bull. Seism. Soc. Am., 96; 2; 637-646; doi: $10.1785 / 0120050085$

Yrigoyen M. R, 1999. Situación de la Argentina en el marco geológico de América del Sur. Geología Argentina. Instituto de Geología y Recursos Minerales.Buenos Aires. Anales 29 (2), 35-39

Zeng, Y., 1991. Compact solutions for multiple scattered wave energy in time domain. Bull. Seism. Soc. Am., 81, 1022-1029.

Zeng, Y., 1993. Theory of scattered P- and S-wave energy in a random isotropic scattering medium. Bull. Seism. Soc. Am. 83, 1264-1276.

Zeng, Y., F. Su and K. Aki, 1991. Scattered wave energy propagation in a random isotropic scattering medium. J. Geophys. Res. 96, 607-619.

Zor, E., E. Sandvol, J. Xie, N. Türkelli, B. Mitchell,. A. H. Gasanov and G. Yetirmishli, 2007. Crustal Attenuation within the Turkish Plateau and Surrounding Regions, Bull. Seism. Soc. Am., 97, No. 1B, pp. 151-161, doi: 10.1785/0120050227. 
